COLUMBIA LIBRARIES UR FIIL
HEALTH SGIENCES STANDARD

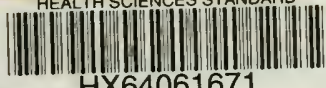

QM23 T65 1898

Descriptive anatomy

\title{
RECAP
}




\section{Crolumbia Ünionergity}

in the Citpaf 筑rmol

THE LIBRARIES

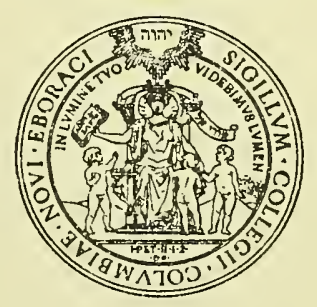

ftledical Iibrary 
1

(1) 

Digitized by the Internet Archive in 2010 with funding from Columbia University Libraries 



\title{
DESCRIPTIVE ANATOMY
}

BY

WIM. B. TOWLES,

FORMER PROFESSOR OF ANATOMY IN THE UNiversity OF Virginia AND IN THE UNIVERSITY OF VERMONT.

\section{REVISED AND ENLARGED}

BY

\author{
W. G. CHRISTIAN,
}

PRESENT Proffessor of ANATOMY in ThE University of Virginia. 
COPYRIGHT,

1898,

ANDERSON BROS.

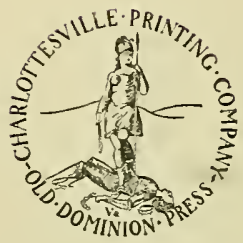




\section{PREFACE.}

Towles' Notes on Anatomy, which forms the basis of the present work, was not the work of a single man, but the outgrowth of a school. This school, founded here, probably, by Prof. Robley Dunglison, who brought the Edinburgh traditions into his teaching, was perfected by Prof. John Staige Davis, who, in his turn, instructed Prof. Towles, from whom the present writer acquired what knowledge of anatomy he possesses. The effort of all of the teachers in this school has been to make the instruction clear, simple, and practical; and these have been the characteristics of the Notes. While endeavoring to increase the amount of information contained in the work and to make it more representative of the lectures, the writer has tried to preserve those characteristics which have always distinguished the School of Anatomy at the University of Virginia. At this late day it is impossible for any one to write an original work on such a subject as anatomy, and originality is not clained for this work. It has simply been the writer's purpose to adopt the best, in his opinion. from all of the other writers he has been able to read; but, at the same time, no statement has been made in this book which has not been verfied by dissection.

The effort has been made to make this a student's book: it is not intended to imitate those works which are designed for practitioners and students alike. In the opinion of the writer a successful work of the kind last mentioned is a manifest impossibility, the needs of the student and the needs of the practitioner being entirely dissimilar. It has been the intention in this work to give to the student a clear, complete statement of the positive truths of anatomy, leaving embryology and histology, so largely treated in other works, to the care of the professors of these subjects. The writer has discussed neither surgery nor practice of medicine, but descriptive anatomy, pure and simple, in such a way as the needs of the student demand.

Universily of Virginia. Augrust, 1898 . 



\section{DESCRIPTIVE ANATOMY.}

\section{OSTEOLOGY.}

The study of the bones which go to form the human skeleton, individually and in relation to one another, with regard to the appearances which they present to the unaided eye, and the uses for which they are intended, constitutes Osteology, as pertaining to Descriptive Anatomy. The chief uses of bones in the living body are: 1st. To give support to the softer tissues. 2d. To form receptacles for important organs. 3d. To form unyielding points of attachment for the muscles. th. To serve as levers, through which muscular action may be utilized.

In order to fulfill these functions, bones present many different points for study. They may be divided in a general way into: 1st. Long Bones, when the extension in one direction greatly exceeds that in the other. 2d. Flat Bones, when the extension in two directions greatly exceeds that in the other. $3 \mathrm{~d}$. Irregular Bones, when the preceding distinctions do not exist. In the study of a bone, notice: 1st. Its position and relation to other bones. 2d. The class to which it belongs. $3 \mathrm{~d}$. Give a description of the appearances on the bone: whether there be surfaces, borders, extremities, shaft, depressions or elevations, and especially note any articular surface, that is a surface produced by contact with another bone. In this connection it is proper to explain two technicalities of frequent use-Internal and External-these terms should be used with reference to the middle line or plane of the body, that is one object is internal to another because it is nearer the middle line. They are often used incorrectly with reference to a cavity; interior and exterior are the terms to be here employed.

The bones are divided into those of the vertebral column: of the Thorax; of the Upper Extremity; of the Lower Extremity; of the skull; the Os Hyoides; the Ossicula Auditus. 


\section{The Vertebral Column.}

The vertebral column or spine, consists of a series of separate bones, classed as irregular, called vertebræ, and extends from the base of the skull to the lower termination of the pelvis. It is flexuous in direction and of unequal size at different places, smallest in the neck, it gradually enlarges to below its middle and then suddenly tapers to a point. It transmits the weight of the head to the lower extremities, giving support on each side to the bones which enclose the thoracic cavity, and indirectly to those of the upper extremities. Towards its lower termination it has expanding from it on either side the bones which form the pelvis. It forms the receptacle for the spinal marrow and its envelopes. In the adult it consists of twenty-six pieces, called vertabræ, which are divided into four classes; in the neck they are called cervical vertebræ, and are seven in number:

In the Thoracic region-Dorsal-twelve in number.

In the Abdominal region-Lumbar-five in number.

In the Pelvic region-Pelvic-two in number.

The last are known as sacrum and coccyx, and before adult life are composed of nine pieces-the sacrum having five and the coccyx four. The vertebræ of the different regions have characteristics which distinguish them from those of other regious, and each vertebra certain less noticeable ones which designate its position in its own region. Each region, however, possesses one or more bones so widely different from the typical vertebra of that region as to deserve the appellation of "Peculiar."

Since there are appearances common to the vertebræ of all regions, these must be first noted.

\section{Vertebra.}

Each vertebra consists of the following parts: 1st, Body; 21, Foramen for the spinal cord; 3d, Laminæ (2); 4th, Pedicles (2) ; 5th, Spinous process; 6th. Transverse Processes (2); 7 th, Articular Processes (4); 8th, Intervertebral Notches (4). 
1st. The Body is the front, thick, massive portion of the bone, flattened above and below to articulate with adjoining bones, indirectly by the intervention of cartilage; it is more or less circular in outline and slightly flattened behind; the edges project somewhat in front and laterally, so as to produce a furrow on the front; its posterior aspect forms the anterior boundary of the spinal foramen.

2d. The Spinal Foramen lies between the body in front and the spinous process behind, and is bounded laterally by the laminæ. It is a large opening, varying in form and size in the different regions.

3d. The Lamina springs, one each side, from the posterolateral aspect of the body, through the medium of the pedicle, and arches back to meet its fellow to form the spinous process, thus inclosing the spinal foramen. It is flattened from side to side, and presents an upper and a lower border.

4th. The pedicle is the more or less rounded commencement of the lamina.

5th. The Spinous Process juts backward from the union of the two lamina.

6th. The Transverse Process projects laterally from near the anterior extremity of the lamina on either side.

7th. The Articular Processes are four, two superior and two inferior. They extend upward and downward from the lamina.

8th. The Intervertebral Notches are four, two superior and two inferior; they are furrows on the upper and lower aspects of the pedicles.

\section{Cervical Vertebra.}

The discrimination of a Cervical Vertebra involves the following points: it is the smallest; its body is smallest, having its transverse diameter greater than the antero-posterior; on its upper aspect the lateral edges are raised into ridges. The spinal foramen is largest and is triangular. The lamina is narrow and long. The spinous process is triangular and bifid at the extremity, generally. The transverse process is perforated at its base by an opening called the vertebral fora- 
men, is grooved above and bifid at its extremity, forming two nodules which are called the anterior and posterior tubercles of the transverse processes. The Articular processes are oblique ; the superior face upward, inward and backward, the inferior down ward, forward and outward.

\section{Dorsal Vertebra.}

To discriminate a typical Dorsal Vertebra the following appearances must be noted:

The body is intermediate in size between that of a cervical and a lumbar vertebra; there is no great preponderance of the one diameter over the other; although in the upper part of the region the transverse is slightly greater, and in the middle portion the antero-posterior. The most characteristic feature is the appearance, on the postero-lateral aspect of the body, of articular half-facets, situated, two on each side, at the upper and lower borders : these are for the reception of the heads of the ribs. The spinal foramen is smaller than in the other regions, and is nearly round. The lamina is short, slopes obliquely from behind, upward and forward, and is almost entirely covered by the origin of the processes which spring from it. The pedicle is deeply notched inferiorly. The spinous process is triangular, and is distinguished from that of a cervical vertebra by not being bifid, it extends downward and slightly backward. The transverse process is peculiarly well marked, being large, long and extending outward and backward, increasing in size as it removes from its origin so as to present an enlargement at its termination; that is, it is "clubbed." It bears on its anterior face, near its termination, an articular facet. The superior articular processes are vertical and look from each other, outward and backward; the inferior are opposite.

\section{A Lumbar Vertebra.}

In the lumbar region the whole bone is more massive than in the cervical and dorsal regions. The body is large, its transverse diameter greater than the antero-posterior. Its anterior surface is concave from above down ward, convex from 
side to side, slightly lipped above and below. The superior and inferior faces present a concave central portion with an elevated circumference. The posterior face although nearly flat, presents a slight concavity from above downward and from side to side with large openings for the veins. No lateral articular facets are seen on the body. The spinal foramen is obscurely triangular and is intermediate in size between the large cervical and small dorsal. The pedicles are short and thick, project nearly backward with but a slight inclination outward and are marked below by deep and wide intervertebral notches. The superior notches are narrow and shallow. The lamina, short and massive, pass downward backward and inward to join the spinous process, and are partly hidden by processes. The spinous process is nearly quadrilateral, strong and thick. It projects straight backward and terminates in a blunt, rough almost clubbed extremity. The transverse processes spring from the junction of the body and pedicles. They are long narrow, thin and curved, resembling a rib in shape. They pass backward and outward with a slight inclination upward. The superior articular processes are cut nearly vertically, as in the dorsal region, but, unlike the latter, face toward each other so that their direction is backward and inward. They are concave from before backward to receive the inferior processes, and are situated at the junction of the pedicles and lamina. The inferior processes, situated entirely on the lamina, are vertical, convex from before backward and face outward and forward so as to be received between the inverted superior processes.

\section{Peculiar Vertebrae.}

The foregoing description of a vertebra in each region is, as stated, of one typical of that region; and this typical vertebra is one from about the middle of that region. Toward the confines of each region the vertebra partake more or less of the characters of vertebra in the adjoining regions, and so far depart from the type as to deserve the appellation "Peculiar", -thus demanding a special description. The departure from 
the typical vertebra is so great in some instances that the bones have received special names.

In the cervical region there are three peculiar vertebræthe first, second and seventh.

\section{The First Cervical Vertebra, or Atlas.}

This vertebra is known as the Atlas because it supports the weight of the head ; it resembles only in a slight degree a typical vertebra, having no body and being scarcely more than a ring of bone. It is described as consisting of four partstwo arches and two lateral masses-surrounding a spinal foramen of remarkable size-much larger than in any other vertebra.

The lateral masses stand one on each side, being connected before and behind by the anterior and posterior arch. The anterior arch is much shorter than the posterior, and forms one-fifth of the circumference of the bone; it presents on the middle of its front an elevation, called the anterior tubercle of the Atlas and opposite this, on the posterior face of the anterior arch, is a circular, concave articular facet for the odontoid process of the next vertebra.

The posterior arch forms about two-fifths of the circumference of the bone. It is convex and rough behind, presenting in the mid line a slight rough eminence called the posterior tubercle of the Atlas, a rudimentary spinous process. The upper border of the posterior arch presents, just behind the lateral mass, a deep groove or notch, the superior intervertebral notch and below, behind the inferior articular process, a similar but slighter groove, the inferior intervertebral notch. It will be seen that the intervertebral notches are behind the articular processes in this vertebra instead of being in front as in a typical vertebra.

Each lateral mass forms about one-fifth of the bony circle, and sustains, above and below, the articular processes. The superior are oval, concave, oblique, articular pits, whose long diameter is directed from in front backward and outward approaching each other more nearly in front than behind, and looking upward, inward, and forward. About their middle 
they are frequenty slightly encroached upon by the non-articular portion of the bone so as to give them something of a kidney shape. The inferior processes are smaller, circular, slightly concave or flat and directed downward, inward and slightly backward. The inner face of each lateral mass is marked, just below the superior articular process, by a slight tubercle intended for ligamentous attachment. The transverse processes project outward and a little down ward. They are large and prominent, perforated at the base but not marked by either groove or tubercle as in the typical vertebra.

\section{The Second Cervical Vertebra, or Axis.}

The body of the Atlas becomes ossified to the Axis and appears on that bone as the odontoid process. This process occupies the whole of the upper surface of the Axis, springing by a slightly constricted portion called the neck, ascending and enlarging for a short distance to again decrease and finally terminate as a blunt point. On either side, just below the termination, there is a rough depression for ligamentous attachment. Below this and on its anterior face, is a smooth circular, convex articular facet to play on a similar surface on the anterior arch of the Atlás. Just opposite to this, on the posterior surface, is a second, smooth surface for the play of the transverse ligament. The body is marked in front by a vertical ridge and on either side of this by deep depression. The superior articular facets appear to be seated on the junction of the pedicles and body. They are circular in form, nearly flat and face upward and outward, possihly slightly forward.

The transervse processes, less prominent than those of the Atlas, are not bifid and pass downward and outward. They are pierced by the vertebral foramen, which runs from below upward toward the superior articular facet and then turns abruptly outward, forming nearly a right angle. The lamina are thick and strong; the spinous process, strong and thick, projects straight backward, is deeply grooved on its under surface and frequently bifurcated at the extremity. There is barely a trace of a superior intervertebral notch, which, when present, lies behind the articular process. The lower portion 
of the bone presents the characteristics of a typical cervical vertebra, but is more massive.

\section{Vertebra Prominens, or Seventh Cervical Vertebra.}

This vertebra derives its sobriquet of prominens from its possession of a remarkably long, thick, non bifid, spinous process, which distinguishes it from the other cerrical vertebræ. It is also larger than the other vertebræ of this region, its spinal foramen is smaller and the lower surface of the body is more like that of a dorsal vertebra.

\section{Peculiar Dorsal Vertebrae.}

The peculiar dorsal vetebræ are the first, ninth, tenth, eleventh and twelfth.

The first presents on each side of its body, above andposterolaterally, a whole facet for the head of a rib; below, in corresponding position, a half facet on each side. The ninth has but one demi-facet on each side, which is on the upper border. The tenth has one whole facet on each side above. The eleventh has one whole facet on each side, but its transverse process has no articular facet. The twelfth has one whole facet on each side; its transverse process has no articular facet and its inferior articular processes are everted.

\section{The Pelvic Vertebrae.}

The pelvic vertebræ, specifically known as sacrum and coccyx, form the lower part of the vertebral column, and derive the appellation of pelvic from the fact that they form the posterior wall of the pelvis in the articulated skeleton. They are described separately.

\section{Sacrum (Sacer-Sacred.)}

The sacrum lies between the fifth lumbar vetebra above and the coccyx below, and between the two ossa innominata. It is triangular in outline, its base being upward and its truncated apex below and backward and tipped by the coccyx. Its anterior face is concave, both from above downward and from side to side, more so in men than in women, this being one means of distinguishing the sex to which the pelvis 
belonged. It is described as consisting of an anterior and posterior face, two lateral borders, a base and an apex.

The anterior face is concave and presents two vertical rows of foramina, of four each, one row on each side of the middle line, called the anterior sacral foramina; the upper two in each row, are considerably larger than the lower two. Connecting each foramen with the corresponding one in the opposite row is a ridge; these ridges marking the line of union of the five separate vertebra of which the sacrun consisted in the young subject. Passing outward from each foramen is a groove for a sacral nerve.

The posterior face of the sacrum is narrower than the anterior, is convex both from above downward and from side to side and is rough. Above, in the mid line, is seen the triangular opening of the spinal canal; below this is a row of rough projections, the rudimentary spinous processes. The upper two of these are usually distinct, while the two lower coalesce. The lower spine is wanting and here the spinal canal is exposed. External to the sacral spines is seen a row of slight projections, the rudimentary articular processes, and between these and the spines is a vertical groove, the sacral, continuous with the vertebral groove above. External to the articular tubercles is a row of foramina, less regular in outline and smaller in size than those on the front, the posterior sacral foramina, four in number like those on the anterior face. External to these again, and nearly on the lateral border, is another row of rough tubercles, the rudimentary transverse processes.

The lateral borders, broad and thick above, narrow, thin and rough below, present superiorly and anteriorly a long, ear-shaped articular facet for the os innominatum, behind this a deep rough depression for ligamentous attachment.

The base, or upper extremity, presents in the mid line an oval articular surface, corresponding in shape and size to the lower surface of the last lumbar vertebra, with which it articulates. On either side of this is a broad, smooth expanded surface, called the wing of the sacrum. Behind the articular 
surface is seen the triangular upper opening into the sacral canal, a continuation of the spinal canal. This canal passes through the sacrum, decreasing in size as it descends, and is open posteriorly below on account of the absence of the last spinous tubercle of the sacrum. On either side of the opening is an articular process. These processes, like the superior articular processes of a lumbar vertebra, face toward each other. In front of the articular processes on either side is a groove corresponding to the intervertebral notch of other vertebræ.

The apex, or lower extremity of the sacrum, presents an oval surface for articulation with the coccyx, and on either side of this an articular nodule, called the cornu. Behind the oval surface is the opening of the sacral canal which terminates the spinal canal.

\section{Coccyx.}

The coccyx forms the lower extremity of the vertebral column; it originally consists of four pieces or vertebræ united, in the adult, into one bone, which is sometimes ossified to the sacrum. It is triangular in shape; its base is above, presenting an oval articular facet for the truncated apex of the sacrum; its apex is below and forward the bone continuing the curve of the sacrum and frequently deviating to one side.

\section{The Vertebral Column as a Whole.}

Viewed as a whole the vertebral column presents two pyramids with their bases opposed, the superior pyramid being composed of all the vertebræ from the second cervical to the last lumbar, the inferior consisting of the nine pelvic vertebræ. On more careful examination it will be found that the superior pyramid may itself be divided into three, one extending from the second cervical to the first dorsal, the second from the first to the fourth dorsal, base upward, and the last consisting of the remaining vertebra with the base at the fifth lumbar. These smaller pyramids are comparatively unimportant. Although the general direction of the vertebral column is vertical, it does not present a straight line. In the cervical 
region, and including the first and second dorsal vertebra, it is convex forward, in the dorsal concave forward, in the lumbar, convex and in the pelvic again concave forward. Thus it will be seen to consist of alternate compensatory antero-posterior curves, an increase in the curvature of one region always causing a corresponding compensation in the region below. Viewed from the front we see the convex bodies of the vertebra, marked by their transverse grooves and separated by the intervertebral disks, the whole presenting the curvatures above described. Posteriorly, in the mid line, will be seen the spinous processes, rudimentary in the first cervical, prominent in the second, decreasing in size until the sixth is reached, well marked and prominent in the seventh and even more prominent in the first dorsal. From this point the spines become longer in the dorsal region until the ninth is reached, but, owing to their downward inclination, less prominent. Again prominent and projecting backwards in the lumbar, they become indistinct in the upper sacral and disappear entirely in the lower sacral region. Sometimes there are normal deviations from the mid line even in health, so that the spines do not form a straight line down the back. On either side of the spines is seen a groove which is broad and shallow in the cervical, deep in the dorsal and, bezoming less marked in the lumbar, finally disappears in the lower pelvic region. Laterally may be seen the sides of the bodies of the vertebra, presenting in the cervical region the transverse processes, gronved by the intervertebral foramina; intervertebral foramina and transverse processes with articular facets for ribs, in the dorsal; and similar foramina and spine-like transverse processes in the lumbar region. The pelvic region shows the lateral border of the sacrum and coccyx.

The length of the vertebral column varies at different periods of life and in different individuals. In the adult it is from twenty-four to twenty-eight inches long.

\section{Sternum.}

The sternum or breast bone, form; the middle portion of the thoracic wall in front, the dorsal vertebra occupying the 
mid-line behind; connecting these on each side are the ribs, which are prolonged by the costal cartilages. Extending outward from the upper end of the sternum on each side is the clavicle. The direction of the long axis of the sternum is downward and forward. Originally consisting of six parts, these in the adult become united into three-the upper or Manubrium, the middle, or Gladiolus, and the lower, Ensiform or Xiphoid cartilage. These are united by cartilage, but later in life may become ossified together, the ensiform cartilage frequently becoming ossified and firmly united to the gladiolus.

The Manubrium or first piece of the sternum has an anterior and posterior surface, upper and lower extremity and two lateral borders. 'The anterior surface is slightly convex from side to side, usually a little concave from above downward, and roughened for muscular attachment. The posterior is concave from side to side and smooth. The upper extremity is marked in the mid-line by a notch known as the inter-clavicular. External to this and located at the junction of the upper extremity and lateral border, is a smooth concavo-convex articular facet for the inner end of the clavicle. This facet is concave from above downward and outward and convex from before backward. The inferior extremity is marked by an oval, oblong articular surface for the upper end of the Gladiolus. The lateral borders present above a pit for the reception of the cartilage of the first rib; below a half pit for the second costal cartilage, converted into a whole pit by articulation with the Gladiolus. Between these the border is rough, narrow and non-articular.

The Gladiolus or second piece of the steruum, presents an anterior surface, slightly convex from above downward and marked by three transverse ridges which indicate the junction of the four bones of which it was originally composed. The posterior surface is concave and smooth. The upper extremity shows an oval articular facet to correspond with the one on the lower extremity of the Manubrium. The lower extremity shows a similar, but 
smaller facet for the Ensiform cartilage. The lateral borders are marked by a demifacet at the upper and lower angles respectively and four whole pits between these two. The upper demifacet is for the second costal cartilage, the lower, for the seventh and those between for the cartilages of the intervening ribs. Between these pits are narrow, rough nonarticular portions of bone. Occasionally the Gladiolus shows a foramen near its lower end.

The Ensiform cartilage presents many varieties in shape and position. Above it has a small oval facet for the Gladiolus, and on its lateral margin a half facet for the cartilage of the seventh rib. It is frequently deflected to one or the other side, and may be turned backward or forward.

Viewed as a whole the Sternum is convex and rough anteriorly, concave and smooth posteriorly, large and broad above, presenting the articular facets for the clavicles and the interclavicular notch, narrow and pointed inferiorly. Laterally it is marked by pits for the reception of the cartilages of the seven true ribs.

\section{The Ribs.}

The ribs are twelve pairs of long bones situated on the side and front of the chest and forming the greater part of the bony wall of the thorax. They are known by numbers from above downward. In describing them a rib from the middle is taken and the characteristics common to all the ribs are described. This is called a typical rib. The peculiar ribs are then described. Of course all the ribs, from any given individual, may be distinguished, but not from different persons.

\section{A Typical Rib.}

A typical rib, being a long bone, has a shaft and two extremities. The posterior or vertebral extremity is sometimes improperly called the head. It consists of a head, neck and tubercle. The posterior, or vertebral extremity has a head which presents two articular facets separated by an antero-posterior ridge. The facets look the one upward and backward, the other downward and backward, are oval in form and articular for the demi-facets found on the bodies of the 
dorsal vertebræ above and below. The ridge is received in the interval between the two vertebræ and is attached to the intervertebral disk. The neck extends outward and slightly backward from the head and terminates at the tubercle. It has an upper and a lower border, anterior and posterior surfaces. The posterior surface is irregular and rough for ligamentous attachment; the anterior, smooth, concave, slopes obliquely from above downward and enters into the formation of the posterior wall of the thorax. The lower border is twice as thick as the upper, is longer and roughened for the attachment of a ligament. The superior border is marked by a prominent crest which extends from the head to near the tubercle. In the lower ribs it rises from behind forward and terminates rather abruptly before reaching the tubercle; in the upper it is less prominent and might be described as a simple ridge marking the upper border.

The anterior, or sternal, extremity, is flattened from before backwards, slightly larger than the shaft and is surmounted by a pit for the reception of the corresponding costal cartilage.

The shaft slopes obliquely from above downward and forward, the degree of obliquity varying with the different ribs. It presents two surfaces, internal and external, and two borders, inferior and superior. The posterior part of the shaft is nearly round and extends from the tubercle to the angle of the rib. Its surfaces look forward and backward. From the angle the ribs slope and the surfaces become internal and external. The external surface is convex from before backward, flat or slightly convex from above downward and is marked at the angle of the bone by an oblique ridge. The internal surface is concave from before backward, convex from above downward and smooth. Near the lower border is seen a long groove which, starting near the angle passes forward to the anterior third where it gradually loses itself on the inferior border. The superior border is twice as thick as the inferior and is slightly roughened for muscular attachment. The inferior border, thin and sharp, overhangs the groove on the inner surface and is itself marked by the same groove near its anterior extremity. 


\section{0}

OSTEOLOGY.

\section{Peculiar Ribs.}

The peculiar ribs are the first, second, tenth, eleventh, and twelfth.

The First Rib departs more widely from the typical rib than any other. Its surfaces look one upward and forward, the other downward and backward, its borders inward and outward.

The superior surface, broad and flat, presents near the middle an oblique ridge which separates two grooves, the internal for the subclavian vein, the external for the subclavian artery. The external border, convex and rough and thicker than the internal border, is not marked by either angle or ridge as is the case with a typical rib. The posterior extremity presents a head with only one articular facet, as this rib articulates with but one vertebra. The neck is flattened from above downward and has no crest. The anterior extremity is thick and rough, being marked on its upper surface by a rough elevation for the attachment of a ligament. The inferior surface is smooth.

The second rib, longer than the first, approaches more nearly the typical rib. It is marked by a crest on its neck and an angle and ridge on the shaft, but both are less distinct than in the typical rib. The tenth, eleventh, and twelfth each have a whole facet, articulating with but a single vertebra. The tenth has a pit at its chondral extremity for the reception of a cartilage which binds it to the cartilage of the ninth and, through it, to the seventh. The eleventh and twelfth are merely tipped at their extremity with cartilage and are called floating ribs, because they are not attached to any other rib. These ribs have no articular facet on the tuberosity for the transverse process.

\section{The Costal Cartilages.}

The costal cartilages are flat pieces of hyaline cartilace attached by one extremity to the ribs and by the other to the pits on the lateral aspect of the sternum. Their surfaces are anterior and posterior and the borders superior and inferior. 
They increase in length from the first to the seventh and each has its separate pit on the sternum down to and including the seventh. From the seventh the cartilages are attached to the cartilage of the seventh rib and through it to the sternum. It is from this arrangement of the cartilages that the upper seven ribs are called vertebro-sternal, the next three, vertebro-chondral and the last two vertebral. The first three cartilages are horizontal, the next seven inclined obliquely upward, the obliquity increasing from the fourth to the tenth, while the last two are horizontal or inclined slightly dorvuward.

\section{The Clavicle.}

The Clavicle or, collar-bone, extends nearly horizontally outward but inclined slightly upward, from the upper end of the sternum on either side. It is a long bone; and like other long bones, is divided for study into a shaft and two extremities. The inner extremity is thick, the bone gradually diminishing in size as it passes from the inner to the outer end. The bone is round or triangular; and bears on its inner extremity a concavo-convex articular surface, convex from above downward and outward, concave from before backward. This surface is intended for articulation with a similar saddle-shaped surface on the upper outer aspect of the first piece of the sternum. Generally, just below this, is found another articular facet to play upon the cartilage of the first rib. The outer extremity is flattened from above down ward, rough and subcutaneous on its upper surface, slightly roughened on its lower surface, and presents at its tip a small articular facet, oval in outline, the long diameter directed from before backward, and looking downward and outward. This is for articulation with the the acromion process of the scapula. The body of the bone presents two surfaces, superior and inferior; and two borders, anterior and posterior.

The inferior surface presents a long shallow groove, the subclavian, terminated internally by the roughened surface which is sometimes a process and sometimes a depression, called the rhomboid impression. Following the groove 
outward, we reach, near the posterior border of the bone, a prominent tubercle, called the conoid; and extending outward and forward from this is a roughened ridge, called the trapezoid ridge. The superior surface is round, convex, smooth except at its inner and outer ends, where it is roughened for muscular attachment, and is subcutaneous. The anterior border is convex for its inner two-thirds, thick and round; concave for its outer one-third, thin and sharp. The posterior border is concave for its inner four-fifths, and is smooth; it is convex for its outer one-fifth, and is rough, thicker than the outer part of the anterior border, but not so thick as the inner extremity of the posterior border itself. The upper surface and anterior border are subcutaneous.

\section{The Scapula.}

The scapula, or shoulder blade, is a flat triangular bone, situated on the upper postero-lateral aspect of the chest, extending over the upper eight ribs with the exception of the first. It consists of a body having two surfaces and three borders, three angles and two processes.

The anterior fuce, or inter, is concave, has ridges running upward and outward, and presents a deep depression towards its upper outer part, which is called the subscapular angle. This face of the scapula is in the main occupied by the subscapular fossa; but it must be remembered that there is a smooth surface at the superior and inferior angles of the bone and along the posterior border, which while belonging to the venter of the scapula, does not belong to the subscapular fossa.

The posterior face, or dorsum, is convex from above downward, presenting a prominent ridge, the spine, which commences at the posterior border and runs upward and outward towards the head, dividing the dorsum into two unequal parts : the smaller above called the supraspinous fossa, and the three-fold larger below called the infraspinous fossa.

The supraspinous fossa is triangular in outline, the apex of the triangle being directed outward, is concave from above downward and from before backward, and smooth. 
The infraspinous fossa ozcupies nearly all of the remaining portion of the dorsum of the scapula ; but there is a strip along the anterior, or axillary, border, and a triangular space at the inferior angle which do not belong to the infraspincus fossa, though they belong to the dorsum of the scapula. The fossa, which is triangular in outline, the apex directed downward and the base upward, presents near its center a prominent bulge, and external to this a broad vertical groove. External to this groove is a prominent ridge which extends as far as the axillary border of the scapula. It is useless to say that the spine furnishes a portion of both supra and infraspinous fossæ.

The spine is triangular and presents three borders, two of which are free, one looking backward and the other outward and forward. The one which looks directly forward, the socalled "attached border," is simply the attachment of the spine to the remaining portion of the bone. The spine itself presents a superior surface which is concave, and enters into the formation of the supraspinous fossa, and an inferior surface which is slightly convex from without inward, slightly concave from above downward, smooth, and forms part of the infraspinous fossa. Its posterior border is rough, commences as a smooth triangular surface near the vertebral border of the bone, rises as it passes forward becomes thicker and rougher, and terminates near the upper outer angle by becoming a roughened projection of bone, known as the acromion process.

This posterior border presents a superior lip, an inferior lip, and an intermediate space intended for muscular attachments.

The external border of the spine is smooth, round, and concave, and is the inner limit of the groove which extends around it and forms the means of communication between the supra and infraspinous fossa. The superior border of the scapula is thin, and is the shortest of the borders. Near its outer extremity it is deeply notched, the notch being known as the suprascapular notch.

It terminates in the superior angle of the bone by joining with the posterior or vertebral border. 
The posertior or vertebral border, commencing at the superior angle passes downward and in ward to the point at which the spine springs from the posterior border. From this point down ward it changes its direction, and is nearly vertical, with a slight inclination outward. This border of the scapula, like the posterior border of the spine, is composed of two lips and a rough intervening space. It is intermediate in thickness be$t$ ween the superior and the axillary borders.

The anterior, or axillary, or outer border is thick. Just below the external angle of the bone it presents a rough impression from one-half to three-quarters of an inch in length, which is intended for muscular attachment. Below this point is seen a vertical groove, which passing downward upon the axillary border winds gradually to the posterior surface of the bone, which it reaches at the junction of the lower third with the upper two-thirds of the axillary border. Starting from the rough impression is a vertical ridge, which is the posterior boundary of the above-mentioned groove. Crossing this ridge at the middle of the axillary border of the scapula is a transrerse groove which transmits an artery from the anterior to the posterior surface of the scapula. Below the termination of the rertical groove the axillary border of the scapula becomes thinner, and is roughened for muscular attachment.

Of the angles of the bone the superior is situated at the junction of the superior and posterior borders, and is about a right angle. It is usually deflected slightly backward; and presents anteriorly a smooth surface, belonging to the venter of the scapula, but not entering into the subscapular fossa.

The inferior angle at the junction of the posterior and axillary borders is acute, having a roughened surface anteriorly and posteriorly. The anterior angle is expanded, and is the thickest portion of the bone. It is frequently called the "head" of the bone; and bears upon its extremity a hollow, oblong, vertical concavity, known as the glenoid cavity. This cavity has its long diameter directed from above downward. It is oval in outline, with the large end below and the small end above. It is supported upon the bone by 
the narrow constricted portion, which is called the "neck" of the scapula.

This neck is distinct anteriorly and posteriorly; but above is obscured by the origin of the coracoid process of the bone, and below by the impression for the attachment of the triceps muscle.

The processes are two: the coracoid process, which springs from the bone just above the glenoid cavity. Its direction is at first upward and slightly forward, but after passing a short distance, about half-an-inch, it abruptly changes its course, and runs nearly outward, with a slight inclination forward. At its origin one surface looks upward and backward and the other downward and forward; but after the change in its direction one surface looks upward and forward and the other one downward and backward. The upper surface is roughened for muscular attachment, while the lower is smooth and concave. The tip is marked by a smooth impression for muscular attachment.

The Acromion Process, is the termination of the spine. It presents one surface which looks upward and backward, one which looks downward and forward, an external border, an internal border, and an apex. The superior surface is rough, irregular and subcutaneous; the inferior surface is rather smooth, is concave, and is pierced by numerous nutrient foramina. The external border is convex and rough for muscular attachment. The internal border is concave; and is marked near its outer extremity by a smooth, oblong articular facet, the long diameter of which extends from before backward; and the surface itself is oblique, looking slightly upward and inward. The apex is roughened for muscular attachment. The upper surface of this process appears to be a continuation of the posterior border of the spine, whose superior lip corresponds to the inner border of the process, the lower lip to the outer border, while the rough intermediate lip expands into the upper surface.

\section{The Humerus.}

The Humerus, (L. Humerus) or skeleton of the upper extremity, is a long bone having a shaft and two extremities. 
The Head, or Upper Extremity, presents for examination a smooth, rounded portion the head proper, forming something less than half a sphere, which looks upward, inward and backward and is articular for the glenoid cavity of the Scapula. External to this is a constricted part of the bone, separating the head from the next appearances, called the anatomical neck. It is narrow and well marked above but becomes indistinct below and behind. External to the anatomical neck there is a large projection called the greater tuberosity. It is prominent and bulging, looks outward and forward and has on its summit three smooth impressions for the attachment of three of the muscles of the scapular group. Internal to the greater is a smaller projection called the lesser tuberosity. It is sharper and more prominent than the greater tuberosity and has on its summit but one muscular impression. Between the two tuberosities is a deep groove, the bicipital, bounded on either side by two prominent ridges, the internal and external bicipital ridues of the humerus. Below the tuberosities the bone is constricted and round, forming the surgical neck.

The Lower Extremity is flattened from before backward, is bent forward, and presents a slight curve of torsion from without inward. On either side is a rough prominence called, improperly, the condyle, internal and external, the internal being the larger of the $t$ wo. From either condyle there passes up to the shaft of the bone a large ridge of which the external is much more prominent and curved than the internal. Proceeding now from the external condyle invard, we next see a rounded articular surface, the eminentia capitata, whose articular surface extends higher up anteriorly than posteriorly. It is for articulation with the cup-like cavity on the upper extremity of the radius. Internal to this is a shallow articular groove for the play of the rim around the head of the radius. Internal to this we encounter two antero-posterior articular ridges, separated by an antero-posterior articular groove the three forming a trochlear surface for the reception of the greater Sigmoid cavity of the Ulna. The internal ridge is 
larger and its articular surface extends higher up in front than the external. Above the trochlea, both before and behind, is a deep pit-like depression. The one in front is for the reception of the coronoid process of the Ulna, the one behind for the Olecranon. The olecranon fossa is deeper and larger than the coronoid. In some bones, the thin septum separating the two cavities is absent and then there is a foramen between the two fossæ. Between the inner condyle and the internal surface of the trochlea, is a deep depression for the passage of an important nerve.

The Shaft of the bone, cylindrical above, prismoid in the middle and below, presents three surfaces and three borders. The borders are anterior and two lateral; the surfaces, posterior and two lateral.

The anterior border begins at the greater tuberosity as the external, or anterior bicipital ridge. Prominent and well marked above, it passes down the mid-line of the bone to termiate at the coronoid fossa, separating the internal from the external surface. The internal border begins above as the inner bicipital ridge. It passes at first downward and backward, then vertically downward to terminate as the internal condyloid ridge. It is prominent above and below, less distinct in the middle of its course. The external border commences at the back of the greater tuberosity and passes straight down to the outer condyle where it becomes the external condyloid ridge. Very sharp and prominent below, rough and well marked above, it is obliterated in the middle by the passage of the musculo-spiral groove.

The internal surface begins as the bottom of the bicipital groove. It passes downward and backward at first and then vertically downward. It is narrow above, wider in the middle and smooth and looking forward and inward below. This surface is usually marked about its middle by the nutrient foramen, though this appearance is often found on the inner border.

The external surface begins at the base of the greater tuberosity, passes straight downward to the middle of the 
bone and then curves forward until it is almost anterior. A bout the middle of this surface there is a large rough surface, with the apex downward, called the deltoid impression from its supposed resemblance to the Greek "Delta" inverted. Below the apex of the impression the musculo-spiral groove crosses to the front, smoothing the outer face, which remains smooth and without appearances throughout. The posterior face is round and smooth above, but becomes broad and flat below. Commencing on the posterior surface and winding downward and outward, is a broad shallow groove, the musculospiral. This groove is iudistinct on the posterior face, obliterates about two inches of the outer border and is finally lost on the external face.

\section{The Ulna.}

The Ulna is a long bone, consisting of a shaft and two extremities, distinguished as upper and lower. It is the inner and more massive of the bones of the fore-arm.

The Upper Extremity is composed of two processes called olecranon and coronoid. The olecranon process is found occupying the summit of the bone and may itself be divided for study into a superior, posterior and anterior surface and two lateral borders. The upper surface, indistinctly quadrilateral in outline, terminates anteriorly in a bluntly pointed projection called the beak, and is marked by a rough surface posteriorly for the attachment of a muscle, while in front is found a transverse groove extending backivard to become continuous with grooves which mark the lateral borders. The posterior surface is triangular in form, the large end of the triangle directed upward, rough and subcutaneous. The anterior surface is concave from above downward, convex from side to side, articular and enters into the formation of the greater sigmoid cavity.

The Coromoid process juts forward from the front of the bone. It is triangular in form, the base of the triangle being attached to the bone while the apex points forward. It presents a superior and an inferior surface and two lateral margins. The superior surface is smooth and articular, convex from 
side to side, concave from before backward, and is continuous with the similar surface on the olecranon process. The inferior surface is very irregular. It is marked near its middle by a rough impression for the attachment of a muscle, the brachialis anticus; at its junction with the shaft, usually, by a slight projection, the tubercle of the ulna, and at its junction with the internal margin by a roughened elevation, also for muscular attachment. The inner margin is roughened for muscular attachment; the external presents a concave, oval, articular facet, the long diameter from before backward, which receives the rim around the head of the radius. It is called the Lesser Sigmoid cavity of the ulna. The Greater Sigmoid cavity is formed by the two processes, is concave from above downward, convex from side to side and might be described as consisting of four depressions, two on either process, to receive the articular ridges on the Humerus, separated by a vertical ridge and a transverse groove. The outer side of this cavity is continuous with the Lesser Sigmoid cavity.

The Lower Extremity of the ulna is much smaller than the upper, and consists of two projections separated by a notch, the inner on the postero-internal aspect of the bone is the smaller and longer, and terminates in a blunt point. It is called the styloid process of the ulna. The outer process is called the capitulum unce(Dim. of Latin caput, head) and is articular upon its summit and around the greater portion of its circumference. The notch which separates the two processes is narrow, and is intended for the attachment of the fibrocartilage which enters into the formation of the wrist joint. The articular surface upon the summit is smoth, slightly concave, and does not directly articulate with the carpus but only through the interposition of the fibro-cartilage. The articular rim which surrounds three-fourths of its circumference is smooth and convex, and intended to play in the cavity which is found upon the lower extremity of the radius.

The Shaft of the ulna is distinctly three-sided; and hence presents three borders and three surfaces. The surfaces are anterior, internal and posterior; the borders are anterior, 
posterior and external, or interosseous. The anterior surface is broad above, narrow and round below. For the upper three-fourths of its course it is usually concave from side to side, presenting a shallow groove. The subcutaneous internal surface is broad, and flattened above, convex and slightly flattened in the middle, round and narrow below. The posterior surface is widest about its middle and presents usually a vertical ridge, which divides it into two unequal portions, of which the inner part is the broader. The upper portion of this surface presents a small triangular space; below it is rounded like the remaining surfaces. Of the borders, the anterior begins above at the inner side of the base of the coronoid process. Prominent and well-marked above, it passes downward to the front of the styloid process, becoming rounder and more indistinct in the lower one-third of its course. The fosteterior border commences above at the apex of the triangle of the posterior surface of the oelcranon process. Prominent, well-marked, and sub-cutaneous above, it becomes round and smooth below, and terminates at the back of the styloid process. The external or interosseous border commences by the convergence of two lines, one from either side of the lesser sigmoid cavity. It is sharp, thin, and well-narked for the middle two-fourths of its course. Below it becomes indistinct or imperceptible, and terminates at the front of the capitulum ulna.

\section{The Radius.}

The Radius (L. radius, a ray, or spoke of a wheel) is the outer of the two bones of the fore-arm; and like other long bones it is divided into a shaft and two extremities.

Its Utper Extremity, unlike the ulna, is much smaller than its lower; and presents for examination a head upon whose summit is an articular, cup-shaped depression for articulation with the eminentia capitata of the humerus. This depression is surrounded by an articular rim; and is supported upon a constricted portion called the "neck," which terminates below on its inner aspect in a prominent projection, called the tuls'rcle or bicifital luberosity of the radius. This tuberosity 
presents posteriorly a prominent rough elevation, for muscular attachment while anteriorly it has a smooth surface for the play of a bursa.

On the large Lower Extremity we find the summit presenting a triangular articular cavity, the carpal, with its apex directed outward, and terminating in a point or projection. called the styloid process. The base is directed inward, and is continuous with the articular facet found on the inner aspect of the lower extremity. The carpal cavity is divided into two portions by a slight antero-posterior ridge. The external portion is continued from the summit of the bone on to the inner face of the styloid process; and is triangular in form. The internal portion, quadrilateral in form, is continuous with the smooth oval, oblong articular facet, whose long diameter is directed from before backward, found on the inner face of the lower extremity, and called the sigmoid cavity of the radius. Between these two, the sigmoid and carpal cavities, is found a non-articular strip, intended for the attachment of the fibro-cartilage. The anterior face of the lower extremity is rough, and projects forward.

The posterior face is rough and irregular. It is marked by five vertical grooves with intervening ridges. Two of these grooves are found upon the styloid process, while of the remaining three, one, very narrow, is separated from the styloid process, by a very prominent projection of the bone, while the other is broad and shallow, and is found near the inner end of the posterior face.

The Styloid Process itself presents an internal and an external face: the internal being articular, and continuous with the articular surface of the carpal cavity; the external rough for ligamentous attachment, and marked by the grooves abore mentioned. Its anterior and posterior borders are roughened, while its tip has a blunt point.

The Shaft of the radius has three surfaces and three borders. The surfaces are anterior, posterior, and external; the borders anterior, posterior, and internal or interosseous. The anterior face is narrow above, slightly convex, becomes 
broader as it approaches the lower extremity of the bone, is slightly concave in the middle and is flat at the lower end. It is marked by an oblique ridge, which will be mentioned later. The posterior face is convex in the upper and lower portions of its course, slightly concave in the middle. The external face is convex throughout its extent, so that the bone appears to be bowed outward. The anterior border begins at the front of the bicipital tuberosity ; it passes downward and outward, crossing the upper one-third of the bone until it reaches its middle third, in which situation it is known as the oblique line of the radius mentioned above.

From this point it continues vertically downward to the front of the styloid process of the radius, where it terminates. The posterior border begins at the back of the neck of the radius, and running vertically downward, more prominent in the middle than elsewhere, terminates at the back of the styloid process. The internal border or interosseons ridge, begins also at the tuberosity near its posterior portion, passes downward, sharp and prominent about its middle, more indistinct below, and terminates at the lower extremity of the bone, by dividing into two lines which enclose the sigmoid cavity of the radius.

\section{The Hand.}

The skeleton of the hand consists of the skeleton of the Carpus, the Metacarpus, and Phalanges.

\section{The Carpus.}

The skeleton of the carpus or wrist, consists of seven small, irregular bones, arranged in two rows one above the other; in the upper row are three bones, arranged in the following order from without in ward: 1st, the Scaphoid; 2nd, the Semilunar; 3d, the Cuneiform, on the front of which plays a sesamoid bone, called the Pisiform. In the lower row in the same order are: 1 st, the Trapezium; 2nd, the Trapezoid; 3d, the Os Masmum; 4th, the Unciform.

\section{The Bones of the Upper Row.}

The bones of the upper row articulate laterally with one 
another; the bones on the inner and outer side respectively have no bones on the inner side of the one and the outer side of the other, and hence there is no articular surface on that aspect of the bones. It must be further noted that viewed from the back there appear to be but three bones in the upper row, placed laterally, the scaphoid, semilunar and cueniform ; but viewed from the front the pisiform is seen perched upon the cuneiform, so that the latter bone has no articular facet on its inner side.

The Scaphoid. This bone has a superior surface, which is rounded, convex, and articular for lower end of Radius; an inferior surface, which is concave and articular for the upper extremity of the os magnum; an outer extremity, which looks downward and outward and is articular for the trapezium and trapezoid; an inner extremity with an oval articular facet, whose long diameter is directed from before backward, for articulation with a similar facet upon the semilunar. Like all the bones of the carpus, the anterior and posterior surfaces are non-articular; but unlike most carpal bones, the anterior surface is broader than the posterior.

The Semilunar, the second bone in the first row, has a superior surface, convex, smooth, and articular for lower end of Radius; an inferior surface having two articular facets, one concave, ova1, with its long diameter antero-posterior, articular for the head of the os magnum, the other long and narrow for the unciform; an outer edge, articular for the scaphoid; and an inner, triangular in shape, larger in size than the outer one, and articular for the cuneiform; anterior and posterior surfaces, roughened and non-articular. The anterior surface is broader than the posterior, as is the case with the scaphoid.

The Cuneiform presents an inferior surface, concavoconvex for articulation with the unciform; an external surface, flat, sligbtly triangular, articular for the semilunar; an antero-external surface, which is articular for the pisiform, while internally it terminates in a blunt projection which is not articular. The posterior surface is broad and rough, the anterior surface is rough and narrow. It is usually said that 
this bone is articular upon its anterior face; but the surface which receives the pisiform bone looks upward more than forward, and is also directed slightly outward.

The Pisiform is a rounded bone; and is in reality only a sesamoid bone, embraced by the tendon of the flexor carpi ulnaris. It is articular upon only one surface, the surface which looks downward and backward. It is generally described as being the only bone of the carpus which is articular upon its posterior surface.

\section{The Bones of the Lower Row.}

The Trapezium, the first bone of the second row, is easily distinguished by the possession of a concavo-convex, or saddleshaped articular surface, which is directed downward and outward. This is its inferior surface. Its superior surface is marked by a smooth, concave articular surface for the reception of the outer end of the scaphoid. The outer surface is rough, prominent and non-articnlar. The inner face is marked above by a smooth concave articular surface for the outer face of the trapezoid, while below this, separated from it by a rough groove, is a flat surface, looking downward and inward for the second metacarpal. Its fosterior surface, broader than its anterior, is rough and prominent, while the anterior surface is easily distinguished by an oblique groove, which runs from above downward and inward.

The Trapezoid can be most easily distinguished by exclusion, having none of the characteristics of the other bones. It has a broad, somewhat quadrilateral posterior surface; a narrow and roughened culerior surface. Its superior surface is concave, and articular for the head of the scaphoid. Its cxternal surface is slightly convex above for articulation with the trapezium. The infrior surface is concave from before backward, and articular for the head of the second metacarpal bone; while the incrnal surface is also concave from before backward, and articular for the lateral aspect of the os magnum.

The Os Magnum presents superiorly a rounded articular head to be received in the cavity which is furnished by the scaphoid and semilunar bones, the articular surface extending 
further on its posterior than on its anterior aspect. Inferiorly it presents a quadrilateral, oblique articular facet, which articulates with the upper extremity of the third metacarpal bone, and which is continuous with the articular facet found upon its, inferior and external lateral aspect for the inner edge of the upper extremity of the second metacarpal bone. The ontersurface presents an articular facet, at the anterior inferior angle, convex from before backward, for articulation with the inner face of the trapezoid. The internal surface presents near its posterior margin an articular facet, broader above than below and somewhat $I_{1}$-shaped, the large end of the $L$ directed upward. This is for articulation with the outer face of the unciform. The posterior surface is broad, rough, and marked by a depression just below the articular head; the anterior face, narrower, than the posterior, is bulging and prominent, and roughened for ligamentous attachment.

The Unciform is more easily distinguished than any bone of the hand. Its upper face looks upward and inward; and is frequently described as its internal face. This face is intended for articulation with the cuneiform; and presents like that bone a concavo-convex articular surface, which is continuous with the upper extremity of the bone, also convex for articulation with the os magnum. Its outer face looks outward and slightly downward; and is marked near the posterior surface by an L-shaped facet, similar to the one found on the inner surface of the os magnum, and intended for articulation with that bone. Its inferior surface is marked by two articular facets, separated by a slight ridge, these facets articulating with the fourth and fifth metacarpal bones. Its posterior face, much broader than the anterior, is roughened for ligamentous attachment. The anterior face will be at once distinguished by a very prominent projection found on the lower inner face of the bone. This projection, named the unciform process, looks forward and slightly outward, being a little curved in its course. It is one of the prominent land-marks of the hand. The remainder of the anterior face is roughened for ligamentous attachment. 
The Carpus as a whole presents us with four separate sets of articulations; itz, The articulation between the upper row of the carpus and the wrist, the articulation between the first and second rows, the articulation between the second row and the metacarpal bones, and the articulations between the individual bones of the carpus. When examined in the articulated skeleton, it is seen that the superior row presents a condyle, oblong, oval and articular. The long diameter of this condyle is transverse; the bones which form it are from without inward: the scaphoid, the semilunar, and the cuneiform, the pisiform not entering into its formation. The articulation betrecn the two rou's of carpal bones is sinuous in outline, or concavo-convex, the trapezium and trapezoid together forming a cavity for the reception of the head of the scaphoid, while the head of the os magnum and the upper end of the unciform form together a convex articular surface received into a concavity which is contributed to by the scaphoid, the semilunar. and the cuneiform bones. The articulation between the bones of the second row and the metacarpal bones consists first of a saddle-shaped articulation, the trapezo-metacarpal; while the remaining articulations vary in form with the varying surfaces found upon the extremities of the metacarpal bones and the corresponding surfaces on the carpal. The articulations between the carpal bones themsclics have been mentioned in the description of those bones. It will be noticed that none of these bones are articular on their posterior and anterior aspects, though it is said that the cuneiform is articular anteriorly and the pisiform posteriorly, but these surfaces do not look directly forward and backward. When viewed from its posterior aspect, the first row presents a set of but three bones, the pisiform not being seen from the posterior face. It will be noticed that the carpal bones taken as a whole form an arch whose concavity is directed to the front, and whose convexity is directed backward. Hence it can be readily seen that all of the bones of the carpus, with the two exceptions mentioned, are broader upon their posterior than upon their anterior aspect. This arch of course gives strength to the hand, while at the same time permitting the necessary flexibility. 


\section{Metacarpus.}

The metacarpus is formed by five bones classed as long bones; four of these, for the four fingers, being nearly parallel, while the fifth-for the thumb-recedes from the others as it descends. The metacarpal bones articulate above with the lower row of the carpus; below with the upper extremity of the first phalanges. At their upper ends they articulate laterally with one another, except the metacarpal bone of the thumb; but at the lower end they do not articulate with one another. The metacarpus, like the carpus, is arched transversely, the convexity being posterior. One of its coustituents may be thus described:

\section{A Typical Metacarpal Bone.}

The Metacarpal Bones are long bones, presenting a shaft and two extremities, the upper extremity called the base and the lower called the head. The upper extremity is quadrilateral; and it presents anterior and posterior surfaces, of which the posterior surface is the broader, these two surfaces being roughened for ligamentous attachment; two lateral surfaces, which are marked by articular facets, intended for articulation with the adjoining metacarpal bones.

The Summit of the bone is marked by a quadrilateral articular facet, for articulation with one of the carpal bones of the second row.

The Lozver Extremity, or head, terminates in an articular surface, oblong antero-posteriorly, and articular higher up in front than behind. On either side of this surface is a depression, and behind this depression is a well-marked tubercle. Posteriorly the lower extremity is flattened and rough, while anteriorly it presents a slight groove, caused by the passage of the tendons of the flexor muscles.

The Shaft is triangular and arched, with the convexity of the arch directed backward. It presents a posterior and two lateral faces, an anterior and two lateral borders. The posterior face at its upper end presents a median lon- 
gitudinal ridge, with a slight depression upon either side of it. This ridge, as it passes downward, divides about the mildle of the bone into two, each, gradually separating, descends to reach the tubercle above the lateral depression on the lower extremity. The triangular space between these diverging ridges is flattened. The lateral faces are concave and smooth from above downward, so that each pair of bones would leave between them a space for the attachment of muscles. These are known as the interosscous spaces. The bone is concave towards its anterior aspect, and marked by the anterior border, which is a rather indistinct vertical ridge separating the lateral faces from one another.

This description applies more or less closely to all the metacarpal bones; but each possesses appearances characteristic of itself, by which it may be discriminated. Each bone is known by the name of the finger which it supports; but the metacarpal bones are also known by numbers from without inward.

The Mctacarpal Bone of the Thumb is the shortest and largest. Its posterior surface is broader and flatter than the remaining bones, and its lateral borders are more distinctly marked while upon its summit it bears a saddle-shaped or concavo-convex articular surface, concave from before backward, and convex from side to side. This with the absence of any lateral articular facet will distinguish it from the other bones of the metacarpus.

The Metacarpal Bone of the Index Finger is the longest, is next in size to that of the thumb, while its base presents three articular facets, two of which are upon the summit, one flattened for articulation witl the trapezium, one concave for articulation with the trapezoid, and one upon the internal lateral aspect for articulation with the metacarpal bone of the second finger.

The Metacarpal Bone of the Second Finger is next in size and in length. On its base it presents an articular facet for the os magnum; on its outer lateral aspect an articular facet for the metacarpal bone of the index finger; on its inner lateral

Des Anat-3 
aspect an articular facet for the metacarpal bone of the ring finger, while its upper outer posterior aspect is prolonged into a styloid process, projecting upward between the trapezoid and the os magnum. On its inner lateral aspect the articular facet is divided into two by a groove.

The Metacarpal Bone of the Ring Finger, next in size and length, is the typical metacarpal bone. On its external lateral aspect a groove divides the articular facet into two for the two corresponding facets on the inner lateral aspect of the metacarpal bone of the middle finger.

The Metacarpal Bone of the Little Finger is the smallest. Its base presents a superior articular facet for the unciform, and a lateral facet on its outer aspect for the corresponding facet on the metacarpal bone of the middle finger. On the inside of the base is a rough and prominent tuberosity with no lateral articular facet, which in itself distinguishes it from the other metacarpal bones.

\section{The Phalanges.}

The Phalanges consist of three rows of long bones, extending from the heads of the metacarpal bones to the tips of the fingers. The rows are numbered from the metacarpal bones, first, second, and third, the third row being usually called ungual.

The Thumb has but two phalanges, the second phalanx taking the place of the characteristic ungual or third.

The first phalanx is the longer, and its shaft is semi-cylindrical. The posterior surface is convex both from above downward and from side to side and is smooth. The anterior surface is slightly curved longitudinally, and is broader above than below. The two lateral borders, well-marked above, terminate in slight tubercles at the lower aspect of the bone. The Base or Upper Extremity presents a smooth round shallow articular facet, whose transverse diameter is slightly greater than the antero-posterior, and it is almost cupshaped. This is for articulation with the head of the metacarpal bone. The Head or Lower Extremity presents an antero-posterior groove, surmounted on either side by an artic- 
ular ridge, constituting a trochlear surface. On either side of the head is a slight depression, marked in front and behind by trifling elevations or tubercles.

The second phalanx presents on its base a receiving surface for the trochlea; that is, a central ridge with lateral depressions to receive the corresponding elevations. Like the first phalanx it is broad and expanded above, narrower below. Its lower extremity presents a trochlea for articulation with the third, or ungual phalanx. The second phalanx with the exception of the head is like the first, only it is shorter and less massive.

The Third, or Ungual Phalanx (L. Uuguis, a nail) presents on its base an articular surface to receive the trochlea of the second. It then becomes rapidly narrow; but just before it terminates, it expands into a broadened surface. The bone is smooth on its posterior, but rough, prominent and flat on its anterior surface. The posterior aspect of this phalanx supports the nail, while the anterior surface supports the tissues which form the pulp of the corresponding fingers. There being but two phalangeal bones for the thumb, the second phalanx for the thumb corresponds to the ungual phalanx for the other fingers.

\section{The Skeleton of the Lower Extremity.}

The skeleton of the lower extremity consists of the skeleton of the hip, thigh, leg and foot.

\section{Os Innominatum.}

The Os Innominatum (L. The un-named bone) haunch bone, is the most important bone in forming the pelvic cavity, and corresponds to the Scapula in the upper extremity. In the foetus it consists of three bones-Ilium, Ischium and Pubis, and, since the bone is very irregular in outline, this division has been retained in describing the bone in the adult.

The Ilium (Deriv. uncertain) or flank bone, is the large, expanded, upper portion of the bone. It is divided for study into and internal and external surface, anterior, posterior, and superior borders, the latter being known as the crest. 
The crest of the ilium is sinuous in outline. Viewed from in front it is seen to be convex for its anterior four fifths, concave for its posterior one fifth. In front and behind it terminates in rough projections, known as the anterior and posterior superior spinous processes. The crest consists of two lips, external and internal, and a rough intervening space or middle lip. The anterior border consists of two projections, anterior superior and anterior inferior spinous processes, with a notch between. The superior is more prominent but less massive than the inferior. Below the inferior is a slight groove separating it from the margin of the acetabulum. The posterior border is less regular in form than the anterior and like it, is marked by two projections with an intervening notch. These are called the posterior superior and posterior inferior spinous processes. They are sharper and less distinctly marked than those on the anterior border, and the notch between is not so deep. Below the posterior inferior spinous process is a deep notch, part of which is found on the ilium and the remainder on the ischium, called the greater sacro-sciatic.

The external surface is curved in outline being concave for the posterior four fifths, convex for the anterior one fifth. On the posterior border is a deep notch, called great sciatic, and commencing in this notch, and curving forward and upward from it, are three lines distinguished as superior, inferior and middle. The superior curved line, the shortest and most distinct of the three, begins near the upper margin of the sciatic notch and passes upward and slightly forward until it reaches the crest near its posterior one filth. The middle, which is the longest, begins at the middle of the sciatic notch and passes upward and forward, markedly curved, to terminate justabove the anterior superior spinous process. The inferior, the least distinct, starts at the lower portion of the notchand, passing forward and slightly upward, reaches the anterior border of the bone a little above the anterior inferior spinous process. The whole surface is roughened for the attachment of muscles and presents the orifices of numerous foramina for the transmission of blood vessels, the largest of which is found near the middle of the bone just below the middle curved line. 
The internal surface, much smoother than the external, is concave for its anterior two thirds both from above downward and from before backward, forming the iliac fossa. Just before the bone unites with the ischium it is crossed by a smooth, elevated ridge which forms a part of the ilio-pectineal line. The portion of bone below this is smooth, nearly flat, continuous with the inclined plane of the ischium, and enters into the formation of the true pelvis. The posterior one third of the internal surface is very irregular in outline. In front this surface is occupied by a rough, ear shaped articular surface which articulates with a similar surface on the sacrum. Behind this is a rough surface, made up of intervening elevations and depressions for the attachment of ligamentous fibres.

The Ischium is the lower, massive portion of the bone. It is divided for study into a body, tuberosity and ramus. The body is triangular and presents for examination three surfaces and three borders. The surfaces are internal, external and posterior; the borders, anterior, posterior and external. Nearly the whole of the external surface is occupied by the articular surface furnished by the ischium to the acetabulum; but a small portion below the rim of the acetabulum is occupied by a groove, the lower border of which is the mark of division between the outer surface and the tuberosity. The internal surface slopes obliquely from above downward and inward, facing inward and backward. It is smooth and concave from before backward and continuous above with a similar surface on the inner face of the ilium. The posterior surface is quacirilateral, forms the posterior wall of the acetabulum and is roughened for muscular attachment. The anterior border is thin and sharp, slopes upward and forward to join the pubes and forms the posterior boundary of the thyroid foramen. The external border is thick and rounded and forms the posterior part of the rim of the acetabulum. The posterior border is long and sharp, nearly vertical in direction and is interrupted a little below the middle by a prominent projection of bone called the spine of the ischium. Above the spine a deep notch is found which enters into the formation of the 
great sacro-sciatic notch; below the spine, a similar, but shallower notch forms the lesser sacro-sciatic notch.

The tuberosity is the rough lower end of the bone on which the body rests when in the sitting posture. It has an inferior surface which is rough and triangular, and just above this, a smooth portion, divided into two by an oblique ridge, the parts above and below the ridge being intended for muscular attachment. Internally it presents a rough, prominent ridge which separates the lower surface from the internal, and abore this ridge a smooth groove.

The Ramus passes forward, inward and slishtly upward. One surface looks outward and forward, the other inward and backward. One border looks outward and forms the anterior bousdary of the obturator foramen, the other looks inward and forms, with the ramus of the pubis, the outer boundary of the pubic arch. This internal border is thick and divided into two lips and an intervering space. The two lips pass downward to become continuous with similar lips on the lower surface of the tuberosity. The internal border is averted, its upper part looking nearly forward. At the junction of the ramus of the ischium with that of the pubes there is sometimes seen a rough line.

The Os Pubis (L. Pubes, hair, or down) is divided for study into a body and a ramus. The body is the horizontal portion of the bone, and is divided into two surfaces, anterior and posterior, two borders, upper and lower, and two extremities, internal and external.

The posterior surface is concave from before backward, convex from above downward and smooth. The anterior surface is convex from before backward, slightly concave from above downward, broad at its inner and narrow at its outer extremity, and rough for muscular attachments. The superior border is marked at its junction with the internal extremity by the angle of the pubis; external to this and running outward and forward is a rough ridge called the crest. The crest terminates after a course of about three fourths of an inch in a prominent projection of bone called the spine. 
Running outward and backward from the spine and separating the superior border from the posterior surface, is a sharp ridge of bone called the pectineal line; in front of which is a triangular surface, limited in front by the anterior surface of the pubis, the pectineal triangle. The base of the triangle, which is directed outward, terminates in a rough prominence known as the pectineal eminence. The inferior border forms the superior boundary of the thyroid foramen and is marked by an oblique groove, which, from within the pelvis, extends forward and inward. The interual extrenity, called the symphysis pubis, is an oval, rough articular surface, the long diameter of which is directed from above downward and backward. It is covered by a series of nipple shaped projections and in the recent state is lined with cartilage. The external extremity is in partattached to the ilium, while the remainder is free and enters into the formation of the acetabulum.

The ramus of the pubes is short and extends downward and slightly outward. Its surfaces look backward and forward, its borders inward and outward. The inner border presents two lips and an intervening space, the two lips being continuous with those found on the ramus of the ischium.

The acetabulum ( $\mathrm{L}$. a vessel for holding vinegar, from a supposed resemblance) is a nearly circular, cup-shaped cavity, one-fifth of which is contributed by the pubes, a little less than two-fifths by the ilium and a little more than two-fifths by the ischium. It is articular around the whole of its circumference except a small portion at the lower and inner part, where a deep notch interrupts the prominent rim which elsewhere surrounds the cavity. This notch, called the cotyloid, leads to a deep non-articular depression in the bottom of the cavity, which in the recent state lodges a mass of fat and, with its margins, grives attachment to the ligamentum teres.

The thyroid or obturator foramen is found below and in front of the acetabulum. It is irregularly oval in outline and its boundaries are formed as follows: Behind by the anterior border of the body of the ischium, in front by the outer border of the ischilo-pubic rami, above by the lower border of the body 
of the pubes, the groove on that bone furnishing nearly the whole of the upper boundary.

\section{Pelvis.}

The Pelvis (L. a basin or laver, from a resemblance to an ancient basin.) The pelvic girdle is far more powerful than the shoulder girdle, the individual bones being more massive and bound more firmly to one another. The cavity has been divided by the ilio-pectineal line, into the true and the false pelvis, though the latter term should not be used, since the false pelvis is simply a part of the abdominal cavity.

The pelvis is the cavity circumscribed by the sacrum, coccyx and two ossa innominata, containing in the living subject some of the small intestine, a part of the large intestine, and the genito urinary apparatus, in part. It is bounded behind by the sacrum and coccyx and throughout the rest of its extent by the ossa innominata. It is divided into two portions, the false pelvis and the true. The false pelvis is that portion between the two iliac fossæ bounded behind by the junction of 5th lumbar and sacrum, while in front it has no bony wall. It extends from the crest of the ilium above to the pectineal line below. This pectineal line, also called linea ilio-pectinea, is a prominent ridge extending around the os innominatum from the symphysis pubis to the sacro-iliac symphysis. At the linea ilio-pectinea the pelvis is suddenly contracted and the true pelvis begins. The plane circumscribed by the ilio-pectineal line is called, the brim or inlet of the pelvis, or superior strait of the pelvis. The true pelvis thus extends from this brim downward to what is called the outlet, or inferior strait of the pelvis. The outlet is limited in front by the lower end of the symphysis pubis; the arch formed by the divergence of the pubic rami being called the pubic, or sub-pupic arch. Behind, the outlet is limited by the tip of the coccyx. Laterally the outlet is bounded by the tuber ischii. The pelvis is so attached to the vertebral column as to render the plane of the inlet oblique, from behind downward and forward, and so great is this obliquity that the top of the symphysis pubis is about four inches 
below the level of the promontory of the sacrum. The socalled axis of the superior strait is an imaginary line, perpendicular to the plane of the brim and cutting it at its centre. The true pelvis is much deeper behind than in front, measuring posteriorly five inches, latterally three and one-half inches, and at the pubes one and one-half inches. Besides these measurements, obstetricians employ others, as of the outlet and inlet, called diameters. The diameters usually given are for the inlet, antero-posterior and transverse.

The objects observable on the exterior of the pelvis are as follows: in front the symphysis pubis and below this the pubic arch; on either side the obturator foramen, farther out the acetabulum; behind this the greater and lesser sciatic notches, separated by the ischiatic spine; on the centre behind are the sacral spines.

The pelvis differs in the two sexes; the characteristic points in the female are as follows: 1st, the thyroid foramen is more distinctly triangular; $2 \mathrm{~d}$, the pelvis is more capacious-measuring more transversely and antero-posteriorly, but less vertically; $3 \mathrm{~d}$, the angle of the pubic arch is greater by $10^{\circ}$, measuring from $90^{\circ}$ to $100^{\circ}$.

The pelvis in the negro is observably smaller than in the Caucasian.

\section{The Femur.}

The Femur (L. Fomur, the thigh) is the longest and most powerful bone in the body. It is divided for study into a shaft and two extremities. The upper extremity presents a head, neck and two tuberosities called trochanters.

The Ltper Extremily consists of the head, neck and two trochanters.

The IIcud, smooth and globular, projects upward, in ward and forward from the neck, and forms about two thirds of a sphere. It is marked a little below and behind its centre by a deep pit which in the recent state lodges the outer end of the ligimentum teres. The neck slopes downward, outward and backward from the head, is fiattened from before backward and is divided into an anterior, and posterior surfuce, an 
upper and a lower border. The anterior surface is flat and slightly roughened; the posterior, convex from above downward, concave from within outward, is smooth and rounded. The upper border, only one half the length of the lower, is broad, round, thicker than the lower and marked at its outer extremity by a deep pit called the digital. It is nearly straight. The lower border is thinner, longer and more oblique than the upper. Below and behind it terminates at the lesser trochanter while the upper terminates at the greater trochanter. The great trochanter is a quadrilateral piece of bone which projects upward from the junction of the neck and shaft. Its outer surface is marked by an oblique ridge; a diagonal, running from the postero-superior angle to the anteroinferior angle. The surface in front of the diagonal is rough, that behind it is smooth. The inner surface is taken up almost entirely by the digital pit. The anterior border is thick and rough, the posterior, thinner and rounded, the upper thin and rather sharp, terminating posteriorly in a blunt projection which overhangs the digital pit. The lesser trochanter is much smaller than the greater, projects from the inner posterior aspect of the bone, is round in form, bluntly pointed at its apex and marked by a single muscular impression for the attachment of the biceps flexor femoris. Extending from the base of the great trochanter downward and in ward to the lesser is a roughened ridge of bone known as the posterior inter-trochanteric line. On the anterior aspect, and starting from the base of the great trochanter, there is a more prominent line which winds around the bone, pissing downward and inward until it reaches the inner face of the bone when it turns and runs outward, called the spiral line of the femur. This line is sometimes incorrectly called the anterior inter-trochanteric line. It passes about half an inch below the lesser trochanter.

The Lower Extremity of the femur is flattened from before backward, so that the lateral diameter exceeds the anteroposterior. It consists of two round or oval articular surfaces, called condyles, two rough projections, one on either side, called tuberosities, and an intervening notch. If the bone is 
held vertically it will be seen that the imer condyle is prolonged down ward considerably below the outer to compensate for the obliquity of the bone caused by the width of the pelvis, which separates the heads of the bone, while the lower extremities approach each other. The long diameter of the articular surface of each condyle is from before backward; but it will be seen upon careful examination that the inner condyle is longer and narrower than the outer, that its articular surface extends higher up behind and not so high in front. The outer condyle is more oral, is shorter than the inner, its articular surface extending higher up in front than behind. The two condyles are separated behind by a deep and roughened notch, called the intercondyloid notch. This notch is marked on either side by a depression for the attachment of the corresponding crucial ligaments of the kneejoint. In front this notch is continuous with a smooth articular surface, concave from side to side, convex from above downward, and forms a trochlea for the opposite surface of the patella. Each condyle presents an internal and an external surface. The external surface of the external condyle is marked by a prominent rough tuberosity, called the outer tubcrosity of the femur; and below this is an antero-posterior groove for muscular attachments. The internal surface of the external condyle is the outer boundary of the intercondyloid notch. The internal surface of the internal condyle is likewise marked by a rough projection, called the internal tuberosity of the femur; while the outer surfuce of the internal condyle is the inner boundary of the intercondyloid notch. The inner surface of the inner and the outer surface of the outer condyles are each marked by nutrient foramina. Above each condyle is seen a slight depression on the posterior aspect of the bone, which is intended for the attachment of the gastrocnemius muscle. Leading upward from either condyle is a ridge. These ridges approach each other above to reach the linea aspera. They are known as the condyloid ridges, and more properly belong to the shaft of the bone. Between these ridges is a triangular roughened space called the Popliteal Triangle. 
The Shaft of the Femur. Like most long bones the femur is triangular upon cross-section; and therefore presents for study three surfaces and three borders. The surfaces are anterior and two lateral; while the borders are posterior and two lateral.

The anterior surface is round, convex from above downward, so that the bone is bowed in outline, with its convexity forward, convex from side to side, and broader above and below than in the middle. Of the two lateral surfaces, the external is slightly concave fromabove downward, and smooth; the internal also slightly concave from above downward, broader than the external, and smooth. The lateral borders are indistinctly marked. The external lateral, commencing at the base of the trochanter major, extends, indistinctlv marked, down to the outer tuberosity of the femur. The internal lateral, commencing at the spiral line of the femur, passes down ward to the inner tuberosity. The posterior border, called linea aspera is the most distinct of the three. It may be considered as consisting of a central portion, called the "body ;" and an upper and a lower portion. The central portion, or body, occupies about the middle two-fourths of the bone. It presents an internal and an external lip, and a rough intervening space, which are intended for muscular attachments. Above it divides into three lines : one winds around about a quarter of an inch below the lesser trochanter to become continuous with the spiral line of the femur; one passes upward to the base of the lesser trochanter; while the vertical passes upward and outward to join the posterior border of the greater trochanter. Below it subdivides into two ridges, which are known as the two condyloid ridges. These two ridges diverge from each other, enclosing the popliteal space, a smooth triangular space found at the lower extremity of the shaft, the base of which is directed downward to the condyles and the apex upward to the linea aspera. Of these two lines the external is the more distinct, the internal being obliterated near its middle by the groove which transmits the femoral artery. A bout the middle of the shaft of the bone, and rising either in 
the linea aspera or upon its external surface close to the linea aspera, is found a large nutrient foramen, which is directed obliquely upward and outward.

\section{The Patella.}

The Patella (L. Patclla, a small pan), or knee-pan, is a sesamoid bone situated on the front of the knee joint, developed in the tendon of the triceps extensor cruris. It presents for examination two surfaces, anterior and posterior, two extremities, superior and inferior, and two borders, internal and external. The anterior surface is rough, marked by a number of vertical lines and a number of nutrient foramina. The poslerior surfuce is smooth and articular. Near its middle it presents a prominent vertical ridge; and on either side of this a depression, also articular, intended for the reception of the condyles of the femur. The posterior face forms the receiving surface for the trochlea. These depressions are dissimilar in size and shape ; and correspond to the dissimilarity of the condyles of the femur, the outer depression being larger and more nearly circular, while the inner is smaller and more oval in outline. The upper extremily of the bone is blunt, rough, and marked by a transverse ridge; the lower extremity, smaller and more pointed, is roughened. Of the borders, the external is thinner and sharper than the inlernal. Both are roughened for the attachment of the tendinous fibres of the triceps.

\section{The Tibia.}

The Tibia (L. Tilia, a flute or pipe) is a long bone, consisting of a shaft and two extremities. It is the larger of the two bones making up the skeleton of the leg.

The Upper Extremily is expanded so that its transverse diameter is much greater than its antero-posterior. It presents upon its summit two shallow articular cavities, called glenoid cavilies, intended to receive the condyles of the femur, and in correspondence with them are dissimilar in shape, the outer being circular and the inner oval. The inner cavity has its long diameter antero-posterior; the ouler cavily is shal- 
lower, as well as being circular in outline. These cavities are separated from one another by a prominent projection, which is called the spine of the tibia. This spine is bifurcated; and is situated nearer to the posterior than to the anterior surface of the extremity. In front and behind the spine is seen a roughened, non-articular space, which is intended for ligamentous attachment. On the front of the bone, about an inch below the articular cavities, is a rough triangular space, below which is seen a projection, called the anterior tubercle of the tibia. This tubercle is smooth above and rough below, the roughened lower portion being intended for muscular attachment. That expanded portion of the bone upon which the glenoid cavities rest is known as the tuberosity of the tibia, internal and external. The internal tuberosity, somewhat more prominent than the external, is rough, and marked near its posterior portion by a deep transverse, or antero-posterior groove. The external tuberosity is roughened, as is the internal, and posteriorly is marked by a flat, circular, articular facet, which is directed downward, outward, and backward, and is intended for articulation with the upper extremity of the fibular. Behind, the tuberosities are both roughened for ligamentous attachment.

The Lower Extremity is four-sided; and is about half the size of the upper. On its inner side there extends downward a rough projection of bone, called the internal malleolus (L. A small mallet). It corresponds nearly to a styloid process. This internal malleoeus presents two surfaces, two borders, and an extremity. Of the surfaces, the internal is rough, and subcutaneous, while the extemal is smooth triangular and articular, entering into the formation of a cavity found upon the extremity of the tibia for articulation with one of the bones of the tarsus. The anterior border of the malleolus is shorter than the posterior, and is vertical in its direction, while the longer posterior border is oblique, extending downward and forward, and has upon it a well-marked groove for the transmission of a tendon. The extremity is blunt; and is marked behind by a pit-like depres- 
sion. On the outer surface of the lower extremity is a transverse depression, sometimes articular, more generally roughened, which receives the lower extremity of the fibula. The long diameter of this depression is directed from before backward; and leading upward from it are two lines, which shortly converge, enclosing between them a rough triangular space for the attachment of interosseous ligaments. The anterior aspect of the lower extremity is roughened for ligamentous attachment, while the posterior, also roughened, is marked by a vertical groove. The summit presents an articular facet, continuous with the one on the outer face of the internal malleolus, and intended for articulation with the upper surface of the astragalus. This facet is broader externally than internally, and broader in front than behind. It is concave from before backward, and nearly flat from side to side.

The Shaft of the bone is three-sided, presenting an internal, an external, and a posterior surface, and an anterior and two lateral borders. The bone is generally somewhat twisted, particularly in the negro race; and is bowed, convexinternally and concave externally. The internal surface is broad above, becoming slightly narrow below; is convex both from above downward, and from before backward; and is subcutaneous. The cxternal face, which is concave from above downward, is marked by a vertical groove close to the external border of the bone. This face in the lower third curves gradually forward to the anterior face of the bone. The posterior face is broad and flat for the upper one-fifth of the bone, becoming more rounded in the middle and flatter and broader in the lower portion. At its upper part it is marked by an oblique ridge, called the Popliteal Linc, which extends downward and inward from the external tuberosity of the bone. Below this ridge the posterior surface is marked about its middle by a vertical ridge, which exists only for about the middle of the bone, disappearing in the lower portion. Near the commencement of the vertical ridge, grenerally on the posterior surface, is found a large nutrient foramen, being in fact the largest in the body, which conveys the blood to the interior of the bone. 
Of the borders the anterior, or shin, is rery sharp and rerp prominent. It is sinuous in outline. more distinct in the upper two-thirds of the bone. While in the lower third it passes to the front of the internal malleolus, and blends with the anterior border of this projection. The intemal border, separating the internal from the posterior surface. begins as an indistinct line at the inne: tuberosity, becomes more prominent about the middle of the boue. and finally passes to the posterior border of the internal malleolus. The external border commences abore at the outer malleoeus. in front of the articular facet for the fibula. It is the interosseous border. It is indistinct above. Well marked in the middle portion of the bone, and at the lower exremity divides into two lines, which form the boundaries of the triangular space mentioned as being ou the lower estremity of the bone.

\section{The Fibula.}

The Fibula (I. Fibula, a clasp) is the outer and more slender of the two bones forming the skeleton of the leg. It is a loug bone, having of course a shaft and two extremities.

The Lpper Extremity, or head, is club-like in outline, and presents on its upper inner aspect an articular facet, the contour of which varies in different bones. It is circular in form usually slightly concare, but it may be flat and looks upward, forward and inward. It is intended for articulation with a similar facet on the outer tuberosity of the tibia. The outer aspect of this extremity is roughened for muscular and ligamentous attachment. and terminates above in a blunt projection. called the str.loid process of the fibula.

The Lo:.er Extremity is usually spoken of as the Extermal Malleolus. It is larger and more pointed than the upper and is indistinctly triangular. It may be described as consisting of internal and exterual surfaces, anterior and posterior borders, and an apes. The outer surface is prominent. conves, rough and subcutaneous. The internal surjace is articular through a part of its ertent: and both abore and below the articular surface is found a roughened space-that abore for the interosseous ligaments which attach it to the fibula, that below for 
ligamentous fibres binding it to the foot. The articular space is intended for the lateral aspect of the astragalus, and occasionally extends upward to the fibula. The posterior border is nearly straight, is much thicker than the anterior border, and is marked by a shallow groove for the passage of tendons. The anterior-border is narrow and convex, and slopes obliquely from above downward and backward. The apex is a blunt, roughened point.

The Shaft of the bone is twisted, presenting what is known as the curre of torsion, so that its faces and borders change their relative positions in the different portions of the bone. The faces may be described as external, internal, and posterior. The extemal face, narrow and convex above, becomes broader about the middle of the bone, and orwing to the twisting of the bone becomes posterior at its lower one-third. The internal face, slightly curverl above, marked by a ridge over the central portion of the bone, twists at the lower third to become anterior. The posterior facc, narrow and convex above, broader and still convex at the centre, becomes flat and internal at the lower one-third of the bone. Of the borders, the anterior is slightly marked above, prominent in the middle of the bone, divides into two lines at the lower one-third, one of which becomes external and passes to the posterior border of the external malleolus, while the other, more slightly marked, passes to the anterior border of the external malleolus, leaving between them a triangular spaca, which is a continuation of the internal surface. The cxternal lorder, commencing at the head of the bone, much more slightly marked than the internal border, becomes posterior in the lower third of its course, and passes to the back of the external malleolus. The internal border commences at the back of the head of the fibula, becomes the interosseous ridge in the middle of the bone, running down upon the internal face, and twists to the front of the bone in the lower portion of its course.

\section{The Foot.}

The foot bears a strong resemblance to the hand: differing essentially from it however in that it is at right angles to the Des Anat-4 
leg; and consequently its surfaces are superior and inferior, instead of anterior and posterior. It consists of an irregular set of bones forming the Tarsus, a set of long bones forming the Metatarsus, and the Phalanges.

\section{The Tarsus.}

The Tarsus consists of seven bones: The Os Calcis, Astragalus, Scaphoid, Cuboid, and three Cuneiform bones, known as External, Middle, and Internal, sometimes as First, Second, and Third. These bones are arranged in two rows: the first row, or posterior row, consisting of the os calcis and astragalus the remaining five bones forming the second row.

\section{The Bones of the First Row.}

The Os Calcis (L. Calx, the heel) forms the posterior abutment of the arch of the foot, and is known as the heel bone. It presents for examination two extremities, anterior and posterior, and four surfaces, superior, inferior, and two lateral. The upper surface consists of two portions, one behind the other. The posterior one-half of the upper surface is roughened, marked by the presence of a few nutrient foramina, and is non-articular. It is convex from side to side. The anterior portion presents two articular surfaces, separated by an oblique groove. Of these surfaces, the larger and more posterior is convex in outline, and oblique in direction. Its long diameter is directed from before downward and outward; and it corresponds to the concave receiving cavity on the inferior surface of the astragalus. The anterior is an oblong articular facet, concave from above downward and outward, the direction of its long diameter ; and intended for the reception of the convex articular facet on the lower surface of the astragalus. The groove which separates the two surfaces is called the Sulcus Calcanei (L. The furrow of the heel), and is directed like the surfaces: that is, downvard and outward. The inferior surface is rough, narrow, and non-articular; and presents near its posterior extremity two tuberosities, of which the internal is the larger. The anterior part of the inferior surface is hardly more than a rough and rounded border. The 
cxtcrnal surface is convex throughout its course, is very rough for the attachment of ligaments; and is marked by two grooves, one above the other, separated by a slight roughened ridge. These gooores are intended for the transmission of tendons. The internal surface is deeply concave; and is overhung in its anterior portion by a projection of bone, which is called the sustentaculum tali (L. A support for the ankle), or lesser process of the os calcis. The deep concavity on the internal surface is caused mainly by this projection of bone, which is grooved on its under surface for the transmission of a tendon, and which bears on its upper surface the greater portion of the anterior one of the two facets on the superior face of the bone. The posterior extremity' is quadrilateral in outline, rough at its lower portion, smooth above for the play of the bursa between itself and the tendon Achillis. The anterior extremity is concavo-convex, forming a saddle-shaped articulation between this bone and the cuboid. This surface is concave from above downward and outward, convex in opposite direction.

The Astragulus, the second bone of the first row, lies between the tibia above, the two malleoli at the sides, and the os calcis below. It presents like the os calcis four surfaces and two extremities, the surfaces, as is the case with the os calcis, being superior, inferior, and two lateral, while the extremities are anterior and posterior. The supcrior surface is divided into two portions, a postęrior articular portion, and an anterior, frequently called the "neck" of the bone, which is non-articular. Thearticular portion is narrow behind and broad in front, a fact which governs some of the movements of the ankle-joint. It is composed of a central depression with two elevated ridges, one upon either side, or, in other words, it is a trochlear surface. The inferior surface is in the main articular, consisting of two facets. The posterior facet is quadrilateral and concave, its long diameter directed downward and outward; and corresponds to the posterior of the two facets on the upper surface of the os calcis. The anterior articular facet is convex. Its long diameter likewise is clirected downward and outward; and it corresponds to the concave facet on 
the os calcis. Between these two articular surfaces is a deep oblique groove, running downward and outward, which is called the Sulcus Tali. This sulcus corresponds in position to the sulcus calcanei; and when the two bones are articulated, the junction of the two sulci forms a canal, which is known as the Sims Tarsi. The internal face is articular to a small extent; that is, at the junction of the internal and superior faces is found an oblong articular facet, its long diameter directed from before backward, continuous with the articular surface on the superior face of the bone, and intended for articulation with the internal malleolus. Below this articular facet is a rough depression for ligamentous attachment; and the remainder of the internal surface is likewise roughened. The external surface is triangular or pyriform, the base upward and apex downward. It is concave from above downward, slightly convex from before backward, and is continuous with the articular facet on the superior surface of the bone. It is intended for articulation with the external malleolus. Below the articular surface is a slight roughness for ligamentous attachment. The anterior extremity, or head of the bone, is oval, convex, and articular ; the long diameter of the head, slightly oblique in direction, is from above downward and invard, and is intended for articulation with the cavity found upon the scaphoid. This head is supported upon a constricted portion of the bone, sometimes called the "neck", a part of which has been already seen as a roughened surface, in front of the articular facet. The remaining portion of the neck is rough and thick. The posterior extremity is hardly more than a narrow border. It is marked by a slight transverse groove just behind the superior articular surface; and by an oblique groove, which extends downward and inward, and is continuous in the articulated foot with the concave inner face of the os calcis.

\section{The Bones of the Second Row.}

The Cuboid lies between the os calcis behind, the scaphoid and external cuneiform to its inner side, and the fourth and fifth metatarsal bones in front. It presents four surfaces, 
superior, inferior, external, and internal, and an anterior and a posterior extremity. The upper surface looks upward and outward, and is rough and irregular for the attachment of ligaments. The inferior surface is very irregular in outline. A little in front of the middle it is divided by a prominent ridge, called the peroneal ridge, into an anterior portion, which is occupied almost entirely by a groove, also called peroneal, and a larger posterior portion, which is roughened for ligamentous attachment. The peroneal ridge terminates externally in the tuberosity, a somewhat rounded blunt projection of bone, which constitutes one of the landmarks of the foot. The internal face near the junction of the inner and superior faces is marked by an oblong articular facet, which is intended for articulation with the external cuneiform bone. Occasionally behind this facet is another smaller facet for articulation with the scaphoid, though usually these bones do not articulate with one another. Below the articular facet the bone is rough for the attachment of the interosseous ligaments. The external face is in reality only a border. It is narrow and roughened, presenting the projection of the peroneal ridge and the commencement of the peroneal groove. The anterior extremity is divided into two articular facets by a vertical ridge. The inner of these facets is quadrilateral in outline; the external, triangular. They are intended for articulation with the fourth and fifth metatarsal bones respectively. The posterior c $x$ tremity is concavo-convex, being concave from above downward and in ward and convex from within outward, to correspond with a similar articular surface on the anterior extremity of the os calcis. At the junction of the inferior and internal surfaces tl:is extremity is prolonged backward and inward, so that the os calcis apparently rests partly upon a prolongation of the cuboid.

The Scaphoid, the second bone of this row, presents an anterior posterior, superior, and inferior surface; and internal and external extremities. The poslerior surface is entirely occupied by a concave smcoth articular facet, whose transverse diameter is greater than its vertical, and is intended 
for articulation with the head of the astragalus. The anterior surface has three articular facets, separated by two slightlymarked vertical ridges. These facets articulate with the three cuneiform bones. The most internal, which approaches the nearest to the inferior surface of the bone, is large and slight1y triangular; the central, quadrilateral; and the external, also nearly quadrilateral in outline, is oblique in its direction. The superior surface is convex from side to side; and roughened for ligamentous attachment. The inferior surface, narrower than the superior, is also roughened for ligamentous attachment. The imner extremity of the bone is a blunt projection, the tuberosity of the Scaphoid which, with the tuberosity of the internal cuneiform, furnishes another of the landmarks of the foot. Theouter extremity is usually roughened; but occasionally presents a small facet for articulation with the cuboid.

The Internal Cuneiform, like the other two, is irregularly wedge-shaped; and lies on the inner side of the tarsus, between the first metatarsal in front, the scaphoid behind, and the middle cuneiform and second matatarsal to the outer side. It is the largest of the cuneiform bones; and unlike all of the others, is broader below than above. Its internal face is rough and convex. The external face is marked along the junction of the superior, external and posterior faces by a reversed $\mathrm{L}_{\mathrm{L}}$-shaped facet, intended for articutation with the middle cuneiform. In front of this facet is a smaller articular surface for the second metatarsal bone. The posterior face is concave, slightly triangular in outline, corresponding to the internal one of the facets upon the scaphoid. The anterior face is articular throughout, slightly convex, and is said to be kidney-shaped. The inferior face, or base of the bone, is very rough ; and terminates posteriorly in the blunt elevation or tuberosity of the internal cuneiform. The superior face is scarcely more than a rough narrow border.

The Middle Cuneiform lies between the internal cuneiform on its inner, and the external cuneiform on its outer side, the scaphoid bone and the second metatarsal in front. 
Its superior surface, much broader than its inferior, is rough and irregular for the attachment of ligaments. The inferior surface, scarcely more than a border, is also roughened. The internal surface presents a reversed L-shaped facet similar to the one found upon the internal cuneiform. The external surface has an articular facet upon its posterior portion ; but is non-articular in front, being roughened for the attachment of the interosseops ligament. The posterior surface is smooth, slightly quadrilateral in outline for articulation with the middle facet upon the scaphoid. The anterior surface is articular, convex slightly from side to side for articulation with the base of the second metatarsal bone. This bone is more distinctly wedge-shaped than the other two cuneiform bones.

The External Cuneiform is intermediate in size between the internal and the middle. It lies with the middle cuneiform to its inner, and the cuboid to its outer side, the scaphoid behind and the third metatarsal bone in front. The bone appears to be slightly bent upon itself, so as to be concave internally and convex externally. Its superior surface is roughened like the preceding tarsal bones; its inferior surface, narrower than the superior, is likewise rough for ligamentous attachment. The internal surface is slightly concave from before backward; and presents an articular facet near its posterior end for articulation with the middle cuneiform. In front of this facet it is roughened for the interosseous ligament. Near the auterior extremity is a very slight facet for articulation with the lateral aspect of the second metatarsal bone. The cxternal surface has an oblong facet near its upper posterior portion, corresponding to the slight facet which is found on the inner face of the cuboid. In front of this the external surface is roughened for ligamentous attachment. Posteriorly it presents a smooth, nearly flat articular facet, which is cut obliquely'so as to face backward and invard, thus distinguishing it from the other cuneiform bones, whose posterior extremities are cut squarely. The anterior surface, or extremity, presents a smooth quadrilateral articular facet for articulation with the third metatarsal bone. 


\section{The Metatarsal Bones.}

The Metatarsal Bones present the same general characteristics as the metacarpal. They are long bones, presenting a shaft and two extremities. Like the metacarpal bones they are five in number; but are numbered from within outivard.

\section{A Typical Metatarsal Bone.}

A Typical Metatarsal Bone presents a posterior extremity, or base, an anterior extremity, or head, and a shaft. The posterior extremity is quadrilateral in outline, marked by an articular facet upon its summit, and an articular facet upon each lateral aspects, its superior and inferior surfaces being rough and square. Its head, much smaller than its base, presents on its summit an articular facet, which is convex, with its long diameter vertical. Behind the head is a slightly constricted portion, which is surmounted on each side, near the dorsal aspect of the bone, by a well-marked tubercle, while a smaller tubercle is found near the plantar aspect. Near the plantar aspect the head is grooved for the transmission of tendons. The shaft of the bone is narrow; and presents a dorsal surface marked by an indistinct ridge, bifurcating below to extend to the two dorsal tubercles. The lateral surfaces are concave, so as to leave an interosseous space between the adjoining metatarsal bones; and the inferior border is rounded and indistinctly marked.

The First metatarsal bone is very massive. Its posterior extremity presents a concavo-convex articular surface; and its anterior extremity, presenting a rounded head, is, unlike the other metatarsal bones, nearly as large as the posterior extremity.

The Second metatarsal, the longest of the metatarsal bones, presents an articular facet on its summit for the middle cuneiform, on its inner side an articular facet for the internal cuneiform, and on its outer side a well-marked articular facet for the third metatarsal, and a somewhat slightly marked facet for the external cuneiform. 
The Third metatarsal bone presents an articular facet on either side, that on the inner side being divided into two by an antero-posterior groove. On its summit is an articular facet for the external cuneiform bone.

The Fourth metatarsal is the typical metatarsal bone; and need be no further described.

The Fifth metatarsal bone has as its peculiar characteristic a blunt projection on the outer side of its base, this projection being subcutaneous, and one of the most important landmarks of the foot. It has only one lateral articular facet.

Certain general characteristics enable us to distinguish between the metatarsal and mctacarpal bones. The most important is that the metatarsal bones are all about the same length, but are slenderer than the metacarpal; and whereas in the metacarpal bones the head is always of nearly the same or greater size than the base, in the metatarsal bones the base more than doubles the size of the head, with the single exception of the first metatarsal, which is distinguished by other marks.

\section{The Phalanges.}

The Phalanges of the toes, like those of the fingers, are arranged in three rows, except that the great toe has, like the thumb, but two phalanges, the place of the second being taken by the ungual. They resemble those of the hand soclosely that a separate description is not necessary. They are smaller and shorter than those of the hand, the second phalanx particularly being scarcely more than a neck of bone separating two articular extremities, while the ungual phalanx is frequently scarcely distinguishable, except that for the first toe. In the fifth, or little toe the ungual and second phalanges are sometimes found ossified together.

\section{The Skeleton of the Head.}

The skeleton of the head, or skull, consists of two portions, the bones of the cranium and those of the face.

\section{Cranium.}

The cranium is the upper posterior division of the skull, and is formed by the union of eight bones-occipital, frontal, sphe- 
noid, ethmoid, two parietal and two temporal. These become securely ossified together in mature life, and can be separated only in the young subject.

\section{Occipital.}

The occipital bone,sometimes called the first cranial vertebra, is situated at the posterior part of the cranium, and presents for examination two surfaces, four angles and four borders.

The posterior surface, frequently erroneously called external, is convex, and presents above its centre an eminence called the posterior occipital protuberance; curving outward from this on either side is a rough ridge called the superior curved line; passing downward from the protuberance to a large aperture-the foramen magnum-is a sharp ridge, the occipital crest; arching outward on either side from the middle of the crest, and concentric with the superior curved line is the inferior curved line. The portion between the curved lines is slightly depressed and rough. At the lower termination of the crest is seen the foramen magnum, a large oval opening, whose antero-posterior diameter is the longer and whose larger end is behind. On either side of the front half of the foramen magnum is seen the condyle for articulation with the superior articular process of the atlas; it is oval, convex, articular, and directed obliquely forward and inward. Just behind the condyle is a depression, the posterior condyloid fossa, with occasionally a foramen opening into it--the posterior condyloid foramen. In front of the condyle is the anterior condyloid foramen. Passing outward from the condyle is a rounded elevated ridge, the transverse process. On the inner aspect of the condyle is a tubercle. In front of the foramen magnum is the basilar process; it is horizontal, quadrilateral, convex, and presents an antero-posterior ridge, the pharyngea1 spine.

The anterior face is concave and presents four ridges, meeting at right angles, about the middle, called the crucial ridge, or occipital cross. Each ridge is called an arm of the cross. Three of these ridges are grooved; while the fourth, which is the inferior, is sharp and not grooved until it approaches the 
foramen magnun1. At the point where the four arms meet there is an eminence called the anterior occipital protuberance. Above each lateral arm of the cross there is a shallow depression, the cerebral fossa; below each lateral arm is another depression, the cerebellar fossa. At the termination of the lower arm is the foramen magnum, and on each side of this the anterior condyloid foramen. In front, and to each side of the foramen magnum is a smooth broad groove for the termination of the lateral sinus. In front of the foramen magnum is the basilar process, which, as on its inferior surface, is nearly horizontal and quadrilateral, but is here concave to sustain the medulla oblongata.

The angles are superior, inferior and two lateral. The lateral angles are each situated where the groove in the lateral limb of the crucial ridge, strikes the border of the bone. The inferior angle is the truncated extremity of the basilar process, and articulates with the body of the sphenoid.

The superior angle juts into the interval formed by the union of the posterior superior angles of the parietal bones.

The borders are two superior and two inferior. Each superior border is serrated for articulation with the parietal bone; and extends from the superior to the lateral angle.

Each inferior border articulates with the temporal bone, mastoid and petrous portions; is serrated below the lateral angle and is interrupted about its middle by a protuberance, the jugular eminence, in front of which is a depression, the jugular fossa.

\section{Parietal.}

The two parietal bones form the upper and most of the lateral wall of cranium, and are situated between the frontal and occipital bones, in front and belind, and the two temporal bones laterally. Fich bone is divided into livo surfuces, four borders, and four angles. The extermal surface is convex and presents a decided bulge about the middle, the parietal emi nence; through this is secn running the temporal ridge, arching across this bone from the frontal; below the ridge, is a nearly flat surface forming part of the temporal fossa. Near 
the posterior superior angle is seen the parietal foramen, which is frequently absent.

The internal surface is mostly concave and is sunken in the middle into a fossa. It is marked by digital pits for lodging cerebral convolutions, and the arborescent furrows worn by the arteries. Along the edge of the superior border is seen half of a groove, completed by the opposite bone, and formed by the longitudinal sinus.

The borders are four. The anterior, for articulation with the frontal bone, and the posterior, for the occipital, are serrated. The superior border, for articulation with its fellow, is straight, serrated and thick. The inferior border, for articulation with the squamous portion of the temporal bone, is short, curved and beveled at the expense of the outer table. The angles are four. The anterior superior and the posterior superior are right angles. The anterior inferior is $10 \mathrm{~g}$, thin and marked on its inner aspect by a furrow formed by the middle meningeal artery. The posterior lnferior is blunt and usually marked internally by the groove for the lateral sinus.

\section{Frontal.}

The frontal bone is situated at the front and base of the cranium. In early life the bone consists of symmetrical lateral halves, separated by a vertical suture, which, however, becomes ossified later in life, though the bone could still be described as consisting of two similar lateral portions. The bone is described as being made up, in each of its lateral halves, of two portions-the vertical, or frontal, and the horizontal, or orbito-nasal portion.

The vertical portion forms the skeleton of the forehead and presents two surfaces and a lateral aspect. Upon the anterior surface, which is convex, there are seen superiorly a smooth, somewhat flattened surface; below this a bulge, called the frontal eminence; below this a broad, shallow, transverse groove; below this a transverse ridge, the superciliary; between this and the opposite ridge, on the middle line, is a prominence called the glabella, or nasal tuberosity. Below the superciliary ridge is seen the margin of the orbit, called 
the supra-orbital ridge, which terminates at the inner extremity in the internal angular process, and at its outer in the external angular process. About the inner third of the supra-orbital ridge is a notch, sometimes formel into a foramen, called the supra-orbital notch or foranen.

The posterior face of the vertical portion is concave and marked by digital pits and arborescent furrows for the arteries. In the middle line this surface presents superiorly a groove for the longitudinal sinus, which, as it descends, terminates in a ridge, at the extremity of which is a small foramen, called the foramen coecum.

The lateral aspect of the bone presents a ridge curving upward and backward from the external angular process, and called the temporal ridge; behind this the surface is sunken and forms part of the temporal fossa. Both the temporal ridge and fossa are only partially seen on the frontal bone, for, in the articulated skull, they are continued on to the parietal and temporal bones.

The horizonlal portion consists of two lateral parts, the orbital plates, separated by a rectangular notch, the ethmoidal fissure. Each orbital plate presents an inferior and a supcrior surface. The inferior face is smooth, triangular and concave, the concavity being greatest behind the external angular process-the lachrymal fossa. It has, just withiu the supra orbital notch, a depression called the fovea trochlearis.

The upper surface of the orbital plate is convex and rough, presenting irregular rough eminences.

The cthmoidal fissure, the margins of which present several half cells, which, when articulated with the ethmoid, complete theethmoidal cells, is the rectangular notch separating the two orbital plates.

In front of it is a roughened interval, between the two internal angular processes, called the nasal notch, descending from the middle of which is a $\operatorname{long}$ pointed process, the nasal spine. Upon the posterior surface of the nasal spine is a vertical ridge. In the front portion of the etlimoidal notch and external to the nasal spine, are the openings of the 
frontal sinuses. The frontal sinus, one on each side of the middle line, is an irregular cavity between the two tables of the vertical portion which gradually increase in size from the time it makes its appearance in early life.

The borders of the frontal bone are two-superior and inferior. The superior border is semi-circular and serrated for articulation with the parietal bones. When examined closely it is seen to be beveled superiorly at the expense of the inner table, and inferiorly at the expense of the outer.

The inferior border is the posterior termination of the orbital plates, interrupted in the middle by the ethmoidal notch. It is straight and articular with the lesser wing of the sphenoid. At the angle of the junction of the two borders, there is a rough triangular surface, the sphenoidal triangle, for articulation with the greater wing of the sphenoid.

\section{Temporal Bone.}

The temporal bone is divided for study into the squamous, mastoid and petrous portions.

The squamous portion occupies the upper front part of the bone and consists of two surfaces, a semi-circular border and a projection called the zygoma. The internal surface is concave from above downward and from before backward. It is marked by irregular, antero-posterior elevations with intervening depressions for the convolutions and sulci of the brain. No distinct line, in the adult, marks the point of separation between the squamous and petrous portions. The external surface may be conveniently divided into two parts-that which lies above and that which lies below the zygoma. The part above is convex from above downward, slightly concave from before backward, rough for muscular attachment and enters into the formation of the temporal fossa. Posteriorly it is separated from the mastoid portion by a rough line, part of the temporal ridge. The rest of this surface is occupied by the zygoma and the glenoid cavity.

The zygoma, or zygomatic process, at its origin from the squamous portion, passes outward with a slight inclination forward; but after a course of about one-fourth 
of an inch, is twisted upon itself so that the surfaces and borders change their relative positions. At its origin the surfaces are superior and inferior, the first occupied by a groove, the second by a roughened tubercle; but in the remainder of its course, where it passes forward with but a slight inclination outward, the surfaces become internal and external, the borders superior and inferior. The external surface is rough, convex both from above downward and from before backward. The internal surface is concave in both directions and smooth. The superior border is longer, thinner and sharper than the inferior, which is thick and rough and short. Anteriorly it terminates in a rough serrated extremity, obliquely cut from above downward and backward, and intended for articulation with the malar. Posteriorly, the zygoma rises by two rough lines called the roots of the zygoma. The anterior root is continuous with the inferior border and terminates in a rough projection called the tubercle of the zygoma, from which a smooth elevated ridge, the eminentia articularis, passes inward as the anterior boundary of the glenoid cavity. The posterior root passes backward, just above the glenoid cavity and the external auditory meatus, and than curving upward forms the continuation of the temporal ridge which separates the squamous from the mastoid portion. Just after it passes above the glenoid cavity, it sends downward a prominent articular projection, sometimes called the middle root of the zygoma, which forms the posterior boundary of the articulation for the lower jaw.

The glenoid carity is concave and oval in outline, its long diameter being directed from without backward and invard. it is bounded in front by the eminentia articularis, a smooth, rounded, oval articular ridge which is a continuation of the anterior root of the zygoma. Posteriorly it is limited by a rough, quadrilateral sheet of bone called the tympanic plate, while in the middle is a well defined slit, the Glaserian fissure, which divides the cavity into an anterior articular portion and a posterior non-articular. The articular portion is limited behind by the middle root of the zygoma, sometimes called the post-glenoid tubercle. 
The semi-circular border is rough, thin and sharp above, strongly beveled at the expense of the inner table; thick and serrated below and slightly beveled at the expense of the outer table.

The Mastoid portion consists of an internal and external surface; superior and posterior borders and a rough nipple like projection, the mastoid process. The internal surface presents but one mark of note. It is smooth and has a deep groove which runs from above downward and backward. It is called the fossa sigmoidea. The external surface is rough throughout and presents near the posterior border a large foramen, called the mastoid, which is sometimes found in the suture between this bone and the occipital and sometimes is altogether wanting.

The Mastoid process is a rough, blunt projection of bone, marked internally by a deep groove, the digastric fossa, internal to which is a shallower groove for the occipital artery and known as the occipital groove. The interior of the mastoid portion is hollowed out into a number of cellular cavities. The superior border is rough, serrated and nearly straight to receive the posterior inferior angle of the parietal. The posterior border, longer than the superior, slopes from above downward and forward and is roughened for articulation with the occipital.

The Petrous portion is a pyramidal projection of bone with its apex directed inward and forward and its base outward and backward. It presents three surfaces, anterior posterior and inferior; and three borders, superior, anterior and posterior. The posterior surface is the simplest of the three. Internal to its middle point it presents an oblique oval opening, the internal auditory meatus. This opening leads into a short canal, directed outward and forward, at the bottom of which may be seen a bony lamina perforated by numerous apertures and crossed by a transverse ridge. External to the internal auditory meatus and near the posterior border, is seen a little slit like aperture which leads to the acqueductus vestibuli. Between the two, and near the superior border, is usu- 
ally seen a slighter depression which lodges a process of the dura mater.

The anterior surface presents near the middle a round elevation which marks. the position of the superior semicircular canal; external to this a smooth concave surface forms the roof of the tympanum; below and in front of the bulge is a groove leading outward and backward to an opening, the hiatus Fallopii, while at the junction of the squamous and petrous portions may sometimes be seen another and slighter groove, the petrosal. Near the apex of the bone is seen a depression for the Gaserian ganglion, while at the apex the roof of the carotid canal is usually wanting and a part of the canal may be seen from the anterior surface.

The inferior surface is rough and irregular and has upon it eleven points which require examination. Near the apex of the bone is a rough, nearly quadrilateral surface which gives attachment to muscular fibres. External to this is a circular opening, the aperture of entrance of the carotid canal. At first this canal passes upward in the bone, but it abruptly turns forward and inward and downward, pursuing a course parallel with that of the petrous bone, to emerge at the apex. External and slightly behind this is a smooth, concave surface, which encroaches on the posterior border of the bone, called the jugular fossa. On the bony partition separating the jugular fossa from the carotid aperture, is a small foramen which transmits Jacobson's nerve : and on the outer wall of the fossa itself is another foramen, smaller in size, for Arnold's nerve. Behind and slightly internal to the carotid aperture, near the posterior border and directly beneath the internal auditory meatus, is a small opening, the aqueduct of the cochlea. External and anterior to the carotid aperture, is a thin, prominent tongue of bone, which extends outward to become continuous with the auditory process, called the vaginal process. At its base this process splits into two sheets, one in front of the other, to enclose a sharp pointed projection, the styloid process. This, the longest of such processes in the body, passes downward and slightly forward and outward. Behind and

Des Anat-5 
external to this is a large foramen in the bone, the stylomastoid; while between the vaginal process and the mastoid process is a slight groove, the auricular fissure. At the junction of the posterior border of the mastoid portion with that of the petrous portion, there is a rough articular surface called the jugular facet.

The borders are three, superior, posterior and anterior.

The supcrior border, the longest of the three, presents along its upper margin a groove for the petrosal sinus, and near the apex of the bone a notch to lodge the fifth cranial nerve. The posterior border, intermediate in length, usually presents near its middle a rough tongue of bone which divides the jugular fossa into two portions. The remainder of the border is rough for articulation with the basilar process of the occipital. The anterior border is the shortest of the three, and is in part articular for the greater wing of the sphenoid. In the receding angle between the petrous and squamous portions there is seen an irregular opening, divided into an upper and a lower portion by a thin shelf of bone known as the processus cochleariformis. The canal above this shelf is for the tensor tympani muscle while that below is intended for the Eustachian tube.

The base of the petrous portion is partly interposed between the other two portions and partly continuous with them. On the free portion is seen the opening into the ear, the external auditory meatus, which is situated between the mastoid process behind and the middle root of the zygoma in front, and between the posterior root of the zygoma above and the auditory process below. The auditory process is a rough curved sheet of bone continuous with the vaginal process, and partly circumscribing the external auditory meatus.

The apex of the petrous portion is rough and presents a large irregular aperture, the aperture of exit for the carotid artery. It is received between the basilar process of the occipital bone and the spinous process of the sphenoid.

\section{The Sphenoid.}

The sphenoid occupies a position near the middle of the base of the skull, and may be considered the key-stone of the cranial 
arch, since it serves to bind together all the bones of the skull and five of those of the face. It has been likened to a bat with the wings spread out, and is divided for study into a body two wings, greater and lesser, on either side, and two projections, or processes, called the pterygoid.

The body presents six surfaces, superior, inferior, anterior, posterior and two lateral.

The upper surface conmences in front in a triangular spine which, from its articulation, is known as the ethmoidial spine of the sphenoid. Behind this projection there may sometimes be seen a line or ridge leading backward and a slight transverse ridge. stretching between the anterior margins of the optic foramina. Behind this is a broad, transverse groove, the optic, and behind this a prominent, rounded elevation, the olivary ridge or process. Behind the olivary ridge is a deep pit-like depression, the sella turcica or pituitary fossa, overhanging which behind is a square projection, the dorsum Ephippii, whose posterior surface, running obliquely downward and backward is called the clivus Blumenbachii. On either side of the mid-line in front of the sella turcica is a slight antero-posterior groove, continuous with one on the upper surface of the Ethmoid, in the articulated skull, for the olfactory bulb. Behind the optic ridge the optic groove extends across to terminate at either end in the corresponding optic foramen. The olivary ridge terminates at its extremities in a small projection, the middle clinoid process, which is sometimes connected by a spicule of bone with the anterior clinoid process of the lesser wing. The sella turcica, deep and pit like, is bounded in front by the olivary ridge, behind by the dorsum ephippii, laterally by the margin of the carotid groove. The dorsum selle terminates at either angle in a rough projection, the posterior clinoid process, sometimes connected to the anterior clinoid process by a spicule of bone. The posterior surface of the dorsum, or the clivus, is a rough, quadrilateral, sloping surface, continuous, in the articulated skull with the upper surface of the basilar process of the occipital. 
The posterior surface is rough and square, presenting many nipple-like projections for articulation with the basilae process of the Occipital. In advanced life it is ossified to thr Occipita1.

The anterior face presents in the middle a prominent, rough vertical ridge called the crest of the sphenoid, for articulation with the posterior border of the perpendicular lamella of the Ethmoid. On either side of the crest the surface is rough and presents near the middle the opening into the sphenoidal sinuses. The margins of the opening are rough for articulation with the os planum and posterior extremity of the lateral mass of the Ethmoid, the latter through the pyramid of IVistar.

The inferior surface is marked in the middle by the continuation of the crest, called the rostrum of the sphenoid. The rostrum is broad behind, narrow and more prominent in front and is received into a fissure on the upper border of the Vomer. On either side of the rostrum is a smooth surface for articulation with thealæ of the Vomer, external to which is the vaginal process for the under surface of each ala, beneath which is the groove called pterygo-palatine, converted into a canal of the same name bv articulation with the palate bone.

The lateral surfaces are almost taken up by the origin of the greater wings. The space just above each wing is occupied by a broad winding groove, the cavernous, which terminates at the lesser wing. In front of this the lateral surfaces are smooth, slightly concave from above downward, and enter into the formation of the sphenoidal fissure and the inner wall of the orbit.

The Lesser Wings. Each lesser wing springs by its perforated base from the junction of the lateral and superior surfaces of the body, and passes outward to terminate in a pointed process near the outer limit of the greater wing. They each present two surfaces, superior and inferior, and two borders, anterior and posterior. The upper surface smooth, slightly concave, broad internally and narrow without, forms a part of theanterior fossa of the skull. The inferior, narrower than the superior, is smooth and slightly convex in outline and forms 
a part of the roof of the orbit and the upper boundary of the sphenoidal fissure. The anterior border is thin, straight and serrated for articulation with the straight border of the Frontal. The posterior, thicker than the anterior, begins internally in a bluntly pointed projection called the anterior clinoid process, which passes backward and inward. This border is concave and smooth, thinner in the middle than at either extremity, and terminates externally in the outer end of the lesser wing. The perforation found at the base of the lesser wing is the continuation of the optic groove of the upper face, is called the optic foramen and leads downward, forward and outward to the cavity of the orbit.

The Greater Wing. The greater wing springs from the lower part of the lateral aspect of the body and passes upward, outward and forward. It is divided into four surfaces, superior, anterior, external and inferior. The circumference of the greater wing will be described later. The upper surface of the greater wing is concave and enters into the formation of the middle fossa of the skull. It is, consequently marked by elevations and depressions corresponding to the sulci and convolutions of the temporo-sphenoidal lobe of the brain which it lodges. It is more deeply concave anteriorly where it is overhung by the lesser wing than at any other part. Near the junction of the wing with the side of the body, and immediately beneath the sphenoidal fissure, there is a circular foramen called, from its shape, the foramen rotundum. Its direction is downward, forward and slightly outward and its anterior opening is found just external to and above the base of the pterycroid process. Behind and external to the foramen rotundum is the foramen ovale, looking directly downward and opening on the inferior face of the greater wing. Behind and external to this is found the foramen spinosum, also opening directly downward. The external face enters into the formation of the temporal fossa. It is broader above than below, slishtly rough for muscular attachment, concave both from above downward and from before backward, and perforated by a number of small foramina. The inferior face is a part of the "ygomatic fossa, is small and quadrilateral 
in shape, looks downward and outward and is slightly roughened for the attachment of muscles.

The greater part of the anterior face looks into and forms a part of, the orbital cavity. It is triangular in form, the apex directed backward and inward and the base outward, concave from above downward, flat from before backward and perforated by one or two small foramina. Just below this portion of the anterior face is a small flat surface of bone forming a part of the pterygo-maxillary fossa and upon which the foramen rotundum opens.

The borders. The circumference of the greater wing may be considered as forming the borders of its four surfaces. From the side of the body to the spine of the sphenoid is the posterior border of the upper surface; from the spine to the point for articulation with the parietal is the outer border of the upper surface; from this point to the body again is the upper border of the superior surface. The outer face, which is quadrilateral in form, has four borders. Its superior border extends from the outer border of the upper face to the anterior face; its anterior border from this point to the pterygoid ridge; its inferior border is the pterygoid ridge, while its posterior border is that part of the outer border of the upper face extending from the pterygoid ridge to the summit of the bone. The anterior face is bounded above, for its outer part, by a serrated edge forming the anterior boundary of the frontal triangle; for its inner part by a part of the upper border of the superior surface; anteriorly or externally, by the anterior border of the outer face and below by a ridge leading from the pterygoid ridge on the base of the pterygoid process. The posterior border of the upper surface is rough in its outer half for the anterior border of the petrous portion of the Temporal; the outer border is serrated for the squamous portion of the temporal; the outer part of its upper border is rough for the Frontal. The upper border of the outer face is articular for the anterior inferior angle of the Parietal; the anterior border is articular for the posterior border of the orbital process of the Malar. The inner part of the upper border of the superior face, and the lower border of the anterior face are non-articular, the 
one entering into the formation of the sphenoidal fissure the other into the spheno-maxillary. The frontal triangle is found at the junction of the upper, outer and anterior surfaces. It is bounded in front by the upper border of the anterior face; externally by the upper border of the outer face; behind by a part of the upper border of the superior surface.

The Pterygoid Process. The Pterygoid Process springs from the junction of the greater wing and the body near the posterior surface of the body. Each process consists of two plates, internal and external, united above and diverging below. Viewed from the front the united plates present a triangular smooth surface above, forming the posterior wall for the pterygomaxillary fossa, pierced above by a round opening, the anterior opening of the Vidian or pterygoid foramen. Below and internal to this is a narrow rough articular strip for the posterior border of the vertical plate of the Palate, terminating below at the divergence of the two plates. Posteriorly the united plates are grooved above and bifurcated below. Just above the groove, more on the posterior border of the greater wing than on the pterygoid process, is the posterior opening of the Vidian canal. Below this the posterior border of the inner plate shows an oblong, shallow fossa, the scaphoid, while internal to this is the opening of a small foramen, the pterygo-palatine. Below and external to the scaphoid fossa is the pterygoid fossa, formed by the junction of the two plates in the separated bone but filled in below in the skull by the tuberosity of the palate. The pterysoid fossa is deeper and broader at its upper portion. The inner plate is longer and narrower than the outer. Its internal surface is smooth and forms theouter boundary of the posterior nares, its outer. smooth and concave, forms the inner boundary of the pterygoid fossa. The anterior border, blended with the outer plate, above, presents below a serrated margin for a groove on the palate. Its posterior border is thin and sharp and terminates below in a thin curved plate of bone, the hamular process, which projects below the level of the outer plate, and is curved outward and backward. The outer plate, broader and more massive than the inner, presents an outer 
face which is concave and rough to form the inner wall of the zygomatic fossa, an internal face, rough and irregularly concave, which forms the outer wall of the pterygoid fossa; an anterior border serrated below for the groove on the Palate, and a posterior border which is thin, sharp and irregular, sloping obliquely downward and backward.

\section{The Ethmoid.}

The Ethmoid lies in the middle of the anterior fossa of the sku1l. It consists of a body and two lateral masses. A portion of the body can be seen by looking into the anterior fossa of the skull, and part of the lateral masses by looking into the orbital cavity; but the larger portion of the bone is concealed by surrounding bones.

The Superior Surface of the body is called the Horizontal or Cribriform Plate; and is perforated by numerous small foramina. These foramina are arranged in three rows, of which the internal and external are fairly distinct, while the middle row is irregular and indistinct. The anterior foramen of the internal row is a narrow, slit-like aperture for the transmission of a nerve. The cribriform plate is a depressed surface, concave from side to side and marked on the mid-line by a projection, called the crista galli (L. A cock's comb). This projection commences near the posterior end of the bone; and rapidly rising in height, terminates near the anterior end of the bone in a blunt, rounded point. Its posterior border is narrow and sharp. Its lateral apects, smooth and slightly concave at the lower portion, are marked above by a bulging projection, which varies greatly in size in different bones. Projecting from the front of the crista galli are two small processes, one towards either side, called the alar processes (L. Ala, a wing).

At each side of the base of the crista Galli is a small fissure called the nasal groove, or slit. Posteriorly the superior surface presents a deep notch, the sphenoidal fissure, for the reception of the ethmoidal spine of the sphenoid bone.

Laterally this surface articulates with the orbital plate of the frontal bone; anteriorly with the frontal bone by the two 
alar processes which complete, posteriorly, an opening called the foramen coecum.

From the lower surface of the cribriform plate there passes downward, on the middle line, a thin sheet of bone called the perpendicular lamella, which aids in forming the septum of the nose, articulating in front with the nasal spine of the frontal and with the nasal bones; posteriorly it articulates above with the beak of the sphenoid, below with the vomer; inferiorly it gives attachment to the cartilage of the septum. Its faces are grooved.

The lateral mass lies to the side of the perpendicular lamella, with a slight interval between; it presents an outer, inner and superior face. The outer surface forms part of the inner wall of the orbit; it is smooth, flat and quadrilateral and is called the os planum; its upper part is marked by tivo grooves, which are the anterior and posterior ethmoidal foramina. The internal surface is convex, rough and fissured behind; this fissure is called the superior meatus of the nose; above this is a curved portion of the bone called the superior turbinated bone; below the superior meatus is another portion called the middle turbinated bone, which is the upper limit of the middle meatus of the nose. The superior surface of the lateral mass presents numerous irregular openings into the cells, of which this part of the bone is made up; these are closed in by the overlapping horizontal plate. Projecting downward and backward from the lower edge of the lateral mass is a thin plate of bone called the unciform process, which articulates with the ethmoidal process of the inferior turbinated and helps to close the opening of the antrum maxillare. From the posterior extremity of the lateral mass there projects backward a triangular, curled, pointed process, which extends into the sphenoidal cell of that side, and, as puberty approaches, becomes detached from the ethmoid and ossified to the sphenoid; it is called the sphenoidal spongy bone, or pyramid of Wistar. The cells, of which the lateral mass consists, are separated by a transverse septum into the two sets, the anterior and posterior ethmoidal cells; the anterior communicate with the middle meatus by a 
tortuous canal called the infundibulum, with which the frontal sinusalso communicates. The infundibulum opens into the upper front part of the middle meatus by a large orifice. At the upper back part of the superior meatus is an orifice which leads into the posterior ethmoidal cells.

\section{The Nasal Bones.}

The Nasal Bones are tivo somewhat flat bones, forming the bridge of the nose, along the centre of which they articulate with each other. They are somewhat quadrilateral; and present for examination two surfaces, anterior and posterior, two borders, internal and external, and two extremities, superior and inferior. The anterior face which looks forward and slightly outward is concave from above downward; and slightly convex from side to side, the anterior surface being a little roughened and marked by nutrient foramina. The posterior surface, which looks inward as well as backward, is convex from above downward; and marked near the internal border by a vertical groove. The superior extremity is thicker and more massive than the inferior; and is roughened and serrated for articulation with the nasal notch of the frontal bone. The inferior extremity is broader and thinner than the superior; and slopes obliquely from within outward and downward, so that when the two bones are articulated, a pearshaped notch is found between them, its apex upward and its. base downward. The internal border is shorter than the external; and presents when articulated with its fellow a welldefined crest for articulation with the nasal spine of the frontal and the perpendicular lamella of the ethmoid bone. The external border, longer than the internal, is serrated, thin, and sharp for articulation with the nasal process of the superior maxillary.

\section{Lachrymal Bone.}

The lachrymal bones are a pair of small bones, one being found on the inner wall of each orbit in front of the os planum. The bone remotely resembles a finger nail and hence acquires its synonym of unguis. It presents two faces, two extremities and two borders. The external face is divided into an anterior 
and a posterior portion, by a vertical ridge, which terminates inferiorly in a hook-like process called hamular. The posterior and larger portion is part of the inner wall of the orbit and is flat and smooth. The anterior portion is a vertical groove, called the lachrymal.

The internal face is rough for articulation with the ethmoid bone, and presents a vertical groove corresponding to the vertical ridge on the opposite side; the portion of the surface in front of this groove enters into the formation of the middle meatus.

The upper extremity articulates with the internal angular process of the frontal bone.

The lower extremity is divided into two parts, the posterior articulating with the orbital plate of the superior maxillary, the anterior projecting farther downward and articulating with the lachrymal process of the inferior turbinated.

The anterior border articulates with the nasal process of the superior maxillary bone.

The posterior border articulates with the os planum.

\section{Inferior Turbinated Bone.}

The inferior turbinated, or inferior spongy bone, is found on the lower part of the outer wall of the nasal fossa; the middle and superior turbinated bones are merely parts of the ethmoid. The bone is divisible into two extremities, two faces and two borders.

The anterior extremity is much the larger and articulates with the inferior turbinated crest of the superior maxillary.

The pesterior extremity is slender and pointed aud articulates with the inferior turbinated crest of the palate bone.

The internal face is convex and marked by apertures and grooves.

The external face is concave and enters into the inferior meatus of the nose.

The inferior border is rounded and free, marking the limit between the middle and inferior meatus.

The superior border articulates from before backward with the superior maxillary, the lachrymal, the ethmoid and the 
palate bones. It presents three processes, lachrymal, ethmoid and maxillary. The lachrymal process is directed upward to articulate with the lower extremity of the lachrymal bone, and with the nasal process of the superior maxillary, aiding to form the lachrymal canal.

The maxillary process is larger than the other two and is a curved plate of bone directed downward and outward from the base of the ethmoidal process and articulates with the superior maxilary by hooking around the orifice of the antrum. The ethmoidal process is behind the lachrymal, and is directed upward to articulate with the unciform process of the ethmoid.

\section{Vomer.}

The vomer is a single bone found separating the nostrils behind and below, forming part of the septum of the nose; the upper part of the septum is the perpendicular lamella of the ethmoid; the anterior part is a plate of cartilage. It presents two faces and four borders.

The faces are lateral and each forms part of the inner wall of a nostril, the bone usually bulging toward one or the other. They are marked by shallow furrows and by the naso-palatine groove, which is directed obliquely downward and forward on each face.

The superior border presents an antero-posterior groove which receives the rostrum of the sphenoid; the edges of the groove are projected outward into lips, or alæ, each of which lies in the groove above the vaginal process of the sphenoid.

The inferior border is received between the averted edges of the palate processes of the superior maxillary bones and of the horizontal plates of the palate bones.

The anterior border presents a longitudinal fissure which receives superiorly the perpendicular lamella of the ethmoid, inferiorly the triangular cartilage of the septum; the lower part is occasionally not fissured but rough.

The posterior border is concave and free, terminating, posteriorly, the septum of the nose. 


\section{Malar Bone.}

The malar bone forms the prominence of the cheek, is somewhat quadrangular and presents for examination four processes and a body with two surfaces and four borders.

The anterior, or external face is convex and has several foramina opening on it, called malar.

The posterior, or internal face is smooth and concave, entering into the temporal fossa above and the rygomatic below.

The frontal process projects upward to articulate with the external angular process of the frontal bone. The orbital process is a smooth projecting lip of bone curving downward from the frontal process, and projecting inward, forming a concavity which enters into the outer wall and floor of the orbit. Its lower surface forms part of the temporal fossa. Superiorly it articulates with the frontal bone. Posteriorly it articulates with the sphenoid bone; internal to which it articulates with the orbital plate of the superior maxillary bone; between these two articulations there is often seen the narrow, rounded, non-articular anterior extremity of the spheno-maxillary fissure. This process presents the openings into one or more small canals, temporo-malar, which extend to the anterior surface of the bone and some usually to the posterior surface.

The maxillary process is rough and triangular, articulating with the superior maxilla.

The zygomatic process extends backwards, is long and narrow and articulates with the zygomatic process of the temporal.

The superior border is concave, smooth and rounded, and forms part of the margin of the orbit.

The inferior border is straight and continuous with the lower edge of the zygoma.

The anterior border is straight and rough to articulate with the superior maxilla.

The posterior border is sinuous and continuous with the temporal ridge above, and below with the upper edge of the zygoma. 


\section{Superior Maxillary.}

The superior maxillary bones form the upper jaw and are, with the exception of the inferior maxillary, the largest bones of the face. Each bone articulates with the frontal and ethmoid, and all the bones of the face except the inferior maxillary. The bone is divisible into a body and four processes.

The body is irregularly quadrilateral, is hollowed out into a cavity called the antrum of Highmore, or antrum maxillare, and presents four faces--anterior, superior, posterior, internal.

The anterior or facial surface is somewhat concave and presents about its centre a depression, called the canine fossa, which is limited internally by a vertical ridge, the canine ridge; on the in ner side of the canine ridge is another depression, the incisive fossa; just above the canine fossa is a foramen, the infra-orbital. This surface is limited internally by the thin concave edge of the nasal fossa: below by the alveolar process; externally by the malar process and a ridge descending from it : superiorly by the margin of the orbit.

The posterior or zygomatic surface is chiefly occupied by a rough bulge, the tuberosity of the superior maxillary bone; it presents numerous small foramina, and at its lower part a rough oval surface; above and internal to this is a smooth spiral groove, which, with a similar groove on the palate bone, forms the posterior palatine canal. The upper limit of this surface, separating it from the orbital face, is a smooth rounded border, on which is a notch, the commencement of the infra orbital canal. The malar process separates this face from the anterior; below it is limited by the alveolar process.

The superior or orbital surface forms the floor of the orbit. It is formed of a thin triangular plate of bone, is smooth, sloping downward and forward, and marked from behind forward, by a groove, the infra-orbital canal, which disappears in the bone to terminate at the infra-orbital foramen on the anterior face. This face is bounded internally by a roughedge for articulation from behind forward, with the palate, ethmoid and lachrymal bones; anteriorly it is limited by the lower 
margin of the orbit, internally, and externally, by a rough border for articulation with the malar bone; externally it is separated from the posterior surface by a rounded border on which begins the infra-orbital canal by a notch; this border forming, in the articulated skull, the lower margin of a fissure, the spheno-maxillary.

The internal or nasal surface aids in forming the outer wall of the nose, and presents a large irregular aperture leading into the antrum of Highmore; this aperture is much reduced by articulations with neighboring bones, palate, ethmoid, lachrymal and inferior turbinated. The antrum is indistinctly triangular; in it are seen numerous vertical grooves and on its floor several conical projections which mark the position of molar teeth. In front of the opening into the antrum is a deep vertical groove, the sulcus lachrymalis. This face is limited above by the nasal process, in front, and behind this by a rough cellular edge for articulation with the lachrymal and ethmoid bones; inferiorly is the horizontal palate process, which may be said to divide it into two portions, the part below terminating in the alveolar process and the part above forming the inferior meatus of the nose. The posterior borber is rough for articulation with the palate bone. The anterior edge is sharp and concave and forms the margin of the anterior nares.

The four processes are nasal, malar, palate and alieolar. The nasal process is a long, thin, triangular projection forming a part of the wall of the nose and presenting threc borders and two surfaces.

The anterior border is convex, thin and serrated for articulation with the nasal bone.

The posterior border is thick and grooved, the posterior margin of the process is rough for articulation with the lacrymal bone; the anterior margin is rounded, continued into the margin of the orbit, and presents below a small tubercle. This groove, in the articulated skull, is formed into a canal for lodging the nasal duct, and is nearly vertical, being slightly oblique backward and outward. The upper border is blunt 
and serrated for articulation with the frontal bone, its internal angular process.

The external face is concave and marked by small foramina.

The internal face is crossed from behind forward by two rough ridges, crista turbinalis inferior and superior, the former articulating with the inferior turbinated and the latter with the ethmoid. Above the superior turbinated crest the surface is rough for articulation with the ethmoid; between the two crests it is smooth and concave, entering into the middle meatus of the nose.

The malar process forms the upper part of the boundary between the anterior and posterior faces. It is concave both in front and behind, triangular in outline and its summit is rough for articulation with the malar bone.

The palate process projects inward from the lower part of the internal surface, forming a portion of the floor of the nose and the roof of the mouth. It does not extend as far back as the body of the bone, but may be said to be deficient behind. Its upper surface is transversely concave and smooth. In front is a foramen which leads in to the anterior palatine canal, which appears on the inner border of the process as a groove. Its lower surface is also concave, but rough, marked by numerous shallow depressions and by a groove externally, which runs from behind forward. The inner border is rough to articulate with its fellow on the opposite side ; it is thicker anteriorly than posteriorly, and its upper edge is raised into a ridge, or crest, which is slightly averted, so as to produce, when the bone is articulated, a narrow groove, for the reception of the vomer. Towards the front the inner border, in its lower part, presents a groove, which sinks into the bone above to communicate with a foramen on the upper surface, the anterior palatine. In front the inner border terminates in a slender, pointed process, which, united with a similar one on the opposite bone, forms the anterior nasal spine.

The anterior border of the process is the thin concave margin of the nose, the nasal notch.

The posterior border is straight and serrated for articulation with the horizontal plate of the palate bone. 
The aveolar trocess projects downwards from the lower, outer part of the bone, and forms about a fourth of the circumference of a circle. It is marked by sockets for eight teeth, with intervening septa.

\section{Palate Bone.}

The palate bone enters into the formation of the orbits, the nasal fossa and the roof of the mouth. It is divided into hor $i$ zontal and íerlical, or perpendicular portions, or plates.

The horizontal portion projects inward, at about a right angle, from the lower limit of the vertical plate, and presents trvo surfaces and three borders. The superior or nasal face is smooth and concave transversely, forming the back part of the floor of the nose. The inferior face is also slightly concave transversely and rough, forming the posterior part of the hard palate. A transverse ridge crosses it posteriorly. The anterior border is serrated for articulation with the palate process of the superior maxillary.

The fostcrior border is smooth, concave and free, having its inner extremity prolonged backwards by a slender, sharp process, which with a similar projection on the opposite bone, forms the posterior nasal, or palate spine. The internal border is thick, rough and serrated for articulation with its fellow; the upper edge of this border is raised and produces, when articulated, a slight groove for the reception of the vomer.

The verlical plate is irregularly quadrangular and presents two faces, internal and external, and three borders, superior, anterior and posterior. The internal surface presents two transverse ridges, the superior and inferior turbinated crests; the inferior to articulate with the inferior turbinated bone, and the superior with the middle turbinated bone of the ethmoid. This surface. below the inferior turbinated crest, is concave and forms the outer boundary of the inferior meatus of the nose; the surface between the two crests is also concave, and forms a part of the middle meatus; above the superior crest is a narrow groove.

The cxtcrnal surface is, to a great extent, rough for articulation with the internal face of the superior maxilla; but at

Des Anat-6 
its upper back part there is a smooth portion, which forms a part of the spheno-maxillary fossa. At the posterior part of this face is a vertical groove, converted into the posterior palatine canal by articulation with the tuberosity of the superior maxilla.

The superior border presents two processes, separated by a deep notch. The notch is called spheno-palatine, and is converted into a foramen of that name by the articulation of the sphenoid bone. The anterior process is known as the orbital: the posterior as the sphenoidal. The orbital process inclines outward as it ascends, is hollow and higher than the sphenoidal, being perched upon a thin plate of bone, its neck. It presents five faces-anterior, posterior, internal, external and superior-the first three being articular, the others nonarticular.

The anterior face articulates with the orbital surface of the superior maxillary.

The posterior face articulates with the sphenoid bone.

The internal face articulates with the ethmoid bone and usually presents the opening into the cellular cavity in the process; but this is sometimes on the posterior face.

The external face forms a part of the inner wall of the spheno-maxillary fossa.

The superior face forms the back part of the floor of the orbit.

The sphenoidal process is a thin plate of bone, inclining inward as it ascends, and presents three faces-superior, external and internal - and two borders - anterior and posterior.

The superior face articulates with the sphenoid bone and presents a groove, converted, by articulation, in to the pterygopalatine canal.

The external face partly enters into the formation of the spheno-maxillary fossa, and partly articulates with the pterygoid process of the sphenoid.

The internal face is free and forms part of the outer wall of the nasal fossa. 
The anterior border of the process is the posterior boundary of the spheno-palatine notch.

The postcrior border of the process articulates with the pterygoid process of the sphenoid bone.

The anterior border of the perpendicular plate is irregular, articulates with the superior maxillary bone and presents, at the intersection of the inferior turbinated crest, a thin process, the maxillary, which aids in closing the opening into the antrum maxillare.

The posterior border of the perpendicular plate is grooved and articulates with the pterygoid process of the sphenoid. At its lower part is a process, the pterygoid process, or tuberosity of the palate bone. It is triangular, is directed downward and backward and outward, and fits into the interval between the two plates of the pterygoid process of the sphenoid. Descending the middle of the tuberosity, posteriorly, is a smooth groove, which, when the bone is articulated, forms part of the pterygoid fossa; on each side of this groove is a rough groove, which articulates with the corresponding plate of the pterygoid process of the sphenoid. Externally the tuberosity is rough and articulates with the superior maxilla. The openings of numerous canals, the accessory posterior palatine canals, are seen on the tuberosity, and it is perforated vertically through its base by the posterior palatine canal.

\section{The Inferior Maxilla.}

The inferior maxilla, or lower jaw bone, is a symmetrical bone, derived from lateral halves, which blend in the middle line-each lateral half consisting of a vertical portion, the ramus, and a horizontal portion, which is one half of the body of the bone.

The Body is of a horse-shoe shape; and presents external and internal surfaces, superior and inferior borders. The surfaces do not look directly outward and inward; but the external inclines outward and forward, and the internal in ward and backward. The exlemal surface is convex from side to side, concave in some portions, from above downward.

In front, on the mid-line of the body, is a vertical ridge, 
the Symphysis Menti (L. Mentum, the chin), or Crista Mentalis, which as it descends spreads out so as to form a triangular projection, the mental process, which forms the prominence of the chin. External to this symphysis is a depression, called the Incisive Fossa; and just beyond this is a large and well-marked foramen, the Mental Foramen. Extending backward and upward from the mental foramen is a prominent ridge of the bone, which becomes continuous with the anterior border of the ramus, called the External Oblique Ridge.

The Internal surface is concave from before backward, irregular in outline from above downward. On the mid-line of the internal surface is a vertical groove corresponding to the symphysis, on either side of this groove are seen two tubercles, one inferior and one superior, both a little below the middle point of the groove; which are known as the Genial Tubercles, superior and inferior, sometimes called the Spince Mentales.

They are very irregular in formation : sometimes all four of them coalesce into a single roughened process; sometimes there are two flat ones on either side of the mid-line; and occasionally the four are distinctly seen. External to the genial tubercles is a slight depression, which in the fresh state lodges the sub-lingual gland. External to and below the tubercles, is a slighter depression, called the Digastric Fossa, serving for the attachment of the muscle of that name. Extending upward and backward from the digastric fossa, and lying nearly opposite to the external oblique ridge, is a prominent ridge of bone, called the Internal Oblique Ridge, or Mylo-Hyoidean Ridge. Below this ridge and parallel to it is a long groove, which is called the Submaxillary Fossa. The upper border of the bone is called the Alveolar Process (L. Dim. of Alveus, a cavity); and presents the depressions for sixteen teeth, these sockets being separated from one another by intervening bony septa. The lower, or basilar, border is slightly everted, strong rounded and smooth. It is the most prominent portion of the bone in the living man. Usu- 
ally about an inch in front of the angle of the jaw this border presents a slight groove, across which passes the facial artery.

The Ramus is a quadrilateral projection of bone, which extends nearly vertically upward, almost at right angles with the body of the bone. Its obliquity varies with the different periods of life. The ramus is divided for study into two surfaces, external and internal, and four borders, anterior, posterior, superior and inferior. The external surface is flat, is slightly roughened for muscular attachment; and presents no other mark of interest. The internal face, slightly concave from before backward, is marked about its centre by a very prominent projecting lip of bone, above which is seen the opening of a canal. Above this opening there is a slight groove. The foramen leads into a canal which extends through the bone, the Dental Canal, and the canal terminates at the mental foramen on the external surface of the body. It is so located as to pass in close contact with the roots of the teeth, and lodge the blood-vessels and nerves which supply the fangs of each one of the teeth of the lower jaw. The projection of bone which guards the foramen below and in front is called the Lingula. The anterior border is thicker than the posterior; and is marked by a nearly vertical groove, which is surmounted by two lips. Of these the external lip. more prominent and sharper than the internal, is the upward continuation of the external oblique ridge; while the inner lip is an abutment that rises from the termination of the alveolar process, and becomes nearly continuous witl the mylo-hyoid ridge on the inner face of the body. The posterior border is more rounded and thinner than the anterior, extending above into one of the proiections of the upper border, and below terminating at the angle of the jaw. The lower border of the ramus should not be described as a separate border, as it is merely a continuation of the basilar border of the body. Where it joins with the posterior border of the ramus the bone is twisted so as to be everted, and forms a prominent projection, which varies greatly with the individual on either side of the face. This angle of the jaw is 
roughened for muscular attachment. The superior border consists of two projections, an anterior and posterior with an intervening notch, the sigmoid. Of these projections the anterior is called the Coronoid Process, while the posterior is described as the Condyle of the Jaw, the notch between the two being called the Sigmoid. The Coronoid Process is thin, triangular in outline; presents an anterior border, which is continuous with the outer lip of the groove. on the anterior borde: of the ramus, a posterior thinner and sharper border, continuous with the sigmoid notch, and a blunt point, the extremity, which is received into the fibres of the Temporal muscle. The sigmoid notch is concave from before backward, its edge thin and sharp. Behind this comes the condyle, which consists of a convex, oval articular surface, mounted upon a constricted portion of the bone, called the "neck."

This condyle is oblique in its direction, its long diameter being from without in ward and back ward. It is articular further down behind than in front; and fits into the Glenoid cavity, which is found on the inferior surface of the squamous portion of the temporal bone. The neck of the condyle, constricted antero-posteriorly, extends laterally, so that the condyle seems to rest on two abutments, one from either side. In sront and to the inner side is found a deep depression beneath each condyle. As life advances the obliquity with which the ramus of the bone ascends increases, so that in extreme old age it nearly prolongs the direction of the body. This, however, is not due to a change in the direction of the bone; but to the loss of the teeth, and the consequent absorption of the alveolar process.

\section{The Articulated Skull.}

The articulated skull is produced by the union of the bones of the cranium and the bones of the face. A description of the articulations, known as sutures, will be found under the head of articulations in general.

The skull is divisible into five regions: superior, inferior, anterior and two lateral. 


\section{Superior Region.}

The superior region or vertex, is bounded in front by the frontal eminences, on each side by the temporal ridge, and behind by the posterior occipital protuberance and the superior curved lines of the occipital bone. It is formed by part of the frontal bone, most of the parietal bones and a part of the occipital bone. It is divided into a superior and an inferior aspect.

The superior surface is seen to be crossed transversely by the coronal suture, extending backward from the centre of which is the sagittal suture, which terminates posteriorly in the lambdoid suture. The parietal foramen may be seen on each side of the sagittal suture near its posterior extremity. On each side is seen the parietal eminence. This surface is markedly convex.

The inferior, or cerebral surface, is concave, and presents the following appearances: on the frontal bone, in the middle line, is seen the indistinct commencement of a groove, for the superior longitudinal sinus, which passes backward, growing broader and more distinct as it travels, first along the line of union of the parietal bones and then down the superior limb of the occipital cross to terminate at the anterior occipital protuberance; along the edges of this groove are seen several pits for lodging the Pacchionian bodies, and external to these digital pits for the convolutions of the brain; numerous arborescent arterial furrows are also seen.

\section{Lateral Region.}

The lateral region is subdivided into four portions-mastoid, temporal, zysomatic and spheno-maxillary.

\section{Mastoid Portion.}

The mastoid portion of the lateral region extends backward from the eminentia articularis, and in it are seen the following appearances: the mastoid foramen and process; the external auditory meatus; the auditory process, the glenoid fossa, which is bounded above by the posterior root of the zygoma, behind 
by the middle root and vaginal process, and in front by the anterior root of the zygoma. It is crossed transversely by the fissure of Glaser, the surface in front of the fissure being articular and that behind rough; where the roots of the zygoma meet is the tubercle.

\section{Temporal Portion.}

The temporal portion of the lateral region is also called the temporal fossa. Its upper and posterior limit is the temporal ridge; this ridge commences at the external angular process of the frontal bone and curving upward and backward, leaves the frontal and passing across the parietal bone, arches downward over the squamous portion of the temporal bone and terminates in the posterior root of the zygoma. In front of the temporal fossa is the external angular process of the frontal bone and the malar bone. Below, it terminates at the zygonia without and the pterygoid ridge within. Its constituents are furnished by the frontal bone, the malar bone, the greater wing of the sphenoid the parietal bone and the squamous portion of the temporal bone.

\section{Zygomatic Portion.}

The zygomatic portion of the lateral region is situated below the temporal portion and is bounded above by the lower surface of the greater wing of the sphenoid, the pterygoid ridge and the squamous portion of the temporal bone; in front by the posterior surface of the superior maxilla and by the malar; internally by the external pterygoid plate and pterygomaxillary fissure; externally, by the zygoma and the ramus of the inferior maxilla. In this region are seen two fissures, one horizontal, the spheno-maxillary, and one vertical, the pterygo-maxillary, the one being at right angle to the other. The spheno-maxillary fissure is seen by looking into the orbit; it is situated at the lower part of its outer wall posteriorly, between the greater wing of the sphenoid above and the orbital plate of the superior maxilla below, terminating, frequently, in the malar boneanteriorly; the posterior termination is at the point where the pterygo-maxillary fissure begins, which descends bet ween the tuberosity of the superior maxillary in front and the pterygoid process behind. 


\section{Spheno-Maxillary Fossa.}

At the point of junction of the two fissures is a small fossa, the size of the end of the little finger, called the spheno-maxillary fossa; it is thus formed: above is the flat surface just at the base of the pterygoid process anteriorly; behind is the pterygoid process, in front is the superior maxilla; internally is the perpendicular plate of the palate bone. There are five forcamina found in this fossa. Three of them are found on the upper postcrior wall, $i . c$., on the flat surface at the base of the pterygoid process; they are-1st, foramen rotundum ; 2nd, pterygoid, or Vidian; 3rd, pterygo-palatine; on the inner wall is seen the 4th, spheno-palatine; and 5 th, inferiorly, is seen the opening into the posterior palatine canal, with accessory posterior palatine canals.

Taking the lateral region of the skull as a whole, it may be seen, from the foregoing description, that it is triangular in outline and is bounded as follows; the base is represented by the sweep of the temporal ridge; the apex is at the angle of the inferior maxilla; the sides may be represented by two lines, meeting at the angle of the inferior maxilla, the one drawn from the external angle of the frontal bone and the other from the mastoid process. The bones entering into its formation are; the mastoid and squamous, and the base of the petrous portions of the temporal bone: part of the frontal bone; part of the greater wing of the sphenoid, part of the parietal, part of the malar, and the constituents of the spheno-maxillary fossa, as given above.

\section{The Inferior Region of the Skull.}

The Inferior Regrion, or Base, of the Skull, presents two surfaces, superior and inferior, or cerebral and basilar.

The Inferior. Surfuce is bounded in front by the alveolar process of the superior maxillary; and from the tuberosity of the superior maxilla it is limited by an imaginary line, which, extending by the styloid process of the temporal bone, passes back to the superior curved line of the occipital, and ends at 
the posterior occipital protuberance. [In describing the appearances found on this face of the skull it is much more accurate to begin with those on the mid-line of the body, which are single appearances, while the double appearances can be given later.] Commencing on the mid-line we find extending from before backward a foramen, called the Anterior Palatine. Behind this foramen is the suture between the two horizontal plates of the two superior maxillary bones, and behind this again the suture between the two horizontal plates of the two palate bones. This suture terminates in the posterior palatine spine, while above and behind this spine is the concave free border of the vomer. Behind this comes the pharyngeal ridge of the basilar process of the occipital, which leads to the foramen magnum, beyond which is found the vertical limb of the occipital cross, which terminates in the posterinr occipital protuberance. On either side the following appearances will be noted : in front, the rough inferior surface of the palatine process of the superior maxilla, behind which lies the suture between this process and the horizontal plate of the palate bones; behind this the nasal cavity, and on the outer side of the nasal cavity, the pterygoid process and pterygoid fossa of the sphenoid. Behind the pterygoid process is the middle lacerated foramen; external to that is the foramen ovale; external to this the foramen spinosum; external to that the spine of the sphenoid; and then the appearances seen on the inferior surface of the petrous portion of the temporal bone. The inferior surface of the petrous portion of the temporal exhibits as the most internal appearance a rough, quadrilateral projection of bone. Behind and external to this is the aperture of entrance for the carotid artery; external to and behind this a thin elongated projection of bone, called here the Vaginal Process. Internal to this is the foramen lacerum posterins; and in the interval between the posterior lacerated foramen and the carotid aperture of entrance is a thin sheet of bone marked by two minute apertures, the one for the transmission of Jacobson's and the other for the transmission of Aruold's nerve. The Vaginal process, as it passes upward and back- 
ward, divides to enclose the next appearance, which is the styloid process, while next to the styloid process is a foramen, the stylo-mastoid foramen. Still further behind is a narrow fissure between the vaginal and mastoid processes called the Auricular Fissure, while internal to the mastoid process is another and larger depression, known as the Digastric Fossa, and internal to that a depression called the Occipital Groorc.

We next encounter the appearances on the occipital boncs: viz., the condyles, with cond yloid fossa behind, and in the bottom of the fossa the openings of the posterior condyloid foramina, when these formina are present. In front on either side of the condyle is seen the anterior condyloid foramen. Behind these appearances is the roughened posterior surface of the occipital bone as far up as its superior curved line.

In describing the appearances on the base of the sliull, it is necessary to give not only those appearances, but their boundarics as well. Thus the two posterior nares are bounded, each internally by the posterior border of the vomer, externally by the inner surface of the internal plate of the pterygoid process, above by the expanded ala of the vomer, and external to this the vaginal process of the sphenoid. Below they are bounded by the posterior concave free border of the horizontal plate of the palate. The middle laccrated foramen is bounded internally by the basilar process of the occipital bone, externally by the apex of the petrous portion of the temporal; in front it is bounded by the posterior border of the upper surface of the greater wing of the sphenoid. In this foramen may be seen the opening of the Vidian canal, leading to the termination of that canal in the spheno-maxillary fossa. Posteriorly it is limited by the junction of the anterior border of the temporal with the basilar process of the occipital. To the outer side of the middle lacerated foramen can be distinctly seen the space between the squamous and petrous portions of the tenporal, in which are lodged the two canals, the upper for the tensor tympani, the lower for the Eustachian tube, separated from each other by a thin process of bone, called the Processus Cochleariformis (L. Cochleare, a spoon; forma, 
shape). The posterior lacerated foramen is bounded in front by the posterior borler of the petrous portion of the temporal, and behind by the junction of the basilar process with the inferior border of the occipital, these two bones presenting notches, which when articulated together form the Posterior Lacerated, or Jugular, Foramen.

\section{The Superior Surface of the Base of the Skull.}

The Superior Surface of the Base of the Skull presents in front the foramen caecum, behind this the crista galli, behind the crista galli the articulation between the ethmoidal spine of the sphenoid and the sphenoidal notch of the ethmoid, behind that a transverse ridge slightly formed bounding the optic groove in front. Next is the groove itself; and behind that is its posterior boundary, the olivary process. Behind the olivary process is a deep depression on the upper surface of the sphenoid, the Sella Turcica (L. A Turkish saddle), behind the sella turcica the Dorsum Ephippii (L. The back of a horsecloth), behind that the Clivus Blumenbachi (L. Clivus, a slope), leading to the grooved upper surface of the basilar process of the occipital bone. Behind the Clivus Blumenbachii is the foramen magmum, behind this the vertical limb of the occipital cross, and at the termination of the upper surface of the base of the skull the anterior occipital protuberance.

To describe the lateral appearances more intelligently it is necessary to divide this portion of the skull, which is so irregular in outline, into three fossæ. These are known as the anterior, middle and posterior; and they descend like steps, the anterior being the highest, the middle next, and the posterior the lowest. The anterior fossa of the skull is bounded in front by the junction of the horizontal and vertical portions of the frontal bone; behind, in the mid-line, by the optic ridge, on either side of which we have the posterior borders of the lesser wings of the sphenoid. Laterally this surface is limited by the vertical portion of the frontal bone. The middle fossa has for its anterior boundaries the posterior boundaries of the anterior fossa; viz., the orbital ridge in the centre and the posterior border of the lesser wings of the 
sphenoid laterally. Posteriorly it is bounded in the mid-line by the basilar suture, laterally by the upper border of the petrous portion of the temporal. The posterior fossa is bounded in front by the basilar suture, on either side by the upper border of the petrous portion of the temporal, behind in the mid-line by the anterior occipital protuberance, and on either side of that by the lateral limbs of the occipital cross. The bones entering into the formation of the anterior fossa are the orbital plates of the frontal, the cribriform plate and crista galli of the ethmoid, the upper surface of the lesser wings of the sphenoid, and the anterior one-third of the upper surface of the body of the sphenoid. Those which form the middle fossa are the posterior two-thirds of the upper surface of the body of the sphenoid, the whole upper surface of the greater wing of the sphenoid, the anterior face of the petrous portion of the temporal, and the internal face of the squamous portion of the temporal. Those bones which form the posterior fossa are the posterior surface of the petrous portion of the temporal, the pnstero-inferior angle of the parietal, and all of the anterior surface of the occipital which lies below the transverse groove for the lateral sinuses upon that bone. The appearances in the anterior fossa are the foramen caecum, the crista galli, the ethmoidal spine of the sphenoid and its articulation with the sphenoidal notch of the ethmoid and the optic ridge forming one of its boundaries. On the mid-line of the frontal bone is seen the beginning of the longitudinal sinus. The lateral appearances are the grooves which are found upon the upper surface of the cribriform plate of the ethmoid, including the slits for the nasal nerves. Still further external is the irregular upper surface of the orbital process of the frontal, three rows of foranina on the upper surface of the ethmoid, and the internal openings of the anterior and posterior ethmoidal foramina. In the middle fossa are seen the optic groove, the olivary process, and the sella turcica, with the dorsum ephippii upon the mid-line extending to the basilar suture, forming the line of separation between the middle and the posterior fossie. Laterally are seen in front the optic 
foramina, the anterior clinoid process, behind and external to this along the olivary ridge the middle clinoid process, external to and slightly in front of this the anterior lacerated foramen, behind and external to that the foramen, rotundum, still further behind and still further external the foramen ovale, and behind and external to that the foramen spinosum, while internal to this external row of foramina is the italicf-shaped groove for the carotid sinus, on the lateral aspect of the body of the sphenoid.

Behind this is the upper opening of the middle lacerated foramen, external to which are the appearances seen on the anterior face of the petrous portion of the temporal, presenting from within outward the digital pit, the groove leading to the hiatus Fallopii, the petrosal groove, the bulge corresponding to the superior semi-circular canal, and the depression which corresponds to the tympanum and the orifice of the carotid canal. The posterior fossa presents the inclined plane formed by the grooved upper surface of the occipital, the foramen magnum, and the lower vertical limb of the occipital cross. Laterally are seen the appearances on the posterior surface of the petrous portion of the temporal; viz, the internal auditory meatus; the opening for the aqueduct of the vestibule, and occasionally a small depression for lodging a process of the dura mater, the posterior border of the petrous portion of the temporal, aiding in the formation of the jugular foramen, marked by the triangular projection of bone which divides this foramen into two portions. External to this is the groove which enters into the formation of the lateral sinus; and which commences at the jugular foramen on the jugular process of the occipital bone, leaves that for the internal face of the mastoid portion of the temporal, leaves that bone to appear momentarily on the postero-inferior angle of the parietal, and then re-appears upon the occipital bone, where it forms the superior boundary of the posterior fossa and terminates in the torcular Herophili, to the side of the anterior occipital protuberance. In the foramen magnum can be seen the posterior openings into the condyloid foramina. Below the lateral limbs of the occipital cross are the fossæ which lodge the cerebellum. 


\section{The Anterior Region of the Skull.}

The Anterior Region of the Skull, or Face, is bounded above by the superciliary ridges, below by the basilar border of the inferior maxilla, and laterally by a line drawn from the external angular process of the frontal bone through the angle of the inferior maxilla. It presents for examination five carities: two orbital, two nasal, and the oral.

The Oral, or Buccal Cavity, has been already described with the base of the skull and with the inferior maxilla itself. In the recent state other appearances than those named should be mentioned; but these are not seen in the dry preparation.

The Nasal Carity, presenting its anterior aperture on the face, is bounded above by the lower end of the two nasal bones, on either side by the anterior border of the nasal process of the superior maxilla, below by the concave sharp free border of the horizontal or palate process of the superior maxilla. Internally each cavity is bounded by a triangular aperture, which in the recent subject is filled by a cartilage of similar shape, but which in the prepared skeleton shows only the anterior border of the vomer and the anterior border of the perpendicular lamella of the ethmoid. These cavities, when examined from in front, exhibit two shelf-like projections, the inferior and middle turbinated bones. The inferior turbinated bone is a separate bone, while the middle is a projection from the lateral mass of the ethmoid. Between the inferior turbinated, which curls over in the cavity, and the upper surface of the palate process of the superior maxilla and the horizontal plate of the palate bone is a narrow long opening, leading to the posterior nares, called the inferior mealus of the nose. Between the inferior and middle turbinated bones is a second canal of the same shape and character, though smaller in size, called the Niddle Meatus of the Nose. The superior mealus. an opening which lies between the superior and the middle turbinated bones, is smaller in size and more oblique in direction than the inferior and middle. It is bounded above by the superior turbinated and below by the inferior turbinaled bone, 
both of them portions of the lateral miss of the ethmoid; and of course belongs more especially to the ethmoid bone than to the cavity of the nose. The middle meatus communicates anteriorly by a tortuous canal, called the Infundibulum, with the anterior ethmoidal cells, and with the sinuses of the frontal bone. It also opens into the antrum-maxillare. Posteriorly the superior meatus receives by the spheno-palatine foramen a communication with the spheno-maxillary fossa, while indirectly it extends through the pyramid of Wister, or sphenoidal turbinated bone, into the posterior ethmoidal sinuses, and occasionally into the body of the sphenoid. The nasal cavities are more completely described among the soft tissues.

The Orbital Cavities. Each orbit is an irregular four-sided pyramid, having of course four surfaces and four angles. The apex of the pyramid is directed backward and inward and the base forward and outward, consequently a line drawn through the centre of each cavity would meet that of the opposite side over the middle of the sella turcica, passing through the optic foramina. The sides of these pyramidal cavities are superior, inferior, internal and external. The angles are supero-internal, supero-external, infero-internal and infero-external. These lines are formed by the articulations of the various bones which enter into the formation of the cavities. The supero-internal line is formed from before backward by the articulation of the internal angular process of the frontal bone, and the upper extremity of the lachrymal; behind this by the superior border of the os planum of the ethmoid and the margins of the rectangular ethmoidal notch on the orbital plate of the frontal; behind that the superior surface of the orbital process of the palate bone articulating with the sphenoid. The supero-external angle is formed by an irregular suture, commencing as an articulation between the external angular process of the frontal and the frontal process of the malar. Behind this is the orbital process of the malar still articulating with the frontal, and behind that the superior border of the anterior surface of the greater wing of the sphenoid, articulating with the orbital process of the frontal. The inferointernal anole is formed by the articulation of the lower ex- 
tremity of the lachrymal with the inner border of the orbital face of the superior maxilla; behind this the lower border of the os planum of the ethmoid with the same face of the superior maxilla; and behind that the maxillary surface of the orbital process of the palate with the angle formed by the junction of the internal and superior surfaces of the superior maxilla.

The infero-external angle is formed by the suture between the maxillary process of the malar and the malar process of the superior maxilla, and behind these by the spheno-maxillary fissure. The superior wall is formed entirely by the inferior surface of the orbital plate of the frontal. The cxtcrnal wall is formed jointly by the internal face of the orbital process of the malar, and the anterior surface of the greater wing of the sphenoid. The inferior face is formed mainly by the orbital, or superior, surface of the superior maxilla; but towards its outer side the grooved portion of the orbital process of the malar enters into its formation. The most posterior portion of the inferior face is formed partly by the orbital process of the palate. The internal face is formed from before backward by the outer surface of the lachrymal, the outer face of the os planum of the ethmoid, and behind that by the orbital face of the orbital process of the palate bone. Entering into this cavity are two foramina, the anterior and posterior cthmoidal, which establish a means of communication between the anterior fossa of the sliull and the cavity of the orbit. Posteriorly, directly at its apex, enters the foramen ofticum, and below this is seen the anterior lacerated foramen, formed between the inferior border of the lesser wing of the sphenoid and the posterior border of the outer surface of the greater wing. This foramen is triangular in outline, its apex directed upward and outward, and its base downward and inward, and communicates at an acute angle with a fissue found at the infero-external angle, called the spheno-maxillary. This fissure is bounded above by the lower border of the anterior face of the greater wing of the sphenoid, interially by the postero-external border of the orbitil face of the superior maxilla. Anteriorly it

Des Anat-7 
is usually bounded by the non-articular portion of the posterior border of the orbital process of the malar; but occasionally the border is articular, when the malar does not enter into the formation of the foramen. On the external wall may be seen a vertical suture extending between the posterior border of the orbital process of the malar and the external border of the anterior face of the sphenoid. Running through this wall are two minute foramina, transmitting branches of the fifth nerve, called the tempero-malar foramina. On the floor of the orbit, near its posterior extremity, is seen a slight groove, which about the middle of the floor is converted in to a foramen by plunging beneath the orbital surface of the superior maxilla. This canal continues its course until it passes beneath the infraorbital margin, and terminates on the anterior surface of the face. It is known as the infraorbital groove, infraorbital canal and iufraorbital foramen in the three respective places. On the roof of the orbit is seen near the internal angular process of the frontal bone a slight projection or sometimes a slight depression, the Fovea Trochlearis. External to this is an aperture which passes through above the margin of the orbit, and is known as the supraorbital foramen. External to this and just behind the external angular process is the most depressed portion of the orbital cavity, forming a fossa in which is lodged the lachrymal gland, the fossa being known as the Fossa Lachrymalis. Below the orbit is the roughened projection of the malar process of the superior maxilla, while external to it are seen the external openings of the tempero-malar foramina. Below the inferior orbital foramen is a depression, called the Incisive Fossa. Above the orbits are seen the supraorbital ridges, and in the mid-line the nasal prominence, or glabella. Below are the roughened surfaces of bone for the attachment of the various muscles of expression.

\section{The Nasal Fossae.}

The nasal fossæ are two irregular cavities situated in the face and extending from the anterior to the posterior nares. The septum between the two is formed, above, by the perpendicular lamella of the ethmoid bone, in its lower posterior part, 
by the vomer and the angular interval left between the two, in front, is filled in by a triangular plate of fibro-cartilage, called the cartilage of the septum. Each fossa presents for examination four walls. The inner wall is formed by the septum. The outer wall is formed by the superior maxilla, the inferior turbinated, the lachrymal, the ethmoid, the palate and the internal pterygoid plate of the sphenoid. The roof is formed by the nasal, the frontal, the ethmoid and the body of the sphenoid. The floor is formed in front, by the palate process of the superior maxilla and completed behind by the horizontal plate of the palate bone. The outer wall of the nasal fossa is very uneven; a considerable bulge in ward is produced, throughout its whole length, by the inferior turbinated bone, and the space between this bone and the floor is called the inferior meatus. Above the inferior is the middle turbinated bone, a part of the lateral mass of the ethmoid, and the space between these two is known as the middle meatus. In the upper part of the inner face of the lateral mass of the ethmoid, is a fissure called the superior meatus, situated between the superior and middle turbinated bones of the ethmoid. Opening into each fossa are eight apertures. Into the superior meatus there are three openings: 1st, the opening of the sphenoidal cells; $2 \mathrm{~d}$, the opening from the posterior ethmoidal cells; $3 \mathrm{~d}$, the sphenopalatine foramen, communicating with the spheno-maxillary fossa. In the middle meatus there are also three openings: 1st, the opening into the antrum maxillare; $2 \mathrm{~d}$, the opening into the anterior cthmoidal cells; $3 \mathrm{~d}$, the opening from the frontal sinus; the last two communicating with the upper front part of the meatus through the infundibulum. Into the inferior meatus there are two openings; 1st, the nasal duct, on its outer wall, and $2 \mathrm{~d}$, the anterior palatine foramen, on the floor. Just prosterior to the outer wall of the inferior meatus is the opening of the Eustachian tube, in fresh subject.

\section{Articulation of the Bones of the Skull-0ccipital Bone.}

The occipital bone articulates by its two condyles with the Atlas; by its superior border with the tzwo parictal bones, forming the lambdoid suture. by the outer half of each inferior 
border with the posterior border of the mastoid portion of the temporal bone, and by the inner half with the posterior border of the petrous portion: by the basilar process with the posterior surface of the body of the sphenoid bone, forming the basilar suture.

\section{Parietal Bone.}

The parietal bone articulates by its upper border with its fellow, forming the sagittal suture; by its lower border with the upper part of the semicircular border of the squamous portion of the temporal bone, forming the squamous suture; by its posterior-border with the upper border of the occipital; by its anterior border, with one-half of the semicircular border of the frontal, forming one-half of the coronal suture; by its anterior inferior angle, with the frontal triangle of the sphenoid, $i$. e., the upper border of the outer surface of the greater wing of the sphenoid; by its posterior inferior angle, with the upper border of the mastoid portion of the temporal bone.

\section{Frontal Bone.}

The frontal bone articulates by its superior, or semicircular border with the anterior border of the two parietal bones; by the posterior border of each orbital plate with the lesser wing of the sphenoid; by the triangular surface $i$. e., the sphenoidal triangle of the frontal, at the junction of the straight and semicircular borders, with the upper border of the outer surface of the greater wing of the sphenoid; by the circumference of the ethmoinal fissure with the lateral and anterior borders of the cribriform plate of the ethmoid, and with the upper surface of the lateral mass of the ethmoid; by the nasal notch with the nasal bones and the nasal process of the superior maxillary; by the nasal spine with the crest of the nasal bones, in front, and with the upper part of the anterior border of the perpendicular lamella of the ethmoid, behind; by the internal angular process with the lachrymal bone; by the external angular process with the frontal process of the malar bone, and by the part just behind this with the orbital process of the malar. 


\section{Temporal Bone.}

\section{Squamous Portion.}

The squamous portion articulates by the upper part of its semicircular border with the lower border of the parietal bone; by the front part of the same border with the posterior border of the outer surface of the greater wing of the sphenoid ; by the lower part of the same border with the outer part of the posterior border of the upper surface of the greater wing of the sphenoid: by the anterior part of the glenoid fossa with the condyle of the inferior maxilla; by the extremity of the zygomatic process with the zygomatic process of the malar bone.

\section{Mastoid Portion.}

By its upper border with the posterior inferior angle of the parietal; by its posterior border with the outer half of the lower border of the occipital bone.

\section{Petrous Portion.}

The petrous portion articulates by its posterior border with the inner half of the lower border of the occipital bone; by its anterior border with the middle part of the posterior border of the upper surface of the greater wing of the sphenoid.

\section{Sphenoid Bone.}

Body: By the posterior surface of the body with the basilar process of the occipital; by the cthmoidal spine with the sphenoidal notch of the cribriform plate of the ethmoid; by the crest with the upper part of the posterior border of the perpendicular lamella of the ethmoid; by the surface on each side of the crest with the posterior extremity of the lateral mass of the ethmoid, throngh the medium of the pyramid of Wistar, by which it also articulates with the posterior surface of the orbital and upper surface of the sphenoidal process of the palate bone; by the rostrum and vaginal prrcesses with the upper border of the vomer.

Grealer Wing. By the posterior border of its upper surface with a portion of the anterior border of the petrous por- 
tion of the temporal; by the external border of the superior surface (or the posterior border of the external surface) with the semi-circular border of the squamous portion of the temporal; by the superior border of the external face with the anterior inferior angle of the parietal, and with the outer part of the sphenoidal triangle of the frontal; by the anterior border of the outer face with the posterior border of the orbital process of the malar; by the outer part of the upper border of the orbital face with the straight border of the frontal bone; by the frontal triangle lying just internal to the upper border of the outer surface with the sphenoidal triangle at the junction of the vertical and horizontal plates of the frontal.

Lesser Wing. By its anterior border with the posterior border of the orbital plate of the frontal.

Pterygroid process. By its anterior border with the posterior border of the perpendicular plate of the palate; by the triangnlar interval between the lower part of its two plates with the tuberosity of the palate; by the inner side of its base with the posterior part of the outer surface, and posterior border of the sphenoidal process of the palate.

\section{Ethmoid Bone.}

Horizontal Plate: By its lateral and anterior borders with the circumference of the ethmoidal fissure of the frontal. By crista galli with anterior border of ethmoidal notch forming foramen coecum. By the spheniod notch with the ethmoidal spine of the sphenoid.

Perpendicular Lamella: By the upper part of its posterior border with the crest of the sphenoid; by the lower part of its posterior border with the upper part of the anterior border of the vomer; by the upper part of its anterior border with the nasal spine of the froutal; by the lower part of its anterior border with the crest of the nasal bones.

Lateral Mass: By its upper surface it is continuous with the lower surface of the horizontal plate, and the half cells on the orbital plate of the frontal, by its posterior extremity with the anterior face of the body of the sphenoid bone, through the medium of the pyramid of Wistar; by its anterior extrem- 
ity with the inner face of the lachrymal bone and with the inner face of the nasal process of the superior maxillary; by the upper border of the os flamm with the horizontal plate, by the lower border of the os planum with the inner border of the orbital face of the superior maxillary bone, and behind that with the orbital process of the palate bone; by the poslerior border of the os flamm with the front of the body of the sphenoid; by the anterior border of the os plamm with the posterior border of the lachrymal bone; by the part below the os flamm with the inner face of the superior maxillary bone; by the unciform process with the ethmoidal process of the inferior turbinated bone; by the anterior cxtremity of the middle turbinated bone with the superior turbinated crest of the nasal process of the superior maxillary; by the posterior cxtremity of the middle turbinated bone with the superior turbinated crest of the vertical plate of the palate bone.

\section{Nasal Bone.}

By its upper border with the nasal notch of the frontal bone; by its ouler border with the nasal process of the superior maxillary; by its imer border with its fellow; by the crest of the two bones, above, with the nasal spine of the frontal; below, with the lower part of the anterior border of the perpendicular lamella of the ethmoid.

\section{Superior Mazillary.}

The superior maxillary bone articulates by its body and processes.

Body: By the lower part of its posterior border with the front of the tuberosity of the palate bone; by the intemal border of its orbital surface, from before backwards, with the lower border of the lachrymal bone, the os planum of the ethmoid and the anterior face of the orbital process of the palate bone. Antero-externally the orbital face articulates with the lower border of the orbital process of the malar; by its internal face the body articulates, by the part posterior to the aperture of the sinus with the anterior border, the maxillary process and most of the outer surface of the vertical 
plate of the palate; above the aperture with the outer surface of the lateral mass of the ethmoid, below the os planum; below the aperture with the maxillary process of the inferior turbinated bone; in front of the aperture with the projection on the anterior border of the lachrymal and the lachrymal process of the inferior turbinated; by the inferior mubinated crest with the anterior extremity of the inferior turbinated.

Malar Process: By the malar process with the maxillary process of the Malar.

Palate Process: By the imner border of the palate process with the inner border of the opposite palate process and with the anterior part of the lower border of the Vomer; by its posterior bordeo with the anterior border of the horizontal plate of the palate.

Nasal Process: By its upper border with the nasal notch and internal angular process of the frontal; by the imer edge of its posterior border with the anterior border of the lachrymal and by the lachrymal tubercle with the hamular process of the lachrymal; by the upper part of its internal surface with the anterior extremity of the lateral mass of the ethmoid; by the superior turbinated crest with the anterior extremity of the middle turbinated bone; by its anterior border with the posterior border of the nasal bone.

\section{Palate Bone.}

The palate bone articulates by its two plates. By its processes and tuberosity.

The Horizontal Platearticulates by its inner border with the opposite bone and with the posterior part of the lower border of the Vomer: by its anterior border with the posterior border of the palate process of the superior maxillary.

The Perpendicular Plate articulates by most of its external surface, its anterior border and maxillary process with the inner face of the body of the superior maxillary, behind the aperture into the antrum; by its superior turbinated crest with the posterior extremity of the middle turbinated bone; by its inferior turbinated crest with the posterior extremity of the inferior turbinated bone; by its posterior border with the ante- 
rior border of the pterygoid process of the sphenoid; by the anterior face of the tuberosity with the lower part of the posterior border of the body of the superior maxillary and by its posterior face with the lower part of the anterior edge of the two pterygoid plates filling in the gap between them; by the anterior face of its orlital frocess with the posterior part of the internal border of the orbital face of the body of the superior maxillary; by its internal face with the external surface of the lateral mass of the ethmoid, below and behind the os planum; by its fostertor face with the front of the body of the sphenoid, through the pyramid of Wista. By the upper surface of the sphenoidal process with the lower surface of the vaginal process of the sphenoid; by its posterior border and the posterior part of its outer face with the inner side of the base of the pterygoid process of the sphenoid.

\section{Vomer.}

The vomer articulates by its borders.

By the grooce on its upper border with the rostrum of the sphenoid ; by its clce with the opposing faces of the vaginal process and lower surface of the body of the sphenoid; by its lover border, posteriorly, with the crested inner border of the horizontal plate of the palate and anteriorly with the crested inner border of the palate process of the superior maxillary; by the upper part of the anterior border with the lower part of the posterior border of the perpendicular plate of the ethmoid.

\section{Inferior Turbinated Bone.}

By its anterior extremity with the inferior turbinated crest of the superior maxillary; by its fosterior ex/remity with the inferior turbinated crest of the palate; by the lachrymal froiess with the edges of the lachrymal sulcus of the inner face of the body of the superior maxillary and with the projection on the anterior border of the lachrymal; by the maxillary troecss with the lower margin of the aperture of the antrum; by the cthmoidal frocess with the unciform process of the ethmoid. 


\section{Lachrymal Bone.}

The lachrymal bone articulates by its upper extremity with the internal angular process of the frontal; by its posterior border with the anterior border of the os planum; by its lower extremity with the front part of the internal border of the orbital plate of the superior maxillary; by the anterior border with the inner edge of the posterior border of the nasal process of the superior maxillary and by the projection from the lower end of this border with the edges of the lachrymal sulcus on the inner face of the boty of the superior maxillary and with the lachrymal process of the inferior turbinated bone and by the back part of the intermul surface with the auterior extremity of the lateral mass of the ethmoid.

\section{Malar Bone.}

The malar bone articulates by its processes and by the anterior border of its body, which articulates with the anterior border of the malar process of the superior maxillary.

By the Frontal Process with the external angular process of the Frontal.

By the Maxillary Process with the malar process of the superior maxillary.

By the Zygomatic Process with the zygomatic process of the temporal bone.

By the Orbital Process; the lower border articulates with the orbital surface of the superior maxillary, antero-externally; by the posterior border, with the outer border of the orbital plate of the greater wing of the sphenoid; and by the upper border with the frontal behind the internal angular process.

\section{Inferior Maxillary Bone.}

The inferior maxillary bone articulates by its condyles with the anterior part of the glenoid fossa of the temporal bone.

\section{The Hyoid Bone.}

The hyoid bone, or lingual bone is placed in the upper, front part of the neck at the base of the tongue. It is $U$ shaped 
and lies horizontally, being a symmetrical bone. It is an isolated bone, but is connected by a ligamentous cord, on either side, with the styloid process of the temporal bone; occasionally this cord becomes ossified and then a complete bony arch is formed between the two temporal bones through the hyoid bone. It is divided for study into a body and four cornua, or projections. The body presents two surfaces, two borders. and two extremities. The anterior surface looks upward and slightly forward and presents four depressions, separated by a crucial ridge; the confluence of the arms of the ridge producing a prominence called the tubercle. The posterior face looks downwards and slightly backwards; is concave, both transversely and vertically, presenting a deep concavity. The upper border faces back ward and is rounded and rough. The lower border faces forward, is protuberant and marked by musclular attachment. The extremity is oval and roughened by cartilaginous attachment. The greater cornu stands backwards from the extremity of the body, on either side, and is rounded, grows smaller, but is terminated posteriorly by a nodular enlargement. It is slightly flattened vertically.

The lesser cornu is, until old age, cartilaginous; it is a mere nodule of cartilage, hook like and about a quarter of inch long; its direction is upward, backward and slightly outward and it articulates by a diminutive joint at the junction of the body, and greater cornu. It becomes calcified and its joint obliterated only very late in life. 


\section{THE LIGAMENTS.}

The study of joints, or articulations, is the study of the relation between bones, more particularly of the surfaces of apposition and the means by which the bones are held together and at the same time permitted to move upon one another. In the study of osteology it has been seen that the articular surfaces of the different bones present widely varying appearances; upon these differences depends the classification of joints. In some joints the opposing surfaces present interlocking, tooth-like processes, so that the union of the bones results in an immovable joint. This form of articulation is called synarthrosis.

The contiguous surfaces may be roughened, showing the attachment of ligamentous fibres passing directly between the bony faces; or the fibres may have been attached through the medium of cartilage, coating the bony surfaces. This indicates a joint in which motion is very slight, consisting of a twisting of the ligimentous fibres; and the articulation is known as amphiarthrosis.

The bony surfaces, lastly, may be smooth and polished, as a result of mutual friction-indicating a freely movable joint of the class called diarthrosis.

The three classes of joints, then are-1st, Synarthrodial, or immovable joints ; 2 nd, Amphiarthrodial, or partially movable ; and 3rd, Diarthrodial, or freely movable joints.

Besides the bones, there are other structures which contribute to the formation of a joint, as follows: In the Amphiarthrodial and Diarthrodial joints, the bones are held together by ligaments. In the Amphiarthrodial these not only pass from bone to bone, exterior to the joint, but directly between the bones, within the joint, forming what are called interosseous ligaments. In the Diarthrodial joints, as a rule, no ligamentous fibres are attached to the articulating surfaces of the bones; the ligaments, more or less completely surrounding 
the joint, are attached to both bones beyond the articular surfaces. The fibres may entirely surround the joint forming a capsular ligament, or they may be gathered into separate bundles.

In the Diarthrodial joints the opposing bony surfaces are coated by encrusting, or articular cartilage, which, on its deep face, is firmly attached to the bone, and presents a perfectly smooth, free surface towards the opposing bone.

In order to lessen friction, and render motion entirely easy, diarthrodial joints possess a secreting, serous-like membrane, called the synovial membrane, which secretes a viscid, glairy fluid, called synovia. The synovial membrane, in very early life, is said to be a closed sac, coating the entire joint; but soon the portion covering the encrusting cartilage is worn away, or becomes inconspicuous.

If joints are subject to very frequent motion, the effect of friction is further guarded against by a plate of fibro-cartilage, called the interarticular cartilage. This is a more or less flat, circular plate of cartilage interposed between the encrusting cartilages of the bony surfaces, and attached only by its circumference to the ligaments. When this exists entire, the joint will hare two synorial membranes, but occasionally the plate is worn through and then, the two membranes communicating, there is virtually but one.

\section{Synarthrodial Joints -4 Kinds.}

The immovable joints are almost confined to the bones of the skull, the articulations between which are generally called sutures.

There are several varieties of suture : 1 st. Dentate, or Serrate, in which edges of the bones present interlocking processes, called dentate when long and tooth-like, and serrate when short, like the teeth of a saw. 2d. Suture by Harmony, in which two roughened surfaces come in contact, as the inner border of the palate processes of the two superior maxillary bones. $3 \mathrm{~d}$. Schindylesis, where the edge of one bone is received in a groove in another, as the articulation between the vomer and the sphenoid. 4th. Gomphosis, where a bone 
is received into a corresponding carity in another. This term is applied to the union between the teeth and their sockets, and is really no articulation.

\section{Diarthrodial Joints -6 Kinds.}

The diarthrodial joints, in accordance with the varying shape of the articular surfaces are dirided into six varieties.

1st. Arthrodial, formed by more or less flat, plane surfaces, so that motion is much restricted.

2nd. Hinge or trochlear, or ginglymoid, characterized by the presence of a pully on one of the articular surfaces. In this joint motion can occur as a rule, in only two directions.

3d. Condyloid, in which one element is a condyle, the other a proper receiving cavity. These joints are generally capable of very free movement.

th. Saddle-shaped, or concaro-convex joints, or joints of reciprocal reception, where both surfaces are saddle-shaped and mutually interlock. The examples of this form of joint are sterno-clavicular, trapezio-metacarpal and calcaneocuboid.

5th. Pivot, or trochoid joints. - There are only two of these, atlo-axoid and radio-ulnar. They present an osseo-ligamentous ring, in which a part of one bone is received; this, in the first example, acting as a pivot around which the other bone revolves; while, in the second case, it rotates on its own axis.

6th. Ball and socket, or enarthrodial joints. - These present a more or less spherical head, as one contribution, while a receiving carity is the other. The two important examples of this variety are the shoulder and hip, in both of which motion is very free.

In describing a joint, the following heads have to be considered:

1st. The class and variety. 2nd. The bony contributions. 3rd. The ligaments. 4th. The synovial membrane. 5th. The inter-articular cartilage, perhaps. 6th. The motions of which the joint is capable. 7th. The muscles which strengthen the joint, if any. 
As numerous as the motions seem to be, they can all be referred to the following:

(a) Gliding, which occurs to some extent in all diarthrodial joints, but is peculiarly characteriste of the arthrodial. It consists of the slipping of 0.12 . more or less, flut ayl plane surface on another.

(b) Flexion-angular morement of a segment of the body in an anters-posterior vertical plane, generally forward, but in some cases backward.

(c) Extension - the reverse of flexion.

(d) Abduction-the morement of a segment away from some established mid line, generally that of the body.

(c) Adduction-the reverse of abduction.

( $f$ ) Circumduction - the partial performance in rapid and regular succession of the four preceling morements, passing. in inward circumduction, from flexion to adduction, to extension, to abduction, and arain to flexion. In outward circumduction the order is reversed. In the performance of this movement, the moving segment circumseribes a coneshaped space, the apex of which is at the joint and the base at the distal extremity of the moving segment.

(g) Revolution, or false rotation. This occurs at but two joints, the atlo-asoid and radio ulnar. In the first case, the atlas revolves around the odontoid process as an axis: in the latter the radius rotates around its own axis in the ring formed by the orbicular liganent and the lesser sigmoid cavity.

(h) Rotation, or true rotation. This is almost limited to two joints, the shoulder and the hip. and, as it happens, can he defined in the same terms for both, riz : movement ontward or inward around a line drawn from the innermast point of the humerus or femur, to the innermost point of the inner condyle of either bone.

6th. A statement of the mechanism of motion-that is, of the changes which occur in the relative positions of the articulating surfaces during the performance of any given motion.

7th. The muscles which are in contact with the liganents of the joint, and which may be considered as imparting strength to the articulation. 
8th. In the important joints, the arteries and nerves distributed to them.

\section{The Articulations of the Vertebral Column.}

The articulations of the spinal column may be divided into. those of the (1st) Column in general; (2d) Atlo-axoid; (3d) Occipito-atloid; (4) Sacro-vertebral.

\section{Articulations of the Spine in General.}

Each vertebra articulates with the vertebra above and the one below by a joint of the diarthrodial class, and arthrodial variety, on each side, formed by the inferior and superior articular processes. The ligaments are capsular around the articulating surfaces, and those which bind the same segments of the different vertebra together. These ligaments are collectively known as the common ligaments of the spine.

\section{Articulation of the Articular Processes.}

The articulation between contiguous articular processes is a diarthrodial joint of the arthrodial variety, presenting a synovial membrane and one ligament, an imperfect capsular ligament.

\section{The Ligaments of the Spine in General-Ligaments of the Bodies.}

The bodies of the vertebræ are connected by means of intervertebral disks and anterior and posterior common ligaments. The intervertebral disk is found lying between the opposing surfaces of two adjacent vertebræ, firmly adherent. to each. In shape it corresponds exactly with the bodies between which it lies; in thickness it varies with the different regions of the spine. Collectively, the disks form about onefourth the length of the column and they are found between all the bodies except the first and second, being absent there because the atlas has no body. It is formed externally, of concentric laminæ of fibrous tissue and fibro-cartilage, the centre of the disk being a soft, pulpy mass.

Some of the fibres extend from the upper surface of the vertebra below to the lower surface of the vertebra above; and 
these fibres are crossed in the form of the letter $\mathrm{X}$, so that when the body bends or twists to either side the cords of the fibres may twist or untwist as the occasion demands.

The Anterior Common Ligament is found descending along the front of the bodies from the axis to the sacrum, widening as it descends. As a matter of fact this ligament extends to the basilar process of the occipital bone; but it has been customary to consider the upper portion extending from the axis to the occipital bone as a separate ligament. It is composed of superimposed layers of fibres, the deepest of which are attached simply to the bodies of adjacent vertebræ, while the next layer of fibres extends over two or three vertebræ, and the most superficial extends over four or five. This ligament widens as it descends, is not attached over the centres of the bodies of the vertebra, where it leaves a slight space between itself and the bodies, but is attached to the lips of the vertebræand to the intervertebral disks. A few scattered fibres continue this ligamentous band around the bodies of the vertebræ to the transverse prozesses.

The Posterior Common Ligument lies within the spinal canal; and is attached to the bodies of the vertebra, extending from the axis to the sacrum. Just as is the case with the anterior common ligament, this in reality extends to the cranium; but the upper portion of it is known under a separate name. Like the anterior it consists of several layers of fibres ; but unlike that ligament it narrows as it descends; and instead of having straight edges, it is scalloped, widening over each intervertebral disk and narrowing over the body. It is thinner and less powerful than is the anterior common ligament.

\section{Ligaments of the Laminae.}

The ligaments that binds the laminae together consist of yellow ligamentous fibres, called the ligamenta sulflava. By their upper extremity they are attached to the anterior surface of the lamina above, and by their lower extremity they are attached to the posterior surface and upper border of the one below. This is necessitated by the imbricated arrangement of

Des Anat-8 
the laminae. They are thicker in the loins than in the back and neck, and the thickest portion corresponds to the position of the spinous process.

\section{Ligaments of the Spinous Processes.}

The spinous processes are connected by two ligaments, the supra and interspinous. The supraspinous is a round cord, which extends from the seventh cervical vertebra to the sacrum, attached to the tips of the spinous processes. This cord is continued upward by a more prominent band, which has received the name of the ligamentum muchae (L. mucha, a nape). This, in man, is composed almost entirely of fibrous tissue, and is simply a division of the supraspinous ligament. The interspinous ligament consists of thin fibres, which are attached to the adjacent edges of the spinous processes; and is found only in the dorsal and lumbar regions.

\section{Ligaments of the Transverse Processes.}

Only in the lower dorsa1 and lumbar regions are the transverse processes held together by a few scattered intermediate fibres, called intertransverse ligaments, extending between the ad jacent edges of the corresponding transverse processes.

The Articulation of the Head with the Spine, and the First with the Second Cervical Vertebra.

\section{The Atlo-Axoid Articulation.}

The articulation between the Atlas and the axis consists of a diarthrodial and arthrodial joint on each side, formed by the articular processes, as seen between other vertebræ, and of a pivot joint, in front, between the posterior face of the anterior arch of the atlas and the front of the odontoid process of the axis.

The anterior atlo-axoid ligament, usually described as two, consists of a broad band of ligamentus fibres attached below to the front of the body of the axis as far up as the base of the odontoid process, and above to the lower border, anterior surface and tubercle of the anterior arch of the atlas. That part occupying the middle line is thicker than the lateral fibres and 
is described as a separate ligament. It is merely a continuation of the anterior common ligament of the spine.

The posterior atlo-axoid ligament is a membrane-like fold closing in the space between the vertebræ behind. It is attached above to the posterior surface and lower border of the posterior arch, extending from the capsular ligament of one side to that of the other and blending with the capsules. Below it seizes the lamina of the axis.

The transierse ligamcnt is a strong band, broader in the centre than at either extremity, attached firmly by either end to the inner face of the lateral mass of the atlas, stretching across the odontoid process and firmly binding the latter to the anterior arch of the atlas. As it passes over the odontoid process a vertical band of fibres crosses it, some of them derived from the transverse ligament, to be attached by one end to the base of the olontoid process and by the other to the basilar process of the occipital. This gives the ligament the form of a cross, hence its name, crucial ligament.

The two capsular ligaments are attached around the margins of the articular facets on the two vertebræ, blending with the posterior atlo-axoid ligament.

There are four synozal sacs connected with the articulation. Two beloug to the joints formed by the articular processes, one is interposed between the front of the ondontoid process and the facet on the anterior arch of the atlas, while one interposes between the posterior face of the odontoid process and the front of the transverse ligament.

\section{The 0ccipito-Atloid Articulation.}

This is a movable joint of the condyloid variety, the articulating surfaces being the two condyles of the occipital above and the superior articular facets of the atlas below. The ligaments, seven in number, are

Two anterior occipito-atloid

Two Lateral Occipito-Atloid
Posterior occipito-atloid Two capsular.

The central part of the anterior occifito-atloid ligament is a rounded cord attached below to the tubercle on the ante- 
rior arch of the atlas and above to the under face of the basilar process of the occipital. It is the upward continuation of the anterior atlo-axoid, and through it of the anterior common ligament of the spine. The lateral portion of the ligament is a broad and thin band attached above to the margin of the foramen magnum and below to the anterior arch of the atlas, extending to the beginning of the capsular ligaments, with which it blends.

The posterior occipito-atloid is a broad and thin membranous band attached above around the margins of the foramen magnum and below to the upper border and posterior surface of the posterior arch of the atlas. It corresponds to the ligaments between the laminæ in the vertebral column, but contains no elastic fibres.

The capsular ligaments are attached around the margins of the condyles above and the receiving surfaces on the atlas below. Behind and in front they blend with the anterior and posterior ligaments.

The two lateral ligaments blend with the sides of the two capsular ligaments and are attached below to the transverse process of the atlas, extending above to the jugular process of the occipital bone.

\section{The Occipito-Azoid Ligaments.}

The occipito-axoid ligament is a broad membranous band which continues the posterior common ligament of the spine upward to the occipital. It is attached below by some fibres to the body of the third cervical vertebra and to the body of the axis. Passing upward it covers over the odontoid process and the crucial ligament and is attached to the anterior margin of the foramen magnum, passing upon the basilar process to blend with the dura.

The odontoid or check ligament consists of three parts. The lateral portions are attached below to the pit on the side of the odontoid process and above to depressions found on the inner face of the condyles. The central portion is attached below to the tip of the odontoid process and above to the margin of the foramen magnum. 
The vertebral artery supplies both of these articulations. The loop between the first and second cervical nerves supplies the articulation between the axis and the atlas, while the suboccipital supplies the occipito-atloid articulation.

\section{Sacro-Vertebral Articulation.}

The joint between the sacrum and last lumbar vertebra, is similar to that between two vertebræ, the articulating surfaces being the articular processes of the two bones. The ligaments are the same as between any two vertebra, with the addition of two ligaments on each side, the ilio-lumbar and lumbo-sacral, which are generally considered among the ligaments of the pelvis. The ilio-lumbar extends from the transverse process of the fifth lumbar vertebra outward to the ilium just above and in front of the ear-shaped articular surface. The lumbo-sacral passes from the transverse process of the fifth lumbar vertebra downward and outward to the wing of the sacrum.

Movements. Movement between the occipital and the atlas is mainly confined to the backward and forward movements, though lateral movement is permitted to some extent. In the nodding movements of the head, flexion and extension, the condyles of the occipital glide backward and forward on the articular surfaces of the atlas. Thus, in flexion the chin is carried to the chest while the condyles pass backward in their articular cavities. In extension the head is carried back'vard and the condyles move forward. In the slight lateral movements the head is carried towards the shoulder and the condyle of that side passes inward while the one of the opposite side passes outward in the corresponding cavity. Motion between the atlas and axis is confined to revolution of the atlas around the odontoid process of the axis. In this movement, the head is turned to one or the other side the articular facet gliding toward that side over the facet on the process, while the inferior facet of the atlas of the side to which motion is made, glides backward on the axis and the opposite facet grlides forward.

Movements of the spine as a whole. The amount of motion which may take place between any two contiguous vertebra 
is but slight, yet the column as a whole enjoys a wide range as well as a great variety of movements. These movements are the four angular movements, flexion, bending forward, extension, bending backward, and right and left lateral movement. In addition to these a considerable amount of rotation, by twisting of the ligamentous fibres is permitted. In flexion the body bends forward, compressing the anterior fibres of the intervertebral disks and putting the posterior fibres on the stretch, the spine becoming convex backward. This movement is limited not only by the compressibility of the disks but by the tension on the posterior fibres, while the supra and interspinous and the sub-flava ligaments are all stretched. The superior articular processes glide upward on the inferior in this movement. Flexion and extension are both free in the cervical and lumbar regions, the vertical direction of the articular processes limiting the movement in the dorsal. Lateral flexion is most free in the cervical region, the inversion of the superior articular processes limiting it in the lumbar, while the attachment of the ribs prevents it in the dorsal, where the shape and direction of the articular processes would indicate that it should be most free. In this movement the articular process of the side away from which motion is made glides upward on the one below, the reverse movement taking place on the side flexed. The lateral margins of the disks would be compressed and the fibres of all ligaments on the opposite side put on the stretch. Rotation of the column takes place by twisting the fibres of the intervertebral disks. It is free in the cervical region, slight or imperceptible in the dorsal on account of the ribs, while the large size of the superior facets as compared to the inferior allows some rotation in the lumbar region in spite of the fitting in of the superior processes between the inferior. Of course in rotation there is a certain amount of gliding of the articular processes, the movement being arrested by the stretching of the vertical fibres of the disks.

\section{The Sterno-Clavicular Articulation.}

This is a movable joint of the saddle shaped variety, or a joint by reciprocal reception. It is formed by the saddle shaped 
articular surface on the inner extremity of the clavicle, which is concave from above downivard and forward and convex from above downward and backward, and a similar surface on the upper angle of the manubrium, aided by a concavity on the cartilage of the first rib. The following ligaments bind the bones together:

Capsular

Interclavicular
Romboid, or Costo-Clavicular

Inter-articular fibro-cartilage.

The inter-articular fibro-cartilage is attached by one end to the upper, posterior part of the inner end of the clavicle and by the other to the junction of the cartilage of the first rib with the articular depression on the lateral aspect of the manubrium. It serves the double purpose of holding the bones together and at the same time divides the joint into two synovial cavities.

The capsnlar ligament is a thick band of fibres completely surrounding the joint but thicker at some points than at others. Thus it is seen to be much more thickened behind than elsewhere, being sometimes described as the posterior ligament of the joint. In front it blends with the tendon of the sterno-mastoid muscle, above with the interclavicular ligament while below it is continuous with the Romboid. The deepest fibres of this ligament pass from the two bones to the interarticular cartilage, and not from bone to bone, while the more superficial fibres pass from the margin of the receiving cavity on the manubrium and cartilage on the first rib to the margin of the articular surface on the clavicle.

The interclaricular ligament is a strong thick band attached to the upper an 1 posterior aspect of the clavicle of one side, blending with the fibres of the capsule, then passes into the interclavicular notch to be attached to the posterior part of the upper border of the manubrium, and rises to be attached to the inner back part of the opposite clavicle.

The romboid, or costo-clavicular ligament is a set of short fibres resembling fibro cartilage which extend from the romboid impression of the clavicle downward, forward and in ward to be attached to the upper face of the cartilage of the first rib. 
There are two synovial membranes, one interposed between the cartilage and the manubrium and the other between the inner extremity of the clavicle and the cartilage. In some cases the cartilage is worn through and the synovial sacs communicate with each other, practically forming one sac.

The blood supply is mainly from a branch of the Interna 1 Mammary. The nerve supply is from the branch to the subclavius.

The movements of the joint, which take place in all directions, are confined within a narrow range and are always of a gliding character. The cartilage accompanies the clavicle in all of its movements except upward. Thus in movement of the shoulder backward, the inner end of the clavicle, with the interarticular cartilage, passes forward; in forward movement of the shoulder, the clavicle passes backward; in upward movement of the shoulder the clavicle passes downward; but in downward movement of the shoulder, the clavicle passes upward, putting the fibres of the cartilage on the stretch and limiting the extent of the movement.

\section{Scapulo-Clavicular Articulation.}

This is a movable joint of the arthrodial variety. The articulating surfaces are an oval facet on the outer extremity of the clavicle and a corresponding facet on the anterior edge of the acromion process of the scapula. It must be observed that the clavicle, in its outward course, passes just above the coracoid process of the scapula, and, while it does not articulate with it, receives a ligament from it.

The bones are held together by two sets of ligaments, a capsular, surrounding the articular surfaces, and a coraco-clavicular binding the clavicle to the coracoid process.

The capsular ligament is a strong but lax band of fibres surrounding the joint and attached to the clavicle beyond the articular facet. Inferiorly these fibres pass as far as the coraco clavicular ligament with which they blend. Above, they are strengthened by fibres of the deltoid and trapezius.

The coraco-clavicular consists of two portions, the conoid and the trapezoid. The conoid ligament is triangular in form 
and is attached by its apex to the coracoid process close to the supra-scapular notch, while its base is inserted into the conoid tubercle and posterior border of the clavicle. The trapezoid, blended internally with the conoid, has its surface looking forward and backward. Below it is attached to a ridge on the upper aspect of the coracoid process, while above it is attached to the oblique ridge running forward and outward from the conoid tubercle to the anterior border of the clavicle.

This joint has an interarticular fibro cartilage, which, however, is often absent. When this is present there are two synovial sacs, otherwise, but one.

The joint is supplied by the acromial thoracic and suprascapular arteries and by the circumflex and suprascapular nerves.

The morements of the joint are confined to a slight gliding of the articular surfaces upon one another. Usually the scapula is the bone which moves; but, of course, when the scapula is fixed, the clavicle may glide upward, downward backward or forward.

\section{Ligaments of the Scapula.}

Stretched between different points on the scapula are two ligaments, coraco-acromial and transverse. The coraco-acromial, thick and triangular, is attached by its apex to the tip of the acromion and by its base to the coracoid process, serving as a protecting arch for the shoulder joint. The transverse ligument passes over the supra-scapular notch, from one extremity to the other, converting it into a foramen.

\section{The Shoulder Joint.}

The shoulder is a joint of the diarthrodial class and enarthrodial or ball and socket variety. The bones entering into its formation are the upper extremity of the lumerus, with its globular head, anatomical neck and two tuberosities; and the glenoid cavity of the scapula, supported by its neck and overhung by the coracoid and acromion processes. The ligaments of the joint are properly but two, the glenoid and capsular, though some accessory bands of the latter are frequently dig- 
nified as separate ligaments. The glenoid ligament is a band of fibro-cartilage, triangular on cross section, which is attached by its base around the margin of the glenoid cavity and serves to deepen, but more especially to widen this cavity. It is connected above with the head of the biceps muscle which passes through the joint, and by its margin is attached to the capsular ligament.

The capsular ligament is a strong but loose sac completely investing the articulation but not holding: the bones in apposition with each other. It is simply a bag of which the upper end is closed by the glenoid cavity and the lower by the articular surface of the humerus. Above, this ligament is attached around the margin of the glenoid cavity, extending on the neck of the scapula further above than below and blending with the glenoid ligament. Below, it is attached around the anatomical neck of the humerus but is prolonged over the tuberosities, between which it is continuous with the transverse band which binds the tendon of the biceps in its groove. Inferiorly it is prolonged over the surgical neck of the humerus to a slight extent. The upper part is very much thicker than any other portion of the ligament. One portion, that extending from the base of the coracoid process to the greater tuberosity of the humerus is the portion individualized as the coraco-humeral ligament. In the interine of the joint there may sometimes be seen a fold in the synovial membrane called the gleno-humeral ligament. The capsule is nearly surrounded by muscles whose tendons, as they pass over it,are blended with the ligament and supply the greater part of its strength.

The synovial membrane is extensive. It lines the interior of the capsule and is reflected over the margins of the articular surfaces, but does not reach the points of greatest pressure. A prolongation of the membrane is continued over the tendon of the biceps muscle and the sac usually communicates with a bursa beneath the subscapularis muscle and frequently with one beneath the infraspinatus.

The joint is powerfully reinforced and protected not only by the numerous muscles which surround it but also by a protecting arch formed by the acromion and coracoid processes and 
the coraco-acromial ligament stretched between them. The muscles are, above the supra spinatus, postero-superiorly the infraspinatus and teres minor, antero-internally the subscapularis: while above, behind, to the outer side and in front the fibers of the deltoid form a cushion over the joint. The first four muscles blend, at their insertion, with the capsule.

The arterial supply is chiefly from the anterior and posterior circumflex, but branches are derived from the dorsalis scapula and suprascapular arteries. The nerves are the suprascapular and circumflex.

Movements and mechanism. The length of the capsular ligament, the shallowness of the receiving cavity and the comparatively large size of the humeral head, permit this joint to enjoy a greater variety and extent of movement than any other in the body. Like all enarthrodial joints it is capable of the four angular movements, their combination, circumduction, and axial rotation. In flexion the humerus with the forearm is carried forward, the head of the bone spinning in the articular cavity around an axis which is drawn from the centre of the head through the centre of the greater tuberosity, to the centre of the glenoid cavity. In extension the reverse movement takes place, with of course a reversal of the mechanism. In abduction the humerus is carried away from the mid-line of the body, the hand raised toward the shoulder, while the head of the humerus glides down ward in the glenoid cavity until it presses on the inferior fibres of the capsular ligament. When the humerus is carried in abduction to a right angle with the body, further movement is arrested by the impact of the bone against the arch of the acromion, further movement in this direction being effected by the gliding of the scapula on the thoracic wall. Adduction is the reverse of abduction. Adduction is limited by the elbow coming in contact with the side and cannot be carried further without first flexing the arm. Circumduction is the combination of these movements, the head of the humerus assuming the position of flexion, abduction, extension and adduction, the arm and forearm describing a cone whose apex is at the shoulder joint. In rotation the head of the humerus moves 
around an axis drawn from the centre of the head downward through the internal condyle, the head moving backward in inward and forward in outward rotation.

The relation of the long head of the biceps to this joint is so peculiar as to demand special mention. Attached to the upper margin of the glenoid cavity, where it is blended with the glenoid ligament, it passes across the upper outer aspect of the joint, pierces its capsular ligament and is covered by a prolongation of the synovial membrane which lines the cavity. This tendon, therefore, becomes a powerful agent in retaining the head of the humerus in apposition with the gleniod cavity and thus strengthens the joint on its upper and outer aspect.

\section{Radio-Ulnar Articulation.}

The articulation between the two bones of the forearm forms a diarthrodial joint of the trochoid or pivot variety. The bones articulate at both the upper and lower extremity, being separated by the interosseous space throughout the shafts.

The ligaments holding the bones together are found at their upper and lower extremities and between their shafts. Above, the two bones are held together by one ligament, the orbicular which forms three-fourths of a ring, the other fourth being the lesser sigmoid cavity of the ulna. The ligament surrounds the articular rim of the radius and is attached by its two ends to the extremities of the lesser sigmoid cavity. The lower fibres are tightly stretched around the radius just below the head, rendering it very difficult to release that bone from its grasp.

The shafts are connected by two ligaments, the interosseous and the oblique. The oblique is a narrow band which passes downward and outward from the ulna, at the base of the coronoid process, to the radius just below the tuberosity. It is of ten wanting.

The interosseous membrane extends from the lower extremities of the bones upward about two-thirds the length of the shafts, leaving an interval,above which is the oblique ligament. Its fibres are directed downward and in ward between the inner border of the radius and the outer border of the ulna. About 
the lower third of the forearm, the membrane is pierced by a foramen for the anterior interosseous artery.

At their lower extremities the bones are held together by two ligaments and the triangular fibro-cartilage. The two ligaments are an anterior and a posterior passing across the two bones. The triangular cartilage tips the summit of the capitulum ulnæ; and excludes it from the wrist joint. By its apex it is attached to the pit between the styloid process of the ulna and the capitulum ulnæ; by its base to the narrow rough surface separating the carpal and sigmoid cavities of the radius.

The movements are two, and in both the radiusalone moves. It can move forward, pronation, or backward, supination, and in both the hand is carried with it. In these movements of rotation, or revolution, the radius rotates around an axis represented by an imaginary line drawn from the centre of the head of the radius through the centre of the capitulum ulnæ; consequently in performing pronation the sigmoid cavity of the radius moves forward on the articular rim of the capitulum ulnæ, the reverse occurring in supination, while the head of the radius rotates in the lesser sigmoid cavity and orbicular ligament.

\section{The Elbow.}

This is a diarthrodial joint of the ginglymoid variety. The bones entering into its formation are the trochlear surface and the rounded eminentia capatata on the lower extremity, of the humerus, and the greater sigmoid cavity of the ulna with the cupshaped upper extremity of the radius. The shape of the articular surfaces furnished by the radius and humerus would render this joint one of the enarthrodial variety; but the movements are so restricted by the close ligamentous union between the ulna and radius, that the joint presents but one of the peculiar movements of the enarthrodial joints-rotation.

The ligaments are anterior and posterior, internal and external lateral. There, as always, some thin fibres which connect the various parts of the ligamentous bands together, completing the capsule around the joint.

The anlerior ligancm is attached above to the humerus just above the coranoid fossa, while below it seizes the lower surface of the coranoid process and the orbicular ligament. 
The posterior ligaments is attached above to the humerus just above the olecranon fossa and below to the groove on the upper surface of the olecranon, and to the bone immediately behind this. It is much thinner and weaker than the anterior.

The external lateral ligament is attached above to a depression beneath the external condyle while its base is attached to the orbicular ligament.

The internal lateral is attached above to the internal condyle while below it expands into two fasciculi with a triangular interval, filled with thin fibres, between them. The most anterior, which may be traced to its attachment to the front of the internal condyle, is attached below to the margin of the great sigmoid cavity on the coronoid process. The posterior, attached above to the back of the internal condyle, is below attached to that margin of the sigmoid cavity furnished by the olecranon process. By means of a few thin, scattered fibres, the lateral ligaments are connected with the anterior and posterior ligaments, thus completing an imperfect capsule.

The synovial membrane is extensive. It lines the whole of the inner face of the capsule, extending upward behind to the olecranon and in front to the coranoid fossa; while below it covers the interior of the orbicular ligament and furnishes the lining for the articulation at the superior radio ulnar articulation.

The muscles which strengthen the joint are in front the brachialis anticus, behind the triceps and anconeus, externally the muscles arising from the external condyle, notably the supinator brevis; and internally those which arise from the internal condyle.

The morements are flexion and extension, to which should be added the rotation of the radius when that bone revolves in the lesser sigmoid cavity. In flexion the greater sigmoid cavity and the cup-shaped articular surface of the radius glide upward on the corresponding articular surface of the humerus, the movement being arrested when the coranoid process impinges upon the coronoid fossa. In extension the reverse would occur and the movement would be arrested by the impact of the beak 
of the olecranon on the bottom of the olecranon fossa. The movement of the radius will be described with the radio-ulnar articulation.

The arterial supply of this joint is derived from communicating branches between the anastomotica magna, the superior profunda and inferior profunda arteries, the anterior, posterior, and interosseous recurrent branches of the ulna, and from the radial recurrent. The nerve supply is derived from the ulna as it passes between the internal condyle and the olecranon, the median is said to furnish two filaments while the musculocutaneous contributes one filament.

\section{The Wrist Joint.}

This is a diarthrodial joint of the condyloid variety. The receiving cavity, oval, transversely, concave both from before backward and from side to side, is furnished by the lower extremity of the radius, aided by the triangular cartilage which shuts out the ulna from participation in the joint. The condyle is provided by three of the bones of the first row of the carpus, scaphoid, semilunar and cuneiform. The ligaments anterior, posterior and two lateral.

All ligaments of this joint blend with the ligaments of the carpus and can with difficulty be separated from them. Thus the antrior ligament is attached above to the margin of the carpal cavity of the radius and to the anterior radio-ulnar ligament, and to the anterior margin of the lower end of the U1na, while below it is attached to the bones of the first row of the carpus, blending with the anterior palmer ligamentous fibres which bind together the first and second rows. The postcrior is, in like manner, attached above to the posterior margin of the carpal cavity and to the posterior radio-ulna ligament and below to the back of the bones forming the first row of the carpus. blending with the dorsal carpal ligaments binding the two rows together. The external lateral ligament is attached above to the styloid process of the radius and below to the tuberosity of the scaphoid and to the trapezium. The internal latcral lisament is attached above to the styloid process of the ulna, below to the inner side of the cunciform and unciform, to the latter through the medium of the internal 
lateral ligament of the carpus. Between these bands are fibres which convert this ligament into a capsule.

The synovial membrane lines the interior of the ligamentous fibres and to a slight extent dips in between the scaphoid and semilunar and between the semilunar and cuneiform. There are no muscles which directly strengthen the joint. Many tendons pass over it but their laxity prevents their adding strength except in certain positions of the joint. Thus in flexion, with the closed fist, the extensor tendons strengthen it behind, and in extension, with the hand open, some strength is given by the flexors. The tendons, also, by the tonic contraction of the muscles, aid in retaining the bones in apposition.

The movements are those usual in condyloid articulations, flexion, extension, abduction, adduction and circumduction. The mechanism will be readily understood if it is borne in mind that in all movements of condyloid joints the distal extremity moves in a direction opposite to that of the condyle. Thus in flexion, the hand passes forward on the forearm while the condyle moves backward in its receiving cavity, pressing against the posterior fibres of the ligament. Exaggerated movement as from a fall on the flexed hand, would produce dislocation. In extension the mechanism is the reverse of flexion. In adduction the hand is carried toward the mid line of body while the condyle passes outward, the movement being limited by the external lateral ligament. In abduction the reverse is true. The combination of these movements makes circumduction, which may be either inward or outward. In the latter the hand would pass from flexion to abduction, thence to extension, then to adduction and again to flexion. The condyle passing first backward, then inward, forward, outward and so to backward again.

The wrist joint gets its arterial supply from the anterior and posterior carpal arches of the radial and ulna. The interosseous arteries, anterior and posterior, as well as some of the ascending branches of the deep palmar arch also contributing to its supply.

The ulna and posterior interosseous furnish the nerve supply. 


\section{Articulation of Carpus and Metacarpus.}

The bones of the carpus, which lie in the same row, are held together by ligamentous fibres passing across them in front and behind, palmar and dorsal ligaments, and by fibres which seize the adjoining surface of opposing bones, called interosseous ligaments. Between the trapezium and trapezoid there is no interosseous ligament. In the same manner the metacarpal bones of the four fingers are held together at their bases. The two rows of carpal bones are held together by ligamentous fibres passing from one to the other in front and behind, palmar and dorsal ligaments, and by two lateral ligaments, the external passing from the scaphoid to the trapezium, the internal from the cuneiform to the unciform.

The metacarpal bones of the four fingers are held to the second row of carpal bones by fibres in front and behind, palmar and dorsal ligaments, and in one situation by interosseous fibres extending from the adjacent parts of the os magnumand unciform to the bases of the third and fourth metacarpal bones.

The synovial membrane between the first and second rows sends prolongations upward and downward between the individual bones of the two rows, as well as between the proximal extremities of the metacarpal bones into the carpo-metacarpal articulations. This is not true of the synovial sac between the metacarpal bone of the thumb and the trapezium.

\section{The Trapezio-Metacarpal Joint.}

This articulation belongs to the morable class and saddleshaped variety. It is invested by a capsular ligament, and allows the four angular movements and their combination, circumduction.

\section{The Metacarpo-Phalangeal Articulation.}

The knuckle joint, or metacarpo-phalangeal, is of the movable class and condyloid variety. The condyle is furnished by the head of the metacarpal bone and the receiving cavity by the top of the corresponding phalanx, aided by a plate of cartilage known as the anterior ligrament of the joint.

Des Anat-9 
The ligaments are two lateral, seizing both bones on their lateral aspect just beyond the articular surface. The place of a posterior ligament is supplied by the extensor tendons of the finger. The anterior is not a ligament in the true sense of the word, being only a mass of fibro-cartilaginous material attached to the front edge of the articulating surface on the base of the phalanx, and increasing the receiving cavity.

To this ligament is attached a band of transverse fibres which stretch between the distal ends of the metacarpal bones and bind them to one another.

\section{Inter-Phalangeal Articulations.}

These joints are of the diarthrodial class and the hinge variety, the trochlear surface being presented by the head of one phalanx and the receiving surface by the base of the other.

The ligaments, auterior and two laterrl, are attached like those of the metacarpo-phalangeal articulations, the extensor tendons here, also, taking the place of posterior ligaments.

The carpal articulations are supplied by the carpal arches and the deep palmar arches. The nerves are derived from the ulnar, median and posterior interosseous. The fingers are supplied by their respective digital branches both for nerves and arteries. The movements of the carpal joints are confined to a slight gliding of one bone on the other, 一the same statement applying to the carpo-metacarpal joint, except the thumb. Movement at the metacarpo-phalangeal joint consists of the four angular movements and their combination, circumduction. Movement is freer at the index and little fingers than at the other two. Movement at the inter-phalangeal articulation is confined to flexion and extension.

\section{Temporo-Maxillary Articulation.}

This articulation is formed by the condyle of the lower jaw and the glenoid cavity of the temporal bone.

This is a diarthrodial joint of the condyloid variety, and presents four ligaments, viz: internal and external lateral, capsular and stylo-maxillary and an inter-articular fibro-cartilage, which is sometimes perforated; when this is the case, 
there is but one synovial sac, otherwise there are two, one between the cartilage and the condyle and the other between the cartilage and the glenoid cavity.

The external lateral ligament is attached to the zygoma above, from its tubercle forward, and, passing downward and backward, is attached, below, to the outer border of the neck of the condyle.

The internal lateral liaament is a specialized piece of the deep cervical fascia, attached above to the spine of the sphenoid and below to the shelf of bone forming the lower margin of the inferior dental foramen.

The capsular ligament consists of fibres of varying thickness, attached above around the articular margin of the glenoid carity and below to the neck of the condyle of the lower jaw. This ligament is slightly thickened at its inner part, while the external lateral ligament is merely a thickening of the capsule. The capsule is attached throughout its circumference to the edge of the interarticular cartilage.

The inter-articular fibro-cartilage is oval in outline, thinner at the centre than at the circumference, concavo-convex on its upper and concave on its lower surface. Its margin is closely attached to the capsular ligament.

The stylo-maxillary is, properly, not a ligament of this joint. It extends from the styloid process of the temporal bone to the angle and posterior border of the ramus of the lower jaw.

The lower jaw receives its nervous supply from the auriculo-temporal branch of the inferior maxillary. The arterial supply is from the temporal.

The joint is strengthened on the outer side by the masseter, internally by the internal pterygoid and above by the temporal.

The movements are elevation and depression, lateral and forward movement. In depression the jaw recedes from the upper and the condyle, when the movement is not extreme, rolls on the inter-articular cartilage around an axis drawn through its own centre. When, however, the movement is 
carried farther, both condyle and cartilage roll forward on the eminentia articularis, exaggerated movement leading to dislocation.

Elevation is the reverse of depression.

In lateral movement the jaw is twisted to one or the other side, the condyle moving backward in the glenoid cavity on the side toward which motion is made.

In forward movement the two condyles are dragged forward on the eminentia articularis, the lower jaw being protruded beyond the upper.

\section{Costo-Sternal Articulation.}

The ribs have attached to their anterior extremities the costal cartilages, which prolong the seven true ribs to the side of the sternum, where the cartilages are received into the pits found there and are held in place by ligamentous fibres passing from them to the sternum in front and behind. The cartilages of the second and third ribs have also each an inter-articular ligament.

\section{The Articulations of the Pelvis.}

The articulations of the pelvis are four, viz: that between the fifth lumbar vertebra and the sacrum, that between the sacrum and coccyx, that between the sacrum and the os innominatum of each side and that between the two ossa innominata.

\section{Sacro-Vertebral Articulation.}

The joint between the sacrum and last lumbar vertebra, is similar to that between two vertebræ; the articulating surfaces being the articular processes of the two bones. The ligaments are the same as between any two rertebræ, with the addition two ligaments on each side, the ilio-lumbar and lumbo-sacral, which are generally considered among the ligaments of the pelvis. The ilio-lumbar extends from the transverse process of the fifth lumbar vertebra outward to the ilium just above and in front of the ear-shaped articular surface. The lumbosacral passes from the transverse process of the fifth lumbar vertebra downward and outward to the wing of the sacrum. 
The Sacro-Coccy'real ligaments are an intervertebral disk, small and insignificant, and an anterior, posterior and lateral ligaments passing directly from one bone to the other.

The Sacro-lliac articulation, symphysis or synchondrosis, is formed by the auricular facets found on the sacrum and ilium, each of which is coated, in the recent state, by a thin plate of cartilage. The ligaments of the joint are an anterior and a posterior and the two sacro-sciatic, greater and lesser.

The anterior sacro-iliac ligament is a thin plane of fibres passing from the front of the sacrum to the inner face of the ilium.

The posterior sacro-iliac ligament is a dense and thick mass of fibres, both oblique and horizontal, passing between the contiguous surfaces of the two bones and filling in the deep depression which exists between them. The fibres are attached to the rough surface on the ilium behind the auricular facet and to the similar depression on the lateral aspect of the sacrum as well as to the posterior face of the latter bone.

The greater sacro-sciatic ligament is attached by its posterior extremity to the posterior inferior spinous process of the ilium, and to the side and posterior face of the sacrum and coccyx. Passing forward it runs below the spine of the ischinm and is attached to the tuberosity of the ischium, its inner edge being free and curved upward upon itself.

The lesser sacro-sciatic ligament is blended with part of the greater at its posterior extremity, being attached to the side of the sacrum and coccyx, slightly in front of the greater, and not extending so high up as the former. In front it is attached to the spine of the ischium.

The lesser sacro-sciatic ligament forms the lower boundary of the greater sacro-sciatic foramen; while that part of the greater sacro-sciatic which extends from the point where the two ligaments separate to the tuberosity of the ischium, forms the lower boundary of the lesser sacro-sciatic foramen.

\section{The Symphysis Pubis.}

The articulation between the ossa innominata is formed between the two pubic bones, and is known as the symphysis 
pubis. Each bone presents an oval articular surface, whose long diameter is downward and backward, coated by a thin plate of encrusting cartilage. The two plates are in contact only at the back part, leaving, in front, a wedge-shaped space filled by fibres passing between the two plates. Besides this interarticular ligament, there are four others-superior, consisting of fibres passing between the two bones above; inferior, fibres passing between the two bones below, and filling in the interval between the two rami, so as to form a smoothlycurved arch; anterior, fibres passing between the bones in front, and posterior consisting of similar fibres behind.

The articulations are supplied by branches from the lateral sacral, ilio-lumbar, middle sacral and internal pudic arteries.

The nerves are received from the cords of the sacral plexus.

\section{The Hip Joint.}

The Hip Joint is one of the ball and socket articulations, of course, belonging to the enarthrodial, or movable variety. The bones which enter into its formation are the three-quartersphere articular surface found on the upper extremity of the femur to correspond to the Acetabulum, a receiving cavity found at the junction of the three bones, the Ilium, the Ischium and the Pubes, which compose the os innominatum. This cavity is articular throughout its entire extent. There is a lower, inner portion, at the bottom of the cavity, a non-articular roughened surface, which in the articulated skeleton serves partly to form the attachment of the ligament which lies in the interior of the joint, and partly serves for the lodgment of the mass of fat, which prevents jarring of the joint, and at the same time lodges the blood vessels which supply the joint and prevents pressure upon these vessels.

The Ligaments which hold the bones together are the Capsular ligament, which surrounds the joint; the Cotyloid, which surrounds the margin of the cavity, and serves to deepen it; the Transverse, which crosses the cotyloid notch; and in, the interior of the articulation, there is a round cord which is known as the Ligamentum Teres. Some anatomists add other ligaments, which are unimportant when con- 
sidered as separate ligaments, being merely portions of $t$ capsular ligament which are developed by the strain upon the joint. One of these, the Ilio femoral ligament, extends from just behind the anterior inferior spinous process of the ilium across to a point close to the Trochanter mijor of the femur, one of the points at which the strain is very great. Another, called the Ischio-femoral, extends from the tuberosity of the ischium; while a third, somewhat less important than the others, extends from near the femoral line of the os pubes, and is known as the Pectineal, or Pubo-femoral ligament. These ligaments are not deserving of a separate name. The proper description is to say that the capsular ligament is strengthened at the superior and inferior extremities of the joint by fibres which come respectively from the spinous process of the ilium, from the ischium, and from the os pubes.

The Cupsular Ligumont, thick and powerful, is attached above to the brim of the Acetabulum. As it passes around downward and backward, it will be noticed that the ligament extends further forward on the Acetabulum; that is, the extent of its attachment increases as we pass around to the posterior aspect of the joint. On reaching the lower portion of the joint, this ligament is found to occupy all the space above the groove on the ischium. On reaching the upper, inner portion of the joint it expands considerably, nearly to the ilio-pectineal line. As the lixament passes downward it completely envelops the joint; and its attachment is again irregular when the attachment to the femur is reached. Superiorly it extends to what is know 11 as thesuperior cervical tubercle, which is found projecting from the upper front part of the neck at its junction with the trochanter major; superiorly to this, it extends behind a part of the Trochanter major; then follows the spirai line, and winds around until it passes just in front of the Trochanter minor; then extends until it passes upward and just misses the digital pit, and reaches the starting-point.

One of the most important of the ligamentary bands composing the Capsular ligament is the so-called "Y Ligrament," which may be seen better in the dry specimen than in the recent. The two branches rise by the "tail" or "stem" of 
the $Y$ from just below and just behind the anterior inferior spinous process of the ilium; and as they pass downward, though there is never any actual interval between them, the central fibres are very thin, and the fibres above and below, particularly above, are very thick, hence the resemblance to a Y. The superior fibres of the Y ligament are attached to the superior cervical tubercle: the inferior fibres are inserted into the spiral line just as they reach the inner aspect of the bone.

The others are of comparatively little importance.

The Ilio-pectineal ligament is a long slender band of fibres, which starts nearly at the pectineal line of the pubes; and passes down to reach the spine, a little lower. The band which comes from the tuberosity of the ischium arises just below the groove; and then stretches across, to be inserted near the digital pit close to the Trochanter major. These ligamentous bands are of comparatively little importance.

The Cotyloid cavity is deepened by a ring of fibro-cartilage, which is described as being nearly a complete circle, but fails to extend all the way around the joint; that is, it is said to be horse-shoe shaped, the direction of the bend in the horse-shoe being downward and inward at the cotyloid notch. What is in reality a continuous ligament, extending all the way around the joint, anatomists have preferred to describe as two ligaments, differentiating between the Cotyloid ligament and the Transverse ligament, which passes across the cotyloid notch, becoming continuous at its extremities with the cotyloid ligament, although the two have the same histiological structure. However, in accordance with general usage they are to be described as two separate ligaments. The Cotyloid ligament is described as being triangular upon cross section, although it is not quite so, the outer face being distinctly convex, the inner upon the cross section proving to be not a straight line, but a curved line, thus forming the concavity which is necessary to receive the convex head of the femur. The thin edge projects away from the bone; the thick edge is attached to the margin of the cavity all the way around.

As above described, the Cotyloid ligament upon reaching 
the cotyloid notch, changes its name, and becomes the Trans''e'rse ligament. which is therefore merely a continuation of the Cotyloid.

The Ligamentum Tcres is a band of ligamentous fibres, holding the bones which form the joint in position. It is attached to the margin of the depression in the bottom of the cavity; its lower fibres are firmly attached to the transverse ligament; and at its lower extremity it passes into the depression on the head of the femur.

In this, as in all other movable joints, there is a synorial membrane, which here presents one peculiarity. Though the Ligamentum Teres has been spoken of as a ligament, it is not a ligament in the true sense of the word. As a rule the synovial membranes disappear as they approach the region of pressure in a bone; but the Ligamentum Teres can be distinctly demonstrated as a synovial membrane at the points of greatest pressure. The synovial membrane starts at the pit on the head of the femur; and passes downward, covering all of the head of the femur. Leaving the bone itself, it appears on the capsular ligament; continues on the cotyloid face until it reaches the cotyloid cavity; then sweeps across the mass of fat lying in this cavity; and then passes all around the Ligamentunı Teres. The Ligamentum Teres, therefore, does not enter into the joint at all, and unless the synovial membrane is first cut, neither the Ligamentum Teres, nor the mass of fat which lies in the bottom of the depression have any communication with the joint.

The Movements of which the hip joint is capable are, of course, all of the movements of the enarthroidal joint; viz., flexion, extension, adduction, abduction, circumduction, and rotation. Any two or more of these movements which are not antagonistic with each other may, of course, be combined; for instance, there may be flexion of the thigh upon the pelvis at the same time with adduction, abduction, or axial rotation.

Flexion, or the movement of the thigh forward, is the most extensive of the movements of the joint. In the prepared specimen flexion may be continued until there is almost a com- 
plete revolution of the joint. The movement consists simply of spinuing the head in the cavity. There is no change of position with reference to the ball in the socket, the ball simply turning in the socket on an axis drawn through the Trochanter major from the centre of the head of the femur. Complete flexion is checked by the powerful band of fibres forming the upper limb of the $\mathrm{Y}$ ligament, though of course in the living man the movement is stopped long before this by the contact between the thick muscles of the abdomen and the muscles of the thigh.

Extension, the movement of the thigh backward, is much less free than flexion. The movement is hindered by the Pectino-femoral band in the dead subject; and is also limited, in the living man, by the mass of muscles passing in front and behind.

In Abduction and Adduction there are complete changes without rotation. In Abduction, which is a movement of the thigh away from the mid-line of the body, as the limb is carried outward, the head of the femur passes downward to the bottom of the acetabular cavity, so that it can be felt through the ligamentous fibres. If the movement is carried to excess in the living man, splitting of the fibres takes place.

Adduction is more limited in this than in almost any other joint in the body. If the body be in the anatomical position, of course the movement is arrested by the limb on the other side; if taken in a position of semi-flexion, adduction can never be equal to abduction, because it is checked by the powerful superior fibres of the capsular ligament.

Circumduction is a combination of all the preceeding movements. In this movement the limb passes from flexion to abduction, from abduction to extension, from extension to adduction, from adduction back to flexion.

Axial Rotation is usually described as the rotation on the line which is drawn from the centre of the head of the femur to the internal tuberosity of the femur. The line is more nearly through the centre of the lower extremity of the femur. 
The muscles which strengthen the joint are as follows:

Above, the rectus and gluteus minimus. In front, the psoas magnus and iliacus internus. Internally, the pectineus and obturator internus. Behind, the outward rotators of the thigh, obturators, internus and externus, pyriformis, the gemelli and the quadratus femoris. Or, beginning at random and naming them in the order in which they lie around the joint, we have, gluteus minimus, rectus, iliacus internus, psoas magnus, pectineus, obturator externus, quaratus femoris, gemellus inferior, obturator internus, gemellus superior and pyriformis.

The arteries supplying the joint are derived from the obturator, sciatic, internal circumflex and gluteal.

The nerve supply for the joint is drawn from articular branches from the sacral plexus, great sciatic, obturator, and a filament from the branch of anterior crural supplying the rectus muscle.

\section{The Knee Joint.}

This is the largest and for many reasons the most important joint in the body. By the formation of the bones it is the weakest joint in the body, its strength consisting entirely in the powerful bands of ligamentous fibres and the muscles by which it is reinforced. It is usually classed as a "hinge joint", being the analogue of the elbow; but it is really no more of a hinge than the elbow is a pure hinge. In formation it is very complex, being a double condyloid joint, combined with a trochlea, and possessing three articulations. Its articulations may be extended to four, as some of the cartilages which enter into the formution of the joint are so arranged as to establish an articulation between themselves and the bones to which they are attached. The condyles and their receiving cavities permit all of the movements belonging to the usual condyloid articulations. The mistake of calling the knee a hinge joint arises from the fact that the classification of joints is based on the movenents of which the joints are capuble and not on the shape of the bones which enter into their formation.

The Bones which compose the knee joint are the two condyles of the femur, the two glenoid cavities on the upper extremity 
of the tibia, and the posterior surface of the patella. The ligaments which bind the bones together are divided into two sets: the external, or exterior ligaments, and the interior ligaments.

The Exterior Ligaments consist of an anterior, a posterior, an external lateral, and an internal lateral.

The Anterior Ligament, or Ligamentam Patella, the most powerful of all the bands of ligamentous fibres attached to the joint, is nothing more than the tendon of the Extensor triceps cruris. This muscle passes downward until it reaches the upper border of the patella. There the fibres become tendinous, and spread out to embrace the patella, passing all around it, some being attached to its upper border, others extending so as to run down upon its lateral borders, while others run upon its anterior surface, the posterior surface being left free.

This tendon passes downward until it reaches the lower portion of the anterior tubercle, where it is inserted, leaving a little space between itself and the upper portion of the tibia,

As the ligamentum patella descends towards its insertion, it does not diminish in size; but it gives off from its lateral aspect tendinous fibres, sometimes spoken of as the lateral patellar ligaments, which pass around until they reach the joint; in other words, they form a complete capsule for the knee joint. This can be stated of every joint which carries a synovial membrane.

The Posterior Ligament of the joint, one of the most peculiar in the body, is a set of fibres running in different directions. In one way or another these are attached above the condyles of the femur. Some are attached at those depressions where the Gastrocnemius and the Plantaris rise; and then extend across the upper margin of the condyloid notch. The central fibres extend further up on the shaft of the bone; but these instead of running downward, run backward and outward. The central and enlarged portion of the attachment of the Semi-menbranus muscle is inserted into the front of the femur, and spreads upward and backward, consequently forming the middle portion of the posterior ligament of the 
joint. Where the central fibres come in touch with the external head of the Gastrocnemius muscle, they blend with the fibres of that muscle. The lower fibres of the ligament are attached to the posterior surface of the tibia.

The Internal Lateral Ligament is attached above to the inner tuberositv of the femur; passes downward and is attached to the internal tuberosity of the tibia; - but the fibres continue down to the internal face of the tibia.

The External Lateral Ligament consists of two bundles of fibres; and is described by some as having the same attachment below and a different attachment above. It is, properly speaking, all one ligament. Some anatomists assert that the muscle which passes over the joint constitutes the greater portion of the ligament, while others say that it does not enter into it at all. The origin of the Popliteus muscle and the origin of the long external lateral ligament of the knee joint are precisely the same thing. They are attached to the little groove on the outer surface of the outer tuberosity of the femur. From that point the external lateral ligament descends to become attached to the styloid process of the fibula.

The remainder of it is nothing further than the tendon of origin of the Popliteus muscle.

The interior of the joinl consists of a set of fibres, which are not ligaments, but are cartilages, without any function in binding the bones together. They serve mainly to deepen the cavity, but are very peculiar in their arrangement, and have an entirely different function to perform. There is a synorial membrane between the cartilages and the tibia; and it is between the semilunar cartilages and the upper extremity of the tibia that the fourth articulation, mentioned above as belonging to the knee joint, takes place. In the interior of the joint there are a pair of ligaments, or cartilages, the Semi-lunar carlilages; running across at the anterior aspect of the joint from one cartilage to the other is a small band forming a Tranverse ligumenl; binding these cartilages down to the bone are a set of very short fibres, known as the Coronary Ligament; then, in the interior of the joint, are seen two 
strong cords which cross each other somewhat in the form of the letter X, one called the Anterior, or External, Crucial Ligament, the other the Posterior, or Internal, Crucial Ligament. In addition we have the folds of synovial membrane, one called the Ligamentum Mucosum, and the other the Ligamenta Alaria.

The attachment of the Semi-lunar cartilages is one of the most important things, both anatomically and surgically, in connection with the knee joint. The External is nearly circular, being but slightly oval in its outline; the Internal, on the contrary, is distinctly oval, being longer from before backward than from side to side. The external is attached just in front of the spine of the tibia; and, in the prepared specimen, is attached just between the spine and the anterior crucial ligament, blending with the anterior crucial. Behind, it is attached between the crucial ligament and the internal cartilage, so that it blends at both extremities with the crucial ligaments. The internal semi-lunar cartilage, which is much longer than the external, passes around so as to be attached in front of the anterior crucial ligament. It does not blend with that ligament at all; and, behind, the internal cartilage is not attached to the crucial ligament. The cartilage can be raised up in any direction, but is attached to the bone by the fibres of the capsular ligament of the joint. In truth it is not really attached to the bone, except just in front and just behind the anterior spine of the tibia. On the contrary, all of the fibres which enter into the formation of the ligaments of this joint, with the exception of those composing the Ligamentum patellæ, become attached to the edges of the semi-lunar cartilages; and from those edges they pass down as short fibres to seek their insertion in the tibia. They bind the cartilages in position, but leave them so as to be movable.

The Anterior Crucial Ligament takes its origin from a point just in front of the spine of the tibia. From that origin it passes upward, outward, and backward; and is inserted into the internal face of the external condyle of the femur.

The Posterior Crucial Ligament arises from behind the spine of the tibia; and is blended with the posterior extremity 
of the external semi-lunar cartilage. From that origin the direction of its fibres is upward, forward and outward; and consequently the two crucial ligaments cross each other in the joint. When they come in contact with each other, the posterior crucial ligament gives off a slip of fibres which decussates with the anterior crucial ligament. The posterior ligament then passes on to its insertion in the external face of the internal condyle of the femur.

The folds of the Symovial Membrane are mixed with fat.

The Ligamentum Mucosum is found attached to the front of the inter-condyloid notch; and passes from that point, lying between the patella and the inter-condyloid notch of the femur, down to become attached to the lower extremity of the patella.

From the lateral edges of the ligamentum mucosum are sent off the fringe-like folds with masses of fat, spoken of as the Ligamenta Alaria. The synovial membrane is the largest in the body, and extends to surrounding structures. The membrane passes down from the articular surface of the femur; and reaches the ligamentum mucosum, which has a few ligamentous fibres. It then ascends and reaches the patella on the posterior surface of the anterior ligament; but a lateral view of it would continue its course down until it reached the upper surface of the tibia. It is a continuous sack. The lateral subdivisions pass downward; and leaving the femur, pass upward as far as the articular surface extends in the mid-line, including the crucial ligaments. Traced forward from the crucial ligaments, the folds pass over the upper surface of the tibia, run over and line the semi-lunar cartilages, and then pass between the cartilages and the tibia, so as to form a sack. As the fold passes downward, the crucial ligaments are left out of the joint, another proof that must at one time have been separate and distinct articulations. While the synovial membrane is all one continuous sack, there is a space above the articular surface of the femur, which covers the non-articular surface, extending to the point where the attachment of the Gastrocnemius commences. The membrane surrounds and embraces the crucial ligaments 
and cuts them off from the joint; it extends down over the patella and the Ligamentum patellæ; passes over the tibia, and in addition lies between the semi-lunar cartilages and the upper surface of the tibia, forming a movable surface between the two. The membrane frequently communicates with little bursæ which are found around this joint.

The External Cartilage, just before it reaches its attachment, extends its transverse band across to become attached to the Internal Cartilage, thus forming the Transverse ligament of the joint.

Between the Ligamentum patelle and the upper surface of the tibia, there is a little sack, which does not communicate with the joint, but forms a bursa, so as to have a freely gliding tendon over the upper extremity of the tibia. At the back of the joint, beneath the two heads of the Gastrocnemius muscle, are found two more bursa. Beneath the tendon on the inner side is found another; and on the outer side, beneath the tendon of the Popliteus, is found another. Any one of these may, and frequently do, communicate with the joint; hence they are known as the Synovial Bursce. Between the skin and the front of the patella is another bursa, which can be easily found in the healthy man and which does not communicate with the joint. It is a mucous bursa.

Actions. This joint never makes the movement of a pure "hinge joint." If placed in a position of extreme flexion, the posterior portion of the condyles rests upon the posterior part of the semi-lunar cartilages. Place the knee in a position of half-flexion, and the central portion of the cartilage then rests upon the central portion of the condyle, the axis of rotation having changed so that the tibia has been carried forward about half an inch. Bring the leg further forward, and the axis of rotation will have been brought further forward. When the joint is drawn into a position of extreme flexion, the toe is bent only slightly forward; when we pass from extreme flexion to extreme extension there is an inward rotation, which brings the toe out until is nearly in a straight line. That is, there is, in addition to the rotation of flexion, an axial rota- 
tion, except for the fact that it takes place on two points instead of one. In addition to the movement between the femur and the tibia, there is, therefore, an actual rotation between the semi-lunar cartilages and the tibia. Besides these, there is an incomplete rotation, a movement between the cartilages and the femur ; and at the same place a constant movement of the patella on the femur. When the limb is in extreme flexion, only the lower portion of the patella is in contact with the femur; when drawn up, the central or bulged portion, is in contact with the femur; and in extreme extension, the upper portion is in contact with the femur. There is a line extending from the centre of the patella vertically and another transversely; and some say there is a third line, which completes the division of the patella into six separate compartments.

The Muscles which strengthen the articulation are, primarily, the Triceps extensor cruris; behind, the two heads of the Gastrocnemius and the Plantaris; on the outer side, the tendon of the Biceps flexor cruris; and on the inner side, the Semi-tendinosus, which is in reality the only one that strengthens the joint internally. The Popliteus powerfully strengthens the joint upon its outer side; and it is a powerful strengthening agent on its posterior aspect.

The arterial supply of the joint is furnished by articular branches from the popliteal, by the anterior and posterior recurrent branches of the anterior tibial, by the anastomotica magna branch of the femoral and by the descending branches from the external circumflex.

The nerve supply is derived from the external and internal popliteal, the obclurator and from the anterior crural.

\section{The Tibio-Fibular Articulation.}

The upper and lower extremities of these bones are held together by ligamentous fibres, while there is a strong interosseous membrane extended between their shafts.

The superior libio-filular joint is of the arthrodial variety and the bones are held together by anterior and posterior ligamentous fiures, passing directly between the contiguous sur-

Des Anat-10) 
faces of the two bones. They are united by a few scattered fibres to protect the synovial membrane, forming an imperfect capsule. The synozial membrane sometimes communicates with the knee joint.

The inferior tibio-fibular joint is an articulation of the amphiarthrodial class. A rough surface is presented by each of the bones for the reception of the powerful interosseous ligament which passes between them. There are also anterior and posterior fibres passing from one bone to the other, forming the anterior and posterior inferior tibio-fibular ligaments. The lower part of each bone presents a small smooth surface, coated with cartilage in the recent state whose synovial membrane communicates with the ankle joint.

The interosseous membrane is composed of oblique fibres directed downward and outward and extending from just below the knee to within a short distance of the ankle. These fibres are attached to the interosseous ridges on the two bones. Above the membrane is seen an oval aperture for the transmission of the anterior tibial vessels. At its lower portion the membrane is perforated for the passage of the anterior peroneal artery.

Movements. A slight gliding movement only is permitted between the two bones above while below there is practically no movement, the little permitted being confined to a slight twisting of the fibres of the interosseous ligament.

\section{The Ankle Joint.}

This joint belongs to the movable class and hinge or, more correctly, the mortise and tenon variety. Its articular surfaces are contributed by the lower extremities of the tibia and fibula, above, and the upper surface of the astragalus below.

The ligaments consist of anterior, posterior and two lateral.

The anterior, thin and fatty, is attached above to the front of the tibia just above its articular surface and below to the upper surface of the neck of the astragalus.

The posterior, more insignificant than the anterior, is mainly for the protection of the synovial membrane. It consists of a few scattered fibres attached above to the tibia and below to the os calcis. 
The internal lateral or deltoid ligament, is a dense and powerful mass of fibres which embraces the tip of the internal malleolus by its apex and by its broad base is attached to the tuberosity of the scaphoid, to the inner face of the astragalus, below the articular facet, and to the os calcis, where it also serves to bind the astragalus and the os calcis together. On section this ligament is seen to consist of two layers.

The external lateral ligament, usually described as one, is really three separate ligaments. The anterior fasciculus, the weakest, is attached above to the anterior border of the external malleolus and passing downward and forward reaches the upper, outer aspect of the astragalus. The middle, passes from the tip of the malleolus vertically downward to the tubercle on the outer face of the os calcis. The posterior is attached externally to a deep depression on the inner face of the malleolus and passes horizontally inward to seize the outer face of the astragalus behind its articular facet.

The synorial membrane is extensive and lines the inner face of the ligaments as well as the bones.

The joint is strengthened behind by the tendo Achilles, which takes the place of a posterior ligament. Antero-internally is the tibialis anticus, postero-internally the tibialis posticus while externally are the two peroneal tendons. The flexor and extensor tendons are too lax to add any strength to the joint.

The malleolar arteries supply the joint with blood while the anterior and posterior tibials give it its nervous supply.

The morements are flexion and extension, although in one position a slight degree of lateral movement is permitter. In flexion the foot is drawn up toward the leg, the articular surface of the astragalus moving slichtly back ward in the receiving cavity. Fxtension is the reverse of flexion. In forced extension the narrow part of the astragalus is brought forward into the broadest part of the receiving cavity, and in this position a slight degree of lateral movement is permitted.

\section{The Articulations of the Tarsus.}

The articulations of the tarsus consist of the articulation between the bones of each row and the union of the two rows. 


\section{Calcaneo-Astragaloid Articulation.}

The two bones of the first row of the tarsus are held together by three ligaments, external, posterior and interosseous.

The external calcaneo-astragaloid ligament extends from the outer side of the astragalus, just behind and internal and as far as the anterior fasciculus of the external lateral of the ankle, to the outer face of the os calcis. It lies between the anterior and middle fasciculi of the external lateral.

The posterior calcaneo-astragaloid passes from the posterior extremity of the astragalus to the contiguous upper aspect of the os calcis and is but a continuation of the protecting fibres, called the posterior ligament of the ankle.

The interosseous is by far the most important bond of union between the two bones. It consists of a large number of fibres, filling the sinus tarsi, and passing directly and obliquely between the surfaces which form that canal.

\section{The Articulation Between the Bones of the Second Row.}

The bones of the second row are held together by fibres passing across the dorsal and plantar faces and by interosseous fibres between opposing surfaces.

\section{The Articulation Between the two Rows.}

The two rows of the tarsus are held together by three sets of ligaments, two sets passing from the os calcis to the cuboid and scaphoid, though the latter does not articulate with it, and one set passing between the astragalus and scaphoid.

\section{Calcaneo-Cuboid Articulation.}

The ligaments binding the os calcis to the cuboid are four, two dorsal and two plantar.

The superior calcaneo-cuboid is thin and narrow and passes between the contiguous surfaces of the bones on the dorsum of the foot.

The internal calcaneo-cuboid, or interosseons, is a thick short band of fibres springing from the depression between the os calcis and the astragalus and inserted into the inner side of the cuboid. At its origin it is closely blended with the superior calcaneo-scaphoid. 
The long mferior calcanco-cuboid ligament is attached behind to the inferior surface of the os calcis from its posterior to its anterior tubercle; and in front to the peroneal ridge on the under surface of the cuboid and to the bases of the metatarsal bones of the second, third and fourth toes. It is much thicker and stronger, as well as longer, than the short inferior calcaneo-cuboid, which lies immediately above it.

The short inferior calcaneo-cuboid ligament is about one inch in length. It is attached to the anterior tubercle of the os calcis and to the bone in front of it as far as the margin of the articular surface; and, by its anterior extremity, to the under surface of the cuboid behind the peroneal ridge.

\section{Calcaneo-Scaphoid Ligaments.}

Connecting the os calcis and scaphoid are two ligaments, superior and inferior calcaneo-scaphoid.

The superior, one arm of the $\mathrm{Y}$ ligament, the other arm being furnished by the internal calcaneo-cuboid, passes forward and inward from the front upper part of the os calcis to the scaphoid.

The inferior calcaneo scaphoid is much stronger than the superior and passes from the inner front aspect of the os calcis, beneath the head of the astragalus, to the lower surface of the scaphoid.

\section{The Astragalo-Scaphoid Articulation.}

The only ligament connecting the astragalus and the scaphoid is the superior astrugalo-scaphoid which is thin and weak and passes from the neck of the astragalus to the upper surface of the scaphoid.

\section{The Tarso-Metatarsal Articulation.}

The first metatarsal bone articulates with the internal cuneiform ; the second, with the middle cuneiform, by its base, and laterally with the internal and external cuneiform bones, being jammed between these and extending farther back than the other metatarsal bones; the third articulates with the external cunciform, and the fourth and fifth with the cuboid.

The tarsus is held to the metatarsus by dorsal and flanlar ligramentous fibres and by three interosseous ligraments-one 
from the internal cuneiform to the second metatarsal and one from the external cuneiform to the third metatarsal.

The metatarso-phalangeal, the inter-phalangeal and the " "intermetatarsal" are exactly similar to those of the hand.

\section{Costo-Vertebral Articulation.}

The parts of the skeleton which enter into a costo-vertebral articulation are the posterior extremity of the rib, the body, and transverse process of a dorsal vertebra and the intervertebral disk.

This articulation is of the diarthrodial class and the arthrodial variety. The ligaments which connect the head of the rib with the vertebral column are the anterior costo-vertebral, capsular and interarticular.

The anterior costo-vertebral is attached to the anterior aspect of the head of the rib, and radiates in three bundles, the upper of which is attached to the body of the vertebra above, the lower to the vertebra below, and the middle to the intervertebral disk. Owing to the divergence of its fibres, this ligament is known as the stellate. The arrangement differs from the foregoing description in the first, eleventh and twelfth ribs, where the ligament is attached to only one vertebra, as the articular facet is furnished wholly by one vertebra.

The capsular ligament consists of a few scattered fibres around the articulation.

The interarticular ligament is attached to the ridge on the head of the riband to the intervertebral disk, thus dividing the joint into two parts, each furnished with a separate synovial sac. For the reason above given, the first eleventh and twelfth have no interarticular ligament and but one synovial sac.

The articulation between the ribs and the transverse processes, known as the costo-transversearticulation, presents three ligaments-anterior, middle and posterior costo-transverse.

The anterior costo-transverse extends from the neck of the rib to the transverse process above; the middle, or interosseous, extends from the posterior surface of the neck of the rib directly backward to the transverse process; the posterior extends from the tubercle of the rib to the apex of the transverse process. 


\section{THE VISCERA.}

\section{The Alimentary Canal and Appendages.}

The alimentary canal begins at the mouth and ends at the anus, the intermediate portions being found in the neck, thorax and abdomen. In the neck are the fances, pharynx and part of the asophagus; in the thorax is the remaining portion of the oesophagus, while the rest of the canal is found in the abdomen.

\section{The Mouth.}

The mouth begins at the lips and terminates behind in a short constricted portion called the fauces. It is bounded above by the hard palate, below by the tongue and on each side by the cheeks.

The lips are two, upper and lower, separated by the transverse labial fissure. Each is formed chiefly by its segment of the orbicularis oris muscle, covered externally by skin and internally by mucous nembrane, a concentric fold of which, called fronum labii, extending in the middle line from each lip to the gum behind. The cheek consists chiefly of the buccinator muscle, covered externally by skin and internally by mucous membrane, which is reflected from it to the gum and presents, opposite to the second molar tooth of the upper jaw, the opening, on a papilla, of Stenson's duct from the parotid gland. The hard palate is formed anteriorly by the meeting in the middle line of the palate processes of the superior maxillary, and posteriorly by the horizontal plate from each palate bone. It is bounded"in front and at either side by the aveolar processes of the superior maxillary bones, containing the teeth of the upper jaw. It is covered by mucous membrane both above and below, forming the floor of the nasal fossie above and the roof of the mouth, below; on the latter aspect the mucous membrane is roughened by grands, called palatal, and is continuerl on to the grums. 
The floor of the mouth is formed by the anterior two-thirds of the tongue, the posterior third the tongue forming the floor of the fauces, and, below that, entering into the formation of the anterior wall of the pharynx.

The tongue is flattened from above downward, is conical in shape and curved in direction, being convex above anteroposteriorly, and extends from the hyoid bone behind to the incisor teeth in front, its base being adherent to the hyoid bone and its apex free and anterior.

The mucous membrane covering the dorsum of the tongue passes around the sides and tip, thus enveloping most of the organ, and leaves the lower surface to become continous with that lining the gum of the lower jaw. In contact with the posterior part of the dorsum is the front of the epiglottis, which is held to it by three folds of mucous membrane, the glosso-epiglottic ligaments, middle and two lateral.

The mucous membrane covering the tongue and mouth is continued back through the fauces to blend with that which lines the gullet.

\section{The Fauces.}

The fauces, or isthmus of the fances, is that narrow part of the alimentary canal which connects the mouth posteriorly with the front of the pharynx. It is about one and one-half inches long, about the same in width, and rather less in depth; the size, however, varying greatly, for its walls are chiefly formed of muscular tissue, which is peculiarly subject to reflex action.

The roof of the fances is the soft palate; the floor is a part of the dorsum of the tongue; the sides are formed by two arching muscles, and, between them, the tonsil glands. The muscle in front is the palato-glossus, forming the anterior pillar of the fauces; that behind is the palato-pharyngeus, forming the posterior pillar of the fauces. The wall, in every aspect, is covered by mucous membrane.

\section{Soft Palate.}

The soft palate, or velum pendulum palati, is thin and flattened from above downward and forward. It is attached 
above to the posterior border of the hard palate and hangs downward and backward, separating the fauces from the upper part of the pharynx. It is prolonged, in the middle line below, by a nipple-like projection, some half an inch long, called the uvula.

\section{Pharynx.}

Food, in order to reach the stomach, passes through four successive portions of the alimentary canal. The first two of these have been described, viz: the mouth and fauces. Leaving the latter, food enters the pharynx, whence it passes to the (esophagus, in which the pharynx terminates, and the nesophagus conveys it to the stomach.

The Pharynx is an oblong tube situated behind the larynx and extending from the lower face of the basilar process of the occipital to the fifth cervical vertebra where it terminates in the nesophagus. By the lower margin of the soft palate it is divided into an upper portion, establishing communication between the nasal cavities and the larynx below, and the same cavities and the middle ear on the side, through the Eustaschian tube; and a lower portion receiving the opening of the fauces above and opening into the esophagus below. By its musculo-aponeurotic fibres is attached to all of the surrounding firm structures, base of the occipital bone, pterygoid processes of the sphenoid, base of the tongue, hyoid bone, inferior maxillary and the larynx. From the occipital down to the attachment to the cartilages of the larynx it is a large cavity always held open by its attachment to the firm surrounding structures; but from the larynx to its termination, the anterior and posterior walls are more or less closely in contact, except during the act of swallowing. The muscular wall is deficient above and behind, where a concave space is seen on either side above the superior constrictor where the pharyngeal aponeurosis is thicker than elsewhere. In the anterior wall, above the soft palate, the openings of the posterior nares are seen on either side; while further out and opposite the posterior extremity of the inferior turbinated bones, may be seen the openings of the Eutaschian tubes. 
Below the soft palate the opening for the fauces ocupies the whole of the anterior wall, while below this is the antero-posterior opening of the larynx, behind which is the transverse, slit like opening into the osophagus. The pharynx is separated from the front of the bodies of the upper five cervical vertebræ by a layer of the deep cervical fascia and some loose connective tissue. It is about four and one half inches in length and tapers as it descends.

\section{Structure.}

The Alimentary Canal is throughout made up of an internal mucous coat, resting upon a thin fibrous layer which is in turn supported by muscular fibres. In the tongue, soft palate and pharynx the muscular coat makes up the bulk of the organ, but the arrangement differs in each and will now be described.

The Tongue consists of two sets of fibres, called intrinsic and extrinsic muscles of the tongue. The extrinsic muscular fibres seek points of attachment to surrounding hard parts, their lingual extremities then passing into the tongue to blend with the intrinsic muscles found there. These muscles are the following:

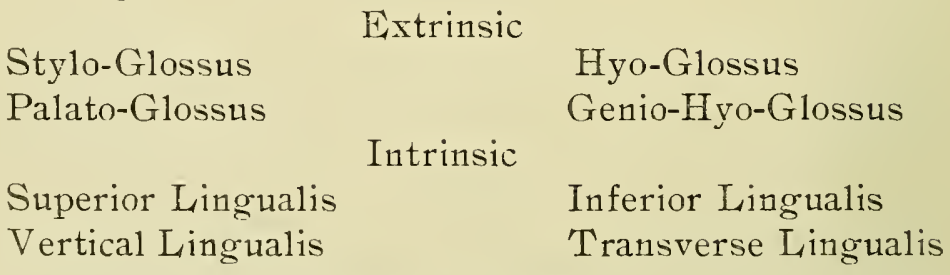

The Stylo-Glossus arises from the anterior and outer side of the styloid process of the temporal bone near its apex, and from the stylo-maxillary ligament, and passing downward, forward and slightly inward is inserted into the side of the tongue, its fibres running as far as the tip where they meet with the muscle of the opposite side. The fibres of this muscle blend with those of the superior and inferior lingualis.

The Hyo-Glossus is a flat quadrilateral muscle which arises from the side of the body and both cornua of the hyoid bone, 
passes directly upward and is inserted into the whole length of the tongue, to the inner side of the Stylo-Glossus, its fibres blending with the vertical lingualis.

The Palato-Glossus is a small muscle found occupying the anterior fold of the fauces. Arising in the soft palate it passes downward and inward and is inserted into the side of the tongue, its fibres blending with the transverse lingualis.

The Genio-Hyo-Glossus is a thin, radiated, fan shaped muscle found lying close beside its fellow near the mid line and extending from base to apex of the tongue. It arises by its apex from the superior genial tubercles and is inserted into the whole length of the tongue, some of its posterior fibres being attached to the body of the hyoid bone. Its fibres blend with the vertical lingualis, running between the fibres of the longitudinal and transverse lingualis.

These muscles are all, except the Palato-Glossus, supplied by the hypoglossal nerve. The Palato-Glossus is supplied by branches from the pharyngeal plexus. The lingual artery is the source of the blood.

The Tongue is divided by a vertical fibrous septum which occupies the middle line and receives the insertion of the transverse lingualis muscle. The intrinsic fibres, largely derived from the extrinsic, are two superficial sets, superior and inferior, which extend from base to apex of the organ, and a transverse and vertical set, the one running from the upper to the lower surface and the other from the septum to the lateral edges. These latter fibres are mingled with each other so as to form a muscular net work extending througliout the tongue.

Aclion. The intrinsic musclescan act only to produce chances in the shape of the tongue, while the extrinsic not only chance the shape but the position as well. The Stylo-Glossus cin draw the tongue upward and backward, or, if one muscle alone acts, upward, backward and toward the side of the muscle acting. The //yo-Glossus draws the tongue downward and backward, increasing the sime of the fauces and aiding the intrinsic fibres in shortening the organ. The Palalo-Glossus widens the tongue and elevates its base, depresses the soft 
palate and constricts the fauces while the Genio-Hyo-Glossus draws the tongue forward by its posterior fibres, shortens it by the approximation of posterior and anterior, while the central fibres depress the organ and make the dorsal face concave. The lower fibres can draw upward and forward the hyoid bone.

\section{Muscles of the Soft Palate.}

Levator Palati

Azygos Uvulæ
Tensor Palati

Palato Glossus

\section{Palato-Pharyngeus}

The Palato-Glossus was described with the muscles of the tongue.

\section{Levator Palati.}

The levator palati arises from the rough surface on the basilar face of the petrous bone, near its apex, and from the cartilaginous portion of the Eustachian tube, and descends to be lost in the soft palate. Its name indicates its action.

\section{Tensor Palati.}

The tensor palati is a small muscle which forms a right angle on itself, hence its synonym of circumflexus. It arises from the scaphoid fossa of the pterygoid process, from the spinous process of the sphenoid bone and from the cartilaginous portion of the Eustachian tube, and, first descending vertically, then turning transversely inward around the hamular process, it spreads out into a broad aponeurosis in the soft palate. Its action is indicated by its name.

\section{Azygos Uंvulæ.}

From the posterior termination of the soft palate there hangs pendulous, in the centre, a short, conical mass called the uvula, which, when dissected, is found to consist, exteriorly, of mucous membrane and, interiorly, of a. pair of minute muscles, each of which, arising from the palate spine, descends beside its fellow and is lost in the mucous membrane of the uvula. These two muscles were formerly considered as one, hence their name. It is their presence in the uvula which accounts for its constant and varied movement. 


\section{Palato-Pharyngeus.}

The Palato-Pharyngeus arises in the soft palate and passes downward and backward to enter the wall of the pharynx, where some of its fibres are lost, the rest being inserted into the posterior border of the thyroid cartilage. Its action is the same as the preceding muscle and its synonym is constrictor isthmii faucium posterior.

\section{The Muscles of the Pharynx.}

The Pharynx is lined by mucous membrane, resting on a thin fibrous coat. External to the mucous coat is a muscular coat consisting, like that of the intestines, of circular and longitudinal fibres, but differing in that the muscular fibres are here red and not pale, and are gathered into bundles which are described as separate muscles. The circular fibres are arranged in flattened bundles on each side, each bundle intersecting its fellow of the opposite side on the middle of the back of the pharynx where they form a raphe. Each pair of bundles overlaps the lower edge of the pair above. When traced from the raphe forward it is seen that these fibres do not encircle the entire pharynx but pass from its sides to seek firm neighboring points of attachment, from which to exert traction, thus leaving the front of the pharynx devoid of muscular fibres, this part of its wall being formed by mucous membrane alone. The larynx resting on the front of the pharynx, is in contact with the mucous membrane and is embraced by these circular fibres. These muscles, from their action, are known as the constrictors of the pharynx; and as there are three separate bundles, they are described as superior, middle and inferior constrictors. The direction of their fibres is not horizontally back ward but back ward and upward.

\section{Inferior Constrictor.}

The inferior constrictor arises from the oblique line on the ala of the thyroid cartilage and surface behind it, from the side of the cricoid cartilage and from the two upper rings of the trachea. It runs backward and upward, its upper fibres 
passing obliquely upward, overlapping the middle constrictor and is inserted into the raphe of the pharynx.

\section{Middle Constrictor.}

The middle constrictor arises from both cornua of the hyoid bone, and from the stylo-hyoid ligament, a fibrous cord extending from the hyoid bone to the styloid process. The fibres radiate somewhat, spreading out on the side of the pharynx, and are inserted into the raphe. The inferior fibres are overlapped by the inferior constrictor, and the superior fibres overlap the superior constrictor. So great is this overlapping that when viewed from behind but little of the superior constrictor can be seen, the raphe of the middle constrictor extending to the basilar process.

\section{Superior Constrictor.}

The superior constrictor lies just beneath the skull and is overlapped by the middle. It arises from the lower one-third of the internal pterygoid plate, from its hamular process, from the pterygo-maxillary ligament, from the extremity of the molar ridge of the lower jaw, and by a few fibres from the side of the tongue. It passes backward and is inserted into the raphe, which is attached to the basilar process. The upper edge of this muscle presents a concave border, thus leaving an arched interval between it and the skull above, over which the fibrous tissue beneath the mucous membrane of the pharynx extends and is here so much thickened as to form a strong fibrous membrane.

The longitudinal fibres of the pharynx are, like the circular, gathered into bundles, of which there are two pairs. These are described as distinct muscles arising from points above the pharynx and inserted into it. One of them, the palato-pharyngeus, has been described as a part of the softpalate; the other is known as the stylo-pharyngeus.

\section{Stylo-Pharyngeus.}

The stylo-pharyngens arises from the inner side of the base of the styloid process and descends to the side of the pharynx, 
entering its wall between the superior and middle constrictors. Some of the fibres are lost in the pharynx. while some are continued to the posterior border of the thyroid cartilage. Action-it elerates and widens the pharynx.

\section{Esophagus.}

The OEsophagus continues the course of the alimentary canal from the termination of the pharyux, at the commencement of the trachea on the front of the body of the fifth cervical vertebra, down the front of the vertebral column, through the neck and thorax, to pierce the diaphragm at the nesophageal opening, opposite the tenth dorsal vertebra, and terminate at the cardiac orifice of the stomach. It is about nine inches long.

In structure the oesophagus resembles the pharynx, but its muscular coat is not arranged in distinct bundles. It is the narrowest part of the alimentary canal, but the most muscular.

\section{Relations of Pharynx and Esophagus.}

In addition to the structures mentioned as opening into or being connected with the Pharynx, it has important relations to structures on the side of the neck. Thus on either side are the muscles attached to the styloid process of the temporal and internal to these the internal jugular vein and internal carotid artery; and, between these two, the ninth, tenth and twelfth nerves, with the sympathetic behind. Below the parotid gland the external carotid artery becomes an external relation, while the lingual lies on the middle constrictor. Below the upper border of the thyroid cartilage the common carotid becomes the external relation.

\section{Relations of the Esophagus.}

The relations of the $C E s o p h a s u s$ must be studied in the neck and in the thorax. The rullet is about nine or ten inches in length, extending from the disk between the fifth and sixth cervical vertebra to the front of the body of the tenth dorsal. It is not a straight tube but presents antero-posterior curves corresponding to the curvatures of the spinal column. There 
are also three lateral curvatures-two to the leftand one to the right. At its commencement it lies on the middle line of the body but curves to the left until it reaches the root of the neck. From this point it curves to the right until it again reaches the middle line, opposite the fifth dorsal vertebra, and from there passes steadily to the left again until it terminates opposite the body of the tenth dorsal vertebra.

In the cervical, and upper part of the thoracic portions, the oesophagus is separated from the front of the vertebral column by a layer of fascia and by the longus colli muscles, particularly the muscle of the left side. In the lower part of the thoracic portion it is more widely separated from the vertebræ. The trachea lies in front of the œesophagus which is thus shut off from contact with the thyroid gland, except that the left. lobe of the latter touches the esophagus on the left side. The left and right common carotid arteries are lateral relations, the left being nearer than the right. At the root of the neck the thoracic duct is a left and slightly posterior relation while the left recurrent laryngeal nerve lies in the groove between the trachea and œsophagus.

In the thorax the œesophagus first lies behind the lower part of the trachea, which separates it from the transverse aorta, and then behind the bifurcation of the trachea and the left bronchus. Below the trachea the pericardium lies on the front of the esophagus to its termination. Laterally the gullet is in contact with the two pleuræ, although the left is a relation for a greater distance than the right. To the right above, and separated from it by the trachea, is the innominate artery, and below this, the vena azygos major is for a moment to the right side. On the left is the left common carotid artery, while the left subclavian is a more distant relation. The descending aorta becomes a left relation at the lower border of the fourth dorsal vertebra and remains a left relation to the upper border of the seventh dorsal, below which point the aorta is behind and finally behind and to the right. Behind are the longus colli muscle above, and the recurrent laryngeal nerve of the left side from the transverse aorta upward. The thoracic duct is a posterior relation throughout the thoracic 
portion, but, below the transverse aorta, lies in the interval between the aorta and the œsophagus. The vena azygos major is a posterior relation, while the azygos minor crosses behind, opposite the sixth dorsal vertebra. The right pneumogastric nerve lies behind while the left lies in front. The relation to the aorta is a fourfold one and should be thus stated, viz; the osophagus first has the transverse aorta in front, then the descending aorta first to its left side, then behind and, at the termination of the cesophagus, the aorta is to the right and behind.

\section{The Abdominal Viscera.}

The cavity of the abdomen is bounded above by the diaphragm, which is a thin arched muscle with its cavity down wards, forming the floor of the chest and the roof of the abdomen. The floor of the abdomen is the floor of the pelvis, $i . e$. the structures which close the outlet of the pelvis. Occasionally the floor is given as the brim of the true pelvis and the iliac fossa, the true pelvis being then considered a separate cavity Laterally and in front, from the lower ribs above to the ilium below, the abdominal wall is formed of soft tissues, muscles, \&c., and is the soft, fleshy front wall of the belly. At its upper part the cavity is partly circumscribed by the lower ribs-the last six or seven; below, by the bouy wall of the pelvis; behind, by the lumbar portion of the vertebral column; while on each side are, above, the floating ribs, below, the pelvic wall, and between the two, the soft tissues. For convenience of description the abdomen is arbitrarily divided into three zones, and each of these into three regions, by two horizonta 1 and two vertical imaginary lines. The upper horizontal line is drawn between the extremities of the ninth ribs: the lower between the anterior superior spinous processes of the ilia. The vertical lines, one on each side, are projected upvard from the middle of the fold of the groin.

The names of the nine regions are as follows: The central region in the upper zone is the Efigastric, the one on either side Mypochondriac, right and left; the central region in the middle zone is the Umbilical, the one on either side Lumbar,

Des Anat-11 
right and left; the central region in the lower zone is the Hypogastric, the one on either side, iliac, right and left.

The contents of the abdomen are the greater part of the alimentary canal, the accessory organs of digestion-liver, spleen and pancreas-and the genito-urinary organs-kidneys and supra-renal capsules, the bladder and its appendages, prostate gland, seminal vesicles and vas deferens. The testicles are considered with the abdominal viscera, although, in the adult, lying in the scrotum, outside the abdominal cavity. In the female there are the uterus and its appendages and the vagina. Enveloping most of these organs, and lining the walls of the cavity, is a serous membrane, called the peritoneum. The following are portions of the alimentary canal contained in the abdomen: 1st, the stomach; $2 \mathrm{~d}$, small intestine; 3d, large intestine. The stomach is the dilated part which succeeds the osophagus and is about twelve inches long. It terminates, about the line which separates the epigastric from the right hypochandriac region in the small intestine. The small intestine is divided into three parts, duodemum, jejumum and ileum. The duodenum is about nine inches long; the jejumm and ileum, together about twenty feet, the jejumum comprising the upper two-fifths and the ileum the lower three-fifths. The duodenum is again subdivided into three portions, viz: first portion, ascending, or oblique duodenum, is about two inches long and terminates at the neck of the gall bladder, on the lower surface of the liver, in the right hypochondriac region in the second portion, called the descending or perpendicular duodenum, which passes vertically downward for about three inches into the right lumbar region, about on a level with the upper border of the fourth lumbar vertebra, where it terminates in the third portion called the transverse duodenum, which is rather more than three inches long and crosses the front of the vertebral column obliquely upward and terminates at the left side of the body of the second lumbar vertebra, in the jejunum.

The large intestine is subdivided into three portions called cocum, colon and rectum. The cacum is the commencing 
two and one-half inches, lies in the right iliac fossa and terminates in the colon at the opening of the small intestine. The colon is divided into the ascending, transierse and descending colon and the sigmoid Hexurc. The ascending passes upward through the right lumbar region into the right hypochondriac. At the under surface of the liver it curves sharply to the left, forming the hepatic flexure of the colon, and becomes the transverse colon. The transverse colon passes down ward and to the left, crosses the abdomen between the epigastric and umbilical regions and rising in to the left hypochondriac region forms another abrupt curve at the lower end of the spleen, called the splenic flexure of the colon, and becomes the descending colon. The descending colon passes downward through the left lumbar region into the left iliac where it becomes the sigmoid flexure.

The sigmoid flexure is that portion of the gut which passes from the left iliac fossa into the pelvic cavity to the middle of the sacrum where it becomes the rectum.

The colon is about four feet in length. The rectum is the last four inches of the large intestine and terminates the alimentary canal at the anus, about the micidle of the floor of the pelvis.

The liver lies in the upper zone of the abdomen, stretching nearly across the cavity just beneath the roof. The spleen is in the left hypochondriac region. The pancreas lies transversely behind the stomach, between the spleen and perpendicular duodenum, crossing the front of the body of the first lumbar vertebra. Each of the two kidncys is beside the lumbar portion of the spinal column, on the front of the posterior abdominal wall. The urinary bladder and its appendagres are found in the pelvis just behind the os pubis. The ulerus and rasina in the female, are interposed between the rectum and bladder.

\section{Reflections of the Peritoneum.}

The peritoncum, being a serous membrane, is a closed sac, one laver covering the viscera, and called the visceral layer, and the other lining the walls and called the parietal layer. 
The existence of the peritoneum renders the motions of the viscera upon one another, and upon the abdominal walls, easy and harmless and furnishes bonds of connection between them and the walls of the containing cavity. Being a closed sac its continuity can be demonstrated by the fact that in following it, transversely or vertically, it can be traced back to the point of departure. The folds it makes from above downward are the most important and will be first stated.

It leaves the lower surface of the diaphragm in two layers, which pass to the upper and lower edge of the posterior border of the liver. The upper layer covers the upper surface of the liver, curves around the anterior border and coats the lower surface as far back as the transverse fissure, where it meets the lower layer, which has covered the lower surface from the posterior border to the transverse fissure. The two layers, having thus enveloped the liver, leave it at the transverse fissure and pass downward to the stomach, forming between these two organs the gastro-hepatic or the lesser omentum. They reach the stomach at its upper border and divide, one passing over the front and one over the back, and meet again at the lower border after furnishing a coat to the stomach. The two layers leave the stomach at its lower border and pass downward to to the transverse colon, pass over it without touching and descend almost to the brim of the pelvis, just behind the anterior abdominal wall; they then reverse their course, run upward, just posterior to their descending course, reach the transverse colon, and, separating, enclose it. That part of the peritoneum extending between the lower border of the stomach and the transverse colon is called the greater omentum. The two layers, having enveloped the transverse colon, meet at its posterior border and pass back to the posterior abdominal wall, where they form the transverse mesocolon, which loosely holds the transverse colon to the posterior abdominal wall. The two layers now finally separate; one goes up the posterior abdominal wall to the lower surface of the diaphragm, where its course was first taken up, thus partially enclosing a space behind the stomach called the lesser cavity of the peritoneum, 
which communicates with the general cavity through an opening behind the oblique duodenum, called the foramen of Winslow; the other layer passes forward to envelope the coils of the jejunum and ilium and returns to the posterior abdominal wall, forming a double layered fold, holding the intestine to the abdominal wall, called the mesentery. The attachment of the mesentery is thus stated: from the left side of the body of the second lumbar vertebra downward and to the right to the right sacro-iliac synchondrosis. After forming the mesentery the peritoneum descends the posterior abdominal wall to the brim of the pelvis passes down its posterior wall, covering the upper half of the rectum completely, leaves the front of the rectum an inch below its middle, strikes the back part of the base of the bladder, in the male, covers the back, sides and posterior half of the top of the bladder, and leaves the bladder to mount on the posterior face of the anterior abdominal wall and pass up it and reach its starting point on the lower surface of the diaphragm.

In the female, when the peritoneum leaves the front of the rectum, it passes to the lower wall of the vagina, covering its upper inch, then strikes the uterus passing up its back and turning down its front whence it passes to the bladder. In mounting over the top of the uterus it also passes over the ovary and its ligament, on each side, thus forming on each side a double layered fold stretching between the sides of the uterus and the lateral walls of the pelvis called the broad ligament of the uterus. Between these two layers are the ovary and its ligament, the round ligament of the uterus and the fallopian tubes.

Followed transversely around the abdomen, about its middle, the peritoneum presents the following folds: commencing on the anterior abdominal wall it can be followed to the posterior abdominal wall, where it reaches the descending colon and passes over it, covering it from half to threefourths around; and, leaving it at a corresponding line on the inner side, it reaches the vertebral column where it turns forward to envelope the folds of the jejunum and ilium and to pass back on itself to the vertebral column, forming the mes- 
entery; it then runs out on the posterior abdominal wall to reach the ascending colon, passes over its front, covering it from half to three-fourths around, and, leaving it, reaches the abdominal wall again along which it runs to the place of departure.

\section{Relations of the Abdominal Viscera-Stomach.}

The stomach lies in the upper zone, in the left hy pochond riac and epigastric regions, generally terminating in the duodenum on the line which separates the epigastric from the right hypochondriac region, but occasionally passing more or less into the latter. Its long axis is directed from above downward, from left to right and from behind forward; one surface looks forward, the other backward; one border, the shorter, or lesser curvature, upward, the other, the long, or greater curvature, downward; the large end is to the left, the small, to the right. It is continuous with the duodenum at the right end. Its upper border is held to the lower surface of the liver by the lesser omentum; its lower border is indirectly held to the transverse colon by the greater omentum; its left end is connected with the spleen by the gastro-splenic omentum. Above it are the diaphragm and the left lobe of the liver; below is the transverse colon, indirectly held to it by the great omentum; to the right is the duodenum; to the left is the spleen, whose concave inner surface is in contact with the convex left extremity of the stomach; behind is the pancreas, immediately above which is the coeliac axis with its branches and the semi-lunar ganglia, and behind these is the abdominal aorta; in front is the anterior abdominal wa1l, partly separated from the stomach by the left lobe of the liver. It has a complete peritoneal coat which forms for it three ligaments, the lesser omentum holding it to the liver, the great omentum suspending the transverse colon and the gastro-splenic omentum binding together the spleen and the stomach.

\section{The Oblique Duodenum.}

The oblique, or ascending duodenum, commences at the right extremity of the stomach, usually on the line which sepa- 
rates the epigastric from the right hypochondriac region, and passes upward and to the right, in the latter region, to terminate in the descending duodenum at the neck of the gall bladder on the lower surface of the liver. It lies in the right border of the lesser omentum and has behind it the hepatic artery, the common bile duct and the portal vein. The duct is furthest to the right, the artery to the left while the vein lies between and behind the two. In front and above is the liver. It is completely invested by peritoneum.

\section{The Perpendicular Duodenum.}

The perpendicular, or descending duodenum, commences at the termination of the oblique duodenum, at the lower surface of the liver, at the end of the gall bladder in the right hypochondriac region, and descends vertically iuto the lumbar region to terminate in the transverse duodenum, about on a level with the upper border of the fourth lumbar vertebra. It lies behind the peritoneum, being covered by it only in front. Behind it has the right kidney and in front the ascending colon; to the left it is adherent to the head of the pancreas.

\section{The Transverse Duodenum.}

The transverse duodemum commences where the oblique terminates, in the right lumbar region, on a level with the upper border of the body of the fourth lumbar vertebra, and passing upward and to the left, across the body or the third lumbar vertebra, terminates in the jejunum at the left side of the body of the second lumbar vertebra. It lies behind the peritoneum, between the diverging layers of the transverse meso-colon, so that it is covered by peritoneum only in front. Above it is the lower border of the pancreas, from which it is separated by the superior mesenteric artery and vein; in front is the transverse colon.

\section{The Jejunum and Ileum.}

The remaining twenty feet of the small intestine, following the duodemum, are thrown into coils called the convolutions of the small intestine, and are found chiefly in the umbilical and hyporgastric regions, falling off, however, into 
surrounding regions, some being always found in the pelvis between the rectum and bladder in the male and the rectnm and uterus in the female. The jejumm comprises the upper two-fifths of the intestine and begins where the duodenum terminates, at the left side of the body of the second lumbar vertebra. The ileum is the lower three-fifths, and terminates in the right iliac region by opening into the large intestine two and one-half inches above its commencement.

The convolutions are completely enveloped by the peritoneum, which holds them by a double-layered fold to the posterior abdominal wall. In front they are separated from the anterior abdominal wall by the great omentum; above is the transverse colon; to the right the ascending, to the left, the descending colon.

\section{The Cœcum.}

The cocum is the commencement of the large intestine. It is two and one-half inches long and terminates in the colon at the opening of the small intestine. It lies in the right iliac fossa or region, and is generally completely invested by peritoneum. Attached to it is the vermiform appendix, a blind tube about the size of a goose quill and from four to six inches long. It opens into the inner back wall of the cœeum, just below the opening of the ileum, and not into the bottom. The appendix has a complete peritoneal coat and lies slightly coiled on the inner side of the cœeum and just below the ileum.

In front, to the outer side and behind the cœeum has the abdominal wall. Internally there are convolutions of the small intestine while to its inner and posterior aspect are the iliac vessels, separated from it, however, by the parietal layer of the peritoneum.

\section{The Ascending Colon.}

The ascending colon commences where the cœcum terminates, in the right iliac region, at the opening for the ileum, and passes upward through the right lumbar region into the right hypochondriac region where it terminates by becoming the transverse colon, forming the hepatic flexure of the colon at the lower surface of the right lobe of the liver, to which 
it is held by a fold of the peritoneum, being covered by it in front from half to three-fourths around. In front and to the outer side is the abdominal wall; to the inner side, the convolutions of the small intestine, and the inferior vena cava; behind, the perpendicular duodenum and right kidney, the duodenum being to the inner and posterior aspect.

\section{The Transverse Colon.}

The transierse colon commences at the termination of the ascending colon, at the lower surface of the liver, in the hepatic flexure of the colon, and passes obliquely downward and to the left, crosses the abdomen between the upper and middle zones, and, rising into the left hypochondriac region, terminates by forming the splenic-flexure of the colon at the lower end of the spleen and becomes the descending colon. Its course is curved with its convexity downward and forward. It is entirely covered by peritoneum, which holds it by a long double-layered fold, the transverse meso-colon, to the posterior abdominal wall and by a small band to the lower end of the spleen. It is indirectly held to the greater curvature of the stomach by the great omentum. In front it is separated from the anterior abdominal wall by the great omentum; behind is the transverse duodenum; above are the liver, stomach and spleen; below, the convolutions of the small intestine.

\section{The Descending Colon.}

The descending colon begins where the transverse colon ceases, in the left hypochondriac region at the lower end of the spleen, and passing downward through the left lumbar region terminates in the left iliac region by becoming the sigmoid flexure of the colon. It lies behind the peritoneum, covered bv it in front from half to three-fourths around. To its front and outer side is the abdominal wall; behind, the left kidney; to the inner side are the convolutions of the small intestine.

\section{The Sigmoid Flexure.}

The sigmoid flexure of the colon begrins by being the continuation of the descending colon in the left iliac region and, 
passing down into the pelvic region terminates on the middle of the third piece of the sacrum by becoming the rectum. The gut presents many differences in position, but, when empty, is usually found passing downward and to the right, then falling over the brim of the pelvis and resting against its own lower portion and the beginning of the rectum. It is completely invested by peritoneum, which holds it by a fold of variable length to the abdominal wall. It crosses the pelvic brim in front of the ureter, from which, as well as from the iliac vessels, it is separated by peritoneum. In the pelvis it lies in front of the internal iliac vessels and sacral plexus of nerves and comes in contact, on its inner side, with convolutions of the small intestines, the side of its own terminal portion and the side of the rectum. In the female it rests against the ovary, uterus and vagina. Between it and the vagina and uterus, in the female, and bladder in the male, are interposed some convolutions of the sma1l intestines.

\section{The Rectum.}

The rectum begins by being the continuation of the sigmoid fixture at the middle of the third piece of sacrum and terminates at the amus. It passes straight down the middle line, conforming to the curve of the sacrum and coccyx, until it reaches the tip of the latter bone when it turns backward, running in this direction downward and backward for about an inch and a quarter when it terminates at the anus. At its commencement the rectum is covered by peritoneum about half way around, but the peritoneum gradually leaves the sides of the gut as it descends, and, after a course of about an inch, leaves the front of the rectum to pass to the bladder in the male, or the back of the vagina, in the female. That portion of the rectum which is covered by peritoneum has upon its front convolutions of the small intestines which separate it from the bladder, in the male, or the uterus and vagina, in the female. Behind, the rectum rests upon the front of the sacrum from which it is separated by the pyriformis muscle, and the lower sacrel nerves, In front, below the peritoneum, it has the base of the bladder, prostate gland, seminal vesicals and 
vas deferens and is crossed on its side by the ureters. In the female the vagina rests upon the front of the gut, at first firmly attached to it but separated from it below by a widening interval filled in by the perineal body. The last inch and a quarter of the rectum is surrounded by fibres of the levator ani muscle and is not properly an abdominal viscus, lying in the ischio-rectal fossa.

\section{The Liver.}

The liver is found in the upper zone of the abdomen, the greater part being in the right hypochoudriac and epigastric regions, but projecting, to a greater or less extent, into the left hypochondriac. Its long axis is transverse; its sharp notched border is in front and below, nearly corresponding to the lower margin of the ribs on the right; its thick border is backwards, resting against the diaphragm and grooved for the inferior vena cava, and also, near the left end, for the œsophagus; its convex surface is above; its large end is to the right.

It is beld in place by five ligaments and is connected to the stomach by the lesser, or gastro-hepatic omentum. Four of its ligaments are formed by the peritoneum while the fifth, called the round ligament, is the remains of the obliterated umbilical vein. The peritoneum reaches the liver by two very short layers, which seize the one the upper the other the lower edge of its posterior border. These layers, on the posterior border, are separated by a considerable triangular space; but at each end they come together and form the right and left lateral ligaments. Between these lateral ligaments the layers surrounding the triangular space form the coronary ligament. The upper layer is diverted forward from the liver, at a point about one-third the length of the liver from its left end, across the upper surface of the liver so as to surround the round ligament by a double-layered fold called the suspensory ligament, or the longitudinal ligament, which holds the liver to the diaphragm. The round ligament is that part of the obliterated umbilical vein which passes backward from the anterior abdominal wall to the notch in the anterior border of the liver, lying between the two layers of the longi- 
tudinal ligament. The peritoneum reaching the liver in the manner described, passes over it, one layer over the upper surface, around the anterior border to the transverse fissure where it meets the other layer, which has run forward on the lower surface from the posterior border, thus furnishing an almost complete peritoneal coat for the organ.

Above the liver is the diaphragm, which separates it from the thorax, containing the heart and lungs; below are, from right to left, the right kidney and supra-renal capsule, lying near the posterior border of the liver; in front of these, and near the anterior border, the hepatic flexure of the colon; behind and to the left of this, near the neck of the gall bladder, the oblique duodenum ; to the left of this the upper border of the stomach, held to the liver by the lesser omentum, and, to the left of this, the spleen, suspended from the under surface of the diaphragm by the suspensory ligament. The spleen is a relation only when the liver or spleen, is much enlarged. The posterior border of the liver has upon it the inferior vena cava, to the left of this the abdominal aorta and still further to the left the oesophagus. On its under surface should be mentioned the gall bladder, considered with the liver itself.

\section{The Spleen.}

The spleen is confined to the left hypochondriac region. Its long diameter is vertical; its thin notched border is anterior; its smallest end is below; its convex surface, external. It has a complete peritoneal coat and is held in position by folds of peritoneum, one fold, called the suspensory ligament, passing from its upper end to the diaphragm, the other, called the gastrosplenic omentum, passing from its inner face to the great end of the stomach. A third small fold extends from its lower end to the splenic flexure of the colon.

Above is the diaphragm from which it is sometimes separated by the liver; below is the splenic flexure of the colon; behind the lower end is the left kidney and its capsule; in front, the anterior abdominal wa11; internal to it are the great end of the stomach and the tail of the pancreas; externally it corresponds 
to the ninth, tenth and eleventh ribs, from which it is separated by the diaphragm, the left pleura and the lower border of the left lung.

\section{The Pancreas.}

The pancreas extends from the inner face of the spleen on the left to the descending duodenum on the right. Its long diameter is transverse; its large end to the right; one surface looks forward, the other backward; one border is above, the other below.

It rests on the front of the posterior wall of the abdomen, crossing the body of the first lumbar vertebra. It is behind the peritoneum, which covers it only in front. To its right is the perpendicular duodenum, to which it is closely adherent; to its left is the inner face of the spleen to which its tail is held by the peritoneum; in front is the stomach; behind its left end is the left kidney; below it is the transverse duodenum, from which it is separated by the superior mesenteric vessels. Its relation to blood vessels are very complex, and may be given as follows: it is separated from the vertebral column by the abdominal aorta, which produces the coliac axis on a level with the upper border of the head of the pancreas while the superior mesenteric artery is emitted just behind the head; the latter descending behind the pancreas, to pass out between it and the transverse duodenum.

The cneliac axis, resting on the upper border of the head of the pancreas, divides into three branches, one of which, the splenic, pursues a very tortuous course along its upper border to the inner face of the spleen. This artery is accompanied by its vein, which passes to the right from the spleen, lying in a deep groove on the posterior face of the pancreas just below its upper horder. Behind the head of the pancreas the splenic vein unites with the superior mesenteric vein, which ascends behind the pancreas as the companion of its artery, the two forming the portal vein. About the middle of its course the splenic vein receives the inferior mesenteric vein which passes behind the body of the pancreas. Surrounding the coeliac axis are the two semi-lunar ganglia of the sympathetic, 
giving off the numerous branches of the solar plexus of nerves, which are in close relation with the head of the pancreas.

\section{The Kidneys.}

The kidneys lie on the front of the posterior abdominal wall, extending from about the eleventh rib down ward and slightly outward to about the crest of the ilium. The right is perhaps a little lower than the left, reaching only to the lower border of the eleventh rib while the left reaches to its upper border. The kidneys correspond to the last dorsal and the upper three lumbar vertebræ. They lie behind the peritoneum, embedded in a considerable mass of loose connective tissue, which usually contains much fat. The peritoneum is loosely connected to the front of the organ by this tissue and can easily be stripped off. The long diameter of the kidney is from above downward and slightly outward; one face looks forward and slightly outward, the other backward and slightly in ward ; the upper end is, perhaps, the larger; the outer border is convex, the inner concave. Through most of its extent the kidney rests on the quadratus lumborum muscle, separated from it by the anterior lamella of the posterior aponeurosis, of the transversalis muscle. Along its inner edge it lies on the psoas magnus muscle, and behind its upper part is the diaphragm, which separates it from the pleura. The diaphragm here frequently presents a fissure of considerable size, where the muscular tissue is wanting, so that, in this event, all that separates the kidnev from the pleura is a little loose connective tissue. Each kidney has upon its upper, inner, front part the suprarenal capsule. The outer border is nearly opposite the outer border of the erect or spinæ muscle, and about corresponds to the junction of the posterior third with the anterior two-thirds of the crest of the ilium.

The right kidney has in front the descending duodenum and in front of that the ascending. colon; along its inner border is the ascending vena cava; its upper end is in contact with the lower surface of the liver, which may also rest on the front of its upper part. The left has on its front the descending colon and, at its upper part, the lower end of the spleen the tail of the pancreas and the great end of the stomach. 


\section{The Bladder, in the Male.}

The urinary bladder occupies the front portion of the pelvic carity, being confined to it when empty or nearly so, but rising out of it according to its state of distension, occasionally reaching the level of the umbilicus. It lies just behind the symphysis pubis with its long diameter from above downward and backward, extending from the upper border of the symphysis pubis, or a point in the linea alba between it and the umbilicus, varying with distension, so that, if prolonged, it would strike the lower part of the front of the sacrum.

The large end of the bladder is below and looks downward and backward. The viscus is retained in position by its ligaments which are ten in number. Five of these are called false ligaments and are furnished by peritoneum, while of the five true ligaments four are processes of fascia and one is the remains of a foetal structure, called the urachus.

The peritoneum leaves the front of the rectum about three inches above the anus, and sweeps in a drooping course to the back part of the base of the bladder. This broad fold, extending from the front of the rectum to the bladder, is spoken of as the two posterior false ligaments. The division between them is purely arbitrary, being the middle line of the body. On each side, however, the fold presents an antero-posterior ridge, produced by the passage of the hypograstic artery from the posterior side of the pelvic wall to the side of the lower part of the bladder, the artery then passing up the side of the back of the bladder to the side of the top, whence it leaps to the anterior abdominal wall and approaching its fellow of the opposite side, makes for the umbilicus.

The peritoneum, reaching the bladder, covers the back part of its base, the back of the bladder, the posterior half of each side and the posterior half of the top, thence passing to the anterior abdominal wall. to which it is guided by the urachus, and the obliterated hypogastric arteries. That part of the peritoneum extending from the middle of the top of the bladder to the anterior abdominal wall is called the superior false 
ligament; and that part on each side, extending from the side of the bladder to the lateral wall of the pelvis, forms the lateral false ligament.

The four remaining true ligaments are formed by the pelric fascia, which lines the pelvic cavity just beneath the peritoneum. From either side of the symphysis pubis a process of this fascia is extended to the lower part of the front of the bladder and prostate gland, these two being called the two anterior true ligaments of the bladder. From the lateral wall of the pelvis, on each side, a process of fascia passes to the side of the bladder, the two being known as the two lateral true ligaments of the bladder.

The front of the bladder is separated from the back of the symphysis pubis only by a little loose connective tissue. The neck of the bladder is received into the back part of the prostate gland and is continuous with the urethra, being about an inch behind and below the pubic arch.

The posterior face of the bladder is separated from the rectum usually by some convolutions of the small intestine. The base of the bladder rests on the front of that part of the rectum which is found descending the front of the sacrum, and is adherent to it. Piercing the posterior part of the base of the bladder on each side is the ureter, the two being about two inches apart each having just internal to it the vas deferens, which, entering the abdomen at the internal abdominal ring passes to the side of the top of the bladder, descends to its posterior face runs forward and inward along its base to terminate at the front of the base by uniting with the duct, which forms the seminal vesicle to produce the ejaculatory duct. The seminal vesicles lie, one on each side, on the side of the base of the bladder. They are pear-shaped, the base being behind and the apex forward and inward at the back of the prostate gland.

In the female the bladder, in general terms, occupies the same position as in the male. There are no prostate glands, no vas deferens, or seminal vesicles. The base of the female bladder rests on the upper wall of the vagina and on the lower 
part of the front of the uterus, which two separate it from the rectum. Otherwise the relations are about the same as in the male.

\section{Description of the Separated Viscera. The Stomach.}

In shape the stomach is a curved cone, with one border shorter than the other and its two sides, called anterior and posterior faces, somewhat flattened. The short border is known as the lesser curvature, the long as the greater curvature. The large end of the cone is to the left and is called the splenic end, because it is hugged by the spleen. At the ieft extremity of the lesser curvature, two or three inches from the left end, is an aperture for the esophagus, called the cardiac orifice. The stlenic end of the stomach is the dilated cul de sac bulging beyond this.

The right extremity of the stomach is much smaller than the splenic end and is called the pyloric extremity, because the opening of this end of the stomach in the duodenum is called the pylorus. It is the smallest part of the alimentary canal, being only an inch and a half in diameter. The position of the stomach is not directly transverse, but somewhat oblique, its long diameter being from above downward, forward, and to the right the cardiac orifice being on a higher level and farther back than the pyloric. When empty, the anterior and posterior faces look almost directly forward and backward respectively.

The longest diameter of the stomach is about twelve inches, and it can receive from one to two quarts at a time.

\section{The Duodenum.}

The duodemum succeeds the stomach. It is about nine inches in length and forms a horseshoe-shaped curve whose convexity is to the right. It is divided into three portions. The first portion, beginning at the stomach, is about two inches in length and is called the olilique fortion. It passes upward, backward and to the right. The second portion, called the descending or perpendicular duodenum, is about three inches

Des Anat -12 
long and passes downward. The third portion, called the transwerse duodenum, begins where the descending terminates and passing across the vertebral column, terminates in the jejunum,or second portion of the small intestine. The duodenum terminates at the left side of the second lumbar vertebra; but the point where the jejunum becomes the ileum is arbitrary and ill defined. The jejumum begins where the duodenum terminates aud comprises the upper two-fifths of the remainder of the gut. The ileum comprises the remaining threefifths and terminates in a suddenly dilated portion called the large intestine. The jejunum and ileum together are about twenty feet long. They lie coiled up chiefly in the umbilical and hypogastric regions, producing an appearance somewhat similar to the upper surface of the brain, from which they are called the convolutions of the small intestine.

The large intestine begins by a sudden dilation just below the termination of the small intestine in the right iliac region, and extends to the termination of the alimentary canal at the ana1 orifice.

The large intestine is about five feet in length. It is sinuous in its course and is divided into three portions, cœecum, colon and rectum.

The entrance of the ileum is not into the extremity of the large intestine but two or three inches above its commencement. The blind pouch or cul de sac, which extends below this orifice is called the cœecum. This is the largest portion of the large intestine; it is about two and a half inches in length, lies in the right iliac fossa, is continuous with the colon above and has projecting from its lower, inner back part a tail-like hollow projection called the vermiform process, or appendix, which is from four to six inches in length and lies just below the terminal part of the ileum, its cavity being continuous with that of the cacum.

The colon is the second portion of the large intestine. It commences at the entrance of the ileum, which is the mark of division between it and the cœcum, in the right iliac region, and passes upward through the right lumbar region to the 
lower surface of the liver, in the right hypochondriac region. This portion of the gut is called the ascending colon. At the lower surface of the liver it makes a bend called the hepatic flexure of the colon, and turns to the left across the abdominal carity. This portion is called the transicrse colon and its course corresponds to the superior horizontal line of the abdomen. At the lower end of the spleen, in the left hypochondriac region, the colon nukes another bend, called the splenic flexure, and turns downward to pass through the left lumbar region to the left iliac fossa, as the descending colon. In the left iliac fossa it makes another turn, first upward and to the right and then downward and to the left, forming the sigmoid flexure, which terminates at the brim of the pelvis, opposite to the left sacro-iliac symphysis, in the rectum. The rectum begins where the sigmoid flexure terminates and passes downward on the front of the sacrum, to terminate at the anus, being approximately straight in its course-hence its name.

\section{Structure.}

The following coats, with slight exceptions, are common to the whole of the alimentary canal found in the abdomen:

1st. The visceral layer of the peritoneum.

2d. The interior coat is mucous membrane.

3d. Between these two are found the longitudinal muscular fibres, lying next to the serous coat, and

th. The circular, next to the mucous coat.

These coats are held to one another by interposed connective tissue, or areolar tissue. Each part, also, presents some point, peculiar to itself.

The slomach has, 1st, the serous coat which, besides investing it, passes off to adjoining parts forming the omenta of the stomach which seem to retain it in its position. It is held to the liver by the gastro-hepatic, or lesser omentum, to the spleen by the gastro-splenic omentum and to the transverse colon by the gastro-colic, or greater omentum.

$2 d$ Just beneath the serous coat is the longitudinal muscular coat. 
3d. Just beneath this is the circular muscular coat, which is thickest toward the pyloric extremity, while the longitudinal is thickest at the lesser curvature. The fourth coat is a partial one, of oblique muscular fibres which diverge from the cardiac end of the stomach beneath the circular fibres and terminate before reaching the pyloric orifice. The fifth coat is the internal mucous coat, which, when the stomach is empty, is thrown into longitudinal ridges, called rugx, which disappear when the stomach is distended. At the pylorus the mucous coat is thickened, and beneath this thickening is an aggregation of the circular fibres so as to produce a sudden contraction of the tube. This appearance is known as the pyloric valve.

The duodenum has four coats: 1 st, serous; $2 d$, longitudinal muscular; $3 \mathrm{~d}$, circular muscular; 4th, mucous membrane. In the commencement of the gut the mucous membrane is smooth, but it is soon thrown into folds, which pass around the gut from three-fourths to five-sixths of its circumference; called valvula conniventes. These valvula conniventes are continued down into the jejunum and ileum, but gradually decrease in size and in the ileum are inconspicuous. They are permanent folds, not affected by distension.

On the lower inner part of the perpendicular duodenum is a prominence of the mucous membrane, called a papilla, on which is seen the aperture for the common bile duct and the pancreatic duct.

The jejunum and the ileum have the same coats, in the same order, as the duodenum. Studding the inner surface of the small intestine are numberless hair-like microscopic projections from the mucous membrane called villi; and besides these, and numerous mucous follicles which have their seat throughout the small intestine, there are some glandular bodies which have special seats.

In the duodenum there are numerous small glands, about the size of a pin's head, lying just beneath the mucous membrane and opening by ducts on its free surface, known as the glands of Brunner. Scattered throughout the small intestine, but much more numerous in the lower part of the ileum, are 
small, round, grayish bodies, in the mucous membrane, which have no duct and are called solitary glands. In the lower part of the ileum, and extending upward ten feet, or more-in a few instances into the dundenum - are found a number of dark, oblong, grayish patches, called Peyerian glands or Peyer's patches. They are twenty to thirty in number. Sometimes there are as many as forty. The long diameter of these patches is in the direction of the long diameter of the gut. They are made up of an aggregation of the solitary glands and become diseased in trphoid fever.

The opening of the small intestine into the large is by means of a horizontal slit-like opening, situated on the inner side of the large intestine two and a half inches above its commencement, and guarded by a valvular arrangement called the ileo-coecal, or ileo-colic valve. This valve consists of two projections into the cavity of the large intestine, one above and the other below the button-hole-like aperture, each segment being a fold of mucous membrane covering circular fibres of muscular tissue.

The large intestine, like the small, has four coats, external serous, internal mucous and, between these, two muscular, outer longitudinal, inner, circular. From the commencement of the gut to the rectum the longitudinal coat is not distributed uniformly around the gut, but the fibres are gathered into three narrow flat bands placed one in front, one on the back and one on the concave, inner side of the gut. These bands are shorter than the other coats of the gut, and in consequence the other tunics are thrown into folds with corresponding depressions. These prominences are known as the saculi of the large intestine. Towards the termination of the colon these bands begin to be diffused, and in the rectum the longitudinal fibres are again distributed and of equal length with the other coats. The mucous membrane, in consequence of the shortness of the longitudinal muscular fibres, is also thrown into saculi. Beneath it, especially in the upper part of the grut, are seen a number of solitary glands which differ from those in the small intestine in having a duct. 
The rectum presents some important departures from the above description. It is divided into two portions. The first portion extends from the commencement of the gut until it reaches the point opposite the tip of the coccyx the second is the lastinch of the gut, which here inclines backward to terminate at the anus. The rectum presents the same four coats, in the same order, with the following exceptions: The serous coat is a partial one, the upper half of the gut being entirely surrounded by peritoneum while the lower half is covered by it for only an inch at its upper front part, from which point it mounts to the bladder. The fibres of the longitudinal muscular coat becomes much more distinct, and of a reddish color, in the lower part of the gut; and, when they reach its extremity, they do notstop short, but turn upward to run along the inner face of the circular fibres, which separate them from the descending longitudinal fibres while the mucous membrane lies between them and the cavity of the gut. These ascending longitudinal fibres are gathered into separate bundles, or columns, which pass up for an inch or two before ceasing and throw the mucous membrane into ridges with intervening depressions called rectal pouches. Towards the lower extremity of the rectum the fibres of the circular muscular coat are aggregated into a thickened ring, internal sphincter ani, and just above this the cavity of the gut is considerably dilated, forming a capacious reservior in cases of long continued constipation.

\section{The Liver.}

The liver stretches across the abdomen just beneath its roof, lying in the right hypochondriac, the epigastric and to some extent, the left hypochondriac region. It is semi-ovoidal in shape, weighs about four pounds, is about twelve inches long, six broad and three thick, at its thickest part; in color it is a dull red with, occasionally, a purplish, or yellowish tinge. It is, in structure, a solid glandular organ. For study it is divided into an upper and a lower surface, an anterior and a posterior border, five ligaments and an excretory apparatus.

The upper surface is smooth and convex, being moulded on the lower surface of the diaphragm. It presents a glistening 
appearance due to the visceral layer of peritoneum, which has been traced. The peritoneum, passing from the diaphragm to the liver in an antero-posterior fold, strikes the liver nearer the left than the right extremity. This fold, the longitudinal ligament, is a mark of division between the two lobes, all that portion lying to the right being known as the right lobe, while the much smaller portion, lying to the left is the left lobe.

The anterior border is thin and sharp and has a notch at the point where the longitudinal ligament intersects it, which also marks the division between the lobes. The anterior border is just above the lower border of the ribs, though, when the liver is enlarged, it may be felt through the abdominal parietes.

The posterior border is thick and rounded and marked by a notch where it is intersected by the longitudinal ligament, a third mark of division between the two lobes. The openings for the hepatic veins are found on this border, and it is grooved for the inferior vena cava.

The lower surface is marked from before backwards by a fissure called the longitudinal, which is just opposite the longitudinal ligament on the upper surface, and extends from the notch in the anterior to that in the posterior border. It is the fourth mark of division between the lobes. The lower surface of the right lobe presents a deep groove, called the transverse fissure, which runs to the right from the longitudinal fissure which it strikes about at its posterior third. In this fissure are found the hepatic duct, to the right, and slighltly in front, the hepatic artery to the left and, between and behind the two, the fortal i'ein. That portion of the longitudinal fissure which is behind the intersection of the transverse fissure, is called the venosus fissure, and the portion in front the umbilical fissure. The latter is frequently crossed by a strip of liver tissue, called the pons hepatis. Lying in front of the transverse fissure, and producincr an impression on the lower surface of the right lobe, is the gall bladder; and between this and the longitudinal fissure 
is a square-shaped portion of liver tissue called the lobus quaratus, whose limits are, in front, the anterior border of the liver, behind, the transverse fissure, to the right the gall bladder to the left the longitudinal fissure. Behind the transverse fissure is another portion of liver tissue, which has received the name of lobus Spigelii. It is three sided, having in front the transverse fissure, to the left the venosus portion of the longitudinal fissure and to the right the groove made by the inferior vena cava.

Running out to the right from the front of the lobus Spigelii, just behind the transverse fissure, is a ridge, called the lobus caudatus. Near the posterior border there is a depression on the lower surface of the right lobe made by the right kidney and its suprarenal capsule.

The liver is held in position by processes of peritoneum, called ligaments, which are five in number.

The longitudinal, or suspensory ligament consists of two layers of peritoneum which pass from the lower surface of the diaphragm to the upper surface of the liver. These two layers separate on reaching the liver and, with two exceptions, completely invest it. One exception is the space on the lower surface, occupied by the gall bladder, for the peritoneum leaps over the gall bladder, investing it, and leaves the contiguous surfaces of gall bladder uninvested. The other exception is found at the posterior border, for the two layers here diverge, to form the right and left lateral ligaments, which are nothing more than the points where the visceral and parietal layers come in contact; and between the two diverging layers, on the posterior border, there is a triangular space which has no serous coat. The two layers, as they skirt this space, are known as the coronary ligament. The fifth ligament is found as a rounded cord in the front edge of the longitudinal ligament. It is the obliterated umbilical vein.

The excretory apparatus consists of a series of ducts, and a reservoir, called the gall bladder.

The gall bladder is a membranous pyriform sac, lying on the lower surface of the right lobe of the liver, its large end lying 
forward and usually falling short of the anterior border. though occasionally projecting beyond it. Its small end lies backward and terminates at the transverse fissure in a neck which becomes continuous with a duct called the cystic. This duct is about one-inch long and unites with the duct from the liver to form the common bile duct. The gall bladder has three coats. The external serous coat is only a partial one, since it passes over the gall bladder from the liver, leaving that portion next the liver uninvested. The next coat is fibro-muscular.

The internal coat is mucous, and, in the neck, it is thrown into spiral folds, so that liquid in following the spiral can flow but slowly.

In the transverse fissnre two ducts, one from the right and one from the left lobe, unite to form the hepatic duct, which is about two inches long and descends to unite with the cystic to form the common bile duct-ductus communis choledochus -which descends for about three inches to open on a papilla on the lower inner part of the perpendicular duodenum.

\section{Structure.}

The external investment of the liver is the nearly complete serous coat. Beneath this is a white fibrous coat which everywhere covers the liver, and gives off numberless processes which pass into the substance of the liver and divide it into minute subdivisions called lobules. These are made up of the proper liver substance.

In the transverse fissure are three sets of vessels which ramify in the liver to fulfil the following offices: 1st. The hepatic artery carries arterial blood to the liver. $2 \mathrm{~d}$. The portal vein also pours a stream of blood into the organ. $3 \mathrm{~d}$. The hepatic duct, resulting from the coalescence of the smaller ducts from the lobules, conveys the bile away from the liver.

When traced in to the liver these vessels are found associated throushout the organ. The venous blood is removed from the liver by means of a fourth set of vessels called the hepatic veins, which result from ramifications associated 
with the other vessels but which open, by three, or four separate apertures, on the posterior border of the liver, into the inferior vena cava.

\section{The Pancreas.}

The pancreas is a pale, lobulated gland. It is six or seven inches long and varies in thickness from an inch and a half to less than half an inch. It weighs about three ounces and lies horizontally behind the stomach, with its large end or head embraced by the concavity of the descending duodendum, and its small end or tail in contact with the inner aspect of the spleen. It crosses the body of the first lumbar vertebra, which renders its posterior aspect concave whereas its anterior is convex. The head is much the larger portion of the organ and sends downward, at right angles to the rest of the organ, a considerable projection from which the gland has been likened to a hammer. The head also furnishes a prominence backward and to the left which is sometimes called the lesser pancreas, and is found lying behind the superior mesenteric vessels.

\section{The Pancreatic Duct.}

The pancreatic juice is collected and conveyed away by a duct called the pancreatic, or canal of Wirsung, which commences in the tail of the organ by a forked origin. The two branches soon unite and the resulting duct, as it passes to the right grows by momentary accessions until it reaches the right extremity of the organ, where it pierces the coats of the perpendicular duodendum to open on the papilla for it and the common bile duct.

Somewhere, just before leaving the pancreas, it receives the duct from the lesser pancreas.

In structure the paucreas is a lobulated gland. It lies behind the peritoneum, and, consequently, has a serous coat only in front.

\section{The Spleen.}

The spleen lies vertically in the left hypochondriac region. It is completely invested by peritoneum, which forms two folds to retain it in position. The first is called the suspensory 
ligament and suspends the spleen by its upper extremity to the lower surface of the diaphragm; the second, the gastrosplenic omentum passes between the inner surface of the spleen, and the contiguous large end of the stomach.

In color the spleen is a dark red, in shape, semi-ovoidal, in consistence, extremely fragile, in size, about six inches long, three broad and one and a half thick, in weight, about seven ounces. It may be divided, for study, into two surfaces, two borders and two extremities. The external face is convex to correspond with the sweep of the abdominal wall; the internal, flat, or perhaps, concave, to hug the great end of the stomach, and marked about its centre by a vertical groove, called the hilum, where the branches of the splenic artery find ingress and the vein egress, and where the gastro-splenic omentum is attached. The upper extremity is much larger than the lower, which is thin and pointed. The posterior border is thick and rounded; the anterior, thin, sharp and marked by one or more notches.

\section{Structure.}

The spleen is invested by two coats, an external serous and, beneath this, a fibro-elastic coat, from the inner face of which are sent off processes, or trabeculæ, in the interspaces of which are found the proper splenic tissure or parenchyma, or splenic pulp. The spleen is a blood vascular, or ductless gland, and whatever it elaborates is carried off without the aid of a special apparatus.

\section{The Kidneys.}

The kidncy's are a pair of organs found, one on each side of the vertebral column in the lumbar region, the left extending from the upper border of the eleventh rib to the crest of the ilium, the right from the lower border of the same rib, being some one-balf an inch lower than the left. They about correspond to the twelf th dorsal and first and third lumbar vertebra, and diverge somewhat as they descend. Each is embedded in a mass of fat behind the peritoneum, which touches them only slightly in front. Perched on the upper, inner part are the two supra-renal capsules. The kidney is about four inches 
long, two broad and one thick. It is peculiar in outline, hence the name reniform. The anterior surface is convex, the posterior, slightly flattened; the upper end is the larger ; the outer border is convex, the inner, concave, and presents a deep depression, called the hilum of the kidney, through which the duct and blood vessels pass, in the following order : the renal vein in front, the duct, or ureter, behind and the renal artery between the two. The hilum leads to a cavity in the organ, called the sinus. The kidney weighs four or five ounces.

\section{Structure.}

The kidney is invested by a fibrous coat, which can be easily stripped off, thus exposing the proper tissue, to study which the kidney should be split longitudinally, beginning at its external border and passing through its width. It is then seen to consist of two portions, an outer layer, red in color, which forms about three quarters of the organ, and within this is a portion of a lighter red forming the remaining fourth. The outer is called the cortical, the inner the medullary portion. The inner portion is made up of conical masses called pyramids of Malpighi; they are arranged with their bases toward the cortical portion and their apices toward the hilum and are from eight to eighteen in number. Each pyramid consists of hundreds of straight tubules, leading from the cortical portion, where the urine is secreted, to the apex or papilla of the pyramid, where they discharge the urine. These pyramids are separated by prolongations of the cortical substance, which projects between them.

The urine, which drops from the papilla, is carried off by coalescing ducts having different names, all of which finally terminate in one duct called the ureter, which in turn opens into the urinary bladder. The course can best be understood by following it from below up. Beginning with the ureter we find that, just before it reaches the kidney, it begins to enlarge forming what is called the pelvis of the ureter, which entering at the hilum, occupies the sinus of the kidney. The pelvis of the ureter soon divides into three tubes called infundibula, one infundibulum collecting the urine from each third 
of the organ. Each infundibulum, after a short course, subdivides into a number of short tubes called calices, each calyx terminating by surrounding the apex of one or more pyramids. The course of the urine, then, after secretion in the cortical portion is (1st.) through the uriniferous tubules, which form the pyramids of Malpighi (2d), dropping from the apex or papilla it falls into (3d) the calyx, which uniting with other calices from its third of the organ, forms (4th) an infundibulum, which combines with the other two infundibula to form (5th) the pelvis of the ureter, which (6th) contracts to the ureter proper which, lastly, opens into (7th) the bladder.

\section{The Ureter.}

The Ureter commences at the kidney in a dilated portion called the pelvis, and, contracting to a small tube-about the size of a crow's quill-passes down beside the vertebral column to the brim of the pelvis, descends in the pelvis behind the bladder, and approaching its fellow, opens into the back part of the base of the bladder. The apertures for the two ureters are about two inches apart, and the tubes pierce the coats of the bladder in an oblique direction. The length of the ureter is from sixteen to eighteen inches.

The ureter-lying behind the peritoneum-consists of three coats, an external fibrous coat, an internal mucous, and, between these, an external longitudinal and an internal circular muscular coat. This description applies to pelvis, infundibulum and calyx. Near the bladder there is another layer of longitudinal muscular fibres lying between the circular fibres and the mucous meinbrare.

\section{The Urinary Bladder.}

The bladder is the reservoir for the urine. It is a membranous sac which lies in the pelvis, just in front of the rectum, in the male, and of the uterus and ragina in the female. Its shape, when distended, is ovoidal, or pyriform, the large end being below. When empty it is flattened against the pubes and is somewhat triangular. The direction of its $10 n g$ axis is downward and backward. The capacity of the bladder is very 
variable, though, in health, the urine is voided when half pint to one pint has been secreted. The upper third of the organ, about, is called the superior fundus, summit or apex; its middle third the body; the lower third the base, or fundus. The channel through which the urine leaves the bladder is called the urethra, whose aperture is seen in the lower front portion. This part of the bladder is called the neck, and lies embedded in the prostate gland. In structure the bladder consists of the following coats: 1st. The internal is mucous membrane. At the neck of the bladder there is a slight prominence, generally absent, called the uvula vesicce, seen just at the commencement of the urethra. Between the uvula vesicce in front, as its apex, and the openings for the ureters, as its posterior angles, there is a triangular space called the trigonum vesicæ, or triangle of the bladder, whose base is formed by a line drawn between the openings for the ureters, and whose sides are formed by a line on either side running from this point to the uvula and represented by a ridge in the mucous membrane made by a bundle of longitudinal fibres from the ureter. This space is by far the most sensitive part of the bladder, and corresponds to a similar space on the exterior of the base. $2 \mathrm{~d}$. The muscular coat is held to the mucous membrane by a layer of areolar tissue. It consists of longitudinal and circular fibres, the longitudinal forming two layers between which are found the circular. The circular fibres, at the neck of the bladder, are aggregated in to a considerable mass, which by their tonic contraction, keep closed the opening of the urethra. $3 \mathrm{~d}$. The external coat is serous and incomplete. In the male it covers the summit, sides, posterior aspect and posterior part of the base of the bladder, leaving uncovered the front and the front part of the base.

\section{The Urethra in the Male.}

The urethra is the last division of the canal which the urine traverses in seeking an outlet from the body. It commences at the neck of the bladder and terminates at the meatus urinarius, its opening on the free extremity of the penis. Its length is variously estimated, owing to the varying length of 
the penis which it tunnels. It is divided into three portions, the prostatic, which begins at the neck of the bladder and pierces the prostate gland to appear at its apex and become the second, or membranous portion, which passes on to enter the bulb of the corpus spongiosum and become the third, or spongy portion. The spongy portion continues through the corpus spongiosum to terminate at the meatus urinarius.

It is the longest portion and the most variable in length. The length of the urethra as a whole is usually given as seven and a half inches of which the prostatic portion occupies about one and one-fourth inches, the membranous threefourths of an inch and the spongy portion the remainder. The prostatic portion is the largest.

\section{The Prostate Gland.}

This gland is a small horse chesnut-shaped body, found in the male, with its base against the neck of the bladder and its apex projecting forvard. Its length is about one and onefourth inches, its breadth one and one-third, and its deptli about one-half inch. It is invested in cellular tissue and has a proper fibrous capsule ; and it consists of interlacing unstriated muscular fibres, in the interstices of which are found the follicles of the gland, which secretes a milky fluid. It is divided into three lobes, two latcral and an inferior, or isthmus. Passing through it, nearer its upper than its lower surface, is the prostatic portion of the urethra. On the floo: of this portion of the urethra is seen a prominence of the mucous membrane, about one-half an inch long, called the veru montanum. On either side of this prominence is a depression in the floor of the urethra, called the sinus prostaticus, in which are found some ten to fifteen minute apertures - the openings of the prostatic follicles; while in front of the veru montanum is a small saccular cavity, projecting backward, called the sinus pocularis, or utriculus prostaticus, or uterus masculinus. Opening on either side of the orifice of this sinus is seen a small aperture, the opening of the ejaculatory ducts, which pass back, one on either side, to two lobulated oblong bodies, one on either side, just behind the prostate gland, called seminal vesicles. 


\section{Seminal Vesicles.}

Each seminal vesicle is a reservoir for the seminal fluid, and is formed of a tube, about the size of a goose quill, five or six inches long, coiled into an oblong mass, which lies on the lower surface of the base of the bladder, its large end projecting backward and outward from its fellow, the small end being in front just behind the prostate gland and approaching its fellow. The posterior extremities are about two inches apart, and a line drawn from one to the other is the base of a triangular space, whose apex is at the prostate gland, which may be designated the intervesicular triangle. This space is devoid of peritoneum. The point where the peritoneum strikes the base of the bladder corresponds to the posterior boundary of this triangle, whose sides are formed by the seminal vesicles with the vas deferens of either side lying internal to them.

\section{Vas Deferens.}

Commencing at the upper extremity of the testicle the vas deferens forms one element of the spermatic cord, the others being blood vessels, nerves, \&c., which ascends to the upper border of the pubes, where it plunges into the anterior abdomina1 wall, through the external abdominal ring, then outward, along the inguinal canal to the internal abdominal ring, where it turns backward through that ring to enter the abdominal cavity. As soon as the cord enters the abdomen its various constituents disperse. The vas deferens passes to the upper part of the side of the bladder, thence down its posterior surface, along the inner edge of the seminal vesicles, at the anterior extremity of which it is joined by the tube, whose convolutions form the seminal vesicles, and the two form the ejaculatory duct.

\section{The Ejaculatory Duct.}

The two ejaculatory ducts lie very near each other and pass forward and upward, through the substance of the prostate gland, for about three-quarters of an inch, to open on the sides 
of the aperture of the sinus pocularis, which is found at the base of the veru montanum, in the floor of the prostatic portion of the urethra.

\section{The Penis.}

The penis consists of three cylinders, two, lying side by side called the corpora cavernosa, and one in a groove between these known as the corpus spongiosum. When dissected out the corpora caveruosa are seen to commence by attachment to bone-the ischio-pubic rami-by a portion called the crus which terminates posteriorly in a pointed extremity; while anteriorly it increases in size to become the corpus cavernosum. The corpora cavernosa do not extend to the extremity of the penis, but stop a little behind the meatus urinarius. The corpus spongiosum begins by a dilated portion called the bulb, situated between the crura, into which the membranous portion of the urethra passes to become the spongy portion. It then passes forward, tunnelled by the urethra, lying between the corpora cavernosa, until it reaches their anterior extremity, when it suddenly dilates into a considerable mass, which covers the extremity of the corpora cavernosa, and projects in a ridge beyond them. This dilated extremity is called the glans penis or head. The glans has its base backward, terminating in a rounded edge, raised above the surface of the corpora cavernosa, which is called the corona glandis, while the constricted portion behind it is called the neck. From the corona the glans slopes to its termination around the meatus urinarius. That portion of the penis extending from the crura, or rather where the three cylinders come in close relation, forward to the head, is called the body of the organ; and the two crura and the bulb constitute the root of the penis.

Surrounding, and loosely adherent to, the body is a thin skin, which in front is formed into a fold, movable over the glans called the prepuce. On the lower aspect of the glans the prepuce is attached by a process, extending forward to the mcalus urinarius. This attached portion is called the franum.

Passing through the corpus spongiosum from the bulb behind to the meatus urinarius in front, is the spongy portion

Des Anat-13 
of the urethra, which, just before its termination at the meatus, presents a considerable dilation called the fossa navicularis. The mucous membrane lining the urethra presents many follicles, called lacunæ, opening into the cana1. When the mucous membrane reaches the meatus it is continued over the glans penis and the deep surface of the prepuce.

The tissue of the three cylinders is that known as erectile tissue. Each cylinder is enveloped by a strong fibrous sheath, that of the corpus spongiosum being more delicate than the others. Within this sheath the structure consists of interlacing bands of fibrous tissue, the interspaces between which contain dilated blood vessels, which when turgid with blood, produce erection. The two corpora cavernosa, at the posterior part of the body of the organ, are separated some little distance from each other, but as they pass forward. come much closer together, for the fibrous tissue between them is thick posteriorly, whereas in front it is thin and presents numerous slit-like interruptions which have obtained for it the name of septum pecteneiform.

\section{The Testicles-Testes.}

The testicles are a pair of small organs whose function it is to secrete the semen. They are found suspended by the spermatic cord, in the bag called the scrotum, separated from each other, although lying side by side. Each testicle weighs from three-fourths to one ounce or more; it is an inch to an inch and a half long, about an inch antero-posteriorly and half an inch transversely. It occupies the back of the scrotum, its position being from above down ward and backward.

The scrotum is thus constituted: 1st, an external covering of skin; $2 \mathrm{~d}$, beneath this a musculo-fibrous covering called the dartos; $3 d$, lining the interior of the dartos, and also enveloping the testicle, a serous membrane called the tunica vaginalis. That portion of the tunica vaginalis lining the dartos is called the parietel, and that lining the testicle the risceral layer.

There are two cavities in the scrotum separated by a septum from the dartos called septum scroti. There is a tunica vaginalis for each testicle. 


\section{Structure of the Testicle.}

When the visceral layer of the tunica vaginalis is removed we find beneath a bluish white fibrous investment of the testicle called the tunica albuginea. This is much thickened at the back part, where it is called the mediastinum.

Lining the inner aspect of the tunica albuginea is a reddish vascular investment called the tunica vasculosa. Lying on the posterior aspect of the testis is a flattened body called the epididymis, which is made up of the convolutions of the tube conveying away the semen. The upper portion of the epididymis is alone permanently connected with the testicle, for it is here that the ducts, which transmit the semen from the testes, emerge and unite to form the epididymis. The upper portion of the epididymis is called the globus major, the lower portion, which terminates in the vas deferens, the globus minor. The intermediate portion is called the body. The semen is secreted in what are called the lobules of the testicles, which number from 250 to 400 , each lobule being separated from those adjacent by septa sent in from the tunica albuginea, which septa, however, are covered on both sides by a layer from the tunica vasculosa.

Each lobule consists of the convolutions of a small tube some one-two hundreth of an inch in diameter, arranged from before backward with the large end of the convoluted mass in front and the small end behind at the mediastinum, where many lobules unite to form a single duct, which, from its comparatively straight course, is called the rectum. In the testis there are from twenty to twenty-five vasa recta, which plunge into the mediastinum and there unite to form from two to twelve ducts, which ascending through the mediastinum in a sinuous course, are called collectively the rete testis. When the ducts reach the upper extremity of the mediastinum, they terminate in from nine to thirty other ducts, called vassa-efferentia, each of which is thrown into convolutions assuming a conical appearance which are known as coni vasculosi and form the globus major. The bases of the cones terminate in large ducts which 
unite in the body of the epididymus into one duct whose convolutions, some twenty feet long, form the body and globus minor of the epididymis and then become the vas deferens.

The continuous course of the semen is then, lobule, which may be composed of as many as three tubes, vasa recta, rete testes, vasa efferentia, coni vasculosi, epididymis, body and globus minor, vas deferens.

\section{The Arial Apparatus.}

The air reaches the lungs from the throat through a tube which has received different names in its various parts. It is first called the larynx, then the trachea, which, opposite the fifth dorsal vertebra, divides into the $t$ wo bronchi.

\section{The Larynx.}

The larynx is formed upon a framework of separate cartilages which require to be studied under individual names.

These cartilages are as follows:

Thyroid

Two Arytenoid

Epiglottis
Cricoid

Two Cornicula Laryngis Two cuneiform

\section{Thyroid Cartilage.}

The thyroid is the upper, the front and the largest cartilage of the larynx. In front it comes to an acute angle and produces the prominence, called the Adam's apple, pomum Adami. From this acute, or receding angle, it passes backward and outward on either side in a quadrilateral plate called the ala of the thyroid cartilage.

Each ala presents an internal and external surface, superior inferior and posterior border. The Outer Surface is slightly concave from above downward, wider above than below and marked by an oblique ridge, running from above downward and forward, terminating at each end in a prominence, or tubercle.

The Internal Surface is nearly flat, slopes outward and backward from the receding angle, and is slightly roughened for muscular attachment. 
The Superior Border is sinuous in outline. It commences in the notch found marking the upper end of the angle, slopes first upward and slightly backward, then runs nearly backward to terminate in a prominent projection called the Superior Cormu. Just before reaching the superior cornu it is marked by a slight depression. This border is rounded and smooth.

The Inferior Border is also sinuous, shorter than the superior, like it round and smooth and terminates in the inferior cornu.

The Posterior Border is rounded and free and prolonged both above and below into processes called the superior and inferior cornua, each terminated by a tubercle. The superior cornu is the longest.

The inferior com projects downward from the posterior border. It is much shorter and thicker than the superior and is marked on its inner face by a round, flat articular facet for a similar surface on the cricoid cartilage.

\section{Cricoid Cartilage.}

The cricoid cartilage is a ring and lies supporting the thyroid, between whose inferior cornua it is grasped. It is narrow in front-not more than one-fourth of an inch deep-but a full inch in depth behind. It presents an upper and a lower border, but, being a ring, surfaces are not ascribed to it. The front portion of its exterior is marked on the mid-line by a slight ridge on each side of which is a small impression for muscular attachment. Behind there is a similar ridge in the middle with well marked muscular impressious on either side. In front of these impressions, and on the lateral aspect, a small, round, flat articular facet is seen on either side intended for the facets on the inferior cornua of the thyroid.

Its Utper Border slopes upward and backward, presenting in the centre, behind, a slight notch, and on either side of this a concavo-convex articular facet, on which is perched another cartilage, the arytenoid. The anterior portion of this border is smooth and rounded for ligamentous attachment. 
The Inferior Border is nearly straight, thick and rounded and sometimes marked by a deep notch.

It is held by ligaments to the first ring of the Trachea.

The interior of the ring is smooth and covered by mucous membrane.

\section{Arytenoid Cartilages.}

The arytenoid cartilages are found upon the cricoid, between the wings of the thyroid occupying the articular facets on its upper border. It is triangular in shape base below and apex above, and is divided into three surfaces, posterior, anterior and internal; three borders, external and two internal; and two angles, anterior and external.

The posterior face is concave for muscular attachment; the external is rough, while the internal is smooth and flat and faces its fellow of the opposite side.

The two internal borders are inconspicious, forming the anterior and posterior boundaries of the inner face.

The external border is rough and slopes upward, inward and backward.

The external angle, points outward and backward and is rough and thick. It is called the muscular process.

The anterior angle, thinner and longer, is know as the vocal process.

The apex points upward and inward and is surmounted by a small cartilage, the cornicula laryngis.

The base, directed downward, is concavo-convex for articulation with the similar facet on the upper border of the cricoid.

The Comicula Laryngis are two small cartilages found perched upon the apex of the arytenoids. The cuneiform are small masses found in the aryteno-epiglottidean fold.

\section{Epiglottis.}

The epiglottis lies just above the receding angle of the thyroid cartilage. It is leaf shaped, its apex downward and forward and its base, which presents a slight notch, upward and backward and lying at the base of the tongue. Its upper end may be sometimes seen by looking down the throat. 
It is covered by mucous membrane which holds it to surrounding structures and, when this is stripped off, is seen to present a convex anterior and a concave posterior surface, the latter marked by depressions for small glands.

\section{Ligaments of the Larynx.}

The cartilages of the Larynx are held together by ligaments some of which, like ligaments at other articulations, serve only the purposes of such fibres; while other so-called ligaments are specialized bands employed in producing sound.

The hyoid bone lies just above the superior border of the thyroid cartilage and passing between them is an unbroken membrane called the thyro-hyoidean, and in each of the posterior edges of this membrane a rounded cord, which passes from the superior cornu of the thyroid cartilage to the posterior extremity of the hyoid bone.

The thyro-hyoid membrane is a thin sheet of fibrous tissue which is attached below to the upper border of the thyroid cartilage, extending from one cornu to the other and filling in the notch above the angle. A bove, it passes beyond the lower border of the hyoid and is attached to the upper border and posterior surface of the body of that bone, extending on either side to the cornua. Between the membrane and the posterior surface of the body of the Hyoid there is a pouch-like space left.

The thyroid and cricoid cartilages are held together by the following ligaments. The extremity of each inferior cornu of the thy roid cartilage is held to the facet on the side of the cricoid by a capsular ligament. The considerable interval which exists in front between the lower border of the thyroid and upper border of the cricoid is closed in by a fan-shaped yellow elastic membrane, called the crico-thyroid membrane, which is attached below to the upper border of the cricoid and above to the lower border of the thyroid for about one-fourth of an inch on each side of the middle line and then presents a free edge, covered by mucous membrane, which posteriorly is attached to the anterior angle of the base of the arytenoid cartilage, forming the lower, or true vocal chords. 
When viewed from above the vocal chords appear to be separate bands of elastic fibres, each arising from the receding angle of the Thyroid and passing backward, diverging from its fellow, to be attached to the vocal process of the Arytenoid. They are called inferior thyro-arytenoid ligaments, or true vocal chords.

Each Arytenoid is bound to the Cricoid by a capsular ligament which holds the cartilages together, forming a concavoconvex articulation lined by a synovial sac. In addition to the inferior thyro-arytenoid ligaments, mentioned above, another pair, the superior thyro-arytenoid, or false vocal chords, extend from just above the base of the Arytenoid to the receding angle of the Thyroid.

Passing now to the epiglottis we find its apex held to the receding angle of the thyroid by a narrow ligament called thyro-epiglottic. It is connected to the hyoid bone by a ligamentcalled hyo-epiglottic, which is attached to the epiglottis on its front edge near its apex. The back of the tongue and the epiglottis are connected by three ligaments, one in the centre and one on each side, called glosso-epiglottic, middle and lateral.

\section{Muscles of the Larynx.}

The movements on each other of the separate cartilages which constitute the larynx are mainly effected by five pairs of small muscles called the instrinsic muscles of the larynx.

Crico-Thyroid Arytenoid
Posterior Crico-Arytenoid Lateral Crico-Arytenoid

\section{Thyro-Arytenoid}

\section{Crico-Thyroid.}

The crico-thyroid is a small triangular muscle lying on the side of the cricoid cartilage and partly covering the cricothyroid membrane, which appears on the mid-line in the interval between the two muscles.

It arises from the front and side of the cricoid cartilage and, passing upward, backward and outward, is inserted into the lower border of the thyroid cartilage from the median line in front to the inferior cornu. 
Action-When the thyroid cartilage is fixed by the thyrobyoid muscle, the crico-thyroid will draw up the front of the cricoid, depressing the back which carries the arytenoid with it. thus elongating and rendering tense the vocal chords.

\section{Posterior Crico-Arytenoid.}

The Posterior Crico-arytenoid arises from the depression on the posterior aspect of the cricoid cartilage and, passing upward, outward and forward, is inserted into the outer angle of the base of the arytenoid.

Action-By drawing on the muscular process, or outer angle of the arytenoid, it rotates that cartilage around a vertical axis, carrying the rocal processes away from each other, widening the glottis and, to some extent, tightening the chords.

\section{Lateral Crico-Arytenoid.}

The Lateral Crico-arytenoid arises from the side of the cricoid cartilage, and, passing upward, outward and backward, is inserted into the outer angle of the base of the arytenoid,

Action-It is the antagonist of the posterior crico-arytenoid. drawing the outer angle of the arytenoid forward, approximating the rocal processes and rendering the rocal chords loose.

\section{Arytenoid.}

The Arytenoid is a single muscle which arises from the concave posterior face of one arytenoid cartilage and passes transversely across the interval between the two to be inserted into the same part of the opposite cartilage.

Action-By drawing the arytenoid cartilages towards each other, it narrows the glottis.

\section{Thyro-Arytenoid.}

The Thyro-arytenoid arises from the receding angle of the thyroid cartilage just beside and external to the attachment of the vocal chords, and, passing backward, parallel with the rocal cord is inserted into the anterior angle of the base of the arytenoid cartilage. This muscle runs parallel with the true rocal chord, into which some of its fibres are inserted.

Action-By drawing the muscular process forward, turning the vocal process inward and forwad, it narrows the glottis 
and relaxes the vocal chord. Those fibres inserted into the vocal chord, change the tension of the chord at different points.

Vessels and Nerves. The superior and inferior thyroid arteries supply these muscles. The superior laryngeal nerve supplies the crico-thyroid. The inferior, or recurrent laryngeal supplies the other muscles.

The larynx is lined by mucous membrane continuous with that of the mouth. Looking into the cavity of the larynx from below, at a certain point, the cavity is suddenly narrowed by two ridges, one on either side, running from before, where they are close together, backward, diverging as they go and leaving a triangular interval between them called the chink of the glottis, or rima glottidis; these ridges are produced by the inferior-thyro arytenoid ligaments, or true vocal chords. Reversing the larynx and looking into its cavity from above, the opening into it from the pharynx is seen to be limited by the epiglottis which projects upward and backward. Over the opening, posteriorly, are seen the arytenoid cartilages; laterally are folds of mucous membrane, the ventricular bands; the opening is heart shaped with its broad end in front. Down in the cavity are seen the ridges on each side, one above the other. The lower pair are those seen from below, the upper correspond to the superior thyro-arytenoid ligaments and are the false vocal chords. As they are much less prominent than the true cords, they cannot be seen when the cavity is viewed from below. The space between the false vocal chords bears no name, but the similar and smaller space between the true chords is known as the rima glottidis. On each side of the glottis is another space formed by a recess in the walls of the larynx, between the true and the false vocal chords, which is called the ventricle of the larynx, and this space is continued up on the outside of the false vocal chords, between it and the wall of the larvnx for some distance. This prolongation of the cavity is known as the sacculus laryngis.

It will be seen, therefore, that the larynx is divisible into three portions by the position of the vocal chords, the upper portion presenting the epiglottis, the folds of mucous 
membrane extending between the arytenoid and the epiglottis and containing the pyriform sacculus laryngis which runs up just internal to its outer wall. This space extends to the superior thyro-arytenoid membrane.

The second subdivision is the space between the vocal chords and contains four openings, viz: one above, leading from the upper to the middle compartment, - being the space between the false chords and sometimes called the false glottis; - one below, the space between the true chords, called the glottis, and leading into the lower compartment; and one on either side, being the opening into each laryngeal pouch. The glottis is the triangular space found between the true vocal chords in front and between the two arytenoid cartilages behind.

The lower compartment is simply the circular space enclosed by the cricoid cartilage, and would, therefore, be the means of establishing communication between the Larynx, above, and the Trachea, below.

\section{Trachea.}

Succeeding the larynx comes the second subdivision of the air tube known as the trachea. Commencing where the larynx terminates, opposite the fifth cervical vertebra, it descends in frout of the vertebral column, from which it is separated by the cesophagus, and terminates by dividing, opposite the fifth dorsal vertebra, into the right and left bronchi. It is a cylindrical tube flattened on its posterior aspect, about four and a half inches long and one inch in diameter. In the female these dimensions are somewhat less. Its appearance, when viewed from the front, is annulose, due to the fact that its largest element is a number-fifteen to twenty-of cartilaginous rings, one lying above the other.

The flattening posteriorly is due to the fact that each ring is wanting in its posterior third, thus leaving this portion of the tracheal wall wanting in cartilage. The rings are not in contact but are separated, and at the same time maintained in position by a fibro-clastic membrane, which covers both surfaces of the rings, for they, in fact, lie embedded in the substance of the membrane. Between the posterior extremities of 
the rings, over the interval left by their imperfection, the membrane is continued and is here strengthened by unstriated muscular fibres, both longitudinal and transverse. The longitudinal are unimportant; but the transverse, passing between the posterior extremities of the rings, can, by their contraction, diminish the diameter of the trachea.

The interior of the trachea is lined by mucous membrane, continuous with that of the larynx above and prolonged below in to the bronchi, bronchial tubes and ultimate air cells of the lungs. Beneath the mucous membrane, between it and the fibro-elastic membrane, is a yellow elastic membrane which is much more distinct posteriorly, where the fibres which compose it are gathered into longitudinal bundles. To sum up its structure we say the trachea is lined by mucous membrane, beneath this yellow elastic fibres, external to this a yellow fibroelastic membrane lying in which are the rings of the trachea. Besides these there are transverse muscular fibres between the posterior extremities of the rings, and, scattered around the tube some unimportant longitudinal fibres.

\section{Bronchi.}

Taking up the air tube again, we find that the trachea, or wind pipe, when it has reached a point corresponding to the front of the fifth dorsal vertebra, forks, the prongs of the fork being known as the bronchi, right and left. Each bronchus extends from its origin to the inner surface of the lung, forming one element of the root of the lung. The two bronchi are not similar and the difference between them may be thus stated: The right is shorter, larger and more nearly horizontal, coming off nearly at right angles to the trachea, whereas the left has a considerable obliquity downward. If the trachea be cut across near its termination and the cavity examined, a slight antero-posterior ridge is found separating the two bronchi. This ridge lies nearer the left than the right side and in consequence a foreign body falling into the trachea is more likely to enter the right than theleft bronchus. The left bronchus is about two inches long, the right about an inch. In structure they exactly resemble the trachea. 


\section{Lungs.}

The lungs are a pair of organs found in the thoracic cavity, one on each side of the middle line, resting upon the diaphram below and having the heart enclosed in the pericardium, ying between them. Each lung is conical in shape with its base below, resting on the upper surface of the diaphragm and concave in shape to correspond with that surface. The apex is above and extends about one and one-half inches into the root of the neck. Besides the base and apex, each lung presents the following subdivisions: the posterior border, long, thick and rounded, contrasting strongly with the anterior, which is thin, short and sharp. The outer surface is convex to correspond with the concave inner face of the chest wall. The inner face, which looks towards its fellow, is marked by a concavity which is caused by the heart, in the pericardium, which lies between the two lungs. The inner surface of the left lung is much more concave than that of the right, owing to the inclination of the heart to the left. A little above and posterior to the centre of each lung on its inner face the root is seen to enter its substance. The root is made up of various elements, nerves, lymphatics, \&c., but its chief constituents are the bronchus, pulmonary artery and two pulmonary veins. From behind forward, the relation which these structures bear to one another is the same for both lungs, viz: Bronchus, artery, vein: but from above down ward the relation is different, in the two lungs. In the right it is bronchus, artery, vein: in the left, artery, bronchus, veins. The reins, on both sides, are the lowest, and the difference is caused by a change in the relation of the artery and bronchus in the left lung, which is accounted for by the downward inclination and greater length of the left bronchus.

Each lung is divided by fissures into lobes, the right into three and the left into two. Commencing about three inches from the apex, on the posterior border of each lung, an oblique fissure passes through the lung down ward and forward to near the lower extremity of the anterior border. In the right lung there is another fissure which begins near the center of the ob- 
lique fissure and runs nearly horizontally forward to the anterior border, thus dividing the right lung into three lobes known as upper, middle and lower. In the left lung there are but two lobes, upper and lower. The right lung is slightly larger than the left, owing to the encroachment of the heart on the left lung. The right, however, is shortened by the right lobe of the liver, which bulges the diaphragm on that side, and the difference is in reality not great--the right lung weighing about twenty-two and the left twenty ounces.

\section{The Pleuræ.}

Enveloping each lung and lining the chest wall is a serous membrane, one on each side.

These two membranes are the two pleuræ. They are separate from each other and each forms a distinct closed sac for its lung, one face of the sack lining the inner surface of the chest wall while the other completely invests the lung. In front, behind the sternum, the two pleuræ approach each other closely, and are sometimes in contact about the centre of the sternum.

In tracing the reflections of the pleura, as it is a closed sac, we can begin at any point and following it around will return to that point. Beginning on the root of the lung we trace first the visceral layer, or pleura pulmonalis, and then the parietal layer, or pleura costalis, the two being continuous. From the front of the root, the pleura passes forward on the inner face to the anterior border of the lung, then around the convex outer surface to the posterior border and then forward on the inner face to the back of the root of the lung and then to the side of the vertebral column, where it becomes the parietal layer, which passes thence along the inner surface of the chest wall to the sternum in front, where it leaves the chest wall and passes backward on the pericardium to the front of the root of the lung to become continuous with the visceral layer which we have just traced. The free surfaces of the two layers secrete a small amount of liquid which renders the movements of the lungs in respiration easy. 


\section{The Mediastinum.}

Between the opposing surfaces of the pleuraand the sternum in front and vertebral column behind, there is a space, called the mediastinum.

This space is of very irregular shape, varying in size at different points. It is conveniently divided into the Superior, Anterior, Middle and Posterior. The Superior Mediastinum extends from Sternum to vertebral column and is all that portion which lies above the level of the pericardium, its lower boundary being a plane extended from the junction of the manubrium and gladiolus in front to the lower border of the fourth dorsal vertebra behind. On either side would be the pleurae, containing the lungs. In this space lie the great ressels springing from the heart and the aorta and the structures passing from the neck into the Thorax.

The anterior mediastium is bounded in front by the sternum and lower costal cartilages of the true ribs, behind by the pericardium and on either side by the pleura. It is oblique in direction, from above down ward and to the left and is larger below than above.

The middle mediastimum is the broadest part of the inter pleural space and contains the heart and pericardium.

The posterior mediastinum is irregularly triangular, being bounded in front by the pericardium and roots of the lungs, behind by the vertebral column and laterally by the pleura. It extends from the fourth dorsal vertebra downward and contains many important cords.

\section{The Heart.}

The heart is a hollow muscular organ found in the cavity of the chest, 1ying between and almost surrounded by the two lungs ; it rests upon the convex upper surface of the diaphragm and is contained in the middle mediastinum. It is contained in a closed cavity, formed by the pericardium, lying, for the Ereater part of its extent, unattached; but, since its function is the maintenance of the circulation of the blood, the vessels which bring this to or carry it from the heart all communicate 
with its upper part or base, and thus maintain it in position. To render its movements free in the pericardium, that structure is lined by a serous membrane which, like all others, is a closed sac, one layer lining the pericardium and the other covering the heart from the origin of the vessels on one side to the same point on the opposite side. The portion lining the pericardium is called the serous pericardium and the membrane which it lines the fibrous pericardium. The heart is conical in shape, its base being above and to the right and its apex below and to the left. It is about five inches long, three and a half inches transversely and two and a half antero posteriorly. Its weight is from nine to twelve ounces, varying in the two sexes and being slightly larger in the male.

The exact position of the heart is thus stated : the base lies behind the sternum, corresponding to a line drawn between the upper borders of the extremities of the third costal cartilages ; the apex is downward and to the left, striking the chest wall at the space between the fifth and sixth ribs, three to three and one-half inches to the left of the middle line, about opposite the gladio-xiphoid joint.

The cavity of the heart is separated into a venous and an arterial half, or apartment, by an obliquely vertical septum, which cuts off all communication between the halves. The position of this septum can be predicted by an inspection of the exterior of the heart, for it is marked by a groove passing from the base anteriorly and to the left downward to the right of the apex to run along the posterior aspect of the heart and terminate at the base, towards its right aspect. An artery is found lying in this groove, which is called, for a reason hereafter given, interventricular. From the position of the groove it is seen that the apex of the heart is formed entirely by the left half, the front of the heart mainly by the right half, while the back is the product, principally, of the left half.

When the heart is laid open its entire cavity is seen to be lined by a serous membrane, continued into the vessels which communicate with it called the endocardium, and that each 
lateral half is subdivided by a partial horizontal septum into two cavities, the upper called the auricle, right or venous, and left, or arterial, the lower, the ventricle, right, or venous and left, or arterial.

The position of the horizontal septum is indicated by a groove passing around the heart called the auriculo-ventricular.

In the adult the venous blood of the entire body, with the exception of that of the heart itself, is returned into the venous auricle by two great veins called venæ cavæ, superior and inferior, the one bringing the blood from the head and upper extremities, the other from the lower extremities and body. The venous blood from the heart is returned to the venous auricle by a separate vein called the coronary, which, just as it is about to enter the auricle, dilates into what is known as the coronary sinus. Besides these channels for the return of the venous blood, there exist numerous minute apertures in every carity of the heart through which its venous blood may enter to a small extent. These are called foramina Thebesii. The venous blood thus collected by the venous auricle is driven by its pulsation into the venous ventricle, with which it communicates by an aperture through the horizontal septum called the venous auriculo-ventricular opening. From the venous ventricle the blood is sent into the pulmonary artery which soón divides into a branch to each lung.

Taking up the appearances seen in the cavities of the heart, we begin with the venous auricle.

The icnous auricle consists of two portions; the larger part is called the sinus, but, communicating with this and projecting forward, is an ear shaped addition, called the appendix auricula-hence the name of the auricles. The sinus of the venous auricle presents the following objects: At its upper backs part, the opening of the superior vena cava; at the lower back part, the opening of the inferior vena cava; between the two openings a slight thickening of the auricular wall called the tubercle of Lower ; between the opening for the inferior vena cava and the auriculo-ventricular opening is the opening

Des Anat-14 
for the coronary sinus, guarded by a valve-like fold of endocardium called the coronary valve; on the septum between the two auricles, an oval depression, called the fossa ovalis, and surrounding this, except below, a ridge called annulus ovalis; extending along the wall of the auricle from the opening of the inferior vena cava to the fossa ovalis, is a ridge, the remains of the Eustachian valve of the fœtus; the lining membrane presents ridges caused by little muscular columns called musculi pectinati; the opening through the horizontal septum into the venous ventricle, and, lastly, foramia Thebesii.

To sum up we have the following: 1st, superior vena cava; $2 d$, inferior vena cava; $3 d$, coronary sinus ; 4 th, foramina Thebesii; 5th, venous auriculo-rentricular ; and six other appearances, viz: 1st, musculi pectinati; 2nd, tubercle of Lower ; $3 d$, coronary valve; 4th Eustachian valve; 5 th, fossa ovalis ; 6 th, annulus ovalis.

The venous ientricle has two openings communicating with it, one for the pulmonary artery and the other from the venous auricle. The venous auriculo-ventricular opening is surrounded by an oval, fibrous ring to which the segments of the valve are attached. To prevent regurgitation of blood when the ventricle contracts, the opening is guarded by a valve consisting of three flaps, formed of folds of endocardium strengthened by fibrous tissue and some muscular fibres, which are triangular in shape with the base attached to the fibrous ring and the apex free. When the ventricle contracts, the blood insinuates itself behind these flaps, which when the ventricle is passive, hang loosely in its cavity, and forces them before it until they come together, their apices meeting in the centre of the opening. The segments, which are quite flexible, are kept from being forced into the auricle by the attachment of a number of muscular and tendinous cords which hold them in the ventricle. The muscular columns are called columnæ carnex and are divided into three sets arranged as follows: some form mere ridges, being attached to the wall of the ventricle throughout their length; the second set are attached to the wall of the ventricle by each end and are free in the middle; while the third are attached by one end only to the ventricular wall, 
terminating at the other in tendinous cords which are themselves attached to the ventricular aspect of the segments of the valve and are only long enough to allow these to close without floating into the auricle. The valve thus formed is known as the tricuspid, and the tendinous cords as chorda tendinea.

The blood, driven by the contraction of the venous ventricle, is forced into the pulmonary artery and, when the ventricle ceases to contract, would again return to it were it not for a valvular arrangement here, called the pulmonary semilunar valve, which guards the orifice of the pulmouary artery. The semilunar valve is three segments arranged around the interior of the pulnonary artery, just at its commencement. Each segment is semilunar in shape, its convex border being attached while the superior border is free and straight. They consist of folds of lining membrane, strengthened by fibrous tissue which, just at the centre of the free edge, is aggregated into a projection called corpus-arantii. In the centre the valve lacks fibrous tissue and presents a lunated space. Behind each serment there is a depression caused by a dilation of the artery, called sinus of Valsalva, and when the ventricle ceases to contract the blood, seeking to re-enter the carity, enters the sinuses of Valsalva and forces the segments together until they meet along their free edges and close the orifice. The corpora arantii have small effect in closing this orifice.

The objects seen in the right ventricle then are: 1st, columna carnex; $2 \mathrm{~d}$, chorda tendinex; $3 \mathrm{~d}$, venous auriculo-ventricular opening; 4th, opening into pulmonary artery; 5 th, tricuspid valve; 6 th, semilunar valve; 7 th, foramina Thebesii.

The arlerial auricle has thicker walls than the venous, being about one-eighth inch thick while the right is only onetwelfth inch. It presents musculi pectinati, which are fewer in number than in the right auricle, being almost confined to the appendix auriculat the arterial auriculo-ventricular opening; the openings for the four pulmonary veins, which return the blood from the lungs. Two of these veins come from each lung, the two from the left sometimes uniting before entering the auricle. 
To sum up, we have 1st openings for four pulmonary veins, 2nd arterial auriculo-ventricular opening, 3rd musculi pectinati.

The arterial ventricle has walls three times as thick as those of the venous, being seven lines thick while the venous is only about two and a half lines. The left side of the heart, therefore, is much more powerful than the right; this being due to the fact that while the venous ventricle has only to drive the blood into the lungs, the arterial has to send it throughout the entire system. On the walls of the arterial ventricle are seen columnæ carneæ much more prominent and more intimately intersecting than in the venous ventricle, but similar to them in arrangement, viz : some attached throughout their length, some at each end and free in the middle, some attached at one end to the wall of the ventricle, while the others terminate in tendinous cords attached to the ventricular aspect of the bicuspid valve. The blood is forced from the arterial auricle into the arterial ventricle, and when this contracts it is forced into the aorta. Both these openings are guarded by valves. The valve guarding the arterial auriculo-ventricular opening is known as the bicuspid, or mitral, and is arranged upon the same principle as the tricuspid, being made up of two segments attached by their bases to the fibrous ring around the opening while their apices are free in the cavity of the ventricle. It consists of folds of endocardium strengthened by fibrous and muscular tissue and receives the attachment of the chorda tendinæ. It differs from the tricuspid valve in being stronger and in having only two segments. The opening into the aorta is guarded by the aortic semiluna valve, which is in every way the counterpart of the pulmonary semilunar, except in being larger and stronger, consist of three segments of lining membrane and fibrous tissue, with corpora-arantii, lunated spaces and sinuses of Valsalva. The mechanism of their action is also the same as in the pulmonary semilunar.

To sum up, there are in the arterial ventricle: 1st, columnæ carneæ; 2d, chordæ tendineæ; 3d, arterial auriculo-ventricular opening; 4th, aortic opening; 5th, bicuspid valve; 6 th, aortic semilunar valve. 


\section{THE MUSCULAR SYSTEM.}

When an incission is made in any part of the body, the following structures, from without in ward, are met with: 1st, skin; 2d, superficial arcolar fascia whose existence allows the free movement observed in almost every part of the integument. It consists of two layers, between which is generally found fatty tissue, varying in quantity in different parts of the body; $3 d$, beneath the superficial fascia, in some parts of the body, are found the muscles with their proper investing fascia; while in others there is interposed between them and the superficial fascia a strong, and more or less thick, membrane, called the investing fascia, or deep fascia which frequently gives off from its deep face partitions, which separate different groups of muscles.

The function of muscles is to produce movement, most of them being attached to bone, some having no bony attachment, while others are attached to bone by one extremity the other terminating in some soft tissue. When a muscle is attached to bone by both extremities a movable joint is almost invariably found between the two extremities, otherwise no movement would occur when the muscle attempted to contract, so that, as a rule, those muscles, which lay hold of bone at at both extremities, are attached to different bones at either end. The two attachments of a muscle are spoken of, one as the origin, the other as the insertion, the origin being usually that attachment susceptible of the least motion, and as a rule, especially true of the limbs, the origin is the point nearest the body, or the mid-line of the body. The action of a muscle consists in the shortening of its length, thus drawing the parts to which it is attached nearer, usually by movement of the part into which it is inserted. Some movements are two complex to be brought about by one muscle and result from the co-ordinated action of several.

The striated muscles are the red, fleshy portion of the body, though generally a muscle consists of two parts: the red 
fibres, gathered into bundles, fasciculi, forming a coarse, or a fine muscle, and a white, tougher, stronger portion called its tendon. With few exceptions the tendon is found at the extremities of the muscle, and, as it is so much stronger, bulk for bulk, the tendon is much the smaller part of the muscle, thus economizing space at the attachments. The portion of muscle between its tendons of origin and insertion is called the belly of the muscle. In a few instances a muscle has two bellies, since it may have a central tendon.

The names of most muscles are derived from one of the following facts: 1 st, the position which it occupies ; 2 nd, its shape; $3 \mathrm{rd}$, the direction of its fibres; 4 th, its attachments : 5 th, its action; 6th, the number of its points of origin.

\section{The Abdominal Muscles.}

The anterior abdominal wall is formed chiefly by six flat, thin muscles, three on each side of the middle line, called the broad muscles of the abdomen. Besides these there are two other pairs, the pyramidales, small and insignificant, and the recti which are long and narrow. The abdominal wall consists of the following structures from without inward, viz: 1st, integument; $2 \mathrm{~d}$, two layers of superficial fascia with fatty tissue interposed; $3 \mathrm{~d}$, the external oblique muscle; 4 th, the internal oblique ; 5 th, the transversalis ; 6 th, the transversalis fascia; 7th, the parietal layer of peritoneum. These muscles all terminate in tendinous fibres as they approach the median line; and, since there is no bone for them to seize, their fibres interlock, forming a white line extending from the ensiform cartilage to the symphysis pubis called the linea alba. The whole line is sunken below the surrounding surface and presents about its centre the umbilicus or navel. The sunken appearance is caused by the bulging of the recti, which lie on either side of it, the width of the linea alba corresponding to the interval between the recti.

\section{External Oblique.}

The external oblique of the abdomen derives its name from the downward and inward direction of its fibres and from its 
position, being external to another oblique muscle. It arises by eight flesny, tooth-like processes or digitations from the anterior surfaces of the eight lower ribs. The upper five digitations interlock with similar processes of the serratus magnus, the lower three with the latissimus dorsi. From their origin the fibres pass downward and inward, the digitations gradually blending to form the belly of the muscle which, toward the front of the abdomen, terminates in a thin, flat tendon, called an aponeurosis, which is inserted into the linea alba from the sternum to the symphysis pubis. The posterior fibres pass almost vertically downward and are inserted into about the anterior one-half or two-thirds of the outer lip of the crest of the ilium; while the intermediate fibres are inserted into the anterior superior spinous process of the ilium and into the spine and pectineal line of the pubes. That portion of the aponeurosis which extends fron the anterior superior spinous process of the ilium to the spine of the pubes is called Poupart's ligament. It is the lower border of the aponeurosis, is free between its points of attachment and folded slightly inward on itself. About an inch from the spine of the pubes Poupart's ligament sends downward some fibres which are inserted into the pectineal ine of the pubis, forming Gimbernat's ligament, which is triangular in shape with its apex within and its base extending for about an inch out ward.

At the lower, inner part of the aponeurosis is seen an openins, near the symphysis pubis, called the external abdominal ring. It is formed by a separation of the fibres of the aponeurosis, is triangular in shape and oblique in direction, its apex being upward and outward and its base downward and inward at the crest of the pubes. The edges of the aponeurosis which form the sides of the ring are called its pillars, internal and external, the external being inserted into the spine of the pubes and the internal interlocking will the internal pillar of the opposite side over the front of the pubes. For a short distance the apex of the ring is obscured by some curved fibres called intercolumnar fibres. The external abdominal ring transmits in the male the spermatic cord and in the feniale the round ligament. 


\section{Internal Oblique.}

The internal oblique muscle of the abdomen derives its name from the upward and inward obliquity of its fibres and from the position it occupies just beneath the external oblique. It arises by a thin tendinous membrane, called the fascia lumborum, from the spinous processes of the lumbar vertebræ, the anterior two-inirds of the middle lip of the crest of the ilium, and from the outer half of Poupart's ligament.

The fascia lumborum terminates in fleshy fibres on the side of the abdomen, and those fibres springing from the crest of the ilium are also fleshy: these radiate somewhat, the general direction, however, being upward and inward, and are inserted, posteriorly, into the cartilages of the four lower ribs; while on the front of the abdomen the fibres become aponeurotic and are inserted into the whole length of the linea alba. The fleshy fibres which rise from the outer half of Poupart's ligament are biended with fibres of the transversalis which arise from the same part of the ligament. These blended fibres arch downward and inward and form, so long as they remain fleshy, the conjoined arch; but as they descend they become tendinous, forming the conjoined tendon which is inserted into the crest and pectineal line of the pubes. By reason of the arched direction of these fibres there is a short space between them above and Poupart's ligament below, their conjoined arch without and conjoined tendon within, where the wall of the abdomen has but one muscular element, viz: the aponeurosis of the external oblique. If an incision were made at this point we would pass through: 1 st, the integument; $2 \mathrm{~d}$, two layers superficial fascia ; $3 \mathrm{~d}$, the aponeurosis of the external oblique; 4 th, transversalis fascia and the parietal layer of the peritoneum. The conjoined tendon descends just behind the external abdominal ring, so that at this point the wall is wanting in only one muscular element-the aponeurosis of the external oblique.

\section{Transversalis.}

The transversalis derives its name from the horizontal direction of its fibres. It lies just beneath the internal oblique 
and is the deepest of the three broad muscles of the abdomen. It arises by means of an aponeurosis trom the transverse and spinous processes of the lumbar vertebra, fleshy from the inner aspect of the cartilages of the six inferior ribs, interlocking with the diaphragm, fleshy from the anterior two-thirds of the inner lip of the crest of the ilium and fleshy from somewhat less than the upper half of Poupart's ligament. The posterior aponeurosis becomes flesliy on the side of the abdomen and these fibres, being reinforced by those which arise from the costal cartilages and crest of the ilium, pass forward, become aponeurotic and are inserted into the whole length of the linea alba. The fibres which rise from Poupart's ligament blend with those of the internal oblique to form the conjoined arch and conjoined tendon which is inserted into the crest and pectineal line of the pubes.

\section{Rectus.}

The rectus abdominis derives its name from the straight course of its fibres. It lies on the front of the abdomen, beside the linea alba, surrounded by the aponeuroses of the three broad muscles of the abdomen. It is flat and ribbon-like in shape. It arises by a flat tendon from the front of the symphysis pubis and crest of the pubes; as it ascends it hecomes fleshy and broader and terminates by three digitations which are inserted into the fifth, sixth and seventh cartilages just beside the sternum. In the upper half of its course the rectus presents three or four tendinous intersections which are called linea transversa. Before the aponeurosis of the external oblique has been remored, the outer edge of the rectus presents a curved ridge called linea semilunaris.

The rectus as it ascends is enclosed between the aponeuroses of the three broad nuscles, and these form what is called its sheath. This sheath is complete in front for the whole length of the muscle, but behind is vanting for its lower fourth. It is thus formed: for the upper three-fourths of the rectus, or from a point half way between the umbilicus and the symplysis pubis upward to its insertion, the rectus has in front the aponcurosis of the 
external oblique and half the aponeurosis of the internal oblique; while for the same distance behind it has the aponeurosis of the transversalis and half of the aponeurosis of the internal oblique, the latter aponeurosis splitting, when it reaches the outer edge of the rectus, sending one layer in front and the other behind that muscle. Of course it also has. behind it the transversalis fascia and the parietal layer of peritoneum separating it from the abdominal carity. For its lower fourth, that is from the point midway between the umbilicus and symphysis pubis, all three aponeuroses pass in front of it; and behind it is separated from the abdominal cavity only by the parietal layer of the peritoneum and the transversalis fascia. Where the sheath ends behind it is curved and is called the fold of Douglas.

\section{Pyramidalis.}

The pyramidalis abdominis is small and derives its name from its pyramidal shape. It is found in the sheath of the rectus, in front of its lower portion, and is frequently absent on one or both sides. It rises from the frout of the crest of the pubes and passes upward, tapering as it ascends to be inserted into the linea alba half way to the umbilicus.

Action-The abdominal muscles are agents of expulsion as in voiding the urine and fæces and in terminating labor. They are also agents of forced expiration, for when they contract they diminish the size of the abdominal cavity, forcing the abdominal viscera against the walls of that cavity and thus driving the diaphragm upward. while at the same time, by drawing down the ribs, they in a measure diminish the size of the thorax.

They can also, acting from below, flex the body on the pelvis; or, acting from above, draw the pelvis and lower extremities upward on the body. One External oblique, acting alone, would draw the body downward and to the side opposite the muscle acting. Of course, if the fixed point be above, it would draw the pelvis upward and towards the muscle which is acting. The internal oblique of one side may act in concert with the external oblique of the opposite side. The trans- 
iersales, acting together, compress the abdominal contents backward and inward. The recti, when the lower attachment is fixed,draw the body downward; or, when acting from above, will draw the pelvis upward. The tyramidales are tensors of the linea alba.

These muscles are supplied by the lower intercostal nerves and the ilio-hypogastric. Blood is received from the lumbar lower intercostal, deep epigastric and internal mamuary.

\section{Diaphragm.}

1st. Definition and position-The diaphragm is the arched muscular septum between the two cavities of the trunk, presenting its upper convex surface as the floor of the thorax and its equally concave under surface as the roof of the abdomen.

2nd. Relations-By its upper surface it supports the pleura on either side, containing the lungs, and the pericardium in the centre, containing the heart. Below it is covered by peritoneum and is in relation with the liver, spleen, stomach, pancreas and kidneys.

3rd. Origin-In front it arises from the posterior surface of the sternum, on each side from the inner surface of the six lower ribs and from a ligamentum arcuatum externum and internum, behind from the front of the vertebral column by two crura.

4th. Its points of origin studied individually.

(a) Between that part which arises from the posterior surface of the sternum and that part on each side which arises from the adjacent ribs there usually exists a fissure for a short distance, the muscular structure here being wanting, the aperture, which would otherwise exist, between the thoracic and abdominal cavities being closed by other structures.

(b) The origin from the inner surface of the ribs is by digitations which interlock with similar digitations of the transi'ersalis abdominis.

(c) The ligumeutum arcualum extermum arches across the quadratus lumborum muscle from the apex of the twelfth rib to the apex of the transverse process of the first or second lumbar vertebra, usually the second. 
(d) The ligamentum arcuatum intermum arches over the psoas magnus muscle from the apex of the transverse process of the first or second lumbar vertebra to be lost in the crus of the same side.

(e) The right crus arises tendinous from the front of the bodies of the second, third and fourth lumbar vertebræ.

(f) The left crus arises tendinous from the front of the bodies of the second and third lumbar vertebæ. It is smaller as well as shorter than the right.

5th. The appearances which it presents.

The muscular fibres of the diaphragm, as they are making for the centre, become tendinous. The central portion, therefore, is called the tendinous portion and is arranged like a clover leaf, presenting a central, a right and a left leaflet, the tendon is therefore called trefoil or central tendon.

The diaphragm is pierced by three large apertures which are for the transmission of the following structures, viz: 1st, descending aorta, vena azygos major, thoracic duct and sometimes the sympathetic nerve; $2 \mathrm{~d}$, the ascending vena cava; $3 \mathrm{~d}$, asophagus and pnemmogastric nerves.

The crura, as stated, arise tendinous from the front of the vertebral column, but as they ascend they become fleshy and leave a slight interval between them. Over the front of the twelfth dorsal vertebræ each crus gives off from its inner side a bundle of fibres which crosses obliquely to join the opposite crus, the one from the right crus being usually in front. This interchange of fibres is known as the decussation of the crura and it leaves between its commencement and the vertebral column an opening which is known as the aortic. This opening is over the front of the body of the twelfth dorsal vertebra, generally slightly to the left of the middle line, but sometimes on it. The opening is bchind the diaphragm and not through it.

The decussating fibres, after passing into the opposite crus, again separate from each other leaving an opening around which they again unite. This is the osophageal opening and 
is situated above and to the left of the aortic opening, between it and the middle leaflet, and about opposite the tenth dorsal vertebra.

The opening for the inferior vena cava is to the right of the middle line, between the right and middle leaflets of the tendinous centre and about opposite the ninth dorsal vertebra.

6th. Action-The diaphragm is a respiratory muscle and an agent of expulsion. When it contracts, as it does eighteen to twenty times a minute, it descends, becoming less arched and thus increasing the size of the thorax while it decreases the size of the abdomen, though the latter is compensated by the bulging of the anterior abdominal walls; but should it be necessary to exert a straining effort, as in voiding the urine or faces or in parturition, the muscles of the abdominal wall contract at the same time that the diaphragm does, so that the abdominal cavity is diminished in size from above downward as well as from before backward, and its viscera subjected to compression. When the diaphram relaxes it again ascends, thus lessening the size of the thoracic cavity.

The diaphragm is supplied by the phrenic nerves. Blood vessels come from the internal mammary and the phrenic branches of the abdominal aorta.

\section{Quadratus Lumborum.}

The quadratus lumborum is a quadrilateral muscle found in the posterior abdominal wa1l, in the lumbar region 1ying beside the vertebral column. It is enclosed between the middle and anterior lamella of the posterior aponeurosis of the transversalis.

It arises from the last rib and descends, being attached by its inner edge to the transverse processes of the lumbar vertebra, to be inserted into the ilium and the ilio-1umbar ligament.

Action-It draws the vertebral column to one side, and acts as an expiratory agent by drawing down the last rib. It also acts as an inspiratory agent by fixing the last rib and preventing the diaphragm frowl drawing it in ward. The two muscles, acting together assist in flexing the body; while either may bend the trunk to its own side. 
The nerves and arteries are from the anterior branches of the lumbar nerves and arteries.

\section{Muscles of the Neck.}

When the skin has been removed from the neck, it reveals a superficial fascia, thin and delicate, continuous below with that which covers the thorax and above with that on the face. Beneath this is the Platysma Myoides muscle, and, on removing this, we encounter the deep or investing cervical fascia.

This fascia is a dense membrane firmly attached behind to the spinous processes of the cervical vertebræ, where it blends with the origin of the muscles in that region. and completely encircles the neck. Below it in part is attached to the clavicle and sternum in part it blends with the deep fascia covering the thoracic muscles while another portion passes into the thoracic cavity where it blends with the pericardium and the fascia supporting the great vessels. Above it is attached to the lower jaw, covers in the parotid gland and gives off some bands considered as ligaments. Like all investing, or enveloping, fascias, it divides into layers, or septa, which form sheaths for neighboring muscles, vessels, nerves and other structures.

Leaving the cervical spines in a single layer, it splits to enclose the trapezius; uniting, at the anterior border of that muscle to cover in the triangular interval between it and the Sterno-matsoid, the two layers again divide and form a sheath for the latter muscle, and, again uniting, they pass forward as a single layer to the midline of the body in front, where the fascia of the opposite side of the neck blends with the one described. Of the numerous septa given off from the deep surface of this fascia are: one which forms a delicate sheath for the infra-hyoid group of muscles; one which envelops the trachea, called pre-tracheal fascia, and one which passes in front of the pre-vertebral muscles and is called the pre-vertebral fascia. From the pre-tracheal and pre-vertebral facias is derived an important layer which forms the sheath of the carotid vessels.

The muscles of the neck are divided into a superficial and a deep group; the former containing two muscles and the latter, 
subdivided into supra and infira-hyoid groups, or elevators and depressors of the hyoid bone, nine.

\section{Superficial Group.}

Platysma Myoides

Sterno-Cleido-Mastoid

\section{Platysma Myoides.}

When the integument and superficial fascia have been remored from the front and side of the neck there is seen a thin pale broad muscle called the platysma myoides. It arises from the fascia covering the pectoralis major and deltoid muscles, and passes upward and inward over the clavicle to cover the side and front of the neck. Its innermost fibres are interlocked with the fibres of the opposite muscle along the upper part of the middle line of the neck; the others are inserted into the inferior maxilla, some passing over it to be lost on the side of the face and some continuing to the angle of the mouth ; these last are joined by some accessory fibres which take their origin on the side of the face from the fascia covering the masseter muscle. These fibres have been considered as a separate muscle called risorius Santorini or laughing muscle.

Action-It is a depressor of the lower jaw. It can draw the angle of the mouth down ward so as to produce a melancholy expression or carry it bacliward as in laughing.

\section{Sterno-Cleido-Mastoid.}

The stcmo-cleido-mastoid is the second muscle in the superficial group and lies beneath the platysma between two layers of the deep cervical fascia. It is one of the most inportant muscles in the body and derives its name from its origin and part of its insertion.

It arises by a forked origin. One fork, a more or less rounded tendon, springs from the upper front part of the manubrium; the other, musculo-tendinous, arises from about the inner one-third or one-half of the clavicle. This origin is very varible. An interval, filled by fibrous tissue usually exists between the two heads. The muscle passes obliquely upward, backward and a little outward on the side of the neck and is inserted into the mastoid portion of the temporal bone 
and adjacent part of the superior curved line of the occipital. Between its two origins there is a fissure which extends upward for some distance.

Action-When both musclesact they bow the head forward; when one acts it draws the head to that side, turning the face somewhat to the opposite side.

\section{Depressors of the Hyoid Bone.}

$\begin{array}{ll}\text { Sterno-Hyoid } & \text { Sterno-Thyroid } \\ \text { Thyro-Hyoid } & \text { Omo-Hyoid }\end{array}$

These muscles are arranged in two layers, the two sternohyoids and omo-hyoids forming the one in front and the sternothyroids and the thyro-hyoids forming the one behind. By their inner edges the sterno-hyoids and thyro-hyoids are in contact, save for a small triangular space just above the sternum. The anterior belly of the omo-hyoid is also, through the greater part of its course, in contact with theouter edge of the sterno-hyoid. After the deep fascia has been removed these muscles are widely separated and show between them much larger triangular spaces than really exist.

\section{Sterno-Hyoid.}

The sterno hyoid derives its name from its attachments. It is ribbon-like in shape and vertical in direction. It arises from the posterior aspect of the manubrium sterui and perhaps from the inner extremity of the clavicle or sterno-clavicular ligament, and ascends the neck beside the middle line to be inserted into the hyoid bone.

\section{Sterno-Thyroid.}

Lying behind the sterno-hyoid and slightly broader than it, is another ribbon-like muscle called, from its attachments, stemo thyroid. It arises from the posterior surface of the manubrium and possibly from the cartilage of the first rib, and, ascending beside the middle line is inserted into the oblique ridge on the ala of the thyroid cartilage. Below it is often traversed by a tendinous intersection as is the sterno-hyoid.

\section{Thyro-Hyoid.}

The thyro-hyoid is a short ribbon-like muscle which continues the course of the sterno-thyroid to the hyoid bone, and 
derives its name from its attachments. It arises from the oblique ridge on the ala of the thyroid cartilage and is inserted into the hyoid bone. Frequently some of the fibres of the sterno-thyroid are continued directly into this muscle.

\section{Omo-Hyoid.}

The omo-hyoid derives its name from its attachments. It is a double-bellied muscle passing with a curve across the side of the neck.

It arises from the upper border of the scapula, near the suprascapular notch, and perhaps from the ligament stretched across the notch; and passes forward, inward and slightly upward until, beneath the sterno-cleido mastoid, it becomes tendinous, its posterior belly ceasing. It then again becomes fleshy, forming the anterior belly, and passes nearly upward but with a slight inclination inward, to its insertion in the hyoid bone at the junction of its body and greater cornu. The central tendon is held down, so as to give the muscle its curve, by a loop of fascia which is said to pass to the cartilage of the first rib.

Action-The depressors of the hyoid bone, as the name indicates, draw the hyoid bone down. The sterno-thyroid draws down the larynx and the two omo-hyoids, acting together, draw the hyoid bone downward and backward. The omohyoid of either side, acting alone, might draw the hyoid bone downward, backward and toward that side. The thyro-hyoids have the important action of drawing the thyroid cartilage, and of course the larynx, uprvard under the hyoid bone, protecting the larynx in swallowing.

Nerve supply from descending noni. Arteriessuperior and inferior thyroid.

\section{Elevators of the Hyoid Bone.}

\section{Digastric \\ Stylo-Hyoid \\ Mylo-Hyoid \\ Genio-Hyoid}

Genio-Hyo-Clossus

\section{Digastric.}

The disastric, as its name indicates, is a double-bellied muscle. It is round in shape, curved in direction and found 
at the upper part of the side of the neck. It arises fleshy from the digastric fossa of the temporal bone and passing downward and forward, becomes tendinous; the portion between its origin and central tendon being called the posterior belly. It then again becomes fleshy, forming the anterior belly, and passing forvard and upward is inserted into the digastric fossa of the inferior maxilla. The central tendon pierces obliquely the belly of a small muscle which lies beside it called the stylo-hyoid, and, after emerging from it, plays through a loop of fascia which binds it down to the hyoid-bone.

\section{Stylo-Hyoid.}

The stylo-hyoid is a small muscle found lying beside the posterior belly of the digastric and deriving its name from its attachments. It arises from the outer side of the styloid process of the temporal bone, and passing downward and forward is inserted into the hyoid bone. Just before its insertion it is pierced by the central tendon of the digastric.

\section{Mylo-Hyoid.}

The mylo-hyoid is a broad, thin, triangular muscle which forms the floor of the mouth. It arises from the whole length of the mylo-hyoid ridge, and, passing nearly invard, with a slight inclination downward, the greater portion of the muscle meets its fellow of the opposite side on the middle line, forming a raphe, while some of its posterior fibres are inserted into the hyoid bone.

\section{Genio-Hyoid.}

The genio-hyoid is a small muscle which slightly increases in size as it descends. It lies beneath the mylo-hyoid just beside the middle line. It arises from the inferior spina mentalis and passes backward and slightly downward to be inserted into the hyoid bone.

\section{Genio-Hyo-Glossus.}

The geni-hyo-glossus is a thin, radiating fan-shaped muscle. It arises by a narrow tendinous origin from the'superior spina mentalis and immediately radiates, fleshy, to be inserted into the under surface of the tongue from its base to near its 
aper, some of its lower fibres being inserted into the hyoid bone. This muscle lies close to the middle line beside its fellow.

Action-The general function is that of elevation of the hyoid bone or depression of the lower jaw. The posterior bellies of the digastrics can carry the hyoid upward and backward, while the anterior bellies carry it upward and forward. The stylo-hyoid acts in concert with the posterior belly of the digastric. The posterior belly of one digastric, and the stylohyoid draw the hyoid upward, backward and to the side of the muscle acting. The mylo-hyoid acts like a swing which by shortening its fibres can elevate the floor of the mouth. The posterior fibres can draw upward and forward the hyoid bone. The genio-hyoid pulls the hyoid bone, and with it the tongue, upward and forward. The genio-hyo-glossus may pull the hyoid upward, slightly, but its main function is in moving the tongue, which it draws forward and upward.

Nerves. Stylo-hyoid and posterior belly digastric by the facial,-genio-hyoid and genio-hyo-glossus by the hypoglossal; mylo-hyoid and anterior belly of digastric by inferior dental.

\section{Surgical Triangles of the Neck.}

The side of the neck presents a quadrilateral surface which is bounded below by the clavicle, above by the body of the inferior maxilla and an imaginary horizontal line passing from its angle to the mastoid process of the temporal bone, in front by the middle line and behind by the anterior edge of the trapezius.

This space, is subdivided bv the sterno-mastoid into a great anterior and a great posterior triangle. The anterior triangolc is bounded as follows : in front by the middle line, behind by the sterno-cleido-mastoid, above by the body of the lower jaw and the imagrinary line. The base is above, the apex below. The posterior triansle has its base below, formed by the clavicle. It is bounded in front by the sterno-cleido-mastoid and behind by the anterior edge of the trapezius.

Each of these triangles is again subdivided into smaller ones. The anterior is divided int) three: 
1st. The inferior carotid triangle, or triangle of necessity, is bounded in front, by the median line; behind, by the anterior margin of the sterno-mastoid; above, by the anterior belly of the omo-hyoid. Its base is internal.

$2 \mathrm{nd}$. The superior carotid triangle, or triangle of election, is bounded behind by the sterno-mastoid; below, by the anterior belly of the omo-hyoid; above, by the posterior belly of the digastric. Its base is external.

3rd. The digastric or submaxillary triangle has its base formed by the lower jaw and the imaginary line and its sides formed, posteriorly, by the posterior belly of the digastric and stylo-hyoid, anteriorly, by the anterior belly of the ligastric.

The great posterior triangle is subdivided into two:

1st. The suboccipital triangle has its base below. It is bounded in frout by the sterno-mastoid; behind, by the anterior edge of the trapezius; below, by the posterior belly of the omo-hyoid.

2nd. The subclavian triangle has its base below. It is bounded above by the posterior belly of the omo-hyoid; in front, by the sterno-mastoid; below, by the clavicle. It contains the subclavian artery and vein, hence its name, and the brachial plexus of nerves. In the posterior triangle the subdivisions are made by the posterior belly of the omo-hyoid; in great anterior triangle by the anterior belly of the omo-hyoid, and the two bellies of the digastric.

\section{The Scaleni Muscles.}

The two scaleni muscles form part of a group called prevertebral.

The two scaleni muscles are distinguished as scalenus anticus and posticus. A small portion of the scalenus posticus is sometimes individualized as the scalenus medius. These muscles are of great importance owing to the fact that they occupy a position in the lower part of the side of the neck, and come into important relation with the subclavian artery and brachial plexus of nerves.

\section{Scalenus Anticus.}

The scalenus anticus, triangular in shape, arises from the anterior tubercles of the transverse processes of the third, 
fourth, fifth and sixth cervical vertebra by tendinous slips, which, uniting as they descend, form the muscle. It is inserted by a narrow tendon into the upper surface of the first rib.

\section{Scalenus Posticus.}

The scalemus posticus arises from the posterior tubercles of the transverse process of all the cervical vertebræ except the first. It descends and divides into two portions, one of which is inserted into the first rib between its tubercle and angleand is the part sometimes called scalenus medius - while the other goes to the same point on the second rib.

The scaleni, acting from above, draw up the first and second ribs, being muscles of inspiration. Acting from below they can draw the neck downward and to the side of the muscles acting; or, the muscles of both sides acting, can flex the spinal column.

The nerve supply is from the lower three cervical nerves.

\section{Prevertebral Group.}

Rectus Capitis Anticus Major Rectus Capitis Anticus Minor
Longus Colli

Rectus Capitis Lateralis

\section{Rectus Capitis Anticus Major.}

This muscle arises from the anterior tubercles of the transverse processes of the third, fourth, fifth and sixth cervical vertebræ by small tendinous fasciculi which terminate in a flattened muscle. The muscular fibres terminate in a flat aponeurosis which is inserted into the basilar process of the occipital, in front of the foramen magnum.

\section{Rectus Capitis Anticus Minor.}

This is a small muscle lying beneath the preceding and further from the mid-line. It arises from the transverse process of the atlas and is inserted into the basilar process of the occipital, slightly external to the preceding.

\section{Rectus Capitis Lateralis.}

This muscle belongs properly to the lateral vertebral region and is an inter-transverse muscle. It arises from the transverse process of the atlas and is inserted into the jugular process of the occipital. 


\section{Longus Colli.}

This muscle might be appropriately described as three. One arises from the transverse processes of the third, fourth and fifth cervical vertebræ and is inserted into the transverse process of the atlas. The second arises from the bodies of the upper three dorsal vertebrae and is inserted into the transverse processes of the third and fourth cervical. The third division arises from the three upper dorsal and four lower cervical and is inserted into the second and third cervical.

The muscles act so as to flex and rotate the head and cervical vertebrae.

Anterior branches from the cervical nerves supply these muscles.

\section{The Muscles of the Back.}

These muscles are arranged in several superimposed layers and are covered by a superficial fascia which varies in thickness in different regions. In some situations, as in the 1 umbar region, it usually contains a large amount of fat; in other portions it is a thick, loosely woven connective tissue, not easily divisible into layers, but everywhere allows free movement of the skin. Many of the muscles arise from, or are covered by, a dense connective tissue forming the vertebral aponeurosis. This fascia is attached to the spines and transverse processes of the vertebræ from which points it sends out septa which not only give origin to muscular fibres, but serve as sheaths for some of the muscles.

\section{First Layer.}

\section{Trapezius}

\section{Trapezius.}

The trapezins arises from the superior curved line of the occipital bone, from the posterior occipital protuberance and from the spinous processes of all the dorsal and cervical vertebra. The fibres converge outward, some ascending obliquely, some descending obliquely and some running horizontally, to reach the insertion into the outer third of the clavicle, the acromion process and whole length of the spine of the scapula. Its insertion is the same as the origin of the deltoid. 
Extending from the posterior occipital protuberance to the seventh cervical rertebra, and attached to the intervening spinous processes, is a fibrous cord called ligamentum nucha; and it is in reality from this cord that the trapezius arises.

Nearly the whole of the origin of this muscle is tendinous. The central portion of the tendon is broad and thin and forms a diamond shaped space between the two muscles.

Action-According to the direction of the fibres which act it can draw the scapula directly backward, downward and backward or upward and backward.

\section{Latissimus Dorsi.}

The lutissimus dorsi arises from the spinous processes of the lower four or six dorsal vertebræ, all the lumbar vertebræ and the spinous tubercles of the sacrum, from the posterior third of the crest of the ilium and by fleshy slips from the three or four lower ribs. The muscle is tendinous at its origin except that part arising from the ribs. As it passes upward and outward the fibres converge, become thicker and fleshy and passing over the lower angle of the scapula, wind around the teres major to be inserted along with it into the posterior bicipital ridge of the humerus. The tendon of the teres major is wider and extends further down on the humerus than that of the latissimus dorsi.

Action-It carries the humerus downward and backward, rotates it inward, is an inspiratory agent, and, when the humerus is fixed, as in climbing or using crutches, it moves the body forward:

The trapezius is supplied by the spinal accessory and branches from the anterior divisions of the third and fourth cervical. The long sub-scapular supplies the latissimus dorsi.

\section{Second Layer.}

Levator Anguli Scapulæ Rhomboideus

\section{Levator Anguli Scapulæ.}

The levator anguli scapule arises by tendinous slips from the posterior tubercles of the transverse processes of the four 
upper cervical vertebre. These slips unite to form the belly of the muscle which descends obliquely outward to be inserted into the posterior border of the scapula from the angle to the intersection of the spine with that border.

This muscle draws the shoulder upward and inward, or, acting from below, inclines the neck and head to that side, rotating them at the same time.

The nerve for the levator is from the anterior divisions of the third and fourth cervical.

\section{Rhomboideus.}

The rhomboideus arises from the spinous processes of the last cervical and four upper dorsal vertebræ, and passing downward and outward is inserted into the posterior border of the scapula as far as the inferior angle. Some make two muscles of this, calling the portion which arises from the cervical vertebra rhomboideus minor and that arising from the dorsal vertebræ rhomboideus major.

Action-It moves the scapula upward and backward, so as to bring the two scapulæ nearer together.

\section{Third Layer}

Serratus Posticus Superior Serratus Posticus Inferior Splenius

\section{Serratus Posticus Superior.}

The serratus posticus superior has a tendinous origin from the spinous processes of the two lower cervical and two upper dorsal vertebræ. It passes downward and outward and is inserted by fleshy serrations into the upper borders of the second, third, fourth and fifth ribs just beyond their angles.

Action-It is an inspiratory agent.

\section{Serratus Posticus Inferior.}

The serratus posticus inferior has a tendinous origin from the spinous processes of the last two dorsal and first two lumbar vertebræ. It passes upward and outward and is inserted by fleshy slips into the lower borders of the last four ribs.

Action-It is an expiratory agent. 


\section{Splenius.}

The splenius arises from the spinous processes of the four lower cervical vertebræ and four or six upper dorsal vertebræ. It ascends and divides into two portions, one, known as the splenius capitis, is inserted into the occipital bone between its curved lines and into the mastoid portion and process of the temporal bone; the other, called splenius colli, is inserted into the posterior tubercles of the transverse processes of the three upper cervical vertebra.

Action-It bends the head and upper part of the spine back and rotates the head toward its side.

\section{Erector Spinæ.}

This powerful muscle occupies a large portion of the vertebral groove, extending from the sacrum upward until its prolongations may be traced through the cervical region. It is held down in position, through a large portion of its course, by the powerful vertebral aponeurosis, call by different names in the different regions. In the lumbar region, where it is most powerful, it is continuous with the aponeurosis forming the sheath of the quadratus lumborum.

The erector spince arises by tendinous fibres from the back of the sacrum, occupying the whole of the sacral groove; from the posterior part of the crest of the ileum: from the great sacro-sciatic ligament and from the spinous processes of the lumbar vertebra. This large mass passes upward and, about opposite the last rib, it divides into two portions, the ilio-costalis, which is inserted into the seven lower ribs and the congrissiums dorsi which is inserted into the transverse processes of the dorsal vertebra and into nearly all the ribs, this portion of the insertion being very variable. Between these two divisions lies an accessory muscle, called musculus accessorizs which, arising from the angles of the six lower, is inserted into the angles of its six upper ribs. Continuing this upward is the cericicalis ascendeus which, springing from the angles of the four upper ribs is inserted into the posterior tubercles of the transverse processes of the fourth, fifth and 
sixth cervical vertebræ. In like manner the longissimus dorsi is continued upward by an inner set of fibres the transversalis colli which springs from the transverse processes of the six upper dorsal and is inserted into the cervical as high as the second ; and the trachelo-mastoid which arises from the transverse processes of the dorsal vertebræ from the second to the sixth is inserted into the mastoid process.

Beside these there are two small muscular masses, the spinalis dorsi, in the lumbar, and spinalis colli in the cervical region which spring from the transverse processes below and are inserted into those above. They are really part of the attachment of the longissiumæs dorsi. The complexus is a somewhat similar offshoot of the trachelo-mastoid arising by slips from the upper seven dorsal and last cervical vertebra to be inserted into the occipital between the curved lines.

The name almost sufficiently gives the action of the erector spinx-it keeping the body in the erect posture. It can also bend the spine backward, or backward and to one side when one muscle alone acts.

This muscle is supplied by the posterior divisions of the spinal nerves in the cervical dorsal and lumbar regions.

Beneath this layer there is another of small muscles which barely deserve mention. The most important being the rectus capitis posticus major and minor, the former springing from the spinous process of the axis and being inserted into the inferior curved line of the occipital; the latter arising from the tubercle of the atlas and being inserted into the occipital internal to the preceding muscle.

\section{The Muscles of the Thorax.}

\section{Triangularis Sterni.}

The triangularis stemi is found on the posterior aspect of the front wall of the chest on either side of the sternum. It arises from the edge of the sternum and from the costal cartilages from the third to the sixth or seventh, and, passing upward and outward, is inserted into the second, third, fourth 
and fifth costal cartilages and corresponding ribs. Its insertion corresponds to that of its antagonist the serratus posticus superior.

Action-It is an expiratory agent.

\section{Intercostal Muscles.}

The interval between two ribs is occupied by two layers of muscular fibres which extend between the edges of adjacent ribs and are known as external and internal intercostal muscles.

\section{Anterior Thoracic Region.}

\section{Pectoralis Major Pectoralis Minor Subclavius}

The muscles of this region are covered by the usual superficial fascia and by a deep fascia which partly blends with that of the cervical and, at the side, with that covering the axillary space.

\section{Pectoralis Major.}

The pectoralis major derives its name from its position on the front of the chest, and from the fact that there is another muscle smaller than it in the same region. It is coarse in structure, triangular in shape with its base within and apex without. It arises fleshy from the sternal two-thirds of the clavicle, from the whole length of the front of the sternum and from the cartilages of five ribs, $i . e$. all of true ribs except the first and seventh (this origin being by fibres which reinforce the muscle as it passes over the ribs) and from the aponeurosis of the external oblique muscle of the abdomen.

The fibres converge as they pass outward. Those from the clavicle pass nearly vertically downward, those from the lower part of the sternum and the aponeurosis of the external oblique pass obliquely upward and outward and the intermediate fibres pass horizontally outward. They all terminate in a folded tendon which is inserted into the anterior bicipital ridge of the humerus. As the fibres are approaching their termination some fold over the others, those from the lower portion of the origin of the muscle passing behind and forming the upper part of the tendon, those from the clavicle passing in front and forming the lower part of the tendon. 
Action-It draws the humerus across the chest slightly rotating the arm inward. If the arm be raised it will aid the latissimus dorsi in drawing it downward, or, acting from above in drawing the body upward. Both it and the next muscle thus act as inspiratory agents.

\section{Pectoralis Minor.}

The pectoralis minor derives its name from its position on the front of the chest, and from the fact that there is another pectoral muscle of larger size. It is found beneath the pectoralis major, is small in size, oblique in direction and triangular in shape. It arises from the front of the third, fourth and fifth ribs, passes upward and outward, fleshy, narrows to a tendon and is inserted into the coracoid process of the scapula.

Action-Rotates the scapula on the thorax and, when the scapula is fixed, aids in expanding the chest.

\section{Subclavius.}

The subclavius derives its name from its position just beneath the clavicle. It is a small round muscle which arises from the cartilage, and possibly the adjacent osseous portion, of the first rib by a tendon, it passes horizontally outward, becomes fleshy, and almost immediately after it ceases to rise, begins to be inserted, its belly being extremely short, into the whole length of the groove on the under surface of the clavicle, This groove occupies about the middle one-third of the clavicle.

Action-It draws the clavicle downward.

\section{Thoracic Surgical Triangle.}

Lying just above the subclavius, which is sometimes given as its upper limit, is a triangular space of surgical importance. It is bounded above by the clavicle, below by the upper edge of the pectoralis minor; its apex is without and is crossed by the auxillary vessels and nerves; its base is within and is formed by an imaginary line drawn from the inner extremity of the clavicle to the commencement of the origin of the pectoralis minor. 


\section{Lateral Thoracic Region.}

\section{Serratus Magnus.}

The serratus magmus is a large thin muscle found upon the side of the chest wall. It derives its name from its extensive origin by means of digitations or serrations.

It arises by nine digitations from the outer surface of the eight upper ribs, the lower five interlocking with serrations of the external oblique, and two arising from the second rib. Its origin is fleshy, and it continues its course, fleshy, outward and backward over the side of the chest, to be inserted into the whole length of the anterior edge of the posterior border of the scapula.

Action-It moves the scapula on the thorax and, when the scapula is fixed, raises the ribs.

\section{Acromial Region.}

\section{Deltoid.}

A single muscle constitutes this region, the deltoid, so named from its supposed resemblance to an inverted Delta. It is a large, coarse muscle, triangular in outline, forming the bulge of the shoulder, and corresponding in a measure to the Gluteus Maximus of the lower extremity. Its base is directed upward and its apex downward. Its origin is extensire, consisting of musculo-tendinous fibres, which arise from the outer one-third of the anterior border of the clavicle, from the acromion process, and whole length of the posterior border of the spine of the scapula, and by some fibres from the intervening fascia covering the infraspinatus muscle. The fibres converge as they descend, the posterior passing for ward and downward, the anterior downward and backward, and the central vertically downward, until they form a short, thick tendon, which is inserted into a $\mathrm{V}$-shaped impression about half-way down the outer surface of the shaft of the humerus.

Action. Its posterior fibres carry the humerus upward and backward its anterior fibres upward and forward. By its central fibres, which act in concert with the supraspinatus, 
the humerus is carried directly away from the body until it is at right angles with the body.

The posterior circumflex nerve and the circumflex arteries supply this muscle.

\section{The Scapular Group.}

Subscapularis

Supraspinatus

Infraspinatus

Teres Minor

Teres Major

The Scapular Group of Muscles consists of the subscapularis, the supraspinatus, the infraspinatus, the teres major and the teres minor (L. Teres, long and round). Three of these muscles, it will be seen, are named from the fossae which they occupy upon the scapula, the other two being named from their supposed shape, "round muscles". In the description of the scapula it was stated that both on the back and on the front the fossae described did not occupy the entire aspect of the bone, hence in studying the origin of these muscles it is unnecessary to point out the exceptions of the anterior lip of the posterior border for instance, but simply to except that part of the fossa from which the muscle fails to rise, and not that part of the anterior or posterior surface of the scapula.

\section{Subscapularis.}

The subscapularis, the first of these muscles, is a coarse, flat muscle, which occupies the entire subscapular fossa; but does not rise from the whole of it, nor from the whole length of the scapula. It rises from the entire subscapular fossa, with the exception of its external one-third; and also from the fascia which invests the entire muscle, and from septa sent down from this fascia between the bundles of muscular fibre to seize the ridges of the subscapular fossa and from an aponeurosis which separates the teres major and form the long head of the triceps.

These fibres converge, the superior one passing nearly directly forward and outward, the central ones having a slightly oblique direction upward, outward and forward, while the inferior fibres run nearly directly upward, outward and forward. It narrows to a tendon, thick and strong, which passes over the 
neck of the scapula, crosses the head of the humerus, and is inserted into the lesser tuberosity of the humerus and into the bone for about an inch below, blending with the fibres of the capsular ligament of the shoulder joint.

Action. It is chiefly an inward rotator of the humerus; but its inferior fibres have a slight effect in drawing the humerus downward when it has been elevated, and carrying it backward after it has reached the vertical position.

Subscapular nerves and arteries supply it.

\section{Supraspinatus.}

The sufraspinatus rises from the whole of the supraspinous fossa, except its outer one-third, and from the intervening fascia, which completely covers the muscle. It is a triangular muscle, which converges as it passes forward and outward, forms a tendon which runs under the acromion process of the scapula, and which is inserted into the highest of the three muscular impressions found on the greater tuberosity of the humerus, the lower fibres of the tendon blending with the capsular ligament.

Action. It aids the deltoid in raising, or abducting, the humerus until it is at right angles to the body; and is to a slight extent an outward rotator.

Suprascapular nerve and artery supply it and the following muscle.

\section{Infraspinatus.}

The Infraspinatus rises from the whole infraspinous fossa, execpt its outer third, from the investing fascia, which covers the muscle, and from the inter-muscular septa sent down between itself and the teres major below and the teres minor above near the axillary border of the scapula. Like its predecessor, the fibres converge to form a tendon, which passing behind the shoulder joint, blends with the capsular ligament, and is then inserted into the middle of the three muscular impressions on the greater tuberosity of the humerus.

Action. Its action is almost entirely confined to external rotation of the humerus. 


\section{Teres Minor.}

The teres minor rises from the upper two-thirds of the axillary border of the scapula, with the exception of the space occupied by the long head of the Triceps. It also rises from the inter-muscular septum between itself and the infraspinatus internally and itself and the teres major externally, and from the fascia which covers the lower portion of the muscle. Its direction is upward and outward. The tendon narrows but slightly and after blending with the fibres of the capsular ligament, is inserted into the lowest of the three muscular impressions on the greater tuberosity of the humerus, and into the bone for a short distance below.

Action. Like its predecessor, its chief action is that of external rotation of the humerus.

Supplied by branch from the circumflex.

\section{Teres Major.}

The teres major lies just below the teres minor, from which, after its origin, it is separated by a widening interval. It rises from the lower one-third of the axillary border of the scapula, and from that portion of the dorsum, called the inferior angle, which does not enter into the infraspinous fossa. 'It also rises from the intervening fascia, which covers the lower portion of the muscle, and from inter-muscular septa between itself and the teres minor above and the infraspinatus below. From this origin it passes upward, outward and forward, its upward obliquity being less than that of the teres minor; and is inserted by a broad, flat tendon into the internal bicipital ridge of the humerus.

Action. It draws the humerus downward and backward, acting here in concert with the latissimus dorsi; and is also an in ward rotator. Supplied by lower subscapvlar.

Between the two teres muscles is a surgical triangle of some importance, the base of which is directed towards the humerus, and the apex towards the scapula. This triangle is bounded above by the lower border of the teres minor, below by the upper border of the teres major, and externally by 
that portion of the shaft of the humerus which intervenes between the teres muscles. This space is subdivided by the long head of the triceps into an external quadrilateral and an internal triangular space. The triangular space, of small importance, is bounded above by the lower border of the teres minor, below by the upper border of the teres major, and anteriorly by the long head of the triceps. The quadrilateral space is bounded behind by the long head of the triceps, in front by the shaft of the humerus, above by the lower border of the teres minor, and below by the upper border of the teres major. Through this space pass important bloodvessels and nerves.

\section{The Muscles and Fascia of the Humerus, or Arm.}

The muscles of the humerus, or arm, are invested by fascia, constituting a portion of the fascia of the upper extremity, which completely envelops the limb. In front it is continuous with the fascia which envelops the pectoral muscles, posteriorly with that which invests the posterior scapular group, internally with the fascia which forms the floor of the axillary space, and externally with the fascia investing the deltoid muscle. As this fascia descends the arm it sends in two septa, one from either side. The internal inter-muscular septum is attached to the internal border of the humerus, the internal condyloid ridge, and to the internal condyle of the humerus. The external inter-muscular septum is likewise attached to the external border, external condyloid ridge, and external condyle of the humerus.

These inter-muscular septa not only serve to separate the anterior from the posterior groups; but they also give a firm and unyielding origin to the various muscles of the arm and forearm.

The Muscles of the Arm are divided into two groups: the Anterior and Posterior Humeral Groups.

\section{Anterior Humeral Group.}

Biceps Flexor

Coraco-Brachialis

Brachialis Anticus 


\section{Biceps.}

The biceps (L. bis, twice; caput, head), as its name indicates, has a double origin, one called the "long" and the other the "short" head. The short head arises by musculo-tendinous fibres from the coracoid process of the scapula, in common with the coraco-brachialis. It descends blended with this muscle for its upper two inches, and then no longer blended, but parallel, with the coraco-brachialis, to the insertion of that muscle, where it is joined by the long head, which arises from the upper border of the glenoid cavity of the scapula, where it blends with the glenoid ligament. By tendinous fibres the long head passes over the shoulder joint in a separate synovial sheath; and blends about the middle of the humerus with the short head to form the belly of the muscle. The belly of the muscle so formed passes down the front of the arm to near the elbow, where it terminates in a tendon, which gives off a strong slip of fascia to blend with the investing fascia of the forearm, while the bulk of the tendon is inserted into the posterior part of the bicipital tuberosity of the radius.

Action. It is a flexor of the forearm; but its short head, if acting alone, may to a trifling extent abduct the forearm, and the long head to some extent can adduct it. With the forearm in a position of pronation, the muscle becomes the most powerful of the supinators of the forearm. It is also a tensor of the deep fascia of the forearm. The biceps is supplied by the musculo-cutaneous nerve.

\section{Coraco-Brachialis.}

The Coraco-brachialis, arises in common with the short head of the biceps from the coracoid process of the scapula, passes down on the inner side of that muscle, adherent to it for about two inches, and then in contact with it, but no longer adherent to it, to the middle of the arm, where it is inserted in the middle of the internal face of the shaft of the hmerus.

Action. It is a flexor of the arm; and can, acting in concert with the short head of the biceps, aid that muscle, though 
acting more powerfully, in carrying the arm inward, or adducting it. It is supplied by the musculo-cutaneous nerve.

\section{Brachialis Anticus.}

The Brachialis Anticus is a bulky mass lying deeper than the preceding muscles on the lower part of the front of the arm. It arises by two digitations, so arranged as to embrace the $T$-shaped insertion of the deltoid, and expanding from that point down the shaft of the humerus to within an inch of the elbow joint, its origin covering the whole front of the humerus between these two points. It also springs from the whole of the internal inter-muscular septum, and from the upper portion of the external inter-muscular septum, being cut off from the latter by the origin of the supinator longus and extensor carpi radialis longior. It narrows to a triangular tendon, which passes over the elbow, and is inserted into the front of the coronoid process of the ulna.

Action. It is a powerful flexor of the forearm upon the arm, though in some positions of the body-as in "chinning" a bar-it flexes the arm upon the forearm, acting, as all muscles do, from either extremity. It is supplied by the musculospiral and musculo-cutaneous nerves.

\section{Posterior Humeral Group.}

\section{Triceps.}

The triceps is a large fleshy mass. It covers the whole of the posterior face of the humerus, being the only muscle found in that situation. It rises by three heads: the middle, or long; the internal, or short; and the external, or intermediate. The long head arises from the upper one-inch of the axillary border of the scapula by tendinous fibres.

The short head arises from the inner one-half of the posterior face of the humerus, and the posterior face of the inter nal muscular septum and the intermediale arises from the external half of the posterior face of the humerus, and the external inter-muscular septum. The three heads blend together to form the belly of the muscle, which passing downward becomes tendinous, the tendinous fibres extending much 
further up on its posterior than on its deep, or anterior, aspect, to be ultimately inserted into the depressed surface on the upper extremity of the olecranon process of the ulna. The muscle can be more briefly described by saying that the inner and the external of the heads rise from the whole of the posterior surface of the shaft of the humerus, the former beginning at the insertion of the Teres major and the latter at the insertion of the Teres minor. Between these is found the long head, arising at the upper inch of the axillary border of the scapula. They all blend to reach the insertion above mentioned.

Action.-This muscle is the extensor of the forearm upon the arm; but its long head being attached to the scapula and passing over two joints, approximates the head of the humerus to the Glenoid cavity, and slightly aids other muscles in drawing the humerus backward. It may also in climbing draw the scapula, and if that be fixed, the body, upward on the humerous.

It is supplied by the musculo spiral nerve.

\section{The Muscles of the Fore-Arm.}

Before reaching the muscles of the forearm we find that portion of the upper extremity enveloped in a strong fibrous membrane,called the Deep or Investing Fascia of the Forearm. This membrane is first attached to the olecranon process of the Ulna and passes downward attached to the posterior border of the shaft of the Ulna. Passing forward on either side, it is attached to the internal and external condyles of the humerus, and then uniting over the front of the limb forms a complete investment. From this investing sheet septa are sent off, which not only serve the purpose of dividing the different layers from one another, but which also serve to give a strong and firm attachment to the muscles which belong to this portion of the upper extremity. Some of these muscles arise from the fascia as a strictly investing membrane, while others arise from the intermuscular septa, which are tongues of fascia sent down between the different muscles or groups of muscles. Thus the whole limb is divided into separate compartments, 
so that could a muscle be dissolved out without affecting the fascia, it would be found to have its separate and distinct compartment, as would be the case for all of the muscles of the limb. This fascia becomes particularly strong and dense at the wrist joint, where it sends across both on the front and the back strong braces, which are respectively known as the Anterior and Posterior Anmular Ligaments.

The Posterior is an oblique band, which passes downward and inward across the back of the wrist, and is attached by one extremity to the lower end and styloid process of the radius and by the other to the cuneiform and pisiform bones. It binds the tendons of the Extensor muscles down upon the bones, and it sends down septa which form six separate compartments through which these tendons pass.

The Anterior Ammlar Ligament is less well marked than is the Posterior. It is attached by one extremity to the front of the styloid process of the radius; by the other extremity it is attached to the pisiform bone, sending off a process which becomes attached to the unciform process of the unciform bone.

\section{The Muscles of the Forearm.}

The muscles of the forearm are divided into those on the front and those on the back; and these are again subdivided by intermuscular septa into a superficial and deep group on the front of the forearm, and into three groups-external or radial, superficial, and deep-on the back. It must be borne in mind that every muscle found upon the front of the forearm is to produce either flexion or pronation and that every muscle upon the back of the forearm produces either extension or supination. Hence the names of the muscles upon the front will be either Flexor or Pronator, on the back either Extensor or Supinutor; and to these names are added the particular function of the muscle, as a Flexor of the radial side of the carpus or an Extensor of the radial side of the carpus.

\section{The Muscles of the Front of the Forearm.}

The muscles of the front are eight in number and are divided into two layers, of which the superficial contains five 
muscles and the deep three. The superficial muscles on the front of the forearm have a common tendon, the origin of which tendon is from the inner condyle of the humerus, the investing fascia which covers the muscle, and the intermuscular septa which are sent down between the muscular fibres. In addition to this, many muscles have other points of origin.

\section{Pronator Radii Teres.}

The Pronator Radii Teres is the first muscle of the superficial group. It takes its origin from the internal condyle of the humerus, from the investing fascia which covers it and from an intermuscular septum between itself and the Flexor carpi radialis. In addition to this, there is a small head, or deep head, of this muscle, which arises from the inner face of the coronoid process of the ulna, these two heads being separated by the median nerve. The two heads unite after a short course, and passing downward and outward, wind around the shaft of the radius, and are inserted into the middle one-third of the outer surface of the shaft of the radius.

\section{Flexor Carpi Radialis.}

The Flexor Carpi Radialis is the second muscle of the superficial group. It arises from a common origin, - the internal condyle of the humerus and investing fascia, and two intermuscular septa, one between itself and the Pronator radii teres and the other between itself and the Palmaris longus. It is a long and slender muscle, which passes downward, with a slight inclination outward for the upper one-third of the forearm. The muscular belly then changes into a tendon, which running vertically downward, passing through a groove in the trapezium, is inserted into the base of the metacarpal bone of the index finger.

\section{Palmaris Longus.}

The Palmaris Longus arises from the internal condyle, the investing fascia, and the intermuscular septa, between itself and the Flexor carpi radialis on one side and the Flexor sublimis digitorum on the other. It is a small and very slender muscle, which passing straight down the middle of the front of the 
forearm, soon becomes tendinous and is inserted into the palmar fascia chiefly, but partly into the anterior annular ligament. The muscle should be called the "middle flexor of the carpus.

\section{Flexor Carpi Ulnaris.}

The Flexor Carpi Uluaris arises from the internal condyle, the investing fascia, and the intermuscular septa between itself and the Flexor sublimis digitorum. It also arises from the inner side of the olecranon process and from the upper two-thirds of the posterior border of the ulna. This muscle remains fleshy until within two inches of the wrist joint. It then becomes tendinous and is inserted into the base of the metacarpal bone of the little finger, its tendous enclosing the pisiform as a sesamoid bone.

\section{Flexor Sublimis Digitorum.}

The Flexor Sublimis Digitorm lies beneath the preceding muscles of the group and is in contact with the investing fascia for only a small portion of its course, frequently not touching that fascia at all. It arises from the inner condyle of the humerus, from the internal lateral ligament of the elbow, from the base of the coronoid process of the ulna, and from the oblique line of the radius for about two-thirds of that line. It descends beneath the superficial layer of muscles, and at the lower third of the forearm divides into four tendons. These tendons, enclosed in a synovial sheath, enter the hand by passing beneath the anterior annular ligament, and then, separating from one another, pass forward to each finger, to be inserted into the side of the shaft of the second phalanx of each of the four lesser fingrers. At the base of the first phalanx, each tendon splits, and through this opening the tendon of the deep Flexor passes. The superficial tendons then unite and forma groove, in which the tendon of the deep Flexor lies. They then split a second time to be inserted in to the sides of the second phalangres.

\section{Second or Deep Layer.}

Flexor P'rofundus Discritorum

Pronator Quadratus

Flexor Longus Pollicis 
The flexor profundus digitorum lies just beneath the sublimis and to the ulnar side of the longus pollicis. It arises from the upper two-thirds of the anterior and inner faces of the ulna; by a strong aponeurosis from the posterior border of the ulna, where it blends with the flexor carpi ulnaris, and from the ulna part of the interosseous membrane.

It divides into four tendons, which enter the palm of the hand and, separating, run one to each of the fingers, pierce the tendon of the superficial flexor at the base of the fingers, and are inserted into the base of the last phalanx.

\section{Flexor Longus Pollicis.}

The flexor longus pollicis lies to the outer side of the preceding muscle. It arises from about the middle two fourth of the front of the radius and from the radial side of the interosseous membrane. It passes downward and, becoming tendinous, runs beneath the anterior annular ligament, passes between the two heads of the flexor brevis pollicis and is inserted into the base of the last phalanx of the thumb.

\section{Pronator Quadratus.}

The pronator quadratus occupies the lower fourth of the forearm and thus prevents the origin of the preceding muscles for that distance. It arises from the inner side of the ulna, receiving some fibres from the front of that bone as it passes over it, and is inserted into the front of the radius as far as the outer side.

\section{Nerve and Arterial Supply.}

The muscles on the outer side of the forearm are supplied by branches from the radial artery; those on the inner side from the ulnar. All of the muscles on the front of the forearm are supplied by the median nerve or one of its branches, except the Flexor carpi ulnaris and one-half of the Flexor profundis digitorum. The branch of the median which supplies the deep muscles is called the interosseons; the nerve supplying the Flexor carpi ulnaris and the inner half of the Flexor profundus digitorum is the ulnar. 


\section{Action of the Muscles of the Forearm.}

The name indicates the action of each of these muscles, but indicates only a portion of the action.

The Pronator radii teres is first a pronator, of the hand; but after having acted to its fullest extent as a pronator, it then becomes, in common with the other muscles of the group, a flexor of the forearm upon the arm.

The Flexor carpi radialis, Palmaris longus, and Flexor carpi ulnaris are primarily flexors of the wrist; but the palmaris longus in addition is a tensor of the palmar fascia, and all of the three flex the forearm upon the arm.

The Flexor sublimis digitomm flexes the second phalanx on each of the four lesser fingers. Having effected this, it will flex the first phalanx upon the hand; continuing to act, it flexes the hand upon the wrist; and acting still further, it flexes the forearm upon the arm.

The Flexor longus pollicis flexes the first phalanx of the thumb; then the second upon the metacarpal bone of the thumb; then the metacarpal bone upon the hand, and the hand upon the wrist, and the wrist upon the forearm.

The Flexor profundus digitorum flexes the first phalanx upon the second; but cannot do this until a superficial flexor first has flexed the second. Continuing its action, it will act in concert with and like the superficial flexor.

\section{Interosseous Membrane.}

The interosseous membrane, mentioned above, is a strong fibrous membrane stretched between the two bones. It economizes weight, while it furnishes as stable an origin for muscles as does bone.

\section{Posterior Region.}

The muscles upon the back of the forearm also have a common origin, viz: external condyle of humerus, investing fascia and intermuscular septa.

They are divided into three groups, each containing four muscles, called radial, superficial and deep groups. These 
muscles, are either extensors or supinators, acting in opposition to those on the front of the forearm.

\section{Radial Group.}

Supinator Longus Extensor Carpi Radialis Brevior
Extensor Carpi Radialis Longior

Supinator Brevis

\section{Supinator Longus.}

The supinator longus derives its name from the facts that it is a supinator of the forearm and that there is another supinator called brevis.

It arises from the upper two-thirds of the external condyloid ridge and from the external inter-muscular septum, and, after descending about two-thirds of its course, terminates in a tendon which is inserted into the outer side of the base of the styloid process of the radius.

\section{Extensor Carpi Radialis Longior.}

The extensor carpi radialis longior derives its name from the action of the muscle and from the fact that there is another radial extensor which is shorter. It arises from the lower one-third of external condyloid ridge and from the external inter-muscular septum, and descends to be inserted by its tendon into the base of the metacarpal bone of the index finger.

\section{Extensor Carpi Radialis Brevior.}

The extensor carpi radialis brevior, deriving its name from the facts stated with the preceding muscle, arises from the common origin, which is the external condyle, the investing fascia and an inter-muscular septum between itself and the extensor communis digitorum. It passes downward, between the extensor carpi radialis longior and the extensor communis digitorum, crosses beneath the tendons of the extensor ossis metacarpi pollicis and extensor primii internadii pollicis, as does the preceding muscle, before reaching the posterior annular ligament, then passes beneath the tendon of the extensor secundi internodii pollicis and is inserted into the radial 
side of the base of the third metacarpal bone. This muscle and the extensor carpi radialis longior, lie in the same groove while passing under the annular ligament.

\section{Supinator Brevis.}

The supinator brevis is a thin flat muscle which encircles the upper third of the radius.

It arises from the external condyle of the humerus, the external lateral and orbicular ligaments from the triangular depressed surface beneath the lesser sigmoid cavity of the ulna, and to some extent from the intermuscular septum between the deep and superficial groups. It winds around the radius and is inserted into the upper third of its oblique ridge.

\section{Back of Forearm-Superficial Group.}

Extensor Communis Digitorum. Extensor Minimi Digiti.
Extensor Carpi Ulnaris. Anconeus.

\section{Extensor Communis Digitorum.}

The exlensor communis digilorm derives its name from its action as the extensor common to the four fingers. It arises from the common origin, external condyle investing fascia and two inter-muscular septa; one between itself and the extensor carpi radialis brevior externally, and one between itself and the extensor minimi digiti, internally. Passing down the back of the forearm the muscle divides into three tendons, which pass beneath the annular ligament in a compartment with the extensor indicis. On reaching the back of the hand the innermost tendon divides into two; and the four tendons, thus formed, pass to the four lesser fingers and are inserted into the backs of the four lesser fingers afler the manner of exlensor lendons. This expression means that opposite the metacarpo-phalangeal joint each tendon becomes narrower and receives the tendon of the lumbricales. Passing to the shaft, each tendon becomes broader and thinner. Upon reaching the phalangeal joints each becomes thicker and furnishes a posterior ligament for those joints. The tendon to the ring finger is usually bound by a slight band to the tendons of the little and middle fingers. 


\section{Extensor Minimi Digiti.}

The extensor minimi digiti is an offshoot of the common extensor and, as its name indicates, has for its function the extension of the little finger. It arises from the common origin (external condyle, investing fascia and septa between itself and the extensor communis externally and the extensor carpi ulnaris internally) and is inserted into the whole length of the back of the little finger along with the slip furnished that finger by the common extensor, after the manner of extensor tendons.

This muscle is really an offshoot of the common extensor. Its claim to individuality is that it has a separate compartment in the annular ligament.

\section{Extensor Carpi Ulnaris.}

The extensor carpi ulnaris derives its name from its action as an extensor of the hand and from its situation on the ulnar side of the forearm. It arises from the common origin (external condyle, investing fascia and the intermusular septa between itself and the extensor minimi digiti externally and the deep group in front) and, by a strong aponeurosis, which blends with that giving origin to the carpal flexor, from the middle third of the posterior border of the ulna. The muscle remains fleshy until just above the wrist joint when it becomes tendinous and, passing through a groove on the back of the styloid process of the ulna, in its own compartment in the annular ligament, is inserted into the base of the metacarpal bone of the little finger on its ulnar side.

\section{Anconeus.}

The anconeus is a small triangular muscle which arises by its apex from the external condyle of the humerus, and is inserted by its base into the olecranon process and triangular surface at the upper extremity of the ulna. It is really an offshoot of the triceps extensor, and its action is to aid that muscle in extending the forearm on the arm. 


\section{Deep Group.}

The four muscles of this group lie beneath those of the superficial group, and all arise from the interosseons membrane as one attachment. As indicated by their names, they are all extensors, three being appropriated by the thumb and one by the index finger.

Extensor Ossis Meta-

carpi Pollicis

Extensor Secundi Inter-

nodii Pollicis
Extensor Primi Inter* nodii Pollicis

\section{Extensor Ossis Metacarpi Pollicis.}

The extensor ossis metacurpi pollicis arises from the radius, ulna, interosseous membrane and a fibrous septum between it and the extensor primi. Its upper attachment to both bones is limited by the supinator brevis. It becomes tendinous and winding around the radius crossing the tendons of the extensor carpi radialis langior and brevior, and passing through the annular ligament, is inserted into the base of the metacarpal bone of the thumb.

\section{Extensor Primi Internodii Pollicis.}

The extensor primi internodii pollicis arises from the radius for about its middle two fourths and interosseous membrane. After becoming tendinous it follows the extensor ossis metacarpi pollicis, lying internal to that muscle, crosses the same tendons, passes through the same groove on the radius and is inserted into the base of the first phalanx of the thumb.

\section{Extensor Secundi Internodii Pollicis.}

The extensor secundi internodii pollicis arises from the ulna for about its middle third and from the interosseous membrane. Its tendon passes through a separate compartment in the annular ligament, crosses the tendons of the extensor carpi radialis langior and brevior after passing through the annular ligament, and is inserted into the base of the last phalanx of the thumb. 


\section{Extensor Indicis.}

The extensor indicis arises from the ulna and interosseous membrane and is inserted into the whole length of the back of the index finger, blending with the tendon furnished that finger by the common extensor. It passes through the annular ligament in a compartment common to it and the extensor communis digitorum.

Action-The names of these muscles in a large measure indicate their action. A carpal extensor extends the hand and draws it to its own side. The radial and ulnar extensors, acting together, bend the hand straight backward. The extensors of the fingers bend the fingers backward and then extend the hand at the wrist. Of the supinators, the brevis is the stronger. It is only when the hand is in full supination that the supinator longus acts to produce supination. With the hand in full supination it becomes a pronator. It may also act as a flexor of the forearm upon the arm.

The supinator longus and extensor carpi radialis langior are supplied by the musculo-spiral nerve; the others by the posterior interosseous.

\section{The Muscles of the Hand.}

The central portion of the palm of the hand is depressed below the level of a prominence on either side, one, extending: from the base of the little finger toward the wrist, called the hypothenar eminence, and the other, considerably more prominent, extending upward from the base of the first phalanx of the thumb toward the wrist, called the thenar eminence.

When the skin has been removed a thick layer of fatty tissue is exposed, which serves as a protection for the vessels and nerves in grasping with the hand; and beneath this is a strong fascia called the palmar fascia. It has a thick central portion and two thinner lateral portions which cover the muscles forming the thenar and hypothenar eminences. Tracing this fascia to the wrist, it is found to be continuous with a strong fibrous band which extends across the wrist 
from one side to the other, binding down the tendons which pass beneath it, known as the anterior annular ligament.

The muscles of the hand consist of two groups, the thenar and hypothenar groups, and four small muscles found in the depressed portion of the hand, called lumbricales.

\section{Thenar Group.}

The muscles of the thenar group are appropriated to the thumb, the movements of which are as follows: ( $a$ ) Abduction, or movement of the thumb outward from the index finger. (b) Adduction, a morement in the opposite direction. (d) Flexion, in which the thumb is put in contact with the palp of any of the fingers. This is effected by putting in motion the metacarpal bone of the thumb by a muscle called opponens. (c) Extension, movement in the opposite direction effected by muscles on the back of the forearm, which have been described.

Abductor Pollicis

Flexor Brevis Pollicis
Flexor Ossis Metacarpi Pollicis. Adductor Pollicis.

\section{Abductor Pollicis.}

The alductor pollicis arises from the trapezium bone and anterior annular ligament, and is inserted in the outer side of the base of the first phalanx of the thumb.

The flexor of the metacarpal bone of the thumb, or the opponens pollicis, arises from the trapezium bone and anterior annular ligament, and is inserted into the whole length of the radial border of the metacarpal bone of the thumb.

\section{Flexor Brevis Pollicis.}

The flexor brevis pollicis consists of two parts, one of which, the superficial portion, arises from the trapezium bone and annular ligament, and the other, or deep portion, from the trapezoid, os magnum and base of the metacarpal bone of the middle finger. It is inserted into both sides of the base of the first phalanx of the thumb, its tendons of insertion having sesamoid bones in them. 


\section{Abductor Pollicis.}

The adductor pollicis is a flat triangular muscle which arises by its base from the whole length of the front of the metacarpal bone of the middle finger. It is inserted into the inner side of the base of the first phalanx of the thumb.

\section{Hypothenar Group.}

Palmaris Brevis

Flexor Brevis Minimi Digiti
Abductor Minimi Digiti

Adductor Ossis Metacarpi

\section{Palmaris Brevis.}

Minimi Digiti

The palmaris brevis is a small pale muscle lying just beneath the skin of the palm. It arises from the palmar fascia and the anterior annular ligament, and passing inward, about an inch wide, is inserted into the skin on the inner border of the hand.

\section{Abductor Minimi Digiti.}

The abductor minimi digiti arises from the pisiform bone and is inserted into the inner side of the base of the first phalanx of the little finger.

Action-It is an abductor of the little finger, $i . e$. , draws the little finger inward away from the ring finger.

\section{Flexor Brevis Minimi Digiti.}

The flexor brevis minimi digiti arises from the unciform process of the unciform bone and the anterior annular ligament, and is inserted into the base of the first phalanx of the little finger.

Action-It is a flexor of the little finger.

\section{Adductor Ossis Metacarpi Minimi Digiti.}

The adductor ossis metacarpi minimi digiti arises from the unciform process of the unciform bone and the anterior annular ligament, and is inserted into the whole length of the metacarpal bone of the little finger.

Action-It draws the metacarpal bone of the little finger outward and forward, carrying the little finger towards the thumb. 


\section{Flexor Tendons of the Fingers.}

It has been seen that the tendons of the flexor sublimis digitorum split at the base of the fingers to allow the passage of the tendons of the flexor profundus, unite and again split to be inserted into the sides of the shaft of the second phalanx of each finger. Before its insertion each tendon gives off thread-like prolongations, called vincula accessoria, which are inserted into the sides of the bones; and both tendons are bound down to the bones by transverse bands of ligamentous fibres called the thecæ.

\section{Muscles of the Lower Extremity.}

The muscles of the lower extremity are divided into those of the hip, thigh, leg and font. The hip is called the gluteal region.

\section{Gluteal Region.}

As in the upper extremity, the muscles are here covered by an investing fascia, which is much thicker and stronger than in the former situation, known in the thigh as the fascia lata or vagina femoris.

The muscles of the gluteal region, nine in number, are arranged in three layers, each of which has a muscle called gluteus.

It receives its nerve supply from the lesser sciatic.

\section{First Layer. Gluteus Maximus.}

The gluteus maximus is a quadrilateral muscle, the largest and coarsest in the body, and forms the bulge of the buttock. It arises from the posterior fifth of the crest of the ilium, from the dorsum ilii between the superior curved line and the crest, from the posterior portion of the crest, from the posterior surface of the sacrum and $\operatorname{coccyx}$, from the greater sacrosciatic ligament and from the fascia covering the gluteus medius. It passes obliquely downward and outward to be inserted into the line leading from the trochanter major to the linea aspera and into the fascia lata.

Des Anat-17 
Action-It is an abductor and outward rotator of the thigh, a tensor of the fascia lata and steadies the pelvis on the femur.

Second Layer.
Gluteus Medius
Gemellus Inferior
Pyriformis
Obturator Internus
Gemellus Superior
Quadratus Femoris

Gluteus Medius.

The gluteus medius is partially covered by the gluteus maximus. It arises from the anterior four-fifths of the crest of the ilium, from the dorsum ilii between the middle curved line and the crest and from the investing fascia, which covers its outer front part. It is inserted into the outer part of the trochanter major. On its outer edge the muscle is continuous with the gluteus maximus.

Action-Its greatest power is shown in abduction; but its anterior fibres can rotate the femur inward while the posterior rotate it outward. It can twist the pelvis on the femur, drawing the body to that side. It steadies the pelvis on the femur and can draw the limb forward in walking.

It is supplied by the superior gluteal nerve.

\section{Pyriformis.}

The tyriformis is a pear-shaped muscle. It arises within the pelvis by three fleshy slips interfosed between the anterior sacral foramina from the first to the fourth and from the adjoining part of the ilium. It escapes from the pelvis through the greater sacro-sciatic foramen, passes downward and outward, and is inserted into the posterior border of the upper extremity of the trochanter major, or into the digital pit.

Action-It is an external rotator of the thigh.

\section{The Gemelli.}

The gemelli are a pair of small muscles lying one above and the other below the tendon of the obturator internus.

The gemullus superior arises from the spine of the ischium, runs horizontally outward and is inserted into the digital pit of the trochanter major. 
The gemellus inferior arises from the tuberosity of the ischium, passes horizontally outward and is inserted into the digital fossa.

Action-Both are outward rotators of the thigh.

\section{Obturator Internus.}

The obturator interms arises within the pelvis from the inner surface of the obturator membrane, from the margin of the obturator foramen and from the inclined plane of the ischium. Its course is downward and slightly backward until, becoming tendinous, it escapes from the pelvis through the lesser sacro-sciatic foramen, and passing horizontally, outward, its tendon winding around the posterior border of the ischium just below the spine, it is inserted into the digital fossa of the trochanter major.

Action-It is an outward rotator of the thigh.

\section{Quadratus Femoris.}

The quadratus femoris, as its name indicates, is a square muscle which lies below the tendon of the obturator internus. It arises from the outer lip of the tuberosity of the ischium, and is inserted across the posterior inter-trochanteric line. Its insertion is rarely marked by the presence of the linea quadrati.

Action-It is an outward rotator of the femur. These muscles are all supplied by branches coming directly from the sacral plexus.

\section{Third Layer.}

\section{Gluteus Minimus}

Obturator Externus

\section{Gluteus Minimus.}

The orluteus minimus lies beneath the gluteus medius and maximus. It is a triangular radiated muscle which arises from the dorsum ilii between the middle and inferior curved lines. It passes down, narrowing as it descends, and is inserted into the anterior border of the trochanter major. In front, it is continuous with the gluteus medius.

Action-It is an abductor and inward rotator of the thigh. Acting from below it steadies the pelvis on the femur.

It is supplied by the superior gluteal. 


\section{Obturator Externus.}

The obturator externus arises from the inner two-thirds of the outer surface of the obturator foramen and from the inner margin of the obturator foramen. It narrows to a tendon, which, passing outward behind the neck of the femur, is inserted into the digital pit of the trochanter major. It lies on the capsular ligament of the hip-joint.

Action-It is an external rotator of the thigh.

It is supplied by the obturator nerve.

\section{Femoral Region.}

The muscles of the thigh are arranged in four groups, viz: posterior, superficial, anterior and internal femoral groups. Investing the thigh, just beneath the skin and superficial fascia, is a strong, thick, fibrous membrane which descending to the knee, invests that joint and passes on to become the investing fascia of the leg. From the hip to the knee it is known by three names, investing fascia of the thigh, vagina femoris, or, generally, fascia lata. It not only forms a firm resisting covering for the muscles, but sends in septa between the groups. Two of these septa, attached to the lips of the linea aspera, are known respectively as the external and internal intermuscular septa. The external intermuscular septum lies between the anterior and posterior femoral groups, and gives origin to muscles of both groups. The internal, lies between the anterior and internal groups, no septum being interposed between the internal and posterior groups. Above, this fascia is continuous, on the front of the thigh, with Pouparts ligament, externally and posteriorly with the fascia covering the gluteal muscles, while internally it is attached to the tuberosity of the ischium and to the ischio pubic rami. Below it seizes the bony prominences around the knee joint and becomes, posteriorly, the popliteal fascia, which covers in the popliteal space.

The fascia lata consists of layers between which are found three muscles of the thigh forming the superficial femoral group. 
Where the fascia covers the posterior femoral region it is thinner than in any portion of its course.

\section{Posterior Femoral Region.}

Biceps Flexor Cruris

Semitendinosus

\section{Biceps Flexor Cruris.}

The biceps, as its name imports, arises by two heads. The long head springs, in common with the semitendinosus, from the tuberosity of the ischium and descends adherent to the semitendinosus for several inches. In then accompanies the semitendinosus, but no longer adherent to it, to the lower third of the thigh; is joined by the short head which arises from the whole length of the outer lip of the body of the linea aspera and from the external intermuscular septum. The muscle thus formed after descending to the lower third of the femur in contact with the semitendinosus, leaves that muscle and makes for the outer side of the knee joint, where it is inserted chiefly into the head of the fibula, but sends some fibres to the outer tuberosity of the tibia and to the fascia of the leg.

\section{Semitendinosus.}

The semitendinosus arises in common with the long head of the biceps, adheres to it for several inches and then descends in contact with it to the lower third of the thigh where it leaves the biceps and passes to the inner aspect of the knee-joint where it is inserted into the inner surface of the shaft of the tibia, below the inner tuberosity sending a slip to the fascia of the leg. This is known as the "goose foot" insertion. It is made up of three muscles, viz: semitendinous, sartorius and gracilis.

\section{Semimembranosus.}

The semimembranosus arises from the tuberosity of the ischium just in front of and external to the preceding muscles and descends, in company with them, to the lower third of the femur, whence it accompanies the semitendinous to the inner side of the knee-joint and receives a three-fold insertion, viz: 
into the horizontal groove on the inner tuberosity of the tibia, into the popliteal fascia and into the posterior ligament of the knee-joint.

These three muscles are spoken of as the hamstring muscles, the biceps being the outer, the semimembranous and semitendinosus being the inner.

Action-It will be seen that these three muscles all pass over two joints. They are, of course, all flexors of the leg upon the thigh; but, in addition, the biceps has a slight influence in rotating the leg outward and the others in rotating it inward. After flexing the leg upon the thigh, they can then extend the thigh upon the pelvis. When acting from below they draw the pelvis downward and backward, keeping the body from falling forward, or aid in restoring it to the erect posture after stooping.

The great siatic nerve supplies the group.

\section{Superficial Femoral Group.}

\section{Tensor Vaginæ Femoris Gracilis Sartorius}

\section{Tensor Vaginæ Femoris.}

The tensor vagince femoris is a short flat muscle found 1 ying on the outer aspect of the thigh between the two layers of the fascia lata. It arises from the outer lip of the crest of the ilium near the anterior superior spinous process, descends with an inclination backward and is inserted into the fascia lata about one-fourth down the thigh.

Action-It is a tensor of the fascia lata and aids in rotating the limb inward.

\section{Sartorius.}

The sartorius is the longest muscle in the body. It arises from the anterior superior spinous process of the ilium and half the notch below, passes obliquely downward and inward across the upper third of the thigh, descends vertically behind the internal condyle of the femur and then turns obliquely forward to be inserted into the upper inner face of the tibia below the internal tuberosity, sending a slip to the fascia of the leg. It forms one of the elements of the "goose foot" insertion, the 
other two being the semitendinous and gracilis. The sartorius is the most superficial at the insertion, then the gracilis and the semitendinosus is the deepest.

Action-It flexes the thigh on the pelvis, the leg on the thigh and carries it inward across its fellow. It may, acting from below, twist the pelvis so as to carry the front of the body towards the opposite side.

\section{Gracilis.}

The gracilis arises by a thin broad aponeurosis from the edge of the symphysis pubis and from the margin of the ischiopubic-ramus. It soon becomes fleshy and passes down the inner aspect of the thigh to be inserted into the upper inner part of the tibia, below the inner tuberosity, sending a slip to the facia of the leg, $i$. e., it is an element of the "goose foot" insertion.

Action-It flexes the leg on the thigh and aids in adducting the thigh.

The tensor vaginae femoris is supplied by the superior gluteal nerve; the sartorius by the middle cutaneous ; the gracilis by the obturator.

\section{Anterior Femoral Region.}

Psoas Magnus

Iliacus Internus
Vastus Externus

Vastus Internus

\section{Rectus Femoris.}

The muscles in this region are usually described as five but can very readily be considered as two, viz: Biceps flexor femoris, consisting of psoas magnus and iliacus internus and the triceps extensor cruris consisting of rectus femoris, vastus internus, and vastus externus. The last three are inserted by a common tendon, called the ligamentum patella, into the anterior tubercle of the tibia, their action being transmitted through the patella to which they are attached.

As might be inferred from their action they all arise from some point perpendicular to their insertion.

\section{Psoas Magnus.}

The psoas magmus is a long spindle shaped muscle lying beside the vertebral column and descending into the thigh. 
It arises from the bodies and bases of the transverse processes of the last dorsal, and all the lumbar vertebræ; from the intervertebral disks between them and from tendinous arches attached to the bodies of the vertebræ, extending from the upper lipped edge to the similar lower edge of each vertebra, thus leaving between it and the central constricted portion of the vertebra an interval through which vessels and nerves pass. The muscle descends beside the vertebral column to the pelvis and, as it pass beneath Poupart's ligament, is joined by the iliacus internus and their blended tendon is inserted into the trochanter minor of the femur and the bone for an inch below.

\section{Iliacus Internus.}

The iliacus intemus arises from the whole of the iliac fossa, from the internal lip of the crest of the ilium, from the anterior superior spinous process backward, from the base of the sacrum, from the ilio-lumbar ligament and by a few fibres from the capsular ligament of the hip joint.

The fibres converge to a tendon which, passing beneath Poupart's ligament, blends with that of the psoas magnus to be inserted into the lesser trochanter of the femur and the bone for an inch below.

Action-Primarily these muscles are flexors of the thigh on the pelvis. Owing to the winding of the tendon around the femur, they are powerful outward rotators, acting just as the biceps does on the radius. With their fixed point below, and the muscles of both sides acting, they bend the body directly forward; but if the iliacus of one side acts alone, it flexes the pelvis and turns the body to the opposite side.

The psoas is supplied by branches of the lumbar plexus, the iliacus by the anterior crural.

\section{Rectus Femoris.}

The rectus femoris derives its name from the straightness of its course. It arises by a forked tendon, one fork springing from the anterior inferior spinous process of the ilium, the other from the upper margin of the acetabulum. The two soon unite, and the tendon thus formed after a short course 
terminates in the belly of the muscle; and that in turn terminates at the lower part of the thigh in a strong tendon, which is inserted into the upper end of the patella. The fibres of this muscle have a bipenniform arrangement.

\section{Vastus Externus.}

The vastus cxternus arises from the base of the trochanter major, from the line leading from the trochanter major to the linea aspera, from the upper half or third of the outer lip of the linea aspera, and from the external intermuscular septum. This muscle is corered over by a powerful aponeurosis from which its fibres arise. At its origin from the linea aspera, and the external intermuscular septum, it is intimately blended with the vastus internus. Its fibres proceed in a general direction downward and inward though the inferior fibres pass more nearly inward. It is inserted into the outer edge of the tendon of the rectus femoris and outer edgre of the patella.

\section{Vastus Internus.}

The icustus internus arises from the whole of the shaft of the femur from the anterior intertrochanteric line downward, from the inner lip of the linea aspera, from the internal intermuscular septum, from the external intermuscular septum and from a part of the external lip of the linea aspera, where it blends with the vastus externus. It is inserted into the inner edge of the tendon of the rectus femoris and inner edge of the patella.

The origin of the muscle is also thus stated: from the inner surface of the shaft of the femur, from the anterior trochanteric line downward, from the front and outer faces, from the internal and external lips of the linea aspera and the internal and external septa.

It is inserted into the inner edge of the tendon of the rectus femoris and into the inner border of the patella. The crureus is here considered a part of the vastus internus.

The insertion of the three muscles should be thus stated: They combine to form a common tendon, the tendon of the triceps extensor cruris, which encloses the patella as a sesa- 
moid bone and is inserted, as the ligamentum patellæ, into the lower part of the anterior tubercle of the tibia, a bursa being interposed between the tendon and the upper part of the tubercle of the tibia.

The vasti muscles envelop the entire femur except the middle lip of the linea aspera, and the $t w o$ extremities of the bone.

Action. This muscle, as its name indicates, is an extensor of the leg upon the thigh. The central portion, or rectus femoris, is also a flexor of the thigh upon the pelvis; or, acting from below, of the pelvis upon the thigh.

\section{Internal Femoral Region.}

Pectineus

Adductor Longus
Adductor Brevis

Adductor Magnus

The muscles of this region are adductors of the thigh, that is, they carry the thigh towards or across its fellow, and consequently they must all arise near the middle line of the body.

\section{Pectineus.}

The pectineus is a flat thin muscle which arises from the pectineal line and triangle of the pubes and the onter face of Gimbernat's ligament. It passes downward and is inserted into the upper part of the line leading from the trochanter minor to the linea aspera.

It would not be improper to describe the remaining muscles of this group as a single muscle with a common insertion, since, at the insertion, their fibres are so intimately blended as to be inseparable without the destruction of fibres. They are arranged in three layers, the adductor longus, which lies on the same plane as the pectineus, in front, next the adductor brevis and last the adductor magnus.

\section{Abductor Longus.}

The adductor longus arises by a small, round tendon from the front of the pubes just below the angle and close to the symphysis. It passes downward and outward, widening as it descends, the tendon terminating in muscular fibres which, 
becoming tendinous in turn, are inserted into the middle third of the linea aspera, the fibres blending with those of the other adductors.

\section{Adductor Brevis.}

The adductor brevis arises from the body and ramus of the os pubis, just below the spine and between the gracilis and obturator externus. It passes downward, outward and backward, rapidly becoming wider as it descends, and is inserted into the whole length of the line leading from the trochanter minor to the linea aspera, its insertion extending further down than that of the pectineus, and blending with the general adductor tendon.

\section{Adductor Magnus.}

The adductor magmus arises from the side of the tuber ischii and from the ischio-pubic ramus. It passes downward and outward in large distinct bundles of fibres and is inserted into the whole length of the linea aspera and by a rounded tendon into the adductor tubercle on the internal condyle of the femur. This muscle is pierced by five apertures, one above the other, which transmit branches of the femoral artery. The lowest is much the largest and transmits the termination of the femoral artery itself, over which the adductor longus and magnus throw a tendinous arch to the vastus internus, forming what is called Hunter's canal.

Action-These muscles are all adductors, or muscles which bring the thighs together; but in aldition, as their origin is in front of their point of insertion, the upper two are flexors. When the thigh has been rotated inward, they become power ful outward rotators. The adductors are supplied by the obturator nerve; the pectineus, usually, by the anterior crural.

\section{Scarpa's Triangle.}

On the upper front part of the thigh there is an important triangle, known as Scarpa's. Its outer boundary is the sartorius, its inner the adductor longus, its base is Poupart's ligament, its apex the intersection of the sartorius and the adductor longus. Entering this triangle at the middle of its 
base and passing through it to disappear at its apex are the femoral artery and femoral vein. The floor of this space is formed by the biceps flexor femoris, pectineus and adductor brevis.

\section{Muscles of the Leg.}

The muscles of the leg are divided into three regions, viz: 1st, anterior tibial; $2 \mathrm{~d}$, posterior tibial ; $3 \mathrm{~d}$, external or fibular.

These muscles are enveloped by a dense fibrous membrane which is found encircling the leg everywhere except on the front. Above it is attached to the head of the tibia and fibula on the sides, and is continuous with the fascia, lata- forming with it the popliteal fascia-behind. Below, it becomes attached to the bony prominences of the foot, forming the anterior and two lateral aunular ligaments. From its deep face it sends off three broad inter-muscular septa, besides smaller ones between individual muscles. Two septa are found on the outer side, one between the peroneal and anterior groups and one between the peroneal and posterior groups. The third is a broad transverse septum passing between the superficial and deep muscles on the back of the leg.

\section{Posterior Tibial Region.}

The muscles in the posterior region are placed in two layers, superficial and deep.

\section{Superficial Layer.}

Gastrocnemius

Plantaris

Soleus

\section{Gastrocnemius.}

The gastrocnemizs is the large muscle which gives shape to the calf of the leg. It arises by two heads from the upper back part of the condyles of the femur and from the ridges which connect the condyles with the linea aspera. The two heads, as they descend, converge to form the lower lateral boundaries of the popliteal space, and unite to form the belly of the muscle along which a groove is contiued for some distance. The belly terminates just below the centre of the leg 
in a large tendon called the tendo Achilles, which is inserted into the lower back part of the posterior tuberosity of the os calcis. It is the largest tendon in the body.

Action-It raises the heel, and continuing to act, raises the foot.

\section{Soleus.}

The soleus is a large fleshy muscle lying beneath the gastrocnemius. It has five points of origin, viz: the head and upper half of the posterior aspect of the fibula, the popliteal line and middle third of the internal border of the tibia and from a tendinous arch stretched between its bony origins. The muscle descends to terminate about the middle of the leg in the tendo Achilles.

Action-Same as the gastrocnemius.

\section{Plantaris.}

The plantaris is an insignificant muscle lying between the gastrocnemius and soleus. It arises from the back part of the external condyle of the femur in common with the external head of the gastrocnemius, the belly of the muscle passing downward and inward between the gastrocnemius and soleus to terminate in a tendon which, escaping to the inner side of the two muscles, passes down either to be inserted into the posterior tuberosity of the os calcis or to be lost in the side of the tendo Achilles.

Action-Same as gastrocnemius.

The three muscles just described form the triceps sura. They are supplied by the internal popliteal nerve.

\section{Deep Layer.}

Popliteus

Tibialis Posticus
Flexor Longus Digitorum Flexor Longus Pollicis

\section{Popliteus.}

The popliteus is a small triangular muscle resembling the anconeus in the upper extremity. It arises by its tendinous apex from a groove on the external condyle of the femur and passes downward and inward, adhering to the head of the fibula, the posterior ligament of the knee-joint and the under 
surface of the fascia which covers it, is inserted into the tibi a above and as low down as the oblique or popliteal line on its posterior face.

Action-It flexes and inverts the leg. "It is supplied by the internal popliteal nerve.

\section{Flexor Longus Pollicis.}

The flexorlongus pollicis arises from the lower two-thirds of the fibula, except the last inch, and from the intermuscular septum. It descends behind the internal malleolus into the sole of the foot, crosses the tendon of the flexor longus digitorum and is inserted into the base of the last phalanx of the great toe.

Action-It flexes the last phalanx of the great toe.

\section{Flexor Longus Digitorum.}

The flexor longus digitorum arises from the lower twothirds of the tibia, except the last two or three inches, and from the intermuscular septum. It passes downward behind the internal malleolus, divides into four tendons, which are crossed by the flexor longus pollicis, and are inserted into the last phalanges of the four outer toes.

Its action is indicated by its name, after flexing the toes it extends the foot on the leg.

\section{Tibialis Posticus.}

The tibialis posticus arises from the whole length of the posterior surface of the interosseous membrane, from the adjacent edges of the tibia and fibula and from the intermuscular septum. It descends behind the internal malleolus and is inserted into the tuberosity of the scaphoid bone and into the internal cuneiform bone.

Action-It is an extensor and abductor of the foot.

The three last muscles all arise from the interosseous membrane; and as the flexor longus pollicis arises from the outer bone of the leg and is inserted into the inner toe, it must cross the flexor longus digitorum. This crossing takes place in the sole of the foot and is known as the decussation of the tendons, which are connected at this point by a small slip. 


\section{The Interosseous Membrane.}

The interosseous membrane is a strong ligamentous membrane which passes from tibia to fibula and is attached to their adjacent edges, after the manner of the interosseous membrane of the forearm. On its posterior aspect it is entirely appropriated by the tibial muscles. The muscles in the deep layer are separated from those in the superficial layer by a strong thick fascia.

\section{Anterior Region of the Leg.}

\section{Tibialis Anticus Extensor Longus Digitorum Extensor Proprius Pollicis}

Of the three muscles in this group two have four points of origin in common. These four points are as follows: 1st, outer tuberosity of the tibia; $2 \mathrm{~d}$, investing fascia of the leg; $3 \mathrm{~d}$, intermuscular septum ; 4 th; interosseous membrane.

\section{Tibialis Anticus.}

The tibialis anticus arises from the common origin (the outer tuberosity of the tibia, the investing fascia, the intermuscular septum between itself and the extensor longus digitorum, and the interosseous membrane) and from the upper twothirds of the outer face of the tibia. It passes downward to terminate in a tendon which runs beneath the anterior annular ligament and is inserted into the inner and under surface of the internal cnneiform bone and into the base of the metatarsal bone of the great toe.

Action-It flexes the foot; and, according as it acts with the tibialis posticus or the peronei, it inverts or everts the foot.

\section{Extensor Longus Digitorum.}

The extcnsor longus digilorum arises from the common origin-outer tuberosity, investing fascia, intermuscular septum between itself and the tibialis anticus internally and the peroneus longus externally, and the interosseous membrane and from the head and whole length of the anterior aspect of the shaft of the fibula. It descends and divides into five tendons, which pass beneath the anterior annular ligament and are in- 
serted as follows: one into the base of the metatarsal bone of the little toe, the other four into the backs of the four lesser toes after the manner of extensor tendons. That part of the muscle inserted into the base of the metatarsal bone of the little toe, and arising from the lower fourth of the fibula, is sometimes called the peroneus tertius.

Action-It extends the toes; and, by its insertion into the metatarsal bone of the little toe, flexes the foot.

\section{Extensor Proprius Pollicis.}

The extensor proprius pollicis is shorter than the two preceding muscles and lies between and is overlapped by them. It arises from the middle two-fourths of the shaft of the fibula and from the interosseous membrane, and terminates in a tendon which passes beneath the anterior annular ligament and is inserted into the base of the last phalanx of the great toe. Its action is indicated by its name.

The anterior annular ligament is a strong oblique ligamentous band which binds the tendons of the three preceding muscles down on the front of the ankle. It consists of an upper part passing from the tibia to the fibula and containing the compartment for the tibialis anticus; and a lower portion, attached above to the inner malleolus and below to the lesser process of the os calcis which forms the swing ligament containing the sheath for the extensor longus digitorum and the extensor proprius pollicis. A small offshoot of this band passes to the plantar fascia.

These muscles are supplied by the anterior tibial nerve.

\section{External Region of the Leg.}

Peroneus Longus

Peroneus Brevis

\section{Peroneus Longus}

The peroneus, or fibularis, longus arises from the head and upper two-thirds of the outer aspect of the shaft of the fibula, from the investing fascia and the intermuscular septa between itself and the extensor longus digitorum on the inner and the posterior group on the outer side-Below another septum is. found between the two peroneal muscles. It descends to 
terminates in a tendon which passes behind the external malleolus, through the lower grooz'e on the outer surface of the os calcis, reaches the sole of the foot and is directed across it obliquely forward and inward, through a groove in the cuboid bone, to be inserted into the base of the metatarsal bone of the great toe.

Action-It is an extensor of the foot.

\section{Peroneus Brevis.}

The peroneus, or filularis, breais derives its name from its position and because its origin and insertion are both short of the preceding muscle, beneath which it lies. It arises from the lower two-thirds of the outer aspect of the shaft of the fibula and from the intermuscular septum, descends behind the external malleolus, through the upper groove on the outer surface of the os calcis, and is inserted into the base of the metatarsal bone of the little toe.

Action-It extends the foot.

The two peronei muscles, as they are passing the outer malleolus, are held down by the external annular ligament

They are supplied by the musculo-cutaneous nerve.

\section{Muscles of the Foot.}

The foot is divided into two regions, dorsal and plantar.

The fascia covering the dorsum of the foot is thin and membranous. Above it is continuous with the anterior annular ligament, while on the sides it becomes attached to the plantar fascia. On either side there is a strong lateral annular lisament each continuous above with the fascia of the leg and below with the plantar fascia. The inlernal ammlar ligament is attached to the internal malleolus above and to the inner face of the os calcis below. It forms grooves for the passage of the flexor tendons.

The cxlernal ammalar liganent is attached to the internal malleolus and to the outer margin of the os calcis forming grooves for the peroneal tendons.

\section{Dorsum of the Foot. Extensor Brevis Digitorum.}

The cxtcnsor brevis digilomm arises from the upper and Des Anat-18 
outer aspect of the os calsis and passing forward and inward divides into four tendons the innermost of which is inserted into the base of the first phalanx of the great toe, the other three into the backs of the next three toes in common with the long extensor tendons.

Its name indicates its action.

\section{The Sole of the Foot.}

\section{The Plantar Fascia.}

When the thick skin on the sole of the foot has been removed, there is brought into view a thick mass of fatty tissue and beneath this a strong fascia called the plantar fascia. It consists of a strong, thick central portion and, on either side, continuous with this, a thinner lateral portion. The central portion begins behind at the under surface of the os calcis, runs forward and just beyond the middle of the sole divides into five branches each of which passes forward to the base of a corresponding toe. The muscles of the sole all lie beneath (or, in the erect position, above) this fascia and are divided into groups in accordance with the subdivisions of the plantar fascia, viz: a central and two lateral groups. The propriety of this division is shown by the fact that the plantar fascia sends up to the bone intermuscular or dividing septa from the line of union of the central aud two lateral portions.

In describing these muscles, however, they are taken in layers, of which there are three. When the plantar fascia, the dissection of which is the first step, has been removed, the first layer, consisting of one muscle in each group, is ex posed. This constitutes the second view in the dissection of the sole.

\section{First Layer.}

Abductor Pollicis

Abductor Minimi Digiti

Flexor Brevis Digitorum.

The three muscles forming the first layer have a common origin, viz; 1 st, the under surface of the os calcis; $2 d$, the plantar fascia which covers them; $3 d$, the intermuscular septa which separate them. Some of the plantar fascia should be 
left on the muscles in dissecting them, since it is a part of their origin.

\section{Abductor Pollicis.}

The abductor pollicis is the innermost of the three muscles of the first layers. It arises from the common origin and is inserted into the inner side of the base of the first phalanx of the great toe.

Its name indicates its action.

\section{Abductor Minimi Digiti.}

The abductor minimi digiti is the outer of the three muscles in the first layer. It arises from the common origin and is inseried into the outer side of the base of the first phalanx of the little toe.

Its name indicates its action.

\section{Flexor Brevis Digitolum.}

The flexor brevis digitom lies in the centre of the first layer. It arises from the common origin, passes forward and divides into four tendons for the four lesses toes. Each tendon splits to allow the passage of the tendon of the long flexor and is inserted into the sides of the shaft of the second phalanx.

Its name indicates its action.

\section{Second Layer.}

Musculus Accessorius

Lumbricales

The second layer, exposed by removing the first, is the third view in the dissection of the sole. It consists of the musculus accessorius, the lumbricales and the tendons of the flexor longus pollicis and flexor longus digitorum. These two tendons, soon after entering the sole from behind the internal malleolus, cross each other forming their decussation and are also connected by slips so that one muscle cannot act independently of the other, but throws the part to which it is attached into slight motion.

\section{Musculus Accessorius.}

The musculus accessorizs arises tendinous and fleshy from the under surface of the os calcis and passing forward is in- 
serted into the outer side and upper surface of the tendon of the flexor longus digitorum just as it splits into its four branches.

Action-It corrects the obliquity which would otherwise be imparted to the four lesser toes by the action of the long flexor.

\section{Lumbricales.}

The lumbricales are four little worm-like muscles, three of which arise from the bifurcation of the tendon of the flexor longus digitorum, while the fourth springs from the inner side of the inner tendon of that muscle. They pass between the toes and are inserted into the tibial side of the extensor tendons of the four lesser toes.

Action--They aid the flexor longus digitorum.

\section{Third Layer.}

Flexor Brevis Pollicis Adductor Pollicis
Flexor Brevis Minimi Digiti Tranversus Pedis

This layer, constituting the fourth view in the dissection of the sole of the foot, is exposed by removing the second layer.

\section{Flexor Brevis Pollicis.}

The fiexor brevis pollicis arises from the cuboid bone, the external cuneiform bone and the expanded tendon of the tibialis posticus. It is inserted into both sides of the base of the first phalanx of the great toe, each tendon having a sesamoid bone in it.

\section{Adductor Pollicis.}

The adductor pollicis arises from the cuboid bone, from the sheath of the tendon of the peroneus longus and from the bases of the adjoining metatarsal bones (second, third and fourth). It is inserted into the outer side of the base of the first phalanx of the great toe.

\section{Flexor Brevis Minimi Digiti.}

The flexor brevis minimi digiti arises from the base of the fifth metatarsal bone and is inserted into the outer side of the first phalanx of the little toe. 


\section{Transversus Pedis.}

The transversus pedis arises by slips from the heads of the four outer metatarsal bones, and passing inward is inserted into the outer side of the base of the first phalanx of the great toe.

Action-It is an adductor of the great toe.

\section{Interosseous Muscles.}

Lying between the metacarpal bones of the hand and the metatarsal bones of the foot are certain small muscles called from their position interosscous. In each member there are seren of these muscles. four dorsal and three plantar. They are bipenniform muscles, arising from the metacarpal or metatarsal bone and inserted into the first phalanx. The first dorsal interosseous of the hand is much larger than the others and is called the abductor indicis.

\section{The Muscles of the Face.}

The muscles of the face are arranged in groups, viz: those connected with the orbit, with the nose, the mouth and, a very unimportant, group connected with the auricle.

\section{Orbital Group-Three Muscles.}

\section{Orbicularis Palpebrarum.}

The orbicularis-palpebram is an elliptical muscle, 1ying just beneath the skin, in front of the orbit, the portion which lies on the lid being thinner and paler than the rest. It arises from the tendo oculi and adjacent bone; and the fibres, forming an ellipse around the orbit, return to the place from which they started. The tendo oculi is a fibrous cord, which is attached by one end to the orbital aspect of the nasal process of the superior maxilla, while the other end bifurcates to be attached to the inner extrenities of the tarsal cartilages.

Externally the orbicularis palpebrarum is attached to the temporal fascia.

Action-It closes the eyelids. - It is one of the sphincter muscles, but in its action differs from most of them in being 
immovably attached at its extremities. When its fibres contract they bring the eyelids togethe. in a line corresponding to its points of attachment, whereas most sphincter muscles close the aperture which they surround by an approximation of all their fibres at the same time, and in the same proportion, towards the centre of the aperture, $e . g$., the orbicularis oris, in the act of whistling, since it has no bony attachment.

\section{Corrugator Supercilii.}

The corrugator supercilii is a small muscle, which arises from the inner extremity of the supercilliary ridge, on each side, passes outward and is lost in the deep face of the orbicularis palpebrarum.

Action-It throws the skin of the forehead into vertical folds, i. e., corrugates the brow.

\section{Tensor Tarsi.}

The tensor tarsi, or Horner's muscle, is very small. It arises from the orbital surface of the lachrymal bone, passes outward and divides into

\section{Cranial Group-One Muscle.}

\section{Occipito-Frontalis.}

The occipito-frontalis of either side, arises from the superior curved line of the occipital bone and the mastoid process. It is tendinous at its origin but becomes fleshy as it passes forward; and then, as it is mounting over the vault of the cranium, it forms a broad aponeurosis and descending on the forehead again becomes fleshy to be lost in the orbicularis palpebrarum and corrugator supercilii, its innermost fibres forming the pyramidalis nasi muscle.

Action-It raises the brows and throws the skin of the forehead into wrinkles.

\section{Nasal Group-Three Muscles.}

\section{Pyramidalis Nasi.}

The pyramidalis nasi is formed by the innermost fibres of the occipito-frontalis, which descending, are lost on the bridge of the nose. 
Action-It draws down the inner extremity of the eyebrow and elevates the nose.

\section{Compressor Naris.}

The compressor naris, triangular in shape, arises from the canine fossa on the superior maxilla by its apex and, mounting on the side of the nose, meets its fellow of the opposite side in a tendinous raphe on the bridge of the nose.

Action-The two are supposed to compress the nostrils.

\section{Depressor Alæ Nasi.}

The depressor alce nasi is found just beneath the mucous membrane of the upper lip. It arises from the incisive fossa of the superior maxilla and is inserted into the ala of the nose. Its name indicates its action.

The muscles of the face are nearly all small and pale and fatty. As a rule they arise from bone and are inserted into soft parts. Their action produces the varying expression of which the face is capable.

\section{Muscles of the Mouth.}

The muscles of the mouth consist of two groups, superior and inferior labial groups and one muscle, the orbicularis oris, which belongs to both groups.

\section{Orbicularis Oris.}

The orbicularis oris is the sphincter muscle of the mouth; as it has hut slight connection with bone, this attachment may be disregarded. It consists of two segments, one in each lip, which meet at the angles of the mouth.

Action-It can close the mouth in two ways, either by bringing the lips together in a horizontal line or by approximating the angles of the mouth at the same time.

\section{Superior Labial Group-Five Muscles.}

\section{Levator Labii Susperioris Alæque Nasi.}

The levalor labii susperioris alceque nasi arises from the nasal process of the superior maxilla and descending divides into two slips, one of which is inserted into the ala of the nose 
while the other continues on to be inserted in the upper lip. Its name indicates its action.

\section{Levator Labii Superioris Propius.}

This muscle arises from the orbital ridge of the superior maxilla and descends to be inserted into the upper lip. Its name indicates its action.

\section{Levator Anguli Oris.}

The levator anguli oris, or canine muscle, arises from the canine fossa of the superior maxilla and passes downward and outward to be inserted into the angle of the mouth. Its name indicates its action.

\section{The Zygomatic Muscles-Two.}

The zygomatic are two small muscles which arise from the zygomatic process of the malar bone and pass downward and inward. The zygomaticus major, the 1nwer one, is inserted into the angle of the mouth and the zygomaticus minor into the upper lip.

Action-They carry the angle of the mouth upward and outward.

\section{Inferior Labial Group-Three Muscles.}

\section{Quadratus Menti.}

The quadratus menti, or depressor labii inferioris, is a square-shaped thin muscle which arises from the oblique line on the front of the lower jaw near the symphysis and, passing upward, is lost in the lower lip. Its synonym indicates its action.

\section{Triangularis.}

The triangularis or depressor anguli oris, arises by its base just external to the preceding and is inserted by its apex into the angle of the mouth. Its synonym indicates its action.

\section{Levator Labii Inferioris.}

The levator labii inferioris lies just beneath the mucous membrane. It arises from the incisive fossa of the inferior maxilla and is inserted into the integument of the chin. Its action is indicated by its name. 


\section{The 0cular Group.}

\section{Levator Palpebræ Superioris}

Superior Rectus

External Rectus

Superior Oblique
Inferior Rectus

Internal Rectus

Inferior Oblique

The muscles forming this group are found within the orbit. All of them, except the levator palpebræ superioris, are attached to the eye-ball, and all, except the two oblique muscles, arise at the apex of the orbit and pass forward flat, straight and widening as they advance toward their insertion.

Four of them, from the direction of their fibres, are called recti and are concerned in noving the eye-ball. They are distinguished as: 1st, superior rectus, because it lies above the eye-ball and draws the front of the eye upward ; 2 d, inferior rectus, because it lies below the eye-ball and drax's its front downward; 3 d, external rectus, because it lies external to the eye-ball and draws its front outward; 4th, internal rectus, because it lies internal to the eye-ball and draws its front inward. By a combined action of these muscles the front of the eye can be moved in all directions intermediate between those mentioned. All the recti arise from the apex of the orbit, that is the margin of the optic foramen, and also receive an origin from the fibrous sheath of the optic nerve. They run forward and are inserted into the scierotic coat about one-fourth of an inch behind the circumference of the cornea. Some give as the origin of three of these muscles the ligament of Zinn, which is a fibrous band attached around the lower portion of the circumference of the optic foramen. This, however, is an unnecessary refinement. These muscles differ but slightly in size and length.

\section{Levator Palpebræ Superioris.}

Lying just beneath the orbit, and between it and the suparior rectus, is a muscle closely resembling the recti called levator palpelore superioris since its action is to raise the upper lid. It arises from the upper margin of the optic foramen and sheath of the optic nerve, passes forward and is inserted into the upper edge of the superior tarsal cartilage. 


\section{Inferior Rectus.}

The inferior rectus arises from the lower margin of the optic foramen and from the sheath of the optic nerve. It passes for ward and is inserted into the under surface of the sclerotic about one-fourth of an inch behind the cornea.

\section{External Rectus.}

The external rectus arises by a forked origin from the outer margin of the optic foramen and from the sheath of the optic nerve. It passes forward and is inserted into the outer surface of the sclerotic coat about one-fourth of an inch behind the circumference of the cornea. It is important to remember its forked origin, since many nerves pass through the interval between its head.

\section{Internal Rectus.}

The internal rectus arises from the inner margin of the optic foramen and sheath of the optic nerve. It passes forward and is inserted into the inner surface of the sclerotic coat about onefourth of an inch behind the circumference of the cornea.

\section{Superior Rectus.}

The superior rectus arises from the upper margin of the optic foramen and from the fibrous sheath of the optic nerve. It passes forward and is inserted in the sclerotic coat onefourth of an inch behind the cornea. It is the thinnest of the recti.

\section{Inferiox Oblique.}

The inferior oblique is a narrow and thin muscle, arising from the orbital surface of the superior maxilla, near the inner side of the orbit. It passes out beneath the eye-ball and inferior rectus to be inserted into the sclerotic near the entrance of the optic nerve, on the outer under aspect of the eye-ball.

\section{Superior Oblique or Trochlearis.}

The superior oblique arises from the inner margin of the optic foramen and sheath of the optic nerve. It passes forward along the upper inner wall of the orbit to its front, where, 
becoming tendinous, it plays through a cartilaginous ring fixed to the fovea trochlearis : thence its tendon passes outward and backward, beneath the superior rectus, to be inserted into the outer aspect of the sclerotic about half way between the circumference of the cornea and the entrance of the optic nerve.

Action-Its action is computed from the pully at the fovea trochlearis-hence it rotates the eye-ball on its antero-posterior axis and draws it forward.

\section{Muscles of Mastication.}

Masseter

Temporal
Buccinator

External Pterygoid

Internal Pterygoid

\section{Masseter.}

The masseter arises from the zygomatic process of the superior maxilla and from the anterior two thirds of the zygomatic arch by a bundle of fibres which pass downward and backward to be inserted into the angle and posterior part of the outer surface of the lower jaw. Beneath these fibres is a smaller bundle arising from the posterior third of the zygomatic arch aud passing downward and forward to be inserted into the outer face of the ramus nearly to the basilar border of the bone.

Action-The two muscles, acting together, draw the lower jaw powerfully upward and press the teeth firmly against the teeth of the upper. The superficial fibres draw the jaw forward while the deep carry it backward.

The masseter is covered by a strong fascia, derived from the cervical fascia, which is attached below to the lower jaw, and blends with the deep cervical fascia, while above it is attached to the zycroma and forms the parotid fascia.

\section{Buccinator.}

The buccinalor, or trumpeter's muscle, is the bulkiest element of the cheek. It arises from the alveolar process of the superior maxilla, from the external oblique ridge of the lower jaw, as far forward as the second bicuspid tooth and 
from the pterygo-maxillary ligament. Its fibres converge to be inserted into the angle of the mouth.

Action-The buccinators compress the cheeks, keeping food between the teeth or expelling air through the lips.

The pterygo-maxillary ligament extends from the hamular process of the pterygoid plate to the posterior extremity of the molar ridge of the lower jaw. In front it gives origin to some of the fibres of the buccinator and behind to the superior constrictor of the pharynx.

\section{Temporal.}

This muscle is found in a fossa, and is covered by a fascia, bearing the same name. The temporal fascia is a dense, shining aponerosis which is attached above to the temporal ridge on the side of the skull; while below it divides into two layers, one seizing the outer and one the inner edge of the upper border of the zygomatic arch. The deep surface of the fascia gives origin to part of the temporal muscle.

The temporal is a radiated muscle occupying the temporal fossa. It arises from the whole of the temporal fossa, from the whole length of the temporal ridge and from the temporal fascia which covers it. It passes downward, its fibres converging, and is inserted into the apex and inner side of the coronoid process of the inferior maxilla.

Action-It is a powerful elevator of the lower jaw, acting in concert with the masseter. Its power is greatest when the jaw is depressed, while the power of the masseter increases as the jaws are approximated.

\section{External Pterygoid.}

The cxternal ptery'goid arises from the lower surface of the greater wing of the sphenoid, from the pterygoid ridge and sphenoidal spine and from the whole of the outer face of the external pterygoid plate. The fibres pass backward and outward and narrow to a tendon which is inserted into the neck of the condyle of the lower jaw and into the inter-articular fibro-cartilage.

Action-The two muscles acting together, will carry the lower jaw directly forward. One muscle alone draws the jaw 
forward and to the opposite side. It is the alternate action of the two muscles which produces the grinding motion necessary for bruising the food.

\section{Internal Pterygoid.}

The internal pterysoid, or internal masseter arises from the inner face of the outer pterygoid plate, from the groored posterior face of the tuberosity of the palate and occasionally by a slip from the tuberosity of the superior maxilla. It passes downward, outward and is inserted into the inner face of the angle and ramus of the lower jaw.

Action-It acts almost exactly like the superficial fibres of the masseter, drawing the jaw forward and upward. Its origin being nearer the middle line, however, it also draws the jaw inward, thus aiding the external pterygoid in grinding the food.

All the muscles of mastication are supplied by the muscular branches of the inferior maxillary division of the fifth, except the buccinator which also receives a branch from the facial. The internal maxillary artery furnishes blood. 


\section{THE VASCULAR SYSTEM.}

\section{The Arteries.}

There are two sets of arteries: one the Pulmonary System, the other the Systemic System. The two systems communicate with one another.

In the Systemic circulation, the blood starts from the heart in the large arteries, and passes on into the branches which spring from those arteries, these branches disminishing in size until the capillaries are reached. Returning, the blood passes through the veins, which increase in size as we approach the heart.

The Pulmonary circulation. The heart is divided into four chambers: two left, one above and one below; and two right, one above and one below. From the chamber on the left, under side, the systemic circulation starts. Entering into the chamber above on the right is the termination of the venous system; and from this chamber the blood is poured into the chamber below by means of two inlets. From there it passes down into a cavity from which the pulmonary artery starts, this artery breaking up into two branches and passing to the lungs. After making the circuit of the lungs, the blood is returned into the upper left side of the heart, from which it drops down into the other left cavity, where the aorta starts.

The arteries all commence with the one great trunk the aorta; but for several reasons we generally begin with one of the arteries belonging to the upper extremity or head and neck.

Arteries decrease in size as they throw off branches; but if we consider the combined area of the two branches into which the artery is divided, we find that this area is greater than that of the original trunk. Similarly, if we consider the combined area of all the branches which spring from any given artery, we find that this area is infinitely greater than that of the original artery. On the other hand, the combined area of the veins, while larger than the combined area of the great 
veins which return the blood to the heart, is much larger than the combined area of the arteries. Consequently, as the blood continues its course in circulating through the body the rapidity of the current decreases in proportion as the distance from the heart increases. As the veins decrease in size, or containing capacity, as they approach the heart, the current becomes swifter; but never anything like as swift as the current in the arteries.

In describing an artery, we not only give the region in which it lies, its course and termination; but its relations, divided for definiteness of description into muscular, visceral and cord relations. Often these relations include a vein, or another artery; and there is usually something else to be noticed, for instance, a tendon. Besides that, arteries have other coverings, skin and fascia; and other relations, which are neither cords, fascias nor muscles. Such, for instance, are the Thyroid gland, the Trachea, the Pharynx, or the Thymus gland. To describe one, for instance. let us take the great vessel on the side of the neck, the Arteria Innominata. Starting at the commencement of the transverse aorta, it passes upward and outward, and terminates in one of the most frequent ways in which arteries terminate, viz., by dividing into two separate branches: one branch, the right common carotid, which runs nearly vertically upward in the neck, the other, the right subclivian, which apparently continues the course of the original vessel, with a slight increase in its obliquity. There are two common carotids, and two subclavians, right and left. To decide the difference between them, notice that the right common carotid and the right subclavian each pass down into the thoracic cavity. There is a combination of the two vessels on the right side; and no junction on the left side, the right springing by a common brauch, the left by separate branches.

\section{The Right Common Carotid.}

The right common carolid artery differs from the left in that it springs from the arteria innominata, whereas the left springs directly from the arch of the aorta, hence the relations of the first portion of the left common carotid would be differ- 
ent from the relations of the right common carotid in this portion. The relations after they have passed from the thoracic cavity, and have reached the back of the sterno-clavicular articulation, become identical; and then the description of one vessel will apply to the other.

The right common carotid commences by being one of the two terminal branches of the arteria innominata. It then passes upward with a slight inclination back ward and outward, lying upon the anterior tubercles of the transverse process of the four lower cervical vertebræ, about on a line drawn from the upper border of the thyroid cartilage. After reaching that point, it terminates by dividing into two branches, which are known as the internal and external carotid arteries.

Covering the vessel we find, first, the skin; then a layer of superficial cervical fascia; then the platysma myoides muscle; then the first layer of deep cervical fascia; then the sternocleido-mastoid muscle ; then the second layer of deep cervical fascia. After pulling that aside, we come to the sterno-hyoid muscle; then to the sterno-thyroid; and anterior belly of the omo-hyoid; then to the last layer of cervical fascia; forming the front of the sheath of the vessel behind which you have the artery itself.

It is more convenient to divide the vessel into two portions, the first portion being considered as lying in the "Triangle of Necessity" or the inferior carotid triangle, the second as lying in the superior, or "Triangle of Election". The first portion commences as the artery itself commences, as being one of the branches of the arteria innominata, and terminates where the vessel is crossed by the anterior belly of the omo-hyoid. The second portion commences where the first terminates; and extends to the termination of the artery at the upper border of the thyroid cartilage. The first portion is deeply seated, difficult of access; and the triangle is for that reason known as the Triangle of Necessity. Throughout the first portion the artery is completely covered by the sterno-cleido-mastoid muscles; it is only at its commencement that it is covered by the sterno-hyoid and thyroid, which rapidly draw away from the vessel and cease to be relations of it. Then as we pass up to 
the second portion of the ressel (the omo-hyoid being the dividing line, belongs partly to each portion), we find that the second portion is covered by superficial and deep fascia, platysma myoides, and the sterno-cleido-mastoid, which in this situation overlaps rather than covers. Throughout its entire course it is covered by the sterno-cleido-mastoid and the platysma myoides; only at its beginning is it covered by the bellies of the sterno-hyoid and sterno-thyroid; and only at the middle of its course is it covered by the omo-hyoid.

The more important things to study in connection with the vessel are the cord relations, these being alwavs of greater importance than anything else, owing to the danger of wounding. Every large artery protects itself by passing as closely as possible to the bone; but another protection is the sheath, which is formed of fibrous tissue, in this case a part of the deep cervical fascia. So powerful is the sheath that many cases could be cited in which vessels have been found floating in pus, the artery remaining entirely uninjured. This vessel possesses one of the largest sheathes in the body; and in the same sheath with it are found the internal jugular vein and the pneumogastric nerve, all lying upon the same plane, the artery being most internal, the vein most external, and the nerve lying between them. The nerve is sometimes described as "lying between and behind" the vein and artery ; but this is not correct: It lies between them and not behind them. Another important structure running downward upon its sheath is the Descendens noni, or Descendens hypoglossi. This nerve passes downward on the front of the sheath of the common carotid: and just before it reaches the upper border of the anterior belly of the ono-hyoid it receives one or two filaments from the cervical plexus of nerves forming, just above the anterior belly of the omo-hyoid, Scarpa's Arch. In addition there is another important cord lying just behind the artery, which has a large bulge upon it near its upper extremity, called the cervical portion of the Sympathetic nerve. It descends upon the prevertebral group of muscles directly behind the sheath.

Des Anat-19 
On the upper portion of the course of the artery are generally seen the superior thyroid veins, frequently two. In the lower portion, about its middle, are seen the middle thyroid generally; and below that generally the anterior jugular, all of these veins being superficial. At the commencement of their course these vessels are deeply seated, and covered by a large number of structures; and they are very close to each other, the distance increasing as we pass upward into the neck. Owing to the projection of the Thyroid cartilage, with the tissues which lie in the neighborhood of it, these vessels appear to be more deeply seated in the second portion than in the beginning of their course. At the commencement of the artery, to its inuer side, it has the Trachea: passing above, the Thyroid gland, passing above that, the cartilages of the larynx; and then, still higher up, the side of the pharynx.

As the Pneumogastric nerve passes downward, it gives off an important branch, which lies over thesubclavian artery, the Recurrent Laryngeal. This branch winds upwards behind the subclavian artery, first passes obliquely inward, and then passes behind the common carotid artery, and runs into the muscles along the side of the neck. As the nerve runs upward, it bears a three fold relation to the common carotid artery : first behind and external, then behind and then to the inner side. A branch of the subclavian, the inferior thvroid is also a posterior relation of the first portion.

Unlike nearly all arteries, the common carotid gives off no branches in its course. The usual arrangement is that the arteries give off branches to those structures among which they lie. The branches of one of its terminal branches supply the upper region of its course, while the branches of the subclavian supply the lower region.

\section{External Carotid.}

The external carotid artery, one of the two terminal branches of the common carotid, begins where that artery bifurcates about on a level with the upper border of the thyroid cartilage and, continuing the direction of the common carotid, passes 
beneath the posterior belly of the digastric and the stylo-hyoid muscles, enters the substance of the parotid gland and terminates in that gland behind the neck of the condyle of the lower jaw by dividing into two branches, the temporal and the internal maxillary arteries. The temporal continues the course of the external carotid to mount over the zygoma and be distributed to the side of the head by two branches, while the internal maxillary passes inward, behind the neck of the condyle of the lower jaw to supply the deep structures of the face.

This artery is divided, for convenience of description into three portions.

Relations. - The first fortion of the external carotid lies in the superior carotid triangle, its length varying as the common carotid divides on a level with, above or below the upper border of the thyroid cartilage, for it extends from the bifurcation of the common carotid to the stylo-hyoid and posterior belly of the digastric. It is covered by the integument and platysma and deep cerrical fascia, is slightly overlapped by the sterno-mastoid and is crossed in front by the hypoglossal or twelfth nerve, near the termination of this portion, and the lingual vein near its beginning. At its commencement the internal carotid lies to its outer side; but by the time the external carotid reaches the termination of its first portion, the internal carotid has passed behind it.

The second portion is that which is crossed by the stylohyoid and posterior belly of the digastric. Its coverings are integument, platysma, stylo-hyoid and posterior belly of the digastric. Thestylo-glossus and pharyngeus muscles, glossopharyngeal and superior laryngeal nerves cross between this and the internal carotid about on a line between the second and third portions.

The third and last portion extends from the stylo-hyoid and posterior belly of the digastric to the termination of the artery behind the neck of the condyle of the lower jaw. It lies embedded in the substance of the parotid gland and is crossed, superficially, near its termination, by the facial nerve and the facial 
and temporo-maxillary veins. The first portion is most superficial, the second the shortest and the third the longest and deepest.

\section{Branches.}

Superior Thyroid

Lingual

Facial
Occipital

Posterior Auricular

Ascending Pharyngeal

Parotidean.

It should be remembered that the common carotid gives off no branches except its terminal ones and hence the structures in its course have to be supplied by other arteries. The external carotid does a part of this work. Besides those into which it divides, the external carotid gives off six single branches, and a set to the parotid gland. The first three arise from the anterior aspect of the artery, low down and in the order given from below upward. The next two rise from the back of the artery higher up.

\section{Superior Thyroid.}

The superior thyroid, which is at first very superficial, being covered only by the platysma and fascia, forms a curve, first passing upward and inward, then downward and inward, crosses beneath the depressor muscles of the hyoid bone, and reaches the upper part of the thyroid gland to which it is distributed. It gives off the following branches:

1st. hyoid, which passes forward just beneath the hyoid bone to be distributed to muscles and anastomose with its fellow of the opposite side.

2nd. superior laryngeal, which passes forward on the thyrohyoidean membrane and pierces it to be distributed to the mucous membrane of the larynx, anastomosing with its fellow.

3rd. crico-thyroid, which passes across the crico-thyroid membrane and gives branches which pierce it to reach the mucous membrane of the larynx anastomosing with its fellow.

4th. muscular or superficial descending branch which passes downward and outward across the common carotid to supply the sterno-mastoid. 


\section{Lingual.}

The lingual artery is divided into four portions: 1st, it descends obliquely inward, over the extremity of the hyoid bone covered only by the skin and platysma; $2 \mathrm{~d}$, it passes forward along and above the hyoid bone, is crossed by the digastric and then covered by the hyo-glossus muscle; $3 \mathrm{~d}$, it ascends to the under aspect of the tongue; 4th, in runs forward on the under aspect of the tongue, under the name of the ranine artery. The first two portions lie on the middle constrictor of the pharynx. Its branches are three:

1st. Hyoid, which runs inward along the upper border of the hyoid bone to be distributed to muscles and anastomose with its fellow.

2d. dorsalis lingua, which ascends to the dorsum of the tongue along the posterior border of the hyo glossus muscle to supply the back of the tongue muscous membrane and soft palate.

3d. sublingual, which runs forward to the sublingual gland from the margin of the hyo-glossus and is distributed to that gland, to the mucous membrane of the mouth and to muscles.

The ranine is the terminal branch of the lingual. It passes forward on the under surface of the tongue, beneath the mucous membrane is ristributed to that organ and anastomoses with its fellow at the end of the tongue.

\section{Facial.}

The facial arlery arises just above the hyoid bone and is divided into two portions, one while it is in the neck and the other after it reaches the face. The first portion passes forward and upward, through the submaxillary gland, to mount over the body of the lower jaw just at the anterior inferior angle of the masseter muscle and about one and one-half inches in front of the angle of the lower jaw. At its origin it is superficial, being covered only by the skin and platysma; but it soon enters the submaxillary wland and is crossed by the stylo-hyoid and posterior belly of the digastric. When it leaves the gland and mounts over the jaw it is again superficial, being covered only by 
the integument and platysma. Its pulsations can here be felt. The second portion of the artery has a tortuous upward and inward course over the face towards the angle of the mouth, thence along the side of the nose to terminate at the inner canthus of the eye as the angular artery. Its branches are divided into those given off below the jaw, five in number, and those on the face, also five in number.

1st. inferior palatine, which ascends to the soft palate, tonsils, Eustacian tube, stylo-pharyngeus and stylo-glossus muscles.

2d. tonsillar, which ascends to the tonsils.

3d. submaxillary, which are from three to five in number and ascend to the submaxillary gland.

4th. submentul, which runs forward beneath the lower jaw and passing over the symphysis menti terminates by inosculating with the inferior labial. It lies on the mylo-hyoid supplies neighboring muscles and anastamoses with the submaxillary before running over the chin.

5th. muscular branches distributed to neighboring branches.

The five branches from the second portion are as follows:

1st. muscular or buccal, which are branches to adjacent muscles.

2d. inferior labail, which runs forward beneath the skin of the lower lip, supplies muscles in its course and anastamoses with the submental inferior coronary and the inferior dental.

3rd. inferior coronary, which skirts the edge of the lower lip, anastamose with its fellow of the opposite side, with the mental branch of the inferior dental and with the inferior labial and supplies muscles and glands in its course.

4th. superior coronary, which skirts the edge of the upper lip, giving off a branch to the septum of the nose, arteria septi. This artery anastamoses with its fellow and supplies structures in its course. The arteria septi passes upward to ramify on the septum of the nose.

5th. lateralis nasi, which is distributed to the side of the nose, inosculating with its fellow of the opposite side.

The angular artery is the continuation of the facial. Passing 
up along side of the nose, it gives off small branches to the cheek and terminates by anastamosing with the nasal branch of the opthalmic.

The inferior labial and inferior coronary very frequently comes off by a common trunk. Sometimes it is a common trunk which divides into the inferior and superior coronary arteries.

\section{Occipital.}

The occipital branch of the external carotid passes upward and backward to the occipital groove of the temporal bone, beneath the muscles attached to the mastoid process, thence mounting on the posterior aspect of the occipital bone and piercing the fascia which connects the cranial attachment of the trapezius with the sterno-cleido-mastoid becomes superficial and is distributed to the back of the head. The hypoglossal nerve winds around, from behind forward-it crosses over the internal carotid artery, the internal jugular vein, the pneumogastric and spiral accessory nerves. Its branches are:

1st. Sterno-mastoid, which, sometimes arising directly from the external carotid, descends to the sterno-mastoid muscle.

2nd. muscular branches to stylo-hyoid and digastric.

3rd. two small branches auricular to the concha and meningeal to the membranes of the brain.

4th. princeps cervicis, which passes down the neck lying deep.

It divides into a superficial branch which passes beneath the splenius and inosculates with the superficial cervical of the transversus calli; and a deep branch which passes beneath the complexus to anastomose with the profunda cervicis.

\section{Posterior Auricular.}

The postcrior auricular passes upward and backward behind the ear and is distributed by an anterior branch to the auricle and by a posterior to the back of the head. It produces three branches, the stylo-masloid which enters the stylo-mastoid foramen to be distributed to the ear; the auricular which is distributed to the back part of the cartilage of the ear-the mastrid which passing over the sterno-mastoid is distributed to the scalp belind and above the ear. 


\section{Parotidean Branches.}

The parotidean branches, four or five in number, are distributed in the parotid gland as the external carotid is passing through it.

\section{Ascending Pharyngeal.}

The ascending pharyngeal branch springs from the externah carotid just at its origin and passes up beside the pharynx to the base of the skull, where it divides into a meningeal branch to enter the cavity of the cranium through the jugular foramen, and a pharyngeal branch to adjacent parts.

\section{The Temporal Artery.}

The temporal artery, one of the two terminal branches of the external carotid, begins where the external carotid forks in the parotid gland, behind the neck of the condyle of the lower jaw, and emerging mounts over the zygoma and divides about one and one-half inches above that point, where it lies on the temporal fascia, into an anterior and a posterior temporal. The anterior passes upward and forward, inosculating with the frontal and supra-orbital the posterior upward and backward to be distributed to the side of the head, and to inosculate with its fellow of the opposite side and with the occipital. These two are called the superficial temporal branches since, as will appear hereafter, there are other and deeper temporal branches.

It gives rise to four branches besides those in which it terminates.

1st. transverse facial which arises below the zygoma, and passes transversely forward on the masseter muscle parallel with Steno's duct. It anastomoses with the facial.

2nd. anterior auricular, to the front of the auricle.

3rd. orbital, which runs forward to the outer angle of the eye.

4th. middle temporal, which plunges into the substance of the temporal muscle where it is distributed. Its name is derived from its situation between the two surperficial temporal arteries and the deep which spring from another trunk. 


\section{The Internal Maxillary.}

The inlemal maxillary is the larger of the two terminal branches of the external carotid. The course of the artery is divisible into three portions. The first portion passes inward, forward and upward behind the ramus of the inferior maxilla, between it and the internal lateral ligament. The second portion passes forward and upward between the internal pterygoid muscle within and the temporal and masseter muscles without. The third fortion disappears into the sphenomaxillary fossa. Its branches are sixteen in number, as follows :

\section{First Portion-Four Branches.}

1st. lympanic which enters the tympanum through the fissure of Glaser.

2nd. meningea media, which ascends to enter the cavity of the cranium through the foramen spinosum.

It then divides into an anterior and a posterior branch. The anterior passes upward and forward over the great wing of the sphenoid, grooves the anterior inferior angle of the parietal and divides into branches which rammify over the dura. The posterior branch passes over the squamous portion of the temporal and divides into branches on the occipital. These branches anastamose with each other and with the anterior and posterior meningeal. They supply the dura and the bone.

3rd. The meningea parva, which enters the cavity of the cranium through the foramen ovale.

4th. inferior denlal, which descends to enter the dental foramen of the inferior maxilla and runs forward in the bone griving a branch to each fang of every tooth in the lower jaw. Just before entering the dental foramen it gives off a branch called mylo-hyoidean; and when it reaches the mental foramen it divides, sending at branch called mentalis through the mental foramen, while another, called the incisive, continues its course in the bone. The mylo-hyoidean passes in the groove of that name, and supplies the mylo-hyoid muscle. 


\section{Second Portion.}

Six branches are derived from this portion of the artery. The deep temporal, two in number, occupy the anterior and posterior portions of the temporal fossa. They anastamose with the superficial temporal arteries and supply the temporal muscle. The pterygoid branches, as the name indicates, supply the pterygoid muscles. The masseteric branch runs through the sigmoid notch and supplies the masseter from its deep surface. The buccal passes forward between the internal pterygoid and the lower jaw and supplies the buccinator. It anastamoses with the facial.

\section{Third Portion.}

1st. superior dental, which descends upon the tuberosity of the superior maxilla and sends its branches through small foramina in the bone to supply the molar and bicuspid teeth.

2nd. Infra-orbital, which runs forward along the canal in the floor of the orbit, sending branches downward to the front teeth of the upper jaw and emerges through the infra-orbital foramen to supply adjacent parts on the face.

3d. descending palatine, which descends along the posterior palatine canal to emerge in the palate through the posterior palatine foramen and send a branch forward in a groove seeu on the side of the hard palate(called the anterior palatine canal) which reaches the floor of the nose through the anterior palatine foramen.

4th. spheno-palatine, which enters the nose through the spheno-palatine foramen and divides into two branches, one being distributed to the septum and the other to the mucous membrane of the outer wall and antrum maxillare.

5th. ptergo-palatine, which runs backward in the pterygopalatine canal to the pharynx, Eustachian tube and neighloring parts.

6th. Vidian, which passes backward along the pterygoid canal and, like the preceding, is distributed to the mucous membrane of the pharynx and Eustachian tube. 


\section{The Right Subclavian.}

The Right Subclavian artery is one of the two terminal branches of the arteria innominata, the other being the right common carotid. It commences upon the upper border of the right sterno-clavicular articulation, arches upward and outward, passing between the Scaleni muscles; then turns downward and outward, and terminates by becoming the axillary at the junction of the outer one-third with the inner two-thirds of the shaft of the clavicle on the outer border of the first rib. It is thus seen that it forms a bow, the highest part of the arch being that which passes behind the Scaleni muscles.

This warrants the sub-division of the artery into three portions.

The first commences by a bifurcation of the arteria innominata and terminates at the inner border of the scalenus anticus; the second lies behind the scalenus anticus; and the third portion extends from the outer border of the scalenus anticus to the termination of the artery in the axilla at the outer border of the first rib. This third portion is itself sometimes subdivided into that part which lies between the scalenus anticus and the clavicle and the part which lies behind the clavicle; but the division is unnecessary.

Relations. The coverings of this artery are the skin, superficial fascia, platysma myoides muscle, one layer of the deep cervical fascia, the sterno-cleido-mastoid muscle, with the second layer of deepcervical fascia, and the posterior belly of the omo-hyoid muscle. It is better to take the relations of the artery as a whole; and then consider them with reference to the various portions of the vessel. Thus, starting from within and passing outward on the anterior face of the artery, we find the pneunorastric nerve, with its recurrent laryngeal branch, external to that the internal jugular vein, then the phrenic nerve. then the scalenus anticus muscle, then the suprascapular vein and artery, the nerve to the subclavius and the subclavius muscle. More superficially, just beneath the skin, are the thoracic branches of the cervical plexus. Below it are its 
vein, the recurrent laryngeal nerve, the lung and pleura, and the first rib. Behind, the recurrent laryngeal, the cervical portion of the sympathetic nerve, the vertebræ, the Scalenus anticus muscle, and the brachial plexus of nerves. Above, the brachial plexus and the omo-hyoid muscle; and to its inner side, the right common carotid.

To consider the different parts separately:

First Portion. The first portion is covered throughout by the integument, platysma, sterno-cleido-mastoid; and, at its commencement, by the right sterno-clavicular articulation, the sterno-hyoid and sterno-thyroid muscles. Superficial branches of the cervical plexus also pass over it. Crossing it in front are the pneumogastric nerve, giving off here its recurrent laryngeal branch; external to this, the internal jugular vein; and external to this, usually, the phrenic nerve. The course of the phrenic is downward and slightly inward upon the anterior face of the scalenus anticus muscle. Occasionally it continues on that muscle until it passes beyond the artery; but generally it drops off from the muscle to the artery to become an anterior relation of the latter. In front near its beginning is the right vertebral vein. Just as the internal jugular vein crosses the front of the artery it communicates with the subclavian vein to form the right Vena Innominata. Hence the junction of these two veins would lie below on a plane anterior and slightly to the outer side of the first portion of the subclavian. The pneumogastric here gives off the recurrent laryngeal, which is first in front, then below, then behind the artery. To the inner side at its commencement is the right common carotid. Behind it is the recurrent laryngeal, and the cervical portion of the sympathetic, the cardiac branches of which cross its front. Below, the artery rests upon the lung and the pleura. The pleura of the right side extends upward into the root of the neck above the level of the first rib, so that the artery makes an impression upon the dome of the pleura, and a portion of that membrane lies behind the artery as well as beneath it.

The Second Portion. The second portion forms the top of the bow; and runs transversely outward. It runs behind the Scalenus anticus muscle; and is co-extensive with its breadth. 
In front are the integument superficial fascia, the platysma first layer of deep cervical fascia, the sterno-cleido-mustoid second layer of deep cervical fascia, with branches of the cervical plexus extending over the clavicle. Occasionally on its front is the phrenic nerve. Thesuprascapular artery and the transversa colli branches of the subclavian pass across the front of the scalenus anticus muscle; and should be considered in all operations in this situation. The brachial plexus of nerves lies behind and above the artery, separating it from the scalenus posticus muscle. Below and behind are found the pleura and the lungs. The subclavian vein is not a relation of the artery, lying some distance below it and separated from it by the scalenus anticus.

The Third Portion. The third portion is the downward and outward part, extending from the outer edge of the Scalenus anticus to the outer border of the first rib, and lying in its lower portion between the clavicle and the first rib. It is covered entirely by the platysma; and to a slight extent, in many cases, by the outer edge of the sterno-cleido-mastoid; and, in a few cases, where this muscle has a redundant origin, the third portion of the artery is covered completely by it. The artery lies in the triangular space bounded above by the omo-hyoid, internally by the sterno-cleido-mastoid, and below by the clavicle. This space is covered over by a rather dense layer of the deep cervical fascia, which is of course one of the coverings of the vesse1. Crosssing in front of it are the suprascapular artery, a branch of the subclavian itself, which passes outward just beneath the upper border of the clavicle. This artery is accompanied by its own vein. Crossing the front of it from above is the external jugular vein, which descends the neck lying just behind the outer edge of the sterno-cleidomastoid muscle; and, after having received on the front of the artery the suprascapular and tranverse veins, it terminates in the subclavian on the outer side of the scalenus anticus.

The subclavius muscle, the descending branches of the cervical plexus, and the nerve to the sub-clavius muscle are also in front. Owing to the curve in the course of the artery, what has heretofore been called its upper aspect now becomes 
its external. The brachial plexus of nerves has been described as being an external relation, though the plexus lies on a plane posterior to the artery as well as to its outer side. The omo-hyoid lies above and to the outer side, in front of the plexus. To the inner side is the subclavian vein, which is in contact with the vessel for only a short distance before its termination. The vein, it will be observed, is a relation of the first and third portions only ; that is, it is in contact with the outer front aspect of the first portion at its commencement, and with the inner front aspect of the third portion at its termination, bearing no relation to the second portion, hence it presents the same relation which a string bears to a bent bow. At the very commencement of this portion it lies upon the pleura, so that it will be seen that the pleura lies below and behind all three of the portions of the artery, though for the third portion the relation is only a momentary one, the first rib quickly separating the two structures. The ligation of this vessel is preferably performed in the third portion of its course.

\section{Branches.}

\section{Vertebral}
Thyroid Axis $\left\{\begin{array}{l}\text { Inferior Thyroid } \\ \text { Suprascapular } \\ \text { Transversa Colli }\end{array}\right.$
Internal Mammary
Superior Intercostal
Profunda Cervicis.

These seven branches usually rise by four trunks: the vertebral, thyroid axis, internal mamary, and the common trunk for the superior intercostal and cervicalis profunda.

\section{Vertebral.}

The vertebral is the first and largest branch of the subclavian. It arises from the posterior aspect of the first portion of the artery; and enters the foramen in the transverse process of the sixth cervical vertebra, usually. Occasionally it passes to the fifth; but, though the seventh is perforated, the vertebral artery never enters it. It passes upward through the foramina of the transverse process, winds backward around the superior articular process of the atlas, enters the cavity of the cranium through the foramen magnum, and at the lower bor- 
der of the pons Varolii unites with its fellow of the opposite side to form a single trunk, called the Basilar artery, which runs upward and forward to the anterior border of the pons, where it divides into four terminal branches, two on either side. At its origin the internal jugular vein and the inferior thyroid artery lie in front of it. On the left side, while lying between the scalenus anticus and longus coli muscles, the thoracic duct passes in front of it. Besides its terminal branches it gives off the following branches:

1st. lateral spinal, which are given off as the artery ascends the neck to enter the intervertebral foramina. They there divide into two branches: one for the bodies of the vertebræ, and the other for the spinal cord and its membranes. That for the spinal cord reaches its along the roots of the spinal nerves, while the branch for the bodies of the vertebræ divides into an ascending and a descending branch, anastomosing with similar branches coming from above and below.

2nd. The muscular branches are unimportant. They come from the lower portion of the artery, and are distributed to the cervical muscles.

3d. The posterior meningeal are small branches distributed to the dura mater of the cranium.

4th. The anlcrior spinal arises near the termination of the vertebral, unites with its fellow of the opposite side, and passes downward on the middle of the spinal cord, where it receives communications from the lateral spinal branches, before mentioned, and forms a continuous vascular channel down the anterior aspect of the cord.

5th. The poslcrior spinal winds around the medulla, unites with its fellow of the opposite side, passes down behind the posterior roots of the spinal nerves, and receives similar branches from the lateral spinal. This also forms a branch passing down the spinal cord.

ith. The posterior inferior cerclicllar arises just before the termination of the vertebral; and passing across the lateral aspect of the medulla, winds around the inferior portion of the cerebellum, to which it is distributed. 


\section{Basilar.}

The Basilarartery is formed by the junction of the two vertebral arteries above the middle of the medulla oblongata; and after passing upward on the lower surface of the pons varolii, it terminates on the upper border of that body by dividing into two branches, the posterior cerebral arteries. It gives off the following branches:

1st. transverse, which are numerous small branches given off to each side of the pons Varolii.

2 nd. anterior inferior cerebellar, or anterior cerebellar, which arises soon after the origin of the basilar; and passing across the pons a little below its centre, reaches the anterior border and lower surface of the cerebellum, where anastomosing with the posterior inferior cerebellar, it is distributed to that organ. It is in reality scarcely more than one of the transverse branches.

In addition to these way-side branches, the basilar artery gives off the following terminal:

1st. superior cerebellar, which passing across the upper border of the pons, passes into the fissure between the upper border of the cerebellum and the cerebrum, and ramifies upon the upper surface of the cerebellum.

2 nd. The posterior cerebral also winds around the outer border of the pons, passing to the inner surface of the posterior lobe of the cerebrum, to which it is distributed, anastomosing with the other cerebral arteries.

These two vessels are formed by the bifurcations of the basilar artery, which just before it divides, gives off numerous small branches, which enter the minute foramina constituting the locus perforatus at the base of the brain.

\section{Thyroid Axis.}

The Thyroid Axis is a short thick trunk, which arises from the upper portion of the subclavian, just at the inner border of the scalenus anticus muscle. It almost immediately divides into three branches; the Inferior Thyroid, the Suprascapular, and the Transversa Colli. 


\section{Inferior Thyroid.}

The inferior thyroid, the branch from which the plexus receires its name, first passes vertically upward for a distance of an inch and one-half or two inches. It then turns inward, crosses behind the sheath of the common carotid artery to reach the thyroid gland. It gives off branches, or twigs, called laryngeal, tracheal, and oesophageal, which are distributed to the structures named: the larynx, the trachea and the oesophagus. Just as it makes its transverse, horizontal turn there springs off a large branch, called the cervicalis ascendens, which passes upward on the anterior tubercles of the transverse processes to be distributed to muscles and glands in the neck.

\section{Suprascapular.}

The suprascupular, the next most constant branch, passes outward across the scalenus anticus muscle, where it is a distant relation of the second portion of the subclavian. It then turns downward until it reaches the clavicle, behind which it passes outward over the scapula, crossing in its course the third portion of the sudclavian. It passes over the transverse ligament of the scapula to reach its dorsum in the supraspinous fossa, where it gives off branches to the bone, to the supraspinatus muscle, and a branch which winding around the anterior border of the spine, enters the infraspinous fossa, to communicate with vessels in that situation, chiefly the dorsalis scapulæ branch of the axillary.

\section{Transversa Colli.}

Next to the inferior thyroid, the transvorsa colli is the largest of the branches of the thyroid axis, and the least frequent of its progeny. It passes across the side of the neck over the scalenus anticus muscle, above the level of the second portion of the subclavian, very frequently passing between some of the cords of the brachial plexus of nerves. On reaching the anterior edge of the trapezius it divides into two branches: the cerviculis superficialis and the poslerior scapular. The cer-

Des Anat-20 
vicalis superficialis runs upward to anastomose with the superficial branch of the arteria princeps cervicis from the occipital. The posterior scapular, much larger than the preceding, passes to the vertebral border of the scapula; and descending along it, anastomoses with the dorsalis scapulæ branch from the subscapular artery.

\section{Internal Mammary.}

The internal mammary arises from the lower aspect of the subclavian at the inner edge of the scalenus anticus, and frequently directly opposite the origin of the vertebral. It passes downward upon the costal cartilages about half an inch from the edge of the sternum to the diaphragm, where it divides into its two terminal branches: the superior epigastric and masculo-phrenic. It is accompanied by satellite veins, 1 ying upon either side. At its origin it is covered by the internal jugular and sub-clavian veins, and has crossing it the phrenic nerve. Its branches are :

1st. The comes nervi phrenici, which arises at the upper portion of the vessel. It is a long and slender branch; and accompanies the phrenic nerve, having the same relations as that nerve, and terminates by anastomosing with the other arteries of the diaphragm.

2nd. The anterior intercostal, which are given off to the intercostal spaces, over which the internal mammary passes. Each passing outward, soon divides into two branches, one for the upper and the other for the lower border of the edges of the ribs. These branches occasionally spring separately from the artery. They first lie between the intercostal muscles and the pleura; and then between the two intercostal muscles.

3rd. perforating branches. These are usually six in number, corresponding to the number of intercostal spaces. They pass forward to the front of the thorax; then running outward, are distributed to the muscles, and in the female, chiefly to the mammary gland.

4th. The mediastinal, small and unimportant branches, which supply the structures of the anterior mediastinum. 
5th. Pericardiac branches, which are about the size of the mediastinal and are distributed to the pericardium.

These are the way-side branches of the vessel. Of the two terminal branches, the larger, the superior epigastric, leaves the thoracic cavity behind the seventh costal cartilage. It enters the sheath of the rectus muscle; and continuing its course downward, anastomoses with the inferior epigastric, a branch which springs from the external iliac. This is the longest anastomosis in the body, establishes a connection between the arteries in the upper and the arieries in the lower extremity, and renders it conceivable that the abdominal aorta might be ligated without immediate death. It gives off branches to the muscles in its course.

The musculo-phrenic follows the curvature of the lower ribs. It passes down ward and outward, giving off intercostal branches similar to those given off from the trunk of the internal mammary itseli, and performing the same service, and in addition giving off a branch to the diaphragm.

\section{Superior Intercostal.}

The superior intercostal and profundus cervicis usually spring by a common trunk from the second portion of the subclavian artery, though occasionally they rise from the third portion, and occasionally by separate trunks. The superior intercostal curves back ward from the apex of the pleura until it reaches the head of the first rib, when it changes its direction, and supplies the first intercostal space after the manner of all intercostal arteries.

\section{Profundus Cervicis.}

The profundus cervicis passes backward between the transverse process of the seventh cervical vertebra and the first rih, or occasionally the transverse process of the first dorsal vertebra, and passes upward in the neck to inosculate with the deep division of the frinceps cervicis branch of the occipital artery, establishing a collateral circulation between the carotid and the subclavian arteries.

\section{The Axillary Artery.}

The Axillary is the second sub-division of the artery of the 
upper extremity. It begins by being a continuation of the subclavian where that artery ceases at the outer border of the first rib, passes downward and outward from the upper lateral aspect of the chest, corresponding to the first four ribs, and down the inner aspect of the arm to terminate at the lower border of the tendons of the latissimus dorsi and teres major muscles by becoming the brachial artery. The direction of the artery will of course vary with the position of the $\operatorname{limb}$; and its relations are studied with the limb in the anatomical position.

Relations. The artery is covered throughout by the pectoralis major, and through part of its extent by the pectoralis minor, and of course by the skin, superficial and deep fascias. Throughout its course its vein lies to its inner front aspect. The brachial plexus of nerves is a continuous relation, lying to its outer side in the beginning, on all three sides in the second portion of its course, while the terminal branches bear various relations to the third portion of the artery. The relationship to the pectoralis minor warrants the subdivision of the vessel into three portions, each of which should be studied in detail. The first portion extends from the beginning of the vessel to the upper border of the Pectoralis minor muscle; and is contained in the triangular space bounded above by the clavicle, below by the pectoralis minor, and externally by the humerus. The second portion commences at the upper border of the pectoralis minor, and terminates at its lower border by becoming the third portion. The third portion, commencing at the lower border of the pectoralis minor, terminates at the lower border of the tendons of the latissimus dorsi and teres major by becoming the brachial artery.

First Portion. The first portion is covered by the skin, superficial and deep fascia, and the pectoralis major muscle. It is crossed by the costo-coracoid membrane; and by the outer anterior thoracic nerves, and the acromio-thoracic and cephalic veins. Internally it has the second serration of the Serratus magnus, the first rib, and the first intercostal muscle. Behind, it lies upon the tendon of the subscapularis. Its vein is internal and in front; while the brachial plexus of nerves 
lies to its outer side and slightly above it. More superficially, in the interval between the pectoralis major and the deltoid, it is crossed by the cephalic rein. Behind it is the respiratory nerve of Bell.

Second Portion. The second portion is covered by the skin, superficial and deep fascias, pectoralis major and pectoralis minor. This is the portion crossing from the chest to the arm; and corresponds to the position of the third serration of the serratus magnus. Behind, it rests upon the subscapularis. The cords of the brachial plexus are here divided into three : internal, external, and posterior, which bear the corresponding relations to the second portion of the axillary, while the vein is still internal and anterior.

Thired Portion. The third portion is covered by the pectoralis major, and a strong fascia, which covers over the axillary space. Posteriorly it rests upon the tendons of the latissimus dorsi and teres major and subscapularis muscles, while external to it is the coraco-brachialis and the short head of the biceps. At the beginning of this portion the brachial plexus breaks up into its terminal branches; and all of these branches bear some relation to the vessel. On the front of its commencement the external and internal cords each give off a branch, which unite to form the median nerve. This nerve immediately drops to the outer side of the vessel; and maintains that relation to its termination. The outer cord also gives off the musculo-cutaneous, which is an external relation for a short distance, then, plunging into the fibres of the coraco-brachialis, ceases to be a relation of the axillary. The inner cord, after contributing one root $\mathrm{t} o$ the median, gives off the ulna and internal cutaneous nerves, both of which are fixed internal relations. To the inner front aspect is the vein. Behind, it rests upon the circumflex nerve and the subscapularartery for a short distance, while the last cord of the plexus, the musculo-spiral, is a continuous posterior relation.

Short Thoracic

\section{The Branches.}

Acromial Thoracic

Long Thoracic

Thoracica Alaria

Subscapular

Posterior Circumflex

Anterior Circumflex 
The point of origin of the branches of the axillary is very uncertain, and no perfectly accurate description applying to all cases can be written. Usually the short thoracic and the acromial thoracic arise from the first portion, the thoracica alaria and long thoracic from the second, and the subscapular and anterior and posterior circumflex from the third. Sometimes they will rise by common branches; and almost any arrangement may be anticipated in the origin of the branches of this vessel.

\section{Short. Thoracic.}

The short thoracic usually arises by a common trunk with the acromial thoracic. It passes inward along the upper border of the pectoralis minor, then between the two pectoral muscles to be distributed to the upper intercostal muscles and a part of the serratus magnus, anastomosing with the intercostal arteries. It frequently sends branches to the pectoral muscles, and occasionally to the mammary gland.

\section{Acromial Throacic.}

The acromial thoracic, usually arising by a common trunk with the short thoracic, arises just above the pectoralis minor, passes directly forward to pierce the costo-coracoid membrane, and divides into three branches, which from their distribution have been called the acromial, the pectoral, and the descending thoracic.

1st. The pectoral passes between the pectoralis major and minor, and is distributed, in the main, to the mammary gland, some branches going to the pectoral muscles.

2nd. The acromial, though the smallest branch, gives its name to the trunk, and passes outward across the coracoid process, sometimes piercing the deltoid muscle, where it anastomoses with the circumflex and suprascapular arteries, forming the plexus around the acromion process.

3d. The descending branch passes into the interval between the deltoid and pectoralis major muscles, and is distributed to the muscles in its course, anastomosing with the plexus formed by the preceding branch.

The thoracica alaria are not infrequently branches from the 
long thoracic. They som atimes arise separately from the trunk; and are distributed minly to the lymphatics, integument and fascia of the axilla.

The long thoracic arises either alone, or by a common trunk with the prezeding, pussing between the pect rral muscles and the serratus magnus to reach the lateral aspect of the chest. It gives branches to the pectorals and to the serratus magnus, anzstomoses with the intercostal arteries, and occasionally gives a large branch to the mammary gland.

The subscapular is usually the largest branch of the axillary artery, arising ganerally opposite the lower border of the subscapularis. It ruus downward and inward, following the edge of that musile, supplies it, the teres major, the latissimus dorsi, an l serratus mignus, and gives branches to structures in the axillary space. At the inferior angle of the scapular it anastomoses with the posterior sapular, while the branches which pass to the thoracic wall anastomose with the intercostal and long thoracic arteries. From an inch to an inch and one-half after its origin it gives off a large branch, called the dorsalis scupule, which passing through the triangular space batween the teres major below, the teres minor above, and long head of the triceps externally, curves around the axillary border of the scapula, and reaches the infraspinous fossa, where it is distributed to that fossa and to the infraspinatus muscle, anastom osing with the suprascapular and the posterior scapular arteries. It also supplies the teres minor, and som times gives a branch to the teres major muscle.

The anterior circumflex, a very small vessel, frequently arises by a common truak with the posterior, or it myy arise from tha subscapular. It passes baneath the coraco-brachialis and short head of the biceps muscle, passes over the bicipital groove; and gives off a branch to the shoulder joint, while the remainder of the artery winds around the neck of the humerus, and anastomoses with the posterior circumflex.

The prostcrior rircumllex, quite a large vessel, arises from the third portion of the axillary below the subscapularis muscle, passes through the quadrilateral space bounded externally by the shaft of the humerus, internally by the long head of the 
triceps, above by the teres minor, below by the teres major, winds around the back of the humerus, and passing beneath the deltoid, breaks up into a large number of branches, to be distributed to that muscle throughout its deep surface. In addition to this work, it gives off branches to the head of the humerus and the shoulder joint, and some axillary branches, while the continuation of the artery anastomoses with the anterior circumflex, completing the vascular zone around the upper extremity of the humerus.

Frequently this artery arises by a common trunk with the anterior circumflex, each artery then pursuing its usual course. The two anastomose with each other, with the suprascapular and the dorsalis scapulæ, thus establishing a complete arterial zone around the shoulder.

\section{Brachial Artery.}

The brachial is the third subdivision of the artery of the upper extremity. It begins by being a continuation of the axillary at the lower border of the tendons of the latissimus dorsi and teres major and passing downward and outward terminates half an inch, or a finger's breadth, below the middle of the front of the elbow-joint by dividing into the ulnar and radial arteries. It lies first on the inner side of the arm and then on the front, so that in order to check the circulation by pressure ; in the first part of its course the force must be directed outward and backward and in the second part directly backward.

Relations.--The muscular relations are as follows: To its outer side, at first, is the lower half of the coraco-brachialis and when this relation is lost by the insertion of the muscle, its place is taken by the biceps, whose belly, when the muscle is bulky, overlaps the artery. At the bend of the elbow it lies beneath the slip given off by the biceps. In the first part of its course it lies on the internal intermuscular septum which separates it from the inner head of the triceps, and in the lower part on the brachialis anticus.

Its relations to cords are as follows:

It is accompanied by satellite veins, one on either side, the one on the inner side being the larger. 
To its inner side are the basilic vein and the internal cutaneous nerve. Both vein and nerve are at first beneath the inresting fascia of the arm; but as the structures pass downward, they both become superficial and are, consequently, internal to and in front of the artery.

The median nerve lies first to the outer side of the artery, then crosses it, usually in front, about the middle of the arm and remains a continuous internal relation.

The ulna nerve is a postero internal relation of the brachial for the upper one-half of its course, when it leaves that artery to pass to the back of the elbow joint. For a few inches the inferior profunda artery, accompanying the ulna nerve, bears also a posterior relation to the brachial. The musculo-spiral nerve and the superior profunda artery are momentary posterior relations above.

\section{Brachial at the Elbow.}

These relations are of such importance that they must be given separately. It lies on the middle of the front of the joint with a satellite vein on either side. On its outer side is the tendon of the biceps muscle; on its inner side the median nerve. In front of it is the bicipital fascia and upon that fascia are found the terminal branches of the internal cutaneous nerve and the median basilic rein, which unites with the common ulnar to form the basilic. The artery here lies in a triangle hounded internally by the pronator adii teres, externally by the supinator longus and above by a line drawn from one condyle of the humerus to the other.

\section{Branches.}

Superior Profunda Inferior Profnnda Anastomotica Magnua.

The superior frofunda arises from the upper part of the brachial, and passes down ward and outward. After piercing the intermuscular septum, it winds around the humerus in the musculo-spiral groove, accompanying the nerve of that name; and on reaching the outer border of the lumerus, divides into two branches. One of these picrees the external inter-muscu- 
lar septum; and continues downward in the interval between the supinator longus and the brachialis anticus to anastomose with the recurrent branch from the radial, while the other branch continues its course behind the external inter-muscular septum to anastomose with the posterior interosseous recurrent and also with the anastomotica magna, the inferior profunda and the posterior ulna recurrent.

The inferior profunda arises a little above the middle of the vessel, just below the superior. It immediately seeks the ulna nerve; and with that nerve pierces the internal intermuscular septum to pass to the inner back part of the elbow joint, where it anastomoses with the anastomotica magna and the posterior ulna recurrent.

The anastomotica magna arises near the termination of the brachial, about two inches above the elbow joint. It runs directly inward to the inner aspect of the arm, anastomoses with the anterior ulna recurrent, pierces the inter-muscular septum, passes outward beneath the triceps, or sometimes through the fibres of that muscle, anastomoses with the inferior profunda and the posterior ulna recurrent on the inner aspect of the elbow joint, with the posterior branch of the superior profunda upon the outer aspect of the elbow joint, and with the posterior interosseous recurrent and with the anterior ulna recurrent. Thus the only branch forming the vascular circle around the elbow joint with which the anastomotica magna does not anastomose is the radial recurrent.

The anastomotica magna is thus the chief artery in completing the chain around the elbow. Passing inward it receives a communication from the anterior ulnar recurrent: then from the inferior profunda and posterior ulna recurrent; then, on the outer side of the arm, from the posterior branch of the superior profunda, whose anterior branch has already inosculated with the radial recurrent.

\section{The Arteries of the Forearm.}

The arteries of the forearm are the radial and ulnar and their branches. 


\section{Radial Artery on the Forearm.}

The radial artery, one of the two terminal branches of the brachial, begins where that artery divides about half an inch below the middle of the front of the elbow, and passes downward with such an inclination outward as to bring it to the outer side of the front of the wrist joint, where it lies on the front of the styloid process of the radius, terminating as the radial artery of the forearm by becoming the radial artery of the zurist.

Relations. To the outer side it has throughout its course the supinator longus, whose belly overlaps it. With this exception the artery is superficial. To its inner side is first the pronator radii teres, and, when that muscle is inserted, its place is taken by the flexor carpi radialis which remains to the inner side.

It is accompanied by satellite veins, one on either side, and in the middle third of its course has the radial nerve to its outer side. The nerve in the upper third is too far a way to be considered a relation, while in the lower third it passes beneath the tendon of the supinator longus and disappears on the back of the forearm.

Behind it lie the tendons of the biceps, the supinator brevis, the insertion of the pronator radii teres, a part of the origin of the flexor sublimis digitorum, a portion of the flexor longus pollicis, and the pronator quadratus. Below these is the front of the shaft of the radius.

\section{Ulnar Artery on the Forearm.}

The uluar artcry', the larger of the two terminal branches of the brachial, begins where that artery terminates about half an inch below the midale of the front of the el bow, by dividing into itself and the radial, and passes first down ward and in ward for the upper third of its course, to the inner side of the front of the forearm and then turns vertically down ward to reach the inner side of the front of the wrist, where it terminates as the ulnar artery of the forearm by becoming the ulnar artery $n f$ the hand. 
Relations. In the first part of its course, while it is passing downward and inward, it lies beneath four muscles, viz: pronator radii teres, flexor carpi radialis, palmaris longus and flexor sublimis digitorum-all the muscles of the superficial group except the flexor carpi ulnaris. When it emerges from beneath these muscles it descends with the tendon of the flexor carpi ulnaris to its inner and of the flexor sublimis digitorum to its outer side. In its upper portion it lies on the brachialis anticus and in its lower portion on the flexor profundus digitorum.

It is accompanied by satellite veins, one to either side, and for its lower two-thirds has to its inner side the ulnar nerve. In its upper part it is crossed by the median nerve which is also an external relation for about an inch.

\section{Ulnar Artery in the Hand.}

When the ulnar artery reaches the wrist it continues its course into the palm of the hand, lying close to and on the outer side of the pisiform bone and on the anterior annular ligament. After passing down a short distance into the palm, it turns outward and runs across the palm of the hand to its outer side, being covered only by the skin and fascia and lying on the flexor tendons and on the origin of the muscles of the little finger, and on the divisions of the median and ulna nerves. Its course across the palm is known as the superficial palmar arch (arcus sublimis) and though its direction is not transverse, it is more or less clearly defined by a transverse line across the palm drawn from the bottom of the interval between the thumb and index finger. The ulnar nerve continues its relation to the artery in the liand, lying to its inner side.

\section{Branches.}

Anterior Ulnar Recurrent

Posterior Tlnar Recurrent
Interosseous

Muscular

The anterior ulnar recurrent arises from the ulnar just below its origin and passes upward to the front of the inner side of the elbow, where it inosculates with the anastomotica magna. The posterior ulnar recurrent rises just below the preceding and passes upward and backward to the internal 
condyle. It passes to the interval between the olecranon and internal condyle and communicates with the anastomotica magna and the inferior profunda.

The interosseous is a large trunk which arises from the ulnar just below the elbow and quickly forks into an anterior and posterior interosseous.

1st. The anterior interosseous descends on the front of the interosseous membrane, between the flexor profundus digitorum and flexor longus pollicis muscles, to the pronator quadratus, passing beneath which it perforates the interosseous membrane and appears on the back of the forearm to inosculate with the posterior interosseous artery. It supplies the muscles in its route and gives off a companion branch to the median nerve. It communicates with the posterior carpal arch and the posterior interosseous.

2 ad. The posterior interosseous, as soon as it is produced, passes between the bones above the interosseous membrane, to reach the back of the forearm and descend on the back of the forearm, between the superficial and deep layer of muscles. to the wrist. Just as it reaches the back of the forearm it gives off a large branch, called the posterior interosseous recurrent, which ascends to the back of the elbow and communicates with the anastomotica magna.

The posterior interosseous artery is distributed to adjacent muscles.

The muscular branches of the ulnar artery are numerous twigs to muscles in its course.

\section{Branches at the Wrist.}

\section{Anterior Carpal}

Posterior Carpal

The antcrior carpal is a branch which passes outward on the front of the wrist to inosculate with the anterior carpal of the radial. From this arch branches pass to the carpal articulations.

The postcrior carpal passes backward and then runs outward on theback of the wrist to anastomose with the posterior carpal of the radial. From this arch are given off branches which supply the ulnar side of the back of the little finger and inter- 
osseous branches to the next two spaces. These branche receive the posterior and anterior perforating arteries.

These branches are very small and the anterior is frequently absent.

\section{Branches in the Hand.}

Communicating

Digital

The communicating branch dips between the abductor minimi digiti and flexor brevis minimi digiti to join the termination of the radial, completing the deef palmar arch.

The thumb and each of the fingers have two palmar arteries, one on either side, and two dorsal, similarly arranged, running from base to tip, called respectively palmar and dorsal collateral digital arteries. The digital arteries now under cousideration are four in number, from the superficial palmar arch. The first is a small branch which supplies the inner side of the little finger; the second supplies adjacent sides of the little and ring fingers, splitting at the cleft between the two; the third does the same for adjacent sides of the middle and ring fingers and the fourth for adjacent sides of the middle and index fingers.

These branches run along either side of the fingers until they reach the tip; where, after giving off small branches which run beneath the nail and communicate with the dorsal arteries they inosculate with one another, completing the loop between the palmar arch and the digital arteries.

\section{Radial Artery of Wrist and Hand.}

When the radial artery reaches the wrist it turns outward, backward and downward beneath the extensor tendons of the thumb, viz: the extensor ossis metacarpi pollicis, extensor primi and extensor secondi internodii pollicis, in the order named, and passing through what is known as the snuff box-the sunken interval between the extensor ossis metacarpi pollicis and extensor primi internodii pollicis on one side and the extensor secundi internodii pollicis on the other-reaches the back of the hand where its appearance is only momentary, for it immediately dips into the palm of the hand by passing between the two heads of the first dorsal interosseous muscle, 
and passing across the palm of the hand from the outer to the inner side, terminates by anastomosing with the communicating branch from the superficial palmar arch. The position of the radial in the palm is deep, lying on the bases of the metacarpal bones and beneath the flexor tendons which separate it from the superficial palmar arch. It is called the deep palmar arch and is situated about half an inch nearer the wrist than the superficial arch.

\section{Branches of the Forearm.}

Recurrent Radial

Muscular

The recurrent radial arises from the radial just below the elbow and passes upward and outward to the outer side of the front of tne joint, where it lies in the space between the supinator longus and the brachialis anticus. It here inosculates with the anterior branch of the superior profunda.

The muscular branches are numerous twigs to muscles in the course of the artery.

\section{Branches at the Wrist.}

Superficialis Volæ

Anterior Carpal

Posterior Carpal
Dorsalis Pollicis

Metacarpea

Dorsalis Indicis

\section{Princeps Pollicis}

The superficialis volce leaves the radial just as it turns outward and passes to the muscles of the thenar group, usually perforating the abductor pollicis. Sometimes it anastomoses with the termination of the ulna, forming the superficial arch.

The antcrior carpal branch passes inward on the front of the joint to form the anterior carpal arch by uniting with the anterior carpal of the ulnar artery.

The posterior carpal passes inward across the back of the wrist to inosculate with the posterior carpal of the ulnar and form the posterior carpal arch. From the posterior carpal arch there pass down on the dorsal interosseous muscle two branches called posterior interosscous besides another which runs along the dorsal aspect of the ulnar border of the hand to supply the ulnar side of the dorsal aspect of 
the little finger. The posterior interosseous pass downward to divide the one at the cleft between the little and ring fingers to form dorsal collateral digital branches for the adjacent sides of those fingers, while the other performs a similar duty for the adjacent sides of the ring and middle fingers.

Just as the radial is passing the root of the thumb it gives off two small branches which course along the sides of the dorsal aspect of the thumb. They are called dorsales pollicis.

The metacarpea is a branch which descends on the second dorsal interosseous muscle to the cleft between the middle and index fingers and there divides to form dorsal collateral digital branches for those fingers.

The dorsalis indicis is a small branch which passes forward to form the dorsal collateral digital branch for the outer side of the index fingers.

\section{Princeps Pollicis.}

The princeps pollicis arises just as the radial dips into the palm of the hand and passes forward and outward to divide and form collateral digital arteries for the palmar aspect of the thumb.

\section{Branches of the Deep Palmar Arch.}

Radialis Indicis

Anterior Interosseous

\section{Perforating}

1st. The radialis indicis, coming off close to the princeps pollicis, runs along the radial border of the index finger to ifs extremity and there anastomoses with the collateral digital artery of the superficial palmar arch. It also anastomoses with the princeps pollicis and gives a communicating branch to the superficial palmar arch thus completing the arch, though sometimes the superficial arch is completed by anastomosing with the superficialis volæ.

The anterior interosseons are three or four small branches which pass forward on the front of the interosseous muscles to unite with the digital branches of the superficial arch.

Besides these the deep arch gives off two sets of unimportant branches called perforating and recurrent. 
The perforating arteries are three in number, passing backward between the heads of the last three dorsal interosseus muscles they inosculate with the dorsal interosseous arteries.

\section{The Aorta.}

The Aortc commences at the base of the arterial ventricle, and terminates by dividing into the common iliac arteries on the front of the body of the fourth lumbar vertebra slightly to the left of the mid-line. It lies first in the thoracic, then in the abdominal cavity. Its direction is first upward, forward, and to the right; then backward, and to the left; and finally it turns downward until it reaches its point of bifurcation. It is divided for description into the Asccnding Aorta, the Transrerse Aorta, and the Descending Aorta, the latter being subdivided into the Thoracic and Abdominal portion of the descending aorta. The old sub-divisions of ascending, transverse, and descending portions of the arch have here been abandoned.

\section{The Ascending Aorta.}

The Ascending Aorta (formerly called the Ascending Portion of the Arch of the Aorta) begins at the base of the arterial ventricle, at a point behind the left edge of the sternum, opposite the third intercostal space, on a plane posterior to the pulmonary artery and anterior to the left auriculo-ventricular opening. It is about two inches in length, is directed upward, forward, and to the right, presenting a slight curve, whose convexity is to the right. Behind the upper border of the second costal cartilage of the right side it terminates by becoming the transverse aorta. Just above its origin it is somewhat triangular on cross-section when distended, and presents three bulging prominences, which correspond to the sinuses of Valsalva. It lies in the fibrous pericardium, about one-fourth of an inch behind the sternum; and is contained in the same fold of serous pericardium as the pulmonary artery.

Relations. On its anterior aspect, at its origin, lies the pulmonary artery; but this vessel quickly winds to the left of the aorta, owing partly to the inclination of the aorta to the

Des 1 nat -21 
right, and partly to the oblique course of the pulmonary artery. Abore the pulmonary, the aorta is separated from the sternum by the pericardium, some loose connective tissue, and the remains of the thymus gland, should any exist. At its origin it is overlapped in front by the right auricular appendix; behind it lies the right pulmonary artery, and other structures which enter into the formation of the root of the right lung, and the left auricle of the heart. To its right at its base is the right auricle; and above this the superior vena cava. To the left, as already seen, is the pulmonary artery.

\section{The Transverse Aorta.}

The Transicrse Aorta begins by being a continuation of the ascending aorta opposite the upper border of the second right costal cartilage; and with a gentle curve in its course, passes from right to left from before backward, and slightly from above downward, to terminate at the left side and lower border of the fourth dorsal vertebra, by becoming the descending aorta. The top of this arch is about an inch below the top of the sternum; and from it arise three large branches: the Arteria Imominata, the left Common Carotid and the Left Sub-clavian.

Relations. In front it is overlapped very slightly by the right pleura, and to a much greater extent by the left pleura and lung, though the two pluræ do not come in contact. It is crossed in the order from right to left by the left phrenic, the cardiac branches of the pneumogastric, and the left pneumogastric nerves. The left superior intercostal vein also crosses the front of the transverse aorta. Behind, it rests upon the trachea, behind that is the osophagus, and in the interval between the $t w o$ the thoracic duct and the left recurrent laryngeal nerve. Above, it is in relation with its three branches before mentioned, from right to left, and from before backward, the arteria innominata, left common carotid, and left subclavian; while above and towards its front aspect is found the great transverse vein of the neck, or the left innominate vein. Below it are the bifurcation of the pulmonary artery, the left bronchus, and the left recurrent laryngeal nerve, while it is 
connected by means of the ductus arteriosus with the pulmonaryartery. The portion formerly described as "the descending portion of the arch of the aorta" will be considered with the thoracic aorta.

\section{Branches of the Ascending Aorta.}

Coronary Arteries.

The right and left coronary branches arise from the ascending aorta. These branches arise from the corresponding sinuses of Valsalva, the right arising from the right anterior sinus, and the left from the left anterior sinus. They descend into the anterior and posterior inter-ventricular furrows, and meet at the apex of the heart. Here they inosculate with one another, while each sends off a branch to supply the auriculoventricular grooves, anastomosing with one another, and forming two vascular circles at right angles with one another, which completely surround the heart.

\section{Branches of the Transverse Aorta.}

Arteria Innominata

Left Common Carotid Left Subclavian

The branches which spring from the transverse aorta are three in number, and bear a definite relation to one another; that is, the first, the Arteria Innominata, arises near the commencement of the transverse aorta; the left Common Carotid at the middle of the transverse aorta; and the left Subclavian near the termination of the transverse aorta.

\section{Arteria Innominata.}

The arteria imominata is the first and doubly the largest branch of the aorta. It arises from the beginning of the transverse aorta, passes upward and to the right for a little less than two inches to terminate behind the upper border of the right sterno-clavicular articulation by dividing into the Right Common Carotid and Right Subclavian arteries. In other words, in it are blended the right common carotid and right subclavian vessels.

Relations. In front is the sternum, from which it is separated by some loose connective tissue, by the prolongation of the 
fibres of origin of the sterno-hyoid and sterno-thyroid muscles, and by the remains of the thymus gland, should there be any. Across the front of it, just at its origin, passes the left innominate vein, or great transverse vein of the neck, and the right inferior thyroid vein. Behind, at its commencement, it rests upon the trachea; but the obliquity of the artery soon carries it to the right of the trachea, which then becomes a left or internal relation to the arteria innominata. On the right side is the right innominate vein, the right pleura, and the right pneumogastric nerve, which is a rather distant relation. On the left at its commencement is the left common carotid artery, which lies to the left and on a plane slightly posterior, separated from the arteria innominata by a triangular interval in which the trachea may be seen. Higher up is the trachea. It usually produces no branches except its terminal branches. The remains of the thymus, and the left inferior thyroid rein are also relations on its left.

\section{Left Common Carotid.}

The left common carotid, the second branch arising from the transverse aorta, occupies a position midway between the innominate and left subclavian arteries.

Relations. Its relations in the thorax are somewhat similar to those of the arteria innominata, but not identical with them. After passing above the left sterno-clavicular articulation, its relations become identical with those of the right common carotid, and a description of one answers for the other. It springs from the highest portion of the transverse aorta, lying with the innominate to its right and on a plane slightly anterior, the left subclavian to its left and on a plane slightly posterior. It is separated from the sternum by the origin of the sterno-thyroid and sterno-hyoid muscles of the left side, some loose connective tissue, and the remains of the thymus gland; and is crossed in front by the great transverse vein, though at a higher level than that at which the same vein crosses the innominate artery. It is overlapped in front by the left lung and pleura. Behind it, at its origin, lies the trachea, which is behind and slightly to its inner side. Crossing obliquely behind 
is the left recurrent laryngeal nerve while behind and to its outer side is seen the thoracic duct. As it ascends, the trachea becomes an internal relation, and the œesophagus a postero-internal relation, the recurrent laryngeal nerve lying in the groove between these two structures. To its inner side at its commencement is the innominate artery; and above this, and internal and posterior to it, the trachea. To its inner side is also the inferior thyroid vein. To its outer side is the left subclavian, which is external and slightly posterior; while in the interval between the subclavian and the carotid is seen the pneumogastric nerve, an antero-external relation of the carotid.

\section{The Left Subclavian.}

The left subclaitinarises from the termination of the transverse aorta. It passes upward and to the left; and its first or thoracic portion terminates at the inner edge of the scalenus anticus muscle by becoming the second portion of the artery. From this point to its termination, the left subclavian is identical with the right.

Relations. The left subclarian is almost enveloped by the left pleura, which covers it in front, to the outer side, and behind. It has the left common carotid lying internal to it and slightly in front of it, while more superficially are the origins of the sterno-hyoid and sterno-thyroid muscles, and the remains of the thymus gland, should there be any. Near its origin, but higher than at the point of crossing of the left common carotid, is found the great transverse rein of the neck, while near the termination of the first portion the internal jugular and the subclavian veins of the left side unite to form the innominate on the front of the subclavian artery. The left vertebral vein is also an anterior relation. The phrenic nerve crosses it near the scalenus anticus muscle, and then passes downward, parallel to the pneumogastric, but external to, and in front of, that nerve. The pneumogastric itself lies in the interval between the subclavian and the left common carotid on a plain anterior to both, but internal to the subclavian, while external to the carotid. Below it, as well as to its outer side, are the pleura and the left lung. The trachea, 
œsophagus, and recurrentlaryngeal nerve might be considered distant internal relations. The relation of the thoracic duct should be carefully noted. It ascends nearly opposite the interval between the left common carotid and the subclavian, passes above the point at which the subclavian arches outward toward the scalenus anticus muscle, then turns to the front of the arterv to empty into the junction of the internal jugular and subclavian veins, so that its relationship to the artery is a three-fold one: being first behind it, then above it, then in front of it, at its termination.

\section{The Descending Aorta.}

The descending aorta commences by being a continuation of the transverse aorta at the left side and lower border of the body of the fourth dorsal vertebra, and terminates opposite the body of the fourth lumbar vertebra by dividing intn the two common iliacs. It consists of the Thoracic and Abdominal Portions.

\section{The Thoracic Aorta.}

The thoracic portion of the descending aorta, commencing at the fourth dorsal vertebra, terminates opposite the body of the twelfth dorsal, slightly to the left of the mid-line, by becoming the abdominal aorta. The course of the thoracic aorta is downward, with a slight inclination to the right. so that from lying first upon the lateral aspect of the body of the fifth dorsal, it changes its relative position to the bodies of the vertebræ to such a degree that at its point of termination it is on the front of the bodies, and but slightly to the left of the mid-line. It presents a curve in its course slightly concave in front to correspond with the dorsal curvature of the spinal column upon which it rests.

Relations. This vessel is covered throughout its course by the pleura; and lies with other important structures in the posterior mediastinum. In front, opposite the body of the fifth dorsal vertebra, is the root of the left lung, consisting mainly of the pulmonary artery, the pulmonary veins, the left bronchus, 1 ymphatics, nerves, and small blood vessels. Below this it lies behind the pericardium; and is completely covered over 
by the left pleura and lung. It bears an important relation to the œophagus, thoracic duct, and pneumogastric nerves. The two pneumogastric nerves course downward, one upon the frontand the other upon the back of the osophagus, and hence hear the same relation to the aorta that this structure does. Owing to the changes in the direction of the oesophagus as well as to the obliquity of the aorta, the nesophagus above lies to the right of the descending aorta. About the middle of the course of the artery it is directly in front of it, while opposite the tenth dorsal vertebra, where the osophagus terminates, it is stili further in front and to the left of the aorta. The thoracic duct is continuously behind and slightly to the right of the aorta, lying usually in the interval between the aorta and the great azygos vein. To the right of the aorta is the great azygos vein, opening into which opposite the sixth dorsal vertebra is the left azygos, which crosses behind the aorta. The extent to which the aorta is covered by the pleura decreases as it descends. In the upper portion of its course, all of the vessel except a small part of its right side, which rests upon the bodies of the vertebra, is enveloped by the pleura, while near its termination the pleura covers only its outer aspect.

\section{Branches.}

Bronchial

Mediastinal

\section{Intercostal}

Pericardiac

\section{Esophageal}

The branches of the thracic anrtu are small, although there are a large number of them.

The bronchial arleries, usually three to the left and one to the right lung, course along the bronchi to the lungs, and ramify with the bronchial tubes. These vessels, it should be understood, have no connection with the pulmonary artery; but simply furnish a nutrient supply for the lung tissue itself. Their method of distribution belongrs to the domain of histrology.

'The a'sophaseal are a number of small twigs, four or five, which are successively furnished to the asophagus as that 
tube comes into relationship with the thoracic aorta, and which communicate with one another and with other œsophageal arteries.

The mediastinal and pericardiac are small twigs furnished to the posterior mediastinum and to the pericardium respectively.

The intercostal, the largest and most numerous of the branches, are ten pairs, which rise from both sides of the thoracic aorta, and run out into the intercostal spaces, the upper space being supplied by the superior intercostal of the subclavian, while the space below the last rib, or sub-costal space, is supplied by one of the terminal branches of the internal mammary. The right intercostal arteries are, of course, longer than the left from having to cross the vertebral column. Each intercostal artery passes outward in its corresponding space to run along the lower border of the rib above in the groove which is formed for lodging the artery and the nerve; and after going some distance, gives off a branch, which descends to course aloug the upper border of the rib below. The arteries of the right side pass behind the vena azygos major and thoracic duct. Further out they pass behind the sympathetic system; and lie first upon the external intercostal muscle, and then, at the angle of the rib, between the two intercostal muscles. They continue their course to finally inosculate with the intercostal branches from the internal mammary for the upper spaces, and those from the musculo-phrenic for the lower spaces. Before terminating each intercostal gives off a dorsal branch, whose distribution corresponds to the dorsal nerves; that is, it divides into a muscular and a spinal branch, the spinal to enter the spinal canal, and the muscular to be distributed to the deep muscles of the back.

\section{The Abdominal Aorta.}

The abdominal aorta commences on the body of the twelfth dorsal vertebra, slightly to the left of the mid-line, being a continuation of the thoracic aorta. It passes behind the diaphragm, through the aortic opening, and curves backward and and to the left, the convexity being to the left. About the middle of its course it changes its direction and curves slightly 
to the right, so that it finally terminates on the body of the fourth lumbar rertebra, usually to the left of the mid-line, by dividing into the two common, or primitive, iliac arteries. This position is indicated by a line drawn from the highest point of the crest of the ilium of one side to the same point of the opposite, or by a point selected half-an-inch below and to the left of the umbilicus. In addition to the curvature to the left, it also is slightly convex forward, conforming to the lumbar portion of the spine. Owing to the large size and great number of the branches given off, it is greatly reduced in size at its termination.

Relations. Posteriorly this artery rests upon the front of the bodies of the lumbar vertebra; but at several points it is separated from these vertebra by intervening structures. Opposite the second lumbar vertebra is the commencement of the thoracic duct, the receftaculum chyli; and throughout its course are found, passing belind it, the left lumbar veins, opposite the corresponding vertebra, as they make for their points of entrance into the inferior vena cava. Above, to the left, are the left crus of the diaphragm and a portion of the sympathetic system, the left semilunar ganglion; to the right, the right crus of the diaphragm and a portion of the sympathetic, the right semilunar ganglion, the right vena azygos, and thoracic duct, while throughout the course of the vessel the inferior vena cava lies upon its right. In front is the stonach. Opposite the apper border of the stomacl one of the largest branches of the abdominal anrta arises, the coliac axis; and this is surrounded by crreds of the sympathetic system, forming the solar plexus of nerves with its two semilunar ganglia. These ganglia are found lying to the front and lateral aspects of the commencerent of the abdominal anrta. Below this comes the head of the pancreas, and inmediately below this the left renal vein, the transverse portion of the duodenum, and the mesentery. $\mathrm{B}$ low this the aorta is covered at the front and at the sides by the peritoneum and the aortic plexus of sympathetic nerves. Aather of its branches, the superior mesenteric, lies for a short distance in front of it; but curres to the left to becone a distant relation of the artery. Convolutions of the small in- 
testines cross over the front of the vessel, and are separated from it by the peritoneum, which covers it throughout, the aorta lying behind the peritoneum.

\section{Branches.}

Parietal $\left\{\begin{array}{l}\text { Phrenic } \\ \text { Sacra Media } \\ \text { Lumbar }\end{array} \quad\right.$ Visceral $\left\{\begin{array}{l}\text { Coeliac Axis } \\ \text { Superior Mesenteric } \\ \text { Inferior Mesenteric }\end{array}\right.$

$$
\text { Genito-Urinary }\left\{\begin{array}{l}
\text { Spermatic } \\
\text { Renal } \\
\text { Suprarenal. }
\end{array}\right.
$$

The branches of the abdominal corta, nine in number, are arranged in three sets: those distributed to the abdominal wall, the Parital Branches; those to the Abdominal Viscera, the organs of digestion, and those accessory thereto; and those distributed to the genito-urinary organs.

\section{The Parietal Branches.}

\section{Phrenic}

Sacra Media

Lumbar

The phrenic are a pair of small arteries, arising from the aorta soon after it enters the abdomen. Their manner of origin is variable. They may arise by a common trunk; they may arise separately from the aorta; or, in some cases, one will arise from the aorta and the other from one of the adjacent arteries, most frequently the renal. Each artery runs backward and outward on the under surface of the diaphragm Below the oesophageal opening they divide into two branches, one of which encircles this opening anastomosing with its fellow of the opposite side, and furnishing the arterial supply for a large part of the central tendon of the diaphragm. The other and larger branch passes outward on the concare under surface of the muscle, supplies the muscular fibre, ard inosculates with the musculo-phrenic branch of the internal mammary, and with the other diaphragmatic arteries.

The lumbar arteries are four or five pairs of small branches. which spring successively from the back of the aorta, and run outward to ramify in the broad muscles of the abdomen. These vessels pass beneath the musculo-tendinous arches 
formed by the tendinous fibres of the psoas magnus and the concave lateral aspect of the bodies of the lumbar vertebra.

They resemble in direction and in distribution the intercostal arteries. Passing in this way beneath the psoas magnus, these vessels divide into an anterior branch, which corresponds to the intercostal artery, and a posterior branch, which corresponds to the dorsal division of the intercostal. These posterior divisions supply the small arteries of the bodies of the vertebræ; and, continuing their course, give off the spinal branches to the contents of the spinal canal, while the remaining portion is distributed to the muscles of the back. The larger anterior division passes forward in the broad muscles of the abdomen, inosculates with other vessels which supply those muscles, mainly, for the upper branches, with the terminal branches of the internal mammary, and for the lower lumbars with the branches which spring from the deep epigastric and ilio lumbar.

The sacra media is a somewhat slender branch, which arises just at the bifurcation of the aorta, though occasionally from one of its forks. It passes down upon the front of the sacrum, gives off branches which anastomose with the lateral sacral arteries, and continues its course to the tip of the coccyx, terminating in the vascular gland found in that situation, which is known as Luschlea's, or the Coccygeal, Gland.

\section{The Visceral Branches.}

Creliac Axis Superior Mesenteric Inferior Mesenteric

The Visceral branches spring from the abdominal aorta by three trunks, the Creliac Axis, and the Superior and Inferior Mesenteric.

The caliac axis is the first and largest of these trunks. It is about half an inch in length. It arises from the front of the abdominal aorta, near its commencentent, and passes directly forward to break up into three terminal branches, the Gastric, the Splenic, and the Hepatic.

Relalions. The artery arises just opposite the head of the pancreas, upon which it rests. In front of it is the lesser omentum; and to the right is the lobus Spigelii, while the cords 
of the solar plexus surround the artery in every aspect, and a semilunar ganglion lies upon either side. Its position corresponds to the level of the upper border of the stomach, while immediately below it and almost in contact with it is the origin of the next branch, the superior mesenteric. On the left side it is relation with the cardiac end of the stomach.

\section{Branches.}

Gastric Splenic Hepatic

The gastric is the smallest branch arising from the coliac axis. It passes upward and to the left until it reaches the cardiac end of the stomach, where it turus to the right, runs along the lesser curvature between the two layers of the lesser omentum nearly to the pylorus, where it inosculates with the pyloric branch of the hepatic artery. Just before making its turn to the right it gives off branches to the large end of the stomach; and in coursing along the upper border, it gives branches which pass downward to both the anterior and posterior faces of that organ.

\section{The Splenic Artery.}

The splenic artery is the largest of the branches arising from the coliac axis. It is very tortuous, runs directly from right to left along the upper border of the pancreas to reach the hilum of the spleen, where it splits into numerous branches to be distributed to that organ. As it courses along the pancreas, it lies behind the stomach and just above the splenic vein, which lies on the posterior surface of the pancreas in a groove a little distance below the artery.

Before reaching the spleen, the artery gives off a number (five or six) of large branches, which pass to the splenic end of the stomach, and, under the nime of the vasa brevia, in osculate with the branches of the gastric artery, and supply the cardiac or splenic end of the stomach.

As it passes along the upper border of the pancreas, numerous small pancreatic branches are given off, which descend into that gland. One of them, of larger size, is frequently individualized as the pancreatica magna. 
One of its terminal branches, the gastro-epiploica sinistra, leaves the artery near the spleen, runs to the right along the greater curvature of the stomach between the layers of the great omentum, and about the middle of the greater curvature of the stomach inosculates with the gastro-cpiploica dextra, an indirect branch of the hepatic artery. From this artery, the gastro-epiploica sinistra, branches ascend to both faces of the stomach, and descend to supply the great omentum.

\section{The Hepatic Artery.}

The hepatic is intermediate in size between the gastric and the splenic. Its usual course is to pass to the right and slightly upward, then make an abrupt bend and pass nearly vertically upward to the under surface of the liver, where it divides into two branches, one to each lobe of the liver, hence known as the right and left hepatic arterics. This artery is accompanied by a duct and a vein. The three structures are in close contact, the duct lying furthest to the right and the artery to the left, while opposite the interval between them, and on a plane posterior to both, is found the portal vein, this vein being formed by the junction of the superior mesenteric with the splenic. The usual manner of expressing the description is: duct to the right, artery to the left, vein between and behind the two. The terminal branches of the artery ramify in the liver, and furnish the arterial blood for nourishing that organ.

Besides these branches it produces the pyloric, which passes to the left along the lesser curvature of the stomach until it meets the gastric artery, inosculates with that vessel, and like it is distributed to both faces of the stomach.

The next branch, generally called the gastro-duodinalis, and described as divided into the pancreatico-duodenalis superior and the gastro-epiploicadextra, is preferably described as the gastro-epiploica dextra. This artery, arising from the hepalic, winds around the greater curvature of the stomach, giving off as a way-side branch, the pancreatico-duodenalis superior. This small branch descends to the interval between the duodenum and the head of the pancreas, and is distributed to both of these structures and anastomoses with the pancre- 
atico-duodenalis inferior, a branch of the superior mesenteric; while the parent trunk continues between the folds of the great omentum until it inosculates with the gastro-epiploica sinistra, forming the loop from which ascending branches pass to both faces of the stomach and descending branches pass to supply the great omentum.

The right branch of the hepatic artery likewise gives off a branch, called the cystic, which passes to the gall bladder and divides into two branches, one running between the gall bladder and the under surface of the liver, is distributed to the gall bladder and duct, the other ramifies on the free surface of the gall bladder. This branch is sometimes described as a third terminal branch of the hepatic artery.

It will be seen therefore that the branches of the cœliac axis freely anastomose with one another, the splenic anastomosing with the gastric at the left end of the stomach, and with the hepatic, through the gastro-epiploica dextra, at the middle of the greater curvature. The hepatic anastomoses by its pyloric branch with the gastric on the upper curvature, and with the splenic on the lower curvature.

\section{Superior Mesenteric.}

The superior mesenteric is a large vessel, which arises from the front of the aorta just below the coliac axis and behind the head of the pancreas. Between it and the cœliac axis are some branches of the solar plexus of nerves. It descends between the lower border of the pancreas and the transverse duodenum to reach the right iliac fossa, presenting in its course a curve, whose convexity is to the left, lying between the layers of the mesentery.

Relations. At its origin this vessel lies behind the head of the pancreas. It then descends for about an inch and a half, the width of the pancreas, covered by that structure. It appears in the interval between the lower border of the pancreas and the transverse duodenum; then crosses in front of the transverse duodenum; and from that point onward continues in the fold of the mesentery, moving with the mesentery in the abdominal cavity. It is accompanied throughout by the superior mesenteric vein. 


\section{Branches.}

Tasa Intestini Tenuis

Ileo-Colic

Colica Dextra

Colica Media

Soon after its origin the superior mesenteric artery gives off a small branch, called the pancreatico-duodenalis inferior, which passing upward between the pancreas and the duodenum, anastomoses with the pancreatico-duodenalis superior, and aids it in supplying the descending duodenum and the head of the pancreas. The remaining branches are divided in to two sets, those which spring from the concave and those which spring from the convex aspect of the vessel. The convexity of this ressel looks downward, to the left, and slightly backward; the concarity looks upward, to the right, and slightly forward.

\section{Vasa Intestini Tenuis.}

Frnm the convex face arise a number of branches, varying from ten to eighteen in number, the i'asa intestini temuis. These branches inosculate so freely with one another that the circulation in the intestinal wall is still abundantly supplied though a large number of the branches may be ligated. They decrease in size from above downward; and their distribution is not quite regular, though the plan of distribution is always the same. Thus each branch in passing outward divides into an ascending and a descending branch. These branches inosculate with the corresponding branches above and below so as to form a series of arches. From these arches are given off a second set of branches, which likewise divide into two, and inosculate with those above and below, forming a second series of arches. This second series, in like manner, forms a third; and in some cases as many as five or six series of arches may be discovered. As a rule, the number of arches does not exceed three; and in some few cases only two may be discovered. No matter what the number of arches, from the terminal arch there proceeds a set of straight vessels, the a'asa recta, which pass outward on both faces of the small intestine, completely encircling the grut and inosculate with each other, so that both in its circumference and 
in its vertical measurement the anastomoses between the vertical arches of the small intestine are free and uninterrupted.

From the concave side of the vessel there are three branches. In the order in which they arise from below upward they are named the Ileo-colic, the Colica Dextra, and the Colica Media. It is sometimes seen that there is no distinct ileo-colic artery; but that its place is taken by the termination of the superior mesenteric itself. As a general rule, however, the superior mesenteric terminates by becoming the last of the vasa intestini tenuis; and the ileo-colic is given off from the concave aspect of the vessel.

\section{Ileo-Colic.}

The ileo-colic, the first of the three branches to arise from the concave aspect, is distributed somewhat after the manner of the vasa intestini tenuis; but with larger arches, or rather with loops instead of arches. Passing down ward and outward toward the right iliac fossa, it divides into an ascending and a descending branch. The descending branch passes back to inosculate with the vasa intestini tenuis or with the termination of the superior mesenteric. The ascending branch passes upward to reach the descending branch of the colica dextra, thus forming two large loops, from which the straight branches are given off to supply the termination of the small intestine and the beginning of the large intestine.

\section{Colica Dextra.}

The colica dextra, in like manner, passing straight outward, divides into an ascending and a descending branch, the descending to anastomose with the ascending branch of the ileocolic, and the ascending with the descending branch of the colica media.

\section{Colica Media.}

The colica media, the highest of the three branches, passes downward and to the right, with the intestine in the anatomical position, and divides into an ascending and a descending, or, more accurately, right and left branches, the descending, or right, branch to anastomose with the ascending branch of 
colica dextra; the ascending, or left, branch to inosculate with the colica sinistra, which is a branch of the inferior mesenteric.

From the loops formed by these three ressels are supplied the termination of the small intestine, the cæcum, or begining of the large intestine, the ascending colon, and the transverse colon to its middle, where the colica sinistra takes up the work.

\section{Inferior Mesenteric.}

The inferior mesenteric is much smaller than the superior. It arises from the front of the abdominal aorta near its termination, and descends obliquely to the left iliac fossa, where it finally terminates by passing over the brim of the pelvis under the name of the superior hemorrhoidal artery, and becoming a relation of the iliac vessels.

\section{Branches.}

Colica Sinistra

Sigmoid

Superior Hemorrhoidal

\section{Colica Sinistra.}

The first and largest branch, the colica sinistra, passes downward and to the left; and divides into an ascending and a descending branch. The ascending branch inosculates with the left branch of the colica media, and the descending with one of the sigmoid branches of the inferior mesenteric. From these two loops are supplied the left end of the transverse colon and the descending colon.

\section{Sigmoid Branches.}

The sigmoid are several branches, varying in number and in size, sometimes there being but a single branch, which passes outward to the sigmoid flexure of the colon, and divides as the colica sinistra does, the ascending branch inosculating with the colica sinistra, while the descending branch, or last branch where there are several, inosculates with the superior hemorrhoidal or termination of the superior mesenteric itself. These branches supply the sigmoid flexure of the colon. 


\section{Superior Hemorrhoidal.}

The superior hemorrhoidaldescends along the posterior aspect of the rectum, lying between the $t$ wo layers of peritoneum which hold the rectum in position. It reaches the middle of the rectum, where it anastomoses with the other hemorrhoidal arteries; but before reaching this point it has received the anastomosis with the sigmoid branches of the inferior mesenteric.

\section{Summary.}

From the foregoing description it will be seen that the stomach is supplied with blood as follows: the lesser curvature by the loop formed between the gastric artery and the pyloric branch of the hepatic; the greater curvature by the right gastro-eploica from the hepatic and the left gastro-eploica from the splenic; the splenic end of the stomach by the vasa brevia from the splenic artery and by branches from the gastric. All of these branches communicate with one another so as to render the stomach a net-work of arterial branches.

The doudenum is chiefly supplied by the pancreatico-duodenalis superior of the hepatic, which vessel is aided in its work by the pancreatico-duodenalis inferior from the superior mesenteric, establishing a communication bet ween the branches of the coliac axis and the superior mesenteric artery.

The small intestine from the duodenum to near the large intestine is supplied by the vasa intestini tenuis of the superior mesenteric. The termination of the small, and the beginning of the large, intestine are supplied by the ileo-colic branch of the superior mesenteric, anastomosing with the termination of the superior mesenteric itself.

The ascending colon is supplied by the loop between the colica dextra and the ileo-colic below, and the colica media above.

The right half of the transverse colon is supplied by the loop between the colica media and the colica dextra, the left half by the loop between the colica media and the colica sinistra, thus establishing a communication between the superior and inferior mesenteric arteries. 
The sigmoid flexure is supplied by the sigmoid branches, after anastomosing with the colica sinistra, and by the superior hemorrhoidal branch of the inferior mesenteric, after anastomosing with the signoid branches.

Thus, beginning with the first visceral branch of the abdominal aorta, we find that the three branches which supply all of the hollow viscera and many of the solid viscera of the abdomen not only communicate with the branches given off from each other, but communication is established between the three by the inosculation between the coliac axis and the superior mesenteric and between the superior mesenteric and the inferior mesenteric.

\section{Genito-Urinary Branches.}

Spernatic (in the male). Renal
Ovarian (in the female). Suprarenal.

\section{Spermatic.}

The Spermatic are a pair of small branches, which arise from the front of the aorta, and descend behind the peritoneum to the brin of the pelvis, one on either side. The left spermatic arises at a lower level, and is consequently a little shorter than the right. Each artery passes across the external iliac close to its termination, enters the deep abdominal ring, and traversing the inguinal canal, leaves the abdomen at the superficial ring to accompany the other elements of the cord to the testicles, where it is distributed. In the female this artery is known as the ovarian.

\section{Ovarian Arteries.}

The ovarian artery arises like the spermatic, and its relations are the same in the upper part of its course; but on reaching the brim of the pelvis, it crosses the common iliac artery, passes into the folds of the broad ligament; then reversing its course, runs outward to be distributed to the ovary, a branch having been griven to the uterus, to the lamina of the broad liganent, and to the Fallopian tube. Other branches continue along the round ligament, through the ingruinal canal, to the integument of the labia and groin. 


\section{Suprarenal.}

The suprarenal are a pair of small arteries, which arise from the aorta above the origin of the renal, and pass outward on either side to be distributed to the suprarenal capsule. These arteries frequently arise from the renal artery itself ; and generally there are two or three on either side of the body.

\section{Renal.}

The renal arteries are a pair of large vessels, which spring at right angles from the abdominal aorta, rising just below the superior mesenteric. They pass transversely outward, with the vein lying in front of them and the ureter behind them. On the rignt side the renal artery passes behind the vena cava. Just as they reach the hilum of the kidney they break up into, usually, three branches, one of which frequently goes in front of the renal vein. They enter the hilum of the kidney to be distributed to that organ. The left renal is on a somewhat higher plane than the right, owing to the relative position of the kidneys.

\section{The Common, or Primitive, Iliac Arteries.}

The common, or primitive, iliac artery of each side begins by being one of the terminal branches of the abdominal aorta, where that vessel terminates a little to the left of the body of the fourth lumbar vertebra, the exact point of division, however, varying slightly, sometimes as much as an inch. From this origin the artery passes downward and outward to the brim of the pelvis to terminate on the side of the disk between the fifth lumbar vertebra and the sacrum by dividing into the external and internal iliac arteries. The average length of each artery is about two inches; but the right is slightly longer than the left, owing to the division of the aorta to the left of the midline of the body.

Their relations are not identical.

\section{Right Common Iliac.}

Relations. The right common iliac, like the left, lies behind the peritoneum. It is usually crossed in front near its termi- 
nation by the right ureter, though this sometimes extends to the external iliac. Convolutions of the small intestine and sympathetic nerves are also found in front of it. In the female it is crossed by the ovarian artery in front. To its outer side is the psoas magnus muscle. Behind it is the left common iliac vein, which joins the right common iliac to the outer side of the beginning of the artery to form the inferior vena cava. Hence its relations to veins are quite complex, having at its commencement the junction of the two veins to its outer side and on a plane posterior to it, then its own vein external and behind, and then the left common iliac vein directly behind it. It lies on the last lumbar vertebra.

\section{Left Common Iliac.}

Relations. The Left common iliac is crossed in front by the sigmoid flexure of the colon, by the superior hemorrhoidal artery, and, near its termination, usually, by the left ureter. External to it is the psoas magnus muscle. Throughout its course its vein is behind and internal to it. It lies on the last lumbar vertebra.

The common iliac arteries give off no branches.

\section{External Iliac.}

The cxternal iliac, one of the two terminal branches of the common iliac, commences where that artery bifurcates on the disk between the fifth lumbar vertebra and the sacrum into itself and the internal iliac. It continues the course of the parent artery down ward and outward; and enters the thigh, to become the femoral artery, by passing beneath Poupart's ligament at a point midway between the anterior superior spinous process and the symphysis. Its course aud that of the common iliac may be indicated by drawing a line from a point just to the left of the umbilicus, the point of bifurcation of the abdominal aorta, to a point midway between the anterior superior spinous process of the ilium and the symphysis pubis.

Relations. To the outer side of the artery is the psoas magnus muscle, separated from it by the iliac fascia, which throws a covering over the artery and vein. In front is the peritoneum, 
the sub-peritoneal fascia, and convolutions of the small intestine. The vermiform appendix usually lies on the front of the artery, separated from it by the peritoneum. Near its termination the artery is crossed in front by the genital branch of the genitocrural nerve, and at the middle by the spermatic artery. In the female of course there is no spermatic artery to cross. Sometimes it iscrossed at its beginning by the ureter, though that structure usually crosses the termination of the common iliac. On the left the vein is throughout an intermal relation to its artery, and on a plane posterior to it until it reaches Poupart's ligament; on the right the vein is at first directly behind, where it unites with the internal iliac vein. Then it becomes internal, and at Poupart's ligament becomes fully abreast of the artery, so that on both sides at Poupart's ligament the vein is internal and abreast of the artery, while on the left side it remains internal and on a plane posterior throughout the remainder of its course; but on the right, when traced from below, it is first abreast, then internal and on a plane posterior, and then directly behind.

\section{Branches.}

Deep Epigastric

Deep Circumflex Iliac

The deep epigastric artery arises from the inner side of the external iliac near Poupart's ligament. It first runs upward and inward on the posterior face of the anterior abdominal wall beneath the peritoneum and the transversalis fascia, which it raises into a ridge. It then enters the sheath of the rectus muscle; passes upward, first between the sheath and the muscle, and then punctures the fibres of the muscle itself, to terminate by anastomosing with the epigastric branch of the internal mammary, thus establishing a communication between the arteries of the upper and the arteries of the lower extremity. Soon after its origin it gives off a communicating branch which descends to the obturator artery; and, in many instances, takes the place of the obturator artery itself. The relations to the abdominal rings will be explained in connection with the obturator artery. In addition to the communicating branch to the obturator, it gives off a 
branch which accompanies the cremaster muscle, muscular branches to the rectus muscle itself, a pubic branch which passes to the back of the pubes to anastomose with its fellow of the opposite side, and cutaneous branches, which pierce the rectus to supply the skin covering that muscle.

The decp circumflex iliac, the second branch of the external iliac, arises from the outer side of the external iliac just above Poupart's ligament, and a little higher up than the epigastric. It lies beneath the peritoneum; passes upward aud outward parallel with and above Poupart's ligament until it reaches the crest of the ilium, where it usually divides into two branches, one of which passes upward and anastomoses with the ilio-lumbar, gluteal and intercostal arteries, the other continues along the crest of the ilium to anastomose with the lowest lumbar artery. In its course it gives off mucular-branches, which aid in supplying the broad muscles of the abdomen, and cutaneous branches, which pierce to the skin and anastomose with other cutaneons branches.

\section{The Femoral Artery.}

The femoral artery is the continuation of the external iliac, commencing where that artery terminates, beneath Poupart's ligament, at a point midway between the anterior superior spinous process of the ilium and the symphysis pubis, where it can be felt pulsating, it descends the front of the thigh to its lower third, where it pierces the insertion of the adductor magnus muscle to reach the back of the thigh and becone the popliteal. As long as it lies on the front of the thigh, that is, for the upper two-thirds of its course, it is known as the femoral artery; as soon as it reaches the lower third and appears on the back of the thigh it is called popliteal.

A line drawn from about the middle of Poupart's ligament to the inner aspect of the internal condyle will indicate the course of the femoral artery, which lies behind the upper two-thirds of the line.

Relations. For the first half or third of its course the femoral artery issuperficial, lying in Scarpa's triangle, having the sartorius to its ruter side and the adductor longus to its inner side. 
It enters the triangle about the middle of the base and passing through the centre leaves it usually about at its apex by passing beneath the sartorius. It then descends, lying on the front of the insertion of the adductor longus, which relation it maintains to within about an inch or so of its termination, and fror that distance it is separated from the posterior face of the sartorius by a tendinous arch which the adductor longus and adductor magnus throw over the artery to the vastus internus; so that the last inch or two of the femoral artery is the deepest portion being covered by the skin, superficial fascia, fascia lata, sartorius and the tendinous arch above mentioned. Throughout its course the artery lies between the sartorius and adductor longus.

In the upper part of its course these muscles lie one on either side, while below the sartorius is in front and the adductor longus behind. For the last inch or two the sartorius is separated from the artery by the tendinous arch.

Relations to Cords. It lies in a sheath which also contains the femoral vein. At Poupart's ligament, the vein is internal and abreast of the artery; but as it descends, passes outward, so as to get first behind and than external. In the upper portion of its course the artery has from one-fourth to one-half an inch from its outer side the anterior crural nerve, which here divides into numerous branches. Of these, the internal cutaneous crosses the front of the artery, as does the middle cutaneous when piercing the sartorius muscle. The crural branch of the genito-crural nerve crosses the artery in front in its upper part. The long saphenous, accompanied by the nerve to the vastus internus, descends as an external relation to the femoral, which relation is maintained until the nerve to the vastus enters the fibres of that muscle. The saphenous, on reaching Hunter's canal, gets directly in front of the vessel but not in its sheath. The two structures then separate, the artery passing to the back of the thigh, while the nerve continues down to the inner side of the knee joint. The nerve to the pectineus, when that musçle is supplied by the anterior crural, passes behind the artery in the upper portion of the vessel. At a variable distance from Poupart's ligament, 
sometimes not more than half an inch, while occasionally more than two inches, there arises a large trunk, known as the Deet Femoral or Profunda Femoris. At its origin this vessel is external to the femoral; but quickly changes its direction to pass downward and inward and become a posterior relation, which position it maintains until the two ressels are separated by the adductor longus muscle. Both the superficial and the deep femoral ressels are accompanied by their veins. The relations between these structures is usually that the two veins are interposed between the two arteries so that a cross-section of the limb would exhibit from before backivard, femoral artery, femoral rein, profunda vein, profunda artery. It should be remembered, howerer, that in a large number of cases (probably as many as one-fourth) the relation is, femoral artery, femoral vein, profunda artery, profunda vein.

\section{Branches.}

Superficial Epigastric Superficial Circumflex Iliac Superficial External Pudic
Deep External Pudic Profunda Femoris Anastomotica Magna

\section{Muscular}

Soon after its commencement the femoral artery gives off four small branches; the Superficial Epigastric, the Superficial Circumflex Iliac, the Superficial External Pudic, and the Deep External Pudic. These are all cutaneous.

The superficiul cpigastric frequently arises by a common trunk with the superficial circumflex iliac. It first passes upward and inward, then continues its conrse nearly directly toward the umbilicus, lying beneath the skin and superficial fascia. It is distributed to the skin near the lower portion of the abdominal wall; and anastomoses with the cutaneous branches of the deep epigastric.

The superficial rircumflex iliac, arising either in common with the superficial epigastric, or separately from the fenoral, prsses upward and outward, parallel with Poupart's ligament, toward the crest of the ilium. It supplies the superficial inguinal glands, the skin from the upper front portion of the thish; and inosculates with the superficial brancles of the deep circnmflex iliac. 
The superficial external pudic arises from the inner aspect of the femoral, and passes in front of the femoral vein toward the genital organs. This artery either passes through the fascia lata or through the cribriform fascia covering the saphenous opening. It passes to the skin covering the pubes and supplies that, one branch passing along the dorsum of the penis, while the other branches are distributed to the sacrotum in the male and the labia in the female.

The deep external pudic sometimes arises in common with the superficial external pudic, runs inward behind the femoral, then passes across the pectineus muscle and perforates the fascia lata close to the symphysis pubis. It inosculates with branches of the superficial perineal artery, and supplies the sacrotum and perineum in the male and the labia in the female.

\section{Profunda Femoris.}

At a variable distance from its commencement, generally from one to two inches though sometimes less and sometimes. more, the femoral artery gives off from its outer posterior aspect a branch called the profunda femoris which is almost as large as the continuation of the femoral itself. The direction of the profunda is for a short distance downward and outward, then curving inward, almost immediately after its origin, it descends vertically lying behind the femoral artery and having no muscle interposed between them until the two reach the upper edge of the adductor longus which muscle thence forward separates them, the femoral passing down on its front and the profunda behind it for some distance and then, perforating the adductor magnus, passes to the back of the thigh where it is distributed.

The profunda artery is contained in a sheath in which lies also its vein, which is in front of the artery. As the profunda descends behind the femoral it is separated from it by two veins, profunda and femoral; so that from the origin of the profunda down to the adductor longus muscle, the structures lie in the following order from before backward: femoral artery, femoral vein, profunda vein, profunda artery. 


\section{Branches of the Profunda.}

\section{External Circumflex Internal Circumflex}

Three Perforating

The extemal circumflex arises eariy from the profunda and passes outward around the upper extremity of the femur, giving off two sets of branches, one ascending to the gluteal region and the other descending to the outer aspect of the thigh and knee-joint. These branches supply the muscles on the outer front aspect of the thigh, anastomosing above with the gluteal, behind with the internal circumflex, perforating and sciatic and below with the superior external articular.

The internal circumflex arises from the profunda near its commencement and is much smaller than the external circumflex. It passes in ward around the upper extremity of the femur and anastomoses with the external circumflex, superior perforating and obturator. In addition to supplying the muscles on the inner aspect of the thigh, this vessel gives a branch to the hip-joint.

The three perforating arleries. As the profunda femoris is descending it gives off in succession three branches, each called perforating, superior, middle and inferior. They derive their names from the fact that they perforate the adductor magnus muscle (the superior perforating the adductor brevis also) to reach the back of the thigh and supply the posterior femoral muscles.

\section{Anastomotica Magna.}

The anaslomolica magna, the last branch of the femoral artery, arises from it just as that artery is about to pierce the adductor magnus and descends toward the knee after dividing into a superficial and a deep branch. The anastomotica magna divides into a supcrficial branch which follows the long saphcnous nerve beneath the sartorius and is distributed to the skin; and a deep branch which, plunging into the vastus internus muscle, anastomoses with the superior internal and external branches of the popliteal and with the recurrent branch of the anterior tibial. 
Besides the six foregoing branches the femoral artery gives off muscular branches to the muscles in its course.

\section{The Popliteal Artery.}

The popliteal artery begins by being a continuation of the femoral, where that vessel terminates at the lower third of the femur by passing to the back of the thigh through the opening in the adductor magnus muscle. Its course is downward and outward (the obliquity being greatest at first) through the popliteal space to the lower border of the poplitens muscle, in front of the tendinous arch of the soleus - a point which corresponds to the junction of the upper fifth with the lower fourfifths of the shaft of the tibia-where it terminates by dividing into the anterior and posterior tibial arteries.

Relations. The artery lies in the popliteal space in relationship with the muscles forming its walls, covered by the popliteal fascia and surrounded by a mass of fat which occupies the space. In entering the space it passes beneath the inner ham-string muscles, and hence enters below the upper angle of the space and on its inner wall. The biceps is to its outer side and diverges from the artery as the latter descends. The heads of the gastrocnemius are distant external and internal relations in the middle of the vessel, but become closer and superficial relations towards its termination owing to their convergence. The artery rests first on the lower third of the femur, then on the posterior ligament of the knee-joint and then on the fascia covering the popliteus muscle.

Relations to Cords. In the popliteal space are found the popliteal artery and vein and the internal popliteal nerve. The artery and vein enter the space together, on its inner side below the superior angle, with the vein behind and external to the artery and closely adherent to it. As the structures descend, the vein winds around the artery and, while remaining behind, becomes also internal. The nerve enters the space at its apex and is, consequently, external to the artery and vein as well as behind. As the artery is inclined outward and the nerve inward this relation changes so that the nerve becomes internal and behind. From behind forward, throughout the 
course of these structures, the order is nerve, vein, artery. In the upper third the vein and nerve are behind and external, the vein closely hugging the artery; in the middle third the vein and nerve are directly behind; in the lower third the vein and nerve are behind and internal. The great sciatic nerve rarely divides at the apex of the popliteal space, so that it is more correct to say that the great sciatic is the posterorexternal relation for the upper third and the internal popliteal is the relation for the lower two-thirds.

\section{Branches.}

\section{Muscular}

Superior External Articular Superior Internal Articular
Inferior Exterual Articular Inferior Internal Articular Azygos Articular

The muscular branches of the popliteal consist of two sets, superior and inferior. The superior are several small branches given off to the ham-string imuscles, while the inferior consist usually of two large branches called the sural which are distributed to the two heads of the gastrocnemius muscle and to the plantaris.

The Articular Arteries, five in number, form a vascular zone around the knee joint, aided by the anastomotica magna from the femoral, the external circumflex from the front of the femur, and a recurrent branch from the anterior tibial. They consist of two sets, superior and inferior, each set consisting of two arteries.

The superior external articular, passes beneath the tendon of the biceps muscle, around the outer aspect of the knee joint above the external condyle, and anastomoses with the external circumflex branch of the profunda, with the terminal branch of the anastomotica magna, the inferior internal and external articular arteries.

The superior internal articular, passing beneath the tendon of the adductor magnus, winds around above the inner condyle of the femur, anastomosing with the anastomotica magna, the superior external articular, and the inferior internal articular. 
The inferior external articular winds around the head of the fibula below the outer tuberosity of the tibia, anastomosing with the superior external articular, the recurrent from the anterior tibial, and the inferior internal articular.

The inferior internal articular passes beneath the internal suberosity of the tibia, anastomosing with the superior internal, and inferior external articular arteries, and, across the patella, with the superior external articular.

The azygos articular consists of one or two small branches, which, rising opposite the posterior ligament of the knee joint, pierce that ligament to pass into the interior of the joint and supply the structures found there.

\section{The Posterior Tibial Artery.}

The posterior tibial artery, one of the two terminal branches of the popliteal, begins where that artery terminates at the lower border of the popliteus muscle, beneath the tendinous arch of the soleus and descends the back of the leg with a steady inclination inward to the inner aspect of the os calcis where it divides midway between the internal malleolus and posterior tuberosity of the os calcis into the internal and external plantar arteries.

Relations. The posterior tibial artery, for about the upper two-thirds of its course, lies deep, covered by the triceps suræ, but for the rest of its course it is surperficial, having emerged to the inner side of the tendo Achilles, becanse of its own in ward inclination and because of the narrowing of the triceps suræ to a tendon. It lies on the tibialis posticus, the flexor longus digit rum, the tibia, an 1 oa the ankle joint.

The artery is accompanied by satellite veins, one on either side, and by the posterior tibial nerve. The nerve for the first few inches of the artery (at the lower part of the upper third of the leg) lies to the inner side of the artery and then crosses it posteriorly to assume a continuous external relation. The nerve crosses about where the peroneal branch arises.

The relations at ankle joint from within outward, first is the tendon of the tibialis posticus, then the tendon of the flexor longus digitorum, these tendons lying in the same groove, 
next comes the artery with a satellite vein on either side, then the posterior tibial nerve and about one-half an inch more external is seen the tendon of the flexor longus hallucis.

\section{Branches.}

Peronea1

Internal Calcanean

\section{Terminal}

Besides muscular branches to the muscles in its course, and its two terminal branches, the posterior tibial produces two branches, peroneal and internal calcanean. One of its earliest muscular branches gives off a recurrent branch which passes upward to the knee-joint.

The peroneal artery is nearly as large as the continuation of the posterior tibial, and arises from that artery high up, near its origin, the distance being about two inches. It first passes obliquely downward and outward to the origin of the flexor longus pollicis muscle and then descends vertically beneath that muscle to terminate on the outer side of the os calcis.

Relations. The peroncal in the upper, oblique, part of its course is covered, like its parent, by the triceps suræ muscle, but after reaching the flexor longus pollicis it is covered by that muscle in addition. In the lower part of the leg the difference between the two arteries in depth is very considerable, since the posterior tibial is here superficial while the peroneal is still covered by the flexor longus pollicis and the tendo Achilles. It lies on the tibialis posticus and flexor longus hallucis.

\section{Branches.}

Just above the posterior tuberosity of the os calcis the peroneal and posterior tibial arteries are connected by a small transverse communicating branch. The peroneal gives muscular branches to the muscles in its course. About the lower third of the leg the peroneal gives off a large branch called the anterior peroneal, which pierces the interosseous membrane to reach the front of the leg. The termination of the peroneal is distributed on the outer side of the heel by branches called cxlcrnal calcancan. 
The internal calcanean branches of the posterior tibial are three or four in number and are distributed to the inner side of the heel. They anastomose with the peroneal, external calcanean, internal malleolar and plantar arteries.

\section{The Internal Plantar Artery.}

The internal plantar, much the smaller of the $t$ wo terminal branches of the posterior tibial, begins where that artery bifurcates midway between the posterior tuberosity of the os calcis and the internal malleolus and passes forward along the inner side of the sole to terminate on the inner side of the great toe by inosculating with the digital branch to that toe, having given off muscular branches in its course.

\section{The External Plantar Artery。}

The external plantar, the larger and more important of the two terminal branches of the posterior tibial, begins where that artery bifurcates midway between the posterior tuberosity of the os calcis and the internal malleolus, and passes forward and outward between the first and second layers of muscles in the sole to the base of the fifth metatarsal bone, where it turns in ward beneath the third layer of the muscles to cross to the inner side of the sole and terminate there at the posterior extremity of the first interosseous space by anastomosing with a communicating branch from the dorsalis pedis artery. That portion of the artery which crosses the sole from the outer to the inner side is called the plantar arch.

\section{Branches.}

Besides muscular branches, the external plantar gives off the following: digital and posterior perforating.

The digital branches are four in number. They run forward to the cleft between the toes and there divide to supply plantar collateral branches to the sides of the toes, except the great toe and the inner side of the second toe. The outermost of the four is appropriated by the outer side of the little toe. Just before the digital arteries divide at the clefts of the toes, they each send up between the metatarsal bones a branch called anterior perforating, to open into the digital branches on the dorsum of the foot. 
The posterior perforating, three in number, pass up between the heads of the three outer interosseous muscles to anastomose with the metatarsea artery on the dorsum of the foot.

\section{The Anterior Tibial Artery.}

The anterior tibial artery begins where the popliteal forks, at the lower border of the popliteus muscle, beneath the tendinous arch of the soleus, and passes directly forward between the tibia and fibula to the front of the leg which it descends, lying, for the upper two-thirds of its course, on the interossenus membrane, and for the lower third on the front of the tibia, to terminate on the middle of the front of the ankle-joint, beneath the annular ligament, by assuming the name of dorsalis pedis.

Relations. The anterior tibial is divided into three portions, upper, middle and lower thirds.

In its upper third it lies on the front of the interosseus membrane between the tibialis anticus internally and the extensor longus digitorum externally. In its middle third it still lies on the interosseous membrane, but has reached the origin of the extensor proprius pollicis and this muscle lies on the outer side, while the tibialis anticus still remains internal.

In its lower third it lies on the front of the tibia and is crossed obliquely by the tendon of the extensor proprius pollicis, which displaces the tibialis anticus and becomes the internal relation, while the extensor longus digitorum again comes into relation with it externally.

The artery is accompanied by satellite veins and the anterior tibial nerve. The nerve is at first to its outer side; then for a short distance in front of it and then agrain to the outer side.

\section{Branches.}

The branches of the anterior tibial artery are muscular, a recurrent branch, which ascends to the front of the knee through the tibialis anticus muscle, and two malleolar branches, external and internal, which surround the tibia just above the ankle-joint and supply that joint.

Des $\Lambda$ nat-23 


\section{The Dorsalis Pedis Artery.}

The dorsalis pedis artery is the continuation of the anterior tibial. It begins beneath the annular ligament, about the middle of the front of the ankle-joint, and passes forward along the inner side of the dorsum of the foot to terminate at the posterior extremity of the first interosseous space by dividing into the dorsalis hallucis and the communicating.

Relations. It has the same relations to muscles and cords as the lower third of the anterior tibial. To its inner side is the extensor proprius pollicis tendon, to its outer side the extensor longus digitorum, and near its termination it is crossed by the tendon of the extensor brevis digitorum to the great toe. It lies between satellite veins and has the anterior tibial nerve to its outer side.

\section{Branches.}

Tarsea

Metatarsea
Dorsalis Hallucis Communicating

The tarsea branch passes outward on the tarsus.

The metatursea branch passes outward on the bases of the metatarsal bones and receives the posterior perforating branches from the plantar arch. The metatarsea sends forward three branches called interosseous, which after receiving the anterior perforating branches of the plantar digital arteries, divide at the clefts of the toes to form collateral digital branches for the dorsal aspect of the fifth, fourth and third toes and the outer side of the second toe.

The dorsalis hallucis is one of the two terminal branches of the dorsalis pedis, and runs forward to supply dorsal collateral digital branches for the great toe and inner side of the second toe.

The communicating passes through the first interosseous space to anastomose with the termination of the external plantar, giving off a branch, the magna pollicis, which passes forward and divides to form plantar collateral digital branches for the great toe and inner side of the second toe. 


\section{Internal Iliac Artery.}

The Internal Iliac, one of the two terminal branches of the common iliac, commences where that artery bifurcates on the side of the disc between the fifth lumbar vertebra and the sacrum, and passes downward into the pelvis over the front of the sacro-iliac symphysis to the upper margin of the great sacro-sciatic notch, where it divides into the anterior and posterior trunks. Its length is variable, and is usually about two inches. It is a short and wide trunk.

Relations. It lies behind the peritoneum, has the ureter upon its front, and rests behind upon the lumbo-sacral cord and the pyriformis muscle and internal iliac vein. On the left side it has resting upon it the end of the sigmoid flexure of the colon.

The distribution of the vessel is to the viscera and walls of the pelvis; and with few exceptions it will be found that the parietal branches spring from the posterior, while the visceral are the offspring of the anterior trunk. The hypogastric artery of the fœtus is a branch of the internal iliac. It is nearly obliterated after birth, becoming an impervious cord from the bladder to its point of attachment to the abdominal wall; but from the internal iliac to the wall of the bladder the artery is pervious, and carries some of the arterial supply of that viscus.

\section{Branches of the Anterior Trunk.}

Hypogastric

Inferior Vesical

Middle Hemorrhoida1
Obturator

Ischiatic

Internal Pudic

\section{Hypogastric.}

The first of these branches, the obliterated hyposastric, passes from the front of the anterior trunk to the posterior surface of the bladder. This artery conveys but a small amount of blood, which is transmitted to the bladder by two small vessels, known as the superior and middle vesical arteries. They are distributed to the apex and greater portion of 
the body of the bladder, while a small twig from one is generally given to the vas deferens.

\section{Inferior Vesical.}

The inferior vesical, springing directly from the anterior trunk is distributed to the base of the bladder, and in the male subject, passes forward also to the prostate gland and to the seminal vesicles.

\section{Middle Hemorrhoidal.}

The next branch, the middle hemorrhoidal, which frequently arises in common with the inferior vesical, passes to the rectum, and supplies the middle portion of that gut, anastomosing above with the superior hemorrhoidal and below with the inferior hemorrhoidal branches of the internal pudic.

\section{Obturator.}

The obturator is the largest of the way-side branches of the anterior trunk. It is very variable in its origin. Generally the product of the anterior trunk, it sometimes springs from the deep epigastric branch of the external iliac; and in a few cases may be found arising from the posterior trunk of the internal iliac. Its usual course is to pass along the wall of the pelvis just below its brim, accompanied by the obturator vein and nerve, the nerve lying above the artery and the vein below. It lies behind the peritoneum, between it and the parieta pelvic fascia, reaches the upper margin of the thyroid foramen, and leaves the pelvic cavity by passing through this foramen in an opening left above the obturator membrane. While in the pelvic cavity it gives off a small branch, which passes in to the iliac fossa to supply the bone, and a minute twig which ramifies upon the posterior face of the pubic arch, anastomosing there with the pudic branch of the deep epigastric. After emerging from the pelvic cavity, it divides into two branches, external and internal. Of these the external, which is the larger, skirts the outer margin of the obturator foramen, and divides into two branches, one of which anastomoses with the internal division of the obturator and the other with the internal circumflex, the artery supplying the muscles around the 
tuberosity of the ischium in its course. The internal branch, skirting the inner margin of the obturator foramen, supplies the muscles around that foramen and the abductor muscles, anastomosing with the internal circumflex and with the external division of the obturator. The external branch also anastomoses with the ischiatic.

After giving off the preceding branches, the anterior trunk divides into two terminal branches, the ischiatic, or sciatic, and the internal pudic.

\section{Ischiatic.}

The ischiatic artery leaves the pelvis through the great sacro-sciatic foramen below the pyriformis muscle. It descends between the trochanter major and the tuberosity of the ischium, crossing the external rotaters of the thigh, and passing downward on the inner side of the great sciatic nerve. It inosculates with the internal circumflex and superior perforating branch of the profunda, and gives off muscular branches to the muscles in its course. Besides these, it gives coccygeal branches within the pelvis, which pierce the great sacro-sciatic ligament to supply the gluteus maximus muscle; and a rather large branch, called the inferior gluteal, passing to the deep surface of the same muscle; an articular branch to the hip joint, given off external to the pelvis; cutaneous branches to the integument of the gluteal region; and a branch which accompanies the sciatic nerve, called the comes nervi ischiatici.

\section{Internal Pudic.}

The internal pudic is the second and smaller of the two terminal branches of the anterior trunk of the internal iliac. It lies in front of the ischiatic; and passes downward over the sacral plexus of nerves, parallel with that artery, to leave the pelvis through the greater sacrosciatic foramen below the pyriformis muscle. It then continues its course to the spine of the ischium, winds around that process, re-enters the pelvis through the lesser sacrosciatic foramen lying about an inch above the tuberosity of the ischium, and passes upward and forward along the ramus of the ischium, or rather the obturator internus muscle, held 
in position by a fold of the pelvis fascia, until it reaches a point opposite the transversus perinæi or near the central point of the perineum, where it divides into a superficial and a deep perineal branch.

The superficial perineal passes forward through the perineum as far as the scrotum. At the upper border of the transversus perinæi it gives off a branch bearing the same name as that muscle, which crosses on that muscle, supplies it and the adjacent portion of the skin, and anastomoses with its fellow of the opposite side.

While the internal pudic is still in the pelvic cavity it gives off inferior hemorrhoidal branches. These branches perforate the pelvic fascia, which holds the artery in position, and pass to the anus, where they supply the sphincter and levator ani muscles, anastomosing with the middle hemorrhoidal and with the vessels of the opposite side.

The deep perineal continues the course of the internal pudic, passing between the two layers of the triangular ligament, and opposite the junction of the crura of the penis divides into two branches, the dorsal artery of the penis and the artery of the corpus cavemosum.

Prior to this division it gives off a large branch, the artery of the bulb, which enters the bulb of the corpus spongiosum and is distributed to that structure. It is a very short trunk, which passes transversely inward between the layers of the triangular ligament to plunge at once into the bulb of the penis.

The artery of the corpus cavernosum, one of the terminal branches, enters the crus penis on its inner aspect, and is distributed in that body.

The dorsal artery of the penis, the other terminal branch, continues upward between the two layers, lying in the substance of the compressor urethræ muscle. It pierces the anterior layer near its apex, and then the suspensory ligament of the penis, and passes along the dorsum of the penis to the corona to anastomose with the artery of the opposite side. In this course it is accompanied by the corresponding nerve, which lies to the outer side of the artery. This artery anas- 
tomoses with the deep external pudic branch of the femoral, which has been known to take its place.

\section{Branches of the Posterior Trunk.}

$\begin{array}{cc}\text { Ilio-Lumbar } & \text { Lateral Sacral Gluteal } \\ \text { Ilio-Lumbar }\end{array}$

The ilio-lumbar is a short recurrent branch, which arising from the postero-external part of the internal iliac, passes upward and outward beneath the common iliac artery. It passes between the lumbo-sacral cord and the obturator nerve, and then beneath the psoas magnus muscle; and divides into two branches, iliac and lumbar.

The iliac branch passes between the iliacus internus muscle and the iliac fossa, is distributed to that muscle, and sends a nutrient artery to the bone.

The lumbar branch, passing beneath the psoas magnus, supplies that muscle and the quadratus lumborum, and sends a spinal branch into the foramen between the last lumbar rertebra and the sacrum.

\section{Lateral Sacral.}

The lateral sacral are a pair of small arteries, the superior of which enters the anterior sacral foramen, where it communicates with branches of the lumbar arteries above, and with branches of the lower lateral sacral below. The inferior pass downward on the front of the sacrum, and send a branch into each anterior sacral foramen, over which they pass, these branches communicating with one another, and also with the superior lateral sacral above.

\section{Gluteal.}

The sluleal artery is a continuation of the posterior trunk of the internal iliac. It passes back ward through the upper part of the great sacro-sciatic foramen above the pyriformis muscle, usually piercing the sacral plexus of nerves. After emerging from the pelvis, it divides into two branches, a superficial and a deep, the superficial passing outward between the gluteus maximus and the gluteus medius muscles to sup- 
ply the former, and, perforating it, to be distributed to the integument.

The deep branch, itself subdividing into two, runs between the gluteus medius and minimus, and gives branches to these two muscles. The superior division of the deep branch anastomoses with the circumflex iliac, and with the external circumflex.

The inferior division anastomoses with the external circumflex.

The superficial branch anastomoses with the posterior branches of the sacral arteries.

\section{Internal Carotid.}

The Internal Carotid artery is one of the two terminal branches of the common carotid; and begins where that vessel terminates on the upper border of the thyroid cartilage opposite the transverse process of the fourth cervical vertebra, and passes upward on the frout of the transverse processes of the three upper cervical vertebra to the carotid aperture on the basilar surface of the petrous portion of the temporal bone. Entering at this foramen, it abruptly changes its course to pass inward and forward, with a slight inclination downward, running in the carotid canal, to an aperture of exit near the apex of the petrous portion of the temporal, where it again changes its direction, passes upward to enter the cavity of the cranium, turns forward, lying in the cavernous groove by the side of the sella Turcica, and finally passes upward beside the anterior clinoid process, and divides into four terminal branches, Anterior Cerebra1, Middle Cerebral, Posterior Communicating, and Anterior Coroid.

Relations. Its relations to muscles and structures throughout its course are as follows: Behind it are the anterior tubercles of the transverse process of the three upper cervicle vertebra, separated from the artery by the origin of the rectus capitis anticus major. In front, it is at first superficial, being covered by the skin, superficial and deep fascia, platysma, stylo-hyoid and digastric muscles, and at its commencement by the anterior border of the sterno-cleido-mastoid. As it ascends, however, 
it passes beneath the parotid gland, the stylo-glossus and stylo-pharyngeus muscles and the stylo-hyoid ligament. To its imer side is the pharynx, and the tonsil, which is a more distant internal relation.

The Rclations to cords are numerous and important. At its commencement the internal carotid is external to the external carotid artery; but as it ascends the neck and reaches the stylo-hyoid muscle and posterior helly of the digastric muscle, the internal passes behind the external and is thenceforward separated from it by the stylo-glossus and stylo-pharyngeus muscles and stylo-hyoid ligament, and by the glosso-pharyngeal nerve, and the pharyngeal branch of the pneumogastric. Through the greater portion of its course the internal jugular vein lies to its outer side, as does the pneumogastric nerve; but near the carotid apertures of entrance the relations are more numerous and more complex. An examination of the skull will show that the jugular foramen is directly behind the carotid apertures of entrance, and that the anterior condyloid foramen is behind and internal to this. Hence the relations which these structures bear to each other. At the point of entrance of the carotid artery the internal jugular vein and the ninth, tenth, and eleventh nerves lie directly behind the artery, while the twelfth nerve is behind and slightly internal. The ninth, tenth and twelfth quickly emerge between the artery and the vein, while the eleventh passes behind the vein. The relation, then, immediately after emergence from their respective foramina would be : the artery the most internal of all the structures lying against the wall of the pharynx, the most external the internal jugular vein, and between these the ninth, tenth, and twelfth nerves. At the lower border of the stylo-glossus muscle the glosio-pharyngeal nerve crosises over the front of the artery, and ceases to be a relation.

The hypo-glossal, or twelfth, nerve now becomes an anteroexternal relation until it reaches the lower border of the stylohyoid and posterior belly of the digastric, when it crosses the artery directly in front to reach its point of distribution, while the pneumogastric, or tenth, continues downivard as an external relation, lying between the artery and the vein. Poste- 
riorly the artery rests upon the cervical portion of the sympathetic, which separates it from the rectus capitis anticus major muscle The interosseous, or petrosal, portion of the artery requires no description, being imbedded in the petrous portion of the temporal bone. The intra-cranial portion, lying in the cavernous sinus, has the sixth nerve lying upon its outer side. The artery is covered by the lining membrane of the sinus; and is not in direct contact with the blood which flows through that sinus.

\section{Branches.}

The first portion gives off no branches. The second portion produces one unimportant branch, the tympanic, which passes to the tympanum. The third portion gives origin to three branches. Two of these, the arteria receptacnli, are unimportant and are distributed to the cavernous sinus and dura mater; the third is an important branch, the opthalmic artery.

\section{The Opthalmic Artery.}

The opthalmic artery springs from the internal carotid about the termination of its third portion and passes forward into the orbit through the optic foramen, first lying below and external to the optic nerve, then crossing to the inner side of the nerve and running forward along the inner wall of the orbit to divide at the internal angle of the orbit into two branches, frontal and nasal.

\section{Branches.}

The branches of the opthalmic artery are ten in number.

The lachrymal artery runs forward along the outer wall of the orbit and after supplying the lachrymal gland passes on to the upper eye-lid. It gives off temporo-malar branches which pass through the bone to the temporal muscle.

The supra orbital runs forward on the levator palpebre muscle and escaping to the forehead through the supra-orbital foramen, is there distributed by a superficial and a deep branch, anastomosing with the frontal.

The anterior and posteriorethmoidal arteries escape through the anterior and posterior ethmoidal foramina, and are distributed to the dura mater, ethmoidal cells and nasal fossæ. 
The palpebral arteries, superior and inferior, arise from the opthalmic near its termination. The superior runs outward on the upper lid near its free edge, between the orbicularis palpebrarum and the tarsal cartilage and is there distributed; the inferior does the same for the lower lid. The two sometimes arise by a common trunk.

\section{Frontal.}

The frontal, one of the two terminal branches of the opthalmic artery, emerges at the inner angle of the orbit and ascends to be distributed along the middle of the forehead, anastomosing with the anterior branch of the temporal, with its fellow and with the supra orbital.

\section{Nasal.}

The nasul, the other terminal branch of the opthalmic artery, emerges at the inner angle of the orbit and is distributed along the bridge of the nose, anastomosing with its fellow and with the facial through its angular and transverse nasal branches.

The arteria centralis retina pierces the optic nerve and runs forward in it to reach the retina and be there distributed.

The opthalmic gives off numerous muscular branches in its course which are divided into two sets, superior and inferior, the superior springing by one trunk and the inferior by another. Together they supply the muscles in the orbital cavity. They are very irregular in number and in point of origin.

The ciliary arteries consist of three sets, viz:

1st. Short ciliary branches, which are numerous small arteries which pierce the sclerotic around the optic nerve and supply the choroid.

2nd. Long ciliury, which are two branches piercing the sclerotic on of posite sides and running forward between it and the choroid to be distributed to the iris.

31. Anterior ciliary branches, which are the offspring of muscular branches and pierce the sclerotic near the front of the eye to reach the iris.

The branches from the fourth or cerebral portion are four. 


\section{Anterior Cerebral Artery.}

The anterior cerebral artery, one of the terminal branches of the internal carotid, runs forward in the longitudinal fissure, ascends the genu of the corpus callosum and runs backward along that body. It distributes branches to the irontal and parietal lobes of the brain, on their inner face, and inosculates with the posterior cerebral. Its branches are frequently given the names of the convolutions which they supply.

Passing from the anterior cerebral of one side to that of the other, soon after their origin, is a communicating branch called the anterior communicating artery.

\section{Middle Cerebral Artery.}

The middle cerebral, arising as one of the terminal branches of the internal carotid, passes outward along the fissure of Sylvius to be distributed to the anterior and middle lobes of the cerebrum and to the convolutions forming the island of Reil. This artery gives off two sets of branches, one to the cortex and one to the ganglia in the interior of the brain. The cortical branches are distributed over the outer face of the frontal parietal and tempora-sphenoidal lobes, and receive the names of the convolutions which they supply. The ganglionic branches pierce the anterior perforated space and are distributed in the interior of the cerebrum. An important fact concerning these branches is that they do not anastomose with each other in the brain.

\section{Anterior Choroid Artery.}

The anterior choroid pierces the base of the brain to enter the descending cornu of the lateral ventricle and form the choroid plexus.

\section{Posterior Communicating Artery.}

The posterior communicating artery, the last of the four terminal branches of the internal carotid, passes backward to anastomose with the posterior cerebral of the basilar artery. 
From this artery are given off ganglionic branches which pass through the posterior perforated spot to the interior of the brain.

Circumscribing the six-sided space at the base of the brain is a series of arteries and their communications forming what is called the vascular circle of Willis. Beginning in front we have the anterior communicating, and, following the arteries back on one side, they come in this order, anterior cerebral, internal carotid, posterior communicating, posterior cerebral and the termination of the basilar artery. Following them forward from the basilar artery they come in the reverse order. 


\section{THE VENOUS SYSTEM.}

The Veins are the vessels which return the blood which the arteries have carried to the capillaries. Consequently the venous current passes in a direction opposite to that of the arterial, or towards the heart, opening into that organ by means of two main trunks, called the Superior and Inferior Venæ Cava, and by small vessels which carry the venous circulation of the heart itself.

The veins are divided into two sets, the superficial and the deep. The superficial veins are found between the layers of snperficial fascia; and ultimately terminate by emptying into the deep veins. The deep veins are found accompanying the arteries, and are arranged in two forms: the smaller arteries are accompanied by satellite veins, one upon either side, while the larger vessels are accompanied by a single vein, bearing usually the name of the artery itself.

The venous system as a whole is very much larger than the arterial system as a whole; and this statement is particularly true of the veins found in the abdominal cavity. These veins are capable of being greatly dilated upon stimulation of the splanchnic nerves, so that it is possible for an animal to bleed to death in the venous channels of the abdominal cavity without a drop of blood being extracted from the body, the veins dilating to such an extent as to be able to contain all of the blood of the system.

As a rule the companion veins of an artery receive accessories corresponding to the number of branches which are emitted by the artery, though there are some notable exceptions to this.

\section{The Veins of the Lower Extremity.}

The Veins of the Lowver Extremity consist of two sets, superficial and deep.

The deep veins, or satellite veins, commence in the sole of the foot on both sides of the plantar arteries, and on its dorsal surface, on both sides of the dorsalis pedis. 
The companion reins of the internal and cxtcrnal flantar arteries, commencing respectively on the inner and outer side of the foot, unite where the posterior tibial artery terminates to form two companion veins for the posterior tibial. These veins ascend, lying one upon either side of the posterior tibial artery, and recieve, about an inch below the beginning of that vessel, the two companion veins of the peroneal artery, which hare united to form a single vein.

The satellite reins of the dorsalis pedis artery, commencing on the dorsum of the foot at the great toe, receive the veins from the tarsal and metatarsal branches of that artery to become at the front of the ankle the companion veins of the anterior tibial. These veins then receive the accessory reins which accompany the branches of the anterior tibial artery, and continue their course up the leg, uniting to form a single vein, which passing above the interosseous membrane, joins the branch formed by the satellite veins of the posterior tibial artery to form the popliteal.

The Popliteal Vein then passes upward accompained by the popliteal artery. It lies superficial to the artery, and between that structure and the internal popliteal nerve, lying at first behind and internal to the artery, directly behind it in the middle portion of its course, and behind and external in the beginning where the femoral artery terminates in the popliteal. It receives the accessory veins which accompany the five articular branches and the muscular branches given off by the popliteal artery, and becomes the femoral vein at the junction of the lower third with the upper two-thirds of the shaft of the femur.

The Femoral Vein then accompanies the femoral artery, receiving in its course the veins which accompany the branches of that vessel. It lies first behind and external to the femoral artery, then shortly before the commencement of that vessel directly behind it, and finally internal to it, so that at Poupart's ligament it is internal and fully abreast of the artery. About an inch and one-half below the commencement of the femoral artery, it gives off its profunda femoris branch, which artery is accompanied by its own vein formed by the various satellite 
veins accompanying branches from the profunda femoris. At the origin of the profunda this vein and the femoral vein unite, forming what is sometimes called the Common Femoral Vein, which lies internal and abreast of its artery as just described. This vein at Poupart's ligament terminates by becoming the External Iliac Vein. Just before reaching Poupart's ligament, the femoral vein, lying then opposite the saphenous opening of the fascia lata, receives the long or internal, saphenous vein and the satellite veins which accompany the superficial branches given off from the femoral in this situation.

After changing its name to become the External Iliac, the vein receives the companion branches of the deep circumflex, and the deep epigastric branches of the external iliac artery. It then passes upward, lying first internal and abreast of the external iliac, and then internal and behind it to the point at which the common iliac artery divides into its two terminal branches, opposite the intervertebral disc between the fifth lumbar vertebra and the sacrum.

At this point it is joined by the internal iliac vein, which is made up of a large number of satellite veius accompanying the numerous branches of the interval iliac artery. The confluence of these two veins forms the Common Iliac, which vein is found lying first directly behind its artery, then external and on a plane posterior, and finaily external to the common iliac artery, at the commencement of which vessel, opposite the body of the fourth lumbar vertebra, it unites with the common iliac vein of the opposite side to form the Inferior Vena Cava.

This description applies only to the common iliac vein of the right side of the body. On the left side the external iliac vein is internal and abreast of its artery at Poupart's ligament, continues internal, but on a plane posterior to it, up to the bifurcation of the common iliac artery, passes here behind the internal iliac, and is joined by the vein accompanying that artery, forming the Common lliac Vein of the left side, which vein is internal to the common iliac artery throughout its course; and finally passes behind the right common iliac artery 
to join with that vein opposite the fourth lumbar vertebra and form the inferior vena cava.

\section{Internal Iliac Vein.}

The internal iliac vein is formed by the coalescence of the veins accompanying the branches of the internal iliac artery,

The veins of the visceral branches are so numerous and intersecting that they form plexuses. The vein thus formed ascends behind its artery and unites with the external iliac vein on the disk between the fifth lumbar vertebra and the sacrum to produce the common iliac vein.

\section{The Inferior Vena Cava.}

The Inferior Vena Cava then continues its course, lying on the right side of the abdominal aorta. As it passes upward, it receives, opposite the centre of the body of each lumbar vertebra, the lumbar veins from the two sides of the body. These veins are usually four or five in number, those from the left side passing behind the abdominal aorta in order to reach the inferior vena cava. About opposite the lower border of the second lumbar vertebra, the renal veins, formed in the hilum of the kidneys, pass at right angles into the inferior vena cava, the vein from the left side passing across the front of the abdominal aorta generally, though occasionally behind it. The vena cava itself receives the suprarenal veins, and continuing its course upward, passes behind, the posterior border of the liver, upon which it makes a deep notch, and there receives the hepatic veins. It then pierces the diaphragm on a level with the ninth dorsal vertebra, but on the right side of the body, through the highest and most anterior of the openings found in the diaphragm; and entering the base of the heart at the lower, inner, and back part of the venous auricle, pours its current into that organ.

\section{The Portal System.}

The blood which is poured into the inferior vena cava on the posterior border of the liver through the orifices of the hepatic veins is derived from a double source, a part of it coming from the veins in which the capillaries of the hepatic artery termi-

Des Anat-24 
nate, while a part of it is the product of the portal system of veins, which drain the circulation of a large portion of the alimentary canal into the liver, where the veins ramify before uniting to form the hepatic veins. It will thus be seen that the liver receives a double blood supply, one portion being that received from the hepatic artery, the ordinary arterial blood, which is intended for the nutrition of the liver tissue itself (parenchyma), the other derived from the alimentary canal contains that blood which has absorbed the products of digestion, and which passes through the liver, not for the purpose of nourishing that organ, but in order to effect the necessary changes in the blood before it is conveyed to the circulation.

This Portal System of Veins is formed in the following manner: the portal vein commences at the upper border of the pancreas by the union of the splenic and the superior mesenteric reins. The splenic vein, before uniting with the superior mesenteric, receives behind the pancreas the inferior mesenteric vein. this vein conveys the return circulation from the sigmoid flexure of the colon, and the descending colon. The superior mesenteric vein carries the return circulation from the transverse colon, descending colon, coecum, and small intestines. Hence it will be seen that the greater portion of the blood from that part of the alimentary canal which serves for the digestion of food is carried through the superior mesenteric vein, but little absorption taking place in that part of the large intestine which lies below the formation of this vein.

After the splenic and superior mesenteric veins have united behind the upper border of the head of the pancreas, the gastric veins, one of which accompanies the gastric artery and the other the arch formed by the inosculation of the gastroepiploica dextra and sinistra, empty into the portal system, which also receives the small vein which accompanies the pancreatica magna artery and the pancreatico-duodenalis superior. The vein which accompanies the pancreatico-duodenalis inferior empties into the inferior vena cava before that reaches the splenic rein. 
The Splenic itself is formed by vessels which convey the return circulation from the spleen; and also receives the small veins accompanying the various branches given to the pancreas and the vasa brevia of the stomach.

The Portal I'ein, formed in this way, is a short, wide trunk, about two inches in length, which passes upward, lying in the right edge of the lesser omentum. It lies behind the hepatic artery and common bile duct; but opposite the interval between these two structures, so that in naming them from right to left the order would be, common bile duct, portal vein, and hepatic artery, the usual statement being, duct to the right, artery to the left, vein between and behind the two. The vein now enters the transverse fissure of the liver, where it breaks up into numerous branches, which, following the lobules of that organ (see histology for description), unite into minute vessels, which in their turn unite again, until finally the hepatic veins are formed.

These veins, three or four in number, open by orifices which remain patulous after death, into the inferior vena cava, as it is coursing along the posterior border of the liver.

\section{The Spermatic Veins.}

The Spermatic I'ins are two in number, right and left. They begin in the scrotum, being formed by the plexus of veins surrounding each one of the testicles. These veins emerge from the back of the testicle; and receiving the tributaries from the epididymus forming a convoluted plexus, called the Pampiniform Plexus (L. Pampinus, a tendril; forma, like). They then pass upward with the other elements of the spermatic cord. Entering at the superficial abdominal ring, they follow the course of the spermatic canal to enter the abdominal cavity through the deep abdominal ring. From this point the courses of the veins on the two sides differ.

On the right side, the vein passes across the external iliac artery, gets to the outer side of that vessel; and passing upward with a steady inclination invard, terminates by entering at an acute angle into the inferior vena cava, the blood from the spermatic vein soing in the same direction as the blood from the vena cava. 
On the left side, it crosses the termination of the internal iliac artery; but ascends vertically, and empties, not into the vena cava, but into the left renal vein, at right angles to that vein, so that the whole current of blood in the renal vein passes across the aperture of the left spermatic and retards the flow of blood from this vein. It is for these anatomical reasons that enlargement of the veins of the left testicle and scrotum so frequently occurs, the surgical disease known as variocele arising from this cause.

\section{The Superficial Veins of the Lower Extremity.}

Returning to the lower extremity, we find that there are certain small veins belonging to the superficial system. Those which have received distinctive names are two in number, the short and the long saphenous. They commence on the dorsal aspect of the foot, the long saphenous on the inner and the short saphenous on the outer side; and the veinlets which compose them inosculate with one another so as to form an arch, extending entirely across the foot near the web of the toes.

The Short Saphenous Vein can be traced back from the outer edge of the little toe behind the external malleolus, where it passes to the postero-external aspect of the leg, accompanying the nerve of the same name. As it passes upward, it gradually winds to the middle of the calf of the leg, and there lies in the groove which is found on the posterior face of the gastrocnemius muscle. It continues its upward course between the two heads of the gastrocnemius, and terminates by emptying into the popliteal vein.

The Long Saphenous Vein commences on the inner side of the great toe. It passes backward along the inner side of the arch of the foot, runs across the front of the internal malleolus, passes upward on the inner face of the calf, behind the internal condyle of the femur, until it reaches the junction of the lower third with the middle third of the thigh, where it runs slightly forward, and continues its upward course along the inner front aspect of the thigh until it reaches the saphenous opening in the fascia lata, where it pierces the cribriform fascia, and terminates by emptying into the femoral vein. Occasionally 
before emptying into the femoral the long saphenous receives the satellite veins of the superficial branches of the femoral artery; and more frequently these branches, four in number, terminate by opening directly into the femoral vein, the apertures which they make through the fascia covering the saphenous opening giving it its cribriform appearance.

\section{The Veins of the Upper Extremity.}

The Veins of the Upper Extremity, like those of the lower, consist of a superficial and a deep set; but unlike the lower extremity, the superficial veins are here so large and so numerous that the satellite veins are small in size, and convey but a small portion of the return circulation.

The deep veins commence in the palm of the hand. They commence as the companion veins of the superficial palmar arch, originating in veins which accompany the digital branches of that arch. This arch receives the interosseus reins; and forms on either side the vena comites of the ulnar and radial arteries.

The companion reins of the radial and ulnar arteries unite with each other below the bend of the elbow, and form the two satellite veins of the brachial artery. That is, the satellite veins of the radial form the external satellite veins of the brachial, while the satellite veins of the ulnar form the internal satellite veins of the same artery. After receiving superficial reins and tributaries from the branches of the brachial, these two satellites pass upward to the lower border of the tendous of the latissimus dorsi and teres major muscles, and there unite to form the Axillary Vcin, which follows the course of the axillary artery, lying to its inner front aspect throughout the course of that vessel, receiving tributaries from the veins accompanying branches of that vessel, the largest and most important of which are the companion veins of the sub-scapular artery, and terminates at the outer border of the first rib by becoming the subclavian vein. This vein then continues its course to join the internal jugular, and form the innominate vein. 


\section{The Superficial Veins of the Upper Extremity.}

Anterior U1nar

Posterior U1nar

Common Ulnar

Cephalic

Median Cephalic
Radial

Median

Basilic

Median Basilic

Subclavian

\section{The Anterior Ulnar.}

The Anterior Ulnar Vein begins on the inner front aspect of the ulnar side of the hand by small tributaries which come from the little finger and inner side of the ring and middle fingers. Continuing its course upward it receives tributaries from adjacent portions of the forearm; and about an inch and one-half below the elbow-joint, unites with the posterior ulnar to form the common ulnar vein.

\section{The Posterior Ulnar.}

The Posterior Ulnar Vein arises near the back of the little finger, passes upward on the postero-internal aspect of the forearm; and joins the anterior ulnar to form the common ulnar.

\section{The Common Ulnar.}

The Common Ulnar Vein ascends to the inner side of the front of the elbow to unite with the median basilic vein to form the basilic vein.

\section{The Radial Vein.}

The Radial Vein commences on the outer side of the back of the hand, receiving tributaries from the thumb, index and middle fingers; and passing upward, winds around to the front of the forearm about at its lower third. It then continues to ascend until it reaches a point about an inch below the elbow joint, where it unites with the median cephalic to form the cephalic vein.

\section{The Median Vein.}

The Median Vein is a small vessel which receives the blood from the middle of the palm of the hand. It is scarcely per- 
ceptible until just above the wrist joint, where the formation of the vein may be said to take place. It then passes directly upward, lying on the middle of the frout of the forearm until it reaches a point about an inch and a half below the midule of the front of the elbow joint. In this situation it divides into two branches, an internal and an external, named respectively the Median Basilic and the Median Cephalic.

\section{The Median Basilic.}

The Median Basilic Vein unites with the common ulnar, the product of the anterior and posterior ulnar veins, to form the basilic.

\section{The Median Cephalic.}

The Median Cephalic Vein unites with the radial vein to form the cephalic vein.

\section{The Basilic Vein.}

The Basilic I'ein, formed below the elbow joint by the union of the common ulnar with the median basilic, passes upward and crosses over the bicipital slip of fascia given off from the tendon of the biceps to the fascia of the inner side of the forearm. It then lies to the inner front aspect of the brachial artery to about the middle of the course of that ressel, separated from it by the deep fascia, where its relation is changed only to the extent of lying beneath the investing fascia. That is, up to the middle of the brachial artery the basilic vein is superficial to the investing fascia; but opposite the middle of that vessel it pierces this fascia, and becomes deeper, lying still to the inner front asp sct of the brachial artery, but more nearly ou the same plane with that vessel. It finally terminates by uniting with the internal satellite vein of the brachial just before the latter unites with the external satellite to form the axillary vein. Some authorities describe the basilic as receiving the two satellite veins of the brachial and becoming the axillary vein.

\section{The Cephalic Vein.}

The Cephalic Vein, formed below the middle of the elbow joint by the union of the radial and the median cephalic, 
passes upward and outward until it reaches the interval between the brachialis anticus and the supinator longus and extensor carpi radialis longior. It then passes upward in a groove found on the outer aspect of the arm, caused by the bulging of the biceps anteriorlv and the triceps posteriorly; and continues its course until it reaches the lower edge of the deltoid muscle, when it turns across the front of the arm to reach the interval between the deltoid and the pectoralis major. After running in the groove between these two muscles, it passes over the fibrous-covered interval between the muscles and empties into the axillary vein just before its termination in the subclavian. Before the cephalic vein enters the axillary it receives a communication from the external jugular vein of the neck.

\section{The Axillary Vein.}

The Axillary Vein is formed by the junction of the brachial satellites and the basilic at the termination of the axillary artery. It passes upward on the inner front aspect of the axillary artery through the axillary space, receives the tributaries which accompany the branches of that vessel; and continually increases in size until it reaches the outer border of the first rib behind the junction of the outer third with the inner twothirds of the shaft of the clavicle, where it terminates by becoming the subclavian vein.

\section{The Subclavian Vein.}

The Subclavian Vein bears the same relation to its artery that the string bears to a bent bow. Commencing at the outer onethird of the clavicle on the anterior border of the first rib, it passes horizontally inward, lying below and on a plane anterior to the corresponding artery, so that it is an antero-internal relation of the third portion of the subclavian, and an antero-external relation of the first portion of the subclavian, being separated from the second portion by the interposition of the scalenus anticus muscle. As this vein passes inward, it receives, first, the companion veins of the suprascapular and transversa colli arteries. These veins usually unite before entering the subclavian; but nccasionally enter by separate openings. The 
external jugular vein, which sends a branch to the cephalic, also empties into the subclarian. The manner of its entrance will be described later. The subclavian then receives the companion branches from the thyroid axis, internal mammary, and vertebral arteries, and terminates behind the sternoclavicular articulation by uniting with the internal jugular to form one of the innominate veins.

\section{The Veins of the Head and Neck.}

The Veins of the Head consist of a set of channels found in the cranium, formed by divisions in the dura mater, and called "sinuses", while the veins of the neck are described under the name of "jugular"' veins (L. Jugulum-diminutive of jugum, the yoke-the throat), of which, however, there are three: the Internal, External, and Anterior Jugular. In addition to these jugular veins there are smaller veins, such as the thyroid and the vertebral, which during a portion of their course are found lying in the neck. Certain veins are also found upon the face, which aid in forming the jugulars.

\section{The Veins of the Diploe.}

Ramifying between the tables of the bones of the skull are numerous veins called diploetic. They take the name of the region they occupy and finally converge to form trunks which open either into the sinuses of the interior or the veins of the exterior. Connecting the sinuses of the interior with the veins of the exterior of the head are several veins which pass through foramina such as the mastoid and parietal.

\section{The Sinuses of the Dura Mater.}

Superior Longitudinal

Straight

Cavernous

Superior Petrosal
Inferior Longitudinal

Lateral

Occipital

Inferior Petrosal

These Sinuses are formed by the uninn of veins found in two positions in the cranium: one set called the veins of the Diple lying between the two tables of which the bones of the skull are composed, while the other set, the Cerebral veins, 
are found ramifying over the surface and in the interior of the encephalon. The Cerebral Veins consist of two sets, superficial and deep. The superficial lie upon the surface of the brain, while the deep cerebral veins lie in the ventricular cavities of the brain, ultimately uniting to form two ventricular veins, the Venæ Galeni, which leave the interior of the brain through the great transverse fissure and terminate in the straight sinus.

\section{The Superior Longitudinal Sinus.}

The Superior Longitudinal Sinus commences at the foramen cæcum, where it communicales with the small veins of the nasal fossa. The groove along which it rnns may be discovered on the dried bones as occupying the mid-line of the frontal bone, and grooving equally the upper border of the two parietal bones. Leaving these bones, it appears on the occipital, grooves it in the mid-line, and passes backward to the torcular Herophili by the side of the anterior occipital protuberance, to diverge on one or the other side into one of the lateral sinuses. This sinus, like other sinuses of the dura mater, is not strictly speaking a vein : it is the space between two layers of the dura; and its resemblance to a vein consists only in two facts; viz, that it conveys venous blood, and that it is lined internally by the endothelial coat, which all blood-ressels contain. As it passes along the groove, this sinus receives the superficial cerebral veins.

\section{The Inferior Longitudinal Sinus.}

The Inferior Longitudinal Sinus is a small sinus, circular on cross-section (unlike the superior, which is triangular on cross-section). It commences near the anterior extremity of the falx major, follows the curvature of that subdivision of the dura mater, and passes bickward to the junction of the falx major with the tentorium cerebelli, where it empties in to the straight sinus.

\section{The Straight Sinus.}

The Straight Sinus runs in the triangular interval between the falx major and the tentorium, to terminate in one of the 
lateral sinuses. Before receiving the inferior longitudinal sinus, the straight sinus first receives the venae Galeni, coming from the ventricles of the brain.

\section{The Occipital Sinus.}

The Occipital Simus is the smallest of the cranial sinuses ; it commences by several small vein around the foramen magnum, and is situated in the attached margin of the falx cerebelli, communicating with the posterior spinal vein, and finally emptying into the torcular Herophili.

\section{The Cavernous Sinuses.}

The Cavernous Sinuses are found on the lateral aspect of the body of the sphenoid. They commence in front by receiving the opthalmatic veins from the sphenoidal fissure. The opthalmic veins, following the course of the opthalmic artery, pass over the sphenoidal fissure, and empty into the cavernous sinuses. These in turn, winding along the lateral aspect of the body of the sphenoid, pass to the junction of that bone with the petrous portion of the temporal, and there join with the petrosal sinuses. Stretching across between the cavernous sinuses are two small transverse vessels, which surround the pituitary body, one in front and the other behind. These together form the Circular Simus.

After the cavernous sinuses have received the superior petrosal and emptied into the inferior petrosal sinus on the side of the basilar process of the occipital bone, they are united by a small rein, extending between the two, which is called the Transicrse Simus.

\section{The Superior Petrosal Sinus.}

The Superior Pelrosul Simus is situated on the upper border of the petrous portion of the temporal. Its inner extremity is connected with the cavernous sinus, while its outer is connected with the lateral sinus, just as that sinus reaches the inner face of the mastoid portion of the temporal, and receives the mastoid veins.

\section{The Inferior Petrosal Sinus.}

The Inferior Pctrosal Simus occupies the groove between 
the posterior border of the petrous portion of the temporal and the inner half of the lower border of the occipital. It receives the cavernous sinus in front; and terminates behind by uniting with the lateral sinus.

\section{The Lateral Sinuses.}

The Lateral Simuses commence at the torcular Herophili, where the longitudinal sinus empties. The torcular is never situated on the mid-line of the body; but to one or the other side, more frequently to the right. The blood from the superior longitudinal sinus pours into the lateral sinus of the right side, while the current of blood from the occipital and straight sinuses usually passes into the opposite lateral sinus.

The lateral sinus of each side arches outward along the anterior face of the occipital bone; but leaves that bone to appear momentarily on the postero-inferior angle of the parietal. Leaving the parietal, it passes downward and inward in a curved direction across the internal face of the mastoid portion of the temporal, forming a deep depression in this bone, the fossa sigmoidea and finally at the posterior extremity of the jugular (the foramen lacerum posterius), it unites with the inferior petrosal sinus to form the internal jugular vein.

It will be seen, therefore, that the right internal jugular conveys the blood received from the superior lougitudinal sinus, which, in its turn, receives the superficial cerebral veins, while the left internal jugular conveys most of the blood carried by the venæ Galeni, and from this by the straight sinus, into the left lateral sinus. The veins of the base of the brain are about equally distributed by means of the petrosal, transverse and circular sinuses into the corresponding jugular veins.

It must never be forgotten that the veins of the diplœ communicate with the sinuses of the dura mater on the one hand, while on the other they communicate with the superficial veins of the cranium. These communicating veins are described as emissary veins, so that any infection of the tissues occupying the surface of the skull may readily pass along the course of the emissary veins into the interior of the skull, and so establish an inflammation of the brain itself. 


\section{The Internal Jugular.}

The Internal Jugular Vein, formed, as above described, by the union of the inferior petrosal with the lateral sinus, is a largely dilated aperture at the base of the cranium; but it quickly contracts to about twice the size of the internal carotid artery. At its origin it lies immediately behind the internal carotid artery, with the twelfth, or hypo-glossal, nerve behind it, and the ninth, tenth, and eleventh cranial nerves emerging in the interval between itself and the internal carotid. It then passes downward as an external relation of the internal carotid to the point at which that vessel is derived from the common carotid artery; then becomes a relation of the common carotid artery, and maintains that relation to the origin of that vessel behind the sterno-clavicular articulation, where it terminates by joining the subclavian vein to form the innominate vein of that side. In its course down the neck, the glosso-pharyngeal and hypoglossal nerves pass forward between the artery and the vein, to cross the front of the artery and cease to be relations of the vein. The eleventh, or spinal accessory, nerve passes behind the vein near the upper portion of its course; but the tenth continues an internal relation of the internal jugular throughout the entire course of that vein.

Its tributaries are the Facial, Lingual, Pharyngeal, Superior and Middle Thyroid Veins.

\section{The Vertebral Veins.}

The Verlebral Veins commence by tributaries at the upper part of the back of the neck, pass down ward in the foramina of the transverse processes, which transmit the vertebral artery; and the two satellites unite, one passing through the sixth and the other through the seventh transverse process, to form a single vein, which empties into the innominate vein of the corresponding side.

\section{The Veins of the Face.}

The Veins of the Face consist of numerous branches, which correspond to the arteries of the face. Of these, the Supra- 
orbital commencing on the forehead, communicates with the temporal vein, and joins the frontal to form the Facial vein. This, after receiving the Nasal, Superior Labial, Transverse Facial, and Inferior Labial, passes over the lower border of the jaw, about an inch in front of the angle, or opposite the internal border of the masseter muscle, receives a communication from the tempero-maxillary vein, and empties into the internal jugular.

\section{The Temporal Veins.}

The Temporal Veins commence on the lateral aspect of the sku11 by numerous tributaries, pass downward in an interval between the internal auditory meatus and the tempero-maxillary articulation, run into the substance of the parotid gland, and receive the internal maxillary vein, made up of tributaries accompanying branches of the internal maxillary artery, and behind the neck of the condyle of the lower jaw forms the tempero-maxillary vein.

This vein divides into two branches, one of which has been already alluded to as uniting with the facial vein to empty into the internal jugular, while the other unites with the posterior auricular to become the external jugular vein.

\section{The External Jugular.}

The External Jugular Vein, commencing in the substance of the parotid gland, receiving the greater portion of the blood from the exterior of the cranium and the deep parts of the face, runs down the neck in the direction of the fibres of the platysma myoides muscle, which covers it. It runs nearly parallel with the posterior border of the sterno-cleido-mastoid muscle until it reaches a point just above the clavicle, where it changes its direction, and passing downward and inward, empties into the subclavian vein.

\section{The Innominate Veins.}

The Imnominate Veins of the two sides differ in direction, though but little in formation.

On the right side the innominate vein has already been described as formed behind the right sterno-clavicular articu- 
lation by the union of the subclavian and internal jugular veins. On the left side the vein is formed in the same way; but usually the internal jugular lies to the outer front aspect of the left subclavian artery, instead of crossing directly orer the front, as is the case on the right side.

The innominate vein on the right passes downward and inward, parallel with and anterior to the innominate artery, and a little to the right side of that vessel. On the left, the innominate rein, also called the Great Transa'crse I'cin of the Veck, crosses successively the thoracic portions of the left subclavian and left common carotid arteries and the arteria innominata, and lying above and on a plane anterior to the transverse aorta. At the commencement of the transverse aorta the two innominate veins unite to form the Superior-I'cua Car'cl.

\section{The Superior Vena Cava.}

The Superior Vena Cara descends anterior and external to the ascending aorta, pierces the fibres of the pericardium, and enters the venous, or right, auricle of the heart on the upper front aspect of that organ.

Besides these veins there are several smaller veins which are found mainly in the thoracic cavity and lower portion of the neck.

Of these, the salcllite veins of the Internal Mammary Artery empty into the corresponding innominate vein.

The Inferior Thyroid Veins, accompanying the artery of the same name, pass likewise into the corresponding innominate veins.

The Superior Inlercostal Veins, returning the blood from the upper intercostal spaces, except the first, empty, the right one into the vena a\%ygos major, the left usually into the left innominate vein.

The Azygros Veins connect the superior and inferior vena cava. They are two in number, the right ayygros, or vena ayygos major, and the left azygos, or vena azygos minor.

\section{The Vena Azygos Major.}

The $A$ zyons Major commences in the abdominal cavity oppo- 
site the first or second lumbar vertebra, sometimes from the first lumbar vein, sometimes by a branch from the renal vein. It enters the thorax through the aortic opening of the diaphragm; and receiving branches from the lower six intercostal spaces, finally terminates by crossing the vertebral column opposite the sixth dorsal vertebra behind the thoracic aorta, and empties into the superior vena cava just before that vessel passes into the heart.

\section{The Vena Azygos Minor.}

Just before the azygos major crosses the vertebral column it is joined by the Vena Azygos Minor, which conveys the blood from the intercostal spaces between the superior azygos, already described as the Left Superior Intercostal, and the highest branches entering into the azygos minor. At its lower extremity this vessel frequently communicates with the inferior vena cava, and thus directly establishes a communication between the superior and inferior venæ cavæ. Occasionally, however, the communication consists entirely of a branch from the renal vein, which in its turn empties into the inferior vena cava, and thus indirectly establishes this communication.

\section{The Spinal Veins.}

The spinal veins consist of three sets, as follows:

\section{Dorsal Spinal Veins.}

The dorsal spinal veins are numerous veins forming a network on the exterior of the vertebræ.

\section{Meningo-Rachidian Veins.}

The meningo-rachidian are four veins perpendicular in direction, which lie within the spinal canal between the bone and dura mater, two in front and two behind.

\section{Medulli-Spinal Veins.}

The medulli-spinal are numerous veins ramifying beneath the arachnoid membrane of the spinal cord. 


\section{The Cardiac Veins.}

The veins of the heart are two, as follows:

\section{The Great Cardiac Vein.}

The great cardiac vein ascends in the anterior ventricular groove, winds around the left auriculo-ventricular groove and opens into the right auricle. The last inch of its course is known as the coronary sinus.

\section{The Posterior Cardiac Vein.}

The posterior cardiac vein is small and ascends in the posterior ventricular groove to epen into the great cardiac vein.

Des Anat-25 


\section{THE LYMPHATIC SYSTEM.}

The lymphatic system consists of numerous small vessels ramifying in nearly every tissue of the body, and of small, reddish, pea-like bodies called lymphatic glands, found at intervals along the lymphatic vessels. The lymphatic vessels are intended for the most part to remove from the tissues the detritus of assimilation, consisting mainly of unexpended plasma; consequently the course of their circulation is from the circumference towards the centre, and in their route they will be found to accompany the veins.

Wherever they are found the lymphatics consist of two sets, superficial and deep. In the extremities the superficial set is found just benæth the skin; the deep in and among the muscles. In the viscera the superficial set ramifies on the surface, while the deep is found in the structure. The greater portion of the lymphatics of the body converge to form one large vessel, the thoracic duct, which emplies in to the venous current near the heart.

\section{Thoracic Duct.}

The thoracic duct begins on the front of the body of the second lumbar vertebra by a considerable dilatation called receptaculum chyli, which, narrowing to a tube the size of a goose quill, ascends the front of the vertebral column behind the descending aorta, passes through the aortic opening of the diaphragm and continues upward as high as the fourth dorsal vertebra, where it inclines to the left and continues thence an oblique ascent behind the arch of the aorta to a point on a level with the seventh cervical vertebra, where it arches forward and downward and opens into the commencement of the left vena innominata. Opening into the commencement of the right vena innominata is another and much smaller lymphatic duct (ductus lymphaticus dexter) which conveys lymph furnished it by the right upper extremity and right side of the head and neck. 


\section{Lymphatics of the Lower Extremity.}

The superficial lymphatics of the lower extremity are found crowding upward in immense numbers along the course of the saphenous veins and, reaching the saphenous opening in the fascia lata, where the vein terminates near Poupart's ligament, they then pass through a set of lymphatic glands. The deep lymphatic vessels accompany the deep veins upward to the groin, where they are connected with a set of glanils, lying beneath the fascia lata and superficial muscles, called the deep lymphatic glands of the groin. Along the course of these vessels are found a few glands, some in the popliteal space and one on the front of the interosseous membrane of the leg. The 1 ymphatic vessels from the external organs of generation and from the abdominal parietes can be traced to a third set of glands, which form a chain along Poupart's ligament, lying superficial and above the glands around the saphenous opening.

All these vessels pass beneath Poupart's ligament to become the external iliac lymphatics which accompany the external iliac ressels and unite with the internal iliac lymphatics to form the common iliac lymphatics, which accompany the common iliac vessels and unite with those of the opposite side to form the lumbar lymphatics. Scattered at intervals along these vessels from Poupart's ligament are lymphatic glands. The lumbar lymphatics ascend along the aorta, constantly increasing in size by accessions from the abdominal viscera, and having passed through numerous glands, and lessened in number as they increased in size, they eventually open into the receptaculum chyli, which is so named from the fact that the lymphatics which it receives from the small intestine are, during the process of digestion, filled with chyle.

\section{The Lymphatics of the Upper Extremity.}

The lymphatics of the upper extremity, like those of the lower, consist of a superficial and a deep set. The superficial ascend in company with the superficial veins and the deep 
accompany the deep veins, both converging to the arm pit where there are some twelve or fifteen lymphatic glands. Besides the vessels from the upper extremity these glands also receive those from the chest wall and mammary gland. A few glands are found along the course of the lymphatics before they reach the axilla, some at the elbow and some along the brachial vessels; these, however, are small and unimportant. From the axillary glands the vessels proceed along the course of the subclavian vein, opening on the left side into the termination of the thoracic duct and on the right into the ductus lymphaticus dexter.

\section{Lymphatics of the Head and Neck.}

The lymphatics of the head and neck consist of vessels which have come from the exterior of the head and others which have converged from veins supplying the internal and external jugular veins, no lymphatics, however, being found in the substance of the brain.

These vessels descend along the external, and especially the internal, jugular veins and on the right side open into the ductus lymphaticus dexter while on the left they join the thoracic duct.

\section{The Lymphatics of the Thorax.}

The lymphatics accompanying the intercostal vessels open into the thoracic duct. Those from the right lung, some portion of the thoracic parietes, diaphragm and even some portion of the upper surface of the liver and the short border of the heart seek the ductus lymphaticus dexter; while those from the left lung, left side of the thorax and most of the heart join the thoracic duct. The vessels from the lungs pass through a set of glands situated around the bifurcation of the trachea called the bronchial glands. 


\section{THE CENTRAL NERVOUS SYSTEM.}

\section{Cerebro-Spinal Centres.}

The chain of nervous centres known as the Cerebro-spinal axis is divided into two portions, one contained in the spinal canal, and called the spinal cord, or Medulla Stinalis (L. Medius, middle), the other contained in the cranium and called the Brain, or Encephalon, the latter name including the brain and its membranes. Enveloping each of these portions and lining its containing cavity are three membranes, lying one within the other, called the Investing Membranes, or Meninges.

\section{The Membranes of the Brain.}

The membranes of the Brain are the Dura Mater (L. Hard mother), lying next to the cranial wall; the Pic Mater (L. Delicate mother), lying next to the brain; and the Arachnoid between the two.

\section{The Dura Mater.}

The Dura Mater is a grayish white, strong, fibrous membrane which lines the inner surface of the cranial wall, adhering closely to the bone, and supplying the place of an internal periosteum. Its internal surface is glistening and smooth because lined by the arachnoid, which performs the functions of a serous membrane. Besides lining the interior of the cranial cavity, the dura mater gives off septa, which passing between the different portions of the brain, serve to separate them from one another and to support the brain in its position, besides carrying blood-vessels which receive and return the circulation from the interior of the cavity. These processes are three in number: the Falx Major, or Falx Corcbri, the Tentorium Cerelelli, and the Falx Minor, or Falx Cerebelli.

The Fulx Major (L. Fulx, a scythe), or Fulx Cercbri, is a sickle-shaped process which lies in the great longitudinal fissure of the brain. It is attached in front to the crista galli and extends along the mid-line to the anterior occipital protuberance, and is attached to the superior surface of the tentorium along its middle line. Along its attachment to the 
cranium, the Falx major divides into two layers, which are attached to the margins of the groove found on the frontal, parietal, and occipital bones. Between these two layers is a triangular space, which lodges one of the important sinuses, the superior longitudinal sinus. The concave lower margin is hollowed out into a small canal, circular on cross section, which extends from the foramen caecum to the attachment of the falx major to the tentorium. This is called the inferior longitudinal simus.

The Tentorinm (L. Tendere, to stretch) commences at the posterior occipital protuberance, and passing transversely forward, extends into the great transverse fissure of Bichat separating the cerebrum from the cerebellum, serving to support the posterior lobes of the cerebrum and at the same time to bind the cerebellum in the cerebellar fossæ. Along its upper surface from before backwards is attached the posterior extremity of the falx major. Its posterior border, divided like the falx major into two layers, is attached along the margin of the lateral limbs of the occipital cross; and leaving this bone, passes forward on the superior border of the petrous portion of the temporal bone, at the apex of which it is joined by the concave free anterior border. This anterior border presents a horseshoe-shaped appearance, and when the two borders, anterior and posterior, meet with one another, they cross, so that the free border continues forward to be attached to the anterior clinoid process, while the attached border reaches the posterior clinoid process. Between these attachments is left an opening through which is transmitted the mid-brain. At the attachment of the posterior border is a sinus, a continuation of the great longitudinal sinus on one side, forming the lateral sinus. Where the tentorium is attached to the upper border of the petrous portion of the temporal is another sinus, the superior petrosal; and where the falx major is attached on the superior surface of the tentorium is found a third sinus, the straight simus passing backward to the torcular Herophili.

The Falx Cerebelli is a small narrow process of the dura, which extends from the margin of the foramen magnum 
uprard to the under surface of the tentorium to the posterior part of which it is attached, at the anterior occipital protuberance. It projects between the two lobes of the cerebellum, and serves to separate them from one another.

\section{The Arachnoid.}

The Arachnoid, like all serous membranes, presents a closed sac, one of its layers lining the inner surface of the dura, and being called the parietal layer, while the other invests the brain, from which it is separated by the pia, and is called the visceral layer. It does not dip into the sulci of the brain; but leaps from convolution to convolution, so that a space is interposed at various portions of the brain between the arachnoid and the pia mater. This space has received the hybrid name of Sub-Arachnoidean. It can be easily demonstrated at some portions of the brain, while at others it is scarcely perceptible. On the inferior surface of the brain, stretching between the two peduncles of the cerebrum, and covering the inter-peduncular space, a considerable distance exists between the pia and the arachnoid; and again in the great transverse fissure of Bichat the space can be easily made apparent.

\section{The Pia Mater.}

The Pia Mater is an extremely thin and very vascular membrane, consisting almost entirely of small interlacing blood vessels, held together by layers of connective tissue. It covers the entire surface of the brain; and not only does this, but dips into every sulcus and every depression of the brain. Thus, it passes through the great transverse fissure of Bichat, covering not only the cerebellum and the under surface of the cerebrum; but continuing through this fissure, it passes even into the interior of the brain itself, so that prolongations of the pia are found in the ventricles, in the interior of the hemispheres, and connecting with the blood vessels which perforate from the base of the brain and supply the structures in the interior of each hemisphere. It serves also in one portion of the brain to form the roof of one of the ventricles. An examination of the brain with its frequent elevations and depressions forming 
the convolutions and sulci would show that if this membrane were stripped off, its area would be enormously greater than the apparent area of the brain itself. Every sulcus into which the membrane dips forms a corresponding fold, so that it bears a close resemblance in its folds to a dress or other garment which has been taken out of a trunk.

\section{The Brain.}

The Encephalon is divided into four parts: the Cerebrum, the Cerebellum, the Pons Varolii, and the Medulla Oblongata, the Pons and its appendages being frequently described as the mid-brain.

The rueight of the entire brain varies with the period of life, with the sex of the individual, with the cause of death, the period after death at which the weight is taken, and with various other circumstances. The average weight is about forty-eight ounces for the male, and about forty-four or fortyfive for the female adult. The intellectuality of the individual bears scarcely any relation to the rveight of the brain, some of the heaviest brains having been those of people by no means distinguished for ability, while comparatively small brains have been found in people of a high order of intelligence. The intellectuality varies more with the variety and number of the convolutions, and consequently with the amount of "gray matter," than it does with the actual bulk of the brain.

The Cerebrum is seven or eight times the size of the rest of the brain, presenting an uneven, convoluted surface. When closely examined these convolutions are seen to be dissimilar on the two sides of the brain, nor do we ever find any two brains with precisely the same convolutions. Certain depressions which separate these convolutions from one another, from the fact that they appear at an early stage of foetal life, are called "primary fissures," and from these primary fissures we get the great subdivisions of the cerebrum. These primary fissures are the Great Longitudinal Fissure, the Great Transverse Fissure of Bichat, the Fissure of Sylvius, the Fissure of Rolando, and the Parieto-occipital Fissure. The 
great longitudinal fissure serves to separate the two lobes of the cerebrum; the transierse fiissure of Bichat to separate the cerebrum from the cerebellum; the fissure of Sylizus to separate the frontal from the tempero-sphenoidal, and by its horizontal limb, the parietal from the tempero-sphenoidal; the fissure of Rolando to separate the frontal from the parietal ; while the paricto-occipital of course separates the parietal and occipital lobes.

\section{The Convolutions of the Cerebrum.}

In studying the convolutions of the cerebrum we begin with the five principal, or primary, fissures.

The Great Longitudinal Fissure commences on the base of the brain, extends upward between the two hemispheres, turns backward toward the posterior extremity, and cutting between the two hemispheres behind, separates them from one another. It will therefore be seen that the two hemispheres of the cerebrum are united in the middle, but are separated in front and behind. The bond of union between them, the corpus callosum, extends further in front than behind and is thicker there than in front.

The Fissure of Sylizus. Commencing on the base of the brain near the great longitudinal fissure, or, more correctly, at the anterior perforated spot, is the fissure of Sylvius. It is first directed outward, forming a means of separation between the frontal and tempero-sphenoidal lobes. On leaving the base of the brain to appear on the outer surface of the hemisphere, changes its direction and passes upward and backward, forming what is called the horizontal limb of the fissure of Sylvius, which separates the tempero-sphenoidal helow from the parietal above. The horizontal limb of the fissure of Silvius extends backward as far as the commencement of the occipital lobe, or the junction of about the posterior one-fifth with the anterior four-fifths of the cerebrum. Just as the main fissure reaches the outer surface of the hemisphere, the vertical $\lim b$ is griven off, which ascends in the convolutions of the frontal lobe, forming in this situation an overhanging apjearance, which is described as the Operalum (L. Operire, to cover.) 
The Fissure of Rolando is situated about the middle of the outer surface of either hemisphere. It begins near the longitudinal fissure and runs first downward and forward about one-half of its course. It then makes an abrupt knee-like bend; and passing nearly vertically downward, terminates near the division of the fissure of Sylvius into its horizontal and its vertical limbs.

The Parieto-occipital Fissure is but slightly seen on the outer surface of the hemisphere, the main portion of the fissure lying on the inner face. It commences beneath the gyrus fornicatus opposite the splenium of the corpus callosum; and passes upward and forward to emerge on the outer surface of the hemisphere, just in the great longitudinal fissure.

By these primary fissures the hemisphere is divided into five lobes, frontal, parietal, occipital, tempero-sphenoidal, and central, or island of Reil.

The Frontal Lobe is all of that portion of the brain situated in front of the fissure of Rolando, hence it would be bounded above by the great longitudinal fissure, on the outer surface, behind, by the fissure of Rolando, on the lower surface, behind, by the main portion of the fissure of Sylvius. It should be noted that the inner face of the various lobes of each hemisphere is not described along with the external and inferior faces, the reason being that the convolutions and fissures on the internal face do not confine themselves to lobes, but extend in many cases throughout the entire length of the hemisphere.

The Parietal Lobe is bounded above by the great longitudinal fissure, in front by the fissure of Rolando, below by the horizontal limb of the fissure of Sylvius, and a line connecting this with the lower end of the superior occipital sulcus, and behind by the parieto-occipital fissure and a line drawn in continuation of that sulcus. This fissure lies mainly on the inner face of the hemisphere, so that on the outer face, the convolutions of the parietal become continuous with those of the occipital lobe.

The Occipital Lobe is cut off on its inner face by the Parieto-occipital fissure from the parietal lobe, this fissure being its anterior boundary. Above it is bounded by the longitudi- 
nal fissure, while below its convolutions run into the convolutions of the parietal and tempero-sphenoidal lobes, there being no fissure to form a separation.

The Temporo-sphenoidal Lobe is that portion which lies in the middle fossa of the skull. On the inferior surface of the base of the brain it is bounded in front by the fissure of Sylvius ; externally, it is bounded above by the horizontal portion of the fissure of Sylvius; while posteriorly it blends around this horizontal limb of the fissure of Sylvius with the occipital and parietal lobes.

The Island of Reil is a cluster of five or six convolutions, situated in the bifurcation of the fissure of Sylvius; and hidden under the fused lower extremity of convolutions which belong to the frontal and parietal lobes.

The Coniolutions and Sulci of the Frontal Lobe are usually described as consisting of two sets, those found on the external surface, and those on the inferior surface, which is frequently described as the Orbilal Lobe. The subdivision is unnecessary, the convolutions being continuous with one another. In brains with a small number of convolutions it will be seen that in front of the fissure of Rolando, and running parallel with it, is a sulcus of the same character, but of less depth. This is called the precontral sulcus. It commences near the bifurcation of the fissure of Sylvius and passes upward nearly to the great longitudinal fissure. This sulcus cuts off a convolution bounded in front by the precentral sulcus and behind by the fissure of Rolando, which is known as the ascending fromlal convolution. Two small sulci extend forward from near the precentral sulcus. Sometimes they run into the precentral sulcus; but as a rule they do not. These extend straight forward to the anterior extremity of the frontal lobe, cutting off short convolutions, which are known from above downward as first, second, and third frontal convolutions, or occasionally as supcrior middle, and inferior frontal convolulions. If these convolutions be traced around to the inferior surface of the frontal lobe, it will be seen that here also are three convolutions, sometimes described as the first, second, and third ortilal convolutions, numbered 
from within outward and backward; but in reality the continuation of the first, second, and third frontal convolutions. On the orbital surface, close to the great longitudinal fissure, is a narrow but deep sulcus, which lodges the olfactory nerve. External to this is another sulcus, less well-marked, which is the external boundary of the first orbital convolution, the continuation of the first frontal. The second sulcus, marking off the second and third orbital convolutions, or middle and inferior frontal convolutions, runs transversely, and is usually divided into two or more limbs, so that the second orbital convolution lies in front of the third.

The ascending frontal convolution begins below near the bifurcation of the Sylvian fissure, and is connected, around the lower end of the precentral sulcus, with the third frontal convolution, forming a part of the operculum. Generally, also, it is connected, around the lower end of the fissure of Rolando, with the ascending parietal convolution; and these two convolutions are nearly always united around the upper end of the Rolandic fissure.

The first, or superior, frontal convolution is limited above by the longitudinal fissure and below by the first frontal sulcus. It passes directly forward from the precentral sulcus, around the upper end of which it is connected with the ascending frontal convolution, and, winding around the anterior extremity of the frontal lobe, becomes continuous with the so called first orbital convolution. On its orbital surface this convolution is marked by a deep antero-posterior sulcus-the olfactory.

The second, or middle frontal convolution is bounded above by the first, and below by the second frontal sulcus. It is shorter than the preceding convolution, with which it fuses around the end of the first sulcus. Passing around the front of the frontal lobe, it becomes continuous with the second orbital convolution, which occupies the antero-external aspect of the orbital surface of the frontal lobe.

The third, or inferior, frontal convolution is cut off from the second by the second frontal sulcus, but fuses, around the 
posterior extremity of that sulcus, with that convolution. It is very short and does not reach the anterior extremity of the hemisphere, but winds around its outer aspect to become continuous with the third or posterior or bital convolution.

The Conzolutions of the Purietal Lobe are the Ascending Parietal, the Superior Parietal, and the Inferior Parietal.

The Ascending Parietal is cut off by the intra-parietal sulcus.

This is one of the most variable sulci on the outer surface of the hemisphere. It consists of three limbs, which may or may rot be joined together. Commencing above the fissure of Sylvius, it passes upward and backward parallel with and behind the fissure of Rolando to near the great longitudinal fissure. From a little above its centre a branch passes nearly directly backward, separating the superior from the inferior parietal convolutions. The vertical portion of this sulcus is named the Post-central; the whole is called the Intraparietal Sulcus. This may consist of three separate and distinct sulci, or any two of these may be united, and the third one exist as a separate sulcus.

That portion of the lobe which is bounded in front by the fissure of Rolando, and behind by the ascending portion of the post-central sulcus is called the ascending parietal convolution. That portion between the great longitudinal fissure above, the horizontal portion of the post-central sulcus below, and the external portion of the parieto-occipital fissure behind, is called the superior parietal convolution. That part which lies between the horizontal limb of the fissure of Sylvius below, and the intra-parietal sulcus above and in front, is called the inferior parietal coniolution. This inferior parictal convolution is frequently divided by a small secondary sulcus into two portions, an anterior lying above the fissure of Sylvius, called the supramarginal, and a posterior, which extends around the horizontal limb of the fissure of Sylvius and blends with the occipital lobe, called the angular syms, or convolution.

It will be seen that the fissure of Rolando reaches neither the great longitudinal fissure nor the fissure of Sylvius, hence 
around the two extremities of this fissure the ascending frontal and ascending parietal convolutions communicate with one another. At the lower extremity of the fissure of Rolando the ascending frontal convolution communicates with the ascending parietal, and also with the third frontal convolution. That portion which connects with the third frontal convolution lies in the interval between the two limbs of the fissure of Sylvius, and forms the overhanging portion of brain substance, the Operculum, which conceals the Island of Reil.

The Convolutions of the Occipital Lobe are three in number, superior, middle, and inferior. These convolutions are separated by two sulci, called the superior and middle occipital sulci.

These sulci are indistinct and poorly marked, each extending backward on the occipital lobe, the convolutions being named from above downward, First, Second, and Third, or Superior, Middle, and Inferior. The superior occipital convolution is connected with the parietal lobe; the middle and inferior with the temporo-sphenoidal lobe.

The Convolutions of the Temporo-Sphenoidal Lobe. This lobe is divided by three sulci into five convolutions, though two of these convolutions are found on the inner surface of the hemisphere. The two sulci found on the outer surface are the superior, or parallel sulcus and the middle, or second temporal, sulcus. The superior, or parallel, sulcus receives its name from being parallel with the fissure of Sylvius; the second, or middle temporal, sulcus is smaller and much less distinctly marked.

The first temporo-sphenoidal convolution is bounded above by the horizontal limb of the fissure of Sylvius, and below by the parallel sulcus. At its posterior extremity it is continuous with the angular gyrus of the parietal lobe. The second temporo-sphenoidal convolution lies between the parailel sulcus and the second temporal sulcus, and is continuous posteriorly with the angular gyrus of the parietal and the second occipital convolution.

The third temporo-sphenoidal, 1ying below the second tem- 
poral sulcus, is continuous posteriorly with the third occipital convolutton.

The Island of Reil. On reaching the operculum a group of five or six convolutions (though as few as three have been observed)are found lying in the interval between the diverging limbs of the fissure of Sylvius. These convolutions constitute the island of Reil. The sulciare only slight depressions, and the convolutions slight elevations. They all run in the same direction, upward, backward, and inward.

\section{Sulci and Convolutions on the Internal Face of the Hemisphere.}

The Calloso-marginal Sulcus commences near the base of the brain beneath the termination of the corpus callosum. It runs at first forward, following the rostrum of the corpus callosum, winds around its genu, thence backward, parallel with the body of the corpus callosum, until it reaches the pnsterior third of the brain, when it abruptly turns upward and passes to the great longitudinal fissure.

The Parieto-occifital, the second sulcus found on the inner face, begins at the splenium of the corpus callosum, and passes upward and back ward to terminate on the external face of the hemisphere.

The Calcarine Fissure, or Sulcus, is the third of the sulci found on the inner face. It commences by a forked extremity near the end of the occipital lobe, and runs nearly directly forward, with a slight inclination downward; and joins the paricto-occipital sulcus at an acute angle.

The Collateral Sulcus. The fourth is a long sulcus which extends across the inner face of the temporo-sphenoidal lobe, separating the fourth and fifth temporo-sphenoidal convolutions. It is called the Collateral Sulcus.

The Denlute Sulcus, the last one, passes beneath the splenium of the corpus callosum to terminate near the commencement of the fissure of Sylvius.

Of these sulci, the calcarine, the collateral, and the dentate all make appearances in the cavities found in the interior of the brain. The calcarine sulcus forms the Hippocampus Minor, 
or Calcar Avis (L. A bird's spur), in the posterior horn of the lateral ventricle. The collateral sulcus forms the eminentia collateralis; and the hippocampal, or dentate, sulcus forms the Hippocampus Major in the descending horn of the lateral ventricle.

These sulci divide the internal surface into a marginal convolution, the gyrus fornicatus, the quadrate lobe, the cuneate, the uncinate gyrus, and the fourth and fifth temporo-sphenoidal lobes.

The Marginal Convolution is the inner face of the frontal lobe. It commences at the anterior perforated space, follows the windings of the calloso-marginal sulcus, then runs forward beneath the rostrum of the corpus callosum, winds upward around the genu, and passes backward until the calloso-marginal sulcus turns upward to the great longitudinal fissure, where the convolution terminates, and is separated from the parietal lobe by the end of this sulcus. It will be seen that this convolution corresponds to the inner face of the first orbital, the first frontal, the ascending frontal and ascending parietal convolutions.

The Gyrus Fornicatus. Below the marginal convolution and between the calloso-marginal sulcus and the corpus callosum is the Gyrus Fornicatus, or convolution of the corpus callosum. It follows the same course as the preceding sulcus until it reaches the posterior extremity of the corpus callosum, where it blends with the quadrate lobe, winding around the splenium of the corpus callosum. Here it changes its name to become the uncinate, or hypocampal, convolution.

The Quadrate Lobe is bounded in front by the upturned portion of the calloso-marginal sulcus, behind by the parietooccipital sulcus. Above, it is limited by the great longitudinal fissure; and below it blends with the gyrus fornicatus. It is the internal face of the superior parietal convolution.

The Cuneate Lobe (L. Cuneus, a wedge) is bounded in front by the parieto-occipital sulcus, and below by the calcarine sulcus; and is the internal face of the first and second occipital convolutions. 
The Hippocampal, or Uncinate, Lobe (L. Uncinus, hooked) is a continuation down ward and forward of the gyrus fornicatus. It is bounded above by the dentate fissure, and below by the collateral fissure. It extends as far forward as the commencement of the fissure of Sylvius; and innerwards to the point where the gyrus fornicatus commences. Here it turus backward upon itself, forming the hook-shaped appearance which gives the convolution its name. It is the fifth temporo-sphenoidal convolution, the fourth temporo-sphenoidal lying beneath the collateral sulcus.

In addition to these convolutions, when the hemispheres are separated by cutting through the corpus callosum we of course see the fibres composing the corpus callosum and the inner face of the optic thalamus.

\section{The Base of the Brain.}

The lower surface, or base, of the brain, is very irregular in outline, because it is fitted into the fossæ of the skull. Anteriorly, on either side, is seen the orbital face of the frontal lobe which is somewhat triangular with the base backward and apex forward. Behind, and prolonged downward to fit in the middle fossa, is the temporo-sphenoidal lobe, and, behind this, the occipital, also triangular with its base forward, flattened by pressure on the tentorium. The numerous appearances found on the base of the brain may be best studied by dividing them into two sets, viz: those which occur in pairs, and lie on either side of the mid-line; and those which are single and occupy the mid-line.

Beginning on the mid-line in front and passing backward, we find the following appearances, viz: The great longitudinal fissure, with the rostrum of the corpus callosum seen at its bottom; the optic chiasm; the pituitary body, infundibulum and tuber cinerium leading the one to the other; the posterior perforated space; the transverse fibres of the pons; the slight median depression on the pons; the fissure between pons and medulla, and the anterior median fissure of the medulla.

The appearances occurring in pairs and described on one side only, are: The orbital face of the frontal lobe with its two sulci

Des Anat-26, 
and three convolutions; the peduncles of the corpus callosum; the groove or sulcus for the olfactory nerve and, the olfactory bulb, and nerve, with the three roots of the latter; the beginning of the fissure of Sylvius; the optic nerve, internal to the roots of the olfactory; the anterior perforated space; the optic tract crossing the crus cerebri, and, internal to this, a corpus albians; and, from this point backward, the roots of the cranial nerves.

The longitudinal fissure and the corpus callosum are elsewhere described. The optic chiasm is formed by the union of the two optic tracts and gives off the optic nerves. It will be described with these nerves. The pituitary body, or hypophysis cerebri, is situated in the sella Trucica, bound down by a process of the dura mater. It consists of two lobes, anterior and posterior, separated by a fibrous lamina, the latter, during fotal life, communicating with the third ventricle through the infundibulum. The infundibulum is a small tube of grey matter attached above to the lamina cineria and below to the infundibulum. It always communicates with the third ventricle. The lamina cineria, or tuber cinerium, is a small mass of grey matter which forms part of the floor of the third ventricle. It is connected with the upper face of the optic chiasm and extends back to the carpora albicantia.

The posterior perforated space is in the triangle formed by the crura on either side and the pons behind. It is made by a number of small vessels which here pass into the optic thalami. The remaining appearances require no further description.

The olfactory bulb (usually destroyed in removing the brain) is an oblong rounded mass of grey matter which lies on the cribriform plate of the ethmoid and gives off numerous filaments to the nose. This bulb contracts to become the olfactory nerve which leads back to the fissure of Sylvius and divides into its three roots, which are described with the other cranial nerves.

The anterior perforated space lies in the commencement of the Sylvian fissure. Antero-internally it is bounded by the optic 
nerve; internally, by the optic chiasm, postero-internally, by the optic tract. Externally are the Sylvian fissure and the outer root of the olfactory nerve, while the middle root runs near the centre of the space. It is crossed by the peduncles of the corpus callosum, is connected with the lamina cinerium, lies beneath the corpus striatum, and receives its name from being perforated by numerous small vessels which pass into the latter body. The optic tract will be described with the cranial nerves. The crus cerabri is a round cord which serves to connect the pons with the cerebrum. It is about three fourths of an inch in length and is composed of two sets of longitudinal white fibres, superficial and deep (or anterior and posterior) and an intermediate bundle of gray matter called locus niger. The superficial white fibres pass through the pons from the anterior pillars of the medulla to the internal capsule. They form what is called the crusta. The deep longitudinal fibres, or tegmentum, pass from the olivary body and lateral and posterior columus of the medulla through the pons to the optic thalamus and cerebral cortex.

Situated at the base of the brain is a six-sided space called the inter-peduncular space. It is bounded in front, on the mid-line, by the optic chiasm; behind, on the mid-line, by the transverse fibres of the pons; antero-laterally, by the optic tract; postero-laterally, by the crura cerebri. The corpora allicantia or mamillaria are a pair of small rounded bodies, situated just behind the tuber cinerium, formed by the anterior pillars of the fornix which pass down to the base of the brain and then turn upward to reach the optic thalami. It is the knuckle formed by reversing their course which forms the corpora albicantia. This space contains the pituitary body, infundibulum, lamina, or tuber, cinerium, corpora albicantia, or mamillaria, and the posterior perforated spot. It, and its contents, form the floor of the third ventricle.

\section{Structure of the Cerebrum.}

When a horizontal slice, an inch or so thick, is removed from the upper surface of one hemisphere, the cut surface shows a central, oval, white portion surrounded, on the exterior, by a 
serrated grayish border; this is known as the centrum ovale minus. When both hemispheres have been cut to the same level, and this level corresponds to the depth of the central portion of the longitudinal fissure, two centra ovalia minora are produced, connected in the centre by transverse fibres, forming a connecting band, called the corpus callosum; and the whole appearance, formed by the two centra ovalia minora and the corpus callosum, is called the centrum ovale majus.

\section{Corpus Callosum.}

The corpus callosum consists almost entirely of transverse fibres passing from one hemisphere to the other. Upon its upper surface there is a shallow groove, extending from before backward along the middle line, called the raphe; bordering each side of the raphe is a slight ridge produced by a bundle of longitudinal fibres, the two ridges being called the nerves of Lancisi. External to these are seen a few other longitudinal fibres, producing slight ridges which are called the lateral longitudinal striæ. When the corpus callosum is bisected longitudinally it is seen to be about four inches long and to bend vertically downward both in front and behind. The bent, rounded, posterior extremity is called the splenium, or pad, or bulb, the central portion the body, and the anterior bent portion the genu, which turns backward beneath the body for a short distance under the name of the rostrum. The rostrum divides into two cords called peduncles, which pass downward and backward to the base of the brain to terminate at the anterior perforated space. Contained in the substance of the brain, between its base and the corpus callosum, is an irregular cavity divided into several parts, two of which are known as the lateral ventricles, while the space is spoken of as the general ventricular cavity.

\section{The General Ventricular Cavity.}

The Ventricular Cavity is a space found in the interior of the brain, divided into two lateral, or first and second ventricles, and third and fourth ventricles, these cavities all communicating with one another by means of small foramina. 
The general ventricular cavity exists between the corpus callosum and velum interpositum above, the interpeduncular space at the base of the cerebrum, and the upper surface of the pons varolii and the medulla oblongata below. The lateral ventricles exist in the two hemispheres of the cerebrum, separated from one another on the mid-line by a thin septum. The third rentricle lies below the two lateral ventricles, and the fourth is below and behind the lateral ventricles, occupying a position on the medulla and the pons.

\section{The Lateral Ventricles.}

The right is usually described as the first, and the left as the second, lateral ventricle. The corpus callosum forms a part of the roof of the ventricular cavity; and upon its removal, the floor, formed of various objects, is brought into view. Each lateral ventricle consists of a body, and three extensions, called the horns of the ventricle, anterior, posterior, and middle. The entire cavity is roofed over in part by the corpus callosum, and in part by the frontal, parietal, occipital, and tempero-sphenoidal lobes. The inner wall is formed in part by a portion of brain tissue called the Septum Lucidum (L. Transparent Septum). The outer wall is the point of junction of the corpus callosum with the fibres of the cerebrum, while the floor is composed of a large number of objects, which will be enumerated later. 'The body' of the ventricle is all of that portion which exists between the anterior horn and the middle and posterior horns. Its upper boundary is the lower surface of the corpus callosum; its inner boundary is, in front, the septum lucidum, and, behind that, the junction of the fornix and the corpus callosum; its outer wall is the junction of the corpus callosum with the fibres of the cerebrum. The floor from before backward is made up of the corpus striatum, the tenia semicircularis, or horny band of Tarinus, the optic thalamus, the choroid plexus, and the fornix. Each of these requires a separate description.

The Corpus. Strialum (L. Striatus, srooved or furrowed) is an oblong, or pear-shaped, gravish mass, with its large end in front. It is divided into two portions, called the intra-and 
extra-ventricular portions, the former being also known as the caudate mucleus and the latter as the lenticular moleus. Between these two, on the outer wall of the ventricle, is a portion of white matter, called the internal capsule. External to this comes the extra-ventricular portion, the nucleus lenticularis (L. lense-shaped); and on the outer face of this again is a mass of white matter, called the etxernal capsule. The white matter which descends between the two portions of the corpus striatum, and on the outer side of the nucleus lenticularis, transmits impulses from the surface of the hemisphere to the medulla oblongata, and through that to the spinal cord.

The Tenia Semicircularis is a rounded cord, which lies in the groove between the corpus striatum and the optic thalamus . It may be traced into the descending horn of the lateral ventricle, where it joins with the anterior pillar of the fornix; and thence backward into the roof of the descending horn, where it terminates in a projection of gray matter, the Corpus Amygdalce.

The Optic Thalamus is a mass of gray matter which projects into the floor of the body of the lateral ventricle, and forms the lateral walls of the third ventricle. It will be described in connection with that ventricle.

The Fornix (L. Fornix, an arch, or vault) is a longitudinal band of white matter lying beneath the corpus callosum; and blended with its fellow of the opposite side behind, but separate in front. Behind it thins out into a broad, flattened band, the outer edge of which is called the corpus fimbriatum (L. Fimbria, an edge, or border). It descenls into the floor of the descending horn, covering the projection in that space known as the hippocampus major. Between the two posterior pillars of the fornix transverse fibres are stretched, which from their supposed resemblance to a harp have given the name of lyra to this portion of the fornix. These posterior fibres blend with the white matter forming the hippocampus major. Anteriorly the two pillars descend to the base of the brain, not connected by transverse fibres, and pass downward to the corpora mamillaria, where they reverse their course and turn upward, 
to be lost in the optic thalamus of the corresponding side. The anterior pillars of the fornix are connected with the peduncles of the pineal gland, and receive fibres from the septum lucidum.

The Choroid Plexus passes along the edge of the most internal object in the ventricle, the fornix. It is a mass of blood ressels arranged in the folds of the pia mater, which here projects into the interior of the brain. These vessels enter the descending horn of the lateral ventricle, pass upward through it, pass through the body of the ventricle, and continue their course into the descending horn, at the bottom of which other small vessels unite with them. The choroid plexus of each side passes beneath the body of the fornix, unites with the choroid plexus of the opposite side, and forms the true roof of the third ventricle. From the under surface of the fornix it passes backward through the transverse fissure of Bichat, joins with the velum interpositum, forming there a portion of the roof of the fourth ventricle; and becomes continuous with the general pia matral covering of the brain.

Of the three horns of the lateral ventricle the descending is the largest, the anterior intermediate in size, and the posterior the smallest.

The Anterior IIorn passes downward, forward, and outward, curving around the anterior extremity of the corpus striatum; and being entirely contained in the frontal lobe of the brain.

The Posterior Horn is curved like a half-bent finger, or cockspur, extends backward into the occipital lobe; and presents on its floor a slight projection, the hippocampus minor, or calcar avis, which is formed by the calcarine sulcus already described, on the inner face of the hemisphere.

The Desconding Horn pursues a tortuous course. It winds around the optic thalamus, passing first outward and backward, then downward and forward; and then, asain changing its direction, it passes downward forward and inward, to terminate near the extremity of the temporo-sphenoidal lobe. This horn of the ventricle is found first in the parietal lobe; but the major portion of it is in the temporo-sphenoidal lobe. 
At the junction of the descending and posterior horns is a slight eminence, frequently absent, called the eminentia collateralis or pes accessorius. Starting from this is a prominent projection, which follows the curvature of the horn itself, is formed by the dentate, or hippocampal, sulcus, and is called the corm ammonis, (L. a ram's horn), or hippocampus major, and terminates in the irregular projections, marked by intervening elevations and depressions, called the pes hippocampi. Lying upon the hippocampus major, and blended with it, is the thin lateral edge of the fornix, called the corpus fimbriatum, while rising behind this is that portion of the choroid plexus which passes into the descending horn. The gray matter in the descending horn covering the edge of the hippocampus major is the investing gray matter from the dentate sulcus, and is known as the fascia dentata.

At the junction of the anterior horn with the body of the lateral ventricle an opening is observed upon either side, which communicates with the third ventricle, and through which the choroid plexus of the lateral unites with the choroid plexus of the third ventricle. This opening is known as the foramen of Munro.

It will be noticed that the descending horn of the lateral ventricle follows the course of the optic thalamus, and hence that that body forms the roof of the descending horn throughout its extent.

\section{The Third Ventricle.}

The Third Ventricle of the Brain is bounded above by the choroid plexus, above which is found the under surface of the body of the fornix. Below its floor slopes from behind downward and forward; and is made up of the structures which are included in the interpeduncular space. The lateral walls are formed by the optic thalami. Instead of a posterior wall there is an openeng, the acqueduct of Sylvius, or the iter a tertio ad quartum ventriculum. The anterior wall is formed by a small bundle of gray matter, which is called the anterior commissure of the brain (L. committere, to join). This anterior commissure is found at the point where the two frontal lobesare 
nearly in contact, and consists of an interchange of fibres be$t$ ween the frontal lobes. It lies just in front of the anterior pillars of the fornix. Stretching across from the optic thalamus of one side to the same body of the opposite side is a second commissure, which is called the middle, or soft, commissure of the brain. This consists of the interchange of fibres between the optic thalami, and is generally broken in the removal of the brain. The posterior commissure, or third set of communicating fibres found in the third ventricle, extends between the posterior extremities of the two optic thalami, and consists of white fibres, the central one being gray, these fibres forming one of the boundaries of the acqueduct of Sylvius. Above the acqueduct of Sylvius is a smail reddishgray body, the Pineal Gland (L. Pinea, a pine cone), from which proceed two processes of gray matter, called the peduncles of the pineal gland. These peduncles pass forward on the upper inner face of each optic thalamus to terminate by blending with these bodies. The two unite behind in the commissure of the peduncles of the pineal gland.

An examination of the third ventricle will show that there are four opcnings communicating with other cavities of the brain. Two are found upon its antero-lateral wall, the foramina of Munro; one in its posterior wall, the acqueduct of Sylvius; and the fourth in the flonr, the Infundibulum. It will be remembered that at the base of the brain, occupying the anterior portion of the interpeduncular space was seen the pituitary body, the infundibulum, the tuber cinereum, and the lamina cineria. Through the last three pass the small opening which leads from the floor of the third ventricle downward to the pituitary body.

The formina of Munro are bounded above and behind by the anterior pillars of the fornix, in front by the anterior commissure. Through these two openings the choroid plexus of the lateral ventricles descends into the third. They present a somewhat $Y$-shaped appearance, the tail of the $Y$ being represented by the union of the choroid plexuses, while the two arms are represented by the foramina of Munro. Thus while the two lateral ventricles communicate with one another, the 
communication is indirect. In order that fluid may pass from one lateral ventricle to the other it is necessary to pass from the right lateral ventricle into the third ventricle, down to the tail of the $Y$; then to ascend through the left foramen of Munro along the second arm of the $\mathrm{Y}$ into the left lateral ventricle.

The Acqueduct of Sylvius, the opening in the posterior wall, passes downward and backward, running beneath the four projections of brain substance, called the corpora quadrigemina (L. fourfold bodies), and pisses into the superior angle of the fourth ventricle. The communication thus established shows the general ventricular cavity to be continuous, the right and left lateral communicating through the intervention of the third and the foramina of Munro, the third in its turn communicating through the acqueduct of Sylvius with the fourth, and the fourth again by the foramen of A rantius communicating with the ventricular cavity found in the spinal cord.

\section{The Fifth Ventricle.}

The Fifth Ventricle is described in the brain as consisting of the minute space between the two layers of the septum lucidum. It should be borne in mind, however, that the fifth ventricle has no connection with the general ventricular cavity; but that the brain being developed from two separate centres, one on either side of the body, unites in the septum lucidum to form the division between the two lateral ventricles. The space between the two layers of which this septum is composed is spoken of as the fifth ventricle ; and it therefore bears no resemblance to the general ventricular cavity.

The fourthientricle, rising behind the pons and the medulla will be described in connection with these portions of the brain.

\section{The Medulla Oblongata.}

Extending from the foramen magnum, or upper border of the atlas to the posterior border of the pons Varolii, is found the medulla oblongata, thus establishing connection between the spinal marrow and the brain. It is conical in shape, with the larger end above and forward, and is about one and one-fourth inches in length. Passing down its centre in front is the ante- 
rior median fissure, and similarly marking it behind is the posterior median fissure; these though not entirely bisecting it, serve to indicate its division into lateral symmetrical halves. Each half is subdivided into four portions by three longitudinal grooves. Lying beside the anterior median fissure, and separated by it from its fellow, is the portion called the corpus pyramidale; just behind this, slightly more bulging and separated from it by a fissure, is a second portion called the corpus olivare, being visible for about half an inch only. Behind this a third portion called the corpus restiforme, separated by a groove from the corpus olivare in front and by another slight groove from the fourth portion, the posterior pyramid, wihch lies behind the corpus restiforme, beside the posterior median fissure by which it is separated from its fellow. The two posterior pyramids are small below, but as they are followed up they suddenly dilate into a bulbous enlargement, and again as suddenly decreasing, separate as they ascend and leave between them an angular interval called the calamus scriptorius-writer's pen. When the medulla oblongata reaches the pons Varolii it seems, on superficial veiw, to stop short, but if the surface fibres of the pons, which are transverse in direction, are peeled off, it is seen that the pons, in its centre, consists of vertical, or longitudinal fibres, which are continuous with the fibres of the medulla oblongata below, and, being gathered into two bands, emerge from its anterior border as the two crura cerebri.

\section{The Fourth Ventricle.}

The Fourth Ventricle is diamond-shaped, having a superior and an inferior angle and two lateral angles. The inferior angle is formed by the diverging posterior pillars and the restiform bodies of the medulla; the superior angle being formed by the peduncles of the cerebellum, The two lateral ancles are formed by the junction of the superior cerebellar peduncles with the inferior. The superior angle is formed by the divergence of the superior peduncles; and the inferior angle by the divergence of the inferior peduncles.

It the superior angle is seen the lower opening of the 
acqueduct of Sylvius; at the inferior angle is seen the opening of the ventricle of Arantius.

The roof of the fourth ventricle is formed above by that small portion of gray matter which stretches across from the peduncle of one side to the peduncle of the opposite side, and which is called the superior medullary velum, or more frequently, the Valve of Vieusscns. The lower portion of the roof is formed by the velum interpositum, above which is found the lower surface of the cerebellum.

The upper one-half of the floor is formed by the posterior surface of the pons, and the lower one-half by the posterior surface of the medulla oblongata. Along the mid-line is seen a fissure, the continuation of the posterior median fissure of the spinal cord. Opposite each of the lateral angles is found a depression, called the fovea anterior; and parallel with the median fissure are two rounded lobes of gray matter, called the fasciculi teretes. Near the central portion of the ventricle is sometimes seen a violet-colored streak, consisting of gray matter, lying just beneath a thin covering of white (which gives the singular color); and called the Touia Violacea (L. Violet-colored band). On the floor of this ventricle may be seen the origin of several of the cranial nerves. A few transverse fibres are seen extending across the ventricle, those above the origin of the eighth nerve called the Auditory Strix, and those below the Lineæ Transversæ.

\section{Corpora Quadrigemina.}

The Corpora 2uadrigemina are situated above the acqueduct of Sylvius, and, as their name implies, are divided into four bodies, two on either side of the mid-line. The anterior pair are called the nates, the posterior the testes. On the posterior extremity, or pulvinar, of the optic thalamus are found two minute projections, called the corpora geniculata, internal and external. From the nates there proceed two small white bands, which pass forward to join the internal geniculate body of either side, while from the testes two more prominent bands pass between the internal and external geniculate bodies. These rounded cords are known as the anterior and posterior 
brachia. From the testes two bundles of fibres proceed down ward, outward, and backward to join the cerebellum. These are the superior peduncles of the corcbellum, or upper lateral boundaries of the fourth ventricle-processus e cerebello ad testes. It is between these two that the superior medullary velum, or valve of Tieussens, extends.

The Pincal Gland, a small reddish projection, lies between the two nates. It consists of two lobes; and in its interior is a minute cavity, which contains a small amount of viscid fluid. It is held in place by four peduncles, or cords, two of which pass downward from the base of the gland to the optic thalami, while the other two have been noticed in the third ventricle as passing forward along the upper lateral aspect of the optic thalami to reach the anterior crura of the fornix.

\section{The Pons Varolii.}

The Pons Varolii consists of superficial transverse fibres: which, passing across the mid-line, enter the lobes of the cerebellum, forming the middle peduncles of that portion of the encephalon, and acting as commissural fibres. Beneath this layer of fibres are two rounded bundles of longitudinal fibres, which pass forward from the medulla to the cerebrum; and emerging from the anterior border of the pons, form the crura of the cerebrum, the postero-lateral boundaries of the interpeduncular space. These crura are found to expand on entering the corresponding hemispheres of the cerebrum, and to assume a fan-shaped appearance, the corona radiata, or bands of white fibres which transmit impulses from the cortex of the cerebrum to the spinal cord. They are separated into trvo bundles by the thin layer of gray matter, called the locus niger.

\section{The Medulla Oblongata.}

Immediately below the pons is seen the medulla oblongrata. which effects the junction between the various portions of the brain and the spinal cord. It is about an inch and one-half in length and three quarters of an inch wide at the widest portion of it; and presents for examination anterior and posterior surfaces, superior and inferior extremities. Many writers 
describe it as having lateral surfaces in addition to the anterior and posterior; but as the lower portion of the medulla is almost completely circular, it is unnecessary to give it more than two surfaces.

The anterior surface, which looks downward as well as forward, rests upon the basilar process of the occipital bone; while the posterior surface, a part of which is flattened and looks upward as well as backward, lies beneath the cerebellum.

Just where the medulla joins the pons there is a deep constriction, or groove, which extends completely around the medulla; but is deeper on the anterior than on the posterior face.

As the medulla transmits the fibres to and from the spinal cord, it must correspond nearly to the arrangement of the cord itself, consequently we find pillars in the medulla corresponding in a great measure to the same pillars found in the cord. They are however, not so numerous, nor are they arranged in precisely the same way. The medulla, like the cord, is divided along its anterior and its posterior face by two rertical fissures, which are continuous with the anterior and posterior median fissures of the cord. Lying by the side of the anterior median fissure, and separated by that fissure from its fellow, is a small portion of the medulla, called the anterior prramid. It is the direct continuation upward of the anterior pyramid of the cord, but also receives fibres belonging to the crossed pyramidal tract of the cord, so that the decussation of these fibres takes place before the cord terminates in the medulla. Behind this anterior pyramid, near the upper extremity of the medulla, is an oblong projection, called the olivary body. This olivary body is concealed in the lower portion of the cord, where the fibres are deeply seated and are covered by other portions of the medulla, and above it is made prominent by the divergence of the anterior pyramid and the fibres forming the restiform body. Behind the olivary body is a large and prominent body, the restiform (L. Restis, a rope; forma, shape) which carries the direct cerebellar tract of the cord, and terminates by forming the inferior peduncles of the cerebellum. 
Next to the restiform body is the fourth and last portion of the medulla, the posterior pyramid, this portion of the medulla carrying the fibres which in the cord are known as the columns of Goll and Burdach. The posterior pyramid, as it passes upward, blends with the restiform body; and the two pass together into the cerebellum to form its inferior peduncles. The anterior pyramids passing through the pons Varolii, enter into the formation of the crura cerebri. The olivary fibres disappear at the upper extremity of the medulla, sinking deeply into the pons, and probably aid in forming the commissural fibres which exist between the two lobes of the cerebellum as the superficial fibres of the pons.

The appearances on the posterior face of the medulla have already been described in connection with the fourth ventricle of the brain.

\section{The Cerebellum.}

The Cercluellum, or Little Brain, is divided into two hemispheres by a deep fissure found on its inferior aspect and a projection found on the superior face. In the adult its relation in weight to the cerebrum is variously stated as one to thirteen and one to twenty, Cruveilhier giving the latter proportion. It is usually described as being divided into three lobes, a middle and two lateral, the middle being generally called the "worm", and the two lateral the "hemispheres". The hemispheres and the worm present a superior and an inferior surface.

When viewed from above, the entire cerebellum seems undivided, the hemispheres being directly continuous through the middle of the worm. They slope from before downward, backward and outward.

On the inferior surface the appearance is entirely different. Here a deep groove separates the two hemispheres; but the groove itself is partially filled by the lower aspect of the worm, which in its turn is subdivided by fissures into three portions. The groove in which the worm lies terminates anteriorly and posteriorly in notches, the incisura cercluclli, anterior and prosterior. The anterior is the wider, and is not so deep as the 
posterior. The worm extends into these notches, but does not completely fill them. The upper aspect of the worm is generally called the superior vermiform process, and the lower the inferior vermiform process. Its sides are attached directly to the hemispheres of the cerebellum; and probably act as commissural fibres. On the upper face the worm has no subdivisions; but when viewed from below it is seen to be divided into a flattened mass of gray matter, the Posterior Medullary Velum, behind that a projection called the Uvula (I. Diminutive of $U v a$, a grape), and behind that the Pyramid.

Each hemisphere of the cerebellum is divided into lobes; but we do not find in the cerebellum the convolutions which appear in the cerebrum. The cerebellum presentsa laminated appearance, the fissures, or sulci, being deep and narrow, while the laminæ overlap one another throughout the entire area of each hemisphere. Certain of these sulci, however, are deeper and more distinctly marked than others, so that it becones possible to divide the cerebellum into lobes which are less distinctly bounded than the lobes of the cerebrum. There names are fanciful; and derived in the main from a supposed resemblaince to other portions of the body.

On the lower surface of each hemisphere, close to the median fissure, is a small projection of the cerebellum cut off by a deep fissure, called the Tonsils from their supposed resemblance to a gland in the throat. Beyond the tonsils, and cut off by the second inferior cerebellar fissure, is a curved lobe, which is called the Digastic. Next to this, and between the second and third cerebellar sulci, is a long narrow, curved lobe, which extends from the median fissure behind the pyramid around to the front of the cerebellum nearly as far as the anterior median fissure. This lobe is very narrow, and is called the Slender Lobe. Behind the slendar lobe, and occupying the posterior extremity of the cerebellum, is another long and slender lobe, which borders the slender lobe, extending from the posterior extremity of the median fissure around to the front of the flocculus. This is known as the posteroinferior Lobe. 
On the upper surface the lobes are very indistinct, and can scarcely be described as separate lobes. There are really but two lobes on the superior surface, the postero-superior and the anterior, or square, lobe. It will be noticed that the inferior vermiform process is that portion of the cerebellum which overhangs the fourth ventricle, and is its indirect roof, the pia mater intervening between the ventricle and the inferior vermiform process.

\section{The Spinal Cord.}

The Spinal Cord is the great connecting link between the brain and the various members of the body. It seems to correspond partially to a mass of insulated wires, a large portion of its work being the transmission of impulses to and from the brain; but it contains nerve centres which in themselves originate impulses, hence it is not merely a bundle of transmitting fibres. It is markedly similar to the brain in the arrangement of its membranes, and to some extent in its functions; but is dissimilar in the arrangement of the mass of gray and white matter.

The Membranes of the spinal cord, like those of the brain, are three in number, and bear the same names as those of the brain, the Dura Mater, the Pia Mater, and the Arachnoid.

The Dura Mater of the spinal cord is, however, unlike that of the brain, much too large for the cord which it envelopes. It lines the interior of the spinal canal, is firmly attached to the bodies of the vertebra, and sends off prolongations for each of the thirty-one pairs of spinal nerves, which accompany these nerves until they leave the spinal column. The inner face of the dura mater is lined by the arachnoid, as is the case in the brain; and it should be borne in mind that the membranes of the spinal cord are all directly continuous with those of the brain.

The Arachnoid, forms here, as in the brain, a serous membrane, covers the entire surface of the cord, and lines the inner face of the dura. It does not closely hug the cord; but between. it and the cord is found a continuation of the mis-named subarachnoidean space. Between the visceral layer covering the

Des Anat-27 
cord and the parietal layer covering the dura is a second space which is known as the sub-dural, and it really lies between the folds of the arachnoid itself.

The Pia Mater covers the cord nearly as it does the brain, but with some slight differences. It sends prolongations to accompany the nerves which leave the spinal cord, it conveys the blood-vessels as in the brain; but it also gives ligamentous fibres, which are not found in the cranium. Between each pair of spinal nerves will be found a wedge-shaped portion of fibrous tissue, which is derived from the pia, and which, passing to the lateral aspect of the cord, becomes attached to it, while the point of the wedge extends outward to blend with the dura. These prolongations form the Ligamentum Denticulatum, and serve the purpose of retaining the cord steadily in position, and of preventing its movement in the large chamber furnished for it by the dura.

The Cord itself is nearly, though not quite, cylindrical, being slightly flattened in the antero-posterior direction. It is seventeen or eighteen inches in length, and varies in weight, as does the brain, with the period of life, cause of death, and even with the period after death when the cord is removed. It corresponds in length to the upper two-thirds of the spinal canal in the adult, though it is longer comparatively in the foetus, extending here to the bottom of the sacral canal; but apparently the vertebræ grow more rapidly than the cord and hence the cord is carried upward in the canal until it reaches about the level of the second or third lumbar vertebra. The cord presents an enlargement in the cervical region, where the branches are given off which supply the upper extremity ; and a similar enlargement in the lower lumbar region, where the branches are given off which supply the lower extremity. Of course, in the living being, its curvature corresponds to the curvature of the spinal column.

When the membranes are removed, it will be seen that the cord presents two Median Fissures, one Anterior, and one Posterior.

The anterior is very wide, but shallow; while the posterior $s$ very deep and narrow. In addition to these two fissures, 
the spinal nerves, rising by two bundles of roots, called the anterior and posterior, makean incomplete and irregular fissure, which extends through the length of the cord, and serves to form the two lateral fissures. Hence it will be seen that the cord is divided into an anterior column, a posterior column, and a lateral column, the last being separated from the other two by the anterior and posterior roots of the spinal nerves. 'There are, however, other columns in the cord, determined more by pathalogical than by anatomical examination.

It will be seen that the cord, unlike the brain, is arranged with all of its gray matter in the centre, and the white matter surrounding it; and on section of the cord in living animals degeneration takes place in two directions. 'Those fibres which convey impulses to the brain degenerate upward, while those which convey impulses from the brain degenerate downward. These pathological changes warrant the division of the cord into a number of tracts, not noted anatomically.

As in all portions of the brain, the two lateral divisions of the cord, separated by the anterior and posterior median fissures, which correspond in fact to the great longitudinal fissure of the brain, communicate across this fissure by means of commissural fibres. The roots of the spinal nerves extend through the white matter of the cord, and communicate with the gray matter in its interior. This gray matter, in addition to furnishing trophic centres, contains centres which act apparently of their own volition without stimulation from the brain. As a matter of course these centres are, however, presided over by the brain, and can be restrained in their action by the higher centres.

The total number of tracts in the cord is large; and we do not find the corresponding tracts in the medulla, but in many cases two or more tracts are blended into one in the transmission from the cord to the brain. Commencing at the anterior median fissure the first tract is called the Anlerior Pyramid, or Anterior Pyramidal Tracl, and next to this is found the Ancrim Ground Zone, or Anterior Rool Zonc, being that portion of the cord in front of the anterior roots of the spinal 
nerves. Then comes behind the spinal nerves a second ground zone, which is, however, called the Lateral bundle, and external to both of these and superficial to them is the Antero-lateral tract, which conveys both ascending and descending fibres to the cerebrum. Behind this tract, also on the superficial surface of the cord, is the Direct Cerebellar tract which extends as far as the posterior root zone; and between the direct cerebellar and the gray matter in the interior of the cord is the Crossed Pyramidal Tract. Behind the posterior root zone are two tracts, the one next to the roots of the nerves being Burdach's Column, or the postero-lateral column, while the one next to the postero-median fissure is the column of Goll, or the postero-median column.

When these columns are traced upward into the medulla, it will be found that the column of Goll and the column of Burdach unite to form the posterior column of the medulla, or, as it is frequently called, funiculus cumeatus (L. Dim. of funis, a cord ; cuneatus, wedge-shaped); that the direct cerebellar tract passes into the restiform body ; that the crossed pyramidal and the direct pyramidal enter into the anterior pyramid of the medulla, when the direct pyramidal will pass upward on its own side, while the crossed pyramidal passes to the posterior side. The antero-lateral or ascending and descending cerebellar tracts, with the anterior root zone, pass upward into the lateral column of the medulla, to ascend with it to form the central fibres of the pons Varolii.

In addition to these white transmitting fibres, the cord contains in its interior the mass of gray matter before alluded to, which is arranged like the letter " $\mathrm{H}$ ', the projections in front of and behind the cross-bar of the " $\mathrm{H}$ " being called the "horns."

The posterior horns are long and slender, while the anterior horns are bluntly pointed. These masses of gray matter contain the fibres furnishing the various centres in the spinal cord.

It will of course be seen that the branches which proceed from the spinal cord are collectively enormously larger than 
the cord itself, and it can be readily understood that large quantities of fibrous tissue, serve in a measure to make up a portion of the bulk of the spinal nerves.

The cord is nourished by the blond vessels which it carries in its pia matral covering; and these blood vessels are derived partly from the anterior and posterior branches of the vertebral arteries, and partly from the branches which enter the spinal canal springing from the intercostal and lumbar arteries.

\section{The Spinal Nerves.}

The Stinal nerves consist of thirty-one pairs, which taking their origin from the spinal cord, pass outward from the spinal canal through the intervertebral foramina, and are distributed throughout the body. These nerves differ materially from the cranial nerves, for, whereas the latter are usually divided into separate nerves, having motor, general sensory, or special sensory functions, the spinal nerves are composed of bundles, performing all nervous functions. Some convey tactile sensations, some painful, some motor, and some various other functions. While these nerves may be traced into the spinal cord, their fibres pass through the various columns of the cord to reach different portions of the brain; and hence while the nerves are described as spinal nerves simply from their origin, they are controlled by the higher centres located in the cerebrum.

They differ from the cranial nerves also in their manner of distribution. The cranial nerves pass out as individual nerves to break up into branches distributed to various portions of the head, face, and viscera, having but slight communications with one another. The spinal nerves on the contrary unite very intimately with one another after their emergence from the spinal column; and form plexuses from which branches are given off to carry out the distribution of the plexus. There are four of these plexuses, called the Corvical, Brachial, Lumbar, and Sacral. Interposed between the brachial and the the lumbar are a set of spinal nerves, the Dorsal, which do not unite to form plexuses, but are distributed individually 
like the cranial nerves. Some writers mention a coccygeal set; but these in reality belong to the sacral plexus.

Each spinal nerve as it emerges from the cord is surrounded by a prolongation of the dura mater, which lines the spinal canal and forms a sheath for the nerve as far as the intervertebral foramen. From this point it becomes continuous with the ordinary fibrous sheath, which envelops every nerve, and ceases to have the characteristics of the dura mater lined by its arachnoid membrane.

Moreover each nerve presents upon it at its emergence an enlargement; and as every nerve arises by an anterior and a posterior root, this enlargement, or ganglion, is confined to one of these roots. The posterior root of a spinal nerve conveys its sensory fibres, while the anterior root conveys the motor fibres. The anterior root also has its trophic centre situated in the spinal cord, so that cutting that nerve causes degeneration downward into the nerve, which has been cut loose from its trophic centre, while cutting the posterior root between the ganglion and the cord causes degeneration upward into the cord, because the trophic centre is located in the ganglion and not in the cord.

It must be always remembered that while these fibres conveying the various impulses exist in the spinal nerves they cannot be demonstrated anatomically.

Each spinal nerve immediately after it emerges from the spinal canal divides into an anterior and a posterior branch. The posterior branches are smali in size and comparatively unimportant. They convey motor and sensory impulses to the muscles found in the back and neck, the posterior fibres of the sacral plexus, the lowest, conveying impulses to the lowest muscles of the trunk, while the limbs are supplied entirely from the anterior cords. It is the anterior cords which enter into the formation of the plexuses.

\section{The Cervical Plexus.}

The first plexus formed by the spinal nerves is called the Cerical. It is the product of the four upper cervical nerves. The plexus is formed by the intercommunication of the ante- 
rior cords of these four cervical nerves; and is found opposite the middle of the sterno-cleido-mastoid muscle. Its principal distribution is to the muscles and integument of the neck.

The plexus itself is formed in the following manner: The first cervicul nerve divides into an anterior and a posterior branch, the posterior branch forming what is known as the Great Occipital Verve (Occipitalis Major), while its anterior division, after receiving a communication from the eleventh and twelfth cranial nerves, sends downward a communication to the second cervical. The sciond cervical, after giving off its posterior branch, sends a communication to the third and the third in like manner sends a communicating branch to the fourth, which in its turn sends a branch to the fifth, which enters into the formation of the brachial plexus. From this loosely formed cervical plexus branches are given off which are distributed to the head, face, neck, and chest. These branches are, for convenience of study, divided into two sets, superficial and deep, the superficial set consisting of three named branches, and certain unnamed branches, which are called descending superficial branches. The deep set consists of muscular branches.

The named branches of the superficial set are the Superfi. cialis Colli, Auricularis Magmus, and Occipitalis Minor.

\section{Superficial Branches.}

\section{Superficialis Colli Auricularis Magnus Occipitalis Minor}

\section{The Superficialis Colli.}

The Superficialis Colli, the first branch, is the product of the sccond and third cervical nerves; and like all the superficial branches from this plexus, it winds around the posterior border of the sterno-cleido-mastoid muscle, about at its middle, and becomes superficial in that situation. It then passes upward and forward, crosses the sterno-cleido-mastoid, runs to the lower border of the inferior maxilla, and spreads out int a fan-shaped set of twigs, which, after communicating with the facial nerve, are distributed to the skin covering the lateral aspect of the face. 


\section{The Auricularis Magnus.}

The Auricularis Magnus is the product of the second and third cervical nerves. After emerging from the posterior border of the sterno-cleido-mastoid, it crosses that muscle, and divides into two branches, mastoid and auricular.

The mastoid branch ascends to the mastoid process of the temporal bone and is distributed to the skin in that region after communicating with the seventh nerve.

The auricular branch divides into several twigs, which are distributed to the skin on the back of the pinna.

\section{The Occipitalis Minor.}

The Occipitalis Minor is derived from the second cervical nerve. It passes upward nearly parallel with the posterior border of the sterno-cleido-mastoid muscle; and gives off three sets of branches, one passing to the back of the ear, one over the mastoid process of the temporal, where it communicates with the auricularis magnus, and the third, the largest, passing to the occipital bone, where it runs over the occipito-frontalis muscle, and is distributed to it and to the skin covering the occipital bone.

\section{The Descending Superficial Branches.}

The unnamed Descending Superficial Branches are produced by the third and fourth cervical nerves. They emerge from beneath the sterno-cleido-mastoid muscle, like the preceding branches, about opposite the middle of its posterior border. They are irregular in size and number. One set passes downward and inward toward the mid-line of the body, crossing the clavicle near its inner extremity and ending in the integument which covers the front of the thorax. The second set passes nearly vertically downward, to cross the middle of the clavicle, and be distributed to the integument covering the pectoral muscles, while the third set, called sometimes the acromial, or deltoid, passes outward, and is distributed to the skin in the neighborhood of the shoulder. 


\section{Deep Branches.}

Muscular

\section{Communicans Noni}

Phrenic

\section{The Muscular Branches.}

The muscular branches are distributed to all of the muscles of the front of the neck with the exception of the depressors of the hyoid bone. They consist of a branch to the sternocleido-mastoid muscle, which arises from the second cervical and communicates with the eleventh cranial in the substance of the sterno-mastoid muscle; a branch to the scalenus medius, coming from the third and fourth nerves; one to the levator anguli scapulæ; and several branches to the trapezius, which are the product of the third and fourth cervical nerves and which communicate with one another.

Besides this, there are some branches, sometimes described as internal, which supply the rectus capitis lateralis, the rectus capitis anticus minor, and the longus colli.

\section{The Communicans Noni.}

The most important communicating branch is the Communicans Noni. This nerve is the product of the second and third cervical nerves. It passes downward, forward, and in ward, and unites with the descending branch of the hypoglossal on the front of the common carotid artery, inmediately above the point at which the omo-hyoid crosses that vessel. After this communication it passes with the descendens noni to be distributed to the muscles of the infra-hyoid group, or depressors of the hyoid bone, with the exception of the thyrohyoid, which is supplied by a branch from the hypoglossal nerve itself.

\section{The Phrenic.}

The last and most important branch of the plexus is the Phrenic Nerve. It is formed by contributions from the third and fourth cervical nerves, reinforced by a small filament from the fifth. The course of the nerve on the two sides of the tody differs. 
On the right side it descends on the front of the scalenus anticus muscle, reaches the inner side of that muscle just before it crosses the subclavian artery, drops off to the inner side, and becomes an anterior relation of the first portion of the subclavian, though occasionally it remains on the scalenus anticus until after it has passed the artery. It then runs in the interval between the subclavian artery and vein, continues its downward course into the thorax, lying to the outer side of the right vena innominata and then the superior vena cava, passes in front of the root of the right lung, then lies on the pericardium between it and the right pleura, and finally pierces the diaphragm, and is distributed to that muscle from its under surface.

The leftnerve, formed in the same way, passes on the scalenus anticus muscle to cross over the front of the first portion of the subclavian, then to descend in front of that vessel and opposite the interval between it and the left common carotid, lying to the outer side and in front of the pneumogastric nerve. Then it crosses the end of the transverse aorta, passes downward on the left side of the pericardium, lying next to the left pleura, and then, like its fellow of the opposite side, enters the diaphragm and is distributed to it from its under surface.

\section{The Brachial Plezus.}

The Brachial Plexus is the second great plexus formed by the anterior cords of the cervical nerves. It is the result of intercommunications between the four lower cervical nerves and a portion of the first dorsal nerve. The manner of its formation differs in different individuals; but it is usually formed in the following way: The fifth and sixth cervical nerves unite to form a single cord; the eighth cervical and the first dorsal unite to form one cord, while the seventh cervical passes out alone. Then the seventh unites with the cord formed by the fifth and sixth, so that at one time there are but two cords in the plexus, one resulting from the union of the fifth and sixth with the seventh, and the other formed by the eighth cervical with the first dorsal. Shortly after, however, each of these two cords gives off a branch; and these branches unite to form 
a third cord in the plexus, this middle cord being the product of all of the cords which enter into the formation of the brachial plexus.

The plexus is triangular in outline, with its base directed towards the spinal column and its apex extending downward. backward and outward towards the axillary space. It passes between the scaleni muscles; and comes into relationship with the subclavian artery in the second portion of its course. Here most of the cords lie above that vessel; but the branch derived from the dorsal nerve lies behind it. In the third portion of the subclavian artery the plexus lies above and to the outer side; but on a plane posterior to it. It then continues forward and outward, coming into relationship with the axillary artery, and lies first to its outer side; but in the second portion of that ressel; viz., that part behind the pectoralis minor muscle, the plexus consists of three cords, one to the inner side, one to the outer side, and one lying behind the axillary artery. Just at the termination of the second portion the inner and the outer cord each give off a branch; and the two branches formed in this manner unite to form the median nerve, so that, on a line drawn from the tip of the coracoid process of the scapula down ward and inward to the lower part of the third intercostal space, it will be seen that the axillary artery is completely surrounded by the cords of the plexns. The outer and inner cords lie on the outer and inner sides of the artery, the posterior cord behind it, and the branches which form the median nerve lie upon its front.

Immediately after the formation of the median, that nerve drops to the outer side of the axillary artery, and maintains that relation to the termination of that vessel, while the remaining cords of the plexus break $\mathrm{np}$ into their terminal branches.

\section{Branches of the Brachial Plexus.}

The distribution of the brachial plexus is chiefly to the upper extremity, supplying the muscles and integunent; but before reaching its point of termination it gives off way-side branches, some muscular and sone cutaneous, which are dis- 
tributed to the lateral aspect of the chest, as well as to the muscles of the scapular group and the skin covering them.

\section{Wayside Branches.}

Above the $\left\{\begin{array}{l}\text { Suprascapular } \\ \text { Rhomboid } \\ \text { Posterior Tho- } \\ \text { racic }\end{array} \quad\right.$ Below the $\begin{array}{l}\text { Clavicle } \\ \text { Subclavian } \\ \text { Scaleni } \\ \text { Lougus Colli }\end{array} \quad\left\{\begin{array}{c}\text { Anterior Tho- } \\ \text { racic, internal. } \\ \text { Anterior Tho- } \\ \text { racic, external. } \\ \text { Subscapular }\end{array}\right.$

\section{The Suprascapular.}

The Suprascapular Uranch is derived from the fifth and sixth cervical nerves. It passes downward and outward parallel with the posterior belly of the omo-hyoid muscle. Reaching the upper border of the scapular it passes through the suprascapular notch beneath the transverse ligament, and consequently below the corresponding artery, enters the supraspinatus muscle, gives motor fibres to that muscle and a twig to the shoulder joint. while the remaining portion of the nerve passes around the anterior border of the spine of the scapular to be distributed to the infraspinatus muscle.

\section{The Rhomboid.}

The branch to the Rhomboideus is a product of the fifth cervical nerve. It passes through the fibres of the scalenus medius, and is then distributed to the rhomboid muscle, or muscles.

\section{The Posterior Thoracic.}

The Posterior Thoracic, or External Respiratory Nerie of Bell, is the product of the fifth, sixth, and seventh nerves. It passes down ward behind the brachial plexus and the first portion of the axillary, and is distributed to the serratus magnus muscle.

\section{Communicating.}

The communication with the cervicle plexus is by means of the fifth cervical, which joins with the phrenic nerve. 


\section{The Subclavian.}

The nerve to the Subclatius muscle is a slender twig, which is derived from the fifth cervical, and crosses the front of the third portion of the subclavian artery to enter the subclavius muscle.

The Branches given below the clavicle, besides the terminal ones, are the external and internal anterior thoracic and the subscapular nerves.

\section{The External Anterior Thoracic.}

The External Anterior Thoracic, derived from the outer cord of the plexus, is distributed to the pectoralis major muscle.

\section{The Internal Anterior Thoracic.}

The Internal Anterior Thoracic, the product of the inner cord, passes between the first portion of the axillary artery and its vein, occasionally perforatiug the vein itself; and after receiving a communication from the external anterior thoracic, is distributed to the pectoralis major.

\section{The Subscapular.}

The Subscupular neries are three in number; and they are derived from the posterior cord. They are known as the upper, middle, and lower. The upper is distributed to the subscapularis muscle alone, the middle accompanies the subscapular artery, and terminates near the latissimus dorsi; while the lower is distributed to the teres major, furnishing some twigs to the subscapularis.

\section{Terminal Branches.}

Musculo-cutaneous

Median

Ulnar
Internal Cutaneous

Lesser Internal Cutaneous Musculo-Spiral

\section{Circumflex}

The terminal branthes of the Brachial Plexus originate from the cords of the plexus as follows: The internal cord produces the internal cutaneous, the lesser internal cutaneous, the ulnar, 
and one head of the median. The external cord divides into two branches: one forms the outer head of the median, while the other is the musculo-cutaneous. The posterior cord divides into the circumflex and the musculo-spiral.

The division of the plexus takes place on the beginning of the third portion of the axillary artery, which is here completely surrounded by the cords of the plexus; but immediatelv after the formation of the median, that nerve drops to the outer side of the axillary artery; and the relation is then, median and musculocutaneous to the outer side, internal and lesser internal cutaneous and ulnar nerves to the inner side, musculo-spiral behind, and circumflex behind as far as the interval between the teres major and lower border of the subscapularis, where it passes to the back of the humerus and ceases to be a relation.

\section{The Musculo-Cutaneous.}

The Musculo-cutaneous Nerve, the product of the onter cord of the brachial plexus, receives fibres which can be traced to the fifth, sixth, and seventh nerves, passes downward as an external relation of the third portion of the axillary artery until it reaches the coraco-brachialis muscle. It then passes through the substance of that muscle, supplying the muscle with branches as it is transmitted through it. Emerging from the coraco-brachialis, it passes between the brachialis anticus and the biceps, runs into the in terval between the brachialis anticus and the supinator longus, where it becomes superficial a little above the elbow joint, and passing across the outer front aspect of that joint, divides into two branches. The posterior branch passes downward and backward; and is distributed to the skin on the postero-external aspect of the forearm as far as the wrist. The anterior branch, supplying the integument on the antero-external face of the forearm, communicates with the radial nerve near the wrist, and extends as far as the skin covering the thenar eminence.

The nerve in its course gives off muscular branches to the coraco-brachialis, the biceps, and the inner portion of the brachialis anticus. 


\section{The Internal Cutaneous.}

The Internal Cutaneons Nerze is the product of the inner cord of the plexus; and is probably derived from the eighth cervical and first dorsal. It is an internal relation of the third portion of the axillary artery to the termination of that vessel ; and then becomes an antero-internal relation to the brachial artery, lying in close companionship to the basilic vein. At the middle of the brachial artery the nerve, with the basilic rein, pierces the investing fascia; and still remaining an antero-internal relation of the brachial artery to its termination, is, for its lower half, separated from that vessel by the deep fascia of the forearm.

At the elbow-joint it breaks up into branches, some of which, lying on the front of the bicipital fascia, are anterior relations of the brachial artery, but separated from it by this fascia. The remaining branches continue their course downward, being divided into an anterior and a posterior set, the antcrior supplying the integument of the inner front aspect of the forearm as far as the wrist, while the posterior supply the integument on the postero-internal aspect of the forearm down to the wrist.

\section{The Lesser Internal Cutaneous.}

The Lesser Internal Cutancous, or Nerve of Wrisberg, arising from the internal cord, receives its branches from the eighth cervical and first dorsal. It is very irregular in its origin, and may be the product of the lateral cutaneous branch of the second intercostal nerve; but, as a rule, it simply receives a communication from that nerve. At first it is an internal relation of the axillary artery, and is separated from that artery by the axillary vein. It continues downward, too distant to be considered a relation of the brachial artery, to th: postero-internal aspect of the arm, and near the elbow turns backward to be distributed to the integument on the 1 wer third of the arm and in the neighborhood of the olecranon process. 


\section{The Ulnar.}

The Ulnar Nerve is the last branch of the internal cord of the plexus; and like the preceding branches, contains fibres from the eighth cervicle and first dorsal nerves. It is first an internal relation of the axillary artery, lying between it and the lesser internal cutaneous nerve. It then becomes an internal relation of the brachial for the upper half of the course of that vessel; and at the middle of this artery it reaches the inferior profunda branch of the brachial. Accompanied by this latter branch, it passes backward, pierces the internal inter-muscular septum, and runs to the space between the internal condyle of the humerus and the olecranon process of the ulnar, forming what is generally known as the "funny bone". The nerve then passes between the two heads of the flexor carpi ulnaris muscle; and at the junction of the upper third with the lower two-thirds of the forearm it becomes an internal relation of the ulnar artery. It then passes downward, lying on the fibres of the flexor profundus digitorum, and maintains a continuous internal relation to the ulnar artery to the wrist joint. At this point it becomes superficial to the anterior annular ligament, passes to the outer side of the pisiform bone, and enters the palm of the hand, where it divides into a superficial and a deep branch.

In the commencement of its course the nerve lies on the anterior face of the internal inter-muscular septum. At the middle of the arm it pierces this septum, and passes downward in the fibres of the triceps muscle, then behind the internal condyle of the humerus, then between the two heads of the flexor carpi ulnaris, separated from the ulnar artery for the upper third of the course of that vessel. Then the nerve lies with the flexor carpi ulnaris to its inner front side, and the flexor profundus digitorum behind it, and with the ulnar artery to its outer side for the lower two-thirds of its course.

The nerve gives off no branches until after it has passed the elbow joint, where it supplies muscular branches to the flexor carpi ulnaris and the inner half of the flexor profundus digitorum. A bout three inches above the wrist joint it gives off a 
cutaneous branch, the dorsal cutaneous branch of the Ulnar, which will be described later; and then a superficial branch which enters the palm of the hand. A few small twigs are given off to the elbow joint.

Superficial Branch. After entering the palm, the superficial branch supplies the palmaris brevis, and the skin covering the hypothenar eminence, a twig to the inner side of the little finger, and a branch which bifurcates to supply the adjacent sides of the little and ring fingers, and lastly a communicating branch to the median nerve.

The distribution of the collateral digital branches to the fingers is accomplished by the median and ulnar nerves. These branches pass to the clefts between the fingers, divide into two, which coursealong superficial to the corresponding arteries, and opposite the posterior extremity of the nail send dorsal branches around which ramify between the nail and its matrix, each nerve communicating with its fellow of the opposite side around both the palmar and the dorsal aspects. The ulnar nerve supplies the little finger and the inner side of the ring finger.

Decp Branch. The Deep Branch of the Ulnar passes between the flexor minimi digiti and the abductor minimi digiti, runs along the palm of the hand accompanyin $x$ the deep palmar arch, supplies the two inner lumbrical muscles, the two outer interosseous muscles, the muscles of the hypothenar eminence, with the exception of the palmaris brevis, and in the thenar sroup, supplies one muscle and a half, viz., the adductor pollicis, and the deep head of the flexor brevis pollicis, the remaining portion of the hand being supplied by the median nerve.

The Dorsal Culaneous Branch of the Ulnar arises three inches above the wrist joint, passes to the back of the forearm, and descends behind the styloid process of the ulnar to the back of the hand. Here it divides into cutaneous branches, the most internal of which is distributed to the ulnar side of the little finger. The second passes to the cleft between the little and ring fingers, where it divides into dorsal collaleral digital branches for these two fingers. The lhird culaneous branch communicates with a branch from the radial nerve, passes to the cleft between the ring and middle Des Anat-28 
fingers, and divides to supply the adjacent sides of these two fingers. These dorsal collateral digital branches communicate in the matrix of the nail with the palmar branches already noticed.

\section{The Median.}

The Median Nerve is the joint product of the internal and external cords of the brachial plexus; and derives fibres from all of the anterior cords entering into the formation of that plexus. It is formed at the commencement of the third portion of the axillary artery by the junction of a branch from the inner with a corresponding branch from the outer cord of the plexus. Formed on the front of the third portion of the axillary artery, it immediately drops to its outer side, and maintains that relation to the termination of the vessel. It then becomes an outer relation of the brachial artery, lying between that artery and the coraco-brachialis muscle. About opposite the insertion of the coraco-brachialis it passes to the front of the brachial artery, crossing it very obliquely, so as to remain on the front of that vessel for the middle one-third of its course. It then arops to the inner side of the brachial artery, and maintains that relation to the termination of that vesse1, a finger's breadth below the middle of the front of the elbow joint. It should here be noted that in cases of high bifurcation of the brachial, the median nerve almost invariably passes between the two branches, the branch in front usually corresponding to the radial artery, and the branch behind to the ulnar. In some cases the median nerve passes behind instead of in front of the brachial artery.

Reaching the elbow joint it lies in the triangular space, the base of which is directed upward, and is formed by a line drawn from the inner to the outer condyle of the humerus, the external boundary being the supinator longus and the inner boundary the pronator radii teres. The nerve exactly bisects this space, passes between the two heads of the pronator radii teres muscle, the deep head of which alone separates it from the ulnar artery. Crossing over the ulnar artery, and separated from it by the deep head of this muscle, it then passes down the middle of the front of the forearm, covered by the 
superficial head of the pronator radii teres and overlapped by the flexor carpi radialis on its outer side and the flexor sublimis digitorum on its inner side, and lying nearly directly behind the palmaris longus. It then passes to the front of the wrist joint, crosses beneath the anterior annular ligament into the palm of the hand, and breaks up into its terminal branches.

Like the ulnar nerve it gives off no branches in the arm. It gives off a few small branches to the elbow joint; and just below the bend of the elbow gives off a large branch, called the anterior interosseous.

The Anterior Interosseous Nerve arises opposite the bicipital tubercle of the radius, runs downward on the interosseous membrane, accompanied by the anterior interosseous artery. as far as the upper border of the pronator quadratus muscle, where after giving branches to the deep surface of that muscle, it pierces the interosseous membrane and communicates with the posterior interosseous nerve.

Immediately below the elbow joint the median gives off branches to all of the muscles on the front of the forearm with the exception of the flexor carpi ulnaris and the inner half of the flexor profundus digitorum. In other words, it supplies muscular twigs to six and one-half out of eight of the muscles of the forearm ; viz., the pronator radii teres, flexor carpi radialis, palmaris longus, flexor sublimis digitorum, flexor longus pollicis, pronator quadratus, and outer one-half of the flexor profundus digitorum.

Just before reaching the anterior annular ligament it gives off cutaneous branches, which are distributed to the integument covering the thenar eminence of muscles, and which communicate with the superficial branch of the ulnar nerve, distributed to the hypothenar eminence.

The renaining branches are divided into two sets. The first set supplies the superficial muscles of the thenar eminence, the abductor pollicis, the flexor ossis metacarpi pollicis, and the superficial head of the flexor brevis pollicis; or, in other words, all of the muscles which arise from the trapezium and the anterior annular ligament, the remaining muscles of the group being supplied by the ulnar nerve. The olher set 
divides into palmar collatera1 branches, of which the first two pass, one to the radial and the other to the ulnar side of the thumb on its palmar aspect, communicating with one another and with dorsal cutaneous branches, which will be described later. The third branch passes to the radial side of the index finger; the fourth to the cleft between the index and middle fingers: and the fifth, after receiving a communication from the ulnar, passes to the cleft between the middle and ring fingers, and supplies the adjacent sides of those fingers, all of these branches communicating with one another and also with the dorsal nerves.

\section{The Musculo-Spiral.}

The Musculo-Spiral, the largest branch of the brachial plexus, arises from the posterior cord ; and is said by some authorities to get fibres from all of the cords of the brachial plexus, though others deny that it receives any from the fifth cervical nerve. It passes downward as a posterior relation of the third portion of the axillary artery. At the termination of that vessel it passes between the internal head of the triceps muscle and the shaft of the humerus; and at the junction of the lower third with the upper two thirds of the shaft of the humerus, pierces the external intermuscular septum, and appears opposite the interval between the brachialis anticus and the supinator longus. At the elbow joint, while still lying between these two muscles, it divides into its two terminal branches, the radial and the posterior interosseus. Before its division it gives off both cutaneous aud muscular branches.

\section{Branches of the Musculo-Spiral.}

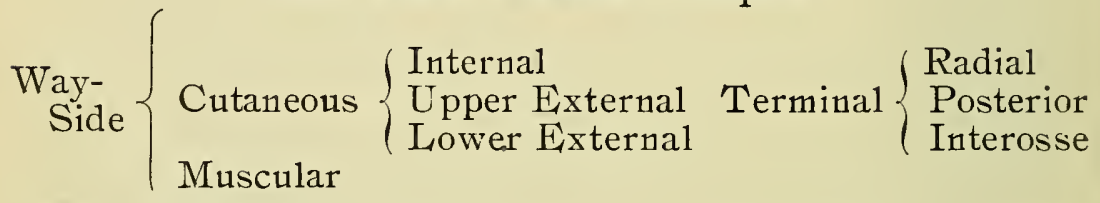

The Internal Cutaneons Branch is distributed to the skin covering the middle of the back of the arm; and extends as far as the distribution of the nerve of Wrisberg, with which it communicates.

The Upper External Cutaneous pierces the external inter- 
muscular septum; and is distributed to the skin covering the outer portion of the biceps muscle.

The Lower External Cutaneous Branch pierces the external intermuscular septum; and is distributed to the skin covering the supinator longus muscle, lying in the interval between the musculo-cutaneous nerve on its outer side, and the posterior branch of the internal cutaneous, on its inner side.

The Muscular Branches are distributed to the three heads of the triceps muscle, a branch to the anconeous, which is a continuation of the triceps, to the brachialis anticus, supinator longus, and extensor carpi radialis longior, that is, to all of the muscles which take their origin above the external condyle of the humerus.

\section{The Radial.}

Of the two terminal branches of the median, the Radial is purely a nerve of common sensation. It commences in the interval between the supinator longus, externally, and the brachialis anticus, internally. It then passes downward; and in the upper part of its course is a distant external relation to the radial artery. In the middle third it is a close external relation of that vessel; but at the junction of the middle and lower thirds of the radius it passes beneath the tendon of the supinator longus and appears upon the back of the forearm, ceasing to be a relation of its artery. Emerging from beneath this tendon, it becomes superficial, gives a branch which divides to supply the radial and ulnar sides of the thumb on their dorsal aspect, a second branch to the radial side of the index finger, a third, which divides to supply the adjacent sides of the middle and index fingers, and a fourth, which after receiving a conmunication from the dorsal cutaneous branch of the ulnar, divides to supply the adjacent sides of the middle and ring fingers.

\section{The Posterior Interosseous.}

The Posterior Interosseous Nerre, the second terminal branch of the musculo-spiral, commencing while the latter nerve lies between the supinator longus and brachialis anticus, passes across the elbow joint to the supinator brevis muscle, and runs directly through the substance of that muscle. It passes through the supinator brevis to the back of the 
forearm; and runs downward on the posterior face of the interosseous membrane to reach the back of the wrist, where it has a small gangliform enlargement upon it, and receives a communication from the anterior interosseous branch of the median. Its distribution is to all of the muscles on the back of the forearm, with the exception of the anconeus, supinator longus, and extensor carpi radialis longior. The muscles supplied are the extensor carpi radialis brevior, the supinator brevis, the extensor communis digitorum, the extensor minimi digiti, the extensor carpi ulnaris, the extensor ossis metacarpi pollicis, the extensor primi internodi pollicis, extensor secundi internodi pollicis, and the extensor indicis.

The above description of this nerve, the usually accepted one, appears to be incorrect. It should be said that the musculospiral nerve supplies all the muscles on the back of the arm and forearm without using the useless term "posterior interosseous nerve;" and the radial should be described as a wayside branch of the nerve, as it really is. The above description is given in conformity with custom.

\section{The Circumflex Nerve.}

The Circumflex Nerve is the last of the terminal branches of the Brachial plexus. Arising from the posterior cord, in common with the musculo-spiral, it passes downward on the subscapularis to the lower border of that muscle, being a posterior relation of the third portion of the axillary artery. It now passes to the back of the humerus, running through the quadrilateral interval bounded above by the teres minor, below by the teres major, internally by the long head of the triceps and externally by the shaft of the humerus. It accompanies the posterior circumflex artery. The nerve now divides into an upper and a lower branch-the upper to accompany the posterior circumflex vessels, winding around the surgical neck of the humerus, beneath the deltoid muscle, which it pierces, supplying that muscle and the skin over its lower part. The lower branch distributes filaments to the teres minor and back of the deltoid and pierces the deep fascia to reach the skin covering the lower part of the deltoid and the long head of the triceps. The nerve gives an articular filament to the shoulder joint. 


\section{Dorsal Spinal Nerves.}

The dorsal spinal nerves are lzelve pairs, the posterior branches of which are distributed to the structures of the back, while the anterior cords form the intercostal nerves, and, unlike other spinal nerves, do not form a plexus but are distributed separately.

\section{The Intercostal Nerves.}

The Intercostal Nerves are the anterior cords of the twelve dorsal nerves, and derive their name from their course forward between the ribs.

The first dorsal nerve sends almost all its bulk to the brachial plexus, and the truelfth usually gives a branch to the lumbar plexus. The intercostal nerves are intended for the supply of the muscles among which they course, and of the integument on the front and sides of the thorax and abdomen. Each intercostal nerve runs forward in the intercostal space between the intercostal muscles to near the edge of the sternum where it turns forward and becomes superficial, being called the anterior cutaneous. It is then distributed to the integument of the front of the chest and abdomen, most of the filaments passing outward in a recurrent course. The six upper nerves appear as the anterior cutaneous through the corresponding intercostal spaces, while the six lower pierce the sheath of the rectus muscle, and are called the anterior cutaneous of the abdomen. After reaching the extremities of the intercostal spaces these last nerves pass on between the broad muscles of the abdomen, and the last one, though called intercostal, is really not one since it lies below the last rib. Whilst running forward the intercostal nerves supply the intercostal muscles and the broad muscles of the abdomen, and about midway in its course each nerve gives off a branch called latcral cutancous, which divides into two, one branch passing forward and the other backward, both being distributed to the integument on the side of the chest or abdomen. The first intercoslal nerie, owing to the fact that most of the cords from which it is derived go to the brachial plexus, gives off no lateral branch, and, to compensate for this delinquency, the lateral cutaneous branch of the second nerve is remarkably large, and not only fulfils the office of the lateral cutaneous branches for itself 
and the first, but under the name of intercosto-humeral, passes into the axilla, where it communicates with the lesser internal cutaneous. The lateral cutaneous branch of the twelfth nerve descends over the crest of the ilium, and is distributed to the integument of the gluteal region.

\section{The Lumbar Spinal Nerves.}

The Lumbar Spinal Nerves are five pairs, the posterior cords of which are distributed to the structures of the back, while the anterior form a plexus, called the Lumbar Plexus, the last cord of the lumbar nerves, however, passing downward to enter into the formation of the sacral plexus.

\section{The Lumbar Plexus.}

The manner of formation of the Lumbar Plexus is generally as follows: The truelfth dorsal nerve gives a branch which descends in the substance of the psoas magnus muscle to unite with the first lumbar. The cord thus formed gives off the ilio-hypogastric and ilio-lumbar nerves, and a branch which descends to unite with the second lumbar. The second lumbar, after receiving this branch, gives off a branch of distribution, the genito-crural, and a communicating branch, which descends to unite with the third lumbar, so that the genito-crural is the product of the first and second lumbar. The cord formed by the union of the branch from the second and third lumbar nerves gives off an external cutaneous branch and a branch which unites with the fourth lumbar, conveying to that nerve some of the fibres derived from the second as well as the third. The union of these branches produces the anterior crural, which, therefore, contains fibres from the second, third, and fourth lumbar nerves, while the last nerve of the plexus, the obturator, is the product of the third and fourth. The remaining portion of the fourth unites with the last lumbar to form the lumbo-sacral cord, which passes down into the pelvic cavity to aid in the formation of the sacral plexus.

The Lumbar Plexus resembles in the manner of its formation the cervical. It is situated in the posterior fibres of the psoas magnus muscle; and its branches, with the exception of the genito-crural, pass outward beneath the posterior surface of that muscle, the genito-crural perforating the muscle and appearing upon its anterior surface. 


\section{Branches of the Lumbar Plexus.}

Ilio-Hypogastric

External Cutaneous

Anterior Crural

Muscular
Ilio-Inguinal

Genito-Crural

Obturator

Communicating

The Branches of the Lumbar plexus, named in the order in which they arise from above down ward are the ilio-hy pogastric. ilio inguinal, external cutaneous, genito-crural, anterior crural, and obturator. Some authorities mention the accessory obturator, which is an infrequent branch. In addition to these the plexus also gives muscular branches, which supply the psoas magnusand quadratus lumborum muscles, and arise irregula. $1 \mathrm{y}$ from the plexus.

\section{Ilio-Hypogastric.}

The Hio-Hypogastric, the product of the first lumbar nerve. appears at the outer border of the psoas magnus, crosses the quadratus lumborum near the upper end of that muscle, passing downward and outward and lying behind the peritoneun, pierces the transversalis muscle, and lies for a short distance between that muscle and the internal oblique until it reaches a point from two to $t w o$ and one-half inches behind the anterior superior spinous process of the ilium, where it divides.

The iliac branch, piercing successively the internal and external oblique muscles, passes downward on the thigh toward the trochanter major, to be distributed to the integument behind the lateral cutaneous branch of the last dorsal nerve.

The hypogastric branch continues the direction of the main trunk, at first lying between the internal oblique and the transversalis, and then between the internal and external oblique muscles, finally piercing the aponeurosis of the external oblique, and becoming superficial, to be distributed to the integument over the lower portion of the abdomen.

\section{Ilio-Inguinal.}

The Ilir-Inguinal Nerve arises from the first lumbar in common with the ilio-hypogastric, and passes downward and outward, parallel with and below that nerve, across the quadratus lumborum muscle. After crossing the iliacus internus, it pierces the transversalis muscle; and hetween that muscle 
and the internal oblique, communicates with the hypogastric branch of the ilio-hypogastric. It then becomes more superficial near the superficial abdominal ring, and leaving the abdominal cavity through that aperture, passes to the thigh, where it is distributed to the skin covering the inner, upper front aspect of the thigh, and to the scrotum in the male and to the labium in the female.

\section{Genito-Crural.}

The Genito-Cmaral is the product of the first and second lumbar nerves. It passes first directly forward through the fibres of the psoas magnus muscle, and descends upon that muscle to just above Poupart's ligament, where it divides into a genital and a crural branch.

The genital branch enters at the deep abdominal ring; and following the course of the spermatic cord, is lost in the male in the cremaster muscle, which it supplies. In the female this branch is rudimentary; but follows the course of the round ligament and is lost upon that structure. It crosses the external iliac artery near the termination of that vessel.

The crural branch passes beneath Poupart's ligament; and runs down on the thigh, to be distributed to the integument of its inner front aspect as far as the middle. It is an anterior relation of the femoral artery.

\section{The External Cutaneous.}

The External Cutaneous Nerve is the product of the second and third Lumbar nerve. It passes downward and outward across the iliacus internus muscle behind the peritoneum, and reaches the notch found below the anterior superior spinous process of the ilium. It here leaves the abdominal cavity, and lies beneath the fascia lata for the upper inch or two inches of its course. It then pierces the fascia lata, and becoming superficial, is distributed to the skin on the outer front aspect of the thigh, its distribution lying in the interval between the iliac branch of the ilio-inguinal and the middle cutaneous branch of the anterior crural. Fibres from this nerve descend as far as the knee joint. 


\section{The Obturator.}

The Obturator Nerve arises from the third and fourth lumbar nerves and occasionally receives fibres from the second lumbar. It passes beneath the psoas magnus muscle, runs over the brim of the pelvis, and crossing along just beneath the pelvic brim, where it lies above the obturator vessels, reaches the upper angle of the thyroid foramen, and piercing that foramen it divides into two branches, an anterior and a posterior. The anterior branch is distributed to the hip joint, to the adductor longus, adductor brevis, the gracilis, sometimes to the pectineus, and has a cutaneous branch which passes to the skin of the inner side of the thigh. The posterior branch also drops a twig to the hip joint, and supplies the obturator externus and adductor magnus muscles.

\section{The Anterior Crural.}

The Anterior Crural Nerve is the largest branch of the lumbar plexus; and receives fibres from the second, third, and fourth lumbar nerves. It passes downward in the interval between the psoas magnus and iliacus internus muscles, and runs beneath Poupart's ligament, where it lies from one-fourth to one-half an inch to the outer side of the femoral artery. After descending for a very short distance, rarely more than one inch and generally about half-an-inch, it divides into branches, which are usually described as superficial, or culcueous, branches, and decp, or muscular branches.

\section{Superficial Branches.}

Middle Cutaneous

\section{Internal Cutaneous}

Internal Saphenous

\section{The Middle Cutaneous.}

The Middle Cutancous branch passes forward superficial to the femoral artery; and is distributed to the integument which covers the front of the thigh, lying between the internal cutaneous and the external cutaneous, which is a direct branch of the lumbar plexus, and communicating with both of these nerves. The middle cutaneous generally perforates the sartorius muscle, and in doing so, gives off branches which supply that muscle.

\section{The Internal Cutaneous.}

The Internal Culcueous is smaller than the middle. It passes 
inward over the femoral artery; and dividing into an anterior and a posterior branch, passes to the inner side of the thigh.

The anterior branch communicates with the middle cutaneous while the posterior branch suns along the edge of the sartorius muscle, pierces the fascia lata, and is distributed to the integument of the inner side of the thigh as low down as the knee joint, communicating in its course with the cutaneous branch of the obturator nerve.

\section{The Internal, or Long, Saphenous.}

The Internal, or Long Saphenous Nerie descends in close coutact with the outer side of the sheath of the femoral vessels until it reaches Hunter's canal, where it becomes superficial to these vessels. In the upper portion of its course it is in close relationship with the muscular branch to the vastus internus muscle, the two nerves bearing almost exactly the same relation to the femoral artery, with the saphenous slightly in front. At Hunter's canal the nerve sometimes passes across that fibrous structure, and is separated by it from the front of the femoral artery; but usually lies on the femoral artery in Hunter's canal, but not in the femoral sheath. At the lower extremity of Hunter's canal the nerve passes beneath the tendons of the sartorius muscle ; and accompanies that muscle downward to its insertion, where it pierces the insertion between the sartorius and the gracilis to become superficial just below the knee. It now comes into relationship with the internal saphenous vein ; and accompanies that vessel to pass in front of the internal malleolus, and then to continue its course on ward to the inner side of the great toe, which it supplies.

It gives off throughout its course cutaneous branches, which are distributed throughout the course of the nerve. At the knee joint it gives one large branch, the patella, which pierces the sartorius, and running downward and outward, aids in the formation of the patella plexus.

\section{Deep, or Muscular Branches.}

The deep, or muscular, branches are a nerve to the iliacus internus, given off while the anterior crural is in the abdomi- 
nal cavity ; branches to the pectineus (usually two in number, though sometimes but one) which pass beneath the femoral vessels ; a separate branch occasionally to the sartorius, though that muscle is usually supplied by the middle cutaneous; and a long branch, already mentioned, the nerve to the vastus internus, which is in close relationship to the femoral artery and the internal saphenous nerve, enters the vastus internus just above Hunter's canal, and there gives branches to the vastus externus and to the rectus, each of these entering the corresponding muscle from its deep surface.

It will be seen, therefore, that the anterior crural nerve by means of one of its superficial branches, the middle cutaneous, supplies one of the muscles of the front of the thigh, the sartorius; while the two vasti and the rectus are supplied by deep branches of the anterior crural. The one remaining muscle on the front of the thigh, the tensor vagina femoris, is supplied by a branch from the sacral plexus of nerves.

\section{The Lumbo-Sacral Cord.}

The last branch of the lumbar plexus is a communicating branch, called the Lambo-Sacral Cord. This cord is the joint product of the fifth lumbar nerve with a branch from the fourth; and passes over the brim of the pelvis to unite with the first sacral nerve, commencing the formation of the sacral plexus.

\section{The Sacral Plexus.}

The Sacral Plexus itself is the product of the lumbo-sacral cord, with the furst, sccond, third, and half of the fourth sacral nerves. The fibres entering into this plexus are more intimately united than in any other nervous plexus of the body. It is triangular in outline, the base of the triangle extending to the anterior sacral foramina, the apex being directed downward and outward to the great sacro-sciatic foramen.

The Lumbo-Sacral Cord, passing behind the common iliac artery, unites with the first sacral nerve, then at a short distance from the anterior spinal foramina the second sacral unites with this cord, then the third, and lastly the upper half of the fourth, so that while lying upon the pyriformis muscle the sacral plexus consists of but a single, broad triangular 
cord, there being no such communication, followed by subdivision, as there is in the case of the brachial plexus.

The plexus, after being formed in this way, extends downward and outward to the upper margin of the great sciatic foramen, lying upon the pyriformis muscle, having the internal iliac artery in front of it, some branches of which vessel pass between the cords entering into the formation of the plexus and having the second portion of the rectum (more correctly, the first portion of the rectum) resting upon the cords as they emerge from the sacral foramina. On the left side, though separated from it by the peritoneum, the end of the sigmoid flexure of the colon would also rest upon the front of this plexus.

\section{Branches of the Sacral Plexus.}

Way-side

Muscular Visceral
Terminal

Gluteal Internal Pudic Lesser Sciatic Great Sciatic

Besides its terminal branches, this plexus also gives off certain wayside, or collateral branches, which are distributed to the muscles and viscera in the pelvic cavity. The muscular branches come in part from the plexus and in part from the cords before they unite to form the plexus. They supply the pyriformis, obturator internus, the gemelli and the quadratus femoris.

The Terminal Branches are the Gluteal, Internal Pudic, Lesser Sciatic, and Great Sciatic Nerves.

\section{The Gluteal.}

The Gluteal or Superior Gluteal Nerve, as it is frequently called, arises from the upper portion of the sacral plexus, and leaves the pelvic cavity by passing above the pyriformis muscle through the greater sacro-sciatic foramen. It then appears in the interval between the gluteus medius and the gluteus minimus muscles, where it divides into two branches, superior and inferior. The superior follows the origin of the gluteus minimus, and is supplied to the gluteus medius from the under surface of that muscle, while the inferior passes downward, forward, and outward, distributing fibres to the gluteus min- 
mus, its terminal branch ending in the tensor vagina femoris.

Inferior Gluteal. The lumbo-sacral cord and the first and second sacral nerves give origin to the second sacral nerve, which frequently arising in common with the one above mentioned, is sometimes distinguished as the inferior gluteal. It also passes above the pyriformis muscle through the greater sacro-sciatic foramen, and enters the deep face of the gluteus minimus muscle, being distributed to it by a number of small branches.

\section{The Internal Pudic.}

The Internal Pudic Nerve arises from the lower part of the sacral plexus, leaves the pelvic cavity through the greater sacro-sciatic foramen, below the pyriformis, crosses the spine of the ischium, and re-enters the pelvic carity through the lesser sciatic foramen. It then courses along the internal face of the ischium ahout an inch above its tuberosity, passes to the ramus of the ischium, and is enclosed in the fibrous sheath called Alcock's canal. It then passes between the two layers of the perineal fascia, and terminates by dividing into two branches, an upper one, the nerve to the dorsum of the penis, and an inferior branch, called the Perineal.

Prior to this division the internal pudic nerve gives off an Inferior Hemorrhoidal Branch, corresponding to the artery of the same name, which is distributed to the sphincter ani muscle and to the integument around the anus.

\section{Terminal Branches of The Internal Pudic.}

\section{Dorsalis Penis}

Perineal

\section{The Dorsalis Penis.}

The Dorsalis Penis Nerve, the branch to the back of the uenis, passes upward and forward along the ramus of the ischium and pubes, accompanying the artery of the same name, pierces the suspensory ligament of the penis, and runs forward upon that organ to be distributed to the integument that covers it and to the corpus cavernosum, while its terminal branches end in the glans penis. In its course along the back of the penis it gives off lateral cutaneous branches, which are distributed to the skin upon the sides of the organ. 


\section{The Perineal.}

The Perineal Nerve, the second branch of the internal pudic, divides into two sets of branches, superficial and deep, or cutaneous and muscular.

The cutaneous branches are two, anterior and posterior, and pass upward and forward, to be distributed to the scrotum, perineum, and under surface of the penis, in which latter situation they communicate with the dorsal nerve of the penis. In the female of course the upper of these two branches would be distributed to the labia majora, the analogue of the scrotum in the male.

The muscular branches are distributed to the muscles of the perineum; that is, the transversus perinei, erector penis, accelerator urinæ and compressor urethræ, these names being given to the muscles in the male subject, each having its analogue in the female. That branch which passes to the transversus perinei corresponds to the transverse perineal artery. Some deeper branches of this nerve also enter the corpus spongiosum, and supply that body, with its attached muscles.

\section{The Lesser Sciatic.}

The Lesser Sciutic Nerve usually arises by two roots. It passes from the pelvic cavity through the greater sacro-sciatic foramen below the pyriformis muscle, and runs down opposite the middle of the interval between the trochanter major and the tuberosity of the ischium, lying beneath the gluteus maximus muscle. It emerges from the lower border of this muscle: and pursuing its course down the back of the thigh, beneath the fascia lata reaches the superior angle of the popliteal space, where it breaks up into its terminal branches, which after being distributed to the skin covering the popliteal space, anastomose with the cutaneous branch from the great sciatic and with the plexus around the patella.

This nerve is almost entirely a cutaneous branch, except for the set of fibres which enters the gluteus maximus muscle, that muscle receiving its nerve supply entirely from the sacral plexus, though some of the fibres pass to it from the inferior 
gluteal while the remaining branches come from the lesser sciatic. The latter branches emerge from below the lower border of the muscle, and then reverse their course to enter it from its deep surface.

The cutaneous branches are distributed to the skin on the back of the thigh as far down as the popliteal space. One branch of large size is individualized as a separate nerve, called the Inferior Pudendal.

The Inferior Pudendal curves forward, running below the tuber ischii, passes to the scrotum in the male and the labia in the female, communicating with the superficial perineal and the terminal branch of the inferior hemorrhoidal.

\section{The Great Sciatic.}

The Great Sciatic is the longest and largest nerve in the body. It appears to be, and in reality is, a continuation of the sacral plexus. Triangularat its commencement, it soon becomes circular in form. It emerges from the pelvic cavity below the pyriformis muscle through the greater sacro-sciatic foramen, being the last of the numerous structures which emerge from this foramen. These structures are seven in number. Two of them pass above the pyriformis, and the remaining five below that muscle. Those which pass above the pyriformis are the gluteal nerve and artery; those passing below it are the internal pudic, the lesser sciatic and great sciatic nerves, the internal pudic and ischiatic, or sciatic, arteries. This does not complete the total number of structures which pass through this foramen, because in addition should be mentioned the pyriformis muscle itself and the companion veins of the arteries named.

After escaping from the pelvis, the great sciatic nerve passes down the middle of the back of the thigh, resting on the adductor masnus muscle, and covered by the muscles which arise from the tuberosity of the ischium. Two of these muscles, the semi-tendinosus and semi-membranosus, quickly pass to its inner side, while the biceps covers the nerve for some distance down the thigh. At the upper angle of the popliteal space (formed externally by the biceps and internally by the

Des Anat-29 
semi-tendinosus and semi-membranosus muscles) the nerve appears between these muscles, called the "ham-string" muscles. It then passes into the popliteal space, and divides into its two terminal branches, the Internal and External Popliteal Nerve.

The point of division is variable, and may exist anywhere between the sacral plexus and the centre of the popliteal space. In a few rare cases the internal and external popliteal nerves come off separately from the sacral plexus; and in such cases they are sometimes separated from one another by the fibres of the pyriformis muscle. In other cases the division takes place below the tuberosity of the ischium, and in still other cases at the middle of the popliteal space. As the nerves are bound together simply by connective tissue, the dissector may split them up to any point between the popliteal space and the origin of the two nerves from the sacral plexus.

The point of termination usually given for the great sciatic is the superior angle of the popliteal space; but it rarely divides before reaching a lower point, so that the great sciatic itself, instead of its internal popliteal branch, becomes a relation of the popliteal artery.

Before dividing into terminal branches, the great sciatic gives off muscular branches to supply the posterior femoral group, cutaneous branches which communicate with the lesser sciatic nerve, and a branch to the adductor magnus.

\section{Branches of the Great Sciatic.}

\section{Internal Popliteal}

External Popliteal

\section{The Internal Popliteal.}

The Internal Popliteal Nerve, the larger of the two terminal branches of the great sciatic, commences below the upper angle of the popliteal space, to pass through that space, and terminate by becoming the posterior tibial nerve. As it enters at the apex of the popliteal space, it necessarily lies to the outer side of, and superficial to, the popliteal artery and vein; but the nerve has an inclination inward, while the inclination of the artery is outward, hence at the middle of the popliteal 
space, opposite the posterior ligament of the knee joint, the nerve is found directly behind the artery, with the vein interposed between itself and the artery, while at the termination of the popliteal space both nerve and vein have become internal to the artery, the vein still lying between the nerve and the artery. At the lower border of the popliteus muscle, at a point which corresponds to the junction of the upper one-fifth with the lower four-fifths of the shaft of the tibia, the internal popliteal nerve terminates by changing its name to the Posterior Tibial.

The nerve, like the other important structures contained in this diamond-shaped space, has the internal ham-string muscles to its inner side above, and the inner head of the gastrocnemius below, while to its outer side are, above, the biceps, and below, the outer head of the gastrocnemius and the plantaris muscles. It is covered by the strong fascia which extends between these muscles and roofs in the entire space.

\section{Branches of the Internal Popliteal.}

\section{Articular \\ Muscular, or Sural \\ Cutaneous}

The Branches of the Internal Popliteal are divided into three sets, articular, muscular, and cutancous.

The Articular Branches are three in number; and are known as Superior and Inferior Internal Articular, and Azygos Articular. They accompany the arteries of the same name; and are distributed in the same way. They anastomose with one another, and with the other nerves which surround the knee joint. passing as far as the patella plexus, while the azygos articular, piercing, with the corresponding artery, the posterior ligament of the knee, is distributed to the synovial membrane in the interior of that joint.

The Muscular, or Sural (L. Sura, the calf of the leg), Branches accompanying the sural branches of the popliteal artery, are distributed to the two heads of the gastrocnemius and to the plantaris muscle.

In addition to these sural branches, the internal popliteal nerve gives off the branches which supply the popliteus muscle. These branches, two in number, pass down to the lower bor- 
der of the popliteus muscle, and, reversing their course, pass upward between that muscle and the posterior surface of the interosseous membrane, to enter the muscle from its deep face.

The only Cutaneous Branch, the External Saphenous, or Communicans Poplitei, passes downward in the interval between the two heads of the gastrocnemins muscle, lies in the groove upon the posterior surface of the belly of that muscle, accompanied by the external saphenous vein; and, about the middle of the calf of the leg, receives a branch of communication from the external popliteal nerve, this branch being called the Communicans Peronei. After receiving this branch it continues downward, with a slight inclination outward, passes behind the external malleolus, and reaching the dorsum of the foot, is distributed to the integument on the back of the leg, the outer, back part of the ankle, and the outer side of the foot as far as the little toe, where it commumicates with a branch of the musculo-cutaneous nerve.

\section{The Posterior Tibial.}

The Posterior Tibial Nerve is a continuation of the internal popliteal; and commences where that nerve terminates at the lower border of the popliteus muscle. It at first lies to the inner side of the posterior tibial artery; but about two inches below the origin of that vessel, crosses it superficially opposite the origin of the peroneal branch, and lies for a short distance in the interval between the peroneal and posterior tibial arteries. It then descends to the outer side of the posterior tibial, and remains a continuous external relation of that vessel to its termination, but lies on a plane posterior to it.

It divides midway between the inner malleolus and the posterior tuberosity of the os calcis into the internal and external plantar branches.

\section{Branches-Way-Side.}

The Branches of the Posterior Tibial Nerve are muscular branches, which supply the tibialis posticus, flexor longus pollicis, and the flexor longus digitorum. At the ankle joint, however, it gives off calcanean branches, which, accompanying 
the corresponding arteries, are distributed to the skin on the inner aspect of the heel, and an articular branch which supplies the ankle joint.

\section{Terminal or Plantar.}

Internal Plantar

External Plantar

The Internal and External Plantar Neries differ from one another, and more distinctly from the internal and external plantar arteries, because while the internal plantar artery is smaller in size than the external, aud has a less extended distribution, the internal plantar nerve is larger than the external. The internal plantar nerve corresponds in in its distribution to the median nerve of the hand; while the external plantar corresponds to the ulnar nerve in the hand. That is, the internal plantar nerve supplies three toes and a half, equal to the three and one half fingers of the median; while the external plantar supplies one toe and a half, the equivalent of the ulnar of the hand.

\section{The Internal Plantar.}

The Internal Plantar Nerve passes forward as the companion of the internal plantar artery, supplies the inner half of the flexor brevis digitorum, then continuing its course it gives a branch to the abductor pollicis and to the two internal lumbrical muscles, and to the flexor brevis pollicis and articular branches to the tarsus and metatarsus.

It then divides into its terminal branches, the most internal of which passes to the inner side of the great toe, the second to the cleft between the second and great toes, where it divides into branches for the inner side of these two toes, the third to the cleft between the second and third, and the fourth, after giving off a brauch of communication to the external plantar, runs to the cleft between the third and fourth toes and supplies the adjacent sides of these toes.

\section{The External Plantar.}

The ExternalPlanlar Neric accompanies the external plantar artery, passes beneath the origin of the flexor brevis digitorum, and lies between that muscle and the Abductor 
minimi digiti. It then runs forward as far as the posterior extremity of the fifth metatarsal bone. It then plunges beneath the second layer of muscles on the sole of the foot, and accompanies the arch formed by the external plantar artery. In this course it supplies the abductor minimi digiti, the outer half of the flexor brevis minimi digiti, the second layer of muscles on the foot, then the flexor accessorious, and the two outer lumbricals, and gives branches to the interosseous muscles occupying the third and fourth interosseous spaces. As it passes between the second and third layers of the foot it supplies all the muscles of the third layer of the sole. Its digital branches are, one to the outer side of the little toe, and one, which after communicating with the internal plantar, divides at the cleft between the fourth and fifth toes and supplies the adjacent sides of these toes.

\section{The External Popliteal.}

The External Popliteal nerve begins as a branch of the great sciatic, near the upper angle of the popliteal s pace, passes downward and outward and is only momentarily a relation of the popliteal artery, lying superficial to it and to its outer side. It then passes behind the external tuberosity of the femur, crosses the head of the fibula, and plunging into the substance of the peroneus longus muscle, terminates there by dividing into the anterior tibial and the musculo-cutaneous nerves. The relation of this nerve to the popiiteal artery is, as before mentioned, only momentary, except in those cases in which the nerve is so closely bound to the internal popliteal as to diverge from that nerve at the middle of the popliteal space.

\section{Branches.}

\section{Way- $\{$ Articular}

Side $\{$ Communicans Peronei

Termi- $\{$ Anterior Tibial

nal Musculo-Cutaneous

In all cases the external popliteal nerve gives off two articular branches corresponding to the superior external and inferior external articular arteries, these branches accompanying the arteries and being distributed in the same way. A recurrent articular branch is also given off which accompanies the anterior tibial artery and supplies the front of the knee. 


\section{The Communicans Peronei.}

Below the knee joint the external popliteal gives off the Communicans Peronei, which unites with the communicans poplitei to form the External Saphenous Nerve and to be distributed with that nerve.

\section{The Anterior Tibial.}

Of the two terminal branches of the external popliteal the Anterior Tibial, after passing through the fibres of the peroneus longus, lies between that muscle and the extensor longus digitorum. It then passes to the front of the interosseous membrane, having passed obliquely beneath the extensor longus digitorum, and becomes a relation of the anterior tibial artery, having the same muscular relations with that vessel; viz., for the upper third to its outer side is the extensor longus digitorum, to its inner side the tibialis anticus: in the middle third the extensor proprius pollicis to the outer side and the tibialis anticus still to the inner side. In the lower third it has the extensor longus digitorum to the outer side and the extensor proprius pollicis to the inner side, which muscle has crossed to the front of the nerve and displaced the tibialis anticus. To the artery it bears a triple relation, lying first to the outer side and resting on the interosseous membrane, then for the middle third of the anterior tibial artery the nerve rests on its front, separated by the artery from the interosseous membrane, and in the lower third of the vessel the nerve drops to the outer side, and maintains that relation to the front of the ankle joint.

At the front of the ankle joint the nerve passes beneath the anterior annular ligament, but does not change its name as the artery does. To the dorsalis pedis artery it maintains the same relation which it bore to the anterior tibial, lying on the outer side of that vessel. It continues as far as the cleft between the great and second tnes, where it divides into two branches to supply the adjacent sides of these two toes, after having communicated with the long saphenous branch of the anterior 
crural nerve on the inner side of the great toe, its outer branch communicating with the musculo-cutaneous.

\section{Branches.}

The Anterior Tibial Nerve in its course over the leg supplies branches to the tibialis anticus, extensor longus digitorum, and extensor proprius pollicis, while on the outer side it has a gangliform enlargement which corresponds to that on the posterior interosseous of the forearm from which are given off branches to supply the flexor brevis digitorum muscle and the tarsal and metatarsal articulations.

\section{The Musculo-Cutaneous.}

The Musculo-Cutaneous Nerve, after being formed by the division of the external popliteal, passes downward, first in the fibres of the peroneus longus muscle, then in the interval between that muscle and the peroneus brevis, then between the two peronei muscles and the extensor longus digitorum. Running across on the front of the external malleolus, it passes on to the dorsum of the foot, where it breaks up into cutaneous branches to supply the toes between the outer face of the second toe and the inner face of the little toe, and gives a twig to the inner side of the great toe.

In its course through the leg it gives off branches which supply the peroneal muscles. As it passes across the external malleolus it gives a branch which corresponds to the external calcanean branches of the anterior tibial artery; and these branches are distributed to the skin on the outer aspect of the heel.

The distribution of the nerve on the dorsum of the foot is very irregular. The most frequent arrangement is that it communicates on the outer side of the little toe with the external saphenous nerve, then gives a branch which passes to the cleft between the little and the fourth toes and supplies them, a third branch to the cleft between the fourth and third toes, and a fourth branch to the cleft between the third and second toes-which branch communicates with the anterior tibial 
nerve, - and a branch to the inner side of the great toe which communicates with the long saphenous.

It will be seen that the distribution of the nerves on both the back of the hand and the back of the foot is quite as irregular as the distribution of the arteries in the same situation. In some instances the anterior tibial nerve has been seen to supply the great, the second, and half of the third toe; and, in other cases, the musculo-cutaneous supplies the little, fourth, third, and second toes; while in but few cases have I been able to trace the internal saphenous to the extremity of the great oe. or the external saphenous to the extremity of the little toe. 


\section{THE CRANIAL NERVES.}

The cranial nerves comprise twelve pairs, one on each side, those of one side having their counterpart on the other.

The definition of a cranial nerve is that it appears at the base of the brain and emerges through an aperture in the skull. The point on the base of the brain where the nerve appears is called its apparent origin, since it may be traced into the interior of the brain to what is called its real, or deep, origin. The real origin of many of the nerves is still a matter of dispute.

The cranial nerves are named numerically, in pairs, from before backward; besides which each pair is known by another or, in some cases, several other names derived from the part to which the nerve is distributed. The following table gives the synonyms of the twelve pairs:

- First Pair, Olfactory.

Second Pair, Optic.

Third Pair, Motores Oculorum.

Fourth Pair, Pathetic (Trochleares).

Fifth Pair, Trifacial (Trigeminus).

Sixth Pair, Abducentes.

Seventh Pair, Facial (Portio Dura).

Eighth Pair, Auditory, (Portio Mollis).

Ninth Pair, Glosso-Pharyngeal.

Tenth Pair, Pneumogastric, Par Vagum, Vagus.

Eleventh Pair, Spinal Accessory.

Twelfth Pair, Hypoglossal.

These twelve pairs were formerly considered as only nine from the fact that, as will be seen, they emerge through nine foramina of the cranium at the base. The classification into nine pairs is known as that of Willis and the one just given as that of Sommerring. The two classifications are similar for the first six pairs, the seventh pair, in Willis' classification, 
consisted of the seventh and eighth, while the eighth consisted of the glosso-pharyngeal, pneumogastric and spinal accessory, and the ninth of the hypoglossal.

A general outline of the cranial nerves successively, from their apparent origin to their point of emergence, will first be given and afterwards each pair will be taken up and described.

The first seen on the base of the brain from before bacleward is the first pair. The first nerve lies on the under surface of the anterior lobe of the cerebrum in a groove a little external to the longitudinal fissure, having its commencement by a three forked origin just, in front of the anterior perforated space. Near the auterior extremity of the anterior cerebral lobe it enlarges into an oval-shaped mass which lies upon the cribriform plate of the ethmoid bone and sends its branches of distribution through the foramina seen there.

The second nerve is first seen under the name of the optic tract, approaching its fellow from the outer aspect of the crus cerebriand passing beside the tuber cinereum and uniting in front of it with its fellow to form the optic chiasm or commissure from which the two optic nerves diverge forward to enter the orbit through the optic foramina.

The third nerve is seen emerging to the inner side of the crus cerebri, just in front of the pons Varolii, and runs forward to leave the cranium through the anterior lacerated foramen.

The fourth nerve has its apparent orign external to the third. on the optic side of the crus cerebri, and also has its exit through the anterior lacerated foramen.

The fifth is the largest of the cranial nerves and is first seen just behind the origin of the fourth. It pierces the lateral aspect of the pons Varolii and splits into three portions, opthalmic, superior and inferior maxillary, which require three apertures of exit from the cranium.

The opthalmic leaves through the anterior lacerated foramen, the superior maxillary through the foramen rotundum, the inferior maxillary through the foramen ovale.

The sixth nerve takes its apparent origrin behind that of the fifth from the upper constricted portion of the medulla oblon- 
gata, just behind the pons Varolii, and passes forward to gain egress through the anterior lacerated foramen, which is thus seen to transmit three entire cranial nerves, viz: third, fourth and sixth, and a part of another, viz: the opthalmic branch of the fifth.

The seventh, eighth, ninth, tenth and eleventh all arise in the order named, from above downward, in the groove between the corpora olivare and restiformia of the medulla oblongata. The seventh and eighth leave the cranium through the same opening, the internal auditory meatus, while the ninth, tenth and eleventh all gain exit through the posterior lacerated foramen.

The twelfth has its apparent origin to the inner side of the five preceding nerves, from the groove between the corpora olivare and pyramidale, by numerous filaments which are speedily collected into two cords, which unite to form the nerve as it emerges through the anterior condyloid foramen.

\section{The First Nerve.}

The first or olfactory nerve arises by three forks or roots, close to the anterior perforated space. The external or long root consistsof white fibres and may be traced along the fissure of Sylvius into the middle lobe of the cerebrum; the middle root is of gray matter and springs from the posterior part of the anterior lobe; the inner or short root is of white fibres and also arises from the posterior part of the anterior lobe. The three roots unite to form the nerve, just in front of the anterior perforated space, which passes forward, triangular in shape and grayish in color, lying in a groove on the under surface of the anterior lobe of the cerebrum a little external to the longitudinal fissure. The nerve swells into an oblong grayish enlargement, called the bulbus olfactorins, which lies beside the crista galli on the cribriform plate of the ethmoid bone. From the under surface of the olfactory bulb are given off some twenty filaments, which descend into the nose through the foramina of the cribriform plate to be distributed to the mucous membrane of the nose as far down as the middle turbinated bone. These filaments are arranged in 
three sets, viz: those to the septum, those to the external wall and those to the roof of the nose.

\section{Second Nerve.}

The second or optic merve has its apparent origin just external to the crus cerebri, whence it may be traced to its deep or real origin from various parts of the optic thalamus and from the corpora quadrigemina. Winding around the outer side of the crus cerebra, it passes forward and inward beside the tuber cinereum and in front of it unites with its fellow to form the optic commissure or chiasm. The portion extending from the origin to the optic chiasm is called the optic tract. From the optic chiasm the two optic nerves diverge anteriorly to enter the orbit through the optic foramina, each piercing the sclerotic and choroid coats of the eye-ball to expand into tine retina.

The connection between the optic tract, chiasm and nerve is as follows: The optic tract is composed of filaments, the outermost of which pass into the nerve of that side, while the innermost curve over, through the chiasm, to form the innermost fibres of the opposite tract, and the middle fibres of the tract of one side pass through the chiasm to form the middle fibres of the nerve of the opposite side; and the innermost fibres of one nerve pass through the chiasm and become the innermost fibres of the opposite nerve, thus accounting for all the filaments.

\section{The Third Nerve.}

The third nerve, or motor oculi, leaves the brain just to the outer side of the crus, near the front of the pons. The deep origin can be traced to the locus niger and tegmentum. It pierces the dura below and to the outer side of the posterior clinoid process. It then passes forward lying in the outer wall of the cavernous sinus, with the fourth and opthalnic division of the fifth below and external to it, and the sixth internal and separating the third from the internal carotid. Continuing its course the nerve reaches the anterior lacerated foramen, or sphenoidal fissure, and splits into two divisions. Both divisions enter the orbit, passing between the two heads 
of the external rectus muscle and being separated from each other by the nasal nerve. In the sphenoidal fissure the third lies on the lower wall of the fissure with the nasal and the sixth, the upper division of the third being most external, then the nasal, lower division of the third and sixth in the order named. The terminal branches are now given off, the nerve supplying all the muscles of the ocular group except the exterual rectus and the superior oblique. The upper division, which passes above the optic nerve, supplies the superior rectus and the levator palpebræ. The lower division passes beneath the optic nerve and supplies the inferior and internal recti and the inferior oblique.

While lying in the cavernous sinus the nerve communicates with the cavernous plexus. Sometimes the superior division receives a communication from the nasal; while the branch to the inferior oblique gives off the motor root of the ciliary ganglion. The branches to the muscles enter on their deep or ocular surface.

\section{The Fourth Nerve.}

The fourth, or patheticus, appears just external to the apparent origin of the third on the outer surface of the crus cerebri. Its deep origin is a nucleus on the floor of the aqueduct of Sylvius. After winding around the crus cerebri the fourth pierces the dura below the posterior clinoid process and runs forward in the sinus with the third above and the opthalmic division of the fifth below it. On reaching the sphenoidal fissure the fourth passes through that fissure at its highest and most internal point. On reaching the orbit it crosses over the origin of the levator palpebræ and enters the superior oblique on the ocular surface of that muscle. It communicates with the cavernous portion of the sympathetic and gives off a small and unimportant branch to the teutorium.

\section{The Fifth Nerve.}

The fifth, or trifacial nerve, appears just behind the fourth, emerging from the side of the pons. It has its superficial, or apparent origin, by two roots which spring from the lateral as- 
pect of the pons, the anterior, consisting of two or three bundles, being the motor, and the posterior the sensory, root. The deep origin is from the floor of the fourth ventricle.

The two roots pass through the dura just above the internal auditory meatus and continue forward to the apex of the petrous portion of the temporal bone, where a gangliform enlargement, called the Gisserian ganglion, is formed on the posterior or sensory root. The motor root passes beneath the ganglion of Gasser without being connected with it.

The ganglion divides into three branches, opthalmic, supcrior maxillary and inferior maxillary. The latter is joined bodily by the small root of the nerve and is, therefore, the only one of the three divisions possessing motor endowments. Besides its terminal branches the ganglion gives off branches to the dura and middle fossa of the skull and receives a communication from the cavernous plexus.

\section{The Opthalmic Nerve.}

The opthalmic branch passes forward and soon divides in to three branches, lachrymal, frontal and nasal, which enter the orbit through the foramen lacerum anterius. This nerve passes through an opening in the dura lying below and external to that for the fourth. It then runs for ward on the outer wall of the cavernous sinus, its place being below and to the outer side of the third and fourth. The terminal branches are given off just before the nerve reaches the sphenoidal fissure.

\section{Branches.}

\section{Lachrymal}

\section{Nasal}

Frontal

The Lachrymal branch of the opthalmic passes through the upper, external portion of the sphenoidal fissure and enters the orbit where it runs along the external wall lying above the external rectus muscle. It continues its course until it reaches the lachrymal gland, which it supplies, and then sends filaments which pierce the palpebral ligament and are distributed to the skin of the upper eyelid and communicates with the facial nerve. 
The Fronta1 is the largest branch of the opthalmic division. It enters the orbit through the sphenoidal fissure, 1ying between the lachrymal, externally, and the fourth, internally. It then runs forward on the levator palpebræ and divides into the supra-trochlear and supra-orbital.

The supra-orbital emerges from the orbit through the supraorbital foramen, gives off branches to the upper eyelid and the muscles and integument of the head as far back as the vertex. While lying in the orbicularis palpebraum, it communicates with the facial nerve.

The supra-trochlear branch advances to the inner angle of the orbit where it emerges above the fovea trochlearis, hence its name, and is distributed to the inner angle of the eye and the integument of the root of the nose and middle of the forehead. It gives a communicating branch to the infra-trochlear branch of the nasal.

The Nasal Nerve enters the orbit between the heads of the external rectus, lies beneath the superior oblique and the levator palpebræ crosses the optic nerve, advances along the inner wall of the orbit, re-enters the cranium through the anterior ethmoidal foramen and is there found lying in the slit beside the crista galli, whence it sinks into the cavity of the nose and divides into a branch for the mucuous membrane of the nose, and another to descend along the posterior aspect of the nasal bone to its lower elge, where it emerges through the notch found there and supplies the integument of the nose to its tip. Just as the nasal nerve is entering the ethmoidal foramen it gives off a branch called the infra-trochlear, which passes forward to emerge at the inner angle of the orbit below the fovea trochlearis and supply the lachrymal sac and inner angle of the eye. Besides the infra-trochlear it gives off in the orbit three other branches; one to the opthalmic ganglion of the sympathetic system and two ciliary branches which pierce the sclerotic coat, pass forward between it and the choroid and are distributed to the iris.

\section{Superior Maxillary Nerve.}

The superior maxillary division of the fifth nerve. like the opthalmic, has a forward course. It gains egress through the 
foramen rotundum, continues its forward direction across the spheno-maxillary fossa, along a groove on the floor of the orbit, sinlis beneath the floor and emerges at the infra-orbital foramen on the face to give sensibility to adjacent parts, viz: the lower lid, cheek, side of the nose and upper lip.

\section{Branches.}

Besides the terminal ones, the branches of the superior maxillary are the following, emitted in the order given:

The orbilal or temporo-malar, branch enters the orbit through the spheno-maxillary fissure and divides into two branches, temporal and malar. The temporal branch enters the temporal fossa through the outer wall of the orbit, while the malar branch continues along the outer wall of the orbit to emerge on the cheek at the outer angle.

The spheno-palatine are two branches of communication which descend to Meckel's ganglion of the sympathetic in the spheno-maxillary fossa.

The chief distribution of the superior maxillary is to the teeth and gums of the upper jaw and this is effected by two branches, anterior and posterior dental. The posterior dental is first emitted and supplies the molar and bicuspid teeth.

The anterior dental leaves the main trunk just as it is about to emerge on the face and supplies the two incisors, the canine and the front bicuspid. The anterior and posterior dental nerves anastomose in the boue and supply each tooth with as many filaments as the tooth has fangs, besides giving twigs to the gums.

\section{Inferior Maxillary Nerve.}

The inferior maxillary dizision of the fifth nerve consists of a brauch from the ganglion of Gasser and the small motor ront of the fifth nerve, which unite and pass downward through the foramen ovale, immediately after emerging from which the nerve divides into an anterior and a posterior trunk. The distribution of each trunk is as follows:

The anterior tmo breaks up into branches which supply the muscles of mastication, each branch bearing the name of

Des Anat-30) 
the muscle to which it is distributed and conveying motor influence.

The masseteric brauch runs out above the external pterygoid muscle, crosses through the sigmoid notch and is distributed to the deep face of the masseter.

The deep temporal branches anterior and posterior, are distributed to the deep face of the temporal muscles.

The buccal branch pierces the external pterygoid, sometimes lying beneath the coronoid process and sometimes running through the temporal muscle, to be distributed by an upper and a lower branch to the buccinator.

The pterygoid branches are one to each pterygoid muscle; that to the external pterygoid being frequently a branch of the buccal nerve.

The posterior trank divides into three branches, inferior dental, gustatory and auriculo-temporal.

\section{Inferior Dental.}

The inferior dental branch curves forward and downward to enter the dental foramen of the lower jaw and run along a canal in the bone giving filaments to all the teeth of the lower jaw. It first lies beneath the external pterygoid muscle and then between the internal lateral ligament and the ramus of the lower jaw.

As it passes the mental foramen it gives off a branch which escapes through that foramen and supplies the integument of the lower lip. Just before entering the dental foramen the nerve gives off a branch called the mylo-hyoidean, which descends along the groove on the inner surface of the inferior maxilla and is distributed to the mylo-hyoid muscle and the anterior belly of the digastric.

\section{Gustatory Branch.}

The gustatory nerve passes downward and forward, first behind the external pterygoid muscle, then between the two pterygoid muscles and then between the internal pterygoid and ramus of the lower jaw, and reaching the side of the tongue splits into many filaments to supply the anterior part of that 
organ. While between the two pterygoid muscles it receives at an acute angle the chorda tympani a branch of the seventh nerve, which it transmits to the submaxillary ganglion of the sympathetic. Beneath the tongue the gustatory gires off a branch of communication to the twelfth.

It distributes branches to the filiform and fungiform papillæ, the mucous membrane of the tongue, mouth, and gums.

\section{Auriculo-Temporal.}

The auriculo-temporal branch arises by two roots. It first passes backward behind the articulation of the lower jaw, then ascends between the meatus auditorius externus and condyle of the lower jaw and emerging from beneath the parotid gland divides into branches called anterior and posterior temporal which supply the integument of the temporal region. It gives off branches to the articulation of the lower jaw, to the parotid gland, to the pinna, to the meatus auditorius and two communicating branches to the facial nerve.

\section{Sixth Nerve.}

The sixth nerve, or abducens, has its apparent origin from the upper constricted portion of the medulla, just behind the pons, while its deep origin can be traced to a mass of gray matter lying in the floor of the fourth ventricle. The nerve, after piercing the dura over the sphenoid, runs through an opening in the dura just below the posterior clinoid process and passes forward in the cavernous sinus, lying internal to the preceding nerve and in close contact with the internal carotid artery. Continuing its course it passes into the orbit through the sphenoidal fissure just external to the opthalmic vein, runs between the two heads of the external rectus and is distributed to that muscle from its deep surface.

\section{Seventh Nerve.}

The seicnth, or facial, nerve (the motor nerve of the face) is the uppermost of the five nerves which spring from the gronve between the corpora olivare and restiformia. Its deep origin is from the floor of the fourth ventricle, where it communicates with the sixth nerve. Just beneath it, in the same 
groove, is another nerve called the portio intermedia which unites with it. If this be counted as a separate nerve, it may be said that there are six nerves springing successively from this groove. It makes for the internal auditory meatus which it enters in company with the auditory nerve; and, when the two reach the bottom of the canal, the facial parts from its companion and enters another canal in the petrous bone called the aqueduct of Fallopius, which conducts it first outward, then backward and then downward to the stylo-mastoid foramen, emerging from which it passes forward through the parotid gland, over the external carotid artery, and divides into numerous branches which are distributed to all the muscles of the face except those of mastication, and of these the buccinator gets a branch.

\section{Branches.}

Like other cranial nerves, its branches may be divided into two sets, viz: communicating and branches of distribution. While in the auditory meatus, it communicates with the auditory nerve. In the aqueductus Fallopii the large superficial petrosal is sent to Meckel's ganglion, the small petrosal to the otic ganglion and the external petrosal to the sympathetic on the middle meingeal artery. After the nerve emerges from the stylo-mastoid foramen, communications are established with the ninth and tenth cranial nerves, with the carotid plexus, with the auricularis magnus of the cervical plexus and with the auriculo-temporal branch of the fifth, All of the divisions of the fifth communicate with the seventh on the face.

The branches of distribution the tympanic and chorda tympani, arising within the aqueductus Fallopii; the posterior auricular, stylo-hyoid and digastric arising outside the stylohyoid foramen, and two terminal branches, the temporo-facial and cervico-facial.

The tympanic, which arises from the facial while it is in the aqueduct of Fallopius and is distributed to a muscle of the tympanic cavity.

The chorda tympani, which is emitted from the facial just 
above the stylo-mastoid foramen and first ascends through the petrous bone and enters the tympanum at the base of the pyramid on its posterior wall, then crosses on the inner surface of the membrana tympani and leaves through the fissure of Glaser, having emerged from which it joins the gustatory branch between the two pterygoid muscles and continues with it to the submaxillary ganglion.

The stylo-hyoid which supplies the stylo-hyoid muscle.

The digastric, which is distributed to the posterior belly of the digastric muscle.

The posterior auricular, which ascends behind the ear and after communicating with the auricular branch of the pneumogastric is distributed by an anterior branch to the auricle and by a posterior to the back of the head.

The temporo-facial is the larger of the two terminal branches of the seventh. It passes through the parotid gland, which separates it from the external carotid artery, and breaks up into three sets of branches, temporal, malar and infra-orbital, the first supplying the attrahens aurem, occipito-frontalis and orbicularis palpebraum muscles; the second the orbicularis and corrugator supercillii, while the last, the largest branch, supplies the pyramidalis nasi, levator labii superioris and levator anguli oris.

The cervico-facial division passes down ward in the substance of the parotid gland, which separates it from the external carotid. It communicates with the auricularis magnus and divides into buccal branches, which supply the buccinator and orbicularis oris; supra-maxillary for the depressor anguli oris; and infra-maxillary', some of which supply the platysma while others pass to the skin over the supra-hyoid regrion, communicating with the branches of the cervical plexus in that region.

\section{Eighth Nerve.}

The eighth nerve, or portio mollis, or anditory, is the second of the nerves arising from the groove between the corpora olivare and restiforme, its deep origin being from the floor of the fourth ventricle. It passes forward and enters, along with the seventh, the internal auditory meatus, at the bottom 
of which it divides into cochlear and three vestibular branches, which are distributed to corresponding parts of the labyrinth.

\section{Ninth Nerve.}

The ninth nerve, or glosso-pharyngeal, is the third nerve arising in the groove between the corpora olivare and restiforme. Its deep origin is from the floor of the fourth ventricle between the eighth and tenth. It escapes from the cranium, along with the tenth and eleventh, through the posterior foramen lacerum, lying behind the internal carotid artery ; it then passes forward between the artery and the internal jugular vein to the outer side of the artery, then crosses the artery in front of the posterior border of the stylopharyngeus, and then crosses that muscle to be distributed by its terminal filaments to the mucous membrane of the posterior part of the tongue.

This nerve presents two gangliform enlargements called superior, or jugular, and inferior, or petrous. The superior which is very small, is situated in the jugular foramen and does not involve all the fibres of the nerve. The inferior ganglion is situated in a depression on the posterior border of the petrous portion of the temporal bone and gives off branches of cummunication to the pneumogastric and sympathetic.

\section{Branches.}

Besides its terminal branches the ninth nerve gives off four branches of distribution.

The tympanic branch, Jacobson's nerve, passes to the tympanum through a canal which opens on the ridge of bone between the carotid and jugular foramina. After reaching the tympanum it is distributed by six branches, as follows:

(a) One to the lining membrane of the tympanum and Eustachian tube.

(b) One to the fenestra rotunda.

(c) One to the fenestra ovalis.

(d) One to the carotid plexus of the sympathetic.

(e) One to the great superficial petrosal nerve.

$(f)$ One to the lesser superficial petrosal nerve. 
A muscular branch which supplies the stylo-pharyngeus.

The pharyngeal branches, which are found on the posterior part of the pharynx aiding to form a plexus called the pharyngeal from which the pharynx is supplied. The other nerves entering into the formation of this plexus are the pharygeal and superior laryngeal of the tenth and the sympathetic.

The tonsillar branches which form a plexus on the tonsils.

The branches of communication of the ninth nerve are as follows:

One to the ganglion of the tenth nerve.

One to the superior cervical ganglion of the sympathetic.

One to the seventh nerve.

Carotid filaments which descend with the carotid artery.

\section{Tenth Nerve.}

The tenth nerve, or pneumogastric, or par vagum, is the fourth nerve arising from the groove between the corpora olivare and restiforme. It escapes from the cranium through the jugular foramen and, descending the neck, enters the thorax through which it passes along with the oesophagus and gains the abdomen where it is found running from left to right along the lesser curvature of the stomach. In the neck the course and relations of the nerve are the same on both sides, $i_{.} e$. the nerve lies first between the internal jugular vein and the internal carotid artery, then between the internal jugular vein and common carotid artery until it reaches the root of the neck and the relations thenceforward vary according to the side. The right nerve at the root of the neck crosses the first portion of the subclavian artery, enters the chest, reaches the posterior aspect of the root of the right luns; descends thence along the posterior aspect of the nesophagus to the stomach and runs along the lesser curvature of that organ, being distributed by its terminal filaments to its posterior face. The left enters the thorax lying on the front of the first portion of the left subclavian, crosses the front of the arch of the aorta, reaches the posterior aspect of the root of the left lung and thence passes down the front of the resophagus to the stomach, runs aloug its lesser curvature and is distributed to its anterin face. 
While in the jugular foramen the nerve presents a gangliform enlargement called the ganglion of the root, and about half an inch below this a second and larger one called the ganglion of the trunk.

\section{Branches.}

The branches are divided into communicating and branches of distribution. The communicating are mainly connected with the two ganglia on the nerve. The ganglion of the root receives branches from the accessory portion of the spinal accessory, the petrous ganglion of the ninth, the auricular branch of the facial and from the superior cervical of the sympathetic.

The ganglion of the trunk is connected with the twelfth, the superior cervical ganglion of the sympathetic and with the loop between the first and second cervica1.

The branches of distribution are

Auricular

Pharyngeal

Superior Laryngeal

Recurrent Laryngeal

Cervical Cardiac
Thoracic Cardiac

Anterior Pulmonary

Posterior Pulmonary

Eisophageal

Gastric

Of these branches the first arises in the jugular fossa, the next four in the neck and the remainder, except the gastric, which comes off in the abdomen, in the thorax.

The auricular, or Arnold's nerve, is a small branch which springs from the ganglion of the root. Passing through a small canal in the jugular fossa it runs through the petrous portion of the temporal, where it communicates with the facial, leaves the temporal through the auricular fissure and is distributed to the back of the pinna, communicating with the auricularis magnus.

The pharyngeal branch arises from the ganglion of the root and descends to the posterior aspect of the pharynx to aid in forming the pharyngeal plexus. It passes across the internal carotid, usually behind to the upper edge of the middle constrictor where it breaks up into branches. It receives its motor fibres probably from the eleventh and communicates with the nith, superior laryngeal and the sympathetic to form the pharyngeal plexus. 
The superior larygeal branch arises from the ganglion of the trunk and descending pierces the thyro-hyoid membrane and is distributed to the mucous membrane of the larynx, giving a filament to the arytenoid muscle. Just below the thyrohyoid membrane it gives off a branch called the external laryngeal, which is distributed to the thyroid gland and cricothyroid muscle and furnishes some filaments to the pharyngeal plexus.

This nerve receives a communication from the eleventh and afterward communicates with the recurrent laryngeal and with the pharyngeal plexus. In its course down the neck it lies by the side of the pharynx and crosses behind the internal carotid artery.

The recurrent or inferior laryngeal branch arises at a different point on each side; on the left it arises as the pneumogastric is crossing the aorta and winds backward around the aorta; on the right it arises as the nerve is crossing the first portion of the subclavian and winds around that artery. After passing around the aorta (or subclavian, as the case may be) each nerve ascends in the groove between the trachea and resophagus, giving filaments to those parts, and is distributed to all the muscles of the larynx except the crico-thyroid.

Each nerve gives off cardiac branches which unite with those from the sympathetic and tenth to form the cardiac plexuses. There are more (xsophageal branches given off from the right than from the left nerve. Other branches supply the mucous membrane of the trachea and some branches pass to the inferior constrictor.

The corvical curdiac branches are divided into superior and inferior. The superior usually join the great cardiac plexus, as does the inferior on the right. The left inferior goes generally to the superficial plexus.

The thoracic cardiac branches come entirely from the right nerve (all the left thoracic coming from the recurrent laryngeal) and pass to the deep plexus.

The posterior pulmonary' branches form an intricate plexus on the back of the root of the lung, whence filaments are sent along the bronchial tubes into the lungs. 
The anterior pulmonary branches form a smaller plexus on the front of the root of the lung.

The esophageal branches are filaments furnished the œsophagus before the nerves are in relation with it.

The two nerves communicate frequently around the tube, surrounding it with numerous anastomoses, thus forming the œesophageal plexus.

The gastric, or terminal, branches are distributed to both faces of the stomach, the right nerve going to the back, and the left to the front, of that organ. The two communicate with each other and with the cæliac, splenic, and hepatic plexuses.

\section{Eleventh Nerve.}

The eleventh nerve, or spinal accessory, is the fifth nerve arising in the groove between the corpora olivare and restiforme and escapes from the cranium through the posterior foramen lacerum along with the ninth and tenth nerves. The nerve consists of two portions: 1st, a cranial or accessory portion which arises from the groove mentioned and, after communicating with the cervical portion, goes to the pneumogastric; $2 \mathrm{~d}$, a spinal portion which arises by successive filaments from the side of the spinal cord as low as the sixth cervical nerve and ascends between the two roots of the spinal nerves. It enters the cranium through the foramen magnum and after communicating with the accessory portion emerges through the jugular foramen, passes down ward and outward, pierces the sterno-cleido-mastoid muscle and reaches the trapezius to which it is distributed, giving, on its way, filaments to the sterno-cleido-mastoid. The two portions of this nerve are never closely united, but after the spinal portion has ascended to the cranium several communicating branches pass between them.

\section{Twelfth Nerve.}

The twelfth nerve, or hypoglossal, arises from the groove between the corpora olivare and pyramidale by ten or twelve filaments which are gathered into two bundles: these pass outward and, just as they are leaving the cranium through 
the anterior condyloid foramen, unite to form the trunk of the nerve. The nerve then descends between the internal jugular vein and internal carotid artery and, when it reaches a point on a level with the jaw, curves forward in front of both internal and external carotid arteries, and after communicating with the gustatory nerve is distributed to the muscles of the tongue.

\section{Branches.}

The branches of distribution, besides the terminal, are two, the descendens noni, a very important branch, and the thyrohyoidean of small importance.

The descendens noni leaves the hypoglossal just as it is crossing the external carotid artery and derives its name from its direction and from the fact that the twelfth nerve was, in Willis' classification, called the ninth. It passes down the neck on the frout of the sheath of the external, carotid artery, and then on the sheath of the common carotid, occasionally descending in the sheath. About the middle of the neck it anastomoses with filaments from the second and third cervical nerves, the communicans noni, forming what is called the arch of Scarpa, which lies in front of the sheath of the common carotid artery about the middle of the neck. From this arch branches are given to the depressor muscles of the hyoid bone, except the thyro-hyoid.

The thyro-hyoidean branch descends from near the termination of the hypoglossal nerve to supply the thyro-hyoid muscle. 


\section{THE SYMPATHETIC NERVES.}

The sympathetic nervous system consists of a vertebral and a cranial portion.

\section{The Vertebral Portion.}

The vertebral portion of the sympathetic consists of two similar series of ganglia, lying one on each side of the vertebral column, extending from the skull to the coccyx, and the nerves which are connected with these ganglia. Of these ganglia there are about twenty-four divided into cervical, of which there are three, dorsal, of which there are twelve, lumbar, of which there are four or five and sacral, of which there are about four. Each ganglion is provided with four sets o $f$ branches: 1st, ascending to connect it with the ganglion above; $2 \mathrm{~d}$, descending to connect it with the ganglion below; 3rd, external to connect it with the contiguous spinal nerve; 4th, interual or branches of distribution. The first three sets of branches are the communicating branches, and each of the connections, viz: with the ganglion above, the ganglion below and the spinal nerves, is affected by two filaments, one of which is gray and the other white. The fourth set of branches, the internal or distributing, pass, as a rule, inward and forward to supply the organs of their various regions; these branches going either singly to their destination, or, as in some cases, several branches from one side meet similar ones from the opposite side and communicating with them on the front of the vertebral column, form plexuses from which the branches of distribution proceed. Three of these plexuses exist, known as the cardiac, solar and hypogastric, these constituting what is called the prævertebral sympathetic. As a rule each ganglion is connected with but one spinal nerve, but to this there are exceptions, which will be found in the cervical portion.

\section{Cervical Portion.}

The cervical portion of the sympathetic is that part of the chain or trunk which is found in the cervical region. Although 
there are eight cervical spinal nerves, there are only three sympathetic ganglia, known respectively as superior, middle and inferior.

\section{The Superior Cervical Ganglion.}

The Superior Cervical Ganglion lies on the front lateral aspect of the second and third cervical vertebræ, is fusiform in shape, varies in size, and is found to be segmented so as to correspond to the four upper cervical vertebræ. It receives communications, by means of its external branches, from the first, second, third, and fourth cervical nerves, and also smaller branches from the glosso-pharyngeal, pneumogastric, and hypo-glossal nerves. This ganglion being situated between the spinal and cranial nerves, receives communications from both sets.

It lies directly behind the internal carotid artery; and the cord which connects it with the middlecervical ganglion passes downward behind the common carotid artery, resting rpon the prevertebral group of muscles.

Its ascending branches pass along the internal carotid artery and are divided into two sets of branches, one entering into the formation of the cavernous and the other into the carotid plexus. The descending branches, white and gray, connect it with the middle cervical ganglion.

Its branches of distribution are three in number, viz., the pharyngeal, which unite with branches of the glosso-pharyngeal and pneumogastric nerves, and with them form a plexus situated on the posterior wall of the pharynx, which divides into numerous minute twigs, distributed to the muscles, bloodvessels, and glands of that organ. The laryngeal branches unite with the superior laryngeal branch of the pneumogastric, and form a plexus distributed to the larynx. The last branch is given to the heart, and is called the superior cardiac, to distinguish it from smaller branches derived from the middle and inferior cervical ganglia. This is a long, slender nerve, which passes down the neck behind the common carotid artery, crossing on the right side, sometimes behind, sometimes in front of the first portion of the subclavian artery, while on 
the left side it passes behind the thoracic portion of the subclavian. The nerve then enters the thoracic cavity. On the right side it joins with the great deep cardiac plexus, while on the left side it unites $w$ ith the great superficial cardiac plexus.

\section{The Middle Cervical Ganglion.}

The Middle Cervical Ganglion is situated opposite the fiftn cervical vertebra, is very small in size, and is frequently entirely absent.

Its superior branch communicates with the upper cervical ganglion, its inferior branch with the lower, while its external branches correspond to the fifth and sixth cervical nerves.

Its internal branches or branches of distribution, are the middle cardiac and thyroia.

The thyroid passes over the inferior thyroid artery just at the point at which it makes its bend to turn inward towards the thyroid gland; and commumicating with the superior cardiac and a branch of the recurrent and external laryngeal nerves, it is distributed to the thyroid body.

The middle cardiac nerve, the largest of the three cardiac branches, arises either from the middle cervical ganglion or from the cord between that ganglion and the superior. It passes down the neck behind the common carotid artery, and at the root of the neck the right nerve passes sometimes in front and sometimes behind the subclavian artery, descends upon the trachea, and, after receiving filaments from the recurrent laryngeal nerve, joins the great deep cardiac plexus. On the left side it enters the chest between the left common carotid and the subclavian arteries, and joins the deep cardiac plexus.

\section{The Inferior Cervical Ganglion.}

The Inferior Ganglion is situated between the base of the transverse processes of the last cervical and the first dorsal vertebræ. It is probably the product of the last two cervical nerves, the two ganglia having coalesced to form a single one. It is not infrequently blended with the first thoracic ganglion.

Its ascending fibres communicate with the middle cervical, and its lower, or descending fibres with the first dorsal ganglion, when that ganglion fails to coalesce with the last cervical. 
Its external fibres are derived from the last two cervical nerres, the seventh and eighth, while its internal branch is the last of the three cardiac nerves. It communicates with the recurrent laryngeal and middle cardiac nerve; and then passing into the thoracic cavity, unites with the deep cardiac plexus.

\section{The Cardiac Nerves.}

It will be seen that there are two large plexuses connected with the heart and supplying the muscular fibres of that organ. These are known as the great deep curdiac plexus and the superficial cardiac plexus.

The Superficial Portion is found lying on the ductus arteriosus, and is the product of the superior cardiac nerve of the left side and the inferior cervical cardiac branch of the pneumogastric.

The Deep Plexus, situated in the bifurcation of the trachea, is formed by the three cardiac nerves of the right side and the middle and the inferior cardiac nerves of the left side.

Thus it is seen that only two cardiac nerves enter the superficial plexus, viz; the left superior cardiac from the sym pathetic and the inferior cervical cardiac branch from the left pneumogastric; while the deep cardiac plexus is formed by the three right cardiac branches from the sympathetic, the left, middle and lower sympathetic, and the cardiac branches of the right pneumogastric.

From these two plexuses branches proceed, which following the course of the coronary arteries, are distributed along these vessels to the muscular coats of the vessels themselves and to the fibres of the heart.

\section{The Thoracic Portion of the Sympathetic.}

The Thoracic, or Dorsal, Sympathctic consists of twelve gauglia on either side of the body, corresponding to the number and position of the dorsal vertebra. These ganglia are uniformly arranged, and consist, like other sympathetic ganglia, of ascending branches communicating with the ganglion above, descending branches communicating with the ganglion below, external, which are usually two branches and communicate 
with the corresponding dorsal nerves, and internal which are branches of distribution.

The Branches of Distribution, coming from the dorsal sympethetic are almost entirely confined to the abdominal cavity. They form three nerves, known as the greater, lesser, and least Splanchnic.

The internal branches of the ganglia are divided into two sets: the upper and small ones are distributed along the branches of the thoracic aorta; while of the lower ones, those between the sixth and tenth ganglia unite to form the great splanchnic nerve, the tenth and eleventh ganglia form the lesser splanchnic, and the least, or renal, splanchnic is the product of the twelfth.

The Great Splanchnic Nerve passes down the front of the vertebral column behind the peritoneum, pierces the corresponding crus of the diaphragm, and enters into the semi-lunar ganglion of its side.

The Lesser Splanchnic Nerve, the product of the tenth and eleventh ganglia, also pierces the crus of the diaphragm. and joins with the coliac plexus; while the smallest, or least Splanchnic terminates in the renal plexus.

The Semi-lunar Ganglia, found on either side of the cœliac axis, is apparently an enlargement of the greater splanchnic nerve. Each ganglion gives off branches which communicate with the ganglion of the opposite side, forming a complete net-work surrounding the lateral and anterior aspects of the abdominal aorta, and completely enveloping the cœliac axis. These numerous branches are collectively known as the Solar Plexus of Nerves.

From this solar plexus large branches follow the three arteries into which the cœliac axis divides; and, forming plexuses along the course of these vessels, known as the Gastric, Hepatic, and Splenic Plexuses, are distributed to the organs which these vessels supply and to the coats of the vessels themselves.

The Lesser Splanchnic, after piercing the diaphragm, enters into the cœliac plexus; and some of its branches accompany the ramifications of the arteries mentioned above, while other 
filaments descend to the renal plexus, where that communicates with the least splanchnic nerve.

The Least Splanchnic, after piercing the diaphragm, enters into the formation of the renal plexus, from which branches pass to the kidney and to the renal artery and vein.

In addition to the filaments accompanying the branches of the coliac axis, the solur plexus also gives off branches which accompany the phrenic, superior mesenteric, spermatic (or o:arian in the female), and inferior mesenteric branches of the abdominal aorta; and are distributed along with these numerous branches. In addition to these certain fibres also accompany the aorta to form the Aortic Plexus of Neries, which after communicating with the inferior mesenteric plexus, passes over the pelvic brim, and aids in forming a plexus on the front of the sacrum, which is called the Hypogastric. From this branches proceed, which are united over the coccyx to form the Ganglion Impar, or terminal ganglion of the sympathetic system.

\section{The Lumbar Portion of the Sympathetic.}

The Lumbar Portion of the Sympathetic System is continuous above with the dorsal by means of the branch which passes from the last dorsal sympathetic ganglion beneath the ligamentum arcuatum to the first lumbar. The lumbar sanglia vary in number, being from three to five.

They receive their branches of communiation from the lumbar spinal nerves, a descending branch of communication from the ganglion above, and give a communicating branch to the ganglion below. These ganglia lie along the inner side of the psoas magnus muscle, where they receive communicating branches from the lumbar nerves.

Of the internal branches of this plexus there are two sets. The upper se't passes to the front of the abdominal aorta, and forms there the Aortic Plexus, which receives branches of communication from the solar plexus and from the semi-lunar gangrion.

This plexus lies between the origin of the superior and the inferior mesenteric arteries, and grives off branches to the in- 
ferior mesenteric and the spermatic arteries and to the inferior vena cava.

The lower internal branches enter into the formation of the hypogastric plexus, which is situated on the promontory of the sacrum between the common iliac vessels, and from which branches are given off to supply the pelvic viscera, communicating with ganglia which correspond to the sacral nerves.

Smaller branches are often given off from the sacral nerves which unite to form the so-called Pelvic Plexus. This plexus, after distributing branches to the lower pelvic viscera, corresponding to the arteries which pass to them, the branches being the Inferior Hemorrhoidal. Vesical, and Prostatic in the male, and Vaginal and Uterine in the female, finally terminates by uniting over the mid-line of the body to form the Ganglion Impar, or termination of the sacral plexus of nerves.

\section{The Cranial Sympathetic.}

The cranial portion of the sympathetic consists of four ganglia, lying in and around the skull, with their branches of communication and distribution, and of the carotid and cavernous plexuses formed by the ascending branches of the superior cervical ganglion. Each cranial ganglion is connected with two cranial nerves or two branches of the same nerve, receiving from one a motor and from the other a sensitive branch, and is also connected with the rest of the sympathetic system by a communicating branch. These various communicating branches form the roots of the ganglion. The four cranial ganglia are the opthalmic, the spheno-palatine, the otic and the submaxillary.

\section{Opthalmic Ganglion, or Ciliary Ganglion.}

The opthalmic or lenticular ganglion is found in the orbit lying on the outer side of the optic nerve close to the optic foramen, with the external rectus on its outer side and itself lying on the opthalmic artery. It is small and reddish gray in color and receives three roots, or branches of communication.

Its sensitive root is furnished by the nasal nerve; its motor root by the branch of the third nerve sent to the infe- 
rior oblique muscle; it's sympathetic root by the cavernous plexus. The sympathetic root frequently blended with the long or sensory root.

Its branches of distribution, known as ciliary, five or six in number, pierce the sclerotic coat around the optic nerve and ramifying between the tunics of the eyeball terminate in the iris.

\section{Spheno-Palatine Ganglion.}

The spheno-palatine, or Meckel's ganglion, is the largest of the cranial ganglia and is found lying in the spheno-maxillary fossa just beneath the superior maxillary division of the fifth nerve, and close to the spheno-palatine foramen.

Its sensitiz'e root consists of two filaments from the superior maxillary nerve: its motor root is derived from the facial nerve through the Vidian; its sympathetic root is from the carotid plexus. The last two roots reach the ganglion together, i.e., they are in the same sheath and form apparently one nerve; but the motor root, coming, from the Vidian, separates from the other branch of that nerve at the termination of the Tidian canal, the other branch, the great superficial petrosal nerve, going to tne facial through the hiatus Fallopii. The branches of distribution of this ganglion are four, palatine pharyngeal, superior nasal and naso-palatine.

The three palatine branches, anterior, middle, and posterior, descend to supply the roof of the mouth, soft palate and tonsils. The anterior palatine passes downward through the posterior palatine canal, emerges upon the hard palate and runs forward and inward to the anterior palatine foramen, communicating with the naso-palatine nerve. In the posterior palatine canal a branch is given off to the nose, the inferior nasal, which supplies membrane of the middle meatus and middle and inferior turbinated bones. Just as it emerges from the canal it gives off branches to the soft palate.

The middle palatine, ofteu absent, gives branches to the tonsils, uvula and solt palate.

The posterior falaline passes through an accessory palatine canal to supply the levator palati and ayygos uvula. 
The superior nasal are four or five branches which enter the nose through the spheno-palatine foramen and are distributed to the superior meatus of the nose.

Thenaso-palatine enters the nose through the spheno-palatine foramen, runs inward to the septum and, after giving branches to it, enters the palate through the naso-palatine canal.

The pharyngeal branches, or pterygo-palatine, passes through the pterygo-palatine canal to reach the pharynx where it is distributed.

The Vidian, one of the communicating branches can be traced back through the Vidian canal to the middle lacerated foramen where it divides into the large superficial petrosal, which enters the cranium through the middle lacerated foramen, passes through the hiatus and aqueductus Fallopii to be connected with the gangliform enlargement of the facial; and the deep petrosal, which enters the carotid canal to join the plexus on the carotid artery.

\section{0tic Ganglion.}

The otic, or Arnold's Ganglion, small and oval-shaped, is situated on the inner side of the inferior maxillary branch of the fifth nerve, just after its exit through the foramen ovale. Its sensitive root comes from the auriculo-temporal branch of the inferior maxillary; its motor root from the nerve to the pterygoideus internus; its sympathetic root from the plexus on the middle meningeal artery. Its branches of distribution are three, as follows:

A branch which passes backward to the tensor tympani muscle.

A branch which passes forward to the tensor palati muscle.

The nervus petrosus superficialis minor, which first enters the cranium through a canal between the foramen ovale and foramen spinosum and passing backward enters the tympanum, to join the tympanic plexus, through a canal on the base of the petrous bone. It gives off a branch (nerve of Longet) which enters the hiatus Fallopii to join the intumescentia gangliformis of the facial nerve. 


\section{The Submaxillary Ganglion.}

The submaxillary ganglion is a minute speck situated near the posterior border of the mylo-hyoid muscle. It receives filaments from the gustatory, from the facial through the chorda tympani and from the sympathetic on the facial artery.

The branches of distribution are distributed to Wharton's duct, the mucous membrane of the mouth and a few to the submaxillary gland. 


\section{THE OUTLET OF THE PELVIS.}

The outlet of the pelvis, which in the recent subject, is closed by various soft parts, in the skeleton is seen to be surrounded by the following parts: in front it is limited by the pubic arch, behind by the tip of the coccyx, on each side, about the centre, by the tuberosity of the ischium and running forward from this the rami of the ischium and pubesand backward from it the greater sacro-sciatic ligament or, in the recent subject, the lower border of the gluteus maximus muscle. In the recent subject the outlet of the pelvis is divided into two portions by a transverse line passing between the anterior edges of the two tuberosities of the ischia, or, in other words, from the indefinite point on one side where the ramus and tuberosity of the ischium meet to a similar point on the opfosite side.

The portion lying in front of this line is called the perineum and that lying behind it the ischio-rectal region.

\section{Ischio-Rectal Region.}

The ischio-rectal region is bounded in front by the transverse line, behind by the apex of the coccyx, laterally by the great sacro-sciatic ligament or the lower edge of the gluteus maximus muscle and the tuberosity of the ischium. It contains three parts to be examined, the anal orifice with its sphincter muscle and, on each side of this, a subdivision known as the ischiorectal fossa.

\section{Anus.}

The anus is the termination of the rectum, and is kept habitually closed by the tonic contraction of muscles called sphincters, internal and external. The sphincter ani internus was described with the structure of the rectum, it being a muscular ring around the gut formed by an aggregation of its circular muscular fibres about an inch above the anal orifice. Surrounding the anal orifice, which is seen in the centre of the ischio rectal region, is a thin elliptical muscle called the 
sphincter ani externus. It arises from and around the tip of the coccyx and passes forward just beneath and closely adherent to the skin to surround the anal orifice, and be inserted in front of it into a point in the centre of the perineal line called the central point of the perineum.

\section{Ischio-Rectal Fossa.}

The ischio-rectal fossa is the triangular or wedge-shaped space lying beside the anus, one on each side. It is about two inches deep, the base being at the surface and its limits being indicated by the anus within, the tuberosity of the ischium without, the transverse line in front and the lower edge of the gluteus maximus muscle behind. The apex is above, and is formed by the coming together of two sides of the space, the inner side being a muscle, called levator ani, which is inserted into the side of the lower part of the rectum, while the outer side is the obturator internus muscle. The levator ani muscle is thin and flat and has a most peculiar origin, to undestand which some preliminary remarks are necessary. The transversalis fascia, which lies between the transversalis abdominis muscle and the parietal layer of the peritoneum, when it reaches the iliac fossa, where it covers the iliacus internus muscle, takes the name of iliac fascia, which, in turn, when it reaches the brim of the pelvis, assumes the name of pelvic fascia, and this descending into the pelvis when it reaches the commencement of the obturator foramen, divides into two layers, one of which, the external, passes over the inner face of the obturator internus, as the obturator fascia; while the other passes to the base of the bladder and the side of the rectum as the recto-resical fascia. Now iust in the interval left by the splitting of the pelvic fascia the levator ani rises, having internal to it the recto-vesical fascia and external, with a widening interval between them, the obturator fascia. Besides this origin from the fascia, it arises posteriorly from the spine of the ischium and anteriorly from the back of the pubes. The ischio-rectal region thus described is found filled with adipose tissue, the absorption of which, in long-continued, debiliating desease, is the cause of the suntren appearince seen there. 


\section{Perineum-In the Male.}

The perineum is that sub-division of the outlet of the pelvis which lies in front of the transverse line. Its limits are as follows: In front the public arch, behind the transverse line, on each side the rami of the pubes and ischium. Crossing along its centre from behind forward, and continued on to the scrotum, is a ridge of the integument called the raphe.

Upon careful dissection the perineum is found to be formed of the following constituents from the surface upward, viz:

1st. Integument.

2d. Several layers of superficial fascia which in no wise differ from the general superficial fascia found beneath the integument everywhere.

3d. A layer of fascia called the superficial perineal fascia, since it is peculiar to the perineum, being attached to the rami of the ischium and the pubes on each side, in front continuous with the dartos of the scrotum and behind attached to nothing but terminating by changing its name, at the transverse line making a bend on itself and running back toward the pubic arch under the name of deep perineal fascia. It is separated from the superficial perineal fascia by the root of the penis and the muscles connected with it.

4th. Removing the superficial perineal fascia there are exposed the root of the penis and its muscles. The root of the penis consists of the two crura attached, one on each side, to the rami of the pubes and ischium, and of the bulb of the corpus spongiosm, which lies between these.

The muscles which are exposed are three pairs-

Transversus perinei, which arises on each side from the spot where the ramus of the ischium is continued into the tuberosity, and passes transversely inward to meet its fellow in the centre. The point where the two perinei are inserted into each other is called the perineal centre. It should be observed that the course of these muscles corresponds with the transverse line.

Accelerator urince. This muscle arises from the perineal centre and from the raphe between it and its fellow. The 
greater portion of its fibres surround the corpus spongiosum to meet the opposing muscle on the top of the corpus, but some of the fibres, posteriorly, are inserted into the deep perineal fascia and ramus of the pubes, while some in front are lost in the corpus cavernosum. Its chief action is to drive the urine forward by contracting on the corpus spongiosum, in which is the urethra.

Erector Penis arises from the rami of the ischium and pubes, and passing forward is lost on the upper aspect of the corpus cavernosum.

After removing these muscles and the root of the penis the deep perineal fascia is displayed. This, as stated, is continuous at tne transverse line with the superficial perineal fascia, the line of reflection being around the posterior aspect of the transverse perineal muscles. From this point it passes forward to the arch of the pubes, attached on either side to the rami of the ischium and pubes. Just below the arch of the pubes it presents a small, round opening for the passage of the urethra. When the deep perineal fascia is dissected it is found to consist of two layers, called the superficial and deep layers of the deep perineal fascia, which is sometimes called the triangular ligament of the pubes. Between the two layers are found blood ressels and nerves and a pair of muscles called compressors urethre. The compressor urethra arises from the point of union between the rami of the pubes and ischium and running transversely inward divides into two fasciculi, which meet those of the opposite side and surround the urethra. 


\section{ORGANS OF GENERATION IN THE FEMALE.}

The organs of generation in the female are divided into those within and those external to the pelvic cavity. The external organs, vulva clitoris, \&c., occupy the perineal space. The boundaries of the outlet of the pelvis are the same in both male and female subjects, but the ischio-rectal region is wider and longer in the female. In the mid-line are seen the opening of the vagina and the anus separated by a mass of tissue about one inch thick called the perineal body. The external organs consist of two labia majora surrounding the aperture of the vagina, composed externally of skin, which is covered with hairs and internally of mucous membrane, and united both in front and behind. Internal to these are two thinner projecting folds, one on each side, called labia minora, which enclose in front a projecting body, the clitoris. Between the clitoris and the mouth of the vagina is a space about one inch long called the vestibule, at the hinder part of which, near the mouth of the vagina, is an opening, the meatus urinarius.

The pelvic organs consist of an egg producing body-the ovary-and a channel to convey the egg to the exterior. This channel is modified to perform the function of hatching-the uterus - and that of copulation - the vagina.

The uterus consists of an upper part or fundus, a middle portion or body, and a lower part or neck. The vagina is a short, bent canal which serves the double function of conveying the seamen to the uterus and of conducting the fotus to the external world. The relations which the uterus and its so-called appendages bear to neighboring structures constitutes the remainder of the study of the female generative organs.

The peritoneum passes over the brim of the pelvis, enveloping the first portion of the rectum completely, and covering the upper one inch of the second portion in front, as in the 
male. From the front of the rectum it passes to the posterior wall of the vagina, which it covers for its upper one inch or inch and a half; passes to the back of the uterus and mounts upon it to its top, where it spreads out upon either side to envelop the appendages of the uterus; descends upon the front of the uterus, covering all of the front except that part contained within the vagina, and thence passes to the bladder, which is covered, as in the male, for its posterior half, the peritoneum leaping from the top of the bladder to the posterior surface of the anterior abdominal wall, forming the false ligaments of the bladder as in the male. That fold of peritoneum which extends transversely outward from the top of the uterus, envelops the appendages of that organ in a double-layered fold which forms a vertical septum extending transversely across the pelvic cavity, striking the lateral pelvic walls a little behind their middle, is known as the broad ligament of the uterus. The folds of peritoneum between the uterus and vagina and rectum posteriorly and the uterus and bladder anteriorly are known as the posterior and anterior ligaments of the uterus respectively, divided by the mid-line into two anterior and two posterior. Another ligament, a round cord, extends, in the folds of the broad ligament, from the lateral aspect of the uterus to the internal abdominal ring, to follow the inguinal canal and be finally lost in the labium majus, under the name of the round ligament. The depression between the vagina and the uterus in front and the rectum behind is known as the pouch of Douglas, the posterior ligaments bounding it on either side.

The broad ligament encloses between its folds the fallopian tube, ligament of the ovary, round ligament and ovary. The fallopian tubes occupy the highest part of the broad ligament, extending outward from the upper angle of the uterus to become closely connected with the ovary. They enlarge as they pass outward and form at the extremity a trumpet-shaped mouth, fringed by diverging processes or fimbriat, one of which is attached to the ovary. The tube is tortuous through the grreater part of its course and when stretched out is about five inches long. A little behind and below the fallopian tube is a small 
round cord, the ligament of the ovary, which attaches the ovary to the upper part of the side of the uterus.

The ovary is about oneand a half incheslong, one inch wide and half an inch thick. One end looks toward the uterus, one end outward, one surface upward and forward, the other downward and backward. It is completely invested by peritoneum, bulging the posterior folds of the broad ligament and appearing not to be in contact with the anterior fold. It is held to the uterus by its ligaments and the ova formed by it are conducted to the uterus by the fallopian tube.

The lower end of the uterus projects into the vagina, which thus surrounds the neck of the uterus, cutting off the lower portion of that organ from relation with surrounding viscera. The vagina extends higher up on the uterus posteriorly than anteriorly.

The rectum is about the same length as in the male, and is divided into three portions for study. Its lateral and posterior relations are the same as in the male, while the anterior relations are different. The first part of the rectum has in front of it the uterus, separated from it by convolutions of the small intestine. The upper inch of the second portion, that part covered by peritoneum in front, is separated, by convolutions of the small intestine, from the posterior wall of the vagina, which in turn cuts off the neck of the uterus. The remainder of the second portion is adherent to the posterior wall of the vagina. The third part of the rectum passes downward and backward, diverging from the vagina, the interval between the two being filled by a triangula - mass of connective tissue forming the perineal body and corresponding to the tissue between the bulb of the corpus spongiosum and the rectum in the male. The ureter bears the same relation to the rectumas in the male, and lies upon the side of the vagina, which cuts it off from relation with the neck of the uterus.

The female bladder occupies the same position as in the male, lying behind the symphysis pubis and bearing the same relation to the peritoneum. The anterior and lateral relations are also the same, except that there is no vas deferens, the position 
of this structure being in part occupied by the round ligament, which lies above and internal to the external iliac artery. The posterior surface of the bladder is separated from the anterior wall of the uterus by some convolutions of the small intestine. The base of the bladder looks more nearly downward than in the male and rests for its anterior three-fourths upon the anterior wall of the vagina, to which it is firmly adherent, its posterior one-fourth being covered by peritoneun. The urethra is nearly straight, but has a slight upward concavity. It lies on the anterior wall of the vagina, is about two inches long, runs through an opening in the triangular liganent about one inch below the symphsis pubis, is surrounded by muscular fibres forming what is called the compressor urethræ, and terminates in the vestibule at the meatus urinarius.

The ragina is the canal of communication between the uterus and the vulva. It is directed obliquely downward and forward, corresponding to the axis of the outlet of the pelvis, while the uterus corresponds to the axis of the brim, the two thus forming a curve whose concavity is forward. It is compressed, so that its anterior and posterior walls are in contact. Its anterior wall is about two and a half inches in length, its posterior about three and a half. It is partly closed in the virgin by the hymen. The relations of the vagina have been given in connection with other structures. 


\section{INGUINAL HERNIA.}

Inguinal hernia is the protrusion of some portion of the abdominal contents through the abdominal wall, making its way along the inguinal canal. The parts concerned are the lower fibres of the broad muscles of the abdomen, their superficial and deep coverings, and the structures composing the scrotum.

There is no deep fascia investing the broad muscles of the abdomen. When the integument is removed, two layers of superficial fascia are seen, separated from each other by the superficial blood vessels and nerves, the deeper of the two being the stronger. The removal of the deep layer exposes the lower portion of the aponeurosis of the external oblique muscle, known as Poupart's ligament, which extends from the anterior superior spinous process of the ilium to the spine of the pubes, the inner portion forming the outer, or lower, pillar of the external, or superficial, abdominal ring. Poupart's ligament is folded upon itself, backward and upward, forming a groove directed upward and inward. To its posterior face is attached the transversalis fascia, where it becomes continuous with the iliac, while its anterior face is continuous with the fascia lata and has adherent to it the deep layer of the superficial fascia. Before reaching the spine of the pubes, Poupart's ligament sends downward and backward a mass of fibres, with its base outward and apex inward, to be attached to the spine and pectineal line of the pubes, under the name of Gimbernat's ligament. Turning down Poupart's ligament we expose the combined origin of the internal oblique and transversalis muscles, which arches upward and inward, muscular, to form the conjoined arch, becomes tendinous and arches downward and inward as the conjoined tendon, which is inserted into the crest and pectineal line of the pubes behind the superficial abdominal ring. The outer abutment of this arch is at the middle of Poupart's ligament, the inner one-half inch 
external to the spine of the pubes, while the centre of the arch is one-half inch above Poupart's ligament. Cutting away these structures we come to the transversalis fascia, behind which is the parietal layer of the peritoneum, the two being separated by some loose connective tissue and fat. In the transversalis fascia is a second opening, called the deep or internal abdominal ring. At the lower, inner, part of the aponeurosis of the external oblique muscle there is a triangular opening, caused by the divergence of the fibres of the aponeurosis, known as the superficial or external abdominal ring; the apex of the opening being directed upward and outward, its base downward and inward and corresponding to the crest of the pubes. The lateral boundaries of this ring are known as its pillars-internal, or superior, attached to the front of the symphsis pubis, external, or inferior, attached to the spine of the pubes. The latter is simply the inner portion of Poupart's ligament. Around the margins of this ring the deep layer of the superficial fascia is attached, forming what is called the inter-columnar fascia. The two layers become continuous below with the dartos of the scrotum. The apex of the trianvile is obscured by a few fibres which cross the others at right angles and prevent a further divergence of the fibres and consequent widening of the triangle. These are called the inter-columnar fibres.

About one and one-half inches above and external to this opening, nearly opposite the centre of Poupart's ligament, there is a hole in the transversalis fascia called the deep or internal abdominal ring, situated a little less than one-half an inch above Poupart's ligament and internal to the outer abutment of the arch. Externally this ring is bounded by the conjoined arch, internally hy the deep epigastric artery, below by Poupart's ligament. The ring does not exist in a healthy subject. Through this ring the testicle descends in the foetus, and to its margins is attached a prolongation of the transversalis fascia, the infundibuliform tube of fascia, which forms one of the coats of the hernia. Extending between the two rings there is a canal, about one and one-half inches long, the inguinal or spermatic canal, which, in health, 
transmits the spermatic cord, in the male, or the round ligament, in the femaie, while, pathologically, it transmits the gut in a hernia.

The spermatic canal is limited internally by the superficial abdominal, externally by the deep, abdominal ring, and its walls are formed as follows: Below by the grooved upper surface of Poupart's ligament; above by the conjoined arch ; in front by the aponeurosis of the external oblique; behind, by the transversalis fascia and parietal layer of peritoneum throughout its extent, while, for about its inner half, the conjoined tendon lies in front of those structures. The canal contains the neck of the infundibuliform tube of fascia, arteries, nerves, 1ymphatics and the duct from the testicle.

On the visceral face of the peritoneum there may sometimes be seen a dimple which corresponds to the deep abdominal ring, and, internal to this, a ridge which is formed by the deep epigastric artery. The space between the ridge and the mid-line of the body is called the internal inguinal fossa, that external to the ridge the external inguinal fossa.

The testicles, in their descent, enter at the deep ring, pass through the inguinal canal and make their exit at the superficial ring. The cause of this descent is not accurately known, but it is aided by the gubernaculum testis, a mass of unstriated muscular fibres, attached by one extremity to the testicle and by the other to the margins of the superficial abdominal ring. In its descent the testicle carries with it the structures closing the two abdominal rings. Thus, it presses upon the peritoneum at the deep abdominal ring, invaginates it and forces along a process of this membrane which becomes the deepest layer investing the testicle and is known as the tunica vaginalis; next it forces along a process of the transversalis fascia, which, after the descent of the testicle, bccomes attached to the deep ring forming the infundibuliform tube of fascia; then, while passing beneath the conjoined arch, muscular fibres, arranged in loops around the cord, are contributed forming the cremaster muscle, which is attached internally to the sheath of the rectus and externally to Poupart's ligament. These loops are attached to each other by fascia called 
the cremasteric fascia. Lastly, the testicle carries with it a process of the intercolumnar fascia, forming the external covering of the cord, which is, of course, covered by the superficial fascia and integument. Shortly after birth the tunica vaginalis is obliterated from the internal ring to within a short distance of the testicle.

When, from weakening of the abdominal wall, or other cause, an inguinal hernia is produced, it usually follows the course of the testicle in its descent, and, hence, its coverings would be similar to those of the testicle. This form is known as an oblique inguinal hernia, subdivided into complete, when it escapes from the superficial abdominal ring, and incomplete, or unbonocele, when it remains in the inguinal canal. A complete inguinal hernia pushes before it the peritoneum closing the deep abdominal ring, which forms the hernial sac, enters the inguinal canal, receiving an investment from the infundibulum tube of fascia and the cremaster muscle, passes through the superficial abdominal ring, carrying with it a covering furnished by the intercolumnar fascia, descends in front of the cord into the scrotum and is invested by the superficial fascia and the integument. In incomplete hernia the coverings cease at the superficial abdominal ring.

When the peritoneal pouch is not obliterated, there is a direct communication between the abdominal and scrotal cavities, and the peritoneal covering of the hernia is the tunica raginalis. This form of hernia constitutes what is known as congrenital hermia.

In infantile hermia the pouch is closed at the deep abdominal ring only, and the gut descends behind the tunica vaginalis, having three peritoneal layers in front, viz: the two layers of the tunica vaginalis and one layer of the proper investing sac of the hernia.

In the encysted form the grut, carrying its own sac, projects into the tunica vaginalis, leaving in front of it but two serons layers, viz: one of its own sac and one from the tunica vagrinalis.

Des Anat-32 
Direct Inguinal Hernia.-Hesselbach's triangle is bounded externally by the deep epigastric artery, internally by the rectus abdoninis, below by Poupart's ligament. It is crossed by the conjoined tendon of internal oblique and transversalis muscles. Hernia may occur either external to or through this tendon. When the hernia is external to the tendon it follows a part of the inguinal canal and has the same coverings as an oblique inguinal hernia, except that the infundibuliform tube is replaced by another prolongation of the transversalis fascia. When through the tendon the latter is pushed before the gut, which has the same coverings as in the oblique form, except that the infundibuliform tube is replaced by another portion of the transversalis fascia, and the conjoined tendon replaces the cremaster muscle. Occasionally the hernia may split the tendon instead of pushing it before it. 


\section{FEMORAL HERNIA.}

DEFINITION. - The protrusion of an abdominal viscus through the femoral or crural ring.

PARTS CONCERNED.-Behind the femoral arch is found the upper border of the pubic bone, marked, from within outward, by the angle, crest, spine, pectineal line, triangle and eminence and the psoas groove. Stretching from the anterior superior spinous process of the ilium to the pubic spine and pectineal line, is Poupart's ligament, forming the femoral or crural arch. Gimbernat's ligament is the portion of Poupart's ligament which is inserted into the pectineal line. The other structures concerned are the iliac artery and vein and their sheath, the peritoneum and fascia closing the mouth of the ring, the fascia lata. cribriform and superficial fascia and the integument.

The space between Poupart's ligament and the upper border of the pubes is filled from without inward by the psoas and iliacus muscles, the anterior crural nerve and the femoral sheath containing the femoral artery and vein and having in it an unoccupied space internal to the vein. This sheath is formed in front by the transversalis, behind by the iliac fascia.

FEMORAL Ring.-The femoral ring is the upper opening of the femoral canal. Internally it is bounded by the outer edgre of Gimbernat's ligament; externally by a septum in the sheath separating the open space from the femoral vein; above is Poupart's ligament; below, the upper border of the pubes and the origin of the pectineus muscle. The femoral canal extends downward in the sheath for about one inch and is lined by a prolongation of fascia which covers the vessels and is known as the fascia propria. The canal terminates by the contraction of the sheath so as to closely hug the vessels. The femoral ring is closed above by the peritoneum and a thin layer of fascia called septum crurale, or femorale, which usually has lying upon it a lymphatic gland. 
Blood Vessels. - Just beneath Poupart's ligament the external iliac vessels terminate by becoming the femoral; but, before its termination, the external iliac artery gives off a large branch called the deep epigastric, which lies above and external to the femoral ring and between it and the deep abdominal ring. The external iliac vein lies to the outer side of the ring, so that in all cases there are blood vessels above and external to the ring. The deep epigastric artery sometimes gives off a branch called the obducator, which may descend next the iliac vein and on the outer side of the ring; but it sometimes passes inward and downward, arching above and internal to the femoral ring, which is thus surrounded by blood vessels on all sides except below. The epigastric also gives off a small pubic branch which lies above the ring, but is, from its size, unimportant.

FAscia Lata. - The investing fascia of the thigh, or fascia lata, presents, about one inch below Poupart's ligament, an oval opening called the saphenous opening. The opening is closed in the recent state. It runs obliquely downward and outward, is about one inch in its vertical by three-quarters of an inch in its transverse diameter; its inner edge is straight and prolonged outward behind the outer edge; its outer is curved, runs downward and outward from Gimbernat's ligament to the inferior cornu and is called Hey's ligament or the falciform process of Burns. The external part of the fascia is known as the iliac portion and is attached to the crest of the ilium and the outer portion of Poupart's ligament, sending inward a spur which is attached to the pectineal line, runs over the femoral vessels and forms the outer boundary of the saphenous opening. The inner part, called the pubic portion, is thin and weak, is attached to the ischiv-pubic ramus and is prolonged beneath the femoral vessels. The saphenous opening is covered by the skin and two layers of superficial fascia, of which the deep, under the name of cribriform fascia, becomes adherent to the margins of the opening. The cribriform fascia is attached more firmly below than above, and hence tends to direct a protruding gut upward. 
CoURSE.-A femoral hernia passes downward through the femoral ring, thence down the canal as far as the saphenous opening, turns forwasd through this opening and then runs upward on the front of the thigh. The main causes of the ascent are the firmer attachment of the cribriform fascia below, the narrowing of the femoral canal and the movements of the thigh.

Coverings.-Femoral hernia pushes before it, first the parietal layer of peritoneum and fat covering the femoral ring, then the septum femorale, fascia propria, cribriform fascia, superficial layer of the superficial fascia and, lastly, the skin. 


\section{THE AXILLA.}

The space known by this name lies between the chest wall internally, the humerus externally and the muscles in front and behind. In the skeleton only the apex of the space is complete, being bounded in front by the clavicle, behind by the upper border of the scapula, internally by the first rib. Below the apex the space corresponds to the upper five ribs internally, the scapula behind and the bottom of the bicipital groove externally. In the recent subject the space has the shape of a three-sided pyramid. The apex is above, the base below and the sharp angle external at the bicipital groove. The whole space is closed in by soft structures. The anterior wall is furnished by the pectoralis major, which covers the whole of the space, while beneath this, forming only the middle of the anterior wall, is the pectoralis minor. The posterior wall is formed by three muscles, subscapularis, teres major and latissimus dorsi; but the order in which they come changes in different parts of the space. Near the inner wall the order, from above downward, is subscapularis, teres major, latissimus dorsi; but near the external wall, owing to the fact that the tendon of the latissimus dorsi winds around the teres major and is inserted above it, the order is subscapularis, latissimus dorsi, teres major. The inner wall is formed by the upper six serrations of the serratus magnus muscle and the intercostal muscles corresponding to the upper five intercostal spaces. The lower border of the muscles which enter into the anterior and posterior boundaries of the axilla form the anterior and posterior axillary folds, of which the posterior is the thicker and more prominent. Stretching across from one fold to the other, and continuous with the investing fascia of the arm, is a strong fascia which forms the floor of the space. The contents of this space are the brachial plexus of nerves and its terminal branches, the axillary artery and vein and their branches, 1ymphatic glands and a quantity of areolar tissue and fat. 


\section{THE ORGAN OF HEARING.}

The organ of hearing is connected with the temporal bone of each side, and properly speaking, consists of two organs, one to each side. In the prepared bone a portion is still preserved, for upon the base of the petrous portion of the temporal bone the bony portion of the external auditory meatus is seen; and on the posterior surface of the petrous portion the internal auditory meatus, at the bottom of which is seen the internal wall of the inner chamber perforated by numerous apertures. The external wall of the inner chamber is seen by looking in to the external auditory meatus. On the anterior face of the petrous portion are seen the bulge of the superior semicircular canal and just external to this a depression corresponding to the roof of the middle chamber, or tympanum, the floor of which is the jugular fossa, on the basilar face of the petrous portion of the temporal.

The organ of hearing is described as consisting of three portions, or chambers, lying the one internal to the other, upon the base and in the substance of the petrous portion of the temporal bone. These three chambers are known from without inward as - 1st, external ear consisting of three portions, (a) pinna, or auricle; (b) meatus auditorius extermus; (c) membrana tympani; 2nd, middle ear or tympanum; 3rd, internal ear, or labyrinth.

\section{The External Ear.}

The external ear consists of three portions named above. Its function is to connect the sonorous vibrations and convey them to the middle ear, which in turn transmits them to the internal ear, where they encounter the sentient nerve of hearing.

The pinna, or auricle is the expanded outer extremity of the external chamber found on the base of the petrous portion of the temporal bone, contracting to its terminati n in tire exter- 
nal auditory meatus. It presents a very uneven surface and the various elevations and depressions have received individual names. The central concavity, leading to the external auditory meatus, is called the concha; the more or less folded margin, or rim, the helix ; the pendant, lower, softer portion, the lobulus; the triangular prominence jutting out in front of the concha, the tragus; behind and somewhat below this, separated from it by a notch, is another projection, the antitragus ; the curved elevation between the helix and the concha, or rather behind the concha, is the anti-helix, which divides superiorly enclosing a depression called the fossa ovalis; the deeply sunken surface between the helix and the anti-helix is the fossa scaphoidea, or innominata.

The pimna varies greatly in different, people, and this is especially true of the lobule. In structure it consists of a plate of fibro-cartilage, whose folds produce the depressions and elevations mentioned. This cartilage is covered by integument and alveolar tissue, supporting various insignificant muscles, intrinsic, intendea to vary its shape, and giving attachment to other small muscles, extrinsic, intended to move the pinna. Of the latter some few people have use, but the former never act to produce visible effect. The cartilage is not continuous throughout the auricle, for it is altogether wanting in the lobule and, besides, presents several fissures which have received names. The pinna is held in position by the integument. by the extrinsic muscles mentioned above and by two ligaments, anterior and posterior. The anterior extends from the helix to the zygoma; the posterior, from the concha to the mastoid process. Besides these there are several small ligaments passing from one part of the cartilage to another, the largest of which is that between the tragus and helix.

The external auditory meatus is the canal which extends from the concha to the tympanum, from which it is separated by the tympanic membrane. It is rather more than an inch in length, its direction being inward and slightly forward and its course somewhat curved, with its convexity upward. It is smaller in the centre than at either end. It consists of an 
external cartilaginous portion, taking up one-third of its length, and an internal, osseous portion, taking the remaining twothirds. The osseous portion terminates internally at an oblique groove for the attachment of the membrana tympani. This portion can be seen in the dried bone, although the septum in the botton is of course absent. The outer extremity is rough and prominent for the attachment of the cartilaginous portion. It is lined throughout by the integument which, toward the exterior, is studded with hairs and contains glands for the secretion of the ear wax. The hairs and wax are intended to obstruct the entrance of insects, \&c.

The membrana tympani is the septum separating the external from the middle chamber, being situated at the inner extremity of the external auditory meatus, attached to the oblique groove there found. It is fragile, semi-transparent. nearly round, oblique in its direction from above downward and inward, striking the floor at an angle of $45^{\circ}$, and is seen to bulge toward the tympanum, having a corresponding concavity externally. Its frame work is fibro-elastic, consisting of radiating and concentric fibres, the latter especially seen near its circumference; it is covered externally by the integuments lining the externally auditory meatus, which is exceedingly sensitive. Internally it is coated by mucous membrane of the typanum.

\section{The Middle Ear.}

The midale ear, chamber, or lymfamm, lies in the petrous portion of the temporal bone, separated, externally, from the meatus auditorius externus by the membrana tympani; and separated, internally, from the inner chamber, or labyrinth by a bony wall and its roof corresponds to the depression on the anterior face of the petrous bone, its floor to the jugular fossa on the basilar face of that bone. Its anterior wall is a bony partition interposed between it and the internal carotid artery and its posterior wall is the cellular substance of the mastoid portion of the temporal bone. Oving to the obliquity of the inner and onter walls, the tympanum is much narrower transversely, below than above. The length of its roof from with- 
out invard is about three lines, the floor being scarcely more than one line. It presents for examination six walls, two of which-floor and roof-have nothing noteworthy. The outer wall has been described as the membrana tympani, having attached to it a chain of bones, which extends to the inner wall.

The internal wall is the bony partition between the tympanum and the labyrinth, upon which are seen the following appearances: (a) a ridge at its upper part corresponding to the acqueduct of Fallopius ; (b) just beneath this a kidneyshaped aperture, opening into one of the apartments of the labyrinth, called the fenestra ovalis. This is closed during life by a membrane to which is attached the foot of the stapes; (c) below this is a bulging surface known as the promontory; (d) beneath this is a second opening oval, or triangular in shape, called the fenestra rotunda, which is likewise closed by a membrane and establishes communication with another part of the labyrinth called the cochlea.

The posterior wall presents several small openings into the mastoid cells and the opening for the entrance of the chorda tympani, iter chordæ posterius, which crosses the tympanum to gain exit on its anterior wall. There is also a conical projection, the pyramid, which has at its summit an opening for the stapedius muscle.

On the anterior wall are seen : (a) the fissure of Glaser ; (b) the opening of exit of the chorda tympani, iter chordx anterius, or canal of Huguier; (c) two small openings, separated by a thin plate of bone, the upper called the opening for the tensor tympani. the lower the opening for the Eustachian tube, and the dividing septum the cochleariform process. Stretching across the tympanum from the membrana tympani, externally, to the fenestra ovalis internally are seen three small bones, malleus, incus and stapes. The malleus is the outermost of the three, resting against the membrana tympani externally and articulating with the incus internally. It consists of a head, for articulation with the incus, a neck and three processes. One of the processes is called the handle, manubrium, and lies embedded in the membrana tympani, half way 
to the floor from above downward. Another, the long process, processus gracilis, passes downward to the fissure of Glaser ; while the third, the short process, bulges outward from the neck. The incus is socalled from its resemblance to an anvil. It consists of a body, for articulation with the head of the malleus, and two processes. The short process passes backward to the opening of the mastoid cells in the posterior wall; the long, passes downward to articulate with the head of the stapes, terminating in an enlargement, called the os orbiculare. The stapes, stirrup, projects inward from the os orbiculare, with which it articulates by a head. Succeeding the head comes the neck and diverging from this are the two crura, one to either side, which are connected at their distal extremity by a plate, called the foot, which is exactly fitted into the fenestra ovalis. When the child is born those bones have attained nearly their full size. They are held together by ligaments and give attachment to several small muscles which are intended to move the bones on one another and thus relax, or tighten the membrana tympani, or the membrane of the fenestra ovalis. This latter membrane is in fact composed of two layers, and the fenestra ovalis is closed successively by the following parts from without inward, viz : 1st, the mucous membrane of the tympanum; $2 \mathrm{~d}$, beneath this the foot of the stirrup; $3 d$, on the labyrinthine surface this foot is coated by the serous membrane lining the labyrinth. The arrangement for closing the fenestra rotunda is similar in consisting of three elements: (1st), a fibrous membrane coated by (2d) the mucous membrane of the tympanum externally and ( $3 d$ ) internally by the serous membrane of the labyrinth. It is constructed so much like the membrana tympani that it has been called the membrana tympani secundaria. The ossicula auditus are held to one another and in position by means of small ligaments. The head of the malleus is held to the body of the incus by a capsular ligament; and another capsular ligament binds together the os orbicularis and the head of the stapes. The ligaments holding the bones torether are three in $n \mathrm{nmber}$ : 1 st, the foot of the stapes is held to the fenestra ovalis by means of ligamentous fibres; $2 \mathrm{~d}$, there is a suspensory ligament of the 
malleus consisting of a few ligamentous.fibres passing between its head and the roof of the tympanum; $3 \mathrm{~d}$, a band of fibres attaches the short process of the incus to the opening of the mastoid cells. These bones, thus permitted motion by means of the ligaments, are moved not only by the vibrations of the membrana tympani but by two small muscles, viz: The tensor tympani which springs from the apex of the basilar surface of the petrous bone and the upper aspect of the cartilaginous portion of the Eustachian tube and enters the opening in the tympanum known by its name, and found just above the processus cochleariformis, which separates it from the opening of the Eustachian tube; it also derives a few fibres from the canal it traverses. When it reaches the anterior wall of the typanum its tendon turns outward and is inserted into the malleus at the junction of the manubrium and long process of the malleus. The stapedius is far the smallest muscle in the body. It arises within the hollow pyramid, on the posterior wall of the tympanum, emerges from the opening on the summit of the pyramid and is inserted by a tendon into the neck of the stapes. The action of the stapedius is to regulate the pressure of the foot of the stapes against the fenestra ovalis.

\section{The Eustachian Tube.}

The Eustachian tube is a canal, which establishes communication between the air in the tympanum and the outside atmosphere. It passes from the anterior wall of the tympanum, where its opening is seen just beneath the processus cochleariformis, downward, forward and inward, to terminate in the pharynx, just behind the opening of the posterior nares. Its lower third is cartilaginous; its upper two-thirds osseous.

\section{The Internal Ear.}

The internal ear, or labyrinth is the innermost of the three chambers, which constitute the organ of hearing and is intended for the terminal ramifications of the auditory nerve, which reaches it through the internal auditory meatus, on the centre of the posterior face of the petrous bone, and for the reception of the communicated vibrations of air, which reach it through 
the tympanum. It consists of an osseous and a membranous labyrinth, the osseous being divided into three chambers, which, however, communicate with one another. These three parts are placed one behind the other: the anterior is called the cochlea, the middle the vestibule, the posterior the three semicircular canals. They are all lined by a serous coat.

\section{The Vestibule.}

The Vestibule is a three cornered cavity, having communication with the tympanum through the fenestra ovalis. Each corner is called a ventricle and the three are known as anterior, superior and posterior. In the anterior are seen the following objects: 1st, a depression, called fovea hemispherica; $2 \mathrm{~d}$, a number of minute apertures, called the macula cribrosa; $3 \mathrm{~d}$, a ridge, the eminentia pyramidalis; 4th, the opening into the cochlea, scala vestibule. In the superior we have: 1st, a depression-the fovea heni-elliptica; 2nd, the two openings of semicircular canals-the dilated extremities of the superior and horizonta1. The posterior presents: 1st, a depressionforea sulciformis; $2 d$, the opening of the aqueduct of the vestibule; $3 d$, the dilated extremity of the oblique semicircular canal, the common aperture for this canal and the superior and the opening of the horizontal canal.

\section{The Semicircular Canals.}

These canals are channels hollowed in the petrous portion of the temporal bone. They are three in number, each about one-twentieth of an inch in diameter, and placed at right angles to one another. Each forms rather more than a semicircle and presents near one extremity an enlargement, called the ampulla, and opens by both ends into the vestibule. The three canals are called: 1st, superior, which is vertical in direction and produces the ridge seen on the anterior face of the petrous bone; 2 nd, the posterior, or oblique, which is also vertical; 3 rd, the external, or horizontal. These three canals present but five openings into the vestibule, owing to the fact that the posterior and superior have a com11non opening into the posterior corner of the vestibule. The other 
openings were mentioned in the description of the vestibulethree being into the posterior and two into the superior corner.

\section{The Cochlea.}

The cochlea lies in front of the vestibule and by its base forms the promotory, seen on the inner wall of the tympanum. When entire it resembles a snail's shell and may be discribed as consisting of a tube divided into two apartments by a longitudinal septum, one end of the tube being closed and much smaller than the open end from which it rapidly tapers. The septum is not complete, for it fails to reach to the small, closed end, thus allowing a communication between the two chambers. This tube is coiled by two turns and a half around a stem, or tapering pillar, called the modiolus. The septum subdividing the two chambers, which are called scalæ, is named the lamina spiralis. It consists of two layers, between which is a narrow space, called scala media, and it terminates in a hook-like projection called the hamulus. When examined closely the lamina spiralis is seen to consist of three structures; the portion next the modiolus is bony, farther out it is membranous and the utermost portion is muscular and sometimes called the cochlearis muscle. The small space, existing between the extremity of the lamina spiralis and the cupola, is called the helicotrema; it establishes communication between the two chambers, one of which is called the scala tympani, the other the scala vestibuli. The scala vestibuli is seen to communicate, at the base of the cochlea, next the vestibule, with the anterior corner of the vestibule. The scala tympani communicates with the tympanum, by means of the fenestra rotunda. The modiolus is the tapering pillar around which the two scalæ wind. In its centre there is a canal called the subulus centralis modioli. Its base is next the vestibule.

\section{The Membranous Labyrinth.}

Beside the parts above described, the labyrinth contains others for, within each semicircular canal is a membranous counter part, two thirds smaller, separated from the bony wall by liquid. The membranous canals present the same dilations at one end, ampulla, as the bony. 
In the iestibule also are two membranons sacs, one above the other. The upper one receives the openings of all the semicircular canals and lies in the fovea hemi-elliptica. It is called utriculus, or sacculus proprius. In the membranous labyrinth, as the above parts are collectively called, are found crystalline particles, carbonate and phosphate of lime, called ear-dust, or otoliths.

The auditory nerve gains the labyrinth through the internal auditory meatus. At the bottom it divides into a vestibular and a cochlear branch, the latter dividing into filaments which run in channels in the modiolus and turn outward to ramify in the lamina spiralis. The vestibular branch divides into three, one for each of the membranous sacs, and one for the semicircular canals. 


\section{THE ORGAN OF VISION.}

The organ of vision is situated in the orbits, two conical cavities found on the face. Like the organ of hearing, it consists of two similar portions, one on each side, called the eye-ball and its appendages.

\section{The Appendages of the Eye.}

The Appendages of the eye, tutamina oculi, are mere accessories to the true organ of vision, the eye-ball, for the purpose of protection and ornament. They are:

Eyebrow is a prominence of the integument, curved, covered with hair and situated above each orbit. They are intended for ornament and protection from dust, perspiration and too vivid light, and to break the force of any blow falling on the front of the orbit.

The eyelids consist of two movable curtains, which are constantly playing over the front of the eye-ball. The aperture, which separates the two lids, one called upper, superior palpebra, the other lower, inferior palpebra, is known as the palpebral fissure and can be extinguished, or dilated at pleasure. The two lids meet externally at an acute angle called the external canthus; internally when they seem about to meet they refrain from doing so and the interval between is prolonged inward towards the nose for some distance before forming the internal canthus. The triangular space thus left between the lids at the inner canthus is called the lachrymal lake, lacus lachrymalis, in which is seen a reddish, conical projection, called the caruncle, which is bounded externally by a curved fold of mucous membrane, called the plica semicircularis. The concavity of this fold is external.

At the commencement of the lachrymal lake, just where the two lids are apparently about to unite, there is a projection from the edge of each lid, called the lachrymal papilla, or tubercle, on the summit of each of which is an opening, the 
punctum lachrymale. The free edge of each lid is provided with several rows of short curved hairs, the eye-lashes, intended for ornament, to render the contact of the lids more intimate and to protect against dust, etc. In structure the eye-lids consist of the following parts: externally there is a layer of thin, loose skin with subjacent areolar tissue; internally, a layer of mucous membrane called the conjunctiva, which not only lines the inner surface of the lids, but is reflected over the front of the ball - palpebral and ocular portions. The palpebral portion is studded with papilla. Just heneath the integument, on the outer surface of the lid, is a pale thin muscle, the orbicularis palpebraum, so named from the fact that it is somewhat round, passes from one lid to the other and surrounds the palpebral fissure, its function is to close the lids. Beneath the orbicularis palpebrarum, in each lid is a curved plate of cartilage, about an inch in length, called the tarsal cartilage. The superior is the larger and is semilunar in shape; the inferior is much the narrower and oval. The two are attached along their circumference to the edge of the orbit by ligamentous fibres. Externally, they are held to the outer angle of the orbit by a ligament called the external palpebral, or tarsal ligament; internally, they terminate in a ligament, called the tendo oculi, which holds them to the edge of the orbit. Lying on the posterior surface of the lids between them and the mucous membrane, and opening on the edge of the lids is a number of vertical, beaded bodies, the Meibnmiam glands, the secretion from which is discharged on the edge of the lids. There are thirty of these glands for the upper lir, but a smaller number for the lower.

Lachrymal apparatus. Lying in the depression, seen. on the orbital plate of the frontal bone, just behind the external angular process, is the lachrymal gland. It is about the size and shape of an almond and rests on the posterior part of the upper tarsal cartilage. The ducts, seven to twelve in number, which convey the secretion of the gland, tears, being discharged on the surface of the conjunctiva, is constantly swept toward the inner canthus of the eye by the frequent movement of the lid; there it disappears in the lachrymal puncta from 
each of which there leads a small canal called the lachrymal canaliculi. The upper first ascends, then bends inward to terminate in the nasal, or lachrymal duct; the lower first descends, then turns inward to terminate in the nasal duct beside the superior. The nasal duct extends a little way above the openings of the canaliculi in a portion, called the lachrymal sac; and from this point the duct extends downward, outward and slightly backward to terminate in the inferior meatus of the nose.

\section{The Globe of the Eye.}

The eye-ball is situated in the frout part of the orbit, embedded in a mass of fat. It is nearly globular, its anteroposterior diameter being one inch and its transverse serentwelfths of an inch. Posteriorly it receives the optic nerve, at a point about one one-tenth of an inch to the nasal side of the centre. The ball consists of concentric layers enclosing a bag of liquid, which forms about four-fifths of it. These coats or layers are sclerotic, choroid and retina.

\section{The Sclerotic Coat.}

The sclerotic, familiarly known as the white of the eye, is a dense fibrous membrane, which serves as a protecting envelope for the rest of the eye-ball. Its anterior one-sixth is wanting, the aperture being filled by a transparent membrane, the cornea. Posteriorly the sclerotic is perforated by the optic nerve, not en masse, for the nerve splits into a number of fibres, which pierce the coat separately, producing the appearance known as macula, or lamina cribrosa, the fibrous sheath of the nerve becoming continuous with the sclerotic, while in front the sclerotic is continuous with the cornea.

\section{The Cornea.}

The cornea forms the anterior one-sixth of the first investment of the eye-ball. It forms a segment of a smaller sphere than the sclerotic and being more convex it causes the increase of the antero-posterior diameter over the transverse. The cornea, instead of being opaque, like the sclerotic, is transparent and its circumference is received beneath the anterior 
bevelled edge of the sclerotic with which it is continuous in some of the structures. It consists of five layers. The anterior surface is an epithelial layer, derived from the conjunctiva ; the posterior layer is also epithelial, the lining membrane of the anterior chamber. Next each of these is found a layer of elastic tissue, the two layers constituting what is called the elastic cornea, and separating these is a layer of fibrous tissue, called the cornea propria, which can be split into sixty layers of spindle-shaped cells with branching intervals, in which circulates the colorless plasna of the blood. It is with this cornea propria that the sclerotic is continuous. The cornea is of varying convexity in different people and at different periods of life. It is more convex in youth and becomes less so in old age, at which period there is often seen around the corvea a yellowish band, called arcus senilis.

\section{The Choroid.}

The choroid lies within the sclerotic, with which it is connected by areolar tissue, sometimes called lamina fusca, which is pierced by the optic nerve. At the point in front where the sclerotic ceases the choroid terminates in a flattened membrane, the iris, which, in its centre presents a round aperture, the pupil. The choroid consists of three layers, the outer two red and the inner black. Between the two red and the black layers some make a fourth layer, the membrana limitans. The external red layer is called the venous layer; the internal red layer the capillary, arterial, layer, or the tunica Ruyschiana. The internal layer, or membrana pigmenti, or tapeta is of a black color, consisting of cells, filled with coloring matter. On the anterior surface of the choroid, just around its anterior margin, is a whitish band, one-fortieth of an inch wide, called the ciliary ligament, which binds together the cornea and sclerotic and the choroid and iris. Extending back, about oneeighth of an inch, on the anterior aspect of the choroid, is a gray circular band, called the ciliary muscle. On the posterior aspect of the front of the clioroid are, about, sixty ridges, diverging from the rim of the choroid, called the ciliary processes. 


\section{The Iris.}

The iris is the thin flat membrane, which continues the choroid in front. In its centre is seen the opening, called the pupil. The various colors of the iris are due to the difference of color of the pigmentary matter found in its cells and to the varying arrangement of the blood vessels. The color is darkest usually near the centre.

Towards the middle of the iris is a circle of little shaggy projections. The foundation of the structure of the iris is fibrous tissue, consisting of circular and radiating fibres interposed between which are pigment cells. In front and behind this fibrous layer is a layer of pigment cells. In the iris are also seen unstriated muscular fibres, circular around the pupil and radiating toward the circumference: by the action of these fibres the pupil is constantly dilating and contracting. Blood vessels and nerves ramify throughout the structure. On the posterior surface the iris consists of a black layer. called the uvea, which is, in fact, a continuation of the membrana pigmenta of the choroid.

Anterior Chamber-Between the iris and pupil behind, and the cornea in front is a small cavity, called the anterior chamber.

Posterior Chamber-Between the iris and pupil in front, and the lens behind is another, smaller cavity, called the posterior chamber. These two contain a liquid, called the aqueous humor, and they communicate through the pupil, after the sixth month of fœtal life, up to which time the pupil, is closed by a membrane, called the papillary. Both chambers are lined by a membrane which secretes the aqueous humor.

\section{The Retina.}

The retina is the innermost of the three coats of the eye. lying just within the choroid. It is wanting in front even for a greater distance than the two preceding layers, for it ceases, as it reaches the ciliary processes in a rough jagged margin, called ora serrata, and is continued thence to the crystalline lens by a vascular membrane, called the suspensory ligament of the lens. The outer surface of the ligament is 
fluted to correspond with the ciliary processes, against which it rests.

The retina is nou-resisting and translucent, being partially formed by the expansion of the optic nerve, with which it is continuous posteriorly.

Exactly in the centre, posteriorly, is a round, yellowish spot, called the macula lutea, and in the centre of this spot a depression called fovea centralis. About one-tenth of an inch to the inner side of this is seen the termination of the optic nerveoptic disk, or papilla-showing in its centre the arteria centralis retinæ, breaking up into branches. From this point forward the retina graaually thins to its termination.

\section{The Vitreous Humor.}

Just within the retina is a bag, called the hyaloid membrane, containing a liquid, called vitreous humor. This is similar in composition to the aqueous humor, being $98 \%$ water. Traversing the centre of this, in the fœtus, is a branch of the arteria centralis retinæ, making its way to the lens, called the capsular artery. It disappears at birth.

\section{The Crystalline Lens.}

Indenting the front of the hyaloid membrane is found a doubly convex transparent body, called the crystalline lens. Its posterior surface is much more convex than the anterior, the latter forming the posterior wall of the posterior chamber. The lens forms only the bulging portion of the wall, the circumference of which is formed by the suspensory ligament of the lens, which extends from the ora serrata of the retina to the rim of the lens. This ligament is formed of two layers and when it lays hold of the lens it leaves a circular canal around it, called the caual of Petit. The lens is invested by a transparent homogeneous, elastic membrane, the capsule of the lens. In structure the lens consists of a series of concentric layers of parallel fibres, which gradually harden towards the centre of the lens. It can also be split in to three triangular segments, which meet by their apices in the centre of the lens.

In youth the lens is more convex and much softer than in old age, as it gradually becomes, with advancing life, firmer and less convex. 



\section{INDEXX.}

Abdomen, boundaries of,$\ldots \ldots . . . \ldots \ldots \ldots \ldots \ldots \ldots \ldots \ldots . . \ldots 1$

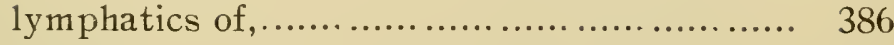

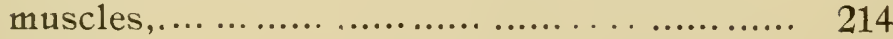

regions, ................................... 161

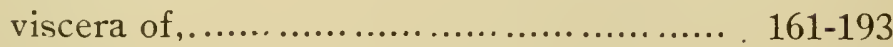

Abdominal aorta,.................................... 328

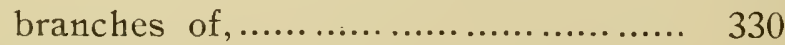

ring, extermal,.......................... 215

" internal,.............................. 495

viscera, relations of,$\ldots \ldots \ldots \ldots \ldots \ldots \ldots \ldots$........... 161-177

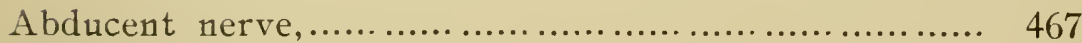

Abductor minimi digiti (hand), ......................... 256

، 6 (foot),........................ 275

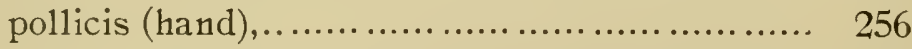

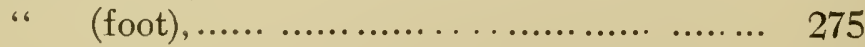

Accelerator urinae, .................................... 488

Accessorius muscle,................................. 275

Acetabulum,.......................................... 39

Acromial muscle,.................................... 237

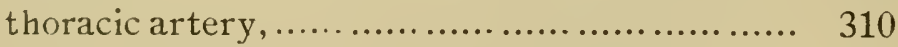

Acromio-clavicular joint, ............................. 120

Acromion process, ...................................... 20

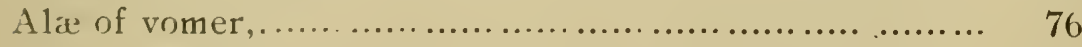

Alar ligaments,...................................... I43

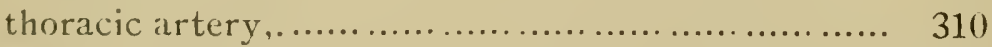

Alimentary canal, ................................ I77-182

Alvenlar process, lower jaw ,.......................... 84

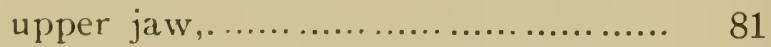

Anconenus muscle, .................................. 252

Angle, jaw, ....................................... 85

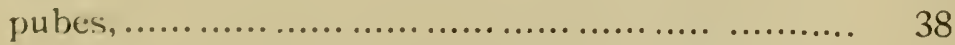

rib, .............................. 14 


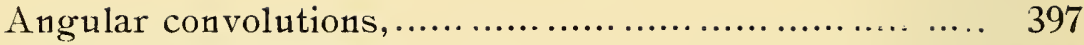

movements, .............................. 111

process, external and internal,.............. 61

Ankle joint, ........................................... 146

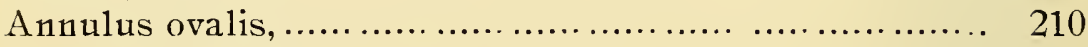

Anterior ethmoidal cells, ............................. 73

fossa skull, ............................... 93

region skull, ................. ............... 95

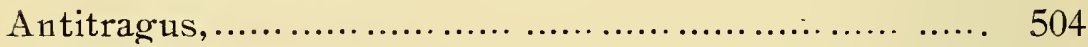

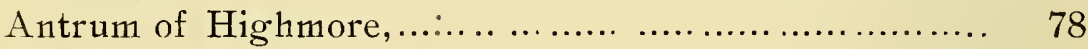

Anus, ............................................ 182

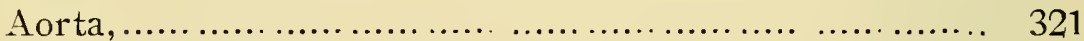

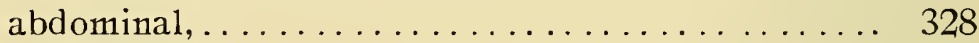

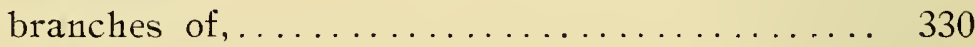

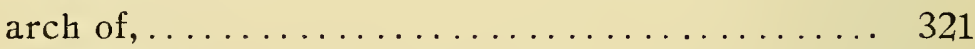

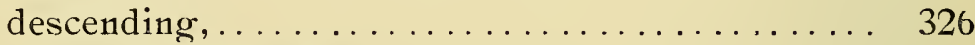

Aortic opening diaphragm, . . . . . . . . . . . 220

semilunar valve $\ldots \ldots \ldots \ldots \ldots \ldots \ldots \ldots \ldots \ldots$

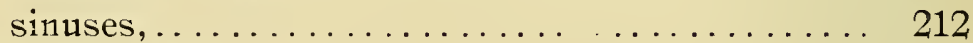

Appendages of eye ...................... 512

Appendix, right auricle, . . . . . . . . . . . . . . . 209

left auricle, ................. 211

vermiform .................... 168

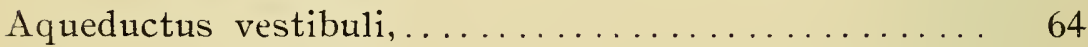

Fallopii, .................... 65

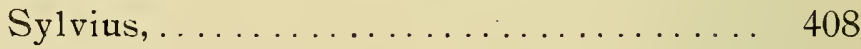

Aqueous humor $\ldots \ldots \ldots \ldots \ldots \ldots \ldots \ldots \ldots \ldots$

Arachnoid of brain $\ldots \ldots \ldots \ldots \ldots \ldots \ldots \ldots$

$\operatorname{cord}, \ldots \ldots \ldots \ldots \ldots \ldots \ldots \ldots \ldots \ldots \ldots$

Arm, arteries of $\ldots \ldots \ldots \ldots \ldots \ldots \ldots \ldots \ldots \ldots \ldots \ldots$

fascia $\ldots \ldots \ldots \ldots \ldots \ldots \ldots \ldots \ldots \ldots \ldots \ldots$

lymphatics...................... 387

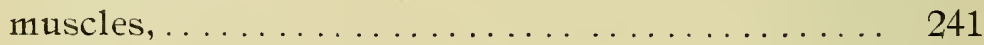

nerves, . . . . . . . . . . . . . . . . . . . 429-438

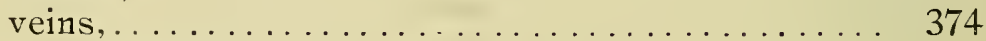

Artery, or arteries:

acromial thoracic $\ldots \ldots \ldots \ldots \ldots \ldots \ldots \ldots \ldots \ldots$

alar thoracic,$\ldots \ldots \ldots \ldots \ldots \ldots \ldots \ldots \ldots \ldots$ 
Artery, or arteries (Contimued):

angular, ................................... 294

anterior cerebral,............................ 364

choroid,............................. 364

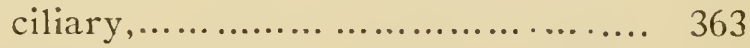

communicating,..................... 364

articular of knee,.......................... 349

axillary,................................... 307

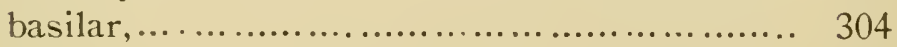

brachial, .................................. 312

calcanean,................................. 351

carotid, common, left....................... 324

“ $\quad$ right. ...................... 287

external,............................ 290

internal.............................. 360

circumflex, arm,......................... 211

thigh,.......................... 347

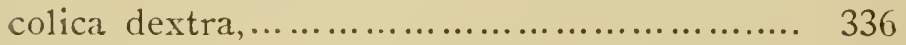

media,............................... 336

sinistra,............................ 337

coronary, heart,.......................... 323

lips, ............................. 294

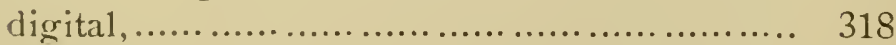

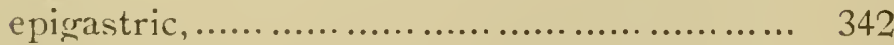

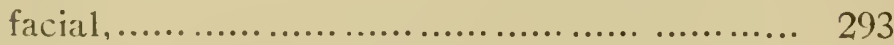

femoral,................................... 343

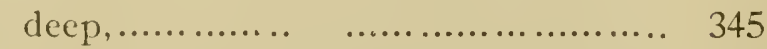

rastric, ................................... 332

gastro-epiploica dextra, .................... 333

sinistra, .............................. 334

gluteal, ..................................... 359

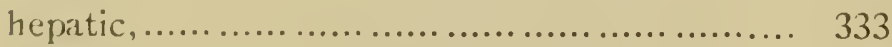

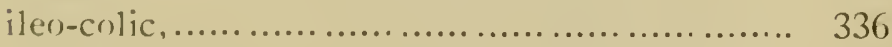

iliac, common,.................... 340

external,.................. 341

internal,................. 355

innominata. .................... 323 
Artery, or arteries (Contimued):

internal mammary,.................... 306

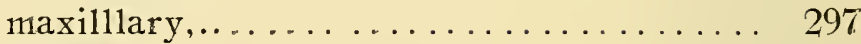

plantar,.................... 352

intestini tenuis, ..................... 335

lateralis nasi,........................... 294

lingual, ......................... 295

long thoracic,....................... 311

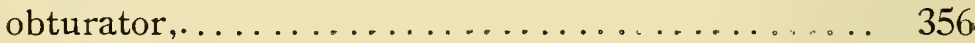

occipital ........................ 295

opthalmic . .................... 362

plantar ........................... 352

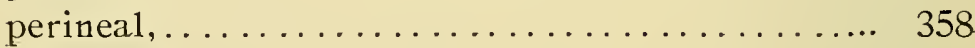

peroneal, ...................... 351

phrenic,....................... 330

radial, forearm,.................... 315

. hand and wrist,.............. 318

renal ...................... 340

sciatic $\ldots \ldots \ldots \ldots \ldots \ldots \ldots \ldots \ldots \ldots \ldots$

temporal, superficial,............... 296

“ deep.................... 298

thyroid axis, ..................... 304

tibial, anterior $\ldots \ldots \ldots \ldots \ldots \ldots \ldots \ldots \ldots \ldots \ldots$

tibial, posterior,................. 350

transversalis colli, ................... 305

ulnar, forearm,................... 315

" hand,................... 316

vertebral, ......................... 304

Articulations in general, . . . . . . . . . . . . . 108

Articulation, ankle,................... 146

atlo-axoid.................... 114

calcaneo-astragaloid,.............. 148

“ cuboid,.................. 148

carpal, ................... 129

costo-vertebral, . . . . . . . . . . . . . 150

costo-sternal, . . . . . . . . . . . . . . . .. 132

elbow, ................... 125 
Articulation, (Contimued):

hip, .

knee,

occipito-atloid,

115

pelvis,

Articulation, radio-carpal, (wrist)

ulnar,

shoulder,

121

sterno-clavicular

118

temporo-maxillary,.

130

tibio-fibular,

145

vertebral column,

112

wrist

127

Arytenoid cartilage

195

Ascending colon, 168

Astragalus,

Atlas,

Auditory nerve,

464

Auricle, of ear...

504

heart.

209-211

Axilla, 502

Axillary artery 307

vein, 366 Axis,

B.

Back, muscles of,

Base of brain,

Basilic vein,

Bicipital fascia,

Bladder, relations in female,

male, 175

description of, 
Bone, or Bones : ................................. 1-107

astragalus, .................................. 51

atlas, .......................................... 6

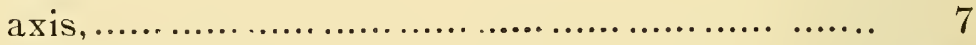

calcaneum, .................................. 50

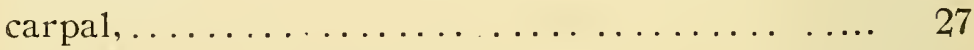

clavicle,...................... 16

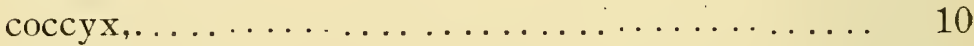

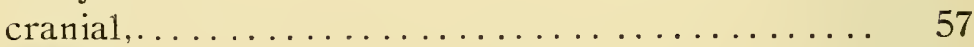

cuboid,..................... 52

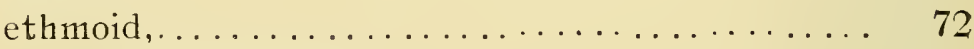

articulations of,$\ldots \ldots \ldots \ldots \ldots \ldots \ldots$

femur,.................. 46

fibula $. \ldots \ldots \ldots \ldots \ldots \ldots \ldots \ldots \ldots \ldots \ldots . \ldots \ldots$

frontal, ....................... 60

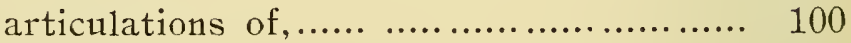

humerus, ....................................... 20

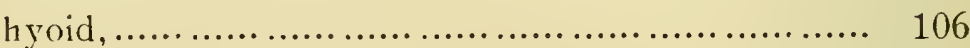

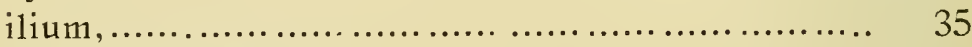

inferior maxillary, .............................. 106

turbinated, .............................. 75

articulations of,$\ldots \ldots \ldots \ldots \ldots \ldots . . . . .105$

innominata, .................................... 35

ischium, ...................................... 37

lachrymal...................................... 74

malar, ........................................ $\quad 77$

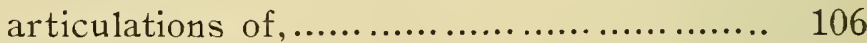

nasal............................................. 74

articulations of,$\ldots \ldots \ldots \ldots \ldots \ldots \ldots \ldots \ldots \ldots . \ldots \ldots . \ldots \ldots$

occipital, .................................... 58

articulations of,$\ldots \ldots \ldots \ldots \ldots \ldots \ldots \ldots \ldots . . \ldots \ldots$

palate, ........................................ 81

articulations of............................ 104

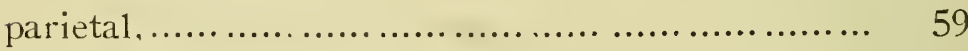

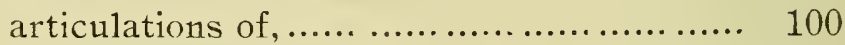

pate1la, ......................................... 45

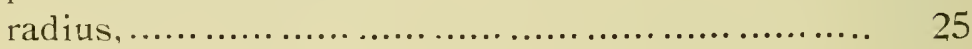


Bone, or bones, (Continued):

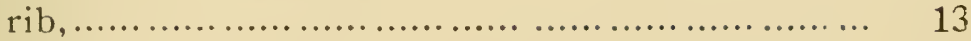

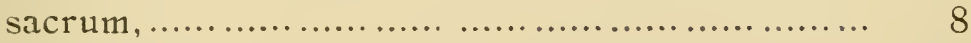

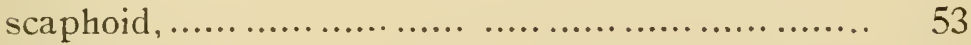

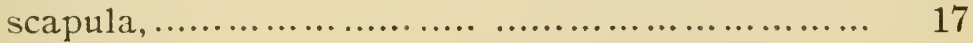

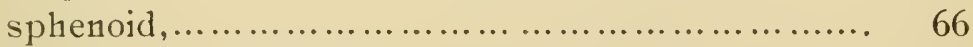

sphenoid, articulations of, .............. 101

sternum,....................... 11

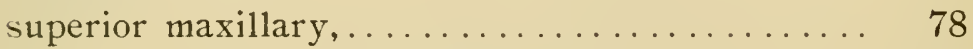

articulations of,$\ldots \ldots \ldots \ldots . \ldots 103$

temporal,.................... 26

articulations of,$\ldots \ldots \ldots \ldots \ldots \ldots \ldots . \ldots 10$.

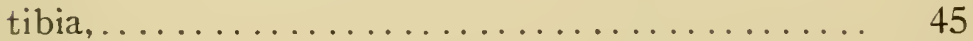

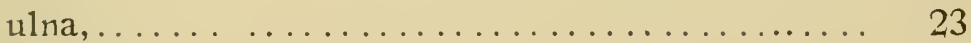

vomer $\ldots \ldots \ldots \ldots \ldots \ldots \ldots \ldots \ldots \ldots$

articulations of,$\ldots \ldots \ldots \ldots \ldots \ldots \ldots$

Brain, . . . . . . . . . . . . . . . . . . . . . . 392

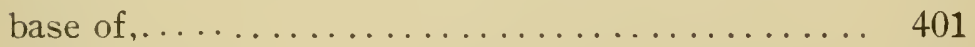

convolutions, ................... 363

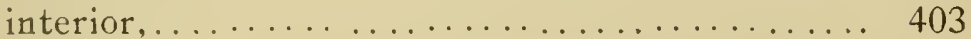

membranes, ..................... 389

Bronchi,............................. 204

C.

Canal, auditory,...................... 504

Capsular ligament, hip, .................... 134

shoulder, . . .......... 121

Cartilage, arytenoid, ................... 201

cricoid, ........................ 197

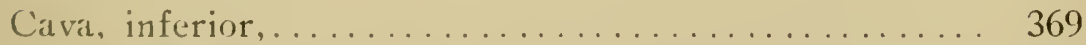

superior....................... 383

Cerebellum, ......................... 415

Cerebrum............................ 392

Clavicle............................ 10

Cireliac axis, ........................ 331

Coracn-acromial ligament, . . . . . . . . . . . . . . . 121

clavicular, ........................ 120

Corpus callosum, . . . . . . . . . . . . . . . . . . 404

fimbriatum, . . . . . . . . . . . . . . . . . 406 
INDEX.

Costal cartilages, . . . . . . . . . . . . . . . 15

Cranial bones, .......................... 57

nerves, . ....................... 458

Cranial nerves, eighth................... 469

eleventh................... . . 474

fifth $\ldots \ldots \ldots \ldots \ldots \ldots \ldots \ldots \ldots$......... 462

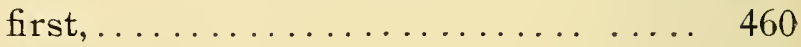

fourth $\ldots \ldots \ldots \ldots \ldots \ldots \ldots \ldots$

ninth, ................ 470

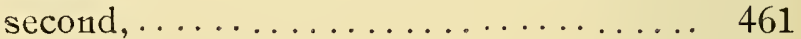

seventh, .................. 467

sixth, ................. 467

tenth,................. 471

third, ................. 461

twelfth, ................ 474

Crystalline lens, ..................... 517

E.

Ear,$\ldots \ldots \ldots \ldots \ldots \ldots \ldots \ldots \ldots \ldots \ldots \ldots \ldots \ldots \ldots \ldots \ldots \ldots \ldots \ldots \ldots$
Elbow, bend of $\ldots \ldots \ldots \ldots \ldots \ldots \ldots \ldots \ldots$

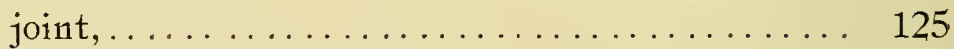

Epididymis, . . . . . . . . . . . . . . . . . . . . 195

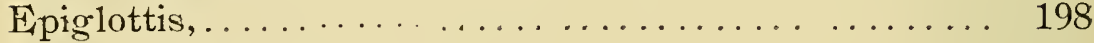

Ethmoid bone,....................... 72

articulations of $\ldots \ldots \ldots \ldots \ldots \ldots \ldots \ldots \ldots \ldots \ldots$

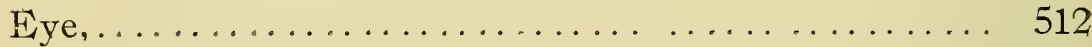

tunics of $\ldots \ldots \ldots \ldots \ldots \ldots \ldots \ldots \ldots \ldots \ldots$

muscles of,$\ldots \ldots \ldots \ldots \ldots \ldots \ldots \ldots \ldots \ldots$

F.

Face, arteries of . ....................... 293

bones, . . . . . . . . . . . . . . . . . . . 74

muscles,..................... 277-285

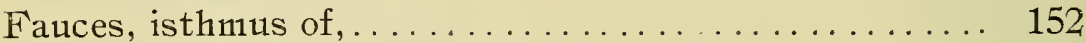

Female generative organs, . . . . . . . . . . . . 490

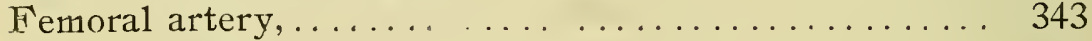

canal,........................ 499

hernia . . . . . . . . . . . . . . . . . . . . . 499

Fifth nerve,$\ldots \ldots \ldots \ldots \ldots \ldots \ldots \ldots \ldots \ldots . \ldots \ldots 2$

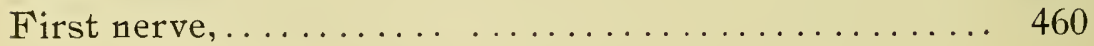


Fissure of brain. 393

Foot, arteries of. 351-351-267

bones, .

muscles,

273

ligaments,

nerves,

Fossa, spheno-maxillary................... 89

of skull, anterior,$\ldots \ldots \ldots \ldots \ldots \ldots \ldots . \ldots \ldots$

middle and posterior, ............. 91

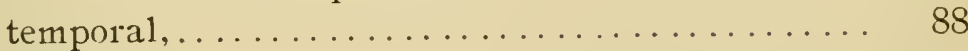

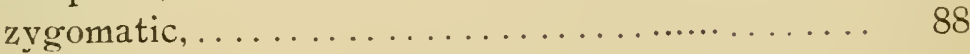

G.

Ganglion, cervical,..................... 476

ciliary, or opthalmic . ............... 482

Meckel's......................... 483

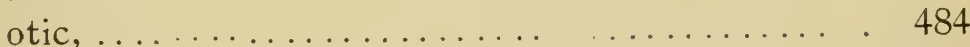

semilnnar........................ 480

H.

Hand, bones of $\ldots \ldots \ldots \ldots \ldots \ldots \ldots \ldots . \ldots \ldots$

ligaments, . ........................ . . 129

Heart.............................. 207

Hernia, femoral ......................... 499

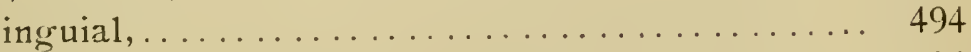

Hip joint, ......................... 134

I.

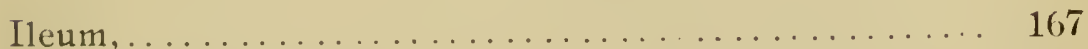

Iliac arteries, common . . . . . . . . . . . . . . . . 340

external,...................... 341

internal,.... ................ 355

veins, external, .................. 368

internal................... 369

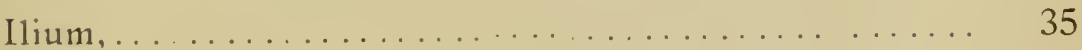

Inferior vena cava, . . . . . . . . . . . . . . . . . 369

Inguinal canal. . . . . . . . . . . . . . . . . 406

hernia,. .................... 494 
Intestine, large, description of, . . . . . . . . . 178 small, discription of,$\ldots \ldots \ldots \ldots \ldots \ldots \ldots \ldots$ relations of ............................ 166

Iris, small divisions of . . . . . . . . . . . . . . 516 Ischio-rectal region $\ldots \ldots \ldots \ldots \ldots \ldots \ldots \ldots \ldots \ldots$ Ischium, . . . . . . . . . . . . . . . . . . . 37 Island of Reil, . . . . . . . . . . . . . . . . . . . . . . . 399

$\mathrm{K}$

Kidney relations of $\ldots \ldots \ldots \ldots \ldots \ldots \ldots \ldots \ldots \ldots$

structure of,$\ldots \ldots \ldots \ldots \ldots \ldots \ldots \ldots \ldots$

Knee joint,.......................... 139

L.

Larynx, cartilages of .................... 196

ligaments of $\ldots \ldots \ldots \ldots \ldots \ldots \ldots \ldots \ldots \ldots$

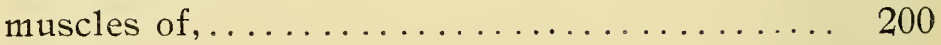

Ligaments:

acromio-clavicular, . . . . . . . . . . . 120

ankle, . . . . . . . . . . . . . . . . 146

atlanto-axoid,....................... 114

calcaneo-astragaloid,................ 148

“ cuboid,....................... 148

“ scaphoid,.................. 149

common vertebral, . . . . . . . . . . . . . 112-114

costo-vertebra1,................. 150

of elbow,..................... 125

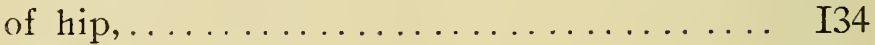

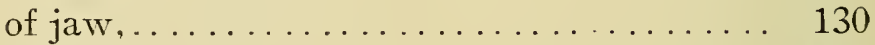

of knee,...................... 139

radio-carpal, ................... 127

radio-ulnar, . . . . . . . . . . . . . . . . . 124

sacro sciatic . . . . . . . . . . . . . . . 133

of shoulder, . . . . . . . . . . . . . . 121

tibio-fibular ................... 145

Liver, relations of $\ldots \ldots \ldots \ldots \ldots \ldots \ldots \ldots \ldots \ldots \ldots \ldots$

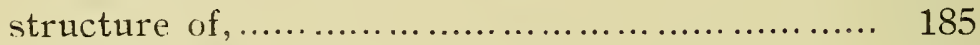

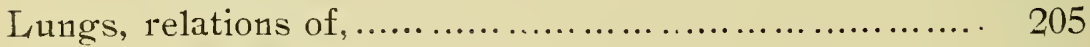

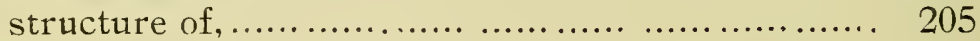


Lymphatics : ...................................... 386

arn1,................................... 387

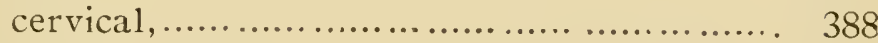

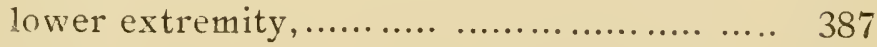

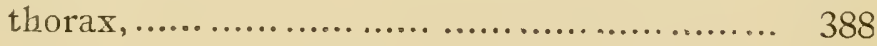

M.

Malar bone,

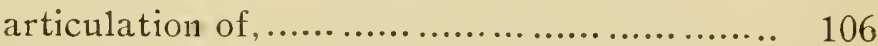

Manubrium ........................................... 12

Mediastinum,........................................... 207

Medulla oblongata, .................................... 410

Membranes of brain, ................................. 380

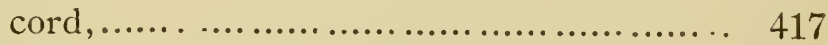

Munro, foramen of .................................... 409

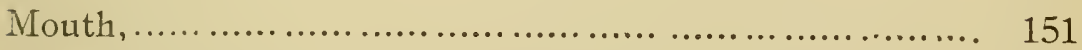

Muscle, or Muscles : .................................... 213

abductor minimi digiti (hand), ............... 256

(foot),$\ldots \ldots \ldots \ldots \ldots \ldots \ldots . . \ldots \ldots$

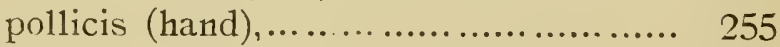

hallucis, ............................ 275

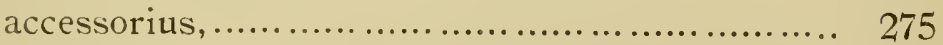

adductor brevis, ............................ 267

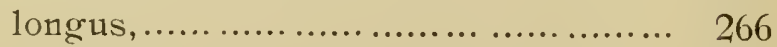

magnus, ............................ 267

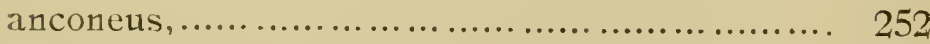

arytænoideus, ............................... 261

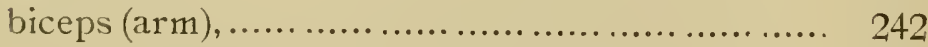

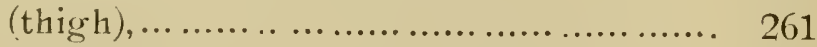

brachialis anticus, .......................... 243

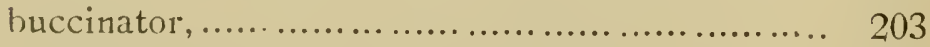

compressor naris............................. 276

constrictor pharyngeus, inferior ............... 157

medius,,$\ldots \ldots \ldots \ldots \ldots \ldots . \quad 158$

superior, .............. 158

coraco-brachialis, ........................... 242

crico-arytonoideus lateralis, .................. 201

posticus .................... 201 
Muscle, or Muscles, (Continued):

crico-thyroid, ................................ 200

deltoid, ..................................... 237

diaphragm,................................. 219

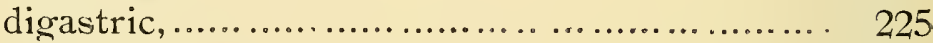

erector spinae,.............................. 233

extensor brevis digitorum,.................. 273

extensor carpi radialis brevior,................ . 250

longior, $\ldots \ldots \ldots \ldots \ldots \ldots \ldots .250$

ulnaris, ....................... 252

communis digitorum, ............... 251

indicis, .............................. 254

longus digitorum, $\ldots \ldots \ldots \ldots \ldots \ldots \ldots \ldots .270$

minimi digiti,..................... 252

ossis metacarpi pollicis, .............. 253

primi internodii pollicis, .............. 253

proprius pollicis, .................... 272

of face,................................... 277

flexor brevis minimi digiti, (hand),............ 256

(foot), ............ 276

digitorum,....................... 275

pollicis (hand), ................. 255

(foot),$\ldots \ldots \ldots \ldots \ldots \ldots \ldots . . \ldots \ldots$

Alexor carpi radialis, .......................... 246

ulnaris,......................... 247

longus digitorum, $\ldots \ldots \ldots \ldots \ldots \ldots \ldots \ldots \ldots, 270$

pollicis (han 1$), \ldots \ldots \ldots \ldots \ldots \ldots \ldots \ldots . \ldots \ldots$

(foot),$\ldots \ldots \ldots \ldots \ldots \ldots \ldots \ldots . . \ldots \ldots$

ossis metacarpi pollicis, ................. 255

profundus digitorum, ................... 248

sublimis digitorum,$\ldots \ldots \ldots \ldots \ldots \ldots \ldots \ldots . \quad 247$

gastrocnemius, .............................. 268

gemellus, superior, ......................... 258

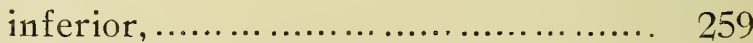

genio-hyo-glossus, .......................... 226

genio-hyoid, ................................ 226

gluteus maximus, ............................ 257

medius, ............................. 258 
Muscle, or Muscles, (Continucd):

minimus, ........................... 259

gracilis................................... 263

of hand..................... 254

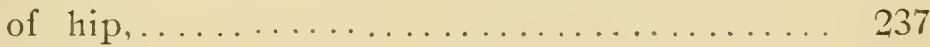

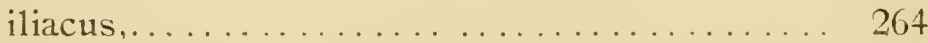

infraspinatus, ................... 239

intercostal . . . . . . . . . . . . . . . . . . 235

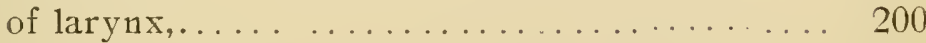

latissimus dorsi,................... 231

levator anguli scapulae................ 231

longus colli, . . . . . . . . . . . . . . . . 229

masseter . . . . . . . . . . . . . . . . . . 283

mylo-hyoid .......... . . . . . . . 226

of neck, . . . . . . . . . . . . . . . 2222-229

obliquus abdominus externus............ 214 internus, . . . . . . 216

obturator externus, .................. 260

internus, . . . . . . . . . . . . . 219

omo-hyoid ...................... 225

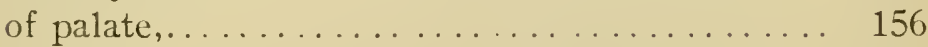

pectineus, .................... 266

pectoralis major $\ldots \ldots \ldots \ldots \ldots \ldots \ldots \ldots \ldots . \ldots \ldots \ldots$

minor $\ldots \ldots \ldots \ldots \ldots \ldots \ldots \ldots 236$

of perineum,.................. 488

peroneus brevis, ................ 273

longus, . . . . . . . . . . . . . 272

plantaris...................... 269

platysma myoides, . . . . . . . . . . . . . . . 223

popliteus, ....................... 269

pronator quadratus, ................ 248

radii teres. .................. 246

psoas maxnus, ............. . .... 263

pterycroid, external,.................. 284

internal,................. 285

pyramidalis abdominis................ 218

pyriformis, . . . . . . . . . . . . . 258 
Muscle, or Muscles, (Continued):

quadratus femoris $. . \ldots \ldots \ldots \ldots . . . . . . .259$

lumborum, . . . . . . . . . . . . 221

rectus abdominis, .................. 217

rectus capitis auticus major, ............ 229

minor............ 229

lateralis, . . . . . . . . . . . . . . . . . . . 229

femoris, . . . . . . . . . . . . . . . 263

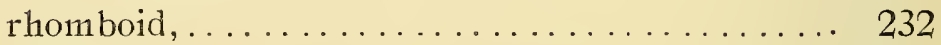

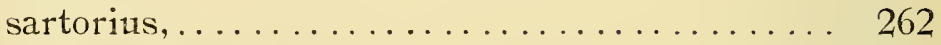

scalenus anticus.................... 228

posticus, . . . . . . . . . . . . . 229

semimembranosus, ................... 261

semitendinosus, ..................... 261

serratus magnus, ................. 237

posticus, inferior,........... 232

superior,$\ldots \ldots \ldots \ldots \ldots 232$

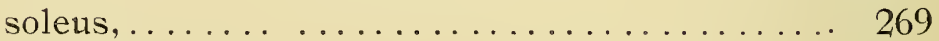

splenius, . . . . . . . . . . . . . . . . 233

sterno-cleido-mastoic.................. 223

sterno-hyoid,....................... 224

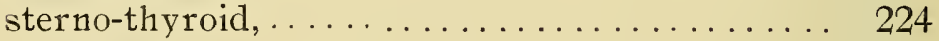

stylo-hyoid, ....................... 226

stylo-pharyngeus, ................. 158

subclavius, .................... 236

subscapularis $\ldots \ldots \ldots \ldots \ldots \ldots \ldots \ldots \ldots \ldots . \ldots \ldots 238$

supinator brevis, . . . . . . . . . . . . . 251

longus, ................ 250

supraspinatus, . . . . . . . . . . . . . . . . 239

temporal,...................... 284

teres major,$\ldots \ldots \ldots \ldots \ldots \ldots \ldots \ldots \ldots \ldots . \ldots \ldots$

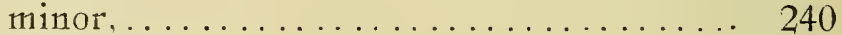

thyro-arytænoideus, . . . . . . . . . . . . . . 201

thyro-hyoid $\ldots \ldots \ldots \ldots \ldots \ldots \ldots \ldots \ldots . \ldots \ldots \ldots$

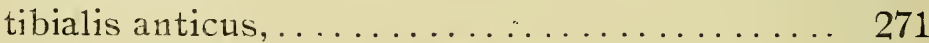

posticus, .................. 270

transversalis abdominis, . . . . . . . . . ... 216

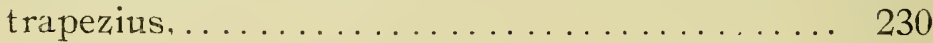


Muscle, or Muscles, (Contimucd):

triceps extensor cubiti,. . . . . ........ 243

cruris, ............ 263

vastus externus.................... . 265

internus, ................ 265

N.

Nares, anterior ............................ 95

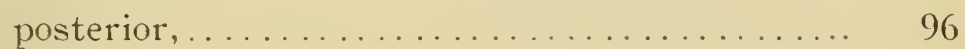

Nasal hones, . . . . . .................. 74

articulations of,$\ldots \ldots \ldots \ldots \ldots . \ldots \ldots$

Neck, muscles of . . . . . . . . . . . . . . 222-229

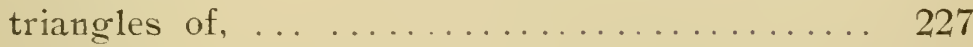

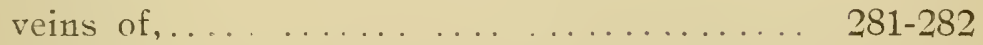

Nerves, or Nerve: ..................... 421

anterior crural, ................... 443

anterior interosseus.......... ...... 435

auditory,..................... 469

auricularis magnus, . . . . . . . . . . . . . 424

chorda tympani, ................. 468

ciliary,...................... 464

circumflex . ..................... 438

cranial, ....................... 458

dental, anterior................... 465

inferior . ...................... 466

posterior.... . . . . . . . . . . . . . . . . 465

descendens noni, ............... 475

dorsal spinal, .................... 439

eighth pair, . . . . . . . . . . . . . . . 469

eleventh pair. ................. 474

external cutaneous, . . . . . . . . . . . . . 442

facial ........................ 467

fifth, ..................... 462

fourth,...................... 462

frontal. ..................... 464

genito-crural, . . . . . . . . . . . . . 442

slossosphary nocal . . . . . . . . . . . . 470)

gluteal, superior . . . . . . . . . . . . . 446

great petrosial . . . . . . . . . . . . $468-484$ 
Nerves, or Nerve, (Contimued):

gustatory,..................... 446

hypoglassal ..................... 474

ilio-hypogastric $\ldots \ldots \ldots \ldots \ldots \ldots \ldots \ldots . \ldots \ldots 41$

ilio-inguinal, ... . . . . . . . . . . . 465

intercosta1,...... . . . . . . . . . . . 439

intercosto-humeral, . . . . . . . . . . . 431-439

internal cutaneous, . . . . . . . . . . . . . . . 443

lachrymal, .................... 463

laryngeal, recurrent,.............. 473

superior $\ldots \ldots \ldots \ldots \ldots \ldots \ldots \ldots 473$

1esser sciatic, ...................... 448

long saphenous, ... . . . . . . . . . . . . . . . . 444

lumbar,....................... 440

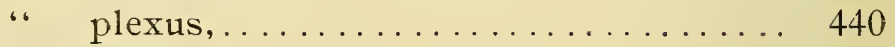

lumbo-sacral, . . . . . . . . . . . . . . 445

maxillary, inferior,$\ldots \ldots \ldots \ldots \ldots \ldots \ldots \ldots$

superior,$\ldots \ldots \ldots \ldots \ldots \ldots \ldots \ldots 464$

median,......................... 434

middle cutaneous, . . . . . . . . . . . . . . . 443

musculo-cutaneous $(\operatorname{arm}), \ldots \ldots \ldots \ldots \ldots \ldots 430$

$(\operatorname{leg}), \ldots \ldots \ldots \ldots \ldots \ldots \ldots \ldots$

musculo-spiral, ................... 436

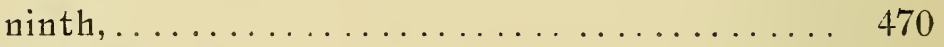

obturator,$\ldots \ldots \ldots \ldots \ldots \ldots \ldots \ldots \ldots \ldots \ldots . \ldots \ldots 43$

occipital, small,................ 424

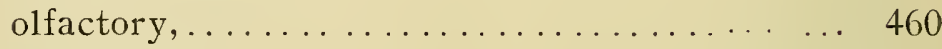

opthalmic, ................... 463

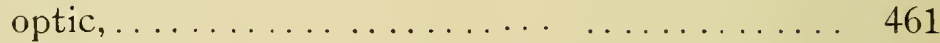

orbital, . . . . . . . . . . . . . . . . . . 464

perineal, .................... 448

peroneal, ..................... 456

phrenic, . . . . . . . . . . . . . . . . 425

plautar, external, ................... 453

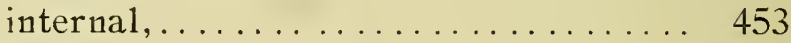

pneumogastric $\ldots \ldots \ldots \ldots \ldots \ldots \ldots \ldots \ldots \ldots \ldots . \ldots \ldots$

popliteal, external,............... 454

internal,......................... 450 
Nerves, or Nerve, (Contiuned):

posterior interosseous, ........................ 437

pudic, internal, .............................. 447

radial,..................................... 437

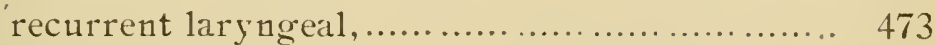

sacral plexus, ............................... 445

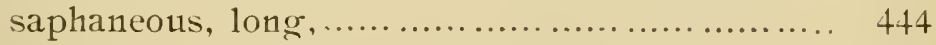

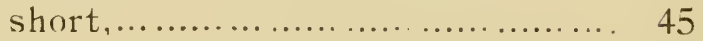

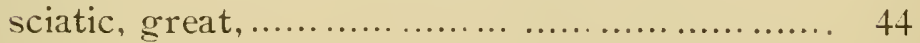

small,................................ 449

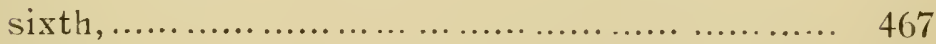

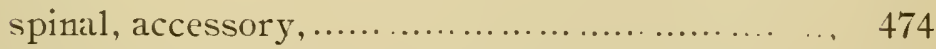

splanchnic, great, ...................... 480

small,$\ldots \ldots \ldots \ldots \ldots \ldots \ldots \ldots \ldots \ldots \ldots \ldots \ldots$

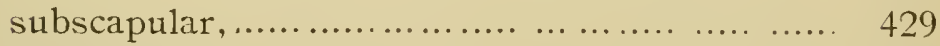

superficialis colli, .......................... $\quad 423$

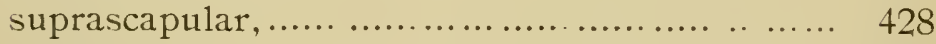

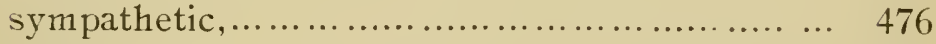

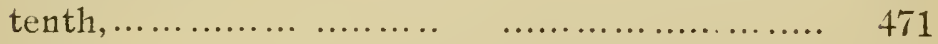

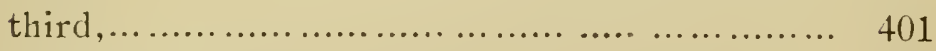

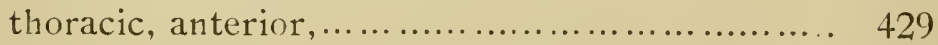

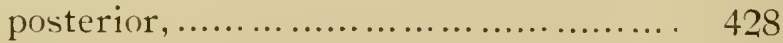

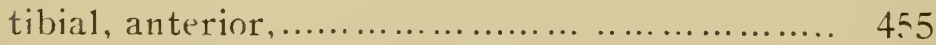

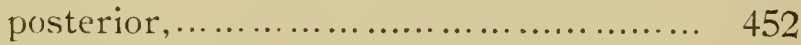

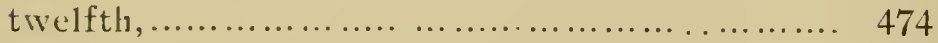

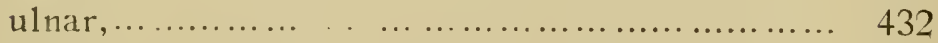

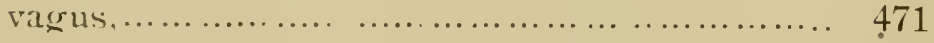

O.

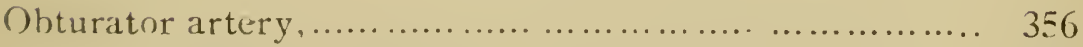

externus muscle.......................... 260

internus, ... . . . .......... 259

()ccipital artery,...................... 295

bonce. ......................... 58

articulations of,$\ldots \ldots \ldots \ldots \ldots$

fossa, ........................ 94

Occipito-atlantal articulation, . . . . . . . . . . 115

Occipito-axial, ...................... 116 
Oesopinagus.. ............................. 159

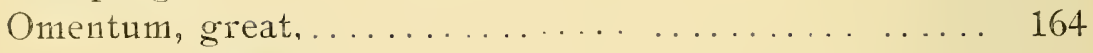

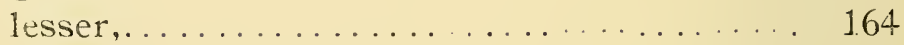

Orbit, ............................. 96

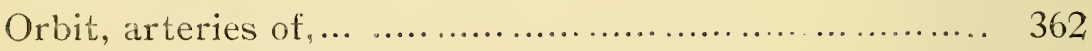

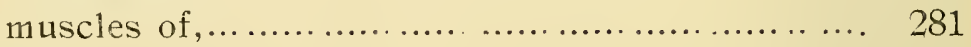

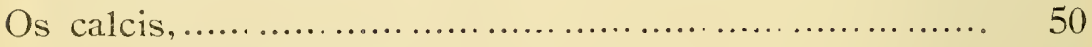

innominatum, .............................. 35

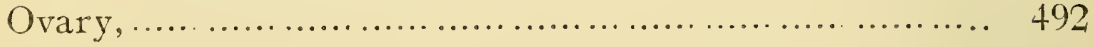

\section{P.}

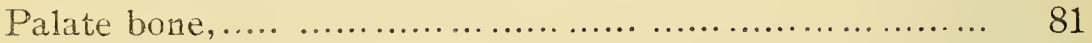

articulations of,$\ldots \ldots \ldots \ldots \ldots \ldots \ldots \ldots \ldots \ldots \ldots \ldots \ldots$

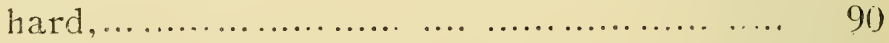

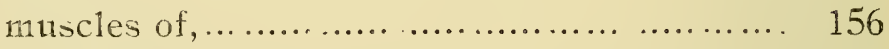

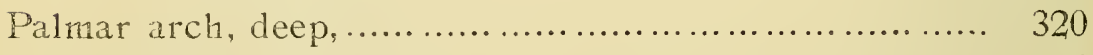

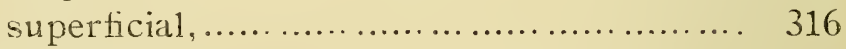

Pancreas, relations of .............................. 173

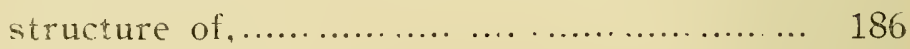

Parietal bones, ...................................... . 39

articulations of,$\ldots \ldots \ldots \ldots \ldots \ldots \ldots \ldots \ldots \ldots \ldots$

Patella,.............................................. 45

Pelvis............................................... 40

Penis, ............................................ 193

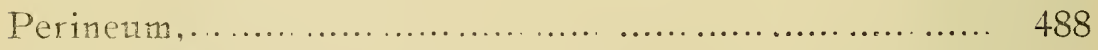

Peritoneum, ....................................... 163

Pharnyx............................................ 153

Pleura, ......................................... 206

Pouch of Douglas, ...... . ........................... 491

R.

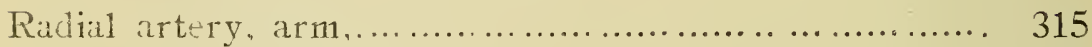

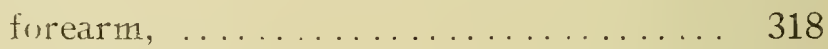

nerve...................................... 437

Radius............................................. 25

Rectum, relations of, female, ....................... 492

male $, \ldots \ldots \ldots \ldots \ldots \ldots \ldots \ldots \ldots \ldots, \quad 170$

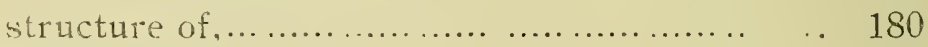

Renal artery, .................................... 340 
Retina,............................................ 516

Ribs, .............................. 13

Ring. abdominal external, .................. 495

internal, . ............... 495

crural, or femoral,............... 499

s.

Sacral arteries... . . . . . . . . . . . . . . . . . . . . 359

foramina $\ldots \ldots \ldots \ldots \ldots \ldots \ldots \ldots$

plexus, ...................... 445

Sacrum............................. 8

Scaleni musclé,.... . . . . . . . . . . . . . . . . 228

Scapula, ... . . . . . . . . . . . . . . . . . . . . . 17

Scarpa ${ }^{\circ}$ triangle,...................... 267

Sciatic artery........................ 357

nerve. great...................... 449

lesser...................... . 448

Shouller joint,....................... 121

Skull, anterior region, . ...... . . . . . . . . . 95

base of, lower surface,............... 89

upper surface, .............. 92

lateral region, .................. 87

vertex $\ldots \ldots \ldots \ldots \ldots \ldots \ldots \ldots \ldots \ldots \ldots$

Soft palate....................... 152

Spermatic artery ....................... 339

cinal. . . . . . . . . . . . . . . . . 495

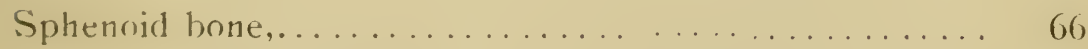

articulations of $\ldots \ldots \ldots \ldots \ldots \ldots \ldots \ldots \ldots \ldots$

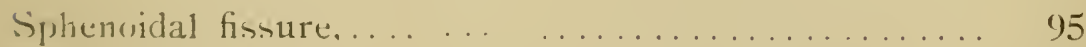

Spinal crord. ........................ . . 417

nerres, ...... .................. 421

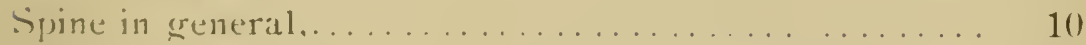

Splanchnic nerves. . . . . . . . . . . . . . . . . 480

Splecen, relations of ................... 172

structure of,$\ldots \ldots \ldots \ldots \ldots \ldots \ldots \ldots \ldots$

sternum, ...................... 11

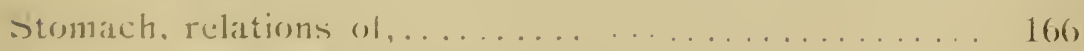

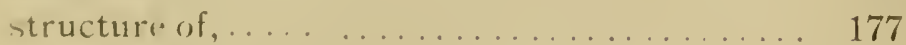


Subolavian artery, left,

Sulci of brain,

Superior maxiliary bone,

articulation of $\ldots \ldots \ldots \ldots \ldots$

Sympathetic nerves,

Tarsus, bones of,

Temporal bone,

Temporo-maxiliary articulation,

Thalami optici,....................... 406

Thyro-hyoid membrane...................... 199

Thyroid cartilage $\ldots \ldots \ldots \ldots \ldots \ldots \ldots \ldots \ldots \ldots . \ldots \ldots$

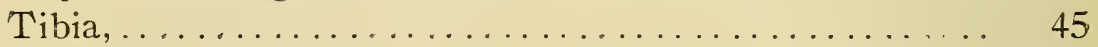

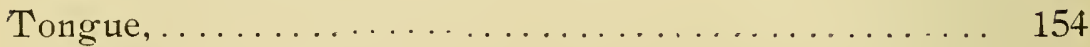

Trachea .............................. 203

Trapezium bone,........................ 29

Trapexoid “ ........................ 29

Triangle of Hesselbach,...................... 298

Scarpa's ...................... 267

Trochanter, greater.................. 42

lesser, . . . . . . . . . . . . . . 42

Turbinated bone, inferior $\ldots \ldots \ldots \ldots \ldots \ldots \ldots \ldots \ldots$

middle, ................. 73

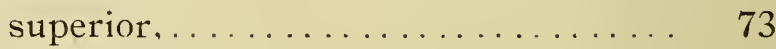

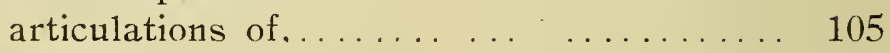

Tympanum,....................... 505

\section{$\mathrm{U}$.}

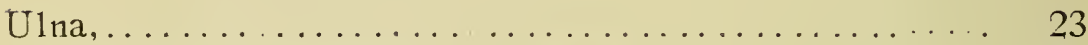

Ulnar artery of forearm ... . . . . . . . . . . . 315

of hand .................... 316

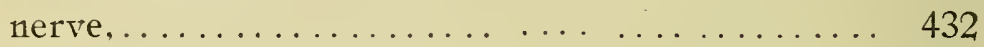

Ureter,$\ldots \ldots \ldots \ldots \ldots \ldots \ldots \ldots \ldots$ 
Urethra; female...................................... 493

male..... .... ................................ 190

Uterus............................................. 490

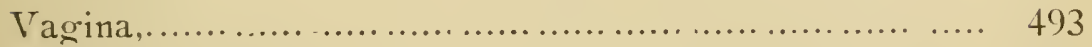

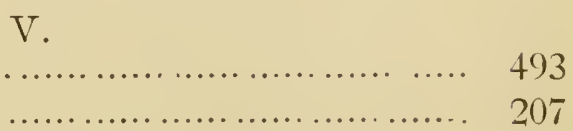

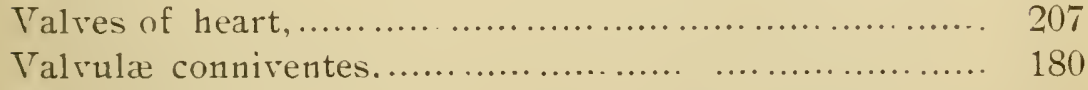

Vas deferens, ........................................ 192

Veins, or Vein : ..................................... 366

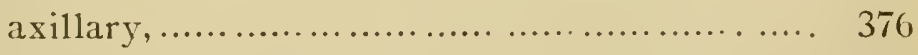

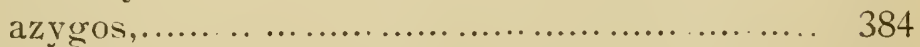

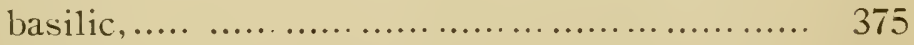

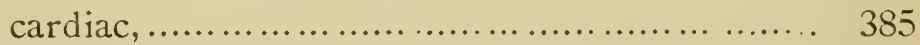

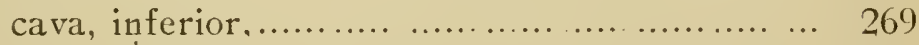

superior............................... 383

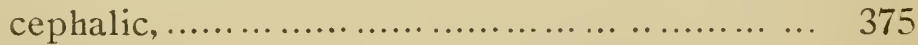

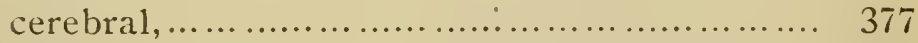

deep or comites, .......................... 360

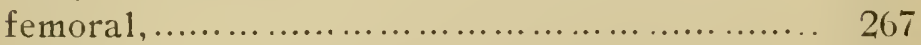

of Galen, ............................... 378

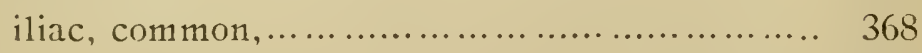

external,.... ........................ 368

internal, .............................. 369

inferior cava, ............................. 369

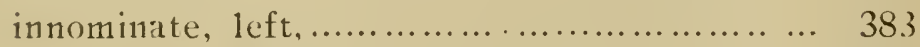

rignt........................... 382

jugrular, anterior, .......................... 377

external,........................... 382

internal,......................... 381

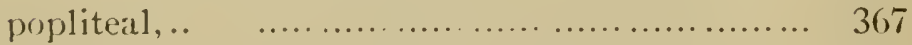

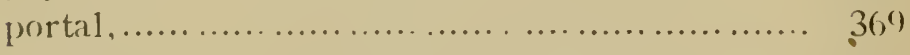

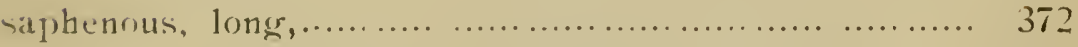

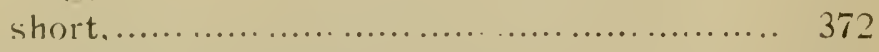

spermatic............................... 371

subclavian, ... ............................ 376

ulnar, anterior.......................... $37 t$

common, ............................. $37 t$

posterior, ............................. 374 
Velum, interpositum,$\ldots \ldots \ldots \ldots \ldots \ldots \ldots \ldots \ldots \ldots \ldots \ldots \ldots . \ldots . \ldots \ldots$

Ventricle of brain, third ............................ 408

fourth ............................ 411

lateral............................. 405

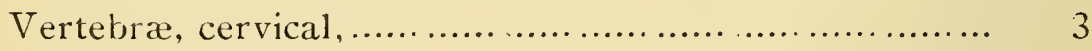

dorsal, .................................. 4

, lumbar, .................................. 4

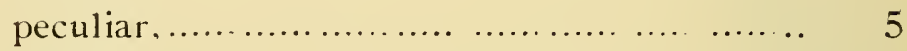

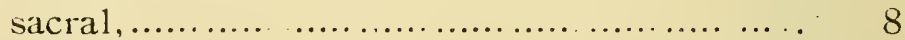

Vesiculæ seminales, ................................ 192

Vocal cords, true, ................................. 199

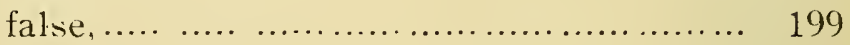

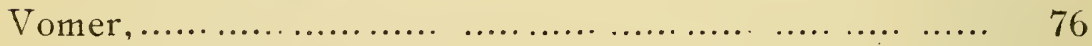

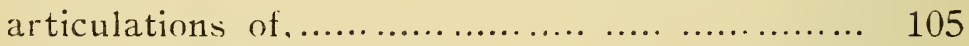

W.

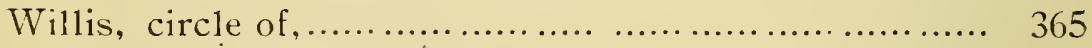

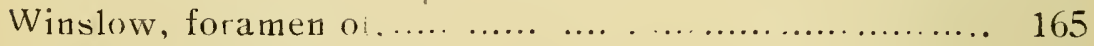

Wrisberg, nerve of $\ldots \ldots \ldots \ldots \ldots \ldots \ldots \ldots \ldots \ldots \ldots \ldots \ldots \ldots, 431$

Wrist juint...................................... 127

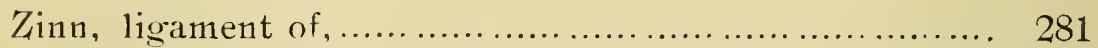

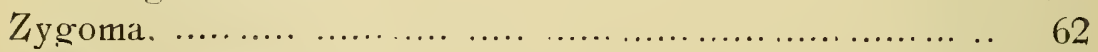

Zygomatic fossa, .................................... 88

muscles, ............................... 280 





\section{COLUMBIA UNIVERSITY LIBRARIES}

This book is due on the date indicated below, or at the expiration of a definite period after the date of borrowing, as provided by the library rules or by special arrangement with the Librarian in charge.

\begin{tabular}{|c|c|c|c|}
\hline DATE BORROWED & DATE DUE & DATE BORROWED & DATE DUE \\
\hline & & & \\
\hline & & & \\
\hline & & & \\
\hline & & & \\
\hline & & & \\
\hline & & & \\
\hline & & & \\
\hline & & & \\
\hline & & & \\
\hline & & & \\
\hline$\therefore \quad:$ & $\because$ & & \\
\hline & ; & & \\
\hline & , & & \\
\hline & & & \\
\hline & & & \\
\hline & & & \\
\hline C28(955) 100MEE & & & \\
\hline
\end{tabular}


COLUMBIA UNIVERSITY LIBRARIES (hSI.Stx) aM 23 T65 1898 C. 1

Descriptive anatomy

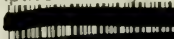

10

-

ก⿻:?

m55

789.8

motiles

Descrintive anatomy.

MR 281957 BINDEN

QM23

T65

1898 


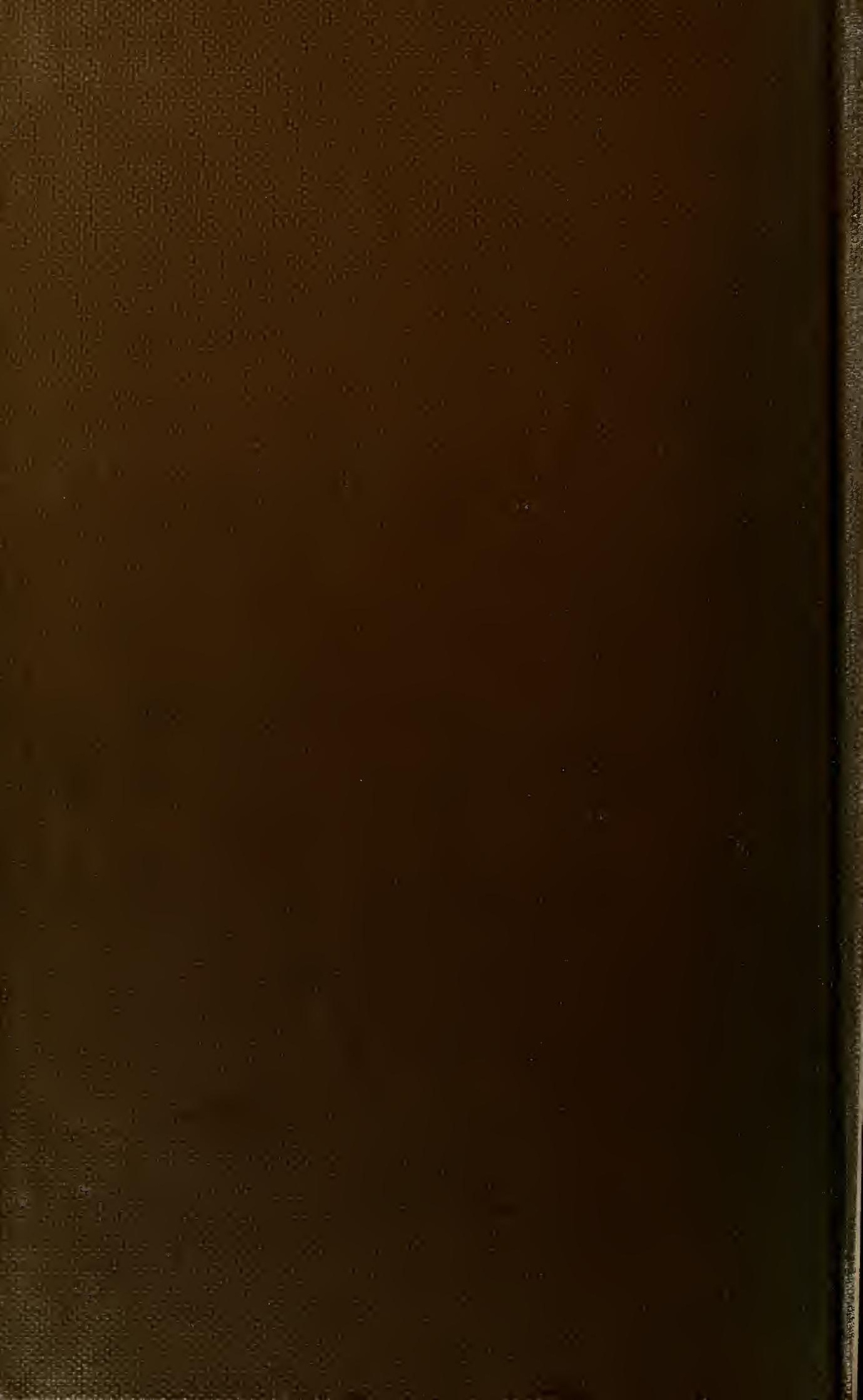

